\section{Poster Session III}

\section{December 8, 2010 5:30PM-7:30 PM}

\author{
1. The Role of the 5-HT6 Receptor in Compulsive Responding: \\ an Investigation Using Viral Mediated Gene Transfer \\ John Neumaier*, Daniel Eskenazi
}

University of Washington, Seattle, WA

Background: The 5-HT6 receptor has been implicated in reward and reinforcement learning, behaviors strongly influenced by striatal circuitry. 5-HT6 receptors are abundantly expressed in the dorsal striatum (caudate-putamen complex), a key brain region for several cognitive and neuropsychiatric-disorders such as obsessive-compulsive disorder (OCD), Tourette Syndrome (TS), and addictive disorders. Our current work investigates the role of the 5-HT6 receptor in compulsive responding.

Methods: We over-trained male Long-Evans rats using random interval schedules of reinforcement to establish compulsive leverpressing. We then employed an omission session in which the rat was rewarded for not pressing the lever (omitted responses) followed by an extinction probe test session to measure responsiveness towards the lever.

Results: Rats that received a post-training, pre-omission session infusion of a herpes-simplex viral vector expressing 5-HT6 receptors demonstrated a decrease in compulsive lever pressing on the extinction test probe compared to rats receiving the viral vector but that were yoked controls during the omission session. Rats receiving an eGFP-only viral vector showed persistent compulsive lever pressing whether they were in the omission or yoked groups. Given that 5-HT6 receptors are present on medium spiny neurons (MSNs) of the direct and indirect pathway (striatonigral and striatopallidal MSNs, respectively), as a way to distinguish the role of 5-HT6 receptor signaling in distinct cell types, we have developed herpes-simplex virus vectors that drive neuronal-subtype specific expression of 5-HT6 receptors using the prodynorphin promotor and the pre-proenkephalin promotor to target direct and and indirect pathway MSNs, respectively.

Discussion: Our hypothesis is that given the opposite signaling between 5-HT6 receptors and dopamine D2 receptors in striatopallidal MSNs, selective over-expression of 5-HT6 receptors in these neurons will be sufficient to replicate our previous finding. We plan to further our investigations into the role of the 5-HT6 receptor in compulsive responding using these newly developed tools.

Disclosure: J. Neumaier: None. D. Eskenazi: None.

\section{A New Animal Model of Psychosis: Chronic Treatment with Lysergic Acid Diethylamide (LSD) \\ Charles Nichols*, David Martin, Zach Daniels, Danuta Marona- Lewicka, David Nichols}

\section{LSU Health Sciences Center, New Orleans, LA}

Background: Schizophrenia is a severe neuropsychiatric disease that at present has no cure. Schizophrenia is a believed to be a complex multifactorial disorder involving genetic, environmental, and developmental mechanisms. Current animal models to study this disorder are problematic because they employ, for example, blockade of the behavioral effects of dopaminergic agonists such as apomorphine or amphetamine, as well as acute administration of drugs whose effects are thought to resemble psychosis, such as phencyclidine (PCP), and do not, in fact, represent what occurs with the onset of psychosis. Therefore, an animal model that more closely mimics the behavioral, neurochemical, and genetic aspects of schizophrenia and psychosis than current models is highly desirable. Rats chronically treated with low doses of the powerful classical hallucinogenic drug, lysergic acid diethylamide (LSD) represent a new and exciting model to study schizophrenia and psychosis. It is generally regarded that acute LSD in humans can reproduce many of the positive symptoms of psychosis, including hallucinations and detachment from reality. We have previously presented behavioral data demonstrating that chronic administration of LSD to rats can produce behaviors highly relevant to both the positive and negative symptoms of schizophrenia (hyperreactivity, social withdrawal, anhedonia, etc.), appearing after about six weeks of treatment. Significantly, these behaviors persist for at least many months after discontinuing LSD treatment. Furthermore, atypical antipsychotic medications can block these abnormal behaviors. We also have previously presented data showing that genes for multiple neurotransmitter systems, including dopamine, serotonin, and glutamate are persistently altered in prefrontal cortex of these rats. Here, we present additional data that further validates the use of our proposed novel model of psychosis. These data include more comprehensive gene expression results from next generation sequencing, proteomic analysis, and analysis of cytokines.

Methods: Male Sprague-Dawley rats were treated with $0.16 \mathrm{mg} / \mathrm{kg}$ LSD every other day for 90 days. Behavioral analysis was performed 30 days after the last LSD treatment to verify persistent behavioral changes in the LSD treated group compared to the saline injected control group. Brains and blood serum were collected and processed for analysis. Total RNA was isolated from the medial prefrontal cortex of each rat and subjected to RNA-seq analysis on an Illumina GAIIx genome analyzer. QPCR also was performed on select genes form the same RNA samples. Protein samples isolated from the mPFC of each rat were analyzed by 2 D-DIGE proteomic analysis. Blood serum from each rat was analyzed by Millipore Milliplex immunoassay to determine cytokine levels.

Results: We found multiple genes relevant to schizophrenia and psychosis that were altered in expression by chronic LSD. These include those for dopamine, serotonin, glutamate, and GABA receptor systems. Expression of additional genes potentially relevant to schizophrenia, but not directly related to neurotransmitter systems also were found to be influenced. Analysis of inflammatory related cytokines revealed that circulating levels of cytokines relevant to psychosis (e.g. IL-4, IL-6) were elevated by chronic LSD. Proteomic analysis has identified a small collection of proteins that also are altered by chronic LSD.

Discussion: Rats chronically treated with low doses of LSD develop behavioral changes potentially relevant to both the positive and negative symptoms of schizophrenia, which persist for many months after the last dose of LSD. Unlike other models of psychosis, where single or short term treatment with a drug leads to acute behaviors that are primarily mediated through acute neurotransmitter modulation, chronic LSD may more closely model the neuroadaptive processes in humans that lead to abnormal brain function and psychosis. In other words, chronic LSD may model the development of schizophrenia, as well as the pathological endpoint. The persistence of abnormal behaviors induced by chronic LSD are not a direct result of LSD because they are present in the absence of drug. Rather, it is the gradual neuroadaptive change in gene and protein expression facilitated by LSD that together lead to the abnormal persistent behaviors. Significantly, we also have found evidence for a chronic inflammatory state in our model that is similar to what has been observed in patients with schizophrenia. How inflammation is related to schizophrenia is presently unknown, but our model may help to address this issue in the future.

Disclosure: C. Nichols: None. D. Martin: None. Z. Daniels: None. D. Marona-Lewicka: None. D. Nichols: None. 
3. The Role of CaMKII $\beta$ Upregulation in Schizophrenia: A New Drug Target for Schizophrenia Treatment

Gabriela Novak*, Philip Seeman

University of Toronto and CAMH, Toronto, ON, Canada

Background: The cerebral frontal cortex of schizophrenia patients shows elevated levels of mRNA for calcium/calmodulin-dependent protein kinase II beta (CaMKII $\beta)$, but not CaMKII $\alpha$. In parallel, animal models of schizophrenia consistently show both an increase in CaMKII $\beta$ mRNA levels and an elevation in levels of dopamine 2 receptors in their high-affinity state (D2High), the major target of antipsychotic medication. In the present study we examined whether an elevation in the levels of CaMKII $\beta$ could lead to an increase in levels of D2High.

Methods: We used quantitative real-time PCR to measure CaMKII $\alpha$ and CaMKII $\beta$ mRNA levels in the frontal cortices and striata of heterozygous and wild type CaMKII $\alpha$ knock-out mice. The heterozygous mice are known to exhibit a 50\% reduction in CaMKII $\alpha$ levels, resulting in an increase of the CaMKII $\beta: \alpha$ ratio. This ratio determines the overall kinetic characteristics of the heteromeric CaMKII enzyme, resulting in an enzyme with higher sensitivity to $\mathrm{Ca} 2+$ signals. We also tested in vitro the effect of a CaMKII inhibitor on D2High states. The level of $\mathrm{D}_{2}$ High was determined by the competition of dopamine with $[3 \mathrm{H}]$ domperidone.

Results: The heterozygous CaMKII $\alpha$ knock-out mice showed the expected reduction in CaMKII $\alpha$ mRNA in the frontal cortex, without an alteration in CaMKII $\beta$ levels. However, they exhibited a $29 \%$ upregulation of CaMKII $\beta$ mRNA in the striatum and a 2.8 -fold increase in the levels of $\mathrm{D}_{2} \mathrm{High}$ receptors. In addition, we found that the CaMKII inhibitor, KN-93, but not its inactive analog $\mathrm{KN}-92$, markedly reduced $\mathrm{D}_{2}$ High states in rat striatum.

Discussion: Elevated levels of CaMKII $\beta$ mRNA in the striatum suggest that the upregulation of CaMKII $\beta$ may increase D2High in animals and, possibly, in schizophrenia itself. Furthermore, its involvement is supported by the reversal of the increase of $\mathrm{D}_{2}$ High levels by a CaMKII inhibitor. This is a novel finding, as no specific molecular pathway has yet been identified, which would control/reverse the levels of D2High. Thus, the inhibition or normalization of CaMKII may provide an important drug target for schizophrenia treatment.

Disclosure: G. Novak: None. P. Seeman: None.

4. Conditional Knockdown of Adult Neurogenesis in Mice Impairs Extinction Learning Following Heroin Self-Administration Kelly C. Wischerath, Richard M. Cleva, Megan P. Hicks, M. Foster Olive $^{*}$

\section{Arizona State University, Tempe, AZ}

Background: Extinction of conditioned responses and behaviors is a process of active learning, and recent studies suggest that neurogenesis in the adult hippocampus is important for certain types of learning and memory. We have previously found that extinction training following intravenous heroin self-administration increases neurogenesis in the dentate gyrus of the rat hippocampus as compared to animals that underwent forced abstinence (Wischerath et al., submitted). The purpose of the present study was to determine if adult neurogenesis mediates extinction learning following heroin selfadministration using a conditional knockdown approach in mice.

Methods: Male mice carrying a transgene consisting of a herpes simplex virus thymidine kinase (HSV-TK) construct that was inserted into the first exon of a mouse glial fibrillary acidic protein (GFAP) promoter cassette (Bush et al., Cell 93:189-201, 1998) were used as subjects. Mice that had been backcrossed from the founder line onto a C57BL/6 background strain for more than 12 generations were obtained from Jackson Laboratories (strain \#005698, B6.Cg-Tg(Gfap-Tk)7.1Mvs/J). In GFAP-HSV-TK mice, proliferating GFAP-positive cells that express the HSV-TK transgene metabolize the antiviral compound ganciclovir
(GCV) to toxic nucleotide analogues which induces cell death. As adult neural stem cells are derived from astrocytes expressing GFAP, administration of GCV to these mice produces a conditional ablation of neurogenesis in the adult brain. At least 14 days of GCV treatment are necessary to achieve significant reductions in adult neurogenesis (Bush et al., 1998). Following implantation of mice with jugular vein catheters and osmotic minipumps (Alzet model 1004, flow rate $0.11 \mathrm{ul} / \mathrm{hr}$ ) containing either saline or GCV (10 mg/kg/day), mice were allowed to spontaneously acquire heroin self-administration $(0.03 \mathrm{mg} / \mathrm{kg} /$ infusion, $20 \mathrm{ul}$ over $1 \mathrm{sec})$ on an FR1 schedule of reinforcement in $3 \mathrm{hr}$ daily sessions. Self-administration chambers (Med Associates) were equipped with an active and inactive lever as well as a stimulus light that was illuminated during each drug infusion. Each heroin infusion was followed by a $20 \mathrm{sec}$ timeout during which additional active lever presses were recorded but did not result in drug infusion. Following 12 days of heroin self-administration, mice underwent 5 extinction training session ( $3 \mathrm{hr}$ each in length), during which presses on the lever that previously resulted in drug infusion no longer had any programmed consequences. Immediately following each of the 5 extinction training session, mice were injected with 2-bromodeoxyuridine (BrDU, $50 \mathrm{mg} / \mathrm{kg}$ i.p.) to label proliferating cells. Ten days following the final extinction session, mice were euthanized and perfused transcardially with $4 \%$ paraformaldehyde, and brains were sectioned and processed for BrDU immunohistochemistry. BrDU-positive cells in the dentate gyrus region of the hippocampus were counted using epifluoresscence microscopy.

Results: GCV treatment had no effect on the acquisition or maintenance of heroin self-administration as compared to salinetreated animals. However, GCV-treated mice exhibited significantly increased numbers of active lever presses on the first 3 days of extinction as compared with saline-treated mice. Immunohistochemical analysis of GCV-induced reductions in neurogenesis showed an approximate $50-75 \%$ reduction in the number of BrDU-positive cells in the hippocampus of GCV-treated mice as compared with saline-treated mice.

Discussion: Our findings indicate that adult neurogenesis contributes to extinction learning following heroin self-administration. Since GCV treatment reduces stem cell proliferation in both the subventricular zone of the forebrain and the subgranular zone of the dentate gyrus, the precise contribution of either of these two regions to reduced extinction learning in GCV-treated mice cannot be currently determined. However, we hypothesize that neurogenesis in the adult hippocampus is the primary brain region involved in extinction learning, as it has previously been demonstrated that ablation of adult neurogenesis by irradiation of the hippocampus impairs extinction learning in rats following cocaine self-administration (Noonan et al., J Neurosci 30:304-15, 2010). Future studies examining whether proneurogenic compounds enhance extinction learning are needed to further characterize the role of adult neurogenesis in extinction. All procedures were performed within NIH Guidelines. All authors have no conflict of interest to declare. Supported by NIH grant DAo24355. Disclosure: K. Wischerath: None. R. Cleva: None. M. Hicks: None. M. Olive: Part 1; Gilead Sciences. Part 4; Gilead Sciences.

\section{The Role of Clock in Ethanol-Related Behaviors \\ Angela Ozburn*, Edgardo Falcon, Shibani Mukherjee, Jaswinder Kumar, Andrea Gillman, Sade Spencer, Colleen McClung}

\section{UT Southwestern Medical Center, Dallas, TX}

Background: Our lab has identified a key role for the circadian locomotor output cycles kaput (Clock) gene, a transcription factor that drives the positive loop of the circadian clock, in the regulation of drug reward. Mice bearing a dominant negative mutation in the Clock gene (Clock $\Delta 19$ mice) exhibit increased cocaine sensitivity and preference. These mice also exhibit increased locomotor activity, reduced anxietylike and depression-like behavior, increased intracranial self-stimulation (ICSS) at a lower threshold, and increased dopaminergic cell 
activity in the ventral tegmental area (VTA; McClung et al., 2005; Roybal et al., 2007). Previous studies in our lab have found that expressing functional Clock in the VTA rescues many of the behavioral phenotypes seen in Clock $\Delta 19$ mice and reducing Clock expression in the VTA of mice via RNA interference recapitulates them (Roybal et al., 2007; Muhkerjee et al., 2010). To further understand the role of Clock in drug reward, we assessed the role of Clock in ethanol-related behaviors.

Methods: We measured alcohol intake in Clock $\Delta 19$ mutants and their wild-type (WT) counterparts using the continuous access two bottle choice paradigm. Differences in ethanol intake can be due to differences in sensitivity to the rewarding or aversive properties of ethanol, acquisition of tolerance, or an increased ethanol metabolism. Therefore, we measured ethanol-induced conditioned place preference (CPP; $2 \mathrm{~g} / \mathrm{kg}$ ethanol dose), conditioned taste aversion (CTA; $2 \mathrm{~g} / \mathrm{kg}$ ethanol dose), acute functional tolerance (two injections of $2 \mathrm{~g} / \mathrm{kg}$ ethanol separated by rotarod recovery and blood alcohol measurements), and metabolism (blood alcohol levels over time after a $4 \mathrm{~g} / \mathrm{kg}$ ethanol dose) in Clock $\Delta 19$ mutants and their wild-type counterparts. To determine if Clock expression in the VTA is important for modulating ethanol intake, we stereotaxically injected AAV-ClockshRNA or a scrambled control (scr) and after a three week recovery period, cohorts of WT mice (from the Clock $\Delta 19$ heterozygous breeding colony) were tested for ethanol intake using a two bottle choice paradigm.

Results: Clock $\Delta 19$ mice exhibited significantly increased ethanol preference and consumption as compared to WT mice (ethanol preference $\mathrm{p}<0.05$; ethanol consumption $\mathrm{p}<0.05$ ). Clock $\Delta 19$ and WT mice developed ethanol-induced conditioned place preference and conditioned taste aversion to a similar extent. Clock $\Delta 19$ mice and their WT littermates acquire ethanol-induced functional tolerance to a similar extent and exhibit similar rates of ethanol clearance. Preliminary results indicate a strong trend for increased ethanol preference and consumption using the two bottle choice in mice that were injected with AAVClock-shRNA as compared with those injected with AAV-scr (ethanol preference $\mathrm{p}=0.07$; ethanol consumption $\mathrm{p}=0.1$ ).

Discussion: Clock $\Delta 19$ mice exhibit increased ethanol preference and consumption, further characterizing them as a hyperhedonic behavioral phenotype. However, it is not likely that the increased ethanol intake in Clock $\Delta 19$ mice is simply due to altered sensitivity to the rewarding or aversive properties of ethanol, or differences in the development of tolerance or metabolism. Importantly, we have identified a significant role for Clock in the VTA as a negative regulator of ethanol intake using mutant mice and RNA interference. Investigations into drug actions in Clock $\Delta 19$ mice suggest they have a hypersensitive glutamatergic response to ethanol and ketamine (data not shown). Future work will focus on testing the effects of acamprosate (FDA approved drug for the treatment of alcohol use disorders) on ethanol intake and other behaviors in these mice. Further examination of the role of circadian genes in alcohol-related behaviors will have exciting translational significance for treatment, as many recovering addicts exhibit circadian disruptions that persist in recovery and contribute to relapse.

Supported by NIDA (T32 DA7290, Ro1 DA023988).

Disclosure: A. Ozburn: None. E. Falcon: None. S. Mukherjee: None. J. Kumar: None. A. Gillman: None. S. Spencer: None. C. McClung: Part 1; GlaxoSmithKline, Pfizer, Servier, Orphagen Pharmaceuticals. Part 4; GlaxoSmithKline.

6. Cognitive Impairment and Decreased Hippocampal Neurogenesis After Treatment with Methotrexate: An Animal Model of Chemobrain Robert Pechnick*, Kevin Reyes, Melanie Das, Liliana Lacayo,

Catherine Farrokhi, Svetlana Zonis, Vera Chesnokova

Cedars-Sinai Medical Center, David Geffen School of Medicine At UCLA, and the Brain Research Institute, Los Angeles, CA

Background: Post-chemotherapy cognitive impairment ("chemobrain") has been most often associated and studied in patients who have received adjuvant treatment for breast cancer. Symptoms of chemobrain include frequent memory lapses, difficulty concentrating, inability to remember details, inability to multitask, and trouble remembering common words or names. Neurogenesis, the formation of new nerve cells, occurs throughout adulthood, and is under the control of cell cycle regulators. Because some chemotherapeutic agents act by inhibiting cell cycle progression, these chemotherapeutic agents might produce cognitive impairment by disrupting neurogenesis in the hippocampus. Methotrexate is used in the treatment of various types of cancer. It is a folic acid antagonist and interferes with DNA synthesis and cellular replication. The objective of the present study was to determine whether methotrexate impairs cognitive function and hippocampal neurogenesis in the mouse.

Methods: Adult female mice were injected once a week for 3 weeks with saline or methotrexate $(3,10$ or $30 \mathrm{mg} / \mathrm{kg} / \mathrm{i} . \mathrm{p}$.). One week after the last injection behavioral testing was carried out. Locomotor activity was assessed using the open field test, and cognitive function was evaluated using spontaneous alternation in the Y-maze, cue- and context-specific fear conditioning and the Barnes Maze. Another cohort of mice did not undergo behavioral testing but were treated with bromodeoxyuridine (BrdU; $50 \mathrm{mg} / \mathrm{kg} / \mathrm{i} . \mathrm{p}$. every $2 \mathrm{hr}$ for a total of 4 injections) and sacrificed $24 \mathrm{hr}$ later.

Results: There were no significant differences in locomotor activity among the treatment groups; however, spontaneous alternation was reduced in the group that received the highest dose of methotrexate ( $30 \mathrm{mg} / \mathrm{kg} /$ i.p.). Neither cue- nor context-specific fear conditioning were affected, but performance in the Barnes maze was impaired in the group that received the highest dose of methotrexate. BrdU incorporation was reduced in the dentate gyrus of the hippocampus only in the group that received the highest dose of methotrexate. In order to determine whether the reduction in BrdU labeling was in neurons, slides were double-stained for BrdU and doublecortin (DCX). Treatment with methotrexate reduced the number of cells that co-expressed BrdU and DCX, indicating that the reduction in proliferation occurred primarily in neural cells, and reflects methotrexate-induced decreases in neurogenesis. TUNEL staining revealed no increase in apoptosis. Glial fibrillary acidic protein (GFAP) is a marker for glia. Immunohistohemical and Western Blot analyses showed that methotrexate increased GFAP expression. Therefore, methotrexate decreased neurogenesis, but triggered gliogenesis in the hippocampus. Discussion: These results support the hypothesis that some chemotherapeutic agents might produce cognitive impairment by disrupting hippocampal neurogenesis. It is plausible that drugs that stimulate hippocampal neurogenesis might be effective in preventing and/or treating post-chemotherapy cognitive impairment.

Supported by Department of Defense Breast Cancer Research Program grant number BC075629.

Disclosure: R. Pechnick: Part 1; Sepracor Inc, Forest Inc, Brain Cell Inc. K. Reyes: None. M. Das: None. L. Lacayo: None. C. Farrokhi: None. S. Zonis: None. V. Chesnokova: None.

\section{Sub-Second Striatal Dopamine Release During The Progression Of Drug-taking Behavior In Rats Ingo Willuhn, Barry Everitt, Paul Phillips*}

University of Washington, Seattle, WA

Background: Dopamine neurotransmission in the ventral striatum is strongly implicated in the acute reinforcing effects of drugs of abuse. After repeated drug intake, the dorsolateral striatum is thought to become increasingly involved in the control of drug taking. In a previous study, we characterized rapid dopamine release encoding drug-related cues in the ventral striatum of rats self-administering cocaine at a single time point. However, it is not known whether such dopamine signaling is expressed in other striatal regions or how it changes with drug-taking experience. Here, we measured rapid dopamine signaling simultaneously in the ventral striatum and dorsolateral striatum repeatedly over the course of weeks. Furthermore, 


\section{S300}

we investigated whether cue-related dopamine release in the dorsolateral striatum is dependent upon ventral striatal circuitry.

Methods: Multiple electrodes for fast-scan cyclic voltammetry were chronically implanted in the striatum of rats outfitted with intravenous catheters for cocaine self-administration. Rats were given access to cocaine for one hour per day for 20 days. During a self-administration session, a nose poke elicited a cocaine infusion (fixed ratio 1 schedule, $0.5 \mathrm{mg} / \mathrm{kg} /$ infusion) that was accompanied by a 20 -second presentation of an audiovisual stimulus (Pavlovian conditioned stimulus; delivery cue), during which additional nose pokes were without consequences (time out). After this time out, a separate cue signaled the availability of additional infusions (instrumental discriminative stimulus; availability cue).

Results: Throughout self-administration training, we observed rapid dopamine release in the ventral striatum associated with the presentation of the delivery cue, consistent with our previous studies. However, dopamine release associated with the availability cue only emerged after repeated training in this brain region. In contrast to the ventral striatum, cue-related dopamine signals in the dorsolateral striatum developed a) only during later stages of training, and b) exclusively in response to presentation of the delivery cue. Importantly, unilateral lesion of the ventral striatum $(0.5-\mu \mathrm{l}$ quinolinic acid, $0.09 \mathrm{M}$ ) did not change drug-taking behavior, but blocked the development of dorsolateral signals in the ipsilateral, but not contralateral, hemisphere.

Discussion: Our results demonstrate that rapid dopamine signaling in the striatum in response to drug cues is dynamic and region specific, developing in the ventral then dorsolateral striatum sequentially. This progression of dopamine signaling to dorsal parts of the striatum requires intact ventral striatal circuitry. Overall, these data demonstrate recruitment of striatal circuitry for dopamine-mediated encoding of drug cues in the development of a drug-taking habit.

Disclosure: I. Willuhn: None. B. Everitt: Part 1; GSK. P. Phillips: Amgen Inc.

\section{Allopregnanolone Upregulates Brain BDNF and Reelin Expression in a Mouse Model of Posttraumatic Stress Disorders Graziano Pinna*}

Psychiatric Institute, Department of Psychiatry, College of Medicine, University of Illinois at Chicago, Chicago, IL

Background: Allopregnanolone (Allo) is a potent positive allosteric modulator of GABA action at $\mathrm{GABA}_{\mathrm{A}}$ receptors that is produced in corticolimbic glutamatergic neurons from progesterone by the sequential action of $5 \alpha$-reductase type I $(5 \alpha$-RI) and $3 \alpha$-hydroxysteroid dehydrogenase ( $3 \alpha-\mathrm{HSD})$. We recently reported that a decrease of CSF Allo levels was correlated with increased PTSD re-experiencing and comorbid depression (Biol. Psychiatry 60, 704-713. 2006). Likewise, a decrease of brain-derived neurotrophic factor (BDNF) has been implicated in the mechanisms underlying the clinical manifestations of PTSD and reelin deficits have been related to an impairment of cognitive functions in psychiatric disorders. A mouse model characterized by behavioral deficits reminiscent of PTSD, the socially isolated mouse (more than 4 week social isolation) shows a downregulation of corticolimbic Allo levels associated with behavioral dysfunctions, including exaggerated contextual fear conditioning and impaired extinction, anxiety-like behavior, and aggressiveness. Corticolimbic Allo levels and behavioral deficits can be normalized by a single dose of selective brain steroidogenic stimulants (SBSSs), including S-norfluoxetine (PNAS 105, 5567-5572. 2008) at doses too low to inhibit serotonin reuptake (Curr Opin Pharmacol 9, 24-30. 2009). Thus, we hypothesized that a deficit of Allo, BDNF, and reelin brain expression could be interrelated in PTSD and would result in a deficit of GABAergic neurotransmission, which triggers behavioral deficits. We tested this hypothesis in socially isolated mice.

Methods: Mice were either socially isolated for a period of 4 weeks or caged in groups of five per cage. BDNF and reelin were measured with
RT-PCR. The levels of Allo in the amygdala, frontal cortex, hippocampus, striatum, and cerebellum were determined by GC-MS in mice killed at termination of the behavioral tests.

Results: We found that BDNF and reelin mRNA expression are downregulated in the medial frontal cortex, hippocampus, and amygdala, but not in the striatum and cerebellum. These mice showed excessive contextual fear responses and altered and incomplete extinction of fear. Allo ( $8 \mu \mathrm{mol} / \mathrm{kg}$, s.c., twice daily, for 2 weeks) or low S-norfluoxetine doses $(1.8 \mu \mathrm{mol} / \mathrm{kg}$, s.c., twice daily, for 2 weeks) that increase the levels of Allo in corticolimbic areas to the levels of group-housed mice normalized PTSD-like behavior and BDNF and reelin mRNA expression in corticolimbic areas, including frontal cortex, hippocampus, and amygdala of socially isolated mice.

Discussion: These observations support the hypothesis that by increasing Allo levels, SBSSs, such as S-norfluoxetine may be involved in the regulation of corticolimbic BDNF and reelin expression and the behavioral dysfunctions that relate to PTSD. Supported by NIMH grant MHo85999-01 to Pinna G.

Disclosure: G. Pinna: None.

\section{Mutation of the Histidine Decarboxylase Gene Leads to Tourette Syndrome-Like Behavioral Abnormalities: Validation of a Recent Genetic Finding in a Mouse Model}

Lissandra Baldan Ramsey, Kuakarun Krusong, Hiroshi Ohtsu, A. Gulhan Ercan-Sencicek, Matthew State, Christopher Pittenger*

\section{Yale University, New Haven, CT}

Background: Tourette syndrome (TS), like many neuropsychiatric conditions, is substantially heritable but genetically complex; identifying contributing or causal genetic variation has been challenging. As has been the case in other common medical conditions, the identification of risk alleles carrying large effects may produce valuable biological insights even if the specific mutations are extremely uncommon in the population. A recent study examining an extraordinary 2-generation pedigree with nine cases of TS identified a rare, highly penetrant, dominant-negative mutation in the histidine decarboxylase (HDC) gene (Ercan-Sencicek et al, NEJM 2010). However, the identification of a putative causal variant in a single, relatively small extended pedigree is inherently correlative and the ability to clarify the biological relevance of the identified mutation is quite limited in the absence of a tractable model system. We have consequently studied HDC knockout mice to investigate the causal association between perturbations in this gene and TS-like phenomenology. We hypothesized that these mice would exhibit functional abnormalities in cortical-striatal circuits strongly implicated in TS and demonstrate behaviors that parallel abnormalities seen in patients with this disorder, including deficits in prepulse inhibition (PPI); spontaneous or induced stereotypies; and abnormalities in striatumdependent habit-like learning.

Methods: HDC protein and histamine were investigated in the forebrain using Western blotting, immunohistochemistry, and mass spectroscopy. Adult male HDC knockout mice, backcrossed multiple generations onto the $\mathrm{C} 57 \mathrm{Bl} / 6$ genetic background, were tested in behavioral assays of specific relevance to TS (PPI; amphetamineinduced stereotypy; striatum-dependent learning in a watermaze paradigm and in an instrumental habit paradigm) as well as in control tasks.

Results: Abnormalities in the striatum, the input nucleus of the basal ganglia, have been found in TS. HDC protein and histamine were found in the striatum at markedly higher levels than in other forebrain structures, reinforcing the importance of histaminergic modulation in this circuitry. HDC knockout mice showed a deficit in PPI, which is seen in TS, and enhanced amphetamine-induced stereotypies, which resemble and may model the tics seen in TS. Performance in striatumdependent learning tasks was equivocal, without a clear deficit. HDC knockout mice showed no elevated anxiety or depression-like behaviors, normal exploratory behavior, and normal spatial learning. 
Discussion: These studies demonstrate that genetic disruption of the HDC gene produces TS-like behavioral abnormalities. This strongly supports the causal importance of the mutation in HDC described in our recent genetic study. While disruption of the HDC gene is clearly rare in TS cases (Ercan-Sencicek et al, NEJM 2010), further investigation of downstream abnormalities in this mouse model, which has clear face and construct validity, may shed light on the pathophysiology of the disorder more generally.

Disclosure: L. Baldan Ramsey: None. K. Krusong: None. H. Ohtsu: None. A. Ercan-Sencicek: None. M. State: None. C. Pittenger: None.

10. The Imbalance between Methionine Oxidation and Reduction May Play a Critical Role in Depression and Related Immune Dysregulation by Disrupting Dopaminergic Pathways in the Frontal Cortex

Marlon Quinones*, Fabio Jimenez, Dianne Cruz, Erica Navaira, Adam Salmo, Martin Javors, Julie Hensler, Arlan Richardson, Seema Ahuja, Consuelo Walss-Bass

\section{UTHSCSA, San Antonio, TX}

Background: Oxidative stress, as indicated by the oxidation of proteins and lipids by reactive oxygen species (ROS), is thought to play a role in the pathogenesis of several psychiatric illnesses including mood disorders. Methionine sulfoxide reductase (MSRA) is an antioxidant enzyme highly expressed in the brain (e.g., cortex an hippocampus) implicated in protection against oxidative stress by repairing free and protein bound oxidized methionine residues. We postulated that impairments of the function of MSRA could play a role in the pathogenesis of depression and its systemic effects.

Methods: We used mice with a genetic inactivation of the MSRA gene, which have been shown to increase sensitivity to oxidative stress.

Results: Supporting our hypothesis, we found that in a murine model system used to assess correlates of depression, namely the forced swim test (FST), genetic inactivation of MSRA was associated with more immobility time, indicating increased baseline depressive behavior. Further, using HPLC we found that this phenotype was associated with a disruption of dopamine metabolism as indicated by lower levels of dopamine and its metabolites DOPAC and HVA in the frontal cortex of MSRA knockouts (KO) compared with age and sex matched WT mice. Demonstrating the specificity of this finding, we observed that frontal cortex serotonin levels were comparable between WT and KO mice. Given the growing body of evidence for a bidirectional link between depression and immune dysregulation in humans, we then tested the hypothesis that the correlates of depression observed in MSRA KO mice could be linked to immune abnormalities. Consistent with this notion, we found that, compared with WT mice, MSRA KO mice presented immune changes suggestive of disruptions in cellular immunity (i.e., increased production of the critical cytokine interferon gamma by $\mathrm{T}$ lymphocytes) and its regulation (abnormal proportion of Regulatory T cells). Further, using two different in vivo model systems, involving either control of an infectious challenge (i.e., intradermal infection with the intracellular pathogen Leishmania major), or the induction of autoimmunity akin to conditions such as Arthritis (i.e., collagen antibody induced arthritis), we found that MSRA KO mice developed an exaggerated inflammatory response.

Discussion: Collectively, our data provides evidence for the previously postulated role of oxidative stress/inflammation in mood disorders. More specifically, disruption of MSRA-regulated processes such as regulation of methionine oxidative state may contribute to the development of depression, possibly due to its negative impact on dopamine metabolism. Our studies also provide a new framework to dissect the potential mechanisms underlying the established effectiveness in the treatment of depression by modulating methionine oxidation/reduction via the oral administration of S-Adenosyl Methionine (SAMe). Further experiments are required to clarify the mechanisms by which MSRA may link depression and immune dysregulation.
Acknowledgements: This work was supported by the National Institutes of Health grants Ko1MHo77777 to CWB, RO1-AR052755 and RO1-AI48644 to SSA; the Stanley Medical Research Institute and NARSAD grants to CWB and MPQ; and The Veterans Affairs Merit Grant to SSA

Disclosure: M. Quinones: None. F. Jimenez: None. D. Cruz: None. E. Navaira: None. A. Salmo: None. M. Javors: None. J. Hensler: None. A. Richardson: None. S. Ahuja: None. C. Walss-Bass: None.

\section{D2 Receptors in Infralimbic Cortex are Necessary for Fear Extinction and Extinction-Related Tone Responses} Devin Mueller, Christian Bravo-Rivera, Gregory J. Quirk*

University of Puerto Rico, San Juan, PR

Background: The D2 family of dopamine receptors is intimately involved in affective, motor, and cognitive functions, many of which are mediated by the medial prefrontal cortex (mPFC). D2 receptor signaling in the mPFC of rats has been linked to working memory, reversal learning, and behavioral flexibility, but their role in extinction of conditioned fear (also mPFC-dependent) is not known. This is interesting in light of a previous report that the $\mathrm{D}_{2}$ blocker sulpiride could facilitate extinction of fear (Ponnusamy et al., 2005), suggesting it might serve as an adjunct to extinction-based therapies for anxiety disorders.

Methods: In the present study, we used raclopride, which is a more selective antagonist of $\mathrm{D}_{2}$ receptors than sulpiride. Raclopride was administered either systemically or infused into the infralimbic (IL) mPFC, shortly prior to extinction training, in an auditory fear conditioning task. Rats were also prepared for single unit recording in IL, to assess the effect of systemic raclopride on IL firing properties. Results: Systemic injection of raclopride given prior to extinction impaired retrieval of extinction when rats were tested drug free the next day, but also induced catalepsy during extinction training. To determine whether impaired extinction was due to impaired motor function or disruption of extinction, we infused raclopride directly into IL. Administered in this way, raclopride did not produce motor deficits, but still impaired recall of extinction when tested drug free. Furthermore, in animals that underwent extinction training, systemic raclopride reduced the tone responsiveness of IL neurons in layer 5, without decreasing average firing rate.

Discussion: The necessity of $\mathrm{D}_{2}$ receptors in IL in extinction suggest that D2 blockers would not useful as adjuncts to extinction based therapies. It also agrees with a recent study in humans, showing that schizophrenic patients (treated with D2 blockers) are deficient in recall of extinction (Holt et al., 2009). We suggest that $\mathrm{D} 2$ receptors facilitate extinction by increasing the signal-to-noise of IL neurons that learn and/or consolidate extinction.

Disclosure: D. Mueller: None. C. Bravo-Rivera: None. G. Quirk: None.

12. This poster has been cancelled. 
vulnerability to development of anxiety disorders in conjunction with other genes or environmental stressors. These hypotheses can be tested via temporal control of CRF overexpression (CRFOE) in mice.

Methods: Using inducible transgenesis based on the doxycyclinedependent rtTA2 factor, we generated mice with strict temporal control over CRFOE. We first evaluated the "state model" of CRF abnormalities by examining whether forebrain CRFOE during adulthood is sufficient to elicit PTSD-like anxiety behaviors. Male and female mice carrying two transgenes, a CaMKII $\alpha$ promoter-driven rtTA2 transgene and a dox-regulated tetO promoter fused to a CRF gene ("CRFOE" group), were treated daily with dox (6 mg dox/g food chow) at 3 months of age until completion of testing. Littermate controls were not treated with dox ( $n=9$ /group). At 21 days after dox initiation, baseline startle, prepulse inhibition (PPI), context/cued fear conditioning, and locomotor activity was assessed.

Results: CRFOE did not affect startle reactivity, PPI, or center entries in an open field. However, CRFOE significantly impaired context, but not cued, fear conditioning, and disrupted locomotor habituation to a novel environment. These effects persisted when mice were re-tested 3 weeks after dox removal. Thus, temporal restriction of forebrain CRFOE in adulthood was sufficient to induce selective and lasting disruptions in tasks involving the hippocampus (context fear conditioning; context habituation), consistent with hippocampal abnormalities reported in PTSD. Restricting CRFOE to adulthood, however, was not sufficient to affect baseline arousal (startle, PPI) and unconditioned anxiety (open field). Thus, the "state model" of CRF abnormalities cannot fully mimic a PTSD-like pathology. We have begun to test the alternative hypothesis that CRFOE during development is sufficient to alter adult emotional behaviors. In a second cohort, CRF was overexpressed during the first 3 weeks of life ( $\left.\mathrm{P}_{2}-\mathrm{P}_{23}\right)$ with testing at 3 months of age. Preliminary data $(n=5-11$ /group $)$ indicate that postnatal dox-induced increases in CRFOE selectively increases startle reactivity and decreases PPI, particularly in females, without affecting fear conditioning, context habituation, or locomotor activity.

Discussion: The results from these two studies suggest that CRFOE restricted to either adulthood or early life is not sufficient to fully account for PTSD-like pathology. However, the timing of the disruption to the CRF system appears to differentially mimic specific features of the PTSD phenotype. CRFOE during adulthood selectively disrupts hippocampal-dependent processes, whereas CRFOE during development selectively disrupts arousal. Perhaps the age-dependent behavioral effects of disturbances to the stress signaling system depend upon the particular brain areas that are developing (or declining) at the time of the disturbance (Lupien et al., 2009). It should also be noted that the phenotypes we have assessed thus far occur after generally mild stress. In future experiments, we will examine the interaction between CRFOE and response to a strong stressor (predator exposure) to determine if CRFOE increases vulnerability to enduring effects of traumatic stress.

Disclosure: J. Gresack: None. C. Scott: None. C. Niemeyer: None. H. Steen: None. E. Vicentini: Part 5; GlaxoSmithKline. L. Mangiarini: None. I. Mansuy: None. E. Merlo-Pich: GlaxoSmithKline. M. Geyer: Part 1; SD Instruments (equity interest). V. Risbrough: None.

13. Transient Forebrain Overexpression of Corticotropin Releasing Factor in Adulthood Selectively Impairs Contextual Fear Memory and Context Habituation

Jodi Gresack, Christine Scott, Christine Niemeyer, Hendrikus Steen, Elena Vicentini, Laura Mangiarini, Isabelle Mansuy, Emilio MerloPich, Mark Geyer, Victoria Risbrough*

University of California, San Diego, La Jolla, CA

Background: Many neuropsychiatric disorders, including post-traumatic stress disorder (PTSD), are linked to chronic increases in corticotropin releasing factor (CRF), a neuropeptide mediating the stress response. It is unclear if CRF hypersecretion occurs after the disorder develops (i.e. state dependent) or if it is a trait that increases

\section{Effects of Social Subordination Stress and Serotonin Transporter (5HTT) Genotype on Brain Volume and White Matter Tracts in Juvenile Female Macaques \\ Brittany Howell, Vasiliki Michopoulos, Zachary Johnson, Xiaodong Zhang, Govind Nair, Xiaoping Hu, Martin Styner, Mark Wilson, Mar Sanchez ${ }^{*}$}

Emory University, Atlanta, GA

Background: This study investigated the effects of an ethologically valid and naturally occurring macaque model of pediatric stress (social subordination) and the modulatory effects of $5 \mathrm{HTT}$ genotype on brain development. Social subordination is associated with dysregulation of 
the hypothalamic-pituitary-adrenal (HPA) axis in adult female rhesus monkeys and with adverse health outcomes that are influenced by 5HTT allelic variants. Social subordination also delays puberty and increases emotional reactivity, effects exacerbated by the short (s) 5 HTT allelic variant.

Methods: The effects of social subordination and ${ }_{5}$ HTT genotype on the development of brain white matter tracts and volume of limbic regions involved in emotional regulation were studied during the pre-pubertal interval, using structural magnetic resonance imaging (MRI) and diffusion tensor imaging (DTI). Subjects were 38 juvenile female rhesus monkeys living in four large social groups. Based on the outcome of aggressive encounters, 18 subjects were considered dominant and 20 subordinate. Half the animals in each rank category had both long promoter length alleles $(\mathrm{l} / \mathrm{l})$ of the gene encoding the ${ }_{5} \mathrm{HTT}$ and half had at least one short allele (s-variant). Animals were scanned between 19-23 months of age. Image analysis of T1-MRI scans (MPRAGE sequence: TI/ $\mathrm{TR} / \mathrm{TE}=950 / 3000 / 3.49 \mathrm{~ms}, \mathrm{FOV}=96 \mathrm{~mm}, 8$ averages, $0.5 \times 0.5 \times 0.5 \mathrm{~mm} 3$ ) involved automatic segmentation of ROIs using an atlas-based approach in which a manually-segmented atlas was first non-linearly registered into each subjects' native space. A Two-Way ANOVA examined the effects of rank (high, low) and 5 HTT genotype (1/l, s-variant -1/s + s/s-) on total brain and regional (ROIs: hippocampus, amygdala, striatum) volumes. DTI data (single-shot double spin-echo EPI sequence, GRAPPA $(\mathrm{R}=3)$, voxel size: $1.3 \times 1.3 \times 1.3 \mathrm{~mm} 3, \quad \mathrm{TR} / \mathrm{TE}=5000 / 86 \mathrm{~ms}$, FOV: $83 \mathrm{~mm}, \mathrm{~b}: 0,1000 \mathrm{~s} / \mathrm{mm}^{2}$, 60 directions, 12 averages) was analyzed using tract-based spatial statistics (TBSS), which generated skeletonized, co-registered fractional anisotropy (FA) maps of the major white matter tracts for all subjects, which was then analyzed using a voxelwise Two Way ANOVA for rank and 5 HTT genotype.

Results: Although no significant effects of social rank or 5 HTT genotype were detected on the volumes of hippocampus, amygdala or striatum, carriers of the s-allelic variant had significantly smaller total brain volumes. A significant effect of rank was also detected in medial prefrontal cortex white matter, with low ranking animals showing increased FA. No ${ }_{5} \mathrm{HTT}$ effects were found in this region. However, a significant rank x 5 HTT genotype effect was detected in: (1) parietal cortex, low ranking, $s$ animals showing lower FA than all other groups; and (2) in superior temporal cortex, where FA was lower in high ranking, $1 / 1$ animals as compared to those with $s$ allele, but FA was higher in low ranking, $1 / 1$ as compared to low, $s$ allele. These initial findings do not account for the effects of relatedness in these individuals. To address this potentially critical variable Monte Carlo significance analyses within this pedigreed population are being performed.

Discussion: The brain volume and FA differences detected reflect overall effects of ${ }_{5} \mathrm{HTT}$ polymorphisms and social subordination on brain development and on structural integrity of white matter tracts connecting cortico-limbic and association cortices. This could affect information transfer between those cortical regions and, therefore emotional processing related to the behavioral alterations reported in this animal model. We are currently examining the correlations between brain structural and behavioral/neuroendocrine alterations. Support: NMIH grant MHo79100 (MW), NICHD 055255 (MS) \& RRo0165 (YNPRC Base grant)

Disclosure: B. Howell: None. V. Michopoulos: None. Z. Johnson: None. X. Zhang: None. G. Nair: None. X. Hu: None. M. Styner: None. M. Wilson: None. M. Sanchez: None.

\section{Antidepressants Decrease Brain Amyloid in Transgenic Mice and} Are Associated with Decreased Human Brain Amyloid

Yvette Sheline*, John Cirrito, Brianne DiSabato, Davinder Hayreh, Jessica Restivo, Deborah Verges, Gina D’Angelo, Tammie Benzinger, John Morris, Zhizi Yan, Tami Curl-Perkins, Rebecca Fierberg, Mark Mintun

\section{Washington University, St Louis, MO}

Background: Currently there are no effective treatment strategies in practice for Alzheimer's disease (AD), the most common neurodegenerative disorder. A growing literature strongly suggests that amyloid- $\beta$ (A $\beta)$ dysregulation may initiate the pathogenesis of $\mathrm{AD}$, augmented by a cascade of downstream factors that exacerbate and propagate neuronal injury. $A \beta$ accumulates in the brain in preclinical $\mathrm{AD}$ as soluble and insoluble conformations, such as toxic oligomers and plaques. We have demonstrated that by the time the earliest symptoms of $\mathrm{AD}$ can be appreciated clinically, 30\%-60\% of neurons in the entorhinal cortex and hippocampus already have been lost.

Methods: Animals: In vivo microdialysis to assess brain ISF A $\beta$ in the hippocampus of awake, freely moving $\mathrm{PS}_{1} \mathrm{APP}^{+/-}$mice was performed similar to previously described (Cirrito \& Holtzman, 2003). Once basal ISF A $\beta$ levels were established, PS1APP mice were treated with either citalopram (10 $\mathrm{mg} / \mathrm{kg}$ body weight, comparable to an adult human dose of $50 \mathrm{mg} /$ day) (Reagan-Shaw et al., 2008), tianeptine $(20 \mathrm{mg} / \mathrm{kg})$, or vehicle (PBS) intraperitoneally or with serotonin $(2 \mathrm{mM})$ by adding the drug to the microdialysis perfusion buffer to infuse the drug directly into the hippocampus. Human Participants: Cognitively normal participants $(n=126)$ were recruited into a study for imaging $A \beta$ plaques by positron emission tomography (PET) PIB scanning in late life. All participants were assessed with the Clinical Dementia Rating scale (CDR) all scoring $C D R=0$, and were excluded for severe or unstable medical disorders or known primary neurological disorders. A blood sample was drawn from each individual. DNA was extracted from blood cells and APOE genotyping was performed using a Pyrosequencing protocol. All participants received a structured interview to assess lifetime use of antidepressants. Amyloid- $\beta$ PET imaging was carried out as has been described in detail. Two-tailed ttests or chi-square analyses as appropriate were conducted to examine differences between participants treated with antidepressants within the past 5 years vs those not treated with antidepressants within the past 5 years.

Results: Animal: Compared to vehicle-treated mice, citalopram visually reduced ISF $A \beta$ levels almost immediately following drug administration with a significant decrease starting 14 hours after treatment. The effect of citalopram was long-lived with ISF A $\beta$ levels remaining reduced by $25 \%$ at 24 hours after drug administration. In contrast, ISF A $\beta$ levels did not change appreciably in vehicle-treated mice compared to basal $A \beta$ levels in each mouse nor with tianpetine treatment. Direct infusion of serotonin reduced $A \beta$ levels by $30 \%$, suggesting that serotonin signaling reduces $A \beta$ generation. Human Participants: A total of 126 participants underwent PET A $\beta$ imaging and ascertainment of antidepressant history. Participants, who had received antidepressant medication for a full 5 years prior to PET scan $\mathrm{n}=15$ (treated), had lower $\mathrm{A} \beta$ mean $\mathrm{MCBP}=0.01,(\mathrm{SD} .07)$ by PET imaging compared to those who had not received medication $\mathrm{n}=111$ in the past 5 years (untreated), $\mathrm{MCBP}=0.13,(\mathrm{SD} .20)$ : unpaired $\mathrm{t}$ - test $\mathrm{p}=0.001$; Wilcoxon rank sum test $\mathrm{p}=0.04$. Treated vs untreated participants did not differ in age, mean $72.1(7.2)$ vs $71.8(4.9)(\mathrm{p}=0.86)$ gender $80 \% \mathrm{~F}$ vs $69 \% \mathrm{~F}(\mathrm{p}=0.40)$, education, $15.4(2.3)$ vs 15.6 (2.6) $\mathrm{p}=0.90$ or ApoE 4 allele status, $20.0 \% \mathrm{E}_{4}+$ vs $20.7 \% \mathrm{E}_{4}+, \mathrm{p}=0.95$. Discussion: These data suggest that antidepressants produced an acute reduction in amyloid in an animal model of $\mathrm{AD}$ and were associated with a reduction in amyloid plaques in cognitively normal elderly. Our mouse studies demonstrate that SSRI antidepressants cause an acute reduction in ISF $A \beta$ levels. Current studies underway are further investigating the mechanism of action of SSRI drugs on ISF A $\beta$ level reduction. A direct causal relation between antidepressants and plaque reduction in humans remains to be shown. A strength of the study's design is that participants with a history of antidepressant use did not differ in age, gender and ApoE status from participants with no antidepressant exposure. It will be important to investigate antidepressant treatment effects further given the proven safety of antidepressants and the window of opportunity for treatment after the detection of amyloid plaques but prior to the onset of clinical AD. Disclosure: Y. Sheline: Part 1; Eli Lilly Speaker's Bureau. J. Cirrito: None. B. DiSabato: None. D. Hayreh: None. J. Restivo: None. D. Verges: None. G. D’Angelo: None. T. Benzinger: None. J. Morris: 
Has served as a consultant, AstraZeneca, Bristol-Meyers Suibb, Elan, Genentech, Lilly, Merck, Novartis, Pfizer, Schering Plough and Wyeth. Z. Yan: None. T. Curl-Perkins: None. R. Fierberg: None. M. Mintun: Avid Radiopharmaceutical. Part 5; Avid Radiopharmaceutical.

\section{A Humanized Mouse Model of the Functional OPRM1 A118G Polymorphism: Role in Alcohol Reward}

Annika Thorsell*, FJ Pavon, Victor Ruiz-Velasco, Hui Sun, Robert

Eskay, T Schoor, Wolfgang Sommer, Loren Parsons, Markus Heilig

NIAAA, NIH, Bethesda, MD

Background: Alcohol use is a major cause of disability worldwide but available treatments have limited efficacy, in part due to the complex pharmacology of alcohol. Sedative, ataxic, and anxiolytic properties of alcohol are primarily mediated through GABA and glutamate signaling, while the rewarding properties of alcohol are in part mediated via endogenous opioids and mesolimbic dopamine (DA). OPRM1 activation in the ventral tegmental area suppresses activity of inhibitory GABAergic interneurons, resulting in disinhibition of DA neurons and DA release from terminals in the ventral striatum/nucelus accumbens (VStr/Nacc). Accordingly, OPRM1 blockade is a treatment for alcohol dependence. Here, we examined whether a functional OPRM1 A118G polymorphism influences dopamine responses to alcohol, as well as behavioral measures of anxiety, alcohol intake, and sensitivity. Two humanized mouse lines, carrying the respective human sequence variant were generated to directly establish the causal role of OPRM1 A118G variation and dopamine (DA) response to an alcohol challenge were examined using microdialysis probes targeting VStr/Nacc. The two mouse-lines were further compared with regard to anxiety-like behavior, locomotor activity, alcohol intake, and alcohol sensitivity.

Methods: Anxiety-related behavior was measured on the elevated plus-maze as well as in the open field. Alcohol intake and preference were measured in a two-bottle choice procedure and alcohol sensistivity was measured in Loss Of Righting Reflex. Dopaminerelease in the NAcc was measured using microdialysis. We also measure receptor affinity, binding, and signaling to confirm functionality of the receptor.

Results: Receptor affinity, binding, and signaling are normal in the $\mathrm{h} / \mathrm{m}$ OPRM1 $118 \mathrm{G} / \mathrm{G}$ and $\mathrm{h} / \mathrm{m}$ OPRM1 $118 \mathrm{~A} / \mathrm{A}$ mouse lines. The $\mathrm{h} / \mathrm{m}$ OPRM1 G/G line displays a bold/anxiolytic-like phenotype in the elevated plus-maze. Alcohol intake is increased in $\mathrm{h} / \mathrm{m}$ OPRM1 G/G while sensitivity to alcohol induced sedation is decreased. The OPRM1 GG allele confers a more vigorous DA response to alcohol in the VStr/NAcc. but not 5-HT response to an ip administration of $2 \mathrm{~g} / \mathrm{kg}$ ethanol is enhanced in $118 \mathrm{G}$ homozygous mice. In summary, the OPRM1 $118 \mathrm{G}$ variant, isolated from possible influence of other polymorphisms, confers several phenotypic traits that resemble those found in subjects at genetic risk for alcoholism.

Disclosure: A. Thorsell: None. F. Pavon: None. V. Ruiz-Velasco: None. H. Sun: None. R. Eskay: None. T. Schoor: None. W. Sommer: None. L. Parsons: None. M. Heilig: None.

\section{Corticotropin-releasing Factor in the Locus Coeruleus Facilitates Behavioral Flexibility \\ Rita Valentino*, Wei-Wen Wang, Kevin Snyder}

The Children's Hospital of Philadelphia, Philadelphia, PA

Background: Corticotropin-releasing factor (CRF), the stress-related neuropeptide that organizes the stress response acts as a neurotransmitter in the major brain norepinephrine nucleus, locus coeruleus (LC) to activate this system during stress. We previously demonstrated that stress- or CRF-induced LC activation underlies the arousal component of the stress response. CRF also shifts the mode of LC discharge from a phasic to a high tonic state and this mode of discharge has been suggested to promote behavioral flexibility (Aston-Jones and Cohen, 2005). Here the effects of CRF administered intracerebroventricularly (i.c.v.) or bilaterally into the LC on different forms of behavioral flexibility were determined in an attentional set shifting task in rats.

Methods: Adult male Sprague Dawley rats were implanted with a cannula guide in the lateral ventricle or with bilateral cannula guides in the LC at least 5 days prior to training and testing for attentional set shifting. Rats were food restricted to $85 \%$ free feeding weight and were first trained to dig in ceramic pots to obtain food reward ( $1 / 4$ peanut butter chip) in their cage and then in the testing arena. On the following day rats were trained in a simple discrimination task to a criterion of 6 consecutive correct trials in which the location of the reward was determined first by odor and then by digging material. Testing on the third day involved the following stages: simple discrimination (SD) in one sensory dimension; compound discrimination (CD), in which distinctions between the second sensory dimension are introduced but are not relevant to the contingency; intradimensional set shifting (IDS), in which stimuli of both dimensions are replaced but the contingency remains based on differences within the original sensory dimension; reversal (REV), in which the contingencies for the same dimension are reversed; and extradimensional set shifting (EDS) where the contingency is based on the alternate dimension. Rats were injected with ACSF ( 3 ul, i.c.v. or $100 \mathrm{nl}$ intra-LC) or CRF (0.03, 0.1, 0.3 ug in 3 ul ACSF i.c.v. or 1,3 , or $10 \mathrm{ng}$ in $100 \mathrm{nl}$ ACSF intra-LC). The number of trials to reach criterion (TTC) for each stage was determined. Dye was injected through the cannula to histologically localize injection sites.

Results: CRF had distinctly different effects on components of the attentional set shifting task when administered i.c.v. vs. intra-LC. Intracerebroventricular administration of the lowest dose of CRF (o.03 ug, $\mathrm{n}=6)$ disrupted EDS $(35 \pm 3$ TTC) compared to ACSF treated rats $(19 \pm 3$ TTC, $\mathrm{p}<0.05)$. At a higher CRF dose $(0.1 \mathrm{ug}, \mathrm{n}=8)$ the effect on EDS was replaced by a disruption of IDS and reversal learning. The mean TTC for CRF-treated rats was $34 \pm 6$ for IDS and $60 \pm 11$ for Rev compared to $16 \pm 3(\mathrm{p}<0.01)$ for IDS and $36 \pm 4$ for Rev $(\mathrm{p}<0.05)$ in ACSF treated rats $(\mathrm{n}=8)$. Only 3 rats were able to complete the task at the highest dose of CRF (0.3 ug). When administered intra-LC no dose of CRF impaired IDS $(\mathrm{F}(3,21)=1.4$, $\mathrm{p}=0.26)$ or reversal learning $(\mathrm{F}(3,21)=1.9, \mathrm{p}=0.16)$. Only three rats administered $10 \mathrm{ng}$ CRF completed the entire task. Although these rats made significantly more errors when learning the initial SD ( $12 \pm 3$ vs. $6.2 \pm 0.2$ TTC, CRF vs. control, $\mathrm{P}<0.001$ ), performance in the remaining tasks was not impaired by this dose and there was a trend for improvement of IDS and reversal learning by this dose. Importantly, intra-LC CRF ( $1 \mathrm{ng}, \mathrm{n}=6$ ) improved EDS with a mean of $14 \pm 2$ TTC to $26 \pm 4$ TTC in ACSF-treated rats $(n=6, p<0.05)$. The overall ANOVA across doses for EDS performance was statistically significant $(\mathrm{F}(3,21)=4.3, \mathrm{p}<0.02)$.

Discussion: Intracerebroventricular CRF could affect many brain regions that impact on set shifting performance. The dorsal raphe nucleus (DRN) is one target of CRF that has been implicated in reversal learning. Inhibition of DRN serotonin neurons by low doses of CRF could account for the observed deficits in reversal learning. Given that CRF activates LC neurons, the ability of intra-LC CRF to facilitate extradimensional set shifting is consistent with other findings implicating LC-norepinephrine projections to prefrontal cortex in this performance. The results are also consistent with the idea that the shift from a phasic to tonic mode of discharge is associated with behavioral flexibility. Importantly, the findings suggest that a function of CRF release in the LC during stress is to facilitate shifting of attention between diverse stimuli in a dynamic environment so that the organism can adapt an optimal strategy for coping with the challenge.

Disclosure: R. Valentino: None. W. Wang: None. K. Snyder: None. 
18. The Autism-Associated SERT Ala56 Variant Causes Hyperserotonemia, Receptor Hypersensitivity, Social Impairment, and Repetitive Behavior in Mice

Jeremy Veenstra-VanderWeele*, Christopher Muller, Hideki

Iwamoto, Jennifer Sauer, L. Anthony Owens, Jordan Cohen, Charisma Shah, Padmananbhan Mannangatti, Tammy Jessen, Brent Thompson, Ana Carneiro, Jacqueline Crawley, Elaine Sanders-Bush, Douglas McMahon, Sammanda Ramamoorthy, Lynette Daws, James Sutcliffe, Randy Blakely

\section{Vanderbilt University, Nashville, TN}

Background: Elevated whole blood serotonin (5-HT) levels in autism were first reported almost fifty years ago, yet our understanding of this biomarker remains incomplete. Genome-wide association data implicates the serotonin transporter gene (SERT, SLC6A4) as a quantitative trait locus for whole blood serotonin levels in males. Furthermore, strong evidence for linkage overlies the serotonin transporter gene in families with two or more children with autism, so long as only males are affected. Within these families with evidence for linkage, multiple rare SERT amino acid variants were detected (Sutcliffe et al. 2005). The most common of these variants, Gly56Ala, was found at elevated levels relative to controls and was associated with rigid-compulsive behaviors. In vitro studies (Sutcliffe et al., 2005; Prasad et al., 2009) demonstrated that the Ala56 variant displays increased basal 5-HT transport, exhibits elevated basal phosphorylation, and is refractory to upregulation through PKG and p38 MAPK pathways.

Methods: To pursue the physiological significance of these findings in vivo, we developed a SERT Ala56 knock-in mouse line. We have used biochemical, electrophysiological, and behavioral assays to compare mice expressing this rare variant to wildtype littermate control mice. Results: The SERT Ala56 variant mice are hyperserotonemic and exhibit enhanced 5-HT clearance in the hippocampus in vivo. Synaptosomes from these mice display elevated basal SERT phosphorylation and do not show any further increase in phosphorylation after stimulation of the PKG pathway. Consistent with an impact of the Gly56Ala variant on 5-HT homeostasis at the synapse, the SERT Ala56 mice show increased 5-HT receptor sensitivity, both pre- and post-synaptically. In slice preparations, 5-HT neurons show a decreased basal firing rate and increased sensitivity to inhibition by 5 -HT. Behavioral testing reveals both repetitive behavior and altered social function. Chronic but not acute treatment with a serotonin reuptake inhibitor partially reverses receptor hypersensitivity and social dysfunction in adult animals.

Discussion: Our efforts establish a new animal model based upon an autism-associated gene variant that manifests autism-relevant social and repetitive behavior, as well as recapitulating the most robust autism biomarker. The response seen to pharmacological treatment suggests that this model may accelerate the development of autism therapeutics. Disclosure: J. Veenstra-VanderWeele: Part 1; Seaside Therapeutics, Roche Pharmaceuticals, Novartis. Part 4; Seaside Therapeutics, Roche Pharmaceuticals, Novartis. C. Muller: None. H. Iwamoto: None. J. Sauer: None. L. Owens: None. J. Cohen: None. C. Shah: None. P. Mannangatti: None. T. Jessen: None. B. Thompson: None. A. Carneiro: None. J. Crawley: None. E. Sanders-Bush: None. D. McMahon: None. S. Ramamoorthy: None. L. Daws: None. J. Sutcliffe: None. R. Blakely: Part 1; Lundbeck Pharmaceuticals, JublilantInnovation, Forest Pharmaceuticals. Part 4; Forest Pharmaceuticals.

\section{Chronic Lithium Improves Cognitive Function in Rats and Decreases Stressor-Induced Increases in Motor Impulsivity: Implications for Clinical Use Catharine Winstanley*, Dominique Levesque, Katrina Yu, Jun-Feng Wang, Allan Young}

\section{University of British Columbia, Vancouver, BC, Canada}

Background: Lithium is a monovalent cation with proven efficacy in the treatment of bipolar disorder (BD), particularly mania. A key component of mania is high impulsivity, which is also associated with increased suicide risk. Recent literature suggests that higher environmental levels of lithium also decrease suicide rates. However, whether lithium acts to directly decrease impulsivity is unclear. Understanding more about lithium's effects on impulsivity could therefore provide valuable insight into the neurobiology underlying successful treatment of $\mathrm{BD}$, and may also provide indications of how to decrease suicidation- a traditionally difficult area of research.

Methods: We determined the effects of chronic lithium administration in rats $(3 \% \mathrm{w} / \mathrm{v}$ added to the diet) on performance of a) the five-choice serial reaction time task $(5 \mathrm{CSRT})$, an analogue of the continuous performance test used clinically, and b) the delay-discounting task of impulsive decision-making in which rats choose between a smaller-sooner vs largerlater reward. During the 5 CSRT, animals learned to respond to a brief visual stimulus presented in one of five locations to earn reward. The accuracy of target detection therefore measures attentional ability. Premature responses made before the stimulus is presented provide an index of motor impulsivity. Once stable performance was established on both tasks, the effects of the drug yohimbine, an alpha-2 receptor antagonist which increases noradrenaline release, was determined. This drug is often used clinically as a pharmacological stressor, and although it can induce impulsivity and mild anxiety in healthy volunteers, it triggers panic in those with anxiety disorders, mania in BD patients, and drugcraving in substance abusers. Manipulations which reduce the maladaptive response to yohimbine may therefore hold promise for reducing the negative impact of stressors on psychiatric prognoses.

Results: Blood levels of lithium were within the therapeutic range ( $0.69 \mathrm{~mol} / \mathrm{L})$ at the time of behavioural testing. Both control and lithiumfed groups learned the 5CSRT within 6 weeks. However, after 8 weeks of treatment, lithium-fed rats were significantly more accurate on the ${ }_{5}$ CSRT than control animals, and this improvement remained stable for the remainder of the experiment. There were no differences in the baseline level of premature responding exhibited by control and treatment groups on baseline. There was also no effect of lithium treatment on the basal level of impulsive choice exhibited on the delay-discounting paradigm. In order to probe the discounting rate more thoroughly, the delays to the large reward were shortened. Lithium-treated rats were faster to alter their choice preferences in response to this change and their behaviour stabilised more rapidly. Furthermore, their choice preference between the first and second sets of delays was internally consistent, whereas rats in the control group showed significant contrast effects. Although yohimbine had little effect on the delay-discounting paradigm, the drug increased motor impulsivity on the 5CSRT in keeping with previous clinical and preclinical reports. Furthermore, this pro-impulsive effect of the drug was significantly attenuated by lithium treatment.

Discussion: These data suggest that chronic lithium may enhance cognitive function and attenuate stress-induced increases in motor impulsivity, findings which could contribute to our understanding of lithium's clinical efficacy. Given that lesions to the medial prefrontal cortex (mPFC) reduce accuracy on the ${ }_{5} \mathrm{CSRT}$ and impair contingency detection in the delay-discounting paradigm, the improvements in these cognitive processes observed here may arise through lithiuminduced modifications in the MPFC. Experiments designed to test this hypothesis are currently underway using brain samples taken from the $\mathrm{mPFC}$, and other regions implicated in impulse control, following chronic lithium treatment.

Disclosure: C. Winstanley: Part 1; Theravance. D. Levesque: None. K. Yu: None. J. Wang: None. A. Young: None.

20. Cognitive Assessment of Murine Models of Alzheimer's Disease with Repeated Acquisition of Incremental Operant Chains Marianne Evola, Katherine Greiner, Sonia Shah, James Deal, Paula Grammas, Alice Young*

Texas Tech University, Lubbock, TX

Background: We assessed the ability of an operant chaining procedure to detect differences in cognitive function among three transgenic 
mouse strains that are used as models of different features of Alzheimer's disease $(\mathrm{AD})$. The procedure required mice to complete repeating chains of signaled operant responses in order to earn an evaporated milk reinforcer (Wenger et al, 2004). Chain length increased sequentially within a session, allowing assessment of performance as attention and memory requirements varied.

Methods: In experimental sessions conducted once per day, mice were placed in a sound-attentuated chamber with three response openings (left, right, center) mounted above a liquid delivery hopper. Mice were required to emit a nosepoke response in the one lit opening (a cued response) to earn milk. Each session began with a single cued response (i.e., left), and successful completion delivered a dipper of milk. Completion of 10 correct responses in a row triggered an increase in the chain length (i.e., right, then left), to a maximum 8-response chain. Mice were exposed to the chaining procedure for 30 days. Different experimental manipulations targeting risk factors for $\mathrm{AD}$ were implemented in the different transgenic models. Tg2576 mice, a transgenic model of $\mathrm{AD}$ that carry the 695 amino acid isoform for human $\beta$-amyloid precursor protein, and their wild-type controls were treated with either saline or streptozotocin $(\mathrm{STZ} ; 40 \mathrm{mg} / \mathrm{kg} / \mathrm{day}$ for 5 days), in order to produce a sustained elevation of blood glucose. ApoE 3 \& 4 mice, another murine model that carry a transgene associated with elevated risk for cardiovascular disease and $\mathrm{AD}$, were fed either normal chow or a diet designed to elevate homocystein (HC), a risk factor for AD.

Results: For Tg2576 and WT mice, the proportion of each group that mastered each chain length did not vary over 30 days of training, whereas the proportion of errors within each chain length progressively decreased. As the length of the response chain increased, the proportion of mice that failed to complete a chain increased, but the proportion of errors during each week of training did not. Most STZtreated $(90 \%)$ and all control $(100 \%)$ mice completed a one-response chain within the first week of training, and errors decreased across training weeks for all groups. Only $70 \%$ of STZ-treated Tg2576 mice could complete a 2-response chain as compared to $100 \%$ of control Tg2576 and all WT mice, but errors decreased similarly across training in all groups. Control WT mice learned the 3-response chain within the first week, whereas fewer than $60 \%$ of STZ-treated WT mice or either group of Tg2576 mice could do so. At the end of training, $\sim 85 \%$ of control Tg2576 mice and STZ-treated WT mice completed the 3-response chain, but no improvement occurred in STZ-treated Tg2576 mice. At a 4 - response chain, only $\sim 40 \%$ of mice in all groups completed the chain, but errors decreased across training in all groups except STZ-treated Tg2576 mice. All apoE 3 \& 4 mice were able to emit a one or two response chain within the first week of training, but apoE 3 \& 4 mice fed the HC diet made more errors $(>40 \%)$ than did mice fed a normal diet $(<30 \%)$. Fewer HC-diet mice were able to emit a 3-response chain $(<40 \%)$ during the first week of training as compared to normal diet mice (80-90\%). However, all apoE 3 \& 4 mice could emit a 3-response chain by the end of training. Few HC-diet mice $(<10 \%)$ could emit a 4-response chain during the first week of training, and even normal-diet mice were challenged $(>40 \%)$. By the end of training, all normal-diet mice and apoE 3 HC-diet mice could accurately complete a 4-response chain. In contrast, apoE4 HC-diet mice exhibited frequent errors on the 4-response chain, although their performance exhibited marked improvement as compared to the first week of training.

Discussion: Comparisons among groups suggested that attention and learning was impaired in all apoE 3 \& 4 mice, as compared to $\operatorname{Tg} 2576$ mice and their WT controls, and that these deficits were exacerbated by the HC diet. Nonetheless, even impaired apoE $3 \& 4$ mice learned the basic task and continued to learn over 5 training weeks, with improvement modulated by diet. These comparisons suggest that one strength of the operant chaining procedure is that the difficulty of the assay progressively increases within a daily training session. These progressive increases allowed both assessment of initial differences in acquisition and, due to the repeated training, assessment of whether a mouse expresses a fixed level of performance - as was exhibited by
Tg2576 and WT mice - or an altered rate of improvement - as expressed by the apoE 3 \& 4 mice. [Supported by USPHS awards AGo15964 (Grammas) and DA03796 (Young).]

Disclosure: M. Evola: None. K. Greiner: None. S. Shah: None. J. Deal: None. P. Grammas: None. A. Young: None.

\section{Drugs That Induce Oxytocin Release Enhance Social Cognition in an Animal Model Relevant to Autism \\ Larry J. Young*, Meera E. Modi}

Yerkes National Primate Center/Emory University School of Medicine, Atlanta, GA

Background: Oxytocin (OT) enhances prosocial behavior in animal models, including social recognition and bonding, and intranasal OT enhances some aspects of human social cognition in both typical and autistic populations. However, the therapeutic potential of oxytocin is limited by its poor penetration of the blood-brain-barrier. An alternative approach to enhance social cognition via the OT system is to pharmacologically stimulate OT release by targeting transmitter systems that regulate OT neurons. Previous studies in rats demonstrated that melanocortin 4 receptors $\left(\mathrm{MC}_{4} \mathrm{R}\right)$ are localized on OT neurons, and that alpha-melanocyte stimulating hormone induces OT release in the brain. Oxytocinergic neurons of rats also express serotonin 1a (5HT-1a) receptors and exogenously applied agonists for this receptor increase peripheral OT levels. To test the hypothesis that peripherally administered compounds that induce OT release facilitate OT-mediated social behaviors, we examined the effects of $\mathrm{MC}_{4} \mathrm{R}$ and ${ }_{5} \mathrm{HT} 1 \mathrm{a}$ agonists on partner preference formation in female prairie voles. Partner preference formation in monogamous prairie voles is an OT dependent complex social cognitive process that involves social information processing and social learning. Typically for a partner preference to form, animals must be allowed to interact socially and mate for at least a $24 \mathrm{~h}$ period and shorter cohabitation periods of only six hours without mating result in insufficient levels of social contact to induce a preference. This abbreviated paradigm, however, can be combined with pharmacological manipulations to test compounds for the ability to enhance social learning and induce a partner preference after only six hours.

Methods: We selected two $\mathrm{MC}_{3} / 4 \mathrm{R}$ agonists, Melanotan I (MTI) and Melanotan II (MTII) and the 5 HT1a partial agonist, busprione (BUS) to test for prosocial effects in the partner preference paradigm. Adult, non-receptive female prairie voles were given i.p. injections of MTI, MTII ( 1 and $10 \mathrm{mg} / \mathrm{kg}$ ), BUS ( 8 or $30 \mathrm{mg} / \mathrm{kg}$ ) or saline and cohabitated with a male partner for $6 \mathrm{hrs}$. Following the cohabitation, the females were tested for partner preference.

Results: Eight out of nine females receiving the high dose of MTII displayed a strong partner preference, spending on average $>10$ times more time huddling with the partner than the novel male $(\mathrm{P}<0.005$, Student's t-test). The females receiving saline, the low dose of MTII and both doses of MTI failed to show a partner preference. BUS at the low dose of $8 \mathrm{mg} / \mathrm{kg}$ induced a significant partner preference in $(p<0.005$, Student's t-test $)$ in 7 out of 9 animals. None of the drugs significantly affected the general locomotor patterns of the animals. Discussion: These results demonstrate that two different classes of drugs that share the common mechanism of acting on receptors on OT neurons to stimulate OT release can enhance OT-mediated social cognitive processes as determined by the development of a partner preference. This suggests that the behavioral results may be due to the induction of OT release by the compounds tested. These compounds may have potential as pharmacological therapies to treat social cognitive deficits in autism spectrum disorders, producing similar results as previously reported for intranasal OT, without the limitations of peripherally administration. These treatments may be particularly effective when used in combination with other behavioral therapies as the effects in prairie voles were achieved with administration during the social learning phase but not testing phase of the paradigm.

Disclosure: L. Young: Part 1; Patent filing related to drugs used in the study. M. Modi: Patent filing related to drugs used in the study. 
22. Childhood Trauma is Associated with Leukocyte Telomere Length Shortening in Young to Middle-aged Adults with PTSD

Aoife O’Donovan, Elissa Epel, Jue Lin, Thomas Metzler, Maryann Lenoci, Thomas Neylan*

\section{University of California, San Francisco, San Francisco, CA}

Background: Post-traumatic stress disorder (PTSD) is associated with an increased risk for age-related diseases and early mortality, but the biological mechanisms mediating such risk remain poorly understood. Leukocyte telomere length (LTL) is an emerging marker of biological age that predicts incidence of age-related diseases and mortality $(1,2)$. To examine if accelerated biological aging could contribute to such patterns of risk, we assessed trauma exposure and LTL in healthy agematched men and women with and without PTSD.

Methods: The present study has a cross-sectional, 2 x 2 design (PTSD/ Control $\mathrm{x}$ Male/Female) with medically healthy medication-free subjects. The sample included 43 individuals with chronic PTSD of at least 3 months duration and 47 age- and sex-matched individuals without PTSD, ranging in age from 20 to 50 years $(\mathrm{M}$ age $=30.55$, $\mathrm{SD}=7.44)$. Exclusion criteria included physical illness, medication use, obesity, alcohol or substance abuse, and pregnancy. Structured clinical interviews were conducted to assess lifetime trauma exposure and the presence of PTSD and other psychiatric disorders. LTL was measured with quantitative polymerase chain reaction.

Results: As predicted, PTSD subjects had significantly shorter ageadjusted LTL compared to controls, $F(1,85)=3.29$, partial $\eta 2=.04$, $\mathrm{p}=.03$. On average, subjects with PTSD had LTL 204 base pairs shorter $(\mathrm{M}=6594.10, \mathrm{SE}=81.50)$ than control participants $(\mathrm{M}=6798.61 .31$, $\mathrm{SE}=77.88$ ). Exposure to childhood trauma was also associated with short LTL, with cumulative exposure to traumatic events in childhood being linearly associated with shortening LTL ( $\mathrm{rho}=-.19, \mathrm{p}=.04)$. In fact, subjects with PTSD had more exposure to childhood trauma than controls and this exposure appeared to account for the PTSD group difference in LTL. There were no significant associations between total CAPS scores and age-adjusted LTL $(r=-.12, p=n s)$. Subjects with PTSD and more than one category of childhood trauma had LTL 344 base pairs shorter than controls, $\mathrm{t}(62)=2.14, \mathrm{p}=.02$. In contrast, subjects with PTSD and one or no categories of childhood trauma were not significantly different from controls in LTL, $\mathrm{t}(61.23)=.34, \mathrm{p}=\mathrm{ns}$.

Discussion: A history of childhood trauma is associated with short LTL in individuals with PTSD, independent of potential confounds. Biological embedding of childhood trauma or chronic exposure to the biological sequelae of traumatic stress could accelerate the rate of leukocyte telomere shortening, resulting in short LTL and increased risk for age-related disease in PTSD. Our findings suggest that exposure to childhood trauma may account for observed group differences in LTL between individuals with PTSD versus controls.

References:

1. Blackburn EH. Structure and function of telomeres. Nature. 1991;350(6319):569-73.

2. Epel ES, Blackburn EH, Lin J, Dhabhar FS, Adler NE, Morrow JD, et al. Accelerated telomere shortening in response to life stress. Proc Natl Acad Sci U S A. 2004;101(49):17312-5.

Disclosure: A. O’Donovan: None. E. Epel: None. J. Lin: None. T. Metzler: None. M. Lenoci: None. T. Neylan: None.

23. AAV-Mediated BDNF Over-Expression in Ventral Tegmental Area: Induction of deltaFosB Expression in Nucleus Accumbens and Prefrontal Cortex, and Long-Term Effects on Social Defeat StressInduced Sensitization

Ella Nikulina*, Junshi Wang, Ernest Terwilliger, Xiangui Ren, Ronald Hammer. Jr.

University of Arizona, Phoenix, AZ

Background: Brain-derived neurotrophic factor (BDNF) is implicated in the functioning of midbrain dopamine neurons, which mediate stress- and drug-induced neuroadaptation. We have shown previously that repeated social defeat stress increases BDNF expression in the ventral tegmental area (VTA) and induces deltaFosB expression in mesocorticolimbic terminal regions that is accompanied by persistent cross-sensitization to amphetamine. We hypothesized that VTA BDNF signaling enhances the effects of social defeat stress via mesocorticolimbic circuitry.

Methods: An adeno-associated viral vector (AAV-10) encoding BDNF and green fluorescent protein (GFP) cDNA or control AAV-GFP was infused bilaterally into the VTA of Sprague-Dawley rats to determine if elevated BDNF levels would induce deltaFosB expression and facilitate social stress-induced sensitization. Rats were exposed to a single episode of social defeat, consisting of a short exposure to an aggressive Long-Evans resident rat, which is known to induce short-term crosssensitization to amphetamine. Control rats were subjected to a handling procedure. Amphetamine $(1.0 \mathrm{mg} / \mathrm{kg})$ challenge was performed two weeks later, when cross-sensitization is no longer present in intact conditions. DeltaFosB was quantified by immunohistochemistry in the prefrontal cortex and nucleus accumbens.

Results: Intra-VTA AAV-BDNF infusion resulted in elevated deltaFosB expression in the anterior cingulate, prelimbic and infralimbic regions of the prefrontal cortex and nucleus accumbens core and shell six weeks after viral infusion. The locomotor activity of rats with BDNF or GFP over-expression was similar in the baseline condition or after saline injection. After social stress exposure, however, intra-VTA BDNF over-expression produced a sensitized locomotor response to amphetamine compared to control groups. Immunohistochemistry confirmed the efficiency of AAV-mediated over-expression of BDNF since labeling increased by nearly $50 \%$ in the VTA. Rats from the BDNF-stress group exhibited significantly greater deltaFosB expression in the nucleus accumbens shell, but not in the nucleus accumbens core or prefrontal cortex regions.

Discussion: These findings indicate an important role for intra-VTA BDNF in modulating long-term effects of social defeat stress on crosssensitization to amphetamine. Furthermore, elevated BDNF level in the VTA together with social defeat stress may interact to create a phenotype vulnerable to psychostimulant abuse. The persistent expression of deltaFos $B$ in the nucleus accumbens shell might be a mechanism for triggering social stress-induced behavioral crosssensitization in individuals with elevated BDNF levels.

Disclosure: E. Nikulina: None. J. Wang: None. E. Terwilliger: None. X. Ren: None. R. Hammer. Jr.: None.

24. Assessment of a Multi-Assay, Serum-Based Biological Diagnostic Test for Major Depressive Disorder: A Pilot and Replication Study George Papakostas*, Richard Shelton, Brianna Bakow, Sam Lipkin, Bo Pi, Linda Thurmond, John Bilello

Massachusetts General Hospital, Boston, MA

Background: The purpose of the experiment was to examine whether a serum-based test could distinguish between patients with major depressive disorder and non-depressed control subjects with minimum sensitivity and specificity of $70 \%$.

Methods: The test involves measuring nine distinct biological markers from one of four domains. Domains include inflammation (Antitrypsin [A1AT], Apolipoprotein CIII [ApoCIII], Myeloperoxidase [MPO], soluble Tumor Necrosis Factor type II [sTNFRII]), the hypothalamic pituitary adrenal (HPA) axis (Epidermal Growth Factor [EGF], Cortisol), neurotrogenesis (Brain Derived Neurotrophic factor BDNF), and metabolism (Prolactin [PRL], Resisitin [RES). These nine assays are used to derive an MDDScore ${ }^{\mathrm{TM}}$ The test was administered to two patient samples (a pilot $n=36$, and replication sample $n=34$ ), as well as 43 non-depressed control subjects.

Results: For the pilot study, of 36 MDD patients, 33 had a "positive" MDDScore $^{\mathrm{TM}}$ test while, of 43 non-depressed subjects, 9 had a "positive" MDDScore ${ }^{\mathrm{TM}}$ test. The test sensitivity and specificity were, approximately, $92 \%$ and $79 \%$, respectively. For the replication study, of 
34 MDD patients, 31 had a "positive" MDDScore ${ }^{\mathrm{TM}}$ test. The test sensitivity and specificity were, approximately, $92 \%$ and $81 \%$, respectively. Discussion: The present findings suggest MDDScore ${ }^{\mathrm{TM}}$ to represent a sensitive and specific blood-based test for differentiating between patients with MDD and non-depressed control subjects. Studies are needed to 1 . further and independently replicate this finding, 2. examine whether it can distinguish between MDD and other axis-I disorders such as bipolar disorder, and 3. examine any prognostic implications of MDDScore ${ }^{\mathrm{TM}}$ with respect to MDD vulnerability, treatment outcome, or long-term illness course.

Disclosure: G. Papakostas: Part 1; Consultant for Abbott Laboratories, AstraZeneca PLC, Eli Lilly Co., GlaxoSmithKline, Otsuka Pharmaceuticals, PAMLAB LLC, Pierre Fabre Laboratories, Shire Pharmaceuticals, and Wyeth, Inc., Honoraria (for consultation and non-speaker bureau talks) from Abbott Laboratories, Astra Zeneca PLC, Bristol-Myers Squibb Company, Eli Lilly Co., GlaxoSmithKline, H. Lundbeck A/s, Otsuka, Honoraria (for consultation and non-speaker bureau talks) from PAMLAB LLC, Pfizer, Pierre Fabre Laboratories, Shire Pharmaceuticals, and Wyeth Inc., Research support from Bristol-Myers Squibb Company, Forest Pharmaceuticals, the National Institute of Mental Health, PAMLAB LLC, Pfizer Inc., and Ridge Diagnostics, In the past but not presently, served on the speaker's bureau for BristolMyersSquibb Co. Part 2; Honoraria for consultations and.or talks (non-speaker's bureau): GlaxoSmithKline, BristolMyersSquibb, Astra Zeneca, Lundbeck Janssen-Cilag, Otsuka. Part 3; Honoraria for consultations and.or talks (non-speaker's bureau): GlaxoSmithKline, BristolMyersSquibb, Astra Zeneca, Lundbeck Janssen-Cilag, Otsuka. Part 4; Research support paid to hospital (not me) from Bristol-Myers Squibb Company, Forest Pharmaceuticals, the National Institute of Mental Health, PAMLAB LLC, Pfizer Inc., and Ridge Diagnostics. Part 5; Not applicable in my case. I am a full-time employee of MGH. R. Shelton: Part 1; Consulting: Eli Lilly, Evotec, Gideon Richter, Janssen, Medtronic, Otsuka, Pamlab, Pfizer, Repligen, Sierra, Grant/research support: Eli Lilly, Forest, Janssen, Novartis, Otsuka, Pamlab, Repligen, St Jude Medical. Part 2; None. Part 3; None. Part 4; Grant/research support: Eli Lilly, Forest, Janssen, Novartis, Otsuka, Pamlab, Repligen, St Jude Medical. Part 5; None. B. Bakow: None. S. Lipkin: None. B. Pi: Ridge Diagnostics. L. Thurmond: Ridge Diagnostics. J. Bilello: Ridge Diagnostics.

\section{The Role of Kalirin in Cortical Spine Morphology and Schizo- phrenia-Related Phenotypes}

Peter Penzes*, Michael E. Cahill, Zhong Xie, Anthony Deo, Michelle Day, Courtney A. Miller, J. David Sweatt, Isaac Goldszer, D. James Surmeier, David Lewis, Robert Sweet

Northwestern University Feinberg School of Medicine, Chicago, IL

Background: Reduced density of dendritic spines in cerebral cortex is one of the most consistently replicated postmortem findings in subjects with schizophrenia, though its basis is unknown. Kalirin is a brain-specific GEF for the Rho-like small GTPases, serving as a critical regulator of dendritic spine formation, plasticity and maintenance. Its mRNA was found to be significantly underexpressed in patients with schizophrenia, and correlated with reduced spine density. The KALRN gene produces several kalirin isoforms generated by alternative splicing. Kalirin-7 is the most abundant isoform in the adult rodent brain, with expression undetectable at birth and increasing during synaptogenesis. Kalirin- 5 and -7 are concentrated in the postsynaptic density and directly activate Rac1. Kalirin-9 and -12 are nonsynaptic, abundant during early postnatal rodent development and activate both Rac1 and RhoA. Based on these findings, we generated and characterized a kalirin-deficient mouse model. In addition, we examined the expression of kalirin proteins in human cerebral cortex. Methods: KALRN null mice were generated from ES cells using standard methods. No gross anatomical abnormalities were detected. Golgi staining was performed using modified Golgi-Cox method. Spines were counted along equivalent length of secondary or tertiary dendritic segments of pyramidal neurons, and quantified using Metamorph. For each individual behavioral test, 8-14 animals of each genotype and treatment group were tested. Matching-to-sample Morris water maze and Y maze was used to test working memory. Trialindependent Morris water maze was used to test reference memory. To test sociability, the preference of a test mouse for an unfamiliar stimulus mouse vs. an inanimate object was assessed using established methods. Prepulse inhibition of acoustic startle responses was measured using the Med-Associates System. Kalirin isoform expression was examined using SDS-PAGE/Western Blot in protein extracted from auditory cortex gray matter of normal human control subjects using rabbit anti-Kalirin spectrin antibody (Millipore \# 07-122) diluted 1:500. Preliminary experiments established the stability of isoform expression over the relevant postmortem intervals in an animal model. Results: KALRN knockout mice have specific reductions in cortical, but not hippocampal, Rac1 signaling and spine density. These mice exhibit robust deficits in working memory, sociability, and pre-pulse inhibition. We found that the behavioral profile of KALRN KO mice was similar to WT mice during early postnatal development, while behavioral dysfunctions and cortical spine deficits were robust in postadolescent mice. Kalirin-7, $-5,9$, and 12 isoforms were all expressed in adult human auditory cortex, with good preservation of expression up to 30 hours postmortem in our animal model and human tissue. Unlike mouse, the relative abundance of Kalirin isoforms in human cortex were Kalirin-12 $>>$ Kalirin-5 $>>$ Kalirin-9 = Kalirin-7.

Discussion: We find that mice lacking the KALRN gene show correlated morphologic and cognitive/behavioral phenotypes that model several schizophrenia endophenotypes, including their onset during adolescence. In wild type mouse, expression of Kalirin-7 in cortex increases dramatically during adolescence, and this isoform is the most strongly expressed of the Kalirins through adulthood. Initial studies in normal humans suggest that examination of Kalirin expression in cortex of subjects with schizophrenia is feasible, but suggest some important differences from rodent models. In particular, the functional balance of the expressed Kalirins in adult human cortex suggests additional roles for Kalirin isoforms other than Kalirin-7. Further studies of Kalirin signaling in the cortex will likely provide critical insight into the neurobiological bases of spine deficits and their relationship with schizophrenia endophenotypes. Our results also have significance for the understanding of other disorders that are associated with alterations in Kailrin expression and spine morphology, such as Alzheimer disease.

Disclosure: P. Penzes: None. M. Cahill: None. Z. Xie: None. A. Deo: None. M. Day: None. C. Miller: None. J. Sweatt: None. I. Goldszer: None. D. Surmeier: None. D. Lewis: None. R. Sweet: None.

26. Altered Glutamate, Dopamine but not Serotonin Transporters in Postmortem Frontal Cortex from Bipolar and Schizophrenic Patients Jagadeesh $\mathrm{R}^{\star}$, Matthew Kellom, Edmund Reese, Kim Hyung-Wook, Stanley Rapoport

\section{National Institute on Aging, Bethesda, MD}

Background: Altered glutamate, serotonin and dopamine neurotransmission has been suggested to contribute to bipolar disorder (BD) and schizophrenia (SZ), but underlying mechanisms of these alterations are not agreed upon. We hypothesized that they involve changes in excitatory amino acid transporters (EAATs), the serotonin reuptake transporter (SERT) and the dopamine reuptake transporter (DAT). Methods: To test this hypothesis, we determined protein and mRNA levels of EAAT subtypes 1-4, the SERT and the DAT in postmortem prefrontal cortex (Brodmann Area 10) from BD and SZ patients and controls.

Results: Compared to control levels, protein and mRNA levels of EAAT1 were increased significantly in cortex from both BD and SZ patients. EAAT2 protein and mRNA levels were decreased significantly in BD but not SZ cortex. EAAT 3 and EAAT 4 protein and mRNA levels were significantly higher in SZ but not in BD. DAT protein and mRNA 
levels were decreased significantly in both BD and SZ cortex. There was no significant difference in SERT expression in either BD or SZ. Conclusions: The decreased EAAT2 in BD may play a role in hyperglutamatergic function in $\mathrm{BD}$, whereas increased EAAT1, 3, and 4 expressions in SZ may contribute to hypoglutamatergic function in SZ. Decreased DAT expression could result in hyperdopaminergic function in both BD and SZ. Differently altered EAATs involved in glutamatergic transmission could be therapeutic targets for treating BD and SZ. This study demonstrates a disparity in glutamate transporters between BD and SZ. Acknowledgements: We thank the Harvard Brain Bank, Boston, MA for providing the postmortem brain samples under PHS grant number R24MHo68855. This research was entirely supported by the Intramural Research Programs of the National Institute on Aging, National Institutes of Health, Bethesda, MD 20892.

Disclosure: J. R: None. M. Kellom: None. E. Reese: None. K. HyungWook: None. S. Rapoport: None.

27. Oligodendrocyte Gene and Protein Expression Is Altered in the Medio-Ventral Prefrontal White Matter Bundle in Major Depression Grazyna Rajkowska*, Dorota Maciag, Abiye Iyo, Mark Austin, Craig Stockmeier

University of MIssissippi Medical Center, Jackson, MS

Background: Recent DTI studies showed alterations in fractional anisotropy and volume of the medio-ventral prefrontal white matter bundle in major depressive disorder (MDD). However, there are no studies at the microscopic level to indicate which cellular and molecular substrates might be involved in this pathology. Our preliminary observations reveal a significant enlargement of oligodendrocytic cell bodies immunoreactive for RIP antigene which is colocalized with CNPase, an enzyme found in oligodendrocytes and their myelin sheaths. The present study investigates gene and protein expression of CNPase in MDD.

Methods: The expression of CNPase protein and mRNA was examined in postmortem tissue dissected from the ventral half of the prefrontal cortex in 20 pairs of MDD and age-matched normal controls. Tissue punches carefully dissected from the medio-ventral prefrontal white matter bundle were used for Western blotting and PCR. CNPase monoclonal antibodies, detecting developing and adult myelin, were used for Western blotting whereas CNPase mRNA expression was measured by quantitative real time PCR.

Results: The protein level of CNPase was significantly reduced in younger subjects with MDD (6o) as compared to non-depressed, agematched controls. In contrast, the expression of mRNA was increased in the entire group of MDD subjects as compared to controls.

Discussion: Increases in the expression of CNPase mRNA parallel the enlargement of oligodendrocytic cell bodies may be a response to increased demand for CNPase synthesis as the protein level is decreased. The divergent expression of CNPase protein and gene could be explained by alterations at the transcriptional and/or translational level in the process of CNPase synthesis. In conclusion, our results reveal alterations in the gene and protein expression for oligodendrocytic markers in MDD.

Supported by RRo17701 and MH054846.

Disclosure: G. Rajkowska: None. D. Maciag: None. A. Iyo: None. M. Austin: None. C. Stockmeier: None.

\section{Elevated Phosphorylation and Impaired Dephosphorylation of Serotonin Transporter Variant Associated with Obsessive- Compulsive Disorder \\ Sammanda Ramamoorthy*, Padmananbhan Mannangatti, Lankupalle Jayanthi}

Medical University of South Carolina, Charleston, SC

Background: The uptake of synaptic 5-HT through serotonin transporter (SERT) is the principal process of terminating serotonergic neurotransmission. Numerous SERT antagonists including the serotonin selective reuptake inhibitors (SSRIs) are effective antidepressants. Documentation of altered SERT expression in various types of psychopathology indicates the importance of SERT expression in maintaining normal brain function. Studies suggest that phosphorylation and dephosphorylation of SERT due to altered protein kinase and phosphatase activities linked to presynaptic receptor modulation may be a common mechanism dictating SERT function in maintaining 5-HT-homeostasis. However, it is not known whether such regulation is important for the normal behavior contributing to 5-HT signaling. Human SERT (hSERT) coding variant, Ile425Val was found in human subjects exhibiting a complex psychiatric phenotype including obsessive-compulsive disorder (OCD) plus Asperger's syndrome or anorexia nervosa. hSERT-Ile425Val variant exhibits higher 5-HT transport with loss of protein kinase $\mathrm{G}$ (PKG)- mediated upregulation. PKG activation increases 5-HT uptake and PKG-mediated SERT upregulation requires Thr276 phosphorylation. The present study is aimed at testing the hypothesis that hSERT-Ile425Val variant interferes with normal PKG- mediated SERT phosphorylation on T276 and dephosphorylation, and intrinsic activity.

Methods: Using heterologous expression cell model systems, the impact of Ile $425 \mathrm{Val}$ mutation on 5-HT transport, SERT phosphorylation and dephosphorylation was analyzed in the presence and absence of PKG phosphorylation of SERT Thr276 and compared with that of wild type hSERT.

Results: In this study, we show that Ile425Val-hSERT exhibits elevated basal phosphorylation and reduced rate of dephosphorylation compared to wild type. When expressed transiently in HeLa cells, Ile425Val exhibited higher 5-HT transport activity and enhanced phosphorylation compared to WT-SERT that could not be further stimulated by PKG activation. Interestingly, gain of transport function and enhanced phosphorylation found in Ile $425 \mathrm{Val}$ could be completely blocked by introducing Thr276Ala mutation. Furthermore, phosphoIle425Val demonstrated reduced rate of dephosphorylation compared to that of phospho-WT.

Discussion: Collectively, our results suggest that Ile425Val, a naturally occurring hSERT coding variant may interact with Thr276 motif and affect normal PKG- mediated SERT regulation and contribute in the development of disorders attributed to compromised 5-HT signaling. Given the value of SERT offering a pharmacological target in the treatment of mental disorders, our study may provide an opportunity for the development of potential pharmacotherapies for the treatment of mental disorders and other disease states resulting from aberrant 5-HT transmission.

Disclosure: S. Ramamoorthy: None. P. Mannangatti: None. L. Jayanthi: None.

29. Use of Cell-Permeable Morpholino Oligonucleotides for Genetic Control of Glutamate Exchanger xCT and Transporter GLT-1 in the Rat Nucleus Accumbens

Kathryn Reissner*, Peter Kalivas

Medical University of South Carolina, Charleston, SC

Background: Current methods which allow for genetic control in the study of rat behavior predominantly include antisense oligonucleotides or RNAi which are virally expressed or continuously infused by osmotic minipump. While these methods have demonstrated effective use, we sought to develop an antisense strategy which might avoid the use of viruses or inclusion of a cumbersome minipump. Morpholinobased antisense oligonucleotides have for many years been effectively employed in genetic embryonic studies, particularly in zebrafish and Xenopus, however this use has not extended to studies in adult brain or rodent behavior. Here we report use of vivo-morpholinos, which provide a $3^{\prime}$ covalently linked octa-guanidine dendrimer delivery moiety, similar in structure to that found in arginine rich peptides, to render oligos cell-permeable, in the control of gene expression in the adult rat brain. Because the glutamate transporter and exchanger 
GLT-1 and xCT have been demonstrated to be regulated by exposure to cocaine, we designed antisense and control sequences against these proteins for a pilot study to determine the feasibility of this approach. Methods: To assess efficacy of genetic knockdown and neural toxicity of morpholinos, intra-accumbens canulae were surgically implanted in male Sprague-Dawley rats. Following recovery, antisense and flipped control morpholinos $(10 \mu \mathrm{M}, 1 \mu \mathrm{L})$ were microinjected once per day for 3 days. Following 4, 7, or 14 days after the last injection, NAc were prepared from individual hemispheres for preparation of a crude membrane subfraction. Levels of GLT-1 and xCT were determined by Western blotting, using PSD-95 as an unchanged and loading control. Slices of NAc were taken from independent animals for histological analysis of morpholino toxicity. Lastly, individual hemispheres were dissected from independent animals following morpholino microinjection for glutamate uptake assays, as a functional measure of protein knockdown.

Results: Intra-accumbens micronjection of antisense or flipped control morpholino oligonucleotides against $\mathrm{xCT}$ and GLT-1 for 3 days had no effect on cellular histology as assessed by Cresyl Violet staining. However, protein levels were significantly reduced seven days following the third antisense injection for both $\mathrm{xCT}$ and GLT-1, compared to flipped sequence controls injected into the contralateral hemisphere. In contrast, one injection was insufficient to achieve significant knockdown. Protein levels were restored to normal by two weeks following the final antisense injection.

Discussion: Results provided here indicate that 3 consecutive days of individual microinjections are sufficient to suppress expression of GLT- 1 and xCT, indicating that this approach can be effectively used at doses which are non-toxic to cells in the nucleus accumbens. Ongoing studies are designed to utilize vivo-morpholinos to look at the behavioral effects of genetic manipulation of these proteins in a selfadministration model of addiction.

Disclosure: K. Reissner: None. P. Kalivas: None.

30. Rapid Adaptations in CRF \& Dynorphin Regulation of Glutamate Release in the VTA During Self-Administration and Stress-Evoked Relapse to Cocaine-Seeking

Benjamin Harlan, Courtney Williams, Art Riegel*

MUSC, Charleston, SC

Background: Addiction to cocaine is characterized by compulsive drug taking behavior. The mechanisms driving this behavior are not well understood, but involve stress. In animal models this is attributed to an interaction between the neuropeptide corticotrophin releasing factor (CRF) and glutamate in the Ventral Tegmental Area (VTA). This work investigates cocaine-induced changes in neuroplasticity in VTA dopamine neurons resulting from an adaptation in presynaptic glutamate synapses. The interaction between CRF and glutamate in dopamine neurons was studied by recording currents mediated by excitatory AMPA receptors. Patch-clamp recordings were performed in acute brain slices from naïve and rats with a history of chronic cocaine self-administration, extinction and yohimbine-reinstatement. We hypothesize that the dopamine neuron plasticity following drug self-administration and stress-evoked relapse results from adaptation of the presynaptic signaling pathways, mediated by CRF-R2. Understanding pathological adaptations in these mechanisms is important because AMPA receptors are important determinants of dopamine neuron firing patterns, which in turn, is important for encoding motivation-relevant stimuli during drug addiction.

Methods: Cocaine self-administration: Rats were trained in operant conditioning chambers to self-administer cocaine, daily $(2 \mathrm{~h})$, on a FR1 schedule, for 10-14 days. Active lever presses infused cocaine $(0.5 \mathrm{ml}$; $2 \mathrm{~s} ; 0.5 \mathrm{mg} / \mathrm{kg}$, iv) and were preceded by a light and tone. Extinction: [GIVE N] rats underwent daily $2 \mathrm{~h}$ extinction sessions, for $>7$ days. Extinction training was conducted in a self-administration chamber. Yohimbine Reinstatement testing: Yohimbine $(2.5 \mathrm{mg} / \mathrm{kg}$; ip) was administered $30 \mathrm{~min}$ prior to testing in the same self-administration chamber. Brain Slices and Whole-Cell Recordings: Horizontal midbrain slices $(220 \mu \mathrm{m})$ containing midbrain dopamine neurons from naïve or self-administration animals were prepared as described previously (Riegel and Williams, 2008).

Results: Excitatory Post Synaptic Currents (EPSCs) were measured in VTA dopamine neurons in tissue from naïve rats. Application of the non-selective agonists CRF or UCN-1 potentiated EPSCs at 1-100 nM, but attenuated EPSCs at higher concentrations (100-300 nM). Receptor subtype-selective agonists and antagonists revealed that the CRFpotentiation was mediated by CRF-R1 and facilitating the actions of presynaptic dopamine D1-receptors. In contrast, attenuation of EPSCs was linked to CRF-R2, facilitating the actions of several inhibitory autoreceptors. Measurements of the paired-pulse ratio and the frequency of sEPSCs localized both CRF-R1/R2 to presynaptic glutamate terminals. To determine whether drug self-administration impacted these mechanisms, experiments were preformed in tissue from rats that chronically self-administered cocaine. Results indicated that whereas the CRF-R2 attenuation was unchanged relative to naive tissue, the CRF-R1 potentiation of EPSCs was greatly increased during self-administration. However, this change was absent in chronic yoked-cocaine rats or rats receiving a single injection of cocaine ( $15 \mathrm{mg} / \mathrm{kg}$, IP), suggesting the self-administration environment is important for the actions of CRF. These CRF mechanisms were also examined during extinction and stress-evoked reinstatement with yohimbine. Unlike tissue sampled during extinction, the CRF-R2 system was up-regulated during reinstatement, facilitating the actions of presynaptic kappa opioid receptors. These effects were not observed in controls experiments evaluating: (i) naïve rats, (ii) yoked-cocaine rats treated with yohimbine in self-administration chambers, (iii) cocaine self-administration rats treated with yohimbine in the home cage. These differences suggest the actions of CRF are modulated by both stress and the environmental context.

Discussion: These results indicate that CRF dynamically regulates glutamate release. The mechanism involves presynaptic CRF-R1/R2 acting to facilitate actions of presynaptic neurotransmitter receptors on glutamate terminals in the VTA. These mechanisms show adaptations during chronic cocaine as well as yohimbine-reinstatement. Such changes would likely affect dopamine neuron excitability. The results of these experiments are expected to help pinpoint novel cellular targets for therapeutic development to treat stress related drug-seeking behaviors. Work was supported by grants from NIDA$\mathrm{NIH}$ ( $\mathrm{P}_{30 D A 028811}$ \& KO1-DA020751) and MUSC pilot grants from the Neurobiology of Addiction Research Core \& the Alcohol Research Center.

Disclosure: B. Harlan: None. C. Williams: None. A. Riegel: None.

31. Full Exome Sequencing Reveals the Genetic Basis of a Case of Idiopathic Hemolytic Anemia and Suggests Candidate Rare Variants for ADHD in a Utah Pedigree

Reid Robison*, Gholson Lyon, Lin Yang, Fred Reimherr, David Nix, Tao Jiang, Peng Zhang, Renhua Wu, Xu Yang, Barry Moore, Jinchuan Xing, W. Evan Johnson, Mark Clement, R. Van Wilk, Joseph Glessner, Hakon Hakonarson, Mark Yandell, William McMahon, Kai Wang

University of Utah, Salt Lake City, UT

Background: ADHD is a common disorder affecting more than 1 in 20 children in the U.S., with as many as $50 \%$ remaining symptomatic into adulthood. Genetic factors are thought to play a large role in the etiology of the disorder, but studies thus far remain inconclusive and inconsistent, accounting for a small portion of the risk for ADHD. It is hypothesized that rare, family-specific genetic variants may account for the remaining missing heritability of ADHD, and advances in nextgeneration sequencing have made whole exome sequencing a potentially viable option for gene variant finding in complex disease pedigrees.

Methods: Whole exome sequencing was conducted on a father and two sons with severe ADHD, combined hyperactive and inattentive 
subtype, all of whom improved with treatment with stimulant medication in an adult ADHD clinical trial. Exome capture was done using a commercially available Agilent SureSelect in solution method. Paired end sequencing was performed using the Illumina Genome Analyzer IIx platform with read lengths of 76 base pairs, providing at least 20x-coverage. Sequence reads were aligned to human reference genome builds hg18 and hg19 using both Novoalign 2.07 and SOAPaligner 2.20. Single nucleotide polymorphism (SNP) calling was conducted using both Maq 0.5.0 and SOAPsnp 1.03. SNP results were filtered as followed: Base quality $>20$, depth from 4-200, copy number estimate $<2$, and distance between two adjacent SNPs no less than 5 . Nonsynonymous SNPs (nsSNPs) were identified using ANNOVAR and USeq Alleler, and insertions/deletions (indels) were detected using SOAPindel. Illumina 610k SNP microarrays were also run on each of the three samples, with copy number variant $(\mathrm{CNV})$ detection using PennCNV.

Results: Approximately 20,000 SNPs were detected in each individual by whole exome sequencing, with $80 \%$ of each exome adequately captured and sequenced. After filtering for nsSNPs that are shared by all three affected individuals and that were not already listed in dbSNP as common variants, $\sim 50$ rare variants remained. The list of candidate SNPs was further narrowed to 18 SNPs by using ANNOVAR to predict functional consequences of each mutation. After manually reviewing the remaining SNPs, nonsynonymous rare variants in 4 brainexpressed genes appeared to be the most plausible candidates for an association with ADHD in this pedigree. Additionally, eight other shared variants (indels, splice site, and UTR mutations) were identified as shared among the three individuals, but did not seem to have major functional relevance. Furthermore, one of the affected sons had recently undergone a splenectomy due to splenomegaly caused by hemolytic anemia, and he was subsequently found to have two rare nonsynonymous mutations in the PKLR gene, thus making him a compound heterozygote for pyruvate kinase deficiency, an autosomal recessive disorder. Biochemical assays confirmed low pyruvate kinase activity in this individual, and these mutations were demonstrated to be inherited from his carrier parents. Lastly, CNV calling of the Illumina 610k microarray data did not reveal any rare CNVs of functional relevance shared amongst the three individuals.

Discussion: Preliminary results show four interesting candidate rare nonsynonymous variants in brain-expressed genes that are shared among 3 individuals (father and two sons) and thus may be associated with ADHD in this family. Validation of these findings is underway in each individual, along with expansion of the pedigree to include DNA sequence and phenotype data from the unaffected mother and other siblings, along with functional characterization of the nsSNPs and PKLR mutations. These preliminary results show that exome sequencing can identify genetic causes of mendelian disorders, as in the case of pyruvate kinase deficiency in one member of this family, and also has potential to discover rare variants associated with complex neuropsychiatric conditions such as ADHD.

Disclosure: R. Robison: Part 1; Clinical Methods LLC. Part 4; Johnson \& Johnson, Novartis, Janssen Pharmaceutica. G. Lyon: None. L. Yang: None. F. Reimherr: Pfizer, Cyberonics, Eli Lilly and Company, Novartis, Otsuka, PGxHealth, Novartis, Bristol-Myers Squibb, Shire. D. Nix: None. T. Jiang: None. P. Zhang: None. R. Wu: None. X. Yang: None. B. Moore: None. J. Xing: None. W. Johnson: None. M. Clement: None. R. Van Wilk: None. J. Glessner: None. H. Hakonarson: None. M. Yandell: None. W. McMahon: None. K. Wang: None.

32. Modulation of Serotonin Transporter Function, Phosphorylation and Cell Surface Expression by Kappa Opioid Receptor Ligands

Toni Shippenberg*, Sammanda Ramamoorthy

NIDA IRP, Baltimore, MD

Background: K-opioid receptor (KOR) agonists produce dysphoria and psychotomimesis in human subjects. They produce prodepressant like effects in rodents. By contrast, antagonists produce anti-depressant-like effects in rodent models and are in Phase 1 testing for the treatment of cocaine addiction. The cellular mechanisms by which KOR ligands produce these effects are not known. KOR agonists pre-synaptically modulate serotonin $(5-\mathrm{HT})$ transmission; an effect that has been attributed to inhibition of 5-HT release. Importantly, however, extracellular 5-HT concentrations are also regulated by the serotonin transporter (SERT), a transmembrane protein that clears ${ }_{5} \mathrm{HT}$ released into the extracellular space. We have previously reported that KOR agonists regulate the dopamine transporter. Whether KOR ligands modulate the serotonin (SERT) transporter is unknown. Such information, however, is important in view of the postulated role of this protein both in affective disorders and in mediating the behavioral effects of cocaine. The present studies used heterologous expression systems and native tissue to determine whether KOR agonists regulate SERT function and the cellular basis of this effect.

Methods: KOR agonist (U69593; salvinorin A)-induced changes in SERT function were quantified in: i) HEK cells co-expressing human or rat KOR and human or rat SERT using live cell, fluorescent confocal microscopy in conjunction with the fluorescent, high affinity SERT substrate, 4-(4-(dimethylamino)-styryl)-N-methylpyridinium: $\left(\mathrm{ASP}^{+}\right)$; and ii) ventral striatal synaptosomes using $\left[{ }^{3} \mathrm{H}\right] \quad 5-\mathrm{HT}$ uptake. Biotinylation and live cell confocal microscopy were used to quantify SERT cell surface expression. $\left[{ }^{32} \mathrm{P}\right]$ orthophosphate labeling was used to quantify SERT phosphorylation state.

Results: The selective KOR agonist, U69593, produced a concentration dependent, KOR antagonist (nor-binaltorphimine)-reversible, decrease in SERT function in cells and native tissue. Analogous effects were observed in response to salvinorin A, a naturally occuring KOR agonist, and in ventral striatal synaptosomes. In both preparations, the down-regulation of function was calcium dependent and prevented by inhibition of CAMK. Inhibition of 338 MAPK, PKC or ERK was without effect. The U69593-evoked decrease in SERT activity was associated with a significant, KOR antagonist-reversible, increase in SERT phosphorylation. SERT cell surface expression decreased and intracellular SERT expression increased.

Discussion: These data demonstrate that KOR activation by synthetic or naturally-occuring agonists decreases SERT function. This effect is physiologically relevant in that it is observed in native tissue. The decrease in function is likely due to agonist- induced alterations in SERT phosphorylation and resulting increases in SERT internalization. We have previously reported that, in contrast to SERT, KOR agonists up-regulate the dopamine transporter whereas norepinephrine function is unaltered. We hypothesize that the opposing effect of KOR agonists on SERT and DAT function may contribute to the pro-depressant and psychotomimetic effects of these agents.

Supported by the NIH/NIDA Intramural Research Program and NIMH grant MHo62612 (S.R).

Disclosure: T. Shippenberg: None. S. Ramamoorthy: None.

33. Amygdala Transcriptome Analysis in Female Major Depression Suggests Altered BDNF Support and Decreased Somatostatin/ GABA-Related Function

Etienne Sibille*, Xingbin Wang, Rama Kota, Gaelle DouillardGuilloux, George Tseng, David Lewis, Jean-Philippe Guilloux

University of Pittsburgh, Pittsburgh, PA

Background: Women are twice as likely to develop major depressive disorder (MDD), display higher symptom number and severity, have frequent co-morbid anxiety symptoms, are more prone to recurring episodes, while their response rate to antidepressant therapies is similar to men. Thus, we predicted that the illness may associate with robust molecular changes, detectable in the postmortem brain of female subjects.

Methods and Results: The investigated cohort was composed of 21 female subjects with MDD paired with age-matched controls (16-72 years old, 14 patients under antidepressant treatment at time of death). We focused our study on the lateral/basolateral complex of the 
amygdala, as a neural hub for mood regulation that is affected in MDD. Using Illumina Beadchip, we investigated large-scale gene expression in this region and confirmed results by real-time quantitative PCR (qPCR) analysis. Using exploratory analytical approaches (parametric, non-parametric and co-factor best-fit model analysis with $\mathrm{p}<0.05$ and $|\mathrm{FC}|>20 \%$ ), we identified over 300 genes with altered transcripts in correlation with the illness. Several glial-related genes were upregulated (GFAP, MBP, MOBP). Downregulated genes included neuropeptide-related transcripts (somatostatin [SST] and its receptor SSTR1, tachykinin, neuropeptide Y, cortistatin), hence confirming our previous observations of low SST in cingulate and prefrontal cortex of MDD patients (Sibille et al, 2010; Tripp et al, 2010; Manuscripts submitted). Additional changes in GABA-related genes (SLC32A1, GABRA1, GABRG2) and signal transduction-related transcripts (RGS4, GARNL4, G3BP2) were observed. These transcript changes were not explained by age, estrogen status, on-going antidepressant treatment or other demographic and technical parameters. Ingenuity $®$ Pathway analysis on the identified gene set confirmed biological networks putatively linked with neuronal and psychological dysfunction. A qPCR survey on adjacent tissue sections yielded highly concordant results (array-qPCR Pearson correlation, $\mathrm{R}=0.83, \mathrm{p}<1 \mathrm{e}-6, \mathrm{~N}=34$ genes), including CORT, MBP, MOBP, NPY, SST, TAC1 ( $\mathrm{p}<0.05$, same direction of changes), together confirming the sampling and technical reliability of the array data. Quantitative Western-blotting confirmed a significant downregulation for SST at the precursor protein level in depressed subjects $(-16 \%, p<0.001)$. Moreover, the pattern of gene changes was strikingly reminiscent of changes observed downstream from decreased BDNF signaling. To test this hypothesis, we overlaid our list of differentially expressed genes with list of genes observed in mice with reduced BDNF signaling (Glorioso et al, Mol Psy, 2006, 11:633-48) and with genes linked with BDNF through systematic literature search (Ingenuity Pathway database), resulting in a current list of 52 BDNF-related genes affected in the amygdala of female subjects with MDD.

Discussion: The observed transcriptome patterns suggest two major trends: altered BDNF signaling and decreased GABA-related function, selectively affecting SST-bearing interneurons. While the BDNF hypothesis of depression is indirectly supported by robust rodent findings, there is limited primary evidence in humans. Our results indirectly suggest that decreased BDNF function in the amygdala may contribute to the molecular pathology of major depression in female subjects. Furthermore, we identified putative mediators (i.e., SST and other neuropeptides) of local microcircuitry dysfunctions potentially underlying altered GABA-mediated function and mediation of symptoms of the illness in the amygdala.

Disclosure: E. Sibille: None. X. Wang: None. R. Kota: None. G. Douillard-Guilloux: None. G. Tseng: None. D. Lewis: None. J. Guilloux: None.

\section{Cortical Expression of an RNA Editing Enzyme Is Increased in Major Depression and Suicide \\ Monsheel Sodhi*, Micah Simmons, James H. Meador-Woodruff}

UAB, Birmingham, AL

Background: Data generated from our laboratory indicates that there may be a previously unexplored pathophysiological pathway to depression and suicide, involving dysfunctional activity of the RNA editing enzyme, ADAR1. RNA editing by ADAR enzymes creates sequence variation in the transcripts of more than 2000 genes, including the transcripts of numerous neurotransmitter receptors in addition to approximately $16 \%$ of all microRNAs. Subtle changes in ADAR activity could create alterations to a substantial proportion of the transcriptome, with profound functional consequences. For example, increased RNA editing of the $5-\mathrm{HT}_{2} \mathrm{C}$ receptor reduces its signal transduction, while reduced editing of the GluR2 subunit of the AMPA receptor in mice results in excitotoxicity in neurons, leading to seizures and premature death. Several postmortem studies have shown consistent increases in $5-\mathrm{HT}_{2 \mathrm{C}}$ receptor RNA editing in mood disorders and suicide, particularly at sites edited by the ADAR1 enzyme. In this study, we report that a more generalized increase in RNA editing may occur in major depression.

Methods: The expression levels of the ADAR1 and ADAR2 enzymes were measured using quantitative PCR (QPCR) in the dorsolateral prefrontal cortex of postmortem subjects from the Stanley Consortium Brain series, which includes subjects with major depression $(n=15)$, bipolar disorder $(n=15)$, schizophrenia $(n=15)$, and a comparison group $(n=15)$. Among the psychiatric cases, 20 subjects were suicide victims. Data were analyzed using the relative standard curve method and gene expression data were normalized to the expression of three housekeeping genes.

Results: ADAR1 expression was found to be increased in major depressive suicide victims $(2.3 \pm 0.4)$ compared with controls $(1.4 \pm 0.5)$ and also when compared with psychiatric patients who did not commit suicide $(1.3 \pm 0.6)$. These results were statistically significant $(\mathrm{T}=3.7, \mathrm{df}=12, \mathrm{p}=0.003)$. No changes in ADAR 1 expression were detected in other patient groups in comparison with controls. There were also no differences in ADAR2 expression levels in any of the psychiatric phenotypes compared to the controls.

Discussion: These data indicate that ADAR1 could play a role in the pathophysiology of major depression. Preliminary tests indicate that similar increases of ADAR1 expression occur in a second group of major depression patients relative to controls, and further studies of specific targets of these enzymes, including glutamatergic and serotonergic transcripts are in progress.

Work funded by the American Foundation for Suicide Prevention Disclosure: M. Sodhi: None. M. Simmons: None. J. Meador-Woodruff: Part 2; ACNP, editor-in-chief of Neuropsychopharmacology.

\section{Dopaminergic Regulation of Brain Architecture and Cell Signaling Systems}

Aliya Frederick, Heather Hollis, Fazal Arain, Gregg Stanwood*

Vanderbilt University, Nashville, TN

Background: Most mental health disorders have developmental etiologies and are produced by alterations in the formation and connectivity of specific forebrain regions including the medial frontal cortex and the striatum. Dopamine and other biogenic amines serve as neurotransmitters in the mature nervous system, and are also prominent drug targets in the treatment of neurological and psychiatric disorders. The dopamine system is expressed early in brain development, prior to the formation of synapses, and pleiotropically modulates decisions related to neuronal differentiation and circuit formation. Early life abnormalities in dopamine receptor signaling, produced by either genetic or pharmacological insults, result in permanent changes in brain architecture and biobehavioral development.

Methods: We have used biochemical, anatomical and immunohistochemical methods to define long-lasting alterations in specific cell signaling pathways following two models of developmental dopaminergic dysfunction: prenatal exposure to cocaine and genetic mutation of the dopamine $\mathrm{D}_{1}$ receptor.

Results: Following prenatal cocaine, there is a long-lasting reduction in $\mathrm{D}_{1}$ receptor expression at the cell-surface and subsequent reductions in G-protein coupling. This produces alterations in the differentiation patterns of both pyramidal neurons and interneurons in dopaminoceptive regions of the cerebral cortex. $\mathrm{D}_{5}$ receptor knockout mice, on the other hand, do not appear to exhibit these changes in cortical connectivity. Biochemical studies reveal altered phosphorylation state of several key downstream molecular mediators of the effects of $D_{1}$ receptor signaling in striatal and cortical extracts in animals exposed to cocaine gestationally and in $\mathrm{D}_{1}$ receptor mutant mice, including DARPP-32, CaMKII $\alpha$, and GluR1. We have observed decreases in basal phosphorylation of CaMKII $\alpha$ at residue $\mathrm{Thr}^{286}$ which we may be due to alterations in a novel class of $\mathrm{D}_{1}$ receptors signaling through 
a Gaq-phospholipase C pathway. Current studies are using a transgenic Drd1-tdTomato reporter line to determine if $\mathrm{D}_{1}$ receptor modulation of neuronal differentiation and signaling is controlled in a cell autonomous manner.

Discussion: Alterations in CaMKII $\alpha$ phosphorylation suggest longlasting disruption of intracellular signaling by dopaminergic challenge during development which may influence circuit connectivity and neurotransmission. $D_{1}$ receptor signaling through $\mathrm{G} \alpha \mathrm{q}$ has recently been described by several laboratories, however, the neurodevelopmental significance of $\mathrm{D}_{1}$ receptors signaling through this pathway remains to be identified. Taken together, our studies will demonstrate the mechanisms by which alterations in dopaminergic activity during development, whether produced by genetic or pharmacological means, alters circuits mediating cognitive and emotional behaviors during critical epochs of development, and may lead to subsequent psychiatric disease later in life.

Disclosure: A. Frederick: None. H. Hollis: None. F. Arain: None. G. Stanwood: None.

\section{Signal Detection in Clinical Trials: A Post-Study Survey of Schizophrenia Trial Sites}

Amir Kalali, Antony Loebel*, Josephine Cucchiaro, Cynthia Siu, David Daniel

\section{Quintiles, San Diego, CA, Sunovion, Inc., Fort Lee, NJ}

Background: Diminishing drug-placebo differences and increasing placebo responses have been observed in recent psychiatric clinical trials, but the reasons behind these trends are little understood. The objective of our survey was to examine key site, subject and trial methodological factors that might systematically affect the success of a controlled clinical trial, defined as the ability to detect a difference between the effects of drug and placebo.

Methods: Questionnaires were sent to principal investigators (PIs) at 42 US sites who had participated in two recently completed lurasidone phase 3, placebo-controlled trials in patients with schizophrenia, conducted between 26 October 2007 and 15 June 2009. The site survey consisted of 24 questions that covered areas of potential relevance to drug detection including: subject recruitment practices and patient population; research staff experience; rater practices including consistency of rater and extent of time spent interacting with subjects; and the importance given by investigators to other specific factors that could influence clinical trial outcome. Internal comparisons and consistency checks were performed to check the reliability and validity of survey responses to questions. Site-specific drug-placebo effect sizes of PANSS total score obtained in 2 lurasidone trials and their associations with site and PI practices were examined using multiple stepwise regression analysis weighted by the inverse variance of PANSS change score.

Results: Of the 42 US study sites, 32 completed the survey, for an overall response rate of $76 \%$. The majority of study site investigators in the sample $(>50 \%)$ recruited patients from outside their own practice. Study sites with a greater number of trials conducted in the past 2 years were more likely to have a larger proportion of research experienced patients $(\mathrm{p}<0.05)$. A larger lurasidone-placebo effect size was observed in study sites with higher percentages of subjects having no prior research experience $(\mathrm{p}<0.05)$ in a stepwise regression model. A larger lurasidone-placebo effect size was also noted in study sites that had a larger percentage of patients with longer illness duration $(p<0.05)$, a lower percentage of subjects derived from advertisements $(\mathrm{p}<0.05)$, and a lower proportion of revenue from pharma-sponsored studies $(\mathrm{p}<0.01)$, after accounting for potential confounding variables. Academic sites $(\mathrm{n}=2)$ followed by private not-for-profit hospital sites $(n=4)$ had greater lurasidone effect sizes compared to private forprofit $(\mathrm{n}=22)$ or public $(\mathrm{n}=2)$ hospitals $(\mathrm{p}<0.01, \mathrm{~F}=7.1, \mathrm{df}=3,10)$ adjusting for significant covariates in CRO quality, illness duration, lack of stable residence proportion $(\mathrm{p}<0.05)$, but the $\mathrm{n}$ for some sites was small. Other relevant factors that showed correlation patterns with drug-placebo effect size included patient selection (diagnostic accuracy), ability to identify a reliable informant, the importance given by investigators to the need for control of placebo response and for investigator and rater experience, plus specific patient characteristics (history of response to non-standard (high dose) treatment, and history of alcohol or substance abuse). The quality, responsiveness and involvement of the sponsor and CRO during a study was rated as an "extremely important" factor contributing to trial success by $72 \%$ and $50 \%$ of PIs, respectively. Illness duration from the survey responses was verified against source data from the lurasidone clinical trial database and showed a correlation trend $(\mathrm{p}<0.10)$, supporting the validity of survey responses.

Discussion: Our findings suggest that site and subject characteristics can influence the likelihood of detecting a treatment efficacy signal in schizophrenia trials. The relevant factors appear to include proportion of research experienced patients at a site, sources of patient referrals, illness duration of subjects with schizophrenia, proportion of site revenue from pharma-sponsored studies, and the importance given by PIs to the need for control of placebo response and for investigator and rater experience. Strengths of the survey include its being conducted shortly after study completion and that site level treatment response data were available to correlate with PI responses. Although analysis of the survey was conducted on a blinded basis, survey response was not anonymous, which may have biased respondents towards perceived "correct" responses. Survey responses did not require use of source records. Further analyses are needed to confirm if these findings are study-specific or are generalizable to other clinical trials.

Disclosure: A. Kalali: Part 1; Cypress Bioscience, Merck, Novartis. Part 5; Quintiles Inc. A. Loebel: Sunovion, Inc. J. Cucchiaro: Sunovion, Inc. C. Siu: Part 1; Paid consultant for Pfizer, Inc.; Dainippon Sumitomo Pharma America; Wyeth (Now Part of Pfizer, Inc.); Memory (Now Part of Roche). Part 2; Paid consultant for Pfizer, Inc.; Dainippon Sumitomo Pharma America; Wyeth (Now Part of Pfizer, Inc.); Memory (Now Part of Roche). Part 3; Paid consultant for Pfizer, Inc.; Dainippon Sumitomo Pharma America; Wyeth (Now Part of Pfizer, Inc.); Memory (Now Part of Roche). D. Daniel: Part 1; UBC. Part 2; UBC. Part 5; UBC.

37. Lurasidone in the Treatment of Acute Schizophrenia: Results of the Double-Blind, Placebo-Controlled, 6-Week, PEARL 3 Trial Antony Loebel, Josephine Cucchiaro, Andrei Pikalov, Kaushik Sarma, Jay Hsu, Amir Kalali, Steven Potkin, Herbert Meltzer*

Vanderbilt University Medical Center, Nashville, TN, Sunovion Inc., Ft. Lee, NJ

Background: Lurasidone is a new psychotropic agent with high affinity for $\mathrm{D}_{2}$ and $5-\mathrm{HT}_{2 \mathrm{~A}}, 5-\mathrm{HT}_{1 \mathrm{~A}}$ and $5-\mathrm{HT}_{7}$ receptors. The objective of this study was to evaluate the efficacy and safety of lurasidone $(80 \mathrm{mg} /$ day and $160 \mathrm{mg} /$ day) in patients with an acute exacerbation of schizophrenia.

Methods: Hospitalized patients who met DSM-IV criteria for schizophrenia and were acutely ill with a PANSS total score $\geq 80$ were eligible for enrollment. Subjects were tapered off psychotropic medication and after a 3-7-day placebo washout period were randomized to 6-weeks of double-blind treatment with lurasidone $80 \mathrm{mg}$, lurasidone $160 \mathrm{mg}$, quetiapine $\mathrm{XR} 600 \mathrm{mg}$ or placebo, administered once-daily in the evening. After 3 weeks, patients were eligible for discharge if sufficiently stable and improved. A mixed model repeated measures (MMRM) analysis was performed for the primary measure, the Positive and Negative Symptoms of Schizophrenia Scale (PANSS) total score, and the key secondary measure, the Clinical Global Impression-Severity scale (CGI-S). Change in negative symptoms was evaluated using the Negative Symptom Assessment Scale (NSA-16). Safety and tolerability measures included adverse events, lipid parameters and prolactin, ECG.

Results: Baseline characteristics were similar among patients randomized to the four treatment groups: lurasidone $80 \mathrm{mg}(\mathrm{n}=125)$; 
lurasidone $160 \mathrm{mg}(\mathrm{n}=121)$; quetiapine $\mathrm{XR} 600 \mathrm{mg}(\mathrm{n}=120)$; and placebo $(n=122)$. Treatment with lurasidone was associated with significantly greater endpoint improvement on the PANSS total score versus placebo $(-10.3)$ among patients in the $80 \mathrm{mg}(-22.2 ; \mathrm{P}<0.001)$ and $160 \mathrm{mg}(-26.5 ; \mathrm{P}<0.001)$ dosage groups. On the CGI-S, significant improvement was observed versus placebo (-0.9), during treatment with both the $80 \mathrm{mg}(-1.5 ; \mathrm{P}<0.001)$ and $160 \mathrm{mg}(-1.7 ; \mathrm{P}<0.001)$ doses of lurasidone. Significant separation from placebo occurred by Day 4 for both lurasidone doses on the PANSS total score, and for the $160 \mathrm{mg}$ dose on the CGI-S. On an LOCF analysis, treatment with lurasidone was associated with significantly greater endpoint improvement on the NSA-16 versus placebo $(-3.4)$ in the $80 \mathrm{mg}(-7.8 ; \mathrm{P}<0.001)$ and $160 \mathrm{mg}$ $(-8.9 ; \mathrm{P}<0.001)$ dosage groups. Quetiapine XR produced significantly greater improvements than placebo on the PANSS total score $(-27.8 \mathrm{vs}$. -10.3; P<0.001), the CGI-S (-1.7 vs. $-0.9 ; \mathrm{P}<0.001)$, and the NSA-16 scale $(-8.6$ vs. $-3.4 ; \mathrm{P}<0.001)$. Discontinuation rates due to adverse events were low on lurasidone $80 \mathrm{mg}(4 \%), 160 \mathrm{mg}(3 \%)$, quetiapine XR $(3 \%)$ and placebo (4\%). No adverse events occurred with an incidence $\geq 10 \%$ on either dose of lurasidone. Treatment with lurasidone $80 \mathrm{mg}$ and $160 \mathrm{mg}$, respectively, was associated with a mean increase in weight that was not significantly different from placebo $(+0.6 \mathrm{~kg}$ and $+0.6 \mathrm{~kg}$ vs. $+0.1 \mathrm{~kg})$ while the mean increase in weight was significantly higher with quetiapine XR $(+2.1 \mathrm{~kg}$; $\mathrm{P}<0.001)$. Median change in total cholesterol was similar during treatment with lurasidone $80 \mathrm{mg}(-4.0 \mathrm{mg} / \mathrm{dL}), 160 \mathrm{mg}(-7.5 \mathrm{mg} / \mathrm{dL})$ and placebo $(-7.0 \mathrm{mg} / \mathrm{dL})$, but was increased during treatment with quetiapine XR $(+6.0 \mathrm{md} / \mathrm{dL})$. Median change in triglycerides was also similar during treatment with lurasidone $80 \mathrm{mg}(-2.0 \mathrm{mg} / \mathrm{dL}), 160 \mathrm{mg}(-9.0 \mathrm{mg} / \mathrm{dL})$ and placebo $(-9.0 \mathrm{mg} / \mathrm{dL})$, but was increased during treatment with quetiapine XR $(+8.0 \mathrm{mg} / \mathrm{dL})$. Small increases in median prolactin levels were observed on lurasidone $80 \mathrm{mg}(+0.8 \mathrm{mg} / \mathrm{dL})$ and $160 \mathrm{mg}$ $(+3.0 \mathrm{mg} / \mathrm{dL})$, while small decreases were observed on quetiapine XR $(-0.3 \mathrm{mg} / \mathrm{dL})$ and placebo $(-0.8 \mathrm{mg} / \mathrm{dL})$.

Discussion: In this study, lurasidone, in once-daily doses of $80 \mathrm{mg}$ and $160 \mathrm{mg}$, was effective for the treatment of patients with an acute exacerbation of schizophrenia. The onset of significant efficacy occurred by Day 4 on the primary outcome. Evening dosing of lurasidone was associated with a low rate of adverse events. In this study, treatment with lurasidone was also associated with no clinically meaningful effect on weight or lipid parameters. Funded by Sunovion, Inc.

Disclosure: A. Loebel: Part 5; Sunovion Inc, Ft. Lee, NJ. J. Cucchiaro: Sunovion Inc, Ft. Lee, NJ. A. Pikalov: Sunovion Inc, Ft. Lee, NJ. K. Sarma: Sunovion Inc, Ft. Lee, NJ. J. Hsu: Sunovion Inc, Ft. Lee, NJ. A. Kalali: Part 1; Cypress Biosciences, Inc. Part 5; Quintiles Inc. S. Potkin: Part 1; Arbor Scientia, AstraZeneca, Bioline, Bristol-Myers Squibb, International Society for CNS Clinical Trials and Methodology, Elan, Lilly, Janssen Pharmaceutica, Merck, Novartis, Otsuka, Pfizer, Roche. Part 2; Bioline, Bristol-Myers Squibb, Elan, Lilly, Janssen Pharmaceutica, Merck, Novartis, Otsuka, Pfizer, Roche, Sepracor DainipponSumitomo; U of So.Calif, UCSF, UCSD, Baylor. Part 3; Bioline, BristolMyers Squibb, Elan, Lilly, Janssen Pharmaceutica, Merck, Novartis, Otsuka, Pfizer, Roche, Sepracor Dainippon-Sumitomo; U of So.Calif, UCSF, UCSD, Baylor. Part 4; Bioline, Bristol-Myers Squibb, Elan, Lilly, Janssen Pharmaceutica, Merck, Novartis, Otsuka, Pfizer, Roche, Sepracor Dainippon-Sumitomo; U of So.Calif, UCSF, UCSD, Baylor, AP. H. Meltzer: None.

38. Therapeutic Drug Monitoring (TDM) and Treatment Response in Major Depression with Comorbid Alcoholism

Ihsan M. Salloum*, James M. Perel, Jack R. Cornelius, Antoine

Douaihy, Levent Kirisci, Joseph C. Helsel, Michael E. Thase

University of Miami Miller School of Medicine, Miami, FL

Background: Therapeutic drug monitoring (TDM), while not routinely used for SSRI's antidepressants has reported utility in monitoring for potential drug interactions, delayed onset of action, and medications compliance. TDM may be particularly relevant for monitoring treatment response and drug interaction. Studies examining serum concentrations of the SSRI fluoxetine, one of the most widely used antidepressants worldwide, and treatment response, have been very scarce. The aim of this study was to examine whether fluoxetine/ norfluoxetine plasma concentrations differentiate treatment nonresponse among patients with major depression and comorbid alcoholism.

Methods: The sample consisted of 80 subjects ( $46 \%$ females) who completed a 24-week randomized, double-blind, placebo-controlled, parallel-group trial evaluating the efficacy of fluoxetine (dose range $20-60 \mathrm{mg} /$ day) \pm naltrexone hydrochloride (dose $50 \mathrm{mg} /$ day) in the treatment of major depression with comorbid alcoholism. We used validated chiral methods (Perel \& Assoc., Cli. Pharmacol. Ther. 59:213, 1996) to measure plasma concentrations for R- \& S- Fluoxetine and R- \& S-Norfluoxetine in 433 samples. After confirmation of eligibility, patients were randomly allocated (1:1) to receive naltrexone or placebo. Post-randomization assessments were undertaken weekly for the first 4 weeks, every two weeks for the subsequent 8 weeks, and every 4 weeks for the remaining 12 weeks of the 24-week study. The baseline assessment battery included the Psychiatric Research Interview for Substance and Mental Disorders (PRISM), the Hamilton Rating Scale for Depression (HRSD-17 \& 25) among others. Fluoxetine and naltrexone plasma concentrations monitoring were conducted at weeks $01,03,06,9,12,18$, and 24. We used chi-square tests for categorical variables, one way analysis of variance, and repeated measures analysis of variance for continuous variables. We examined whether fluoxetine/ norfluoxetine plasma concentrations differentiated treatment resistance (defined as less than $25 \%$ improvement from baseline on the HAMD-17) for each assessment point.

Results: Plasma concentrations were statistically significantly lower for the treatment resistant group compared to the treatment responsive group on total fluoxetine + norfluoxetine $($ mean $=154.7 \mathrm{ng} / \mathrm{ml}$, $\mathrm{sd}=120.6$ vs. $223.8 \mathrm{ng} / \mathrm{ml}, \mathrm{sd}=179.1, \mathrm{p}=0.0001)$, total fluoxetine $(\mathrm{mean}=$ $76.4 \mathrm{ng} / \mathrm{ml}, \mathrm{sd}=80.6$ vs. mean $=119.2 \mathrm{ng} / \mathrm{ml}, \mathrm{sd}=104.7, \mathrm{p}=0.0004)$, and total norfluoxetine (mean $82.3 \mathrm{ng} / \mathrm{ml}$, sd $=57.4$ vs. mean $111.2 \mathrm{ng} / \mathrm{ml}$, $\mathrm{sd}=92.9, \quad \mathrm{p}=0.0010)$. This difference was pronounced in the placebo group; total fluoxetine + norfluoxetine concentrations (mean $=$ $161.8 \mathrm{ng} / \mathrm{ml}, \quad \mathrm{sd}=116.9$ vs. $252.9 \mathrm{ng} / \mathrm{ml}, \mathrm{sd}=208.4, \mathrm{p}=0.0002)$, total fluoxetine concentrations $($ mean $=81.8 \mathrm{ng} / \mathrm{ml}, \mathrm{sd}=86.5$ vs. mean $=$ $134.4 \mathrm{ng} / \mathrm{ml}, \mathrm{sd}=121.5, \mathrm{p}=0.0016$ ), and total norfluoxetine concentrations (mean $83.4 \mathrm{ng} / \mathrm{ml}$, sd $=52.8$ vs. mean $128.1 \mathrm{ng} / \mathrm{ml}$, $\mathrm{sd}=109.4, \mathrm{p}=0.0002$ ). In the naltrexone group, there was an overall decrease of fluoxetine/ norfluoxetine serum concentrations, raising the possibility of a fluoxetinenaltrexone medication interaction. The average fluoxetine dose did not differ between the two treatment groups. Average fluoxetine dose was $31.8 \mathrm{mg}(\mathrm{sd}=14.9)$ for the fluoxetine + naltrexone group compared to an average fluoxetine dose of $33.4 \mathrm{mg}(\mathrm{sd}=14.8)$ for the fluoxetine + placebo group. Also, the two groups did not differ on several indices of medication adherence. The information derived from the individual enantiomer data will be reported separately.

Discussion: These results provide suggestive evidence of potential relationship between treatment non-response and fluoxetine/norfluoxetine plasma concentrations. Specifically, a minimal decrease in the Hamilton Rating Scale for Depression (HAMD-17) from baseline (less than $25 \%$ ) was found to be associated with lower plasma concentrations of total fluoxetine + norfluoxetine, total fluoxetine, and total norfluoxetine. To our knowledge, this is the first study examining fluoxetine/norfluoxetine plasma concentrations and treatment nonresponse for major depressive disorder with co-occurring alcoholism. Future studies are warranted to further elucidate the role of therapeutic drug monitoring in treatment resistant depression. TDM may be particularly indicated in the treatment of comorbid conditions with polypharmacy, which may uncover yet unknown interactions between commonly prescribed medications. Source of Funding: Supported by USPHS Grants Ro1 AA11929and in part by Ro1AA015385; Ro1 DA019992; Ro1 DA-019142, Ro1AA13370, NIDA Ko2-017822, NIDA CTN; \& VA MIRECC grant. 
Disclosure: I. Salloum: Part 1; Jnassen Pharmaceutica. Part 2; AstraZeneca. J. Perel: None. J. Cornelius: None. A. Douaihy: None. L. Kirisci: None. J. Helsel: None. M. Thase: None.

\section{Treatment of PTSD with Elevated Doses of Escitalopram: an Open Label Study}

Arieh Shalev*, Yevgeni Levin, Yael Ankri, Yossi Israeli - Shalev, Members of the Center for Traumatic Stress Study, Hadassah University Hospital

\section{Hadassah University Hospital, Jerusalem, Israel}

Background: Chronic PTSD is tenacious and treatment resistant. Studies of specific serotonin inhibitors (SSRIs) have shown consistent but limited effects on PTSD symptoms. Elevated doses of SSRIs have been used in other anxiety disorders (e.g., OCD). This open-label study evaluated the tolerance to and the therapeutic effect of an elevated daily dose of escitalopram in adults with chronic unremitting PTSD. Methods: Adult civilian trauma survivors with chronic unremitting PTSD were recruited from psychiatric outpatient clinics in Jerusalem. Participants with lifetime history of psychotic disorder, bipolar disorder, opiate or stimulants abuse, with prior sensitivity to SSRIs, or with medical or neurological condition precluding the use of an SSRI were not included.

Instruments: The clinician administered PTSD scale (CAPS) evaluated PTSD and PTSD symptoms at study onset and treatment end. Additionally the revised Posttraumatic Symptom Scale (PSS-SR) and the back depression inventory (BDI) evaluated symptoms of PTSD and depression at each session. Procedure: Eligible participants gave written informed consent and were invited for treatment. The treatment consisted of four weekly sessions followed by four biweekly sessions (12 weeks in total). Escitalopram was started at an initial daily dose of $20 \mathrm{mg}$ and increased to $40 \mathrm{mg}$ per day within four weeks of starting treatment.

Results: Fifty-one participants were enrolled, including 26 (51\%) male and 25 females. Participants' mean age was 43.53 ( \pm 12.89 ). Forty $(78.4 \%)$ were survivors of motor vehicle accidents, five $(9.8 \%)$ survived terror attacks, $4(7.8 \%)$ had work accidents and 2 (3.9\%) had other traumatic events. The mean duration of PTSD was $29.80 \pm 18.93$ months (Range: 5-92). Four enrolled participants (7.8\%) left the study after the initial evaluation. One participant did not meet initial DSM IV PTSD diagnostic criteria. Forty-four participants (86.3\%) started the study and received at least one week of treatment. Fifteen participants received escitalopram before starting the study at an average daily dose of $19.6 \mathrm{mg}$. Five participants received other antidepressants. Thirty-six (81.8\%) participants completed all treatment sessions. The mean prescribed daily dose across study session was of $32.70( \pm 9.93) \mathrm{mg}$. The mean dose actually taken was of $31.58( \pm 10.24)$ mg. The mean prescribed daily dose at treatment end was $38.36 \pm 4.82 \mathrm{mg}$ and the dose actually taken was $37.78 \pm 5.04 \mathrm{mg}$. All the participants met initial PTSD diagnostic criteria. The mean initial CAPS score was $71.81 \pm 14.29$ (range $48-100 ; 95 \%$ CI: 67.00-76.64). Eleven participants $(30.6 \%$ of $n=36)$ did not meet PTSD diagnostic criteria at the end of treatment. The mean endpoint CAPS score was $60.08 \pm 25.85$ (range $60-127,95 \% \mathrm{CI}=51.33-68.83$ ), yielding a significant within sessions effect $(\mathrm{F}(\mathrm{df}=35)=12.21, \mathrm{p} .<0.001$. Treatment effect size (first to last visit Cohen's d) was 0.55). Similar decrease of symptoms was seen in patients who received a lower daily dose of escitalopram before treatment (from $72.58 \pm 15.4$ to $60.33 \pm 25.48, \quad \mathrm{~F}(\mathrm{df}=11)=4.676, \mathrm{p} .=0.053)$ There was remarkable heterogeneity in treatment outcome with $16.7 \%$ of the participants losing above 50\% of their initial symptoms (range: $55 \%-72 \%$ ) while others showing more limited improvement or slight worsening.

Discussion: As reflected by the limited dropout rate, $40 \mathrm{mg}$ of escitalopram daily was well tolerated by chronic, unremitting PTSD patients. The treatment was associated with significant reduction in PTSD symptoms, including among participants who received lower doses of SSRIs before beginning the study. The effect of treatment did not differ, however, from those seen in previous studies of regular doses of SSRIs. Remarkable heterogeneity in treatment outcome should lead to further exploration of specific sensitivity to the effect of SSRIs among chronic PTSD patients.

Disclosure: A. Shalev: Part 1; Lundbeck Pharmaceuticals Ltd. Part 4; Lundbeck Pharmaceuticals, Ltd. Y. Levin: None. Y. Ankri: None. Y. Israeli - Shalev: None.

40. Olanzapine vs. Risperidone Effects on Appetite and Ghrelin in Chronic Schizophrenic Patients

Robert C. Smith*, Isabella Kanellopoulou, Saumya Rachakonda, Jean-Pierre Lindenmayer, John M Davis

NYU Medical School, Manhattan Psychiatric Center, and NKI, New York and Orangeburg, NY

Background: Weight gain is an important side-effect of many first and second generation antipsychotics, with the greatest effect on weight gain reported with olanzapine and clozapine treated schizophrenic patients, and reportedly somewhat less with risperidone. The mechanisms by which these drugs induce weight gain have not been clearly established. One mechanism may be though increasing appetite and subsequent increased food consumption. Several studies suggest that higher ghrelin levels may be related to meal hunger stimulation in humans and fall in ghrelin after eating may be related to satiation after feeding, and these effects may be mediated by action through receptors on hypothalamic neurons. We report of a randomized clinical trial investigating these issues.

Methods: Hospitalized chronic schizophrenic patients who had been treated with multiple antipsychotics in the past, were randomized to 5 months of treatments with either olanzapine (mean dose $25.2 \mathrm{mg} /$ day $\mathrm{SD}=10.1$ ) or risperidone (mean dose $6.1 \mathrm{mg} /$ day $\mathrm{SD}=1.8$ ). Weight and glucose and lipid metabolic measures were assessed monthly. Appetite, hunger, and food craving was assessed at baseline and at and during the study using 2 scales, the Visual Analogue Scale (VAS) and the Eating Behavior Assessment (EBA), in interview assisted assessments. These are Lilly developed scales which have been described in detail in previous publications. A fatty meal test was administered at baseline and 2 months of drug treatment and, glucose, lipids, and total serum ghrelin was collected and assayed at pre-meal baseline (o hrs), and 1 and 4 hours after the ingestion of the fatty meal.

Results: Although there was a slight increase in patients weight over the 5 months of the study $(2-4, \mathrm{Kg}$, Time effect, mixed model, $\mathrm{F}=3.51$, $\mathrm{P}=.046)$, there was no difference in weight increase between patients treated with olanzapine or risperidone (Drug $x$ Time effect: $\mathrm{F}=1.53$, $\mathrm{P}=0.1813$ ), Neither olanzapine or risperidone increased appetite over the five months of study drug treatment, and there was no difference between the effects the two drugs; there was a trend ( $\mathrm{P}$ 's =.07-.10) for decrease in appetite scores over time. On the EBA scale olanzapine patients had consistently higher appetite scores both at pre-study baseline and during the 5-months of study drug, and on the VAS appetite scale olanzapine patients had higher scores on month 2 assessments, but not at months 3 or 5 . Change in VAS or EBA total or component scores were not correlated with weight change in the whole group, but for olanzapine patients there was a small to moderate correlation between increase in EBA scores at 5 months compared to baseline and change in weight $(\mathrm{r}=+.46, \mathrm{P}=.028)$ whereas risperidone patients showed a trend for a negative correlation $(r=-.34$, $\mathrm{P}=.118$ ). However most of the positive correlation in the olanzapine patients seemed to be due to a decrease in weight among those patients who had decrease in appetite. There was no difference in pre-meal total Ghrelin or change in fasting ghrelin in olanzapine compared to risperidone patients. Ghrelin deceased $1 \mathrm{hr}$ after ingestion of a fatty meal in both olanzapine and risperidone treated patients and there were no significant differences between the two drugs in ghrelin response over $4 \mathrm{hrs}$ after fatty meal. 


\section{S316}

Discussion: Although we did not have objective measures of food consumption, our results suggests that olanzapine does not increase subjective appetite in chronic schizophrenics who have a long history of treatment with lst and 2nd generation antipsychotic medications. The lack of difference between fasting ghrelin levels, or ghrelin response to fatty meals in olanzapine vs risperidone patients, provide a physiological measure related to appetite increase and satiation, which parallel our results on the subjective appetite changes. We do not have a control group to be able to assess whether these ghrelin responses are different from those found in non-psychotic obese or lean subjects. It is possible that younger patients or those drug-naïve to olanzapine or second generation antipsycothcis, might show a different pattern of change in appetite and ghrelin response to the drug, since these are the patients who tend to show the largest weight increase during the lst 6-12 months after starting olanzapine.

Disclosure: R. Smith: Part 4; Eli Lilly, Takeda, Pfizer. I. Kanellopoulou: None. S. Rachakonda: None. J. Lindenmayer: Part 1; Janssen, Eli Lilly. Part 4; Eli Lilly, Bristol-Meyers, Squibb,, Janssen, Eli Lilly, Pfizer. J. Davis: None.

\section{Effectiveness of Ziprasidone in Bipolar Disorder Patients Primarily Taking Complex Pharmacotherapy \\ Shefali Srivastava*, Pichai Ittasakul, Po Wang, Shelley Hill, Meredith Childers, Terence Ketter}

\section{Stanford University, Stanford, CA}

Background: Efficacy studies have informed the treatment of bipolar disorder (BD) patients with ziprasidone as monotherapy or added to lithium or valproate, but have excluded patients with complicated comorbidities and/or taking complex pharmacotherapy. We reviewed measurement-based care clinical treatment records of such patients in order to address questions that clinicians are likely to encounter with respect to the effectiveness of ziprasidone in bipolar disorder patients with challenging illness.

Methods: We naturalistically administered open ziprasidone to Stanford University Bipolar Disorders Clinic patients assessed with the Systematic Treatment Enhancement Program for Bipolar Disorder (STEP-BD) Affective Disorders Evaluation, and monitored longitudinally with the STEP-BD Clinical Monitoring Form. We addressed the following questions: $\{1\}$ When is ziprasidone started?; $\{2\}$ How is ziprasidone initially dosed and titrated, and what is the average dose on which patients are maintained?; $\{3\}$ What concurrent therapies are present?; $\{4\}$ What clinical benefits are seen?; $\{5\}$ How often and when are subsequent additional pharmacotherapies added?; $\{6\}$ Why are subsequent additional pharmacotherapies added?; $\{7\}$ How often and when is ziprasidone discontinued?; $\{8\}$ Why is ziprasidone discontinued?; and \{9\} What adverse effects are seen with ziprasidone? Results: 82 patients ( $40 \mathrm{BD}$ I, $38 \mathrm{BD}$ II, 4 BD NOS, mean \pm SD [median] age $40.8 \pm 12.1$ [ 41.4 ] years, $76.8 \%$ female, and $72.0 \%$ with at least one lifetime comorbid psychiatric disorder) received ziprasidone, initiated at Stanford in 71 patients, and initiated prior to Stanford in 11 patients. \{1\} Baseline mood state was most often syndromal or subsyndromal depression $(53.7 \%)$ or euthymia $(31.7 \%)$. \{ 2$\}$ In 71 trials started at Stanford, mean initial dose was $67 \pm 26(80) \mathrm{mg} /$ day, and after a mean of $79 \pm 268$ (17) days, mean maximum dose was $180 \pm 79$ (160) mg/day, most often given at dinner and/or with a snack at bedtime. For all 82 trials, mean ziprasidone final dose was $133 \pm 80$ (140) $\mathrm{mg}$ /day and mean trial duration was $532 \pm 618$ (290) days. $\{3\}$ For all 82 trials, ziprasidone was combined with a mean of 3.6 (at least 2 in $85 \%$ of patients, at least 3 in $74 \%$ ) other prescription psychotropic medications and a mean of 1.2 prescription non-psychotropic medications. $\{4\}$ Final compared to baseline mean Clinical Global Impression-Severity of Illness (CGI-S) score in 44 patients with baseline syndromal or subsyndromal depression improved by $0.9(\mathrm{p}=0.01)$, in all 82 patients improved by $0.3(\mathrm{p}=0.04)$, but did not change significantly in 12 patients with baseline syndromal or subsyndromal elevation or in 26 patients with baseline euthymia. Also, in 70 trials started at Stanford, mean weight decreased from $195 \pm 50(191)$ lbs at baseline to $183 \pm 47$ (179) lbs at final visit taking ziprasidone $(\mathrm{p}=0.002)$, with $34.3 \%$ of patients achieving at least $7 \%$ weight loss. $\{5\}$ For all 82 trials, subsequent psychotropic was added in $48.8 \%$ after a mean of $203 \pm 252$ (125.5) days, with a mean overall trial duration of $859 \pm 709$ (885.5) days, and no subsequent psychotropic was added in $51.2 \%$ with a mean trial duration of $221 \pm 272$ (98) days. \{6\} Subsequent additional pharmacotherapies were added due to depressive symptoms in $22.0 \%$ of patients, anxiety or insomnia in $18.3 \%$, mood elevation symptoms in $2.4 \%$, weight control in $2.4 \%$, and for other reasons in $3.7 \%$. \{7\} Ziprasidone was discontinued in $57.3 \%$ of all 82 trials, after a mean of $208 \pm 364$ (94) days, and not discontinued in $42.7 \%$, with a mean duration of $968 \pm 625$ (939) days. $\{8\}$ Ziprasidone was discontinued due to side effects (in $26.8 \%$, primarily central nervous system), due to inefficacy for mood (in $23.2 \%$, primarily depression), and for other reasons in $7.3 \%$. $\{9\}$ Despite a $26.8 \%$ discontinuation rate due to side effects, mean adverse effect severity ratings did not change significantly from baseline to last visit taking ziprasidone, with the notable exception of "increased appetite," which significantly decreased $(\mathrm{p}=0.04)$.

Discussion: In $\mathrm{BD}$ outpatients primarily taking complex pharmacotherapy, ziprasidone not only decreased overall bipolar illness severity, with the effect being most evident in patients who had syndromal or subsyndromal depression at baseline, but also yielded clinically significant weight loss in $34.3 \%$ of patients. However, an even higher percentage of patients discontinued ziprasidone (albeit on average after 208 days), consistent with the challenging nature of this population. Further studies are needed to assess the effectiveness of ziprasidone and other medications in bipolar disorder patients taking complex pharmacotherapy.

Disclosure: S. Srivastava: None. P. Ittasakul: None. P. Wang: Part 1; AstraZeneca Pharmaceuticals LP, Bristol-Myers Squibb Company, GlaxoSmithKline, Pfizer, Inc., Synosia Therapeutics, Abbott Laboratories, Inc., Cephalon, Inc., Eli Lilly and Company, Sepracor, Inc. Part 2; AstraZeneca Pharmaceuticals LP. Part 3; AstraZeneca Pharmaceuticals LP. Part 4; AstraZeneca Pharmaceuticals LP, Eli Lilly and Company, Sepracor, Inc. (via Quintiles, Inc.). S. Hill: None. M. Childers: None. T. Ketter: Part 1; Abbott Laboratories, Inc., AstraZeneca Pharmaceuticals LP, Bristol-Myers Squibb Company, Cephalon Inc., Eli Lilly and Company, GlaxoSmithKline, Pfizer, Inc., Astellas Pharmaceuticals, Dainippon Sumitomo Pharmaceuticals, Forest Labs, Janssen Pharmaceeutica Products, LP, Merck \& Co., Inc., Sepracor, Inc., XenoPort, Inc., Johnson \& Johnson (spouse). Part 2; GlaxoSmithKline, Johnson \& Johnson (spouse). Part 3; Johnson \& Johnson (spouse). Part 4; AstraZeneca Pharmaceuticals, LP, Eli Lilly and Company, Sepracor, Inc. (via Quintiles, Inc.). Part 5; Johnson \& Johnson (spouse).

42. Long-Term Safety and Tolerability of Lurasidone in Subjects with Schizophrenia: Results of a 6-Month, Open-Label Study

Stephen Stahl*, Josephine Cucchiaro, Doreen Simonelli, Antony Loebel

University of California, San Diego, Carlsbad, CA

Background: Lurasidone has a unique chemical structure that differs from conventional and atypical antipsychotic agents. It has high affinities for dopamine $\mathrm{D}_{2}$, serotonin $5-\mathrm{HT}_{7}, 5-\mathrm{HT}_{2 \mathrm{~A}}, 5-\mathrm{HT}_{1 \mathrm{~A}}$, and noradrenaline $\alpha_{2 C}$ receptors. Lurasidone exhibits little or no affinity for histamine $H_{1}$ or acetylcholine $M_{1}$ receptors. The objective of this study was to evaluate the long-term safety and tolerability of lurasidone, and to determine if improvement during the acute DB phase of treatment was sustained.

Methods: Subjects who successfully completed a 6-week, double-blind (DB), placebo-controlled trial evaluating the efficacy of fixed-doses of lurasidone $40 \mathrm{mg}$ and $120 \mathrm{mg}$, with olanzapine $15 \mathrm{mg}$ as an active arm (to confirm assay sensitivity), were continued in a 6-month, open-label extension phase which is summarized here. After completing a single- 
blind, 3-day placebo washout, subjects in all DB treatment arms were switched to lurasidone $80 \mathrm{mg}$ daily in an open-label fashion. Flexible dosing was permitted, after one week, in the dosing range of 40$120 \mathrm{mg} /$ day. Safety and tolerability measures included adverse events, body weight, lipid parameters, prolactin, and ECGs. Efficacy assessments included the Positive and Negative Symptoms of Schizophrenia Scale (PANSS) total and subcale scores, and the CGI-Severity scale.

Results: A total of 254 subjects were enrolled in the open-label extension, of whom 246 were analyzed for safety and efficacy. The mean (SD) PANSS total score, for all subjects continuing into the open-label phase, decreased from 96.6 (10.9) at the baseline of the DB phase to $66.6(16.9)$ at the baseline of the open-label phase. During open-label treatment, subjects showed further improvement in the PANSS total score, with a mean (SD) score of 54.9 (16.0) at the end of the extension phase. Improvement from open-label baseline to Month 8 was also observed for the PANSS positive subscore $(-2.6$; 95\%-CI: $-3.5,-1.8)$, negative subscore $(-2.5 ; 95 \%-\mathrm{CI}:-3.3,-1.7)$ and general psychopathology subscore $(-3.6 ; 95 \%-C I$ : $-5.0,-2.2)$. The CGI-Severity score also continued to decrease from 3.2 (o.9) at open-label baseline to $2.7(1.0)$ at the end of open-label. The modal dose of lurasidone was $80 \mathrm{mg}(63 \%)$, followed by $120 \mathrm{mg}(27 \%)$ and $40 \mathrm{mg}(10 \%)$. Two adverse events occurred with an incidence $>10 \%$ : akathisia $(13.0 \%)$ and insomnia (11.0\%). An adverse event was rated as "severe" by $7.3 \%$ of subjects; and a total of $12.2 \%$ of subjects discontinued due to an adverse event during 6 months of open-label treatment. There were no clinically meaningful changes in vital signs, or laboratory and ECG parameters; one subject $(0.4 \%)$ reported $\geq 60 \mathrm{msec}$ increase in QTcF, and no subject had a QTcF interval $>500$ msec. Body weight and BMI remained relatively stable during the open-label extension, except for subjects who had been randomized in the initial DB phase to olanzapine $15 \mathrm{mg}$ : after the switch to open-label lurasidone, there was a mean (SD) reduction of -1.8 (4.9) $\mathrm{kg}$ in weight. There were no clinically meaningful changes, from open-label baseline to endpoint, in cholesterol $(-7.1 \mathrm{mg} / \mathrm{dL})$, LDL $(-2.6 \mathrm{mg} / \mathrm{dL})$, triglycerides $(-18.3 \mathrm{mg} / \mathrm{dL})$, insulin $(-2.4 \mathrm{mU} / \mathrm{L})$, or whole blood HbAic $(-0.06 \%)$. Prolactin, which had increased during the DB phase of the study $(+3.2 \mathrm{ng} / \mathrm{mL}$ on combined lurasidone; $+3.4 \mathrm{ng} / \mathrm{mL}$ on olanzapine), showed an overall median decrease $(-1.3 \mathrm{ng} / \mathrm{mL})$ during the open-label extension.

Discussion: Flexibly dosed lurasidone (40-120 mg, daily) was welltolerated and maintained efficacy in treating subjects with schizophrenia during this open-label extension study. Up to 8 months of treatment with lurasidone was associated with a low potential for weight gain and dyslipidemia.

Disclosure: S. Stahl: Part 1; Adams Coogler, Alkermes, Allergan, Arbor Scientia, Arena, Astra Zeneca, Azur, BioMarin, Bionevia, Biovail, Boehringer Ingelheim, BMS, Cambridge University Press, CME Solutions, Covance, Cypress Bioscience, Dianippon Sumitomo, Eisai, Eli Lilly, Endo, Finegan Henderson, Forest, Genomind, GSK, GlaxoSmithKline, Great Point Partners, Health and Wellness Partners, HealthLogix, Pierre Fabre, Innovision, Interactive Forums, Ionevia Pharma, Janssen, Jazz Pharmaceuticals, LaboPharm, Lundbeck, Marinus, Meiji, Merck, Neuronetics, Neuroscience Education Institute, Novartis, Noven, Nuvis, Ogilvy, Otsuka, PamLabs, Paragon, Pfizer, PGX Health, Propagate Pharma, Rexahn, Sanofi, Schering Plough, SciMed, Sepracor, Servier, Shire, SK Corporation, Solvay, Somaxon, State of California, Vanda, Vivus, Wyeth. Part 2; Arbor Scientia, Astra Zeneca, Biomarin, Boehringer Ingleheim, Cambridge University Press, Dianippon, Eisai, Eli Lilly, Endo, Forest Laboratories, Genomind, Innovision, Janssen, Labopharm, Lundbeck, Meiji, Neuroscience Education Institute, NOvartis, PamLabs, Pfizer, Pierre Fabre, Sanofi, Schering Plough, Seporacor, Servier, Shire, SK Corporation, Somaxon, Vanda, Wyeth. Part 3; Arbor Scientia, Cambridge University Press, Neuroscience Education Institute, PamLabs. Part 4; Alkermes, Astra Zeneca, Boehringer Ingleheim, BMS, Cephalon,, Dainippon Sumitomo, Eli Lilly, Forest, Janssen, Merck, Novartis, Organon, PamLabs, Pfizer,
PGX Health, PriMed, Sanofi, Schering Plough, Sepracor, Servier, Shire, Takeda, Somaxon, Torrent, Vanda, Wyeth. J. Cucchiaro: Part 5; Sunovian. D. Simonelli: Sunovian. A. Loebel: Sunovian.

43. Trajectories of Response to Treatment with Atypical Antipsychotic Therapy in Patients with Schizophrenia Pooled from 6 Doubleblind, Randomized Clinical Trials

Virginia Stauffer*, Michael Case, Sara Kollack-Walker, Haya AscherSvanum, Tamara Ball, Shitij Kapur, Bruce J Kinon

\section{Eli Lilly and Company, Indianapolis, IN}

Background: Patient response to antipsychotic therapy varies. Understanding patterns of response could help clinicians more proactively manage patients with schizophrenia. Distinct patterns of response to antipsychotic therapy have been identified in patients with chronic schizophrenia who were enrolled in trials of up to 12 weeks duration. In this analysis, we identified trajectories inherent in pooled data from 6 randomized clinical trials of 24 weeks duration and assessed for baseline patient characteristics associated with these trajectories.

Methods: We pooled data on 1990 patients with chronic schizophrenia from 6 randomized, double-blind, comparator trials of at least 24 weeks duration who were treated with olanzapine, ziprasidone, risperidone, quetiapine, or aripiprazole. Growth mixture modeling (GMM) was used to identify homogeneous subpopulations within the larger heterogeneous population. Baseline patient demographics were compared between the identified latent classes.

Results: Five distinct response trajectories based on Positive and Negative Syndrome Scale (PANSS) Total score over 24 weeks were identified. On average, 9 out of 10 patients $(n=1802 / 1990,90.6 \%)$ belonged in Class 2, characterized by a moderate level of illness at baseline (PANSS Total $=90$ ), with a mean improvement of $20 \%$ by Week 4, and little further improvement. Patients in Class $1(n=47 /$ 1990, 2.4\%) were severely ill at baseline (PANSS Total=124), but responded with rapid (51\% reduction in mean PANSS Total score by Week 3$)$ and sustained improvement. Classes $3(n=32 / 1990,1.6 \%)$. and $4(\mathrm{n}=28,1.4 \%)$. both showed minimal response initially, followed by loss of that initial gain. Patients in Class 4 (baseline PANSS Total score $=102$ ) improved rapidly between Weeks 8 and 12, with little change afterward; whereas patients in Class 3 (baseline PANSS Total score $=95)$ showed maximal improvement slightly later (at Week 12), followed by gradual worsening thereafter. Class $5(n=81 / 1990,4.1 \%)$ was characterized by marked to severe illness at baseline (PANSS Total $=113)$, an initial delay in response $(11 \%$ improvement over 8 weeks), and noticeable improvement in the remaining 4 weeks (baseline-to-endpoint improvement $=28 \%$ ). Significant differences between classes were noted for gender, age, race, baseline illness severity, specific symptom types, weight, extrapyramidal symptoms, and discontinuation rates (all $\mathrm{p}<.05$ ).

Discussion: GMM analysis of response to antipsychotic therapy over 24 weeks in a large, pooled, heterogeneous population of patients with schizophrenia revealed 5 distinct trajectory classes. Most patients demonstrated a minimal, but sustained degree of improvement regardless of their baseline illness severity. Smaller subsets of patients showing rapid and dramatic improvement, delayed improvement, and improvement which could not be sustained were also identified. Baseline differences associated with these trajectories may help clinicians identify patients more likely to follow specific response patterns.

Disclosure: V. Stauffer: Part 5; Eli Lilly and Company. M. Case: Eli Lilly and Company. S. Kollack-Walker: Eli Lilly and Company. H. Ascher-Svanum: Eli Lilly and Company. T. Ball: Part 1; i3 Statprobe. S. Kapur: Eli Lilly and Company, AstraZeneca, Bristol-Myers Squibb, GlaxoSmithKline, Bioline, Janssen, Lundbeck, Otsuka, Organon, Pfizer, Servier, Solay Wyeth. B. Kinon: Part 5; Eli Lilly and Company. 
44. Long-Term Safety and Tolerability of High Dose Donepezil $(23 \mathrm{mg} /$ day) in Moderate to Severe Alzheimer's Disease: A 12-Month Open-Label Study

Pierre Tariot*, Jane Yardley, Margaret Moline, Elimor BrandSchieber, Heng Zou, Hsu Timothy, Andrew Satlin

Banner Alzheimer's Institute, Phoenix, AZ

Background: Alzheimer's disease (AD) is characterized by progressive neurodegeneration; a patient's clinical condition eventually declines even with continuous pharmacotherapy. Approved pharmacotherapy options for patients with moderate to severe $\mathrm{AD}$ have been limited. To address this issue, a double-blind, global study was conducted recently to determine whether patients with moderate to severe $\mathrm{AD}$ who were already taking donepezil $10 \mathrm{mg} /$ day could benefit from an increased dose in a new formulation with a longer $\mathrm{T}_{\max }$. Patients who were treated for at least 3 months on donepezil $10 \mathrm{mg} /$ day were randomized 2:1 either to once-daily donepezil $23 \mathrm{mg}$ tablets or to continue on oncedaily $10 \mathrm{mg} /$ day tablets for 24 weeks ${ }^{1}$. On the basis of results from that study, the FDA recently approved the use of $23 \mathrm{mg} /$ day donepezil in patients with moderate to severe AD.

Methods: The present study was a 12-month, open-label extension of the double-blind study. Patients were eligible to enroll if they completed the double-blind study without any ongoing or studydrug-related serious adverse events (SAEs). All patients received $23 \mathrm{mg} /$ day donepezil. Safety was assessed at each study visit (Months 3, 6, 9 and 12) and included treatment emergent adverse events (AE), laboratory tests (hematology, clinical chemistry, and urinalysis), vital signs and body weight, and 12-lead ECG findings. Safety analyses were descriptive; the denominator for rates of all events was the Safety Population (i.e., patients who took at least one dose of the open-label study drug and had at least one post-baseline safety assessment).

Results: Approximately $83 \%$ of the 1084 patients who completed the double-blind study enrolled in the extension trial and were included in the Safety Population $(\mathrm{N}=902)$. The Safety Population comprised 570 patients $(63.2 \%)$ who received donepezil $23 \mathrm{mg}$ both in the double-blind study and the present, open-label study (23-23 group), and $332(36.8 \%)$ patients who received donepezil $10 \mathrm{mg}$ in the doubleblind study and $23 \mathrm{mg}$ in the present study (10-23 group). Patients were $74.3 \pm 8.58$ (mean $\pm S D$ ) years old, $63.7 \%$ were female, $74.7 \%$ were White and $15.5 \%$ were Asian/Pacific. The Mini-Mental State Exam score at open-label study baseline was $13.8 \pm 5.9$ (mean \pm SD). The mean and median durations of treatment during the open-label study were approximately 10 and 12 months, respectively. Overall, $74.7 \%$ of patients each reported at least one AE. The AEs with the highest incidence ( $>5.0 \%$ of subjects) were weight decreased $(11.2 \%)$, fall $(8.8 \%)$, agitation $(6.8 \%)$, urinary tract infection $(5.9 \%)$, and aggression (5.8\%). As expected, cholinergic AEs were reported more frequently in patients whose dose was increased from $10 \mathrm{mg} / \mathrm{day}$ to $23 \mathrm{mg} /$ day at the start of the open-label study than in patients who were already taking $23 \mathrm{mg}$ (nausea: $6.0 \%$ [10-23 group] v. $2.1 \%$ [23-23 group]; vomiting: $4.5 \%$ [10-23] v. $1.6 \%$ [23-23]; diarrhea: $5.7 \%$ [10-23] v. $3.5 \%$ [23-23]). Similarly, the discontinuation rate due to AEs (13.6\% overall) was higher in the $10-23$ group $(17.5 \%)$ than in the $23-23$ group (11.4\%), mostly due to the cholinergic-related events (e.g., nausea: $1.5 \%$ [10-23] v. $0 \%$ [23-23]; vomiting: $1.2 \%$ [10-23] v. $O \%$ [23-23]). After an initial increase in AEs, generally cholinergic-related, associated with the upward dose titration, the incidence of patients with newly occurring AEs declined rapidly and remained low throughout the duration of the study. Similarly, the majority of discontinuations due to AEs occurred shortly after the dose increase. The AEs were mostly of mild or moderate severity. The SAE rate was $15.1 \%$. The most frequently occurring SAEs in the entire population $(>0.5 \%)$ were syncope $(1.3 \%)$, urinary tract infection $(1.2 \%)$, fall (1.0\%), cerebrovascular accident and pneumonia (o.8\% for each), and dehydration (o.6\%). The death rate was $2.1 \%$ $(n=19)$. With one exception (attributed to hematemesis), none of these deaths were considered by the investigators to be related to the study drug. Laboratory tests, vital signs, and ECG did not reveal any new safety signals.

Discussion: In both the double-blind and open-label label studies, a dose increase from $10 \mathrm{mg} /$ day to $23 \mathrm{mg}$ /day initially led to slightly higher rates of cholinergic-related AEs. Overall, long term open-label treatment of moderate to severe AD patients with donepezil $23 \mathrm{mg}$ was generally safe and well tolerated, with no new safety signals.

Disclosure: P. Tariot: Part 1; Abbott, Acadia, AstraZeneca, Baxter, Bristol-Myer Squibb, Eisai, Elan, Genentech, GlaxoSmithKline, Janssen, Pfizer, sanofi aventis, AVID, Wyeth, Lilly, Forest, Merck, Medivation, Medavante, GE Healthcare. Part 4; AstraZeneca, Baxter, Bristol-Myer Squibb, Elan, Janssen, Lilly, Merck, Medivation, Pfizer, Wyeth, AVID, GE Healthcare. J. Yardley: Part 5; Employed by Eisai. M. Moline: Employed by Eisai. E. Brand-Schieber: Employed by Eisai. H. Zou: Employed by Eisai. H. Timothy: Employed by Eisai. A. Satlin: Employed by Eisai.

\section{Brain Volume Changes With Divalproex Sodium In Alzheimer's Disease}

Adam Fleisher, Pierre Tariot ${ }^{*}$, Diana Truran, Jacqueline Mai, Paul Aisen, Jeffrey Cummings, Lon Schneider, Clifford Jack, Michael Weiner, Jessica Langbaum, Ronald Thomas

Banner Alzheimer's Institute, Phoenix, AZ

Background: ivalproex sodium was examined in a clinical trial of people with mild to moderate Alzheimer's disease(AD) based in part on its neuroprotective potential. Volumetric MRI's were performed in a subset of participants.

Methods: A subset of 89 of 313 volunteers enrolled in a 24 month randomized, placebo-controlled, parallel-group trial of divalproex received volumetric MRI scans at baseline and 12 months. Clinical outcomes were assessed at 6 month intervals. Semi-automated segmentation was used to extract bilateral hippocampal volumes. Longitudinal change in whole brain volume and ventricular size were assessed using a boundary shift integral technique. Wilcoxon rank sum tests were used to compare interval MRI volume percent changes between treatment and placebo groups. Group differences and change from baseline in all clinical outcomes were assessed at each timepoint. Results: 88 individuals had MRI scans that passed quality control for hippocampal measures at both timepoints ( lacebo $=45$, divalproex $=43$ ), 66 for whole brain measures (placebo $=35$, divalproex $=31$ ), and 71 for ventricular volumes $($ placebo $=38$, divalproex $=33$ ). There were no significant baseline differences in age, education, brain volumes, cognitive scores or APOE4 carrier status. Sex was unbalanced, with more females in the placebo group, and associated with 12 month volumetric changes, and therefore used as a covariate in ANCOVA models. The divalproex group showed greater decline in hippocampal and brain volumes, and greater ventricular expansion $(\mathrm{p}<0.001)$. There were no significant betweengroup differences over 24 months on cognitive or functional measures in this subset. However, unlike the ADAS-cog and CDR, MMSE scores showed a more rapid decline in the divalproex group through month 12 $($ placebo $=-2.0 \pm 4.3$, divalproex $=-3.9 \pm 4.0(p=0.037)$ ).

Discussion: Divalproex appeared to accelerate brain volume loss in $\mathrm{AD}$ patients. This was not associated with decline in the chief clinical outcomes over 24 months, although the MMSE data suggest possible transient acceleration of cognitive decline. These results are consistent with reports of reversible brain atrophy and cognitive impairment in children and young adults. It is unclear if the brain volume loss and cognitive decline we observed result from direct drug effects such as osmotic shifts, other toxicity, or influences on AD pathology, or whether these effects are reversible or clinically relevant.

Disclosure: A. Fleisher: Part 1; AVID, Baxter, Elan, Janssen, Pfizer, Wyeth. Part 4; AVID, Baxter, Elan, Janssen, Pfizer, Wyeth. P. Tariot: Part 1; Abbott, Acadia, AstraZeneca, AVID, Baxter, Bristol-Myer Squibb, Eisai, Elan, Forest, Genentech, GlaxoSmithKline, Janssen, Lilly, Medavante, Medivation, Merck, GE Healthcare, Pfizer, Wyeth, AC Immune. Part 4; AstraZeneca, AVID, Baxter, Bristol-Myer Squibb, 
Elan, GE Healthcare, Janssen, Lilly, Medivation, Merck, Pfizer, Wyeth. D. Truran: None. J. Mai: None. P. Aisen: Part 1; Neurophage, Eisai, Elan, Wyeth, Schering-Plough, BMS, Lilly, Merck, Roche, Amgen, Genentech, Abbott, Pfizer, Novartis, Bayer, Baxter, Astellas, Dainippon, Biomarin, Solvay, Otsuka, Daichi, Medavante. Part 4; Baxter, Pfizer. J. Cummings: Part 1; Abbott, Acadia, Acceris, ADAMAS, Astellas, Avenir, BMS, Comentis, Eisai, Envivo, Forest, GSK, Janssen, Lilly, Lundbeck, Medivation, Merck, Merz, Myriad, Neuren, Neurokos, Novartis, Orion, Pfizer, Prana, reMYND, Schering-Plough, Signum, Sonexa, Takeda, Toyama, Wyeth. L. Schneider: Abbott, AC Immune, Baxter, Forest, Johnson and Johnson, Lilly, Myriad, Novartis, Pfizer, Takeda, Wyeth, Accera, Allergan, Allon, BMS, Elan, Exonhit, GSK, Lundbeck, Medavante, Merck, Roche, Sanofi, Servier, Schering-Plough, Schwabe, TEVA, Toyama, Transition Therapeutics, Voyager. Part 4; AstraZeneca, Baxter, Elan, Forest, Johnson and Johnson, Lilly, Myriad, Novartis, Pfizer, Takeda, Wyeth. C. Jack: Part 1; Baxter, Elan, Lilly, Pfizer, GE. Part 4; Baxter, Pfizer. M. Weiner: Part 1; AVID, Merck, Bayer, Lilly, Comentis, Neurochem, Eisai, SIRA UCSD, Aegis, Allergan, Genentech, BMS, Forest, Pfizer, Mitsubishi, Novartis, Synarc. Part 4; AVID, Merck. J. Langbaum: None. R. Thomas: None.

\section{Predictors of Relapse or Recurrence in Bipolar I Disorder Elisabeth Degenhardt, Jennifer Gatz, Jayanthi Jacob, Mauricio Tohen*}

\section{UT Health Science Center, San Antonio, TX}

Background: Bipolar disorder represents a major public health concern and despite treatment the condition is characterized by recurring episodes of mania, depression, or mixed states. Prevention of relapse and recurrence is a primary treatment objective in the management of the disorder. The objective of the current study was to identify predictors of relapse/recurrence in patients with bipolar I disorder.

Methods: Data from four clinical studies were pooled for this post-hoc analysis. Patients with bipolar I disorder achieving remission after pharmacological treatment and entering randomized double-blind maintenance therapy with olanzapine, lithium, divalproex, olanzapine plus mood stabilizer, or placebo for 44 to 72 weeks were included in this analysis. Cox Proportional Hazard models and Kaplan-Meier analyses were used to determine predictors of relapse/recurrence for the pooled data and within each treatment group.

Results: A total of 929 adult patients were included in this analysis and 427 patients (46.0 \%) experienced symptomatic relapse/recurrence during the follow-up period. HAMD total score, gender, rapid cycling and treatment emerged as significant predictors of relapse/recurrence within the pooled patient population. Patients with HAMD $<4$ at entry into maintenance phase showed a significantly longer time to relapse than those with a score of $4-8(p<0.001)$.

Discussion: The relative prophylactic effects of olanzapine, lithium, divalproex, olanzapine plus mood stabilizer and placebo were assessed and baseline predictors of relapse/recurrence from combined and individual treatment groups were identified. Findings from this study suggest that HAMD score $<4$ may be a better indicator of remission in bipolar I disorder than HAMD $<8$.

Disclosure: E. Degenhardt: Part 5; Eli Lilly and Company. J. Gatz: Eli Lilly and Company. J. Jacob: Eli Lilly and Company. M. Tohen: Part 1; Shareholder, Consultant; Eli Lilly and Company. Part 4; Shareholder, Consultant; Eli Lilly and Company.

47. Efficacy of Asenapine in Bipolar I Disorder as Monotherapy and Adjunct Therapy Compared With Other Atypical Antipsychotics Based on Meta-analyses of Placebo-Controlled Trials

Hein Fennema, Bernhard Slaap, Craig Karson, Armin Szegedi*

Merck, Rahway, NJ

Background: Asenapine is an atypical antipsychotic indicated in the United States in adults for acute treatment of schizophrenia and of manic or mixed episodes associated with bipolar I disorder with or without psychotic features. The superiority of flexible-dose asenapine monotherapy ( 5 or $10 \mathrm{mg}$ twice daily [BID]) vs placebo in the treatment of mania in patients with acute manic or mixed episodes of bipolar I disorder was demonstrated in 2 randomized, double-blind, placeboand olanzapine-controlled 3 -week trials. Superiority of flexible-dose asenapine ( 5 or $10 \mathrm{mg}$ BID) vs placebo in patients with acute manic or mixed episodes as adjunctive therapy to lithium or valproate was shown in a separate randomized, double-blind, placebo-controlled trial. Here, we present results of the asenapine clinical trial program compared with other atypical antipsychotics based on available placebo-controlled trials using meta-analyses.

Methods: For all included studies, the primary endpoint was change from baseline on the Young Mania Rating Scale (YMRS) total score after 3 weeks of treatment versus placebo. Data from the asenapine clinical program in the effective dose range of 5 or $10 \mathrm{mg}$ BID used the prespecified primary analysis, with last observation carried forward to impute missing data; treatment differences were estimated using analysis of covariance adjusted for baseline YMRS score and (pooled) investigative site. Data for comparators (aripiprazole, olanzapine, quetiapine, risperidone, and ziprasidone) were obtained from findings of monotherapy $(n=18)$ or adjunctive treatment $(\mathrm{n}=9)$ trials that were published between 1999 and 2009, with additional olanzapine data being included from the two 3-week asenapine monotherapy trials. All meta-analyses used the randomeffects model described by DerSimonian and Laird (Control Clin Trials 1986;7:177-188); Cochran's Q statistic was used to test study homogeneity.

Results: Combining the asenapine monotherapy studies, the estimated difference in the change in YMRS total score from baseline to endpoint for asenapine vs placebo was -4.7 YMRS points $(95 \% \mathrm{CI}$, -6.6 to -2.7$)$, which was statistically superior to placebo $(\mathrm{p}<0.0001)$. The results across asenapine studies were homogeneous $(\mathrm{Q}=1.1$, $\mathrm{df}=1, \quad \mathrm{p}=0.30)$. The effect size of asenapine vs placebo was comparable to the overall effect size of all active comparators vs placebo of -4.9 YMRS points ( $95 \%$ CI, -6.0 to -3.8 ). For this analysis there was considerable heterogeneity $(\mathrm{Q}=70.3, \mathrm{df}=20, \mathrm{p}<0.001)$. However, after exclusion of results from 1 risperidone study (Khanna et al. Br J Psychiatry 2005;187:229-234) with a markedly higher change from baseline than other agents and aripiprazole studies (for which the lowest changes from baseline versus placebo were observed), data from the remaining monotherapy studies were markedly homogeneous $(\mathrm{Q}=21.7, \mathrm{df}=14, \mathrm{p}=0.09)$, leading to an overall effect size of -5.0 YMRS points $(95 \% \mathrm{CI},-5.8$ to -4.3$)$. The efficacy of asenapine vs placebo as adjunctive therapy to lithium or valproate revealed a treatment effect of -2.4 YMRS points $(95 \% \mathrm{CI},-4.3$ to -0.5). This effect size was comparable to the overall effect size vs placebo of -2.7 YMRS points (95\% CI, -3.3 to -2.0 ) observed across all compounds included in the meta-analysis. The results of the adjunctive therapy studies across compounds were markedly homogeneous $(\mathrm{Q}=8.2, \mathrm{df}=9, \mathrm{p}=0.51)$.

Discussion: The asenapine clinical program reveals statistically superior results compared with placebo in the treatment of acute bipolar mania, considering both the meta-analysis of the 2 monotherapy studies as well as the result of the single asenapine adjunctive therapy study in patients showing incomplete response to lithium or valproate monotherapy. When treating patients, the choice is not between active treatment and placebo, it is a choice among available options that are generally well tolerated and that demonstrate efficacy superior to placebo. When considered in light of published clinical trials indicating that asenapine appears to be well tolerated in patients with bipolar mania, these meta-analyses are instructive in revealing that, within the class of atypical antipsychotics, the efficacy of asenapine is similar to that of other treatment options compared with placebo for both monotherapy and adjunctive therapy.

Disclosure: H. Fennema: Part 5; Merck Sharp \& Dohme. B. Slaap: Merck Sharp \& Dohme. C. Karson: Merck. A. Szegedi: Merck. 
48. Efficacy and Safety of the Novel Triple Re-Uptake Inhibitor EB-1010 in the Treatment of Patients with Major Depressive Disorder

Pierre Tran*, Phil Skolnick, Pal Czobor, Nuoyu Norman Huang, Mark Bradshaw, Anthony McKinney, Frank Bymaster, Maurizio Fava

Euthymics Bioscience Inc., Cambridge, MA

Background: EB-1010 (formerly known as DOV21,947) is a novel, unbalanced triple monoamine uptake inhibitor with a relative potency to inhibit serotonin, norepinephrine, and dopamine uptake of $\sim 1: 2: 8$, respectively. It has shown antidepressant-like activity in relevant animal models. The present study tested the hypothesis that the broad spectrum mechanistic profile of EB-1010 also translates into unique antidepressant activities in humans, with perhaps enhanced efficacy such as improvement of anhedonia (a core symptom of depression), presumably linked to a deficit in mesocorticolimbic dopaminergic function, as well as a reduced liability to induce sexual dysfunction, weight gain, and cognitive dysfunction, adverse events typically associated with selective serotonin reuptake inhibition.

Methods: The study was a 6-week, multicenter, randomized, doubleblind, parallel, placebo-controlled study of 63 patients with Major Depressive Disorder (MDD). Eligible patients (HAMD-17 $\geq 22$ at baseline) were randomized to receive either EB-1010 $25 \mathrm{mg}$ b.i.d. for 2 weeks and then $50 \mathrm{mg}$ b.i.d. for 4 weeks or placebo. Key outcome measures included the MADRS (primary), the HAMD-17, the CGI-I, and the Derogatis Interview for Sexual Functioning Self-Report (DISF-SR).

Results: The intent-to-treat (ITT) population $(n=56)$ showed the following combined (placebo and EB-1010) mean baseline scores on the main outcome measures: MADRS (31.4) (primary); HAMD-17 (29.5) (secondary); and DISF-SR (25.8). At the end of the double-blind treatment (Week 6), the estimated LS mean change from baseline (MMRM or mixed model repeated measures) in the MADRS total scores was statistically significantly superior for EB-1010 when compared to placebo $(-12.5$ vs. $-10.2 ; \mathrm{p}=0.028)$, with an overall statistical effect size of -0.63 (Cohen's $d$ ). When assessed with the CGII, a global impression scale sensitive to clinically relevant changes in improvement status, treatment with EB-1010 was also statistically significantly superior to placebo $(\mathrm{p}=0.03$; Week 6; MMRM). An anhedonia factor score grouping Items 1 (apparent sadness), 2 (reported sadness), 6 (concentration difficulties), 7 (lassitude), and 8 (inability to feel) of the MADRS demonstrated a statistically significant difference in favor of EB-1010 in comparison to placebo ( $p=0.049$ ). EB-1010 was relatively well tolerated. Two patients in each treatment group discontinued the study early due to AEs but no serious AEs were reported. The EB-1010 group reported modest and clinically insignificant increases in heart rate, consistent with norepinephrine uptake inhibition. Rates of sexual dysfunction (as assessed with the DIS-SF) and weight changes were similar between the two treatment groups.

Discussion: The results of this small placebo-controlled study suggest that EB-1010 is efficacious in the treatment of patients with MDD and well-tolerated at a dose of $100 \mathrm{mg} /$ day. Consistent with its triple reuptake inhibition properties, patients treated with EB-1010 showed improvement in anhedonia, in addition to the overall core symptoms of depression. Furthermore, treatment with EB-1010 does not appear to be associated with weight gain or sexual dysfunction. Further studies in MDD will be conducted to confirm these findings.

Disclosure: P. Tran: Part 1; Cortex Pharmaceuticals. Part 5; Euthymics Bioscience Inc. P. Skolnick: Part 3; DOV Pharmaceutical. P. Czobor: DOV Pharmaceutical. N. Huang: DOV Pharmaceutical. Part 5; QRx Pharma. M. Bradshaw: Part 3; Euthymics Bioscience Inc. Part 5; GCPMB Consulting LLC. A. McKinney: Euthymics Bioscience Inc. F. Bymaster: Euthymics Bioscience Inc. M. Fava: Part 1; Abbott; Alkermes; Aspect Medical Systems; AstraZeneca; BioResearch; BrainCells; Bristol-Myers Squibb; Cephalon; Clinical Trials Solutions; Clintara, Covidien; Eli Lilly; EnVivo; Forest; Ganeden Biotech; GlaxoSmithKline; Johnson \& Johnson, Lichtwer Pharma; Lorex Pharmaceuticals; NARSAD; NCCAM; NIDA; NIMH; Novartis;
Organon; PamLab; Pfizer; Pharmavite; Roche; RCT Logic; SanofiAventis, Shire; Solvay; Synthelabo; Wyeth-Ayerst; Abbott; Affectis Pharmaceuticals; Amarin Pharma; Aspect Medical Systems; AstraZeneca, Auspex Pharmaceuticals; Bayer AG; Best Practice Project Management; BioMarin Pharmaceuticals; Biovail; BrainCells; BristolMyers Squibb; CeNeRx BioPharma; Cephalon, Clinical Trials Solutions; CNS Response; Compellis Pharmaceuticals; Cypress Pharmaceutical; Dov Pharmaceuticals; Eisai; Eli Lilly; EPIX Pharmaceuticals, Euthymics Bioscience; Fabre-Kramer Pharmaceuticals; Forest.; GenOmind; GlaxoSmithKline; Gruenthal GmbH; Janssen; Jazz Pharmaceuticals, Johnson \& Johnson; Knoll Pharmaceuticals; Labopharm; Lorex Pharmaceuticals; Lundbeck.; MedAvante; Merck, Neuronetics; Novartis; Nutrition 21; Organon; PamLab; Pfize; PharmaStar; Pharmavite; Precision Human Biolaboratory; Prexa Pharmaceuticals, PsychoGenics; Psylin Neurosciences; Rexahn Pharmaceuticals; Ridge Diagnostics; Roche; RCT Logic; Sanofi-Aventis; Sepracor; ScheringPlough, Solvay; Somaxon Pharmaceuticals; Somerset Pharmaceuticals; Synthelabo; Takeda Pharmaceutical; Tetragenex Pharmaceuticals, TransForm Pharmaceuticals; Transcept Pharmaceuticals.; Vanda Pharmaceuticals; Wyeth-Ayerst; Adamed, Co; Advanced Meeting Partners; American Psychiatric Assn, American Society of Clinical Psychopharmacology; AstraZeneca; Belvoir Media Group; Boehringer Ingelheim; Bristol-Myers Squibb; Cephalon; Eli Lilly; Forest, GlaxoSmithKline; Imedex; MGH Psychiatry Academy/Primedia; MGH Psychiatry Academy/Reed Elsevier; Novartis; Organon; Pfizer; PharmaStar; United BioSource, Wyeth-Ayerst; Compellis, Patent for SPCD \& patent application for a combination of azapirones \& bupropion in MDD, copyright royalties for the MGH CPFQ, SFI, ATRQ, DESS, \& SAFER. Part 4; Bristol-Myers Squibb, PamLab, GlaxoSmithKline, Johnson \& Johnson, Pfizer, AstraZeneca, Novartis, Healthcare Technologies, BrainCells, Eli Lilly, Sanofi Aventis.

49. Glycine Transporter Type 1 (GLYT1) Inhibitor RG1678: Positive Results of the Proof-of-Concept Study for the Treatment of Negative Symptoms in Schizophrenia

Daniel Umbricht*, Kisook Yoo, Eriene Youssef, Ernest Dorflinger, Meret Martin-Facklam, Alexander Bausch, Richard Arrowsmith, Daniela Alberati, Stephen Marder, Luca Santarelli

\section{F. Hoffmann-La Roche Ltd, Basel, Switzerland}

Background: Deficient signaling through the N-methyl-D-aspartate (NMDA) receptor has been hypothesized to be a key factor underlying many signs and symptoms of schizophrenia. Thus, increasing NMDA receptor function pharmacologically is thought to compensate for the deficient receptor signaling and to provide a way to better manage the symptoms of schizophrenia. Targeting the allosteric glycine site of the NMDA receptor has been proposed as an approach to enhance NMDA receptor functioning. RG1678 is a potent and non-competitive inhibitor of glycine transporter type 1 (GlyT1) with more than 1000fold selectivity for human GlyT1 versus GlyT2, and at least 30o-fold greater selectivity over other targets. Preclinically, RG1678 shows efficacy in assays of NMDA receptor functioning and schizophrenia models, and increases cerebrospinal fluid glycine levels in a dosedependent fashion.

Methods: The effects of RG1678 on negative symptoms of schizophrenia were investigated in a Phase IIb proof-of-concept study. Clinically stable patients with predominant negative symptoms were randomized equally to 8 weeks of treatment with three doses of RG1678 (10 mg, $30 \mathrm{mg}, 60 \mathrm{mg}$ ) or placebo once daily as adjunct to ongoing treatment with second-generation antipsychotics. Efficacy parameters were change from baseline in Positive and Negative Syndrome Scale (PANSS) negative symptom factor score (NSFS), proportion of responders defined as patients showing $\geq 20 \%$ improvement in NSFS, and Clinical Global Impression (CGI)-Improvement in Negative Symptoms. Functional effects were assessed with the Personal and Social Performance (PSP) scale. Populations analyzed included 
intent-to-treat (ITT) and per protocol (PP; patients who completed 8 weeks of treatment without any major protocol violations).

Results: 323 patients were randomized (male, 64\%; Caucasian, 66\%; mean age, $39.9 \pm 10.1$ [SD] years; mean duration of illness, $11.7 \pm 8.9$ years; PANSS total, 79.2 \pm 9.3; PANSS NSFS, $26.1 \pm 3.9$; PANSS positive symptom factor score, $17.7 \pm 3.6$ ). The ITT population included 312 patients and the PP population 231 patients (placebo, $\mathrm{n}=61 ; 10 \mathrm{mg}$, $\mathrm{n}=60 ; 30 \mathrm{mg}, \mathrm{n}=57 ; 60 \mathrm{mg}, \mathrm{n}=53$ ). Overall, efficacy parameters were consistently improved in the $10 \mathrm{mg}$ dose group, to a lesser extent in the $30 \mathrm{mg}$ dose group and not so in the $60 \mathrm{mg}$ dose group. The NSFS showed a significantly greater decrease from baseline in the $10 \mathrm{mg}$ and $30 \mathrm{mg}$ dose groups $(\Delta$ in both dose groups $=25 \%$ ) than in the placebo group $(\Delta=19 \%)$ in the PP population (10 mg, $\mathrm{p}=0.049 ; 30 \mathrm{mg}$, $\mathrm{p}=0.034)$ at week 8 . In the ITT population, the reduction in NSFS was slightly weaker and approached significance at endpoint in both dose groups (10 mg: $\mathrm{p}=0.071 ; 30 \mathrm{mg}: \mathrm{p}=0.090$ ). Effect sizes were $\sim 0.3$ for both dose groups in both populations. The percentage of responders in the PP population was significantly higher in the $10 \mathrm{mg}$ dose group than in the placebo group ( $65 \%$ vs $43 \% ; p=0.013$ ). The same measure approached significance in the ITT population $(58 \%$ vs $44 \%$; $\mathrm{p}=0.078)$. Differences in the distribution of the CGI-Improvement in Negative Symptoms were significant between the $10 \mathrm{mg}$ dose group and the placebo group in both populations (ITT, $\mathrm{p}=0.021$; PP, $\mathrm{p}=0.025$ ) as more patients in the $10 \mathrm{mg}$ dose group were rated as 'much improved' or 'very much improved' than in the placebo group at endpoint. The same comparison approached significance for the $30 \mathrm{mg}$ dose group in the PP population $(\mathrm{p}=0.061)$. Compared with placebo, there was a trend towards functional improvement as evidenced by an increase in PSP score from baseline to week 8 in the $10 \mathrm{mg}$ dose group in the PP population $(\mathrm{p}=0.072)$. The $60 \mathrm{mg}$ dose group did not differ from placebo in any outcome measure. RG1678 was well tolerated. The percentage of patients withdrawn due to adverse events ranged from $0 \%$ (placebo) to $9 \%(60 \mathrm{mg})$. The percentage of withdrawals for any reason was similar in all four treatment groups $(13-20 \%)$.

Discussion: RG1678 demonstrated a consistent and clinically meaningful reduction in negative symptoms that was accompanied by the emergence of a positive effect on functionality. This proof-of-concept study provides clinical support for glycine reuptake inhibition and enhancement of NMDA signaling as a therapeutic approach for negative symptoms in patients with schizophrenia, further strengthening the NMDA hypothesis of schizophrenia. Additionally, to our knowledge RG1678 is the first compound in clinical development to demonstrate convincing efficacy against negative symptoms. If these findings are confirmed in Phase III trials, this agent has the potential to expand the therapeutic options for patients with schizophrenia. Reference: 1. Marder et al. J Clin Psychiatry 1997; 58: 538-546.

Disclosure: D. Umbricht: Part 5; F. Hoffmann - La Roche, Ltd. K. Yoo: F. Hoffmann - La Roche, Ltd. E. Youssef: F. Hoffmann - La Roche, Ltd. E. Dorflinger: F. Hoffmann - La Roche, Ltd. M. Martin-Facklam: F. Hoffmann - La Roche, Ltd. A. Bausch: F. Hoffmann - La Roche, Ltd. R. Arrowsmith: F. Hoffmann - La Roche, Ltd. D. Alberati: F. Hoffmann La Roche, Ltd. S. Marder: Part 1; F. Hoffmann - La Roche, Ltd., Wyeth, Pfizer, Schering Plough, Otsuka, Sanofi Aventis, Lundbeck, BristolMeyers Squibb. Part 2; Medavante. L. Santarelli: Part 5; F. Hoffmann La Roche, Ltd.

50. ITI-007, an Investigational New Antipsychotic Drug with a Novel Pharmacological Profile, is Safe and Well-Tolerated with Early Clinical Signs for Efficacy in Patients with Stabilized Schizophrenia Kimberly E. Vanover*, Robert E. Davis, Larry Ereshefsky, Lev Gertsik, Amir E. Ettekal, Paul Greengard, Sharon Mates

Intra-Cellular Therapies, Inc., New York, NY

Background: ITI-007 is an investigational new drug for the treatment of schizophrenia and related neuropsychiatric disorders with potential efficacy in depression and insomnia, based on animal models and emerging clinical data. ITI-007 is a potent $5-\mathrm{HT}_{2} \mathrm{~A}$ receptor antagonist with activity as a mesolimbic/mesocortical selective dopamine phosphoprotein modulator (DPPM), consistent with presynaptic D2 receptor partial agonism and postsynaptic D2 receptor antagonism in vivo. ITI-007 also demonstrates mesolimbic selectivity as it stimulates the phosphorylation of NMDA NR2B receptors downstream of $\mathrm{D}_{1}$ receptor activation. Additionally, ITI-oo7 inhibits reuptake of serotonin. This pharmacological profile is predicted to translate clinically as an antipsychotic drug with broad spectrum efficacy against positive and negative symptoms. Reduced affinity for off-target receptors such as alpha1, $\mathrm{H}_{1}$, and $\mathrm{mACh}$ receptors predicts improved tolerability compared to currently available antipsychotic drugs. Previous Phase II clinical data demonstrated that low doses of ITI-007 (1 - 10 mg) improved sleep in patients with insomnia. The purpose of the present study was to determine the safety, tolerability and pharmacokinetic profile of ITI-007 in patients with stabilized schizophrenia. Exploratory measures to assess signals of clinical efficacy included the Positive and Negative Syndrome Scale (PANSS) and the Calgary Depression Scale for Schizophrenia (CDSS).

Methods: The design was an inpatient, single site, double-blind, randomized, placebo-controlled study. Patients with schizophrenia or schizoaffective disorder, who were stable with regard to their psychotic symptoms, were washed off their previous antipsychotic medication for at least 4 days before randomization to ITI-0o7 ( $N=8 /$ cohort; 6:2, drug:placebo). The starting dose was $30 \mathrm{mg}$ ITI-007. Doses were increased in successive cohorts (i.e., 30, 40, 60, 80, 100, and $140 \mathrm{mg}$ ITI-007) after review of safety data from previous cohort(s). Subjects received ITI-007 (or placebo) once daily in the morning for 5 consecutive days. The primary objective was to determine the safety and tolerability of ITI-007 in the target patient population. Safety assessments included vital signs, clinical laboratory tests (hematology, serum chemistry, urinalysis), 12-lead electrocardiograms (ECGs), and the Columbia-Suicide Rating Scale. Additionally, the Simpson-Angus Scale and Barnes Akathisia Rating Scale were included at the highest dose levels. Tolerability was assessed by reported or observed adverse events for all subjects throughout the study. Serial blood samples were collected on Study Day 1 and Study Day 5 for pharmacokinetic analysis. Exploratory efficacy measures included the PANSS and the CDSS.

Results: The trial met its primary objective by demonstrating that ITI007 at doses up to and including $140 \mathrm{mg}$ was safe and well-tolerated with repeated administration in patients with stabilized schizophrenia. There were no drug-related serious adverse events. There were no safety concerns with respect to vital signs, 12-lead ECGs, or clinical chemistries. Interestingly, total cholesterol and triglyceride levels decreased following withdrawal from previous antipsychotic medications and while being treated with ITI-007. All adverse events were mild to moderate. Notably, there were no extrapyramidal side effects including no akathisia. ITI-007 plasma concentrations increased with increasing doses. Exploratory analyses indicated that total PANSS scores improved with ITI-007 administration. Furthermore, patients who had been randomized to ITI-007 also exhibited improvements in symptoms of depression.

Discussion: The present results demonstrate that ITI-007 is safe and well-tolerated across a wide range of doses in patients with stabilized schizophrenia. The lack of extrapyramidal side effects with ITI-007 is consistent with its mesolimbic/mesocortical selectivity. Surprisingly, after only 5 days of administration, ITI-007 showed signals consistent with efficacy with improved total PANSS scores and improved symptoms of depression on the CDSS. Although the present study was not powered to measure statistically significant effects on these measures, the data are encouraging and will be evaluated more fully in future efficacy studies. Given the pharmacodynamic effects across a wide range of doses and sequential engagement of pharmacological targets as the dose increases, further exploration of dose-response relationships will guide future dose selection for the potential treatment of a number of psychiatric and neurological disorders. 


\section{S322}

Disclosure: K. Vanover: Part 5; Intra-Cellular Therapies, Inc. R. Davis: Part 1; Intra-Cellular Therapies, Inc. Part 5; 3-D Pharmaceutical Consultants. L. Ereshefsky: PAREXEL International and California Clinical Trials Medical Group. L. Gertsik: California Clinical Trials Medical Group. A. Ettekal: California Clinical Trials Medical Group. P. Greengard: Part 1; Intra-Cellular Therapies, Inc., Envoy Therapeutics, PsychoGenics, Neurologix. Part 2; Intra-Cellular Therapies, Inc., Envoy Therapeutics, PsychoGenics, Neurologix. S. Mates: Part 5; IntraCellular Therapies, Inc.

\section{The MARQ-AD: A New Measure of Interview Quality in Alzheimer's Disease Trials}

Christopher Weber, Louis Kirby, Janet Williams*

\section{MedAvante, Inc., Hamilton, NJ}

Background: Clinician-administered cognitive assessments in Alzheimer's disease are used to detect treatment effects, but can also diminish or obscure a treatment effect when scale rating variability is introduced. It has been demonstrated that interview quality is directly related to better detection of a treatment effect. A quality measurement tool to assess and improve test instrument administration would be a desirable goal given the high stakes in Alzheimer's disease clinical trials. The MedAvante Analysis of Rating Quality - Alzheimer's Disease (MARQ-AD) was developed specifically for Alzheimer's rating scales to quantify critical domains of rating/interview quality and identify administration and scoring deviations encountered in $\mathrm{AD}$ assessments in randomized clinical trials. This poster presents the development of the MARQ-AD scale and a review of clinician training on the scale. The MARQ-AD is an integral component of the MedAvante quality control process, which mirrors the successful use of a similar process applied to psychiatric trials.

Methods: The MARQ-AD was developed for Alzheimer's rating scales from the Rater Applied Performance Scale (RAPS), a scale developed for psychiatric ratings3. Seven doctoral level psychometricians with at least 7 years of AD assessment experience ("Trainers") were selected to perform independent rater evaluations using the MARQ-AD. Each Trainer learned the administration and scoring parameters of the MARQ-AD as applied to the MMSE, ADAS-cog, CDR, ADCS-ADL, and ADCS-CGIC. Trainers then reviewed and rated a series of videos of outside clinicians performing actual patient and/or caregiver interviews. The Trainers independently scored the interviews along the specified MARQ domains, and the scores were collated. After each set of videos, the group of trainers compared scores and discussed their ratings. Variability of ratings prompted revisions to the MARQ-AD that were then tested in the next set of interviews. We present data from a calibration exercise, where Trainers viewed and scored the MMSE, ADAS-cog, CDR, ACDS-ADL, and ADCS-CGIC with accompanying MARQ-AD scores for an AD patient presenting with mild dementia symptomatology. An average domain score for each MARQ$\mathrm{AD}$ was calculated, and the assessment was assigned a "Meets Criteria" or "Does Not Meet Criteria" rating, the latter of which may indicate the need for rater remediation.

Results: The average MARQ-AD scores $(n=105)$ were examined for each completed assessment (35 MMSE, 35 ADAS-cog, 35 CDR, 35 ADCS-ADL, and 35 ADCS-ADL), and the independently-derived MARQ-AD scores showed good agreement across the 7 Trainers. An algorithm for determining whether or not a site rater needs remediation ("Meets Criteria" or "Doesn't Meet Criteria") was established. Trainers indicated high agreement on the Meets Criteria/ Does Not Meet Criteria on all 5 assessments. Training and calibration data (e.g., "Meets Criteria" or "Doesn't Meet Criteria") are presented across scales and MARQ-AD domains. Data collection is ongoing and poster will include updated results and analysis.

Discussion: The MARQ-AD is derived from the RAPS, a validated instrument for assessment of the quality of administration of psychiatric rating scales. After group calibration, qualified Trainers who participated in MARQ-AD training achieved nearly $100 \%$ agreement when reviewing a recording of common $\mathrm{AD}$ scales. The MARQ-AD could become integral to an ongoing quality control process incorporated into an $\mathrm{AD}$ clinical trial with the goal of reducing assessment scale variability, making detection of a true treatment effect more possible.

Disclosure: C. Weber: Part 1; MedAvante, Inc. Part 2; MedAvante, Inc. Part 3; MedAvante, Inc. Part 5; MedAvante, Inc. L. Kirby: Part 1; Neuraltus Pharmaceuticals. Part 2; Neuraltus Pharmaceuticals, Critical Path Institute, Phoenix Neurology. Part 3; Neuraltus Pharmaceuticals. J. Williams: Part 1; MedAvante, Inc. Part 2; MedAvante, Inc. Part 3; MedAvante, Inc. Part 5; MedAvante, Inc.

\section{Weight Change and Metabolic Effects of Asenapine in Placebo- or Olanzapine-Controlled Studies \\ Jun Zhao*, Paul Van Hoek, Pilar Cazorla, Joep Schoemaker, Mary Mackle, John Panagides, Craig Karson, Armin Szegedi}

Merck, Rahway, NJ, Merck Sharp \& Dohme, Oss, Netherlands

Background: The weight change and metabolic effects of atypical antipsychotics vary considerably. We present results of post hoc analyses of the weight and metabolic effects of the atypical antipsychotic asenapine ( 5 or $10 \mathrm{mg}$ twice daily [BID]) in adults.

Methods: Data were from a pooled database of 1748 patients (asenapine, $\mathrm{n}=989$; placebo, $\mathrm{n}=759$ ) who participated in trials that included a placebo control and 3430 patients (asenapine, $\mathrm{n}=2067$; olanzapine, $\mathrm{n}=1363$ ) who participated in trials that included an olanzapine control (other active comparators were not included in these analyses). A total of 13 schizophrenia and 4 bipolar disorder trials were included, of which 4 trials (2 schizophrenia and 2 bipolar disorder) included both placebo- and olanzapine-controls. Study duration ranged from 1 to 6 weeks in trials with a placebo control and 3 to $>100$ weeks in trials with an olanzapine control (including extension studies). In $15 / 17$ trials, asenapine doses were 5 or $10 \mathrm{mg}$ BID (2-20 $\mathrm{mg}$ BID in 2 clinical pharmacology studies) and olanzapine doses were 5-20 mg QD. Assessments included change from baseline to endpoint and other intermittent time points for weight, body mass index (BMI), and fasting lipid and glucose levels. Inferential analyses used an analysis of variance model with terms for protocol and treatment. $P$-values were independently assessed for asenapine versus placebo or asenapine versus olanzapine using only the data relevant for each comparison. Categorical analyses of these changes based on predefined baseline categories were also conducted.

Results: Baseline demographics were generally similar between treatment groups in trials with a placebo control or trials with an olanzapine control. In trials with a placebo control, mean asenapine dose was $16.2 \mathrm{mg} / \mathrm{d}$ (modal dose, $20 \mathrm{mg} / \mathrm{d}$ ); mean (median) treatment duration was 25.1 (21.0) and 24.8 (21.0) days for asenapine and placebo, respectively. In trials with an olanzapine control, mean dose was $15.3 \mathrm{mg} / \mathrm{d}$ for asenapine (modal dose, $20 \mathrm{mg} / \mathrm{d}$ ) and $15.0 \mathrm{mg} / \mathrm{d}$ for olanzapine (modal dose, $20 \mathrm{mg} / \mathrm{d}$ ); mean (median) treatment duration was 207.3 (96.0) and 226.2 (168.0) days, respectively. At endpoint, the least squares (LS) mean \pm SE weight change with asenapine was significantly greater than with placebo $(1.2 \pm 0.2$ vs $0.1 \pm 0.2 \mathrm{~kg}$; $P<0.0001)$ and significantly less than with olanzapine (0.9 \pm 0.1 vs $3.1 \pm 0.2 \mathrm{~kg} ; P<0.0001)$. LS mean changes in BMI and weight across BMI categories at endpoint in trials with a placebo control and trials with an olanzapine control were consistent with observed weight changes, with the exception that the BMI change in patients with baseline BMI $<18.5 \mathrm{~kg} / \mathrm{m}^{2}$ was not significantly greater with asenapine than placebo due to small sample size. LS mean change in total cholesterol, low-density lipoprotein (LDL), and high-density lipoprotein (HDL) at endpoint was not significantly different between asenapine and placebo; LS mean \pm SE change in triglycerides significantly differed for asenapine versus placebo $(1.8 \pm 6.3$ vs $-12.2 \pm 5.9 \mathrm{mg} / \mathrm{dL} ; P<0.05)$. Percentages of patients with clinicallyrelevant lipid value changes in categorical analyses tended to be higher with asenapine than placebo, with the exception of LDL for which no 
differences were seen. LS mean \pm SE change was significantly lower with asenapine than olanzapine for total cholesterol $(-0.4 \pm 1.1$ vs $6.2 \pm 1.2 \mathrm{mg} / \mathrm{dL}, P<0.0001)$, LDL (-0.3 \pm 1.1 vs $3.1 \pm 1.2 \mathrm{mg} / \mathrm{dL}, P<0.05)$, and fasting triglycerides (-0.9 \pm 5.4 vs $24.3 \pm 5.8 \mathrm{mg} / \mathrm{dL}, P<0.0001) ; \mathrm{LS}$ mean \pm SE change in HDL was significantly greater with asenapine than olanzapine $(1.3 \pm 0.4$ vs $-0.2 \pm 0.4 \mathrm{mg} / \mathrm{dL}, P<0.01)$. Lipid changes for asenapine versus olanzapine in categorical analyses reflected the mean treatment group changes in nearly every category. At endpoint, LS mean \pm SE change in fasting glucose was significantly higher for asenapine versus placebo $(1.9 \pm 1.7 \mathrm{vs}-1.6 \pm 1.5 \mathrm{mg} / \mathrm{dL} ; P<0.05)$ but was numerically lower for asenapine versus olanzapine $(2.0 \pm 1.3$ vs $3.3 \pm 1.3 \mathrm{mg} / \mathrm{dL}$ ). Categorical analyses of fasting glucose changes were consistent with the mean treatment group findings in trials with a placebo control and trials with an olanzapine control.

Discussion: These post hoc pooled analyses support published reports of individual clinical trials and suggest that, on average, asenapine was associated with moderate weight gain and increased fasting triglyceride and glucose levels compared with placebo. Compared with olanzapine, asenapine had a lower propensity to induce weight gain or to increase serum lipids including triglycerides, LDL, and cholesterol.

Disclosure: J. Zhao: Part 5; Merck. P. van Hoek: Merck Sharp \& Dohme. P. Cazorla: Merck. J. Schoemaker: Merck Sharp \& Dohme. M. Mackle: Merck. J. Panagides: Merck at the time this research was conducted. C. Karson: Merck. A. Szegedi: Merck.

53. Effect of Genetic Variation in $\mathrm{KCNH} 2$ on Antipsychotic Treatment in Schizophrenia

Fengyu Zhang*, Jose Apud, Kristin Bigos, Heather Decot,

Radhakrishna Vakkalanka, Daniel Weinberger

National Institutes of Health, Bethesda, MD

Backgroud: $\mathrm{KCNH} 2$ (7q36.1) encodes Herg1, a voltage-gated potassium channel that plays a key role in regulating excitability and repolarization of cardiac muscle and of neurons. Multiple genetic variants in $\mathrm{KCNH}_{2}$ are associated with cardiac arrhythmias and sudden death and antipsychotic drugs bind to Herg channels and produce QT interval prolongation as a result. Recently, several SNPs in $\mathrm{KCNH}_{2}$ that predict expression of a novel, brain and primate specific isoform of $\mathrm{KCNH}_{2}$ with unique physiologic properties have been associated with risk for schizophrenia and also with cortical physiology, and cognition, suggesting that this isoform of $\mathrm{KCNH}_{2}$ might be involved in the therapeutic actions of antipsychotic drugs [1]. We evaluated the impact of genetic variation in $\mathrm{KCNH}_{2}$ related to expression of this novel isoform in a pharmacogentic study of response to antipsychotic drug therapy.

Methods: Three independent cohorts of patients undergoing antipsychotic treatment trials were included in this study. The first two cohorts consisted of 69 Caucasian individuals from the NIMH Clinical Brain Disorder Branch (CBDB) inpatient antipsychotic response study who completed a double blind placebo controlled cross-over design: Subgroup $1(\mathrm{~N}=34)$ of these patients received sequentially 4-weeks of placebo and then four weeks of standard antipsychotic drug therapy; subgroup $2(\mathrm{~N}=17)$ received the inverse sequence, i.e. standard antipsychotic treatment for four weeks followed by placebo. Positive and Negative Symptom Scale ratings (PANSS) were assessed once or twice a week to evaluate treatment response. The second cohort consisted of patients randomly assigned to olanzapine during the Phase $1 / 1 \mathrm{~A}$ of the Clinical Antipsychotic Trials of Intervention Effectiveness (CATIE) Study [2-4]. Primary outcomes in the CATIE sample included time to medication discontinuation, mostly due to lack of efficacy or side-effects, and PANSS ratings. We genotyped three SNPs (rs3900779, s748693 and rs1036145) in KCNH2 which have been associated with schizophrenia and with expression of the novel $\mathrm{KCNH} 2$ isoform in human brain (1) and analyzed the data using a general linear model, log-rank test and COX proportional hazard model while potential covariates were controlled.
Results: All three SNPs affected PANSS outcome ratings of treatment response in all three datasets. In the CBDB dataset, we observed very significant effects of rs3800779, rs748693 and rs1036145 (all p values $<0.001$ ) on negative symptoms in subgroup 1 and modestly significant (all p values <0.05) in the smaller group 2 sample. No effect of these SNPs on positive symptoms was found in either subgroup of patients. The findings were replicated in the Caucasian sample $(\mathrm{n}=89)$ from CATIE. $r s 3800779(p=0.0684$ two tailed $), \operatorname{rs} 748693(p=0.0431)$ and rs1036145 $(\mathrm{p}=0.0755)$ were associated with negative symptom response, but not with positive symptoms. Log-rank test of time to discontinuation of medication showed that individuals with rs 748693 genotype GA were less likely than those with AA genotype to discontinue treatment $(\mathrm{p}=0.0317)$, while at $\mathrm{rs1036145}$ genotype TT $(\mathrm{p}=0.0078)$ was less likely than CC to discontinue. After controlling for other covariates including age, sex and drug clearance in the Cox model, the Hazard ratio of GA versus AA at rs748693 is 0.255 $(\mathrm{p}=0.0308)$, and TT vs CC at $\mathrm{rs1036145}$ is $0.209(\mathrm{p}=0.0335)$. In the African American sample $(n=47)$ from the CATIE trial, significant associations with positive $(\mathrm{p}<0.05)$ and suggestive association with negative symptoms $(\mathrm{p}<0.08)$ response were found for both $\mathrm{rs} 3000779$ and for rs748693 genotype, but not for rs1036145 genotype. No SNP was associated with time to discontinuation of medication in the African American sample.

Discussion: We found consistent evidence in three independent cohorts that genetic variation in $\mathrm{KCNH}_{2}$ associated wtih schizophrenia and with brain function and with expression of a novel brain specific Herg channel also affect antipsychotic treatment response in patients with schizophrenia. These data raise intriguing questions about the role of Herg channels in the mechanism of action of antipsychotic drugs and in a possible novel target for drug discovery. Further analysis of the full CATIE sample is ongoing.

Disclosure: F. Zhang: None. J. Apud: None. K. Bigos: None. H. Decot: None. R. Vakkalanka: None. D. Weinberger: None.

54. Results from Two Double-Blind, Placebo-Controlled Studies of the Novel NK1 Receptor Antagonist Casopitant in Patients with Major Depressive Disorder

Emiliangelo Ratti*, Kevin Bellew, Paolo Bettica, Heather Bryson, Stefano Zamuner, Graeme Archer, Lisa Squassante, Alan Bye, John Travers, David Trist, Sofia Fernandes, Ranga Krishnan

\section{GlaxoSmithKline, Verona, Italy}

Background: Clinical study results for neurokinin (NK) receptor antagonists in the treatment of depression have been mixed, with Phase III studies failing to fulfill the early promise demonstrated in Phase II studies. Casopitant, a selective $\mathrm{NK}_{1}$ antagonist shown to achieve nearly complete receptor occupancy was studied in two randomized, placebo-controlled, double-blind, Phase II trials in a total of 694 depressed outpatients to test the hypothesis that nearly complete $\mathrm{NK}_{1}$ receptor occupancy is required to achieve antidepressant efficacy.

Methods: Study 092 utilized an interactive voice response system to recruit depressed patients with baseline Hamilton Depression (17-item, HAMD17) total scores $\geq 24$ who were randomized to fixed dose casopitant $30 \mathrm{mg} /$ day, $80 \mathrm{mg} /$ day or placebo for 8 weeks. Study 096 required Carol Depression Scale-Revised self-assessment (CDS-R) scores of $\geq 24$ for randomization to casopitant $120 \mathrm{mg} /$ day, paroxetine $30 \mathrm{mg} /$ day (both reached via forced titration) or placebo for 8 weeks. Results: In Study 092, casopitant $80 \mathrm{mg}$ but not $30 \mathrm{mg}$ achieved statistically significant improvement vs. placebo on the primary outcome measure, Week 8 LOCF change from baseline HAMD17 (difference $=-2.7,95 \%$ CI -5.1 to $-0.4, p=0.023$ ). In study 096, neither casopitant nor paroxetine achieved statistical separation from placebo at end point on HAMD17 (casopitant difference $=-1.7,95 \%$ CI -3.8 to $0.4, \mathrm{p}=0.282$ ). Post hoc analyses of patients with baseline HAMD17 $\geq 20$ indicated a significant difference from placebo for casopitant 


\section{S324}

but not paroxetine. Casopitant and paroxetine were generally well tolerated.

Discussion: These studies suggest $\mathrm{NK}_{1}$ antagonists that have nearly complete receptor occupancy have the potential for efficacy in the treatment of depression.

Disclosure: E. Ratti: Part 5; GlaxoSmithKline. K. Bellew: GlaxoSmithKline. P. Bettica: GlaxoSmithKline. H. Bryson: GlaxoSmithKline, AstraZeneca. S. Zamuner: GlaxoSmithKline. G. Archer: GlaxoSmithKline. L. Squassante: GlaxoSmithKline. A. Bye: GlaxoSmithKline. J. Travers: GlaxoSmithKline. D. Trist: GlaxoSmithKline. S. Fernandes: GlaxoSmithKline. K. Krishnan: Part 1; GlaxoSmithKline, Cenerex, Orexigen, Cypress Bioscience, Sonexa, Alta, Roche, Eisai.

55. New Model Curriculums for Teaching Psychopharmacology to Residents, Medical Students, and Primary Care Prescribers Bruce Lydiard, Richard Balon*, Ira Glick

Wayne State University, Detroit, MI

Background: Over the last decade, there has been an explosion of new knowledge in neuroscience and clinical psychopharmacology. There has been a drastic drop in funds for psychiatric education in general and for psychopharmacology education (e.g., lectures) in particular. Other than the ASCP curriculum for teaching psychiatric residents, there has been no materials for teaching medical students or primary care physicians (who do a large share of prescribing). We present here the 6th edition of the resident curriculum and 1st editions for medical students and primary care M.D.'s.

Methods: A committee of the ASCP composed of directors of both resident education as well as medical student education educators have developed materials related to the "what, why, and how" to teach and evaluate. In addition, for each curriculum we included a core series of lectures developed by experts in their fields.

Results: Model Resident Curriculum; Medical Student Curriculum; Primary Care Curriculum.

Discussion: For teaching medical students, there has never been a generally accepted curriculum or set of teaching materials specifically designed for them. There is a great deal to teach in the four-year curriculum and medical students have widely divergent career paths. This curriculum has 22 lectures. For primary care physicians, we have developed a concise and useful curriculum and set of lectures. This curriculum has 18 lectures. For residents, the curriculum is now in its 6th edition and has 82 lectures and 4000 slides.

Disclosure: B. Lydiard: None. R. Balon: None. I. Glick: Part 1; Merck, Novartis, Janssen, BMS, AstraZeneca, Pfizer. Part 2; Johnson \& Johnson. Part 4; Lundbeck, GlaxoSmithKline, BMS, Pfizer.

\section{Dopaminergic Influences on Emotional Decision-Making in Euthymic Bipolar Patients}

Katherine Burdick*, Raphael Braga, Serge Sevy, Alison Gilbert, Walter Stearns, Anil Malhotra

\section{Zucker Hillside Hospital-NSLIJHS, Glen Oaks, NY}

Background: Dopamine is critical to normal cognitive function and reward-based learning. Although several studies in animals and humans demonstrate beneficial cognitive effects of pro-dopaminergic agents on measures of attention and working memory, there are also reports of rare but concerning side effects which include the induction of impulse control disorders in individuals with no prior history. Multiple case reports in Parkinson disease (PD) patients have implicated dopamine agonists, such as pramipexole, in the emergence of risk-seeking behaviors like pathological gambling. There are no published data on the effects of pramipexole on reward-based activities in other clinical samples.

Methods: We assessed reward-dependent decision-making in 30 euthymic patients with bipolar I disorder (BPD) randomized to either pramipexole or placebo. A comprehensive neurocognitive battery was administered at baseline and again at week 8, including a rewarddependent decision making task, the Iowa Gambling Task (IGT). The IGT requires subjects to choose cards from among four decks (2 risky and 2 conservative) and is designed to promote the subject's learning to choose from the advantageous (conservative) decks over time (5 blocks). IGT performance was analyzed first using repeated measures ANOVA with risky choice preference assessed across the 5 blocks. Subsequent analyses were carried out using the expectancyvalence model outcomes (recency, attention to gains vs. losses, and consistency) to determine strategies used on a trial-by-trial basis.

Results: 30 strictly defined euthymic bipolar I patients completed the IGT at both time points (17 placebo and 13 pramipexole). Patients had a mean age of $41.2+/-11.9$ years and were $63.3 \%$ female, $63.3 \%$ Caucasian and had a premorbid $\mathrm{IQ}=97.0+/-13.1$. Mood state was euthymic (mean Hamilton Depression rating $=4.1 /-2.4$; Clinician Administered Mania rating $=2.2=+/-2.0$ ). Baseline performance on the IGT did not differ significantly by drug condition $(\mathrm{F}=0.94 ; \mathrm{p}=0.46)$; however, at week 8 , BPD patients on pramipexole demonstrated a significantly greater tendency to make increasingly high-risk, high-reward choices across the five blocks of the test, while the placebo group's pattern was similar to that of healthy controls (treatment $\mathrm{x}$ time $\mathrm{x}$ block interaction $\mathrm{p}<0.05)$. Moreover, when evaluating the trial-by-trial approach used to make choices, the pramipexole group's performance was characterized by a change from baseline to week 8 which favored an abnormal attentional bias toward gains vs. losses. The opposite pattern of was noted in the placebo group (treatment $\mathrm{x}$ time interaction; $\mathrm{F}=4.7$; $\mathrm{p}=0.04$ ). There were no significant changes in mood symptom ratings from baseline to week 8 and no increased propensity toward maniclike behaviors were reported.

Discussion: Our results suggest that the enhancement of dopaminergic activity influences decision-making performance in euthymic BPD. Bipolar patients administered a dopamine agonist, pramipexole, displayed a greater propensity to use a high-risk, high-reward strategy in the context of a standardized gambling paradigm. These data suggest that dopaminergic tone may be critical in the risk-reward circuitry of patients with BPD.

Disclosure: K. Burdick: Part 1; Merck. R. Braga: None. S. Sevy: Abbott. A. Gilbert: None. W. Stearns: None. A. Malhotra: Eli Lilly, Janssen, Vanda Pharmaceuticals, Sunovion. Part 2; Merck, BMS, PGx Health.

\section{Neural Responses as a Function of Cognitive Coping Style During Emotion Processing \\ Astrid Veronika Rauch*, Jochen Bauer, Patricia Ohrmann, Carsten Konrad, Udo Dannlowski, Boris Egloff, Walter Heindel, Volker Arolt, Thomas Suslow}

Department of Psychiatry and Psychotherapy, University of Muenster, Muenster, Germany

Background: Coping is the process of attempting to control threat relevant sources and to regulate the emotional demands created by threatening situations. The model of coping modes introduces repression and sensitization as basis constructs for the description of dispositional habitual behavior tendencies in threatening situations. Repression designates coping strategies that aim to shield the organism from distressing stimuli by disregarding their aversive characteristics. In contrast, sensitization comprises coping strategies that are employed to reduce situational uncertainty such as analyzing the environment. So far very few studies exist in which brain activation has been examined as a function of coping style. In previous functional Magnetic Resonance Imaging (fMRI) studies, sensitizers tended to exhibit stronger neural responses in the amygdala to fearful faces compared with repressors, whereas they presented enhanced responsivity to angry faces in prefrontal areas. Repressors were cortically more responsive to fearful and happy facial expressions. During the identification of sad intonations repressors showed larger left than right hemisphere activation in temporo-parietal regions. Repressors manifested a discontinuity of memory processing for 
emotional information (encoding, storage, retrieval). The neural correlates of this discontinuity have not been fully investigated. The present study was designed to examine these neural correlates using fMRI.

Methods: Study participants were selected from a sample of 150 female students on the basis of their scores on the German version of the Mainz Coping Inventory. 20 repressors and 20 sensitizers participated in this study. All subjects were right handed and had no history of psychiatric or other relevant medical diseases. The event-related $3 \mathrm{~T}$ fMRI paradigm consisted of different emotional face conditions (angry, fearful, happy and neutral). Every emotional condition consisted of 30 trials. A fixation cross of $500 \mathrm{~ms}$ preceded the emotional face stimuli which were presented for $3000 \mathrm{~ms}$ followed immediately by a time interval $(3500 \mathrm{~ms})$ in which subjects were requested to evaluate the facial emotion expression. The subjects were instructed to memorize faces and evaluate the expression on a 4-point scale ranging from -1.5 to +1.5 (threatening or non-threatening) by button press. $\mathrm{T}_{2}{ }^{*}$ functional data were acquired at a 3 -Tesla scanner (Gyroscan Intera 3T, Philips Medical Systems, Best, NL). fMRI data were analyzed using Statistical Parametric Mapping (SPM5, Wellcome Department of Imaging Neuroscience, London). The emotion face conditions were contrasted with the neutral condition. Subjects performed two sessions of face recognition: after 30 minutes and after 3 days.

Results: The behavioral data showed no main or interaction effect of coping style for memory retrieval over time. There was a main effect of time for the two memory tasks. As could be expected retrieval of the original faces was better in the first memory task than in the second. The evaluation task did not show any significant group differences. Compared to sensitizers repressors exhibited a greater activation of frontal, parietal and temporal-occipital areas during encoding of angry, happy and fearful faces. The most obvious activation differences between repressors and sensitizers were found in case of angry faces.

Discussion: During encoding repressors did not withdraw their attention from emotional stimuli. Instead, they showed an increased activation in cortical areas. This heightened activation of frontal, parietal and temporal-occipital areas during encoding of emotional faces can be interpreted as an enhanced initial processing of emotional information in repression supporting the discontinuity hypothesis. But, the findings of the behavioral performances of the face recognition tasks are not consistent with this hypothesis. This could be due to the relatively small sample size (and a limited power to detect group differences) or the sole use of facial stimuli. Previous behavioral studies have investigated the discontinuity hypothesis with a larger number of participants, emotional verbal and pictorial stimuli. Knowledge about the neurobiology of cognitive coping style and its influence on emotion processing might be useful for a better understanding of development and therapy of psychiatric disorders, such as depression and anxiety disorders.

Disclosure: A. Rauch: None. J. Bauer: None. P. Ohrmann: None. C. Konrad: None. U. Dannlowski: None. B. Egloff: None. W. Heindel: None. V. Arolt: None. T. Suslow: None.

\section{Cognition and Behavioral Changes Occur Prior to Subjective Cognitive Impairment (SCI) in the Evolution of Brain Aging and Alzheimer's Disease (AD): $>25$ Years Prior to the Advent of Mild Dementia}

Barry Reisberg*, Melanie Shulman, Carol Torossian, Isabel Monteiro, Istvan Boksay, Tariq Gill, Rinku Chakraborty, Mohammad Zeeshan, Wei Zhu

\section{New York University School of Medicine, New York, NY}

Background: Alzheimer's disease (AD) is recognized to be a gradual process with a progressive course. The overt dementia of $\mathrm{AD}$ can last $>13$ years. In 1988, we coined the terminology "mild cognitive impairment” (MCI) (Reisberg et al., Drug Dev Res, 1988) for Global
Deterioration Scale (GDS) (Reisberg et al., Am J Psychiatry, 1982) stage 3, lasting $\sim 7$ years (Reisberg et al., Geriatrics, 1986) prior to the dementia of AD. This MCI stage is marked by subtle, but manifest, clinical deficits. We subsequently characterized MCI in many ways (see Reisberg et al., Int Psychogeriatr, 2008, for a review). We have long identified a putative subjective cognitive impairment stage (SCI) (GDS stage 2) prior to the advent of MCI. In a 9 year longitudinal study, we have validated our 1986 estimate of a 15 year duration for the SCI stage prior to the MCI of AD (Prichep et al., Neurobiol Aging, 2006; Reisberg \& Gauthier, Int Psychogeriatr, 2008). Other studies in our subjects have demonstrated neurometabolic decrements (Mosconi, et al., Biol Psychiatry, 2008) and increments in urinary cortisol (Wolf et al., Neurobiol Aging, 2005), in SCI subjects in comparison with age matched, no cognitive impairment (NCI) (GDS stage 1) subjects. We have also recently shown a hazard ratio of decline to MCI or dementia of 4.5 for SCI vs. NCI cohorts (Reisberg et al., Alzheimer's \& Dementia, 2010). We now investigate whether cognitive and behavioral changes antedate SCI.

Methods: Consecutive subjects who were otherwise healthy with $\mathrm{NCI}$, enrolled during the period from $1 / 1 / 1984$ to $12 / 31 / 1997$, were studied at baseline and followed over mean intervals of 2 years, until 12/31/2001. Baseline evaluations included the MMSE, Brief Cognitive Rating Scale (BCRS), Hamilton Depression Scale (Ham-D), Behavioral Pathology in AD assessment (BEHAVE-AD), and a psychometric test battery (see Reisberg et al., Alzheimer's \& Dementia, 2010, for a detailed description). The first follow-up period with decline prior to 2002 was used for analyses. Decline was defined as a change to SCI or a more severe global cognitive status. The accelerated failure time model was used to determine the time to decline, controlling for baseline age, gender, education and follow-up time. The additional contribution of other variables to decline time was examined.

Results: Of 67 NCI subjects enrolled, $47(78 \%)$ were followed over a mean of $6.7 \pm 3.1$ years. For followed subjects, the mean $( \pm S D)$ baseline age was $64.1 \pm 8.9$ years, mean education level was $16.1 \pm 2.4$ years, and the mean MMSE at baseline was $29.6 \pm 0.8$ years. There were $2.8 \pm 1.5$ follow-up visits. At follow-up, 10 subjects (21\%) remained NCI and $37(79 \%)$ declined. Of decliners, at the time of the first observed period of decline, 29 (78\%) were SCI, 7 (19\%) were MCI, and 1 was AD $(3 \%)$. Mean time to decline was $3.6 \pm 1.6$ years. Baseline variables contributing to time to decline were: WAIS, digits forward $(p<0.01)$; WAIS, digits backward $(\mathrm{p}<0.01)$; BCRS Axis V (functioning) $(\mathrm{p}<0.05)$; Ham-D, middle insomnia $(\mathrm{p}<0.01)$; and BEHAVE-AD, verbal outbursts $(\mathrm{p}<0.05)$

Discussion: Changes in cognition, sleep and behavior antedate the advent of SCI symptomatology in eventual AD. One implication is that cognitive changes might profitably be addressed at the present time in treatment studies throughout the SCI stage of brain aging and dementia. In the future, cognitive and behavioral changes should be addressed even prior to the advent of SCI.

Disclosure: B. Reisberg: None. M. Shulman: None. C. Torossian: None. I. Monteiro: None. I. Boksay: None. T. Gill: None. R. Chakraborty: None. M. Zeeshan: None. W. Zhu: None.

\section{Novel Analyses of Hierarchical Early Information Processing Deficits in a Large Cohort of Schizophrenia Patients \\ Anthony J. Rissling*, Joyce Sprock, Marlena Pela, Neal Swerdlow, David Braff, Gregory Light}

\section{University of California San Diego, La Jolla, CA}

Background: The parsing of early information processing deficits and their neural and genomic substrates is a key issue in understanding schizophrenia. EEG measures of auditory mismatch negativity (MMN), $\mathrm{P}_{300}\left(\mathrm{P}_{3} \mathrm{a}\right)$ and the reorienting negativity ( $\left.\mathrm{RON}\right)$ are neurophysiological probes of the "chain" of serial, temporally defined automatic sensory discrimination, orienting of attention, and the reorienting of attention following stimulus changes. Several previous studies have 


\section{S326}

reported deficits in $\mathrm{MMN}$ and $\mathrm{P}_{3} \mathrm{a}$ in schizophrenia patients versus normal controls. However, no studies have reported schizophrenia related deficits in the RON. The present study aimed to examine whether RON is: 1) elicited in the context of a passive duration deviant oddball paradigm; 2) deficient in schizophrenia patients relative to normal controls; and 3) a dissociable process that independently contributes to our understanding of schizophrenia related deficits beyond MMN and $\mathrm{P}_{3}$ a deficits.

Methods: In large groups of Schizophrenia patients $(n=429)$ and normal controls $(n=286)$ cortical event-related potentials were recorded while participants watched a silent video and were presented binaurally with $1-\mathrm{kHz}$ tones $500 \mathrm{~ms}$ apart, including standard duration tones $(\mathrm{P}=.90,50-\mathrm{ms}$ duration $)$ and deviant tones $(\mathrm{P}=0.10,100-\mathrm{ms})$. Results: MMN, $\mathrm{P}_{3}$ a and RON amplitudes were significantly reduced in schizophrenia patients, with large between-group effect sizes (MMN, $\mathrm{d}=1.05 ; \mathrm{P}_{3} \mathrm{a}, \mathrm{d}=0.87 ; \mathrm{RON}, \mathrm{d}=0.77$ ) at frontocentral electrodes. While the MMN, $\mathrm{P}_{3} \mathrm{a}$, and RON response complex showed evidence of intercorrelations among components, covariate analyses revealed distinct deficits in schizophrenia patients on each of the components. Discussion: The present study demonstrates the parsing of hierarchical, time dependent information processing abnormalities during the earliest stages of automatic sensory information processing. MMN, $\mathrm{P}_{3} \mathrm{a}$, and RON can be differentiated from one another and represent dissociable deficits in schizophrenia patients. To our knowledge, this is the first report to specifically examine and report dissociable (from MMN and $\mathrm{P}_{3}$ a) deficits in the automatic reorienting of attention in schizophrenia patients using a passive duration oddball paradigm. Future studies will examine the genomic and neural architecture supporting these separate and dysfunctional early information processing operations and their relationships to higher-order cognitive and functional outcomes.

Disclosure: A. Rissling: None. J. Sprock: None. M. Pela: None. N. Swerdlow: Part 1; Allergan. D. Braff: Part 4; MHo65571; Department of Veteran's Affairs VISN-22 Mental Illness Research, Education, and Clinical Center. G. Light: Part 1; Allergan; Hoffmann-La Roche. Part 4; MHo79777.

6o. MTHFR Genotype Influences Error-Related Anterior Cingulate Activation in Two Schizophrenia Cohorts

Joshua Roffman*, Adam Nitenson, David Brohawn, Caitlin Carey, Marlisa Isom, Jesse Friedman, Kara Dyckman, Yigal Agam, Donald Goff, Dara Manoach

\section{Massachusetts General Hospital, Charlestown, MA}

Background: Response monitoring, which involves detecting errors, evaluating what went wrong, and adjusting behavior, is an executive function that is often disrupted in schizophrenia. Intact response monitoring relies on the dorsal anterior cingulate cortex (dACC), which is functionally and structurally abnormal in schizophrenia. We examined whether a common, functional variant in the MTHFR gene, ${ }_{677 \mathrm{C}}>\mathrm{T}$, contributes to the well-replicated finding of reduced dACC activation following error commission in schizophrenia patients. MTHFR is a key regulator of folate metabolism, and the lowfunctioning $677 \mathrm{~T}$ variant has been consistently associated with elevated risk for schizophrenia, as well as with more severe negative symptoms and increased perseverative errors on the Wisconsin Card Sort Task in schizophrenia patients. We hypothesized that blunted error-related dACC activation would be more pronounced in patients who carry at least one copy of the $677 \mathrm{~T}$ allele.

Methods: Two cohorts $(\mathrm{n}=18$ and 23) of medicated schizophrenia outpatients were recruited from an urban mental health clinic. Each cohort performed a different variant of the antisaccade paradigm during functional magnetic resonance imaging (fMRI) in a $3 \mathrm{~T}$ Tim Trio magnet (Siemens). Antisaccades require inhibition of the prepotent response of looking toward a suddenly appearing visual target and the substitution of a gaze in the opposite direction. Eye position was monitored using the fMRI Remote Eye Tracking
Laboratory (ISCAN). Finite impulse response (FIR) models, implemented in FS-FAST, provided unbiased estimates of the event-related hemodynamic responses at each time point for each trial type. Patients were genotyped for MTHFR $677 \mathrm{C}>\mathrm{T}$ using the MassArray plaform (Sequenom). We compared error-related activation, which is based on the contrast of error minus correct antisaccade trials at $6 \mathrm{~s}$, in $\mathrm{C} / \mathrm{C}$ homozygotes versus $\mathrm{T}$ allele carriers $(\mathrm{C} / \mathrm{T}+\mathrm{T} / \mathrm{T})$. For region-ofinterest analyses, the dACC was functionally constrained to those vertices that showed error-related activation at $\mathrm{p}<.05$ when all subjects within the cohort were averaged.

Results: In the first cohort, the main effect of MTHFR genotype on dACC activation across error and correct conditions approached significance $(C / C>T$ carrier, $p=.07)$. A significant genotype $x$ condition interaction was observed $(\mathrm{p}=.009)$, which reflected that $\mathrm{C} / \mathrm{C}$ patients exhibited significant error-related activation $(\mathrm{p}=.03)$ and $\mathrm{T}$ carriers did not $(\mathrm{p}=.57)$. In the second cohort, the findings were similar: while there was no main effect of MTHFR genotype on dACC activation $(p=.45)$, a genotype $x$ condition interaction was again observed $(\mathrm{p}=.005)$, indicating significant error-related activation in $\mathrm{C} / \mathrm{C}$ patients $(\mathrm{p}=.009)$ but not $\mathrm{T}$ carriers $(\mathrm{p}=.92)$. Group dACC activation differences were not related to error rate, which did not differ significantly by genotype in either cohort.

Discussion: Reduced error-related dACC activation has been associated with an increased error rate in both patients and healthy individuals in previous studies. Here, we observed more extreme blunting of dACC error-related activation in schizophrenia patients who carry the MTHFR $677 \mathrm{~T}$ allele. To the extent that error-related dACC activation reflects response monitoring, greater blunting following error commission in $677 \mathrm{~T}$ allele carriers may exacerbate difficulties in flexibly modifying behavior, and may contribute to our previous observation of increased perseverative errors among $677 \mathrm{~T}$ patients. The $677 \mathrm{~T}$ allele has also been related to abnormal dopamine signaling, which has been hypothesized to mediate error-related dACC activation through a phasic reduction in mesencephalic dopamine release. The present finding thus suggests several avenues through which heritable alteration in folate metabolism contributes to cognitive deficits in schizophrenia.

Disclosure: J. Roffman: Part 4; Fellowship support from the HarvardMIT Division of Health Sciences Clinical Investigator Training Program, which receives funding through unrestricted educational grants from Merck and Pfizer. A. Nitenson: None. D. Brohawn: None. C. Carey: None. M. Isom: None. J. Friedman: None. K. Dyckman: None. Y. Agam: None. D. Goff: Part 1; Consultant/advisor: Xytis, Forest Labs, Pfizer, Indevus Pharmaceuticals, H. Lundbeck, Schering-Plough, Eli Lilly, Takeda, Biovail, Solvay, Hoffman- La Roche, Cypress, and Dainippon Sumitomo. Part 4; Pfizer, Janssen, Novartis, and GlaxoSmithKline. D. Manoach: Part 1; Consultant for Sepracor, Inc. Part 4; Sepracor, Inc.

\section{Impact of Increasing Adult Hippocampal Neurogenesis On Cognition And Mood}

Amar Sahay*, Kimberly Scobie, Alexis S. Hill, Colin M. O’ Carroll, Mazen A. Kheirbek, Nesha S. Burghardt, Alex Dranovsky, René Hen

Columbia University and NYSPI, New York, NY

Background: Understanding how neural circuit-plasticity mechanisms may be harnessed to improve cognition and mood is essential for developing novel treatments for psychiatric disorders. Adult hippocampal neurogenesis is a unique form of plasticity that generates new neurons in the dentate gyrus throughout life. Levels of adult hippocampal neurogenesis are elevated by interventions associated with beneficial effects on cognition and mood such as exercise and chronic antidepressant treatment. These properties of adult hippocampal neurogenesis suggest that it may be harnessed to improve hippocampal functions. However, despite a substantial number of lossof-function studies demonstrating an involvement of adult-born neurons in mediating select cognitive functions and some of the 
behavioral effects of antidepressants, it is unknown whether stimulation of adult hippocampal neurogenesis is sufficient to improve cognition and mood. Addressing this question is pivotal to determine the potential therapeutic impact of strategies designed to specifically stimulate adult hippocampal neurogenesis.

Methods: Here, we developed a novel genetic gain-of-function strategy with which we can inducibly augment the number of adultborn neurons in a cell autonomous manner. Specifically, we generated mice in which the pro-apoptotic gene, Bax, is ablated selectively in neural stem cells in the adult brain (referred to as iBax mice). iBax mice have a two-fold expansion in the population of young excitable adult-born dentate granule neurons and exhibit enhanced survival and functional integration of adult-born neurons. Since iBax mice phenocopy the enhancing effects of chronic antidepressant treatment on survival of adult-born neurons and neurogenesisdependent synaptic plasticity, it represents a powerful tool to investigate whether increasing adult hippocampal neurogenesis is sufficient to modify dentate gyrus dependent functions in cognition and mood.

Results: We show that inducible genetic expansion of the population of adult-born neurons in mice improves performance in a specific cognitive task in which an animal must distinguish between two similar contexts. Mice with increased adult hippocampal neurogenesis show normal object recognition, spatial learning, contextual fear conditioning and extinction learning but are more efficient in differentiating between overlapping contextual representations, suggestive of enhanced pattern separation. In contrast, increasing adult hippocampal neurogenesis, on its own, does not produce an anxiolytic or antidepressant-like behavioral response. However, genetic expansion of adult hippocampal neurogenesis, when combined with an environmental intervention like voluntary exercise, increased exploratory behavior and decreased anxiety-like behavior in the open field test.

Discussion: The improved performance of iBax mice in differentiating between similar contexts is consistent with the proposed role of the dentate gyrus in pattern separation, a mnemonic process crucial for formation of new episodic memories. These findings suggest that strategies designed to specifically increase adult hippocampal neurogenesis, by targeting cell death of adult-born neurons, may have therapeutic potential for reversing age-related impairments in pattern separation and for treating disorders involving excessive contextual generalization such as Post-Traumatic Stress Disorder (PTSD) or panic disorder. The finding that iBax mice do not show anxiolytic or antidepressant-like behaviors reveals a previously unexpected dissociation between the effects of increasing adult hippocampal neurogenesis on cognition and mood. Our results suggest that other antidepressant-dependent modifications of neural circuits may act in concert with changes in levels of adult hippocampal neurogenesis to produce the behavioural effects of antidepressants. Consistent with this possibility, genetically increasing adult hippcoampal neurogenesis, when combined with environmental interventions such as voluntary exercise, potentiates exploratory behavior and produces anxiolytic effects in the open field test.

Support for A.S: 2009 NIMH Grant 1K99MH86615-01, NARSAD and the Sackler Institute of Columbia University.

Disclosure: A. Sahay: None. K. N. Scobie: None. A. S. Hill: None. C. M. O' Carroll: None. M. A. Kheirbek: None. N. S. Burghardt: None. A. Dranovsky: None. R. Hen: Part 2; Brain Cells, Inc.

\section{Are Sleep Complaints Associated with Subsequent Mood Variability in Bipolar Disorder?}

Erika Saunders*, Julio Fernandez-Mendoza, Mahvash Sheikh, Scott Langenecker, Alexandros Vgontzas, Melvin McInnis, Alan Gelenberg

Penn State College of Medicine, Hershey, PA

Background: Sleep disturbances are core manifestations of depressive and manic episodes, which characterize bipolar disorder (BP).
Inter-episode sleep disturbances in BP impact symptom presentation, overall quality of life, and lifetime depressive episodes. Self-reported circadian rhythm dysfunctions are thought to be a potent predictor of daytime functioning in euthymic patients with BP. We investigated the relationship between subjective sleep reports, mood variability, and impact of illness in BP.

Methods: At baseline of a longitudinal study on BP, subjects with BP were administered the Diagnostic Interview for Genetics Studies, the Pittsburgh Sleep Quality Index (PSQI), the Hamilton Depression Rating Scale (HDRS), and the Young Mania Rating Scale (YMRS). Subjects completed the Patient Health Questionnaire-9 (PHQ-9) self rating scale for depression and the Altman Self-Rating Mania Scale (ASRM) every 2 months for 3 years. Mood variability was defined as a ratio of how often subjects had changes in the bimonthly PHQ-9 and ASRM that fell into the highest quartile. Scores for all mood scales were calculated without sleep items to reduce redundancy. Spearman's rho correlations were used to identify relationships between the indexes of depression and mania mood variability with sex, age, HDRS, YMRS, PSQI individual sleep items, total and composite scores, history of rapid cycling, mixed episodes or psychosis and impact of illness on functioning. Linear regression was used to control for severity of depression and mania at baseline on all variables of interest.

Results: Two hundred eight subjects with BP [BPI $(n=161)$, BPII $(\mathrm{n}=27)$, BPNOS $(\mathrm{n}=13)$ and schizoaffective disorder, BP type $(\mathrm{n}=7)$ ] completed all measures listed above. At baseline, subjects had mild depression (HDRS mean: $7.6+/-6.7$ ), were not manic (YMRS mean: $2.7+/-4.4$ ), and had poor sleep quality (PSQI total score mean: $9.1+/-3.4)$. Poor sleep quality was significantly correlated with depression variability $(\mathrm{p}=.007)$, but the association did not hold after controlling severity of depression and mania at baseline $(p=.930)$. There was no significant association between poor sleep quality and mania variability. Severity of depression at baseline significantly correlated with mood variability in depression $(p=.008)$; severity of mania at baseline correlated with mood variability in mania $(p=.001)$ and mood variability in depression $(\mathrm{p}=<.0001)$. A history of rapid cycling reported at baseline was correlated with prospective mood variability in mania and depression $(p<.045)$. Daytime dysfunction due to sleepiness correlated significantly with depression variability $(\mathrm{p}=.003)$. Use of sleep medications was significantly correlated with mood variability in mania $(p=.016)$. Subjective sleep duration, sleep latency, sleep efficiency, sleep disturbance, insomnia symptoms, breathing pauses while sleeping, coughing and snoring were not significantly correlated with mood variability in mania or depression. History of rapid cycling was associated with sleep disturbance $(p=.014)$. Psychosis was not associated with any mood or sleep variables. A history of mixed episodes had a trend to correlate with trouble staying awake $(p=.081)$. Impact of illness was associated with sleep efficiency $(\mathrm{p}=.019)$.

Discussion: This preliminary study shows a significant association between daytime dysfunction due to sleepiness and subsequent mood variability in depression in BP patients. Previous studies have shown a relationship between both subjective and objective measures of sleep disturbance and relapse in BP. In our study, subjective sleep efficiency was only associated with impact of illness, whereas sleep duration, sleep disturbance, and sleep latency were not associated with mood variability. Interestingly, sleep disturbance was associated with a history of rapid cycling. Understanding the relationship between subjective sleep reports and subsequent mood variability in depression and mania can help elucidate factors involved in the physiopathology, course of illness, and treatment of BP.

Disclosure: E. Saunders: None. J. Fernandez-Mendoza: None. M. Sheikh: None. S. Langenecker: None. A. Vgontzas: None. M. McInnis: None. A. Gelenberg: Part 1; Eli Lilly, Pfeizer, BestPractice, AstraZenica, Wyeth, GalaxoSmithKline, ZARS Pharma, Jazz Pharmaceuticals, Lundbeck, Takeda, E-Research Technology (ERT) Inc., Dey Pharma, PGxHealth, Myriad Genetics. Part 4; Eli Lilly, GalaxoSmithKline. 
63. Implicit Learning of Socio-Emotional Stimuli in Schizophrenia: Behavioral and fMRI Findings

Barbara Schwartz ${ }^{\star}$, Chandan Vaidya, James Howard, Jr., Stephen Deutsch

\section{VA Medical Center, Washington, DC}

Background: Social and emotional deficits are characteristic in schizophrenia. These deficits have a significant impact on interpersonal communication and daily functioning. Although studies in nonpsychiatric subjects have identified the processes important for perceiving and remembering the social actions of others, the translation of these findings to clinical populations has been limited. Here we examine automatic or implicit learning in schizophrenia. Previously, we found that subjects with schizophrenia automatically react to threat-related stimuli (gaze direction in fear faces), despite an inability to explicitly identify emotional expression (Schwartz, Vaidya, Howard, \& Deutsch, in press). However, it is not understood how processing of socio-emotional events is modified by experience. This research aimed to determine whether implicit learning of socioemotional stimuli is normal in schizophrenia, despite the wellestablished deficit in explicit memory for past events. We present findings concerned with two forms of implicit learning: repetition priming and stimulus-response rule learning. Repetition priming refers to the finding that repeated stimuli are identified faster and more easily than novel stimuli. Enhanced processing of repeated stimuli reflects changes in a perceptual-based learning system, subserved by sensory-perceptual cortical regions. The gradual learning of stimulus-response associations reflects a form of procedural learning dependent on striatal regions. Each form of learning can occur in the absence of conscious recollection.

Methods: Repetition priming: In study one, 22 patients with DSM-IV diagnoses of schizophrenia or schizoaffective disorder and 18 matched non-psychiatric controls viewed a continuous sequence of fear faces and neutral faces and judged whether each face was male or female; faces were presented twice within the sequence of trials. fMRI (block design) was conducted on an additional 8 patients and 8 controls using the same task. Stimulus-response rule learning: In study two, 30 patients and 22 matched controls performed a probabilistic classification task in which subjects learned to associate faces with one of two outcomes according to a probabilistic rule. The task was administered over 80 trials and on each trial feedback on the accuracy of responses was provided.

Results: Repetition priming: Behavioral results showed that male/ female judgments were faster for repeated faces $(M=691 \mathrm{~ms})$ relative to novel faces $(M=710)$, revealing significant levels of repetition priming for emotional expressions, $\mathrm{P}<.0001$. Furthermore, there was no difference in priming between the groups, $\mathrm{F}<1$, whereas explicit recognition of expressions was impaired in schizophrenia. Neuroimaging results yielded the predictable finding of a decrease in neural response for repeated items. Repetition of faces elicited a reduction in neural activation in the right and left inferior occipital gyrus (BA 18) and in the posterior area of the fusiform gyrus (BA 19) [thresholded at $\mathrm{P}<.005$, uncorrected; cluster-level threshold, $\mathrm{P}<.05$ ]. Although the group comparison did not show a difference in repetition effects between the groups, individual group maps revealed that controls activated a more anterior region of the fusiform gyrus (BA 37) involved in face recognition, whereas patients did not. The behavioral findings indicate that perceptual priming is intact in schizophrenia. However, brain imaging results suggest that regions of the occipito-temporal cortex crucial for supporting abstract facial representations may be impaired in patients, even under implicit learning conditions.

Stimulus-response rule learning: Subjects learned to associate faces with outcomes according to a probabilistic rule, $\mathrm{P}<.0001$. There was no difference in total learning between the groups, $\mathrm{P}>.05$, and no difference in the pattern of learning across blocks of trials, $\mathrm{F}<1$. In contrast, there was a trend for patients to have less explicit knowledge about stimulus-response associations on post-test questioning,
$\mathrm{P}=.069$. The results suggest that striatal-based learning of associations is unimpaired in schizophrenia.

Discussion: Social cognition depends on a number of cognitive components and brain systems. This research indicates that individuals with schizophrenia benefit from experience and learn basic perceptual features and specific stimulus-response associations for socio-emotional stimuli. However, these results suggest that stimulus repetition may not enhance abstract aspects of face-processing. These problems could contribute to poor explicit memory for social experiences.

Disclosure: B. Schwartz: None. C. Vaidya: None. J. Howard, Jr.: None. S. Deutsch: None.

64. Cannabis Use Disorders and Information Processing in DecisionMaking

Serge Sevy*, Sui Kwong Li, Christina Dougherty, Edward Nunes

The Zucker Hillside Hospital, Glen Oaks, NY

Introduction: Little is known about how individuals with cannabis use disorders process information when making decision. The purpose of this study is to assess in these individuals the processing of outcome attributes when making choices.

Methods: Twenty-five subjects (19 males and 6 females) with a current DSM-IV diagnosis of cannabis abuse or dependence were compared with 21 healthy subjects ( 17 males and 4 females) for cognition and decision-making. Attributes of outcomes we measured using two computerized tasks: the Iowa Gambling Task (IGT) (frequency and magnitude of rewards/losses) and U-Titer (magnitude, timing, and probability of outcomes). To control for the effects of cognition on decision-making, subjects were also assessed for premorbid intelligence, attention, working memory, verbal fluency, processing speed, executive function, and motor performance using the following tests: WRAT-3, CPT-IP, COWAT, WAIS-III, CVLT, Trail Making A \& B, WCST, Stroop Test, Finger Tapping, and Grooved Pegboard. Decisionmaking was assessed using the Iowa Gambling Task and U-Titer.

Results: The average age at onset of cannabis use was $15 \pm 3$ years and the average age at onset of cannabis abuse or dependence was $18 \pm 4$ years. The average duration of cannabis use was $12 \pm 7$ years (range 4-28). CUD subjects were using cannabis on average $4 \pm 2$ days per week at the time of the screening. They were sober for at least one week and had negative urine toxicology at the time of testing. None of the subjects reported withdrawal symptoms. Groups did not differ for age $(27 \pm 8$ (SD) in both groups), sex ( $76 \%$ vs. $81 \%$ males), level of education ( $13 \pm 2$ vs. $14 \pm 2$ years of education), but CUD subjects had a lower premorbid intellectual functioning (WRAT3 standard score $92 \pm 11$ vs. $100 \pm 10)$. They did not differ for other measures of cognition. At the Iowa Gambling Task, compared to healthy subjects, CUD subjects have higher attention to gains (significant on a onetailed Mann Whitney test; $\mathrm{Z}=1.66, \mathrm{p}<.05$ ) and a preference for choices resulting in more frequent rewards. They did not differ for other measures of decision-making (choice strategies, values, timing, and probabilities of outcomes). In the CUD group but not in healthy subjects, there was a negative correlation between performance at the IGT (measured with total score) and time for completing the Trail Making Test (TMT) part B (spearman's rho $=-0.54, \mathrm{p}<0.01$ ).

Discussion: Our results suggest that subjects with cannabis use disorders are more sensitive to frequent rewards compared to healthy subjects. This increased salience for rewards could be related to a dysfunction of the mesolimbic dopamine secondary to a decrease in prefrontal cortical input. Despite cannabis use for long period of time and large amount, we did not find differences between groups for cognition. Performance at the TMT has been associated with frontal activation and the negative correlation between IGT and TMT performances in CUD subjects suggests lower frontal activation associated with decreased IGT performance.

Disclosure: S. Sevy: Part 1; Abbott. S. Li: None. C. Dougherty: None. E. Nunes: None. 
65. A High Gamma Oscillation Deficit During Object Perception in Schizophrenia

Kevin Spencer*, Shahab Ghorashi

VA Boston Healthcare System/Harvard Medical School, Boston, MA

Background: Abnormalities in gamma oscillations $(30-100 \mathrm{~Hz})$ in the scalp-recorded electroencephalogram (EEG) have been proposed to reflect neural circuitry abnormalities in schizophrenia. Gamma oscillations are thought to play an important role in visual perception, mediating the binding of visual features into coherent objects. Recent evidence suggests that "high" $(60-100 \mathrm{~Hz})$ gamma oscillations may be particularly involved in object perception, as opposed to "low" $(30-60 \mathrm{~Hz})$ gamma oscillations which are more involved in attentional processes. In the present study we examined whether schizophrenia patients would show deficits in high gamma activity in a visual Gestalt perception task.

Methods: Subjects (14 healthy controls [HC], 17 chronic schizophrenia patients [SZ]) discriminated between illusory Kanisza squares and nosquare control stimuli, indicating their judgment with a manual button press. In contrast to previous studies by our laboratory, stimuli were presented briefly (100 ms duration, in white on a black background) and the EEG was recorded from a dense electrode array ( 72 electrodes, $\mathrm{DC}-100 \mathrm{~Hz}, 512 \mathrm{~Hz}$ sampling rate) and average-referenced. Timefrequency decomposition of the EEG was based on the Morlet wavelet transform. Time-frequency maps of phase locking values were calculated for stimulus- and response-locked oscillations. Statistical non-parametric mapping was used to find time-frequency clusters that reflected Group (HC/SZ) X Stimulus (IS/NS) interaction effects and main effects of Stimulus in each subject group.

Results: HC and SZ did not differ in overall reaction time or error rate. Increased high gamma oscillations were elicited by perceived illusory squares compared to no-squares in HC but not SZ. These oscillations were phase locked to the manual response and occurred in the high gamma band $(80-99 \mathrm{~Hz})$ at fronto-central electrodes shortly preceding the time of the response, suggestive of a late stage of object processing. Decreased high gamma phase locking for illusory squares compared to no-squares was found in HC in stimulus-locked oscillations and in SZ in response-locked oscillations. Increased phase-locking in the alpha band $(7-10 \mathrm{~Hz})$ was found for illusory squares relative to no-squares in both $\mathrm{HC}$ and SZ at frontal and posterior electrodes. No low gamma activity differed between stimulus conditions for either group.

Discussion: In this study schizophrenia patients demonstrated a deficit in frontal high gamma activity associated with visual object perception. In contrast, oscillatory activity in the alpha band was not affected in patients. These findings suggest that high gamma oscillatory activity in the frontal lobe is disturbed in schizophrenia, a deficit which may be related to impaired integrative processes in object perception in this disorder.

Disclosure: K. Spencer: Part 1; Galenea, Inc. S. Ghorashi: None.

\section{Risk And Reward In Obsessive-compulsive Disorder}

Emily Stern*, Kate Fitzgerald, William Gehring, Robert Welsh, Joseph Himle, James Abelson, Stephan F. Taylor

University of Michigan, Ann Arbor, MI

Background: Previous research has suggested that symptoms of obsessive-compulsive disorder (OCD) are related to heightened perceptions that "something is wrong", which is supported by neuroimaging studies identifying an increased error response in patients with OCD. However, the repetitive nature of obsessions and compulsions may also be perpetuated by impairment in the experience of satiety or reward associated with successful task completion. Furthermore, anticipated risk of negative outcomes may also contribute to repetitive behaviors that aim to ensure that a bad event has not occurred. Given the overlap between neural regions implicated in OCD and those involved in reward and risk processing, such as posterior medial prefrontal cortex ( $\mathrm{pMFC}$ )/anterior cingulate cortex
(ACC), orbitofrontal cortex/ventromedial prefrontal cortex (VMPFC), and caudate nucleus, it is important to investigate whether altered neural responses to risk and reward could be contributing to the phenotype of OCD.

Methods: Event-related fMRI measured BOLD response while 39 OCD patients (20 unmedicated, 19 medicated) and 38 matched controls (20 unmedicated, 18 medicated due to history of depression) performed a speeded reaction time task where incentive cues presented prior to flanker stimuli indicated whether correct responses would be associated with no change in money (null trials), a failure to lose money (loss trials), or a gain (gain trials). Cue phase activity compared anticipation on risky trials where there was potential for gain or loss with that on null trials. Analysis of responses to correct feedback focused on activations across incentive types in order to probe for group differences during the attainment or completion of task goals, as well as the comparison between gain and null outcomes to specifically investigate reward processing.

Results: Both OCD and control groups activated large regions of pMFC, dorsolateral prefrontal cortex, and caudate nucleus when anticipating stimuli on risky trials (gain or loss) as compared to null trials, with few differences between groups. While OCD patients did show more activity than controls in subgenual cingulate for loss as compared to null cues, this effect appeared to be driven primarily by less activity in OCD for null cues rather than a difference in processing risk on loss cues. In response to correct feedback, OCD patients showed less activity in the left parahippocampal gyrus and bilateral midbrain than controls. For correct trials associated with rewarding outcomes (gain > null corrects), OCD patients showed less activation than controls in midbrain and medial frontal regions including VMPFC, subgenual cingulate, and rostral portions of ACC.

Discussion: Whereas few group differences emerged during risk anticipation, correct feedback elicited greater activation in controls than OCD patients in neural regions associated with emotional/ motivational processes, particularly on trials with rewarding outcomes. These data suggest that a reduction in reward signaling may contribute to the phenomenology of OCD.

Disclosure: E. Stern: None. K. Fitzgerald: None. W. Gehring: None. R. Welsh: None. J. Himle: None. J. Abelson: None. S. Taylor: Part 1; St. Jude Medical.

\section{Impulsivity: Evidence for Compensatory and Sensitization} Mechanisms in Bipolar Disorder and Antisocial Personality Disorder Alan Swann*, Marijn Lijffijt, Scott Lane, Kimberly Kjome, Joel Steinberg, F. Gerard Moeller

University of Texas Health Science Center at Houston, Houston, TX

Background: Bipolar disorder and antisocial personality disorder (ASPD) overlap in clinical characteristics and behavioral consequences. Impulsivity is prominent in both, but there is little information on how specific mechanisms of impulsivity differentiate, bridge, or underlie the disorders. Substance-use disorder or increased recurrence in bipolar disorder may contribute to impulsivity in the combined disorders.

Methods: Subjects were controls $(n=78)$, bipolar disorder without cluster B personality disorder $(\mathrm{n}=55)$, ASPD without bipolar disorder $(n=34)$, and bipolar disorder with ASPD $(n=24)$. Diagnoses, including substance-use disorders, were rendered using SCID-I and -II. Impulsivity measures were the Immediate Memory Task (IMT), a continuous performance test of response inhibition measuring ability to evaluate a stimulus before responding, and the Two Choice Impulsivity Paradigm (TCIP), a choice between smaller-sooner and larger-later reward. Course of illness was determined retrospectively. Data were analyzed using general linear models analysis.

Results: Subjects with bipolar disorder had more IMT omission errors and slower reaction times than controls. Commission errors (inhibitory failures) per correct detection were higher in combined bipolar disorder and ASPD than in controls. Reaction times were faster 
with combined diagnoses than in bipolar disorder alone. TCIP responding in either diagnosis alone resembled controls, but was more impulsive in combined disorders. In combined ASPD and bipolar disorder, increased reaction speed, liberal response bias, and reward-delay impulsivity occurred independent of substance-use disorder, but increased commission errors were associated only with comorbid substance-use disorder. In bipolar disorder, the effects of ASPD and substance-use disorders on rapid-response impulsivity were entirely accounted for by many episodes of illness, but effects of ASPD and substance-use disorder on reward-delay impulsivity were independent of illness course.

Discussion: Impulsivity was increased in the combined disorders over either disorder alone. Results were consistent with compensations for impulsivity in uncomplicated ASPD or bipolar disorder, where commission errors are reduced at the expense of increased omission errors and slowed responses. This resembles effects of postsynaptic ${ }_{5} \mathrm{HT} 1 \mathrm{~A}$ receptor activation. This compensation is lost when the disorders are combined. Recurrent illness course had differential effects on rapid-response and reward-delay impulsivity.

Disclosure: A. Swann: Part 1; Cephalon, Merck, Astra Zeneca, Sanofi Aventis, Abbott Laboratories, Pfizer, Alexca. M. Lijffijt: None. S. Lane: None. K. Kjome: None. J. Steinberg: None. F. Moeller: None.

68. Memantine and Amphetamine Effects on Neurocognition and Sensorimotor Gating in Healthy Subjects

Neal Swerdlow*, Gregory Light, Susrutha Thanam, August Slater, Jo Talledo

UCSD School of Medicine, La Jolla CA

Background: We reported that the NMDA antagonist, memantine (MEM: $20 \mathrm{mg}$ po), increases sensorimotor gating (prepulse inhibition (PPI) of acoustic startle) in healthy individuals (Neuropsychopharm 34:1854-64, 2009), and that the stimulant d-amphetamine (AMPH: $20 \mathrm{mg}$ po) has both PPI-increasing and decreasing effects in healthy subjects, depending on their baseline PPI and personality phenotypes (Psychopharm 204: 165-75, 2009). Our group and others have also reported that PPI correlates significantly with specific neurocognitive functions, including working memory (WM). Both MEM and AMPH have pro-cognitive effects in humans. We are now testing the prediction that MEM and AMPH PPI-enhancing effects will be accompanied by increased WM performance, as a prelude to studies in patient populations.

Methods: These preliminary data are part of an ongoing double blind, cross-over, placebo-controlled study of the effects of MEM (20 mg po) and AMPH $(20 \mathrm{mg})$ on PPI and WM. At present, 23 subjects have completed a full within-subject cross-over design (mean (range) age $=24.6$ y 18-35), education $=14.8$ y (12-18)): 8 men tested with MEM and 15 subjects tested with AMPH $(\mathrm{M}: \mathrm{F}=11: 4)$. Carefully screened subjects lacking medical or psychiatric illness completed two full test days, separated by 1 month. Participants completed PPI testing at time points previously shown to be maximally sensitive to drug effects (210 min post-MEM or $40 \mathrm{~min}$ post-AMPH), using our established procedures. Briefly, bilateral EMG recordings of orbicularis oculi contraction were acquired during a $250 \mathrm{~ms}$ window after a $115 \mathrm{~dB}(\mathrm{~A})$ $40 \mathrm{~ms}$ noise startle stimulus alone, or preceded $10,20,30,60$ or $120 \mathrm{~ms}$ by a $20 \mathrm{~ms}$ prepulse that was $15 \mathrm{~dB}$ over a $70 \mathrm{~dB}(\mathrm{~A})$ background. Subjects also completed the MATRICS Consensus Cognitive Battery (MCCB), which includes specific measures of WM; the primary dependent measure was the $\mathrm{T}$-score for each cognitive domain. Personality dimensions were assessed using the Tridimensional Personality Questionnaire (TPQ).

Results: Bioactivity of both MEM and AMPH were confirmed by autonomic measures and subject ratings. ANOVA of MCCB T-scores revealed a significant interaction of drug $\mathrm{x}$ placebo/active condition for the WM domain $(\mathrm{p}<0.035)$; this differential drug effect reflected significant WM-increasing effects of MEM $(p<0.003)$ but not AMPH. There were no significant interactions for other MCCB domains (processing speed, attention/vigilance, verbal or visual learning, reasoning and problem solving, or social cognition). Analysis of test day revealed significant overall practice effects $(\mathrm{p}<0.001)$, reflecting specific domains of processing speed $(\mathrm{p}=0.002)$, visual learning $(\mathrm{p}<0.002)$ and reasoning and problem solving $(\mathrm{p}<0.03)$ but not other domains. ANOVA of PPI revealed the predicted pattern of drug condition $\mathrm{x}$ prepulse interval $(\mathrm{p}=0.10)$ reflecting a significant increase in PPI at long $(120 \mathrm{~ms})$ prepulse intervals after active drug $(p<0.035)$, primarily due to the effects of AMPH $(\mathrm{p}<0.015)$; PPI-increasing effects of MEM were also detected but reached only trend levels with this small sample. Subgroup analyses based on baseline PPI levels and personality measures will also be presented.

Discussion: These preliminary data extend our published results of MEM- and AMPH-induced changes in PPI. More importantly, at this dose, MEM appears to increase WM performance while AMPH does not. This design controlled for practice effects, which could not account for the observed WM-enhancing effects of MEM. If these results are confirmed in ongoing studies, they will suggest that MEM, but not AMPH, may be a useful adjunct to WM-dependent therapeutic modalities such as cognitive interventions in patients with impaired sensorimotor gating and WM. Supported by $\mathrm{MH}_{59803}$ and DA027483. Disclosure: N. Swerdlow: Part 4; Allergan, Inc. G. Light: Part 3; Hoffman-La Roche, Inc. Part 4; Allergan, Inc. S. Thanam: None. A. Slater: None. J. Talledo: None.

\section{Selective Effects of Long-term Delta9 ${ }^{9}$-Tetrahydrocannabinol Administration on Spatial Working Memory Performance in Adolescent Rhesus Monkeys \\ Christopher Verrico, David Lewis}

\section{University of Pittsburgh, Pittsburgh, PA}

Background: Although heavy cannabis use is known to impair working memory functioning in adults, few studies have examined the effects of cannabis in adolescents. Indeed, cannabis use during adolescence has been associated with an increased risk of schizophrenia, a disorder characterized by working memory impairments. Moreover, substantial refinements in the cortical circuits that mediate spatial, but not object, working memory occur during adolescence in humans. Thus, adolescents exposed to delta ${ }^{9}$-tetrahydrocannabinol (THC) may be more susceptible to spatial working memory impairments. However, it is difficult to control for potential pre-exposure differences between users and non-users in intellectual, cognitive, and psychological attributes that could confound interpretation of epidemiological studies of the effects of cannabis in humans. Rhesus monkeys are well-suited for modeling the cannabis-induced phenotypes observed in humans because both species have a protracted period of adolescence when homologous neural circuits, which underlie adult-levels of performance on spatial working memory tasks, mature.

Methods: Thus, we determined the effects of long-term (7 months), repeated (5 days/week) intravenous THC administration during adolescence on the performance of two working memory tasks. Fourteen male rhesus monkeys ( $\sim 28$ months of age at first exposure) were paired according to 1 month of baseline performance on a spatial delayed-response (SDR) task and an object-familiar, delayed match-tosample (DMTS) task. Subsequently, the acute dose-dependent effects of THC administration on task performance were determined for the 7 monkeys assigned to receive THC, within each pair, across an escalating dose range $(0.03-0.3 \mathrm{mg} / \mathrm{kg})$. The selected dose range was based on published literature concerning the self-administration of THC by drug-naïve monkeys and heavy use in humans. The dosedependent effects of THC were used as a guide to determine chronic doses by selecting doses that acutely induced a similar magnitude of working memory impairments. Therefore, based on these data, 3 monkeys received a chronic does of $0.12 \mathrm{mg} / \mathrm{kg} /$ day while the other 4 monkeys received a chronic does of $0.24 \mathrm{mg} / \mathrm{kg} / \mathrm{day}$. Importantly, the chronic data presented here were collected $\sim 23$ hours after the last 
exposure to THC (i.e., each day, THC or vehicle was administered after the monkeys completed both tasks).

Results: Preliminary analyses revealed that, as expected, performance of all 14 monkeys on both tasks was delay-dependent and, relative to baseline performance, response accuracies improved on both tasks over the 7-month period. Strikingly, however, the age-related increase in accuracy of the vehicle-exposed monkeys on the SDR task was nearly 2 -fold greater than that in the THC-exposed subjects. In contrast, DMTS task performance improved in a similar fashion in both groups.

Discussion: These findings suggest that long-term, repeated exposure of monkeys to THC selectively impairs the normal adolescence-related improvement in spatial working memory performance, but does not alter the improvement in object working memory performance. These findings provide strong experimental support for human epidemiological data indicating that cannabis use during adolescence has adverse effects on working memory. However, given the extended halflife of THC it seems particularly important that future studies determine whether the selective memory impairments persist following an appropriate washout period and whether they are accompanied by alterations in the maturing neural circuits of the dorsolateral prefrontal cortex that mediate adult level proficiency of spatial working memory. Nonetheless, the selective deleterious effects of THC on spatial working memory performance during adolescence suggest that the effects of THC may be exacerbated when exposure coincides with a developmental stage during which the neural circuits that mediate a function are actively maturing.

Disclosure: C. Verrico: None. D. Lewis: Part 1; BMS Foundation, Bristol-Myers Squibb, Curridium Ltd, Pfizer.

\section{Neural Correlates of Model-Based Reinforcement Learning Parameters in Healthy Volunteers and Patients with Schizophrenia James Waltz ${ }^{*}$ Zuzana Kasanova, Michael Frank, Thomas Ross, Betty Jo Salmeron, Pradeep Kurup, James Gold, Elliot Stein}

Univ. of Maryland School of Med. Baltimore, MD

Background: Computational models of reinforcement learning have helped to capture specific aspects of learning, which, in turn, can be linked to underlying neural substrates in the context of neuroimaging studies. Learning rate parameters, for example, vary across individuals and characterize the impact of prediction errors on learning.

Methods: In a 3T MRI scanner, we gave 24 individuals with schizophrenia (SZ) and 17 controls (CT) a probabilistic reversal learning (PRL) task, involving the presentation of eight pairs of fractal patterns, each for a block of 50 trials. In each pair, the choice of one pattern was rewarded $(+5 \phi) 80 \%$ of the time, while the other was rewarded $20 \%$ of the time. For analyses of behavior, we modeled sequences of responses by individual participants according to a Q-learning algorithm with subject-specific parameters for learning rate $(\alpha)$ and temperature $(\beta)$, and trial-by-trial estimates of prediction error. We investigated brain responses, in the context of this task, to unexpected feedback and to stimuli evoking behavior shifts in healthy controls and SZ patients. Correlation analyses were used to determine whether individual differences in $\alpha$ were predicted by neural responses to brain areas during choice and reinforcement events, and whether these relationships are disrupted in SZ. We had previously modeled BOLD responses by categorizing feedback events according to their valence (gain or loss) and their validity (e.g., invalid "probabilistic errors" vs. valid "reversal errors"). We also categorized choice events as either "stays" (choice of the same stimulus as on the last trial) or "switches". Regressor functions used these events of interest, along with head motion vectors. We derived functional regions of interests (ROIs) based on the results of second-level, whole-brain linear mixedeffect (LME) analyses, using a voxel-wise threshold of $p<0.001$ and selecting clusters on the basis of their spatial extent (minimum of 15 voxels $/ 405 \mu \mathrm{l}$ ). We identified ROIs based on the following contrasts, done for the entire sample: 1 ) areas showing significantly greater activation for negative than positive feedback; 2) areas showing significantly greater activation for positive than negative feedback; and 3) areas showing significantly greater activation for shifts than stays. Results: Brain regions showing significantly greater activation for negative than positive feedback included ventrolateral PFC, dorsal anterior cingulate cortex (ACC) and the inferior and superior parietal lobules. Areas showing significantly greater activation for positive than negative feedback included ventral striatum, parahippocampal gyrus/ amygdala, rostral ACC and posterior cingulate. Areas showing significantly greater activation for shifts than stays included mPFC, bilateral VLPFC and inferior parietal lobule, and right FPC, dorsolateral prefrontal cortex (DLPFC) and premotor cortex (PMC). Analyses based on modeling of behavioral data revealed that, in controls, $\alpha$ correlated significantly with neuronal responses to negative feedback in inferior and superior parietal lobule, as well as responses to choice stimuli followed by shifts (regardless of the preceding feedback), in medial PFC. These systematic relationships between brain activity to task-related events and learning rate were not observed in SZ patients.

Discussion: These results suggest that healthy controls who show more learning from prediction errors show stronger responses to salient events in brain regions implicated in attentional updating and cognitive control processes, often attributed to parietal cortex and $\mathrm{mPFC}$, respectively. The absence of these systematic brain-behavior relationships in SZ patients may partly explain abnormalities in learning and decision-making associated with the disorder.

This work was supported by NIH grants 5K12RRo23250-02, 5Ro1MHo80066-03, Contract HHSN271200599091C, and the National Institute of Drug Abuse, Intramural Research Program, National Institute of Health (NIDA/IRP/NIH).

Disclosure: J. Waltz: None. Z. Kasanova: None. M. Frank: None. T. Ross: None. B. Salmeron: None. P. Kurup: None. J. Gold: Part 1; Neurocog Trials, Inc., Merck Pharmaceuticals, AstraZeneca Pharmaceuticals, Solvay Pharmaceuticals, GlaxoSmithKline. E. Stein: None.

\section{Time Perception Deficits In Patients With Frontotemporal} Dementia

Josh Woolley*, Nikhil Murthy, Tal Shany-Ur, Jonathan Pai, Pardis Poorzand, Bruce Miller, Katherine Rankin

UCSF, San Francisco, CA

Background: The ability to correctly estimate, predict, and produce time intervals plays a critical role in behavior. However, the neural mechanisms of time perception are not well understood. Frontotemporal dementia (FTD) is a neurodegenerative disease characterized by progressive behavioral, mood and personality changes, including impaired decision making, disinhibition, and impulsivity. Patients with FTD frequently exhibit behaviors consistent with altered time perception and have atrophy of brain regions implicated in time perception, including the orbitofrontal cortex and the dorsal striatum. However, there have been no systematic studies of time perception in FTD. Characterizing time perception in patients with FTD may lead to a better understanding of behavioral changes in FTD, as well as the normal neural circuitry of time perception.

Methods: We examined differences in time perception in 21 healthy controls (HC), 13 patients with FTD, and 9 patients with Alzheimer's disease $(\mathrm{AD})$ as a dementia control group. Subjects performed two time estimation and two time production tasks. To examine short-term time estimation, subjects viewed an animated skier moving across the screen at a constant velocity and were asked to estimate the duration of the skier's travel. Durations ranged from 2 to 9 seconds. To examine long-term estimation, subjects were asked at the end of testing to estimate how much time has passed since the beginning of the roughly 30 minute session. To examine time production, subjects 1 ) pressed a computer key once per second for 5, 10, and 15-second intervals in a pacing task, and 2) started and then stopped a covered stopwatch after they guessed 10 seconds had elapsed. 


\section{S332}

Results: Patients with FTD significantly overestimated short-term intervals compared to $\mathrm{HC}$ and patients with $\mathrm{AD}(\mathrm{p}<0.05)$. Patients with FTD overestimated intervals by $143.6 \% \pm 23.5 \%$ ( \pm S.E.) (i.e., a 2-second interval was estimated to be approximately 5 seconds on average) whereas $\mathrm{HC}$ overestimated intervals by $28.3 \% \pm 5.9 \%$ and patients with $\mathrm{AD}$ overestimated intervals by $58.3 \% \pm 6.9 \%$. FTD patients were significantly more variable in their estimates of intervals (mean S.D. $=86.6 \%$ ) than $\mathrm{HC}$ (mean S.D. $=38.3 \%$ ) and $\mathrm{AD}$ (mean S.D. $=40.0 \%)$. Patients with FTD were no less accurate on long-term estimation than $\mathrm{HC}$ or patients with $\mathrm{AD}$, overestimating intervals by $15.3 \% \pm 42.0 \%$ ( \pm S.D.) (HCs overestimated by $7.7 \% \pm 28.7 \%$, ADs overestimated by $15.6 \% \pm 84.7 \%)$. On the pacing task, patients with FTD pressed significantly faster than patients with $\mathrm{AD}$; pressing the key every $737.1 \pm 62.5 \mathrm{~ms}$ (AD: $1269.1 \pm 842 \mathrm{~ms}$ ). Patients with FTD exhibited a non-significant trend towards pressing the key faster than HCs, who pressed it every $933.8 \pm 10.7 \mathrm{~ms}(\mathrm{p}<0.1)$. Patients with FTD produced significantly shorter intervals on the 10 second stopwatch task than HCs and AD patients, producing an average interval of $7.0 \pm 1.63$, whereas HCs produced an average interval of $9.65 \pm 2.31 \mathrm{~s}$ and patients with $\mathrm{AD}$ produced an average interval of $9.95 \pm 3.91 \mathrm{~s}$.

Discussion: Our data demonstrate that patients with FTD have an altered perception of time intervals compared to HCs and a dementia control group of $\mathrm{AD}$ patients. Specifically, it appears that patients with FTD have a faster short-term internal clock, as shown by their overestimation, faster pacing, and underproduction of short time intervals with intact long-term time estimation. This accelerated subjective time may contribute to the impaired decision-making and impulsivity seen in patients with FTD. In particular, an accelerated sense of time may explain why patients with FTD choose smaller immediate rewards instead of larger delayed rewards and are therefore impulsive. The data also suggests that atrophy in FTD-specific brain regions may be responsible for a faster internal clock. In our study, patients with $\mathrm{AD}$, a neurodegenerative disease that primarily affects posterior cerebral cortices and hippocampi, did not exhibit the same deficits in time perception as patients with FTD, who typically have damage to more anterior structures such as the insula, prefrontal cortex, and striatum. Thus, the present data taken together with previous research suggest that time perception is subserved by a fronto-striatal circuit. We plan to use quantitative imaging techniques to further explore relationships between patterns of brain atrophy and deficits in time perception. This data may ultimately lead to a more neurologically specific understanding of behavioral changes in FTD and the normal neural mechanisms underlying time perception.

Disclosure: J. Woolley: None. N. Murthy: None. T. Shany-Ur: None. J. Pai: None. P. Poorzand: None. B. Miller: None. K. Rankin: None.

72. Impairments in Bottom-Up and Top-Down Visual Processing in Schizophrenia: An ERP and Time-Frequency Analysis

Jonathan Wynn*, Ian Mathis, Brian Roach, Daniel Mathalon, Judith Ford, Michael Green

\section{VA Greater Los Angeles Healthcare System/UCLA, Los Angeles, CA}

Background: Schizophrenia patients exhibit deficits in several visual processing tasks, though most of those tasks are limited to examining bottom-up processes. What is less clear is if schizophrenia patients exhibit deficits in later, top-down stages of visual processing, that rely on cortico-cortico processing. Event-related potentials (ERPs) and time-frequency analysis (TFA) of the EEG are uniquely suited to examining both bottom-up and top-down processes during visual processing.

Methods: We utilized a novel object substitution backward masking task, designed to specifically impair top-down of processing of visual stimuli. Targets consisted of squares with a notch cut in the top, side or bottom. The mask (which also served as the cue as to which square to identify) consisted of an array of 4 dots that surrounded but did not touch the target. In object substitution masking, as the SOA between the target and mask increases, target identification decreases due to the 4-dots being substituted for the target. 88 schizophrenia patients and 66 healthy controls participated. Four different stimulus onset asynchronies (SOAs) were presented, with accuracy typically seen to decrease as SOA increases. ERPs, evoked activity and phase locking in the gamma band were examined.

Results: While both groups showed the expected pattern of poorer performance with increasing SOA, schizophrenia patients performed worse than controls on the backward masking task across all SOAs. The P100 and N100 were reduced in amplitude in the schizophrenia patients compared to healthy controls across all SOAs. Healthy controls had a larger $\mathrm{P}_{300}$ when targets were easily identifiable at the two earliest SOAs, with $\mathrm{P}_{300}$ amplitude decreasing as SOA increased. Schizophrenia patients' $\mathrm{P} 300$ amplitude was lower overall compared to controls and did not change in amplitude with changes in SOA. Finally, schizophrenia patients exhibited reduced evoked activity and phase locking in the beta and gamma bands compared to the healthy controls.

Discussion: Both bottom-up (P100 and $\left.\mathrm{N}_{100}\right)$ and top-down $\left(\mathrm{P}_{3} 00\right)$ visual processing is impaired in schizophrenia patients. Additionally, the gamma-band results imply dysfunctional cortico-cortico processing during visual processing in schizophrenia. In summary, our results reveal that top-down visual processing is also impaired in schizophrenia.

Disclosure: J. Wynn: None. I. Mathis: None. B. Roach: None. D. Mathalon: Part 1; Astra-Zeneca. J. Ford: Astra-Zeneca. M. Green: None.

\section{Substantia Nigra Hyperactivity and Reduced Functional Connectivity with the Prefrontal Cortex in Schizophrenia Jong Yoon*, Michael Minzenberg, Cameron Carter}

\section{UC Davis, Sacramento, CA}

Background: The dopamine hypothesis remains one of the most enduring theories explaining psychosis in schizophrenia. While plausible models of prefrontal dysfunction leading to excess subcortical dopamine release have been proposed, direct evidence identifying the functional neural circuitry underlying this relationship has been lacking. Here, using event-related fMRI, we demonstrated the transient hyperactivity of the substantia nigra, one of the main sources of neural dopamine, during the response phase of working memory in schizophrenia. The hyperactivity occurred in the context of impaired task performance, prefrontal and striatal hypoactivity and diminished functional connectivity within this prefrontostriatalnigral circuit. In a sample of neuroleptic-naive subjects, we replicated nigral hyperactivity and diminished nigral-prefrontal connectivity. The magnitude of hyperactivity was highly predictive of positive, negative and disorganized symptoms. These results are consistent with the hypothesis that impaired prefrontal-striatal regulation of evoked substantia nigra activity could be a proximal cause for excess dopamine neurotransmission in schizophrenia.

Disclosure: J. Yoon: None. M. Minzenberg: None. C. Carter: None.

74. Nicotine and an Alpha4Beta2 Nicotinic Agonist, but not an Alpha7 Nicotinic Agonist, Attenuate Scopolamine-Induced Disruption in a Mouse Continuous Performance Test

Jared Young*, Jessica Meves, Mark Geyer

\section{University of California, San Diego, La Jolla, CA}

Background: The link between cognitive dysfunction and functional outcome observed in several psychiatric disorders has driven the need to develop pro-cognitive treatments. Treatment development has been slow, however, due to the 'translational bottleneck' related to the paucity of validated cross-species tasks that can assess putative cognition enhancers in rodents. Attention/vigilance in humans is commonly assessed using the continuous performance test (CPT). Thus, the NIMH-funded MATRICS initiative chose the CPT-identical pairs as the task from which evidence of drug-induced enhancement is 
required for a drug to obtain FDA approval as a pro-attentive treatment in schizophrenia. We have recently created and validated a mouse version of the $\mathrm{CPT}$ (the $5 \mathrm{C}-\mathrm{CPT}$ ). Pharmacological validation has included scopolamine-induced disruption at $1 \mathrm{mg} / \mathrm{kg}$ and subchronic nicotine-induced improvement of normal performance at $10 \mu \mathrm{g} / \mathrm{kg}$. Here we examine whether nicotine, a non-selective agonist at the nicotinic acetylcholine receptor (nAChR), the alpha7nAChR selective agonist PNU 282987, and the alpha4beta2 nAChR selective agonist ABT-418 could attenuate the disruption of performance produced by scopolamine in mice tested in the ${ }_{5} \mathrm{C}-\mathrm{CPT}$.

Methods: $\mathrm{C} 57 \mathrm{BL} / 6 \mathrm{~N}$ mice $(\mathrm{n}=44)$ were trained to perform the ${ }_{5} \mathrm{C}-\mathrm{CPT}$. Treatments used to reverse the $5 \mathrm{C}-\mathrm{CPT}$ deficits produced by $1 \mathrm{mg} / \mathrm{kg}$ scopolamine included: 1) nicotine (3,30, 300 $\mu \mathrm{g} / \mathrm{kg}) ; 2$ ) PNU 282987 $(1,3,30 \mathrm{mg} / \mathrm{kg})$; and 2$)$ ABT-418 $(12,40,120 \mu \mathrm{g} / \mathrm{kg})$. Nicotine $(300 \mu \mathrm{g} / \mathrm{kg})$ was included as a positive control in experiments 2 and 3. A 2-week washout occurred between experiments during which animals were counter-balanced into drug groups. During acute drug challenges, performance was further challenged using an extended session duration (250 rather than 120 trials) and variable stimulus duration $(0.75,1.25$, and $2 \mathrm{~s}$, rather than a fixed $1.5 \mathrm{~s}$ duration). Performance was analyzed using a one-way ANOVA with drug group as the between subject factor and post hoc analyses subjected to a Bonferroni post hoc correction.

Results: In experiment 1 , scopolamine impaired performance as measured by $\mathrm{d}$ prime while nicotine at $300 \mu \mathrm{g} / \mathrm{kg}$ attenuated that deficit $(\mathrm{F}(4,32)=7.7, \mathrm{p}<0.0001)$. In experiment 2 , scopolamine again impaired performance in the $5 \mathrm{C}-\mathrm{CPT}$; this effect was attenuated by $300 \mu \mathrm{g} / \mathrm{kg}$ nicotine but not by any dose of PNU $282987(\mathrm{~F}(5,31)=5.5$, $\mathrm{p}<0.005)$. In experiment 3 , scopolamine-induced disruption of $\mathrm{d}^{\prime}$ was again attenuated by $300 \mu \mathrm{g} / \mathrm{kg}$ nicotine, an effect that was also observed after pretreatment with $12 \mu \mathrm{g} / \mathrm{kg}$ ABT-418. These effects on d prime were not accompanied by changes in the subjects' accuracy of responding to lit vs. unlit cue stimuli, because scopolamine-induced impairment in accuracy was not attenuated by these nAChR agonists. Moreover, the effects observed were unlikely to be attributable to motoric abnormalities during performance given the finding that neither scopolamine alone nor scopolamine in combination with any of the nAChR agonists affected mean correct latencies to respond.

Discussion: Here we demonstrated that while scopolamine can impair mouse $5 \mathrm{C}$-CPT performance, this deficit can be attenuated reliably by acute nicotine pretreatment. This attenuation of scopolamine-induced attentional disruption was also observed for the selective alpha4beta2 nAChR agonist ABT-418, but not the alpha7 nAChR agonist PNU 282987. Considering that scopolamine impairs human CPT performance, future clinical studies could examine the efficacy of drug-induced attenuation of scopolamine effects as a biomarker for whether that drug may produce the same effect in man as in mice. Therefore, these data provide a) a clear preclinical to clinical pathway by which drugs can be assessed and b) evidence that selective alpha4beta2 $\mathrm{nAChR}$ agonists may reproduce the nicotine-induced improvements observed in attention.

Disclosure: J. Young: Part 4; Cerca Biotech. J. Meves: None. M. Geyer: Part 1; Wyeth, Scientific Advisory Board, Omeros Pharma, Scientific Advisory Board, Acadia Pharma, Program Evaluation Group, Cenomed Pharma, consultant, Cerca Insights, consultant, Johnson and Johnson Pharma, consultant, Amylin Pharma, consultant, Medivation Pharma, consultant, Merck, consultant, Sepracor, consultant, Takeda, consultant, Teva, consultant. Part 2; San Diego Instruments, Inc., 33\% equity, Wyeth, Scientific Advisory Board, 2008, Omeros Pharma, Scientific Advisory Board and equity. Part 4; Intra-Cellular Therapies; Service Agreement to UCSD.

\section{Nucleus Accumbens Anticipatory Responses to Reward are Associated with Stress-Induced Dopaminergic Activity Tiffany Love, Christian Stohler, Jon-Kar Zubieta*}

University of Michigan, Ann Arbor, MI

Background: There are extensive interactions between stress and motivational systems particularly at the level of the mesolimbic circuitry. Activity within this pathway has been shown to be critical in the encoding and modulation of salient stimuli, both rewarding (e.g. food, money) and aversive (e.g. stress). However, whether neural responses to rewarding stimuli are related to stress-induced neural activity in humans has yet to be been explored. This an important question given the relationship between stress and development or maintenance of drug abuse and other compulsive behaviors such as pathological gambling. Here, we sought to determine whether brain activity observed during the performance of a monetary incentive delay task (MID) was associated with stress-induced dopaminergic activity.

Methods: Fifty-four healthy participants (22 male, 32 female) were scanned with Positron Emission Tomography (PET) using [11C] raclopride, a selective $\mathrm{D}_{2} / \mathrm{D}_{3}$ receptor radiotracer at baseline and during an experimental stress challenge, moderate levels of sustained pain over 20 minutes. Measures of receptor binding (nondisplaceable binding potential) were calculated at full equilibrium using a bolus-continuous radiotracer infusion protocol and the cerebellum as reference region. The activation of the $\mathrm{D}_{2} / \mathrm{D}_{3}$ system was calculated as the difference between a non-painful control condition and the pain-stress condition. Subsequently, each subject underwent functional magnetic resonance imaging (fMRI) while performing a monetary incentive delay task specifically designed to examine the neural circuitry responsive to the anticipation of rewards.

Results: For the fMRI data, consistent with previous studies, whole brain group level analyses of the monetary incentive delay task indicated task effects centered in the left (BOLD-LNAc, $t=7.51$, $\mathrm{p}<0.0001$, FDR corrected) and right (BOLD-RNAc, $\mathrm{t}=8.78$, $\mathrm{p}<0.0001$, FDR corrected) nucleus accumbens and the anterior cingulate (BOLD-ACING, $\mathrm{t}=7.41, \mathrm{p}<0.0001$, FDR corrected). Functional data from these regions were extracted using Marsbar and introduced into voxel-by-voxel regression analyses of the PET data (e.g. three simple regression analyses with blood oxygenation level dependent (BOLD) activity in each of the ROIs as the dependent variable). Voxel-by-voxel analyses indicated significant negative correlations between the magnitude of stress-induced dopamine release within the left (DA-LNAc) and right (DA-RNAc) nucleus accumbens and BOLD responses to anticipatory reward stimuli. Specifically, BOLD-LNAc activity was negatively correlated with DALNAc $(\mathrm{t}=4.79, \mathrm{p}=0.026$, FDR corrected $)$ and DA-RNAc $(\mathrm{t}=3.41$, $\mathrm{p}=0.044$, FDR corrected). Similar negative relationships were observed for BOLD-RNAc with DA-LNAc $(\mathrm{t}=4.59, \mathrm{p}=0.019$, FDR corrected) and DA-RNAc $(\mathrm{t}=3.33, \mathrm{p}=0.029$, FDR corrected $)$. No significant correlations were noted for BOLD-ACING activity.

Discussion: Stress is a known risk factor for the development and maintenance of drug addiction as well as mood and anxiety disorders. Using two imaging modalities, this study establishes links between anticipatory neural responses to reward and dopaminergic responses to a stressor in healthy humans. Greater BOLD responses during the anticipation of a reward in the nucleus accumbens was associated with lower stress-induced dopaminergic activity within the nucleus accumbens. These results underline the significance of the nucleus accumbens in the processing of stressful and rewarding stimuli and are consistent with an interactive view of stress and motivational systems in humans.

Disclosure: T. Love: None. C. Stohler: None. J. Zubieta: Part 1; Eli Lilly Co, Merck. Part 2; Eli Lilly Co. Part 3; Eli Lilly Co.

\section{Perception Of Affect In Biological Motion Cues In Anorexia Nervosa}

Nancy Zucker*, Ashley Moskovich, H. Ryan Wagner, Cynthia Bulik, Rhonda Merwin, Joseph Piven, Kevin LaBar

Duke University Medical Center, Durham, NC

Background: Individuals with anorexia nervosa (AN) seem to be perpetually in motion as evidenced by excessive exercise routines, 
compulsive body-checking behavior, and profound psychomotor agitation. Herein, we develop a proposal suggesting that such acute awareness of the motive state of their body may have implications for the capacity of those with AN to empathically attune to others. Burgeoning evidence supports that empathic attunement extends well beyond capacities to perceive and label the experience of others The experience of empathy demands a sensual embodiment of the experience of others via a virtual reenactment of their felt sense. If true, then individual differences in the experience of one's body or in the intensity with which different emotional states are experienced would correspond with differing capacities to understand or be empathic with those experiences in others.

Methods: We examine the capacities of adult women with AN (21 with current AN (AN-C), 22 weight-restored (WR)) to perceive affect in body motion relative to 23 healthy adult women (CNTL). Participants viewed Pointlight Walkers (PW), animated displays in which the human form is reduced to small patches of light located at major joints. Participants indicated the emotional state portrayed by the image from five options (happy, sad, afraid, angry, neutral). Statistical analyses were based on a series of OLS regression models regressing the PW scale averaged over the five emotion subscales on a three-level proxy variable denoting diagnostic status. In a second series of analyses, we used tendency to engage in driven exercise for weight loss as a proxy for degree of motor movement. The effects of exerciserelated weight control on the PW subscale for sadness were tested.

Results: Age, level of education, and verbal IQ were not significantly different between groups. BMI was significantly lower in AN-C subjects relative to both WR and CNTL subjects, but WR subjects were not significantly different from CNTL subjects. The main effect for Group in the PW total score was marginally significant $\left(\mathrm{ChiSq}_{(\mathrm{df}=2)}=5.87 ; \mathrm{p}=0.0532\right)$; contrasts between levels of the group proxy indicated that average total PW scores were significantly decreased in the $\mathrm{AN}-\mathrm{C}$ group relative to the other two groups. The main effects for Group in models re-estimated after stratifying on the PW subscales were significant among two of the five conditions: Sadness $\left(\mathrm{ChiSq}_{(\mathrm{df}=2)}=12.74 ; \mathrm{p}=0.0004\right)$ and Anger $\left(\mathrm{ChiSq}_{(\mathrm{df}=2)}=\right.$ $6.69 ; \mathrm{p}=0.0352)$. In the former instance, scores for subjects with a diagnosis of AN were significantly decreased after viewing sad images relative to both WR subjects and to CTRL subjects based on post-hoc contrasts. Post-hoc evaluation of scores among AN-C subjects were significantly increased compared to both WR subjects and to CNTL subjects after viewing angry images. Both the main effects for Group $\left(\mathrm{ChiSq}_{(\mathrm{df}=2)}=15.64 ; \mathrm{p}=0.0004\right)$ and for the dichotomous proxy variable endorsing exercise-related weight control $\left(\mathrm{ChiSq}_{(\mathrm{df}=2)}=\right.$ 56.03; $\mathrm{p}=0.0250$ ) were significant. Subsequent post hoc contrasts involving the Group proxy indicated that PW Sadness scores among the AN-C group were significantly reduced relative to scores among subjects in either the WR or CNTL groups. Levels of the PW Sadness measure were also significantly lower among subjects who endorsed exercise as a means of weight control. A test of the interaction term crossing the exercise proxy with Group was not significant indicating the pattern holds irrespective of group membership.

Discussion: AN-C individuals had greater difficulty deciphering affect from motion cues, with particular difficulty with sadness. However, they demonstrated an advantage in deciphering anger. There was a significant main effect for participation in driven exercise to be associated with reduced capacity to recognize sadness. AN is a state of physical threat and, like any starved animal, the ability to perceive threat can be advantageous as it facilitates escape and adaptive protective mechanisms. Deciphering sadness would be of little advantage as starved organisms are not in a position to care for others. Thus, current results are comprehendible as a biological adaptation to starvation. Findings may also have clinical significance. Families of those with AN are in distress. Yet, those struggling with AN can appear seemingly indifferent to family pleas to engage in health changes. Current findings have implications for study of distributed neural circuitry related to action understanding in others and increase knowledge of the phenomenology of starvation in AN.
Disclosure: N. Zucker: None. A. Moskovich: None. H. Wagner: None. C. Bulik: None. R. Merwin: None. J. Piven: None. K. LaBar: None.

\section{Impact of Lurasidone and Olanzapine on Framingham Ten-Year Coronary Heart Disease Risk Estimate in Schizophrenia \\ John Newcomer*, Antony Loebel, Andrei Pikalov, Phillip Sarocco, Josephine Cucchiaro, Cynthia Siu}

\section{Washington University School of Medicine, St. Louis, MO}

Background: Patients with severe mental illness are at significantly increased risk for cardiac disease-related mortality. Strategies for the prevention of cardiovascular diseases recommended by the Third Adult Treatment Panel of the National Cholesterol Education Program (NCEP ATP III) guidelines are based primarily on the 10-year absolute Coronary Heart Disease (CHD) risk estimates determined from the Framingham Risk Score (FRS) function (Wilson et al., 1998). We tested the significance of treatment effects on changes in 10-year CHD risk from baseline to Week 6 endpoint in a randomized, double-blind, placebo-controlled study of lurasidone and olanzapine in patients with acute schizophrenia(PEARL 2).

Methods: Demographics, patient's medical history including tobacco use and treatment for hypertension or diabetes were recorded at the screening visit. Diabetes was considered present if at least one of the following criteria was met: 1) fasting blood glucose $>=126 \mathrm{mg} / \mathrm{dL}, 2$ ) receiving treatment for diabetes, or 3 ) HbA1c $>=6.5$. Hypertension was considered present when subjects had hypertension stage I (systolic 140 to $159 \mathrm{~mm} \mathrm{Hg}$ or diastolic 90 to $99 \mathrm{~mm} \mathrm{Hg}$ ), or hypertension stage II-IV (systolic $>=160 \mathrm{~mm} \mathrm{Hg}$ or diastolic $>=100 \mathrm{~mm} \mathrm{Hg}$ ). Vital signs and fasting laboratory measurements were assessed at baseline and over the course of the 6-week doubleblind treatment period. Subjects were randomized to receive oncedaily fixed doses of lurasidone $(40 \mathrm{mg} / \mathrm{d}$ or $120 \mathrm{mg} / \mathrm{d}$ ), olanzapine $15 \mathrm{MG} / \mathrm{D}$, or placebo. Analysis of covariance model, with terms for treatment, gender, treatment-by-gender interaction, and baseline value, was applied to compare treatment effects on assessment of 10-year CHD risk and individual risk factors at Week 6 endpoint. FRS estimate of 10-year CHD risk was the primary outcome measure in this post-hoc analysis. Secondary outcome measures included assessments of individual risk factors in the FRS model.

Results: Baseline lipid data were available for 439 of 475 subjects ( 343 male, 96 female) who participated in the PEARL 2 study. Mean age of this cohort was 37.8 years (SD 10.9, range 18 to 68 ). The analysis sample used to calculate the 10-year CHD risk (FRS sample) comprised 315 $(72 \%)$ subjects who met the FRS model age criterion (30 years or older). In the overall baseline sample, twelve percent were diabetic (male $13 \%$, female $8 \%$ ), $22 \%$ were hypertensive (male $21 \%$, female $25 \%$ ), $45 \%$ had low HDL ( $46 \%$ male $<=44 \mathrm{mg} / \mathrm{dL}, 43.5 \%$ female $=240 \mathrm{mg} / \mathrm{dL}$ (male $16 \%$, female 19\%). The mean baseline total cholesterol was 202 (SD 43) $\mathrm{mg} / \mathrm{dL}$ and HDL was 49 (SD 14) $\mathrm{mg} / \mathrm{dL}$. The baseline prevalence rate for smoking was significantly higher in males than females (overall $68 \%$; male $75 \%$ vs. female $47 \%$, $p<0.001$ ). The baseline mean 10-year CHD risk was higher in males at $9 \%$ (SD $7 \%$ ) compared to females at 5\% (SD 5\%) according to Wilson et al. (1998). Average risk ratio (10-year CHD absolute risk relative to normal reference risk) was 2.3 for male and 1.4 for female. The overall 10-year CHD risk estimates were $8.3 \%$ at Week 6 from the baseline of $8.4 \%$ for lurasidone, compared to $7.2 \%$ (baseline $6.6 \%$ ) for placebo, or $10.3 \%$ (baseline $8.5 \%$ ) for olanzapine respectively. Men treated with olanzapine had significantly high 10 -year CHD risk (12\% at Week 6 from baseline 9.4\%) compared to 9.3\% at Week 6 from baseline $9.4 \%$ $(\mathrm{p}<0.001)$ in men receiving lurasidone, or to $8.3 \%$ at Week 6 from baseline $7.6 \%(\mathrm{p}<0.001)$ in men receiving placebo. There were no significant differences in 10-year CHD risk estimates between treatment groups for women receiving lurasidone $(5.0 \%$ at Week 6 from baseline $5.0 \%)$, olanzapine $(5.0 \%$ from $5.8 \%)$, or placebo $(3.9 \%$ from $3.7 \%$ ) (treatment-by-gender interaction effect, po.05). There was a significant increase in fasting total cholesterol level in men receiving 
olanzapine $(+6.8 \mathrm{mg} / \mathrm{dL})$ compared to those receiving lurasidone $(-9.2 \mathrm{mg} / \mathrm{dl})$ or placebo $(-10.6 \mathrm{mg} / \mathrm{dL})(\mathrm{p}<0.05)$. Hypertension status was worse in males receiving olanzapine compared to placebo $(p<0.05)$, with no differences found in other treatment groups.

Discussion: These results suggest that the acute effect of lurasidone on 10-year CHD risk is comparable to that of placebo in patients with schizophrenia, based on these 6-week study results. Olanzapine was associated with higher risk compared to placebo or lurasidone treatment. Further investigation including longer-term exposure data is warranted to confirm these results.

Disclosure: J. Newcomer: Part 1; AstraZeneca Pharmaceuticals, BioVail, Consult to LItigation, H. Lundbeck, Janssen Pharmaceutica, Obecure, Otsuka Pharmaceuticals, Pfizer, Inc., Sepracor, Inc., Solvay Pharma, Inc., Vanda Pharmaceutica, Wyeth Pharmaceuticals. Part 2; Washington University School of Medicine, Compact Clinicals/Jones and Bartlett Publishing, Dainippon Sumitomo Pharma America, Inc., Vivus, Inc., Consult to Litigation. Part 4; NARSAD, Bristol-Myers Squibb, Pfizer, Inc., Wyeth, Sidney R. Baer Jr. Foundation, NIMH. Part 1; Bristol-Myers Squibb. A. Loebel: Part 5; Sunovion, Inc. A. Pikalov: Sunovion, Inc. P. Sarocco: Sunovion, Inc. J. Cucchiaro: Sunovion, Inc. C. Siu: Part 1; Pfizer, Inc., Dainippon Sumitomo Pharma America, Wyeth, Memory. Part 2; Pfizer, Inc., Dainippon Sumitomo Pharma America, Inc., Wyeth, Memory. Part 3; Pfizer, Inc., Dainippon Sumitomo Pharma America, Wyeth, Memory.

\section{Change in Cardiometabolic Risk Indicators in Children During 3 Months of Initial Antipsychotic Exposure}

Ginger Nicol*, Michael Yingling, Karen Flavin, Julia Schweiger, John Newcomer

\section{Washington University, St. Louis, MO}

Background: The US prevalence of childhood-onset obesity and type 2 diabetes, both predictors of cardiovascular risk, has increased to epidemic proportions in recent decades. Children with mental illness who are treated with antipsychotic medications are at additional risk for obesity and related risk conditions, evidenced by recent reports of increased rates of adverse cardiovascular events in this population. Recommendations for metabolic screening and monitoring in adults treated with antipsychotic medications were published in 2004 . However, rates of metabolic screening and monitoring for patients treated with antipsychotics remain low overall, with the lowest rates of glucose and lipid monitoring in children taking antipsychotics compared to other age groups. Early results from the Metabolic Effects of Antipsychotics in Children study (MEAC; PI Newcomer, MHo72912) indicate that 12 weeks of initial antipsychotic treatment is associated with increases in directly-measured adiposity and insulin resistance, a cardiometabolic profile change predictive of CVD and diabetes risk. The present analysis aims to provide information regarding changes in clinically recommended pediatric screening parameters for cardiometabolic risk, including body weight and fasting lipid profiles, for children during initial antipsychotic exposure.

Methods: Antipsychotic-naïve participants ages 6-18 were assessed before and after 3 months of randomized antipsychotic therapy with both gold standard and clinical measures of adiposity, cardiometabolic risk and insulin sensitivity. Clinical measures include anthropomorphic assessment (height, weight, BMI\%ile and waist circumference), fasting lipids and glucose, and C-reactive protein and liver enzymes. Clinical measures of adiposity, including waist circumference, height and weight, with calculated BMI and BMI percentile, are obtained at baseline, 6 and 12 weeks. For this preliminary analysis $(\mathrm{N}=113)$, mean change in clinical indicators of risk, including BMI $\%$ ile, fasting lipids and glucose were evaluated and compared to National Health and Nutrition Examination Survey (NHANES) and Integrated Healthcare Information Services (IHIS) national averages. Results: Preliminary data show that antipsychotic naïve children (mean age 11.5 +/- 2.7 years) entered the MEAC study with a baseline prevalence of overweight or obesity of $34 \%$ (13\% overweight, $21 \%$ obese). Baseline prevalence of "at-risk" status based on fasting lipid fractions of total cholesterol $>165 \mathrm{mg} / \mathrm{dl}$, LDL cholesterol $>130 \mathrm{mg} / \mathrm{dl}$ and triglyceride $>150 \mathrm{mg} / \mathrm{dl}$ was $16.8 \%, 6.2 \%$, and $1.8 \%$, respectively. After 3 months of antipsychotic treatment, $51 \%$ of the population met criteria for overweight $(24 \%)$ or obesity $(27 \%)$. The number of children with "at-risk" triglyceride values increased to $5.3 \%$. No appreciable changes in fasting LDL, total cholesterol or glucose values were observed.

Discussion: Preliminary data from the MEAC study indicates that children with mental health conditions have baseline prevalence of overweight and obesity that is very similar to the $32 \%$ rate of overweight (15\%) and obesity (17\%) in the general population, based on 1999-2006 NHANES data. Following 3 months of initial antipsychotic exposure in the MEAC study, rates of overweight and obesity increased to $51 \%$. Baseline rates of elevated lipid fractions in the MEAC study were also similar to that in the general population, based on 1999-2004 NHANES and 2003-2006 IHIS information: total cholesterol 9.6-10.7\%, LDL cholesterol 5.2-6.6\% and triglyceride $13.2-14.2 \%$. Following 3 months of antipsychotic exposure, a notable increase in fasting triglyceride, a surrogate marker for insulin sensitivity, was detected. These combined results do not support the idea that mental illness itself is a predictor of cardiometabolic risk in this pediatric sample, and strongly suggest that antipsychotic treatment rather than illness is responsible for adverse changes in body weight and fasting triglyceride.

Disclosure: G. Nicol: Part 1; NARSAD, Dana Brown Charitable Trust, Sidney R. Baer Jr. Foundation. Part 2; Washington University School of Medicine. Part 4; NARSAD, Dana Brown Charitable Trust, Sidney R. Baer Jr. Foundation. M. Yingling: None. K. Flavin: None. J. Schweiger: None. J. Newcomer: Part 1; AstraZeneca Pharmaceuticals, BristolMyers Squibb, BioVail, Consult to Litigation, H. Lundbeck, Janssen Pharmaceutica, Obecure, Otsuka Pharmaceuticals, Pfizer, Inc., Sepracor, Inc., Solvay Pharma, Inc., Vanda Pharmaceutica, Wyeth Pharmaceuticals. Part 2; Washington University School of Medicine, Compact Clinicals/Jones and Bartlett Publishing, Dainippon Sumitomo Pharma America, Inc., Vivus, Inc., Consult to Litigation. Part 4; NARSAD, Bristol-Myers Squibb, Pfizer, Inc., Wyeth, Sydney R. Baer Jr. Foundation, NIMH.

\section{Tardive Dyskinesia Among Patients Being Treated for a First Episode of Schizophrenia \\ Carlos Garcia-Ribera, Natasha Bennett, Melissa Naraine, Serge Sevy, Delbert Robinson*}

\section{The Zucker Hillside Hospital, Glen Oaks, NY}

Background: Although occurring less frequently than with treatment with first generation antipsychotics, treatment with second generation antipsychotics is associated with the development of tardive dyskinesia. This study examined abnormal involuntary movement rates and clinical correlates with a group of subjects with first episode schizophrenia spectrum disorders who were initiating treatment with the second generation antipsychotics olanzapine or risperidone.

Methods: One hundred twelve subjects (70\% male; mean age 23.3 $(\mathrm{SD}=5.0$ years $))$ with first-episode schizophrenia $(75 \%)$, schizophreniform disorder $(17 \%)$ or schizoaffective disorder $(8 \%)$ were randomly assigned to treatment with olanzapine $(2.5$ to $20 \mathrm{mg}$ daily) or risperidone ( 1 to $6 \mathrm{mg}$ daily). Subjects were required to have taken antipsychotics for a cumulative total of 12 weeks or less during their lifetime before study entry. All subjects were given the opportunity of being treated and assessed for 3 years. Subjects who met response criteria after 4 months of treatment with their assigned medication were continued on their successful medication for their remaining time in study. Subjects who did not meet response criteria, who developed intolerable side effects or who relapsed were removed from controlled treatment, treated based upon best clinical judgment and had continued longitudinal assessments. 


\section{S336}

Results: Eighty-seven (78\%) subjects had never taken any antipsychotic medication before study entry; fifteen (13\%) had taken 1 to 7 days of antipsychotic medication in their entire lifetime. Mean length of follow-up was 110.0 (95\% CI: 98.2, 121.2) weeks. The mean weeks before starting another antipsychotic was 77.1 weeks (95\% CI: 60.4, 93.8) for subjects randomized to olanzapine and 87.4 weeks (95\% CI: 70.4, 104.6) for subjects randomized to risperidone. During the study, 57 subjects had one or more ratings on the global item of the Simpson Dyskinesia scale indicative of abnormal movements. Of these 57 subjects, 2 had at least one rating of moderate severity on the global item of the Simpson Dyskinesia scale and 11 subjects had at least one rating of mild severity. The most severe rating for the remaining 44 subjects was questionable severity. Comparison of subjects who had any global Simpson Dyskinesia ratings of questionable or more severe with those who did not showed that younger subjects were more likely to have abnormal movements $(\mathrm{p}<0.05)$ but no effect of substance misuse or family history of schizophrenia. Nine subjects met Schooler and Kane research criteria for tardive dyskinesia. Of these nine subjects, one (who had never taken any antipsychotics) had abnormal involuntary movements at baseline assessment. Time to meeting research criteria for tardive dyskinesia for the remaining 8 subjects varied from week 36 to week 128 of treatment. Total antipsychotic medication treatment (both before and after study entry) before meeting tardive dyskinesia criteria for the 8 subjects were: 1 subject took risperidone only, 4 took more than one second generation antipsychotic and 3 took several second generation antipsychotics along with very brief treatment with haloperidol. Within the study, only 1 subject developed tardive dyskinesia while taking their originally randomized medication; the other 7 subjects had been switched from their randomized medication before developing tardive dyskinesia.

Discussion: Abnormal movements are not rare during treatment of first episode patients with current medication treatments. Younger age may be a risk factor for developing abnormal movements. The finding that subjects in our study who developed tardive dyskinesia almost always had been switched from their randomized antipsychotic suggests further study of abnormal movements as a marker for poor response to antipsychotic treatment by patients with early phase schizophrenia spectrum disorders.

Disclosure: C. Garcia-Ribera: Part 1; GlaxoSmithKline, Almirall, Otsuka, Sanofi. N. Bennett: None. M. Naraine: None. S. Sevy: Abbott. D. Robinson: Janssen, Bristol-Myers Squibb. Part 4; Janssen, BristolMyers Squibb.

8o. Skeletal Deficits and Recovery in Methylphenidate Treated Rats Panayotis Thanos*, James Swanson, Michelle Mary, Haden Janda, Christine Snearly, Winfred Abrams, Lisa Robison, Mala Ananth, Tim Wigal, Michael Hadjiargyrou, Gene-Jack Wang, Nora Volkow, David Komatsu

\section{NIAAA, Upton, NY}

Background: Up to $9 \%$ of American youths are diagnosed with Attention Deficit Hyperactivity Disorder (ADHD). A high percentage $(\sim 90 \%)$ are treated with stimulant medications; namely amphetamine and methylphenidate (MP). Treatment is usually initiated in childhood, but increasingly treatment continues or is started in adolescence or adulthood. This rat study examined the effects of oral MP at clinically relevant doses beginning in adolescence so as to test the hypotheses that long-term treatment is detrimental to skeletal development and cessation of treatment leads to recovery of normal skeletal characteristics.

Methods: Male 4-week adolescent rats $(n=72)$ were randomly divided into three treatment groups (water, MP 4/10, MP 30/60) and two protocols (standard, recovery) resulting in 12 rats per treatment and protocol. Rats within the standard protocol group received daily in their drinking water one of the following treatments: water, MP 4/10, or MP $30 / 60$ for thirteen weeks. Following the 13 weeks of treatment, rats were sacrificed. Rats within the recovery protocol group received daily in their drinking water one of the following treatments: water, MP 4/10, or MP $30 / 60$ for 13 thirteen weeks. Following 13 weeks of treatment, rats underwent a five week recovery period, during which they received only water and were then sacrificed (18 weeks after treatment initiation). Following sacrifice, multiple biologic and biomechanic outcomes were measured based on the femur, tibia, vertebral body, and total vertebrae. Weights were recorded daily during the treatment and recovery (if applicable) periods.

Femora and tibiae were harvested for: DXA determination of bone mass density (BMD), Bone Mineral Content (BMC), and area; caliper measurements of length, anterior-posterior (AP) and medial-lateral (ML) diameter; and 3-point bending tests for energy to failure (EF), stiffness (SF), and ultimate force (UF), as well as histologic and gene expression analyses. Serum was also collected for biomarker analyses. One-way ANOVA and Dunnett's tests were used to identify significant differences $(\mathrm{p}<0.05)$.

Results: Weight gain during treatment in MP high rats was $15 \%$ less than Veh rats. Femoral and tibial BMD and BMC were lower in MP high compared to Veh (femur: 6\% \& 9\%; tibia: 5\% \& 9\%, respectively). In addition, femoral AP diameter was smaller in MP low (5\%) and MP high (9\%) as compared to Veh. Biomechanically, EF was lower in MP low (25\%) and MP high (31\%) compared to Veh, and UF was $14 \%$ lower in MP high. In the Recovery rats, none of these bone parameters were significantly different. Serum alkaline phosphotase (ALP) was $31 \%$ higher in MP high rats compared to Veh, and similarly elevated in Recovery rats.

Discussion: In summary, MP treatment resulted in less weight gain and weaker, less mineralized, smaller bones, particularly at high doses. However, these effects were ameliorated within 5 weeks of MP discontinuation. Higher serum ALP in MP high rats suggests increased osteoblast activity that continues despite MP discontinuation, but bone specific ALP remains to be quantified. Also, ongoing experiments to assess osteoclast activity, histologic and gene expression studies need to be completed to determine how MP impairs skeletal development. Clinically, these data suggest that adolescents treated with high dose MP may have weaker bones and an increased fracture risk.

Disclosure: P. Thanos: None. J. Swanson: None. M. Mary: None. H. Janda: None. C. Snearly: None. W. Abrams: None. L. Robison: None. M. Ananth: None. T. Wigal: None. M. Hadjiargyrou: None. G. Wang: None. N. Volkow: None. D. Komatsu: None.

81. RDC-0313 (ALKS 33), a Novel Opioid Receptor Modulator, Reduces Olanzapine-Induced Weight Gain in Female Rats

Mark Todtenkopf ${ }^{*}$, Krystal O’Neill, Sean Kelly, Katherine Richie, Reginald Dean, David Eyerman, Daniel Deaver

\section{Alkermes, Inc., Waltham, MA}

Background: Metabolic dysfunction and weight gain are associated with antipsychotic (ATAP) treatments. Increases in BMI (body mass index) can be observed in as little as 1 week after initiating treatment (Kluge et. al., 2009). Recent evidence suggests that endogenous opioids may be involved in regulating some types of weight gain (Yuan, et. al., 2009). Using a rat model of olanzapine (OLZ)-induced weight gain in female rats (Davoodi, et. al., 2006), we examined the effects of RDC0313, a new opioid modulator that acts as an antagonist at $\mu$ opioid receptors, with mixed agonist/antagonist activity at $\kappa$ and $\delta$ receptors We have characterized unique pharmacological properties of RDC-0313 using rodent models of reward-related disorders and RDC-0313 is currently under development for central nervous system (CNS)-related disorders. Preliminary data from separate studies suggests that circulating levels of the gut hormone, ghrelin, would be attenuated after administration of RDC-0313. Thus, we hypothesize that RDC-0313 may be having non-CNS related effects that could play a role in ATAPrelated weight gain and may limit fasting ghrelin conditions.

Methods: The first objective was to examine potential effects of RDC0313 on OLZ-induced weight gain. Three groups of female rats $(n=8 /$ 
group) were used for this study: 1) OLZ only; 2) OLZ with RDC-0313; and 3) vehicle control. Rats were assigned to treatment groups using a random block design based on initial body weight. The OLZ group was given PO twice daily ( 6 hours between doses) at a dose of $1 \mathrm{mg} / \mathrm{kg}$ (in $1 \%$ methylcellulose, for 10 consecutive days). RDC-0313 (2 mg/kg, SC) was administered concurrent with the afternoon administration of OLZ. The second objective was to examine potential effects of RDC0313 on the efficacy of OLZ. OLZ administration attenuates amphetamine-induced locomotion. Rats were co-administered amphetamine and OLZ with or without RDC-0313 and open-field activity was measured to determine if RDC-0313 affects OLZ's ability to reduce amphetamine-induced activity. In addition, we measured nucleus accumbens (NAc) dopamine (DA) release in response to OLZ with or without pretreatment with RDC-0313 to determine any neurochemical effects interacting with OLZ administration.

Results: In the first study, while all rats gained weight, OLZ alone caused greater increases in weight gain and the increased gain was apparent by day 5 . Weight gain in rats receiving RDC-0313 was similar to those in vehicle-controls rats, demonstrating the ability of RDC-0313 to block OLZ-induced weight gain. In the second study, pretreatment with RDC-0313 did not affect OLZ attenuation of amphetamineinduced hyperactivity. Finally, pretreatment with RDC-0313 did not affect NAc DA levels when co-administered with OLZ; however we observed decreases in DOPAC and HVA suggesting DA turnover may have been attenuated in rats pretreated with RDC-0313.

Discussion: Based on these data, RDC-0313 was able to reduce OLZassociated weight gain and may potentially offer an adjunct therapy to patients with antipsychotic-related weight gain. Importantly, RDC-0313 given in conjunction with OLZ did not inhibit OLZ efficacy in the amphetamine-induced hyperactivity model. Studies are ongoing examining potential mechanisms by which RDC-0313 may be affecting ATAP-related weight gain.

Disclosure: M. Todtenkopf: Part 5; Alkermes, Inc. K. O’Neill: Alkermes, Inc. S. Kelly: Alkermes, Inc. K. Richie: Alkermes, Inc. R. Dean: Alkermes, Inc. D. Eyerman: Alkermes, Inc. D. Deaver: Alkermes, Inc.

82. An Investigation of an Association between Psycho-Pharmacological Intervention and Insurance Status

Tanya Alim*, Elaine Graves, Fredric Lombardo, Marlon Daniel, Ashanta Brady, Candace Giles, William Lawson

\section{Howard University, Washington, DC}

Background: Treatment of mental illness is seen as an unmet need for a vast majority of Americans and disproportionately impacts minority populations. Studies have shown that minorities are less likely to visit mental healthcare facilities as well as comply with prescribed pharmacological therapies. Researchers have examined numerous factors that may explain the gap between access and delivery in minority populations. Few studies have evaluated how provider, drug coverage, and demographic factors explain the disparities in treatment of psychiatric disorders. In most urban settings there is a less developed health infrastructure and poor health literacy that further impacts members of the community and may increase the likelihood for a patient to have under-treated mental disorders. In a previous study conducted at Howard University, patients were approached to undergo a cross sectional questionnaire evaluating personal exposure to trauma in primary care offices. In the present study, researchers examined the disparities in treatment of psychiatric disorders (Posttraumatic Stress Disorder-PTSD, Major Depressive EpisodeMDE, and other anxiety disorders) based on differences in insurance type, education, and other demographic criteria. Patients with private insurance were hypothesized to be more likely to receive selective serotonin reuptake inhibitors- SSRIs as compared with those with public insurance.

Methods: Patients were approached in the primary care offices at Howard University Hospital to participate in a cross sectional study assessing lifetime personal exposure to trauma. Patients who provided written consent to participate in the study and completed the questionnaire were then called back to undergo a DSM-IV structured clinical interview if they self reported at least one traumatic event. Approximately $69 \%$ of the patients reported at least one traumatic event. Further analyses were conducted to describe the treatments received, type of insurance(public vs private), socioeconomic factors (i.e. income, geographical location, and educational level), as well as demographic characteristics (i.e. race/ethnicity, religiosity/spirituality, age, gender, marital status, and family home). Public Insurance was operationalized as any health insurance plan that was government funded (i.e. Medicaid, Medicare). Appropriate medical treatment was determined by use of the APA Treatment Guidelines for PTSD. SAS 9.1.3 (SAS Institute, Cary, NC) was used to describe the constructs and variables in the study by means and frequencies to identify factors with potential association with a patient seeking mental health care. Odds Ratios were calculated to quantify the strength of association between the outcome of SSRI prescription and Insurance type.

Results: Analysis was performed on a subset of patients who had either public or private insurance $(n=288)$. Bivariable association was examined between those patients receiving an SSRI and Insurance type (public vs. private insurance). There was no statistically significant association found between the two insurance categories $(\mathrm{OR}=1.096$, $\mathrm{P}=0.8690$ ).

Discussion: Public insurance can be viewed as a corollary of low income. Based on our results, insurance type does not appear to be a factor in disparity of pharmacological treatment. Our findings suggest that income may not contribute significantly to lack of access to quality care. We will explore other socio-demographic variables to determine other potential associations of disparity in health care.

Disclosure: T. Alim: Part 4; Astra Zeneca. E. Graves: None. F. Lombardo: None. M. Daniel: None. A. Brady: None. C. Giles: None. W. Lawson: AstraZeneca, Pfizer, Reckitt Benckiser. Part 1; AstraZeneca, Pfizer, Reckitt Benckiser.

\section{MTHFR Maternal Influenza and Other Respiratory Infections Increase Risk of Bipolar Disorder in Offspring}

Alan Brown*, Raveen Parboosing, Yuanyuan Bao, Catherine Schaefer

\section{New York State Psychiatric Institute, New York, NY}

Background: Accumulating evidence from epidemiologic studies suggests that maternal respiratory infections, including influenza, are involved in the etiology of schizophrenia. To our knowledge, however, no studies have utilized prospective data from individual pregnancies to determine whether these infections increase the risk of bipolar disorder among offspring.

Methods: In a follow-up of the Child Health and Development Study (CHDS), a large population-based birth cohort, we investigated the relationship between prospectively documented maternal exposure to respiratory infections and risk of bipolar disorder. Cases with bipolar disorder were identified by registry linkages between CHDS and Kaiser Permanente Medical Plan (KPMCP) data on bipolar disorder diagnoses and use of medications to treat bipolar disorder. Potential cases were interviewed with the SCID for DSM-IV-TR and consensus diagnoses were made by three psychiatrists; 60 cases were diagnosed and were compared to 8,919 non-cases. Prospectively collected data on maternal respiratory infections from the CHDS database were utilized.

Results: Maternal influenza infection during pregnancy was associated with a greater than fivefold increased risk of bipolar disorder among offspring $(\mathrm{RR}=5.24,95 \% \mathrm{CI}=1.87-14.67, \mathrm{p}<.01)$. Maternal respiratory infection during the periconceptional period was related to a greater than twofold increased risk of bipolar disorder $(\mathrm{RR}=2.69,95 \%$ $\mathrm{CI}=1.32-5.48, \mathrm{p}=.01)$. The associations were not confounded by maternal age, race, education, smoking, and birthweight.

Discussion: These findings suggest that exposure to influenza and other respiratory infections during pregnancy may increase the risk of bipolar disorder in offspring. Hence, this exposure may not be specific 


\section{S338}

to schizophrenia among major psychiatric disorders. The findings add to a growing body of evidence of shared etiologic factors in schizophrenia and bipolar disorder. Further studies of biomarker based assays of maternal sera in relation to bipolar disorder risk are planned. If confirmed, this work may have implications for the prevention of bipolar disorder by influenza vaccinations and other measures to treat and prevent respiratory infections during pregnancy. Moreover, this work may inspire translational animal models of maternal immune activation and exposure to infection in order to shed light on pathogenic processes by which these infections alter fetal neurodevelopmental processes, leading to bipolar disorder phenotypes.

Disclosure: A. Brown: None. R. Parboosing: None. Y. Bao: None. C. Schaefer: None.

\section{Measuring Addiction Propensity and Severity: The Need for a New} Instrument

Kevin Conway*, Janet Levy, Michael Vanyukov, Redonna Chandler, Joni Rutter, Gary Swan, Michael Neale

\section{National Institute on Drug Abuse, Bethesda, MD}

Background: Drug addiction research requires but lacks a valid and reliable way to measure both the risk (propensity) to develop addiction and the severity of manifest addiction. This paper argues for a new measurement approach and instrument to quantify propensity to and severity of addiction, based on the testable assumption that these constructs can be mapped onto the same dimension of liability to addiction. The case for this new direction becomes clear from a critical review of empirical data and the current instrumentation.

Methods: The instruments in this review were identified through a literature search of several databases (e.g., PubMed) for key terms such as "drug abuse severity", "drug addiction severity", "substance abuse severity", "substance addiction severity". To be included, an instrument had to be explicitly designed to measure severity of drug or alcohol addiction. Excluded were instruments that measure nicotine addiction, screening instruments, and those that measure only one specific domain of addiction (e.g., craving). The review includes the purpose and content of each instrument and other information on the applicability of the instrument for liability measurement.

Results: The many assessment instruments in use today have proven utility, reliability, and validity, but they are of limited use for evaluating individual differences in propensity and severity. Existing instruments have several limitations: (1) most fail to measure the hallmark characteristic of addiction (compulsivity in seeking and using drugs despite harmful consequences); (2) the content of existing measures is unlikely to reflect the full range of addiction severity; (3) only one extant instrument is currently available to measure propensity to addiction, let alone both the propensity and the severity of addiction on the same metric; (4) most instruments were constructed using classical test theory methodology, which has its own psychometric limitations that directly bear on severity measurement.

Discussion: The conceptual and methodological shortcomings of instruments currently used in research and clinical practice can be overcome through the use of new modern psychometric approaches to develop a reliable, valid, and standardized assessment instrument(s) to measure and distinguish individual variations in expression of the underlying latent trait(s) that comprises propensity to and severity of drug addiction. The plausibility of a single common (versus drugspecific) liability dimension (latent trait) and the feasibility of its measurement are supported by clinical, neurobiological, genetic, and statistical findings. Item Response Theory (IRT) appears particularly suited for the derivation of an index of liability to addiction and, when coupled with sophisticated computer technology (e.g., IRT-based computerized adaptive testing [CAT]), will enable measurement covering the full phenotypic scale, with maximum flexibility, accuracy, and efficiency. Such instrumentation would enhance our capacity for drug addiction research on linkages and interactions among familial, genetic, psychosocial, and neurobiological factors associated with variations in propensity and severity. It would lead to new opportunities in substance abuse prevention, treatment, and services research, as well as in interventions and implementation science for drug addiction.

Disclosure: K. Conway: None. J. Levy: None. M. Vanyukov: None. R. Chandler: None. J. Rutter: None. G. Swan: None. M. Neale: None.

\section{Clinical Characteristics of Children Receiving Antipsychotic Medication}

Robert Findling*, Sarah Horwitz, Boris Birmaher, Robert Kowatch, Mary Fristad, Eric Youngstrom, Thomas Frazier, David Axelson, Neal Ryan, Christine Demeter, Judith Depew, Benjamin Fields, Mary Kay Gill, Elizabeth Deyling, Brieana Rowles, L. Eugene Arnold

\section{Case Western Reserve University, Cleveland, $\mathrm{OH}$}

Background: Recent reports indicate an increase in the prescription rates of antipsychotics to children. In addition, data suggest that a substantial number of children may be receiving antipsychotic medications "off-label" and for reasons not supported by scientific evidence. Specifically, concerns have been raised about the prescription of atypical antipsychotics to children with attention-deficit/ hyperactivity disorder (ADHD) and, despite speculation that antipsychotics may be prescribed to treat disruptive behaviors, this possibility has not been explicitly investigated. A plausible but unexplored contributor to the increased prescription of antipsychotics could be the increase in the diagnosis of bipolar disorder in children and adolescents, considering that antipsychotics may be prescribed for these youths. This study explored the demographic and diagnostic features of children who were currently receiving antipsychotic medications compared to children who were prescribed other psychotropics in an epidemiologically ascertained sample of children with and without elevated symptoms of mania. In addition, this study specifically examined whether children who met diagnostic symptom criteria for ADHD without comorbidity were receiving antipsychotics. All diagnoses were based on semi-structured research interviews using formal DSM criteria.

Methods: Study participants were recruited from 10 child outpatient mental health clinics associated with four universities in Ohio and Western Pennsylvania under the auspices of the NIMH-funded Longitudinal Assessment of Manic Symptoms (LAMS) study. Guardians with children between 6-12 years who presented for new clinical evaluations completed the Parent General Behavior Inventory-10 Item Mania Scale (PGBI-10M). All children who scored $\geq 12$ on the PGBI$10 \mathrm{M}$ and a select demographically matched comparison group of patients who scored $\leq 11$ were invited to participate in the study. Children were divided into two medication groups: those receiving at least one antipsychotic medication and those receiving other psychotropics. The groups were compared on demographics, diagnoses, psychiatric symptoms, functioning, and past hospitalizations. Results: Of the 707 children enrolled in the LAMS study, $443(63 \%)$ were currently prescribed medication at baseline. Of these, $157(35 \%)$ were currently receiving an antipsychotic and $286(65 \%)$ were prescribed other psychotropics. Children with a diagnosis of psychosis $(p=0.016)$ or bipolar disorder $(p<0.001)$ were more likely to receive an antipsychotic than other medications. However, children with a diagnosis of ADHD without comorbidity $(\mathrm{p}=0.010)$ or ADHD plus another diagnosis $(p<0.001)$ were more likely to be treated with other medications. Multivariate results indicated that being prescribed antipsychotics was related to: being White; having been previously hospitalized; having a psychotic or bipolar I disorder; and the site where the child was receiving services $(\mathrm{p}<0.001)$. The ADHD group who were prescribed antipsychotics $(\mathrm{N}=109)$ had significantly more diagnoses at baseline compared to the ADHD group not prescribed an antipsychotic $(\mathrm{N}=252)(\mathrm{p}<0.001)$. Of the 62 diagnosed with ADHD without comorbidity, $13(21 \%)$ were receiving an antipsychotic. Youth 
diagnosed with ADHD being treated with antipsychotics were more likely to have a comorbid diagnosis of a bipolar disorder $(p<0.001)$ than any other comorbid diagnosis. However, if a child with ADHD was diagnosed with a comorbidity other than a bipolar diagnosis, the child was more likely to be treated by a non-antipsychotic medication $(\mathrm{p}<.001)$. Specifically, those children with ADHD being treated with a medication other than an antipsychotic were more likely to have a comorbid disruptive behavior disorder $(\mathrm{p}=0.003)$.

Discussion: This study finds that in a sample mostly from new referrals to university affiliated outpatient mental health clinics, enriched for symptoms of mania, it is relatively common for a child to be prescribed an antipsychotic medication. However, the only diagnoses associated with a greater likelihood of being treated with an antipsychotic were psychotic disorders or bipolar I disorder.

Disclosure: R. Findling: Part 1; Abbott, Addrenex, AstraZeneca, Biovail, Bristol-Myers Squibb, Forest, GlaxoSmithKline, Johnson \& Johnson, KemPharm, Lilly, Lundbeck, Neuropharm, Novartis, Noven, Organon, Otsuka, Pfizer, Sanofi-Aventis, Sepracore, Schering-Plough, Shire, Solvay, Supernus Pharmaceuticals, Validus, Wyeth. S. Horwitz: None. B. Birmaher: Forest Laboratories, Inc., Schering-Plough. R. Kowatch: None. M. Fristad: None. E. Youngstrom: None. T. Frazier: Shire. D. Axelson: None. N. Ryan: None. C. Demeter: None. J. Depew: None. B. Fields: None. M. Gill: None. E. Deyling: None. B. Rowles: None. L. Arnold: Abbott, Celgene, Lilly, McNeil, Novartis, Neuropharm, Organon, Shire, Sigma Tau, Targacept.

\section{Relationship between Adverse Childhood Experiences and Alcohol-Related Outcomes in Recently Detoxified Alcoholics Melanie Schwandt*, Julnar Issa, Ashley Smith, Linda Doty, Tom Lionetti, Dave Spero, Christopher Geyer, Daniel Hommer, Markus Heilig, D. Ted George, Vijay Ramchandani}

National Institutes of Health Bethesda, MD

Background: There is growing evidence that adverse childhood experiences are associated with a range of adverse outcomes in adults, including alcohol and substance use disorders as well as other psychiatric disorders. The objectives of this study were to: (1) examine the prevalence of early life trauma and stressful experiences in a sample of recently detoxified alcoholics, and (2) to evaluate the relationship between these adverse childhood experiences and alcoholrelated outcomes, including co-morbid psychiatric diagnoses.

Methods: Data were obtained from 172 alcohol-dependent patients (53 females and 119 males) undergoing inpatient detoxification in the NIAAA treatment research program. Adverse childhood experiences were assessed using the Early Life Stress Questionnaire (ELSQ) and the Childhood Trauma Questionnaire (CTQ). Alcohol dependence severity was assessed using the Alcohol Dependence Scale (ADS), and recent and lifetime drinking history were assessed using the Timeline Follow Back (TLFB) and Addiction Severity Index (ASI). Psychiatric diagnosis was assessed using the Structured Clinical Interview for DSM-IV-TR diagnoses (SCID).

Results: $148 / 172$ patients $(86.0 \%)$ reported stressful events on the ELSQ. Results from the CTQ indicated that $77 / 172$ patients $(44.8 \%)$ experienced childhood emotional abuse, 82/172 (47.6\%) experienced physical abuse, 46/172 (26.7\%) experienced sexual abuse, 86/172 (50.0\%) experienced emotional neglect, and 55/172 (32.0\%) experienced physical neglect. Women were more likely to report both emotional (Chi-Square $=9.48, p=0.002)$ and sexual abuse (Chi-Square $=34.86$, $\mathrm{p}<0.0001)$. Patients who reported ELSQ events ( 1 or more event) had an earlier age of onset of alcohol use $(\mathrm{F}=9.5, \mathrm{p}=0.002)$ and significantly higher scores on the $\operatorname{ADS}(\mathrm{F}=7.2, \mathrm{p}=0.008)$. In addition, there was a significant positive relationship between the number of ELSQ events and ADS score $\left(\mathrm{R}^{2}=0.05, \mathrm{p}=0.02\right)$. Analysis of results from the CTQ revealed an earlier age of onset of alcohol use among patients who experienced childhood physical abuse $(\mathrm{F}=13.0$, $\mathrm{p}=0.0004)$ and sexual abuse $(\mathrm{F}=6.0, \mathrm{p}=0.02)$, while higher ADS scores were found for patients who reported emotional abuse $(\mathrm{F}=15.7$, $\mathrm{p}=0.0001)$ and emotional neglect $(\mathrm{F}=11.6, \mathrm{p}=0.0008)$. There were no effects of early life stress exposure on drinking frequency or quantity in the 90 days prior to admission. Logistic regression analyses indicated increased risk of both co-morbid anxiety disorders $(\mathrm{p}<0.001$, odds ratio $=1.40,95 \% \mathrm{CI}=1.18$ to 1.56$)$ and mood disorders $(\mathrm{p}=0.001$, odds ratio $=1.28,95 \% \mathrm{CI}=1.11$ to 1.49$)$ with increasing frequency of ELSQ events. Increased risk for anxiety disorders was also found for patients who specifically experienced childhood physical abuse $(\mathrm{p}=0.02$, odds ratio $=2.87,95 \% \mathrm{CI}=1.23$ to 6.71$)$, while increased risk for mood disorders was found for patients who specifically experienced childhood sexual abuse $(\mathrm{p}=0.04$, odds ratio $=2.94,95 \% \mathrm{CI}=1.03$ to 8.39 ). Increased risk for developing a substance use disorder (other than alcohol) was associated with childhood emotional neglect $(\mathrm{p}=0.05$, odds ratio $=3.00,95 \% \mathrm{CI}=1.01$ to 8.86 ).

Discussion: Adverse childhood experiences were highly prevalent in our sample of recently detoxified alcoholics. While patients who experienced early life stress or trauma did not differ from patients lacking these experiences in their recent drinking history, they did exhibit earlier onset of alcohol use as well as a higher level of alcohol dependence severity. In addition, adverse childhood experiences were associated with a higher risk of co-morbid anxiety, mood, and substance use disorders. Our findings are consistent with previous research demonstrating the impact of adverse childhood experiences on adult alcohol-related and behavioral health outcomes. Future investigations will examine the moderating role of sex and of genetic factors on the effects of early life stress exposure in this population. Disclosure: M. Schwandt: None. J. Issa: None. A. Smith: None. L. Doty: None. T. Lionetti: None. D. Spero: None. C. Geyer: None. D. Hommer: None. M. Heilig: None. D. George: None. V. Ramchandani: None.

87. The Association between Glutamate System Genes and Brain Volume Alterations in Children with OCD

Paul Arnold*, Ke Wu, Frank MacMaster, Philip Easter, James Kennedy, Gregory Hanna, David Rosenberg

Hospital for Sick Children/University of Toronto, Toronto, ON, Canada

Background: Obsessive-compulsive disorder (OCD) is a common and often debilitating neuropsychiatric condition that affects $1-3 \%$ of the population worldwide. Individuals with OCD display regional volumetric brain abnormalities, which provide promising intermediate phenotypes of the disorder. Multiple lines of evidence have suggested that dysfunction in glutamate neurotransmission may play a role in OCD, and our own preliminary work revealed positive associations between glutamate system genes and ventral prefrontal and thalamic volume (Arnold et al., 2009). In this study we expanded our previous work and conducted a more comprehensive study to examine the association between glutamatergic candidate genes and regional brain volumes in children with OCD.

Methods: Volumes of brain regions selected for a priori evidence of association with OCD (thalamus, anterior cingulate cortex (ACC), orbitofrontal cortex (OFC), and caudate) were measured using structural magnetic resonance imaging (MRI) in 24 psychotropicnaïve pediatric OCD patients. Single nucleotide polymorphism (SNP) genotype data for nine glutamatergic candidate genes (DLGAP1, DLGAP2, DLGAP3, GRIN2B, SLC1A1, GRIK2, GRIK3, SLITRK1 and SLITRK5) and their nearby regions were selected for analysis based on either: 1) previous reported association with OCD in humans, or 2) evidence from animal models of OCD. Each volumetric phenotype was tested for association with the SNP genotypes using a linear regression model that assumes an additive contribution of each minor allele. All analyses included age and intracranial volume as covariates. Secondary analysis (left vs. right) was also performed if the corresponding total volume was significantly associated with a SNP after correcting for multiple comparisons. 


\section{S340}

Results: SNPs in several candidate genes showed nominal association $(\mathrm{P}<0.05)$ with multiple volumetric measures. After correcting for multiple comparisons, several SNPs remained significantly associated with volumetric alterations. The rs11842338 SNP near SLITRK5 was associated with decreased total, left and right thalamus volume. The rs4653112 SNP in DLGAP3 was associated with increased total and left, but not right OFC volume. Two SNPs in DLGAP1, rs1116345 and rs342483, were associated with increased ACC volume.

Discussion: These preliminary results suggest that the glutamate candidate genes such as $\mathrm{DLGAP}_{3}, \mathrm{SLITRK}_{5}$ and DLGAP1 may be associated with regional volumetric alterations in thalamus, OFC and ACC. We are currently collecting a larger sample in order to confirm our findings.

Disclosure: P. Arnold: None. K. Wu: None. F. MacMaster: None. P. Easter: None. J. Kennedy: None. G. Hanna: None. D. Rosenberg: None.

88. 5-HT3 Receptor Subunit Type A Gene (HTR3A) Polymorphism Is Associated with Anxiety and Increased Amygdala Responsiveness in Healthy Controls and Irritable Bowel Syndrome (IBS) Patients Lisa A Kilpatrick*, Jennifer S Labus, Kristen Coveleskie, Christian Hammer, Johanna Jarcho, Jim A McRoberts, Gudrn Rappold, Joshua Bueller, Brandall Suyenobu, Jenny C Papp, Sugandha Dandekar, Kirsten Tillisch, Beate Niesler, Emeran A Mayer

Center for Neurobiology of Stress, UCLA, Los Angeles, CA

Background: $5-\mathrm{HT}_{3}$ receptor $\left(5-\mathrm{HT}_{3} \mathrm{R}\right)$ antagonists are effective in treating patients with several stress-sensitive persistent pain disorders (including IBS and fibromyalgia) and have anxiolytic effects. The therapeutic effect in IBS is related in part to reduced amygdala engagement during expected visceral pain (Berman et al, Gastroenterology 2002). The HTR $3 A$ single nucleotide polymorphism (SNP) c. $-42 \mathrm{C}>\mathrm{T}(\mathrm{C} 178 \mathrm{~T}$; rs1062613) is associated with altered amygdala reactivity during processing of neutral facial stimuli in healthy control subjects (HCs) (Iidaka et al, J Neurosci 2005). We aimed to evaluate the influence of this particular $\mathrm{HTR}_{3} A \mathrm{SNP}$ on amygdala reactivity to emotional facial stimuli and non-emotional visual stimuli in female HCs and IBS patients.

Methods: Brain responses during a validated emotional reactivity task were measured in 55 female subjects ( 26 diagnosed with IBS) using fMRI (3.0 T). Subjects attended to the emotional expression (angry or fearful) of a target face in the Match Emotion condition (ME) and the shape of a target geometric form in the Match Form condition (MF). A region of interest analysis in $\mathrm{SPM}_{5}$ was used to examine the effect of the $\mathrm{HTR}_{3} \mathrm{~A}$ c. $-42 \mathrm{C}>\mathrm{T}$ genotype ( $\mathrm{C} / \mathrm{C}$ vs. $\mathrm{T}$ carrier) on amygdala responsiveness in HCs and IBS patients.

Results: Fifteen out of 29 HCs and 16 out of 26 IBS patients carried the C allele in a homocygous manner, nine HCs and seven patients carried the heterozygous $\mathrm{C} / \mathrm{T}$ genotype, and five $\mathrm{HCs}$ and three patients carried the homozygous $\mathrm{T} / \mathrm{T}$ genotype. Subjects carrying the $\mathrm{T}$ allele in either a homocygous or heterocygous manner $(\mathrm{C} / \mathrm{T}+\mathrm{T} / \mathrm{T})$ were combined into a single group ("T carriers") for analyses. Chi-square test indicated no significant association between $\mathrm{T}$ carrier status and diagnosis $(\mathrm{p}=.588)$. An ANOVA revealed a significant interaction of diagnosis and $\mathrm{T}$ carrier status on anxiety symptoms, with IBS patients reporting higher anxiety ratings, and $\mathrm{T}$ carriers (IBS and HCs combined) reporting lower anxiety ratings $(\mathrm{F}(1,51)=4.20, \mathrm{p}=.046 ; \mathrm{F}(1,51)=4.37, \mathrm{p}=.042$, respectively). Within IBS patients, $\mathrm{C} / \mathrm{C}$ genotype subjects demonstrated greater overall symptom severity $(\mathrm{t}(24)=2.102, \mathrm{p}=.046)$ and abdominal discomfort (bloating) ratings $(\mathrm{t}(24)=2.654, \mathrm{p}=.014)$ but did not differ in abdominal pain ratings $(\mathrm{p}>.05)$. Consistent with previous studies, the emotional reactivity task activated bilateral amygdala across all subjects, while no diagnosis or genotype effects on amygdala emotional reactivity (ME-MF) were found. However, C/C genotype subjects, regardless of diagnosis, demonstrated greater amygdala activity during both the ME and MF conditions when considered separately ( $p$ 's $<.05$, small volume corrected). This indiscriminate pattern of response suggests a generalized hyper-responsiveness of the amygdala. Further analyses revealed a subgroup of IBS patients with C/C genotype with unusually low differential amygdala activity during ME relative to MF. These subjects, compared to other $\mathrm{C} / \mathrm{C}$ genotype subjects demonstrated exceptionally high amygdala activity during MF (p's $<.05$ ).

Discussion: Regardless of diagnosis, the C/C genotype of the c. $-42 \mathrm{C}>\mathrm{T}$ polymorphism, compared to $\mathrm{T}$ carrier status is associated with increased anxiety and a generalized hyper-responsiveness of the amygdala. While this particular genotype is not required for a diagnosis of IBS, it plays a role in the expression of symptom severity. In addition, a subset of IBS patients with $\mathrm{C} / \mathrm{C}$ genotype demonstrated an extreme pattern of generalized amygdala hyper-responsiveness, failing to discriminate between emotional and neutral stimuli due to heightened responses during the non-emotional condition. Our findings suggest that the $\mathrm{C} / \mathrm{C}$ genotype predisposes individuals to a state of indiscriminant hypervigilance, especially in IBS patients. Subtyping of IBS patients based on gene variants of central receptors modulating emotional arousal may therefore improve the outcome of future clinical trials.

Disclosure: L. Kilpatrick: None. J. Labus: None. K. Coveleskie: None. C. Hammer: None. J. Jarcho: None. J. McRoberts: None. G. Rappold: None. J. Bueller: None. B. Suyenobu: None. J. Papp: None. S. Dandekar: None. K. Tillisch: Part 1; Avera. B. Niesler: None. E. Mayer: GSK, Avera.

89. Putative Psychiatric Risk Genes and Neonatal Brain Structure Rebecca Knickmeyer*, Sandra Woolson, Robert Hamer, Thomas Konneker, Weili Lin, J. Keith Smith, Guido Gerig, Martin Styner, John Gilmore

\section{UNC-Chapel Hill, Chapel Hill, NC}

Background: Studies in adolescents and adults have demonstrated that polymorphisms in putative psychiatric risk genes are associated with differences in brain structure. These findings are of great interest, but because the studies were carried out on older children and adults, they cannot address when in development these changes arise. In addition, gene-brain relationships may be confounded by medication, years of environmental and gene-environment interaction effects on the brain, and the disease process itself. In order to fully understand how genetic variants alter neurodevelopment, it is necessary to perform studies at much earlier periods. The present study tests whether specific genetic polymorphisms that have been implicated in psychiatric illness and structural brain phenotypes in older children and adults predict brain structure in neonates.

Methods: 373 neonates received structural MRI scans on a Siemens head-only $3 \mathrm{~T}$ scanner with MP-RAGE T1-weighted, and TSE, dualecho (proton density and T2 weighted) sequences. Brain tissue was classified using an automatic, atlas-moderated expectation maximization segmentation tool. Parcellation of each subject's brain into regions was achieved by non-linear warping of a parcellation atlas template. The lateral ventricles were segmented using InsightSnap (SNAP). 10 polymorphisms, representing 7 putative psychiatric risk genes, were genotyped using Sequenom $(\mathbb{R})$ iPLEX $($ Gold Genotyping Technology. This included variants in BDNF, COMT, APOE, ESR1, GAD1, and NRG1. We tested for significant relationships between genotypes and brain tissue volumes using mixed models, with gestational age at birth and intracranial volume as covariates.

Results: Nominally significant relationships with specific tissue volumes were observed for polymorphisms in DISC1, ESR1, GAD1, NRG1, and $A P O E$. The most consistent findings across different analyses were relationships between $\mathrm{rs} 9340799$ in ESR1 and total and parietal gray matter volumes, rs821616 in DISC 1 and frontal and parietal gray matter volumes, rs35753505 and rs6994992 in NRG1 and frontal gray matter volume, and rs2270335 in GAD1 and prefrontal gray matter volume. Differences between genotypes groups were on the order of $3 \%$. The observed relationships for ESR1 and GAD1 are consistent with previous studies in adults. No effects were observed for COMT or BDNF.

Discussion: While we cannot rule out the possibility of false positives, our study suggests that polymorphisms in ESR1, DISC1, NGR1, and GAD1 are 
associated with individual differences in brain tissue volumes in the first month of life. Participating children are returning at ages 1, 2, 4 and 6 years for follow-up scans and detailed developmental assessments so that, ultimately, we can test how these variants impact the trajectories of brain development throughout childhood.

Disclosure: R. Knickmeyer: Part 4; Pfizer. S. Woolson: None. R. Hamer: Part 1; Acadia, Alpharma, AstraZeneca, Cenerx, Corcept, EnabledMD, Epix, J \& J, Novartis, SAS, Sanodfi-Adventis, Wyeth. Part 2; BMS, Amgen, Lilly, Genetech, Proctor and Gamble, Sepracor. T. Konneker: None. W. Lin: None. J. Smith: None. G. Gerig: None. M. Styner: None. J. Gilmore: Part 4; Sepracor.

90. A Family-Based Association Study of the Serotonin Transporter Gene in Schizophrenia

Bhaskar Kolachana*, Alixandra Sebrechts, Jens Wendland, Qiang

Chen, Fengyu Zhang, Daniel Weinberger

NIMH, Bethesda, MD

Background: The serotonin transporter (SERT) gene has long been implicated in the pathogenesis of major psychiatric disorders. SERT is known to influence mood, emotion, cognition and efficacy of antidepressants, particularly that of selective serotonin uptake inhibitors. Atypical antipsychotic treatments are thought to exert their effects partially through the $5 \mathrm{HT}$ receptors. The $44 \mathrm{bp}$ ins/del variant $(5 \mathrm{HTT}-\mathrm{LPR}$ ) regulates the expression of SERT gene and a snp (A/G) within this polymorphic locus, rs25531, has been shown to alter SERT expression. A third variant, Stin2, a $17 \mathrm{bp}$ repeat in the second intron, has been shown to be a transcriptional enhancer. Previous genetic studies have yielded inconsistent evidence for the association of SERT and schizophrenia, perhaps in part because most studies have been unable to control for population stratification in the case-control design and these variants show marked population divergence. The biological and therapeutic evidence suggests that alterations in the function of this SERT protein could be involved in the development of schizophrenia.

Methods: In this study we examined the association of these three markers with risk for schizophrenia in two independently collected samples of Caucasian (CBDB: $\mathrm{N}=408$ cases, NIMH GI: $\mathrm{N}=139$ cases) and African American (NIMH GI: $\mathrm{N}=36$ cases) origin using a "caseparent" trio design. The data were analyzed using FBAT for familybased association analysis.

Results: Overall, no significant association was observed for any of these three markers with schizophrenia. However, in CBDB dataset, significant associations were found for markers STin2 $(\mathrm{p}=0.041)$ and 5 HTT-LPR $(\mathrm{p}=0.040)$ with schizophrenia in Caucasian females only (the short allele of the 5 HTT-LPR and 12 -repeat of the STin2). Haplotype analysis also suggests a preferential co-transmission of risk alleles (short and 12 repeats) to the affected female offspring of Caucasian probands $(\mathrm{P}=0.0269)$. In African American females, only the ${ }_{5}$ HTT-LPR short allele showed a trend of association with illness $(p=0.09)$.

Discussion: These results add to prior evidence that the serotonin transporter gene play a role in the pathogenesis of schizophrenia, at least in females. Additional analyses are underway to examine the gender-specific risk architecture of this gene.

Disclosure: B. Kolachana: None. A. Sebrechts: None. J. Wendland: None. Q. Chen: None. F. Zhang: None. D. Weinberger: None.

\section{Interaction of Specific Genetic Variants with Treatment E} ffectiveness in Disulfiram Pharmacotherapy for Cocaine Dependence David Nielsen, Wen Huang, Mark Harding, Sara Hamon, Jan Lindsay, Thomas Kosten*

Baylor College of Medicine, Houston, TX, Baylor College of Medicine Michael E. DeBakery VA Medical Center, Houston, TX

Background: Disulfiram is a promising pharmacotherapuetic agent for the treatment of cocaine addiction. Disulfiram acts by blocking dopamine hydroxylase $(\mathrm{D} \beta \mathrm{H})$. $\mathrm{D} \beta \mathrm{H}$ inhibition increases dopamine levels, and decreases peripheral and central norepinephrine levels. Furthermore, disulfiram inhibits plasma cholinesterase as well as plasma and microsomal carboxylesterases that degrade cocaine systemically. Disulfiram can reduce subjective measures of craving produced by IV administered cocaine.

Methods: Ninety-one cocaine and opioid codependent (DSM-V) subjects were stabilized on methadone for one week. They were randomized into the disulfiram $(n=45)$ and placebo groups $(n=46)$. During weeks 2-13, subjects were in a randomized, double-blind placebo-controlled trial receiving either o or $250 \mathrm{mg} /$ day disulfiram. Treatment effectiveness score was assessed by the number of cocainefree urines during weeks 2-13. Urines were collected three times per week. Fourteen genetic variants that have been shown to be involved in drug addiction were genotyped and evaluated for their role in the treatment of cocaine dependence with disulfiram (ADRA1A rs1048101, $A D R A 1 D$ rs223655, ANKK1 rs1800497, BDNF rs6265, CHRNA5 rs16969968, COMT rs468o, CSNK1E rs1534891, DBH rs1611115, DRD2 rs6277, MAOB rs1799836, OPRK1 rs6473797, POMC rs1866146, TPH1 rs1799913, and $\mathrm{TPH} 2$ rs4290270).

Results: Using generalized estimating equations, we determined if there was significant evidence for differences in treatment effectiveness scores (TES) between specific variants over time. The most significant findings (point-wise $P<0.01$ ) for the interaction of the variant with TES of each study week in the disulfiram treatment group were with variants in the $B D N F$ and $C S N_{1} E$ genes. In the placebo group, significant interactions were found with variants in the OPRK1,COMT, and $\mathrm{CHRNA}_{5}$ genes.

Discussion: These results suggest that the genetic background of subjects should be taken into account in the treatment of cocaine addiction with disulfiram. In addition, the involvement of these genes in the treatment of cocaine addiction with disulfiram may reveal new treatment strategies.

Disclosure: D. Nielsen: None. W. Huang: None. M. Harding: None. S. Hamon: None. J. Lindsay: None. T. Kosten: Part 1; Reckitt Benckiser. Part 2; Catalyst Pharmaceutical.

92. Genetic Association Studies of Relapse After Citalopram Response in Major Depressive Disorder

Gonzalo Laje*, Silvia Paddock, A. John Rush, Francis McMahon

NIMH, Bethesda, MD

Background: Major depressive disorder (MDD) will become the second greatest cause of disability in the next decade. Antidepressant treatment is effective, but of those patients who respond to treatment about one third relapse. Some predictors of relapse have emerged, such as incomplete remission and stressful life events, but to date genetic predictors have been difficult to identify.

Methods: The Sequenced Treatment Alternatives to Relieve Depression study $\left(\mathrm{STAR}^{\star} \mathrm{D}\right)$ offered up to one year of follow up treatment for those patients who improved on a specific treatment strategy $(\mathrm{n}=862)$. We defined relapse as those patients who reported a QIDS-SR total score $<11$ at the first follow-up visit, had at least one additional follow-up visit, and reported a QIDS-SR score of 11 or more at any follow-up visit $(\mathrm{n}=255)$. Those who's QIDS-SR score remained $<11$ at all follow-up visits were used as the comparison group $(n=430)$. We conducted a candidate gene study with 780 observed SNPs covering common variation in 68 genes in neurotransmitter, neurotrophin, and other pathways, and a separate genome-wide study based on a published set of genotypes (Garriock et al 2010) that we cleaned and imputed using the latest methods. Analyses were adjusted for population stratification and corrected for multiple testing.

Results: In the candidate gene study, the marker rs2251388 was associated with relapse (OR: $1.82, \mathrm{X}_{2}=4.12$, unadjusted p-value: $3.8 \mathrm{e}-5$, adjusted p-value: 0.03 ). Consistent results were found in the genomewide analysis, although no markers achieved genome-wide significance. This marker resides within an intron of the gene GRIK1, which 


\section{S342}

encodes the gluR5 subunit of the glutamate-kainate receptor, known to be regulated by stress and corticosteroids.

Discussion: We conclude that genetic variation in GRIK1 may be associated with relapse of depressive symptoms in patients who had initial response to citalopram treatment.

Disclosure: G. Laje: None. S. Paddock: None. A. Rush: Part 1; Advanced Neuromodulation Systems, Inc, AstraZeneca, Best Practice Project Management, Inc, Bristol-Meyers Squibb Company, Cyberonics Inc., Eli Lilly \& Company, Forest Pharmaceuticals Inc, Gerson Lehman Group, GlaxoSmithKline, Healthcare Technology Systems Inc., Jazz Pharmaceuticals, Magellan Health Services, National Institute of Mental Health, Neuronetics, Ono Pharmaceutical, Organon USA Inc, Otsuka, Pamlab, Personality Disorder Research Corporation, Pfizer Inc, Robert Wood Johnson Foundation, Stanley Medical Research Institute, Urban Institute, Wyeth-Ayerst Laboratories Inc. Part 2; Pfizer Inc, Guilford Publications, Healthcare Technology Systems Inc., UT Southwestern Medical Center. Part 1; Merck \& Co. Inc. F. McMahon: None.

\section{Genome-wide Association Study of Antipsychotic-induced Weight Gain in Previously-untreated Patients}

Todd Lencz ${ }^{\star}$, Christoph Correll, John Kane, Anil Malhotra

\section{The Zucker Hillside Hospital, Glen Oaks, NY}

Background: Weight gain and related metabolic abnormalities are a significant side effect associated with antipsychotic drug treatment. Treatment studies comprised of chronic patients may underestimate the severity of this side effect, as prior treatments may have caused weight gain and obscure the true weight liability of the current drug treatment. In a recent study (Correll et al. 2009, JAMA), we showed that pediatric patients without significant prior exposure to antipsychotic medications experienced marked weight gain at 12 weeks of treatment with each of the study drugs; the weight gain was far greater than reported for previously treated patients and the weight change was highly variable. Therefore, we have focused our pharmacogenetic efforts on the identification of genes that predict weight gain in this largely antipsychotic-naïve population of subjects.

Methods: We have comprehensively characterized a cohort of antipsychotic-naïve (or minimally-exposed) pediatric patients undergoing initial clinical treatment with second generation antipsychotic drugs including risperidone, aripiprazole, and quetiapine (Correll et al. 2009). Subjects were confirmed to be receiving antipsychotic drug by plasma blood levels, and were weighed at baseline, 4, 8 and 12 weeks of treatment. DNA was collected via blood sample and genotyping conducted with the Illumina $1 \mathrm{M}$ OmniQuad platform. Regression analysis of BMI change at 12 weeks of treatment versus baseline was conducted using both additive and recessive models.

Results: QTL analysis revealed a region of the genome significantly associated with antipsychotic induced weight gain. The top SNPs that achieved genome-wide significance under the recessive model are located near a previously identified candidate gene for antipsychotic induced weight gain.

Discussion: Pharmacogenetic studies of antipsychotic-induced weight gain may be most powerful if comprised of previously untreated patient populations with documented adherence to treatment. Our preliminary data suggest that specific candidate genes may be associated with antipsychotic induced weight gain in these populations. Replication studies are underway to confirm and extend these results.

Reference: Correll CU, Manu P, Olshanskiy V, Napolitano B, Kane JM, Malhotra AK.Cardiometabolic risk of second-generation antipsychotic medications during first-time use in children and adolescents.JAMA. 2009 Oct 28;302(16):1765-73.

Disclosure: T. Lencz: Part 1; Cowen \& Co., Eli Lilly, Merck, Clinical Data, Inc., Golden Helix, Inc., Guidepoint Global. C. Correll: Actelion, AstraZeneca, Boehringer-Ingelheim, Bristol-Myers Squibb, Cephalon, Eli Lilly, IntraCellular Therapies, Ortho-McNeill/Janssen/J\&J, GSK,
Hoffmann-La Roche, Lundbeck, Medicure, Otsuka, Pfizer, ScheringPlough, Sepracor/Sunovion, Supernus, Takeda, Vanda. Part 2; AstraZeneca, Bristol-Myers Squibb, Cephalon, Ortho-McNeill/Janssen/J\&J, Otsuka, Pfizer. Part 3; AstraZeneca, Bristol-Myers Squibb, Cephalon, GSK, Ortho-McNeill/Janssen/J\&J, Otsuka, Pfizer. J. Kane: Part 1; Otsuka, Takeda, Merck, Janssen, Intracellular Therapeutics, Proteus, Novartis, Rules Based Medicine, Targacept, J\&J, BMS, Boehringer. Part 2; Lilly, Otsuka, BMS, AstraZeneca. Part 1; Medavante, Lilly, AstraZeneca. A. Malhotra: Lilly, Merck, Clinical Data, Inc., Wyeth, Arbor Scientia (Sepracor Inc).

\section{Genomewide Association Study of Schizophrenia Identifies Novel Loci in an Arab Israeli Family Sample with Independent Confirmation}

Anna Alkelai, Sara Lupoli, Yoav Kohn, Fabio Macciardi, Bernard Lerer*

Biological Psychiatry Laboratory, Hadassah-Hebrew University Medical Center, Jerusalem, Israel

Background: Using genome-wide linkage in a genetically homogeneous sample of Arab Israeli families multiply affected with schizophrenia, we previously reported significant evidence for a schizophrenia susceptibility locus at chromosome 6q23.2-q24.1 and suggestive evidence for loci at chromosomes 10q22.3-26.3, 2q36.1-37.3 and 7p21.1-22.3 (Lerer et al, Mol Psychiatry 2003). To identify susceptibility genes within the $6 \mathrm{q} 23$ region, fine-mapping was performed and the most significant genetic association with schizophrenia was found within a $500 \mathrm{~kb}$ genomic region that harbors the AHI1, MIR548H4 and C6orf217 genes (Amman-Zalcenstien et al, Eur J Hum Gen 2006). The associations were subsequently replicated in an Icelandic case control sample (Ingason et al Eur J Hum Gen 2007) and in a large European sample (Ingason et al, Hum Mol Gen 2010) and further supported by our own fine mapping study (Torri et al FASEBJ 2010). While the use of population-based samples in genome wide association studies (GWAS) is a common approach, there has been difficulty in the identification of loci that are significantly and replicably associated with psychiatric disorders. To identify additional schizophrenia susceptibility genes in our Arab Israeli sample we implemented a family-based GWAS strategy. To determine replicability of observed significant associations with schizophrenia beyond this unique Arab population, we genotyped our top SNPs (withstanding correction for multiple testing) in a validation case-control sample of Jewish Israelis.

Methods: Our Arab-Israeli family sample is drawn from an ethnically homogenous population that originated less than 30 generations ago with a limited number of founders and is characterized by a high birthrate, an unusually high level of consanguinity and a low rate of intermarriage with other population groups. It consists of 58 nuclear families and encompasses 99 affected subjects out of 198 genotyped individuals. Our Jewish, Israeli case-control sample consists of 261 patients with schizophrenia and 237 controls. Genotyping of the ArabIsraeli sample was performed with the Illumina HumanCNV370-Duo BeadChip and of the Jewish case-control replication sample with the Sequenom MassARRAY system. Association analyses were performed with PBAT and PLINK software. QVALUE was used to calculate false discovery rate (FDR)-based q-values.

Results: We found genome-wide significant association (q-value $<0.05$, the best p-value $=2.24 \times 10^{-12}$ ) with 17 SNPs. One of them is in an intron of the AHI and MIR548H4 overlapping genes, previously associated with schizophrenia in our sample (6q22.33-q24.1 linkage area). Of particular interest are a group of 5 SNPs within and flanking the transcriptional repressor LRRFIP1 gene (2q36.1-q37.3 linkage area). Additional significant SNPs were found in the 2q36.1-q37.3, 6q22.33-q24.1 and 7p22.3-p21.1 linkage areas identifying further novel candidate genes for schizophrenia. In our Jewish Israeli case-control sample we found replication of association in the predicted promoter region of the $S L C 29 A 4$, also named plasma membrane monoamine transporter, gene (7p22.1).

Discussion: Overall, this family-based GWAS strengthens and significantly extends the results of our previous studies. It focuses interest on several novel candidate genes all of which are highly 
plausible functional candidates for schizophrenia. Of particular note are the transcriptional repressor gene $L R R F I P_{1}$ (2q37.3) and the plasma membrane monoamine transporter gene, SLC29A4 (7p22.1) which was replicated in our Jewish Israeli case-control sample. Our findings clearly demonstrate the considerable advantage of using genetically homogeneous family-based samples in GWAS of psychiatric disorders. Supported in part by the Israel Science Foundation and by the 7th EU project POCEMON (FP7-ICT-2007-216088).

Disclosure: A. Alkelai: None. S. Lupoli: None. Y. Kohn: None. F. Macciardi: None. B. Lerer: None.

95. Association Analysis of Variants in the SLC6A4 and HTR2A Gene and Treatment Response to Venlafaxine XR in Generalized Anxiety Disorder

Falk Lohoff*, Tiffany Richardson, Paul Bloch, Rachel Hodge, Glenn Doyle, Bijan Etemad, Robert Gallop, Karl Rickels

University of Pennsylvania, Philadelphia, PA

Background: Generalized Anxiety Disorder (GAD) is a highly prevalent chronic psychiatric disorder with significant morbidity and mortality. Currently, antidepressant drugs are the preferred choice for acute and chronic treatment. Treatment response is often variable, with some patients responding well to medications while others fail treatment. Growing evidence suggests that genetic factors might be involved explaining treatment response and tolerability to antidepressant medication. Several studies in major depression have implicated a role of the serotonin transporter gene (SLC6A4) and serotonin receptor gene (HTR2A) in treatment response to antidepressants. In this study we tested the hypothesis that the genetic variant in the promoter region of the SLC6A4 gene and $\mathrm{HTR}_{2} \mathrm{~A}$ gene predict treatment outcome in GAD patients treated with venlafaxine XR.

Methods: Treatment response was assessed in 118 patients (EuropeanAmericans $\mathrm{n}=84$; African-Americans $\mathrm{n}=31$; others $\mathrm{n}=3$ ) that participated in a 6-month open label clinical trial of venlafaxine XR for GAD. Primary analysis included HAM-A reduction at 6 months, response was defined as HAM-A reduction of $>50 \%$ and remission was defined as HAM-A $<7$. Genotypes were obtained using standard procedures. Genotype and allele frequencies were compared between groups using chi-square contingency analysis.

Results: We observed a trend for an association of the 5-HTTLPR-Lallele and response at 6 months in our European-American sample $(\mathrm{n}=84)$, with responders having an L-allele frequency of $60 \%$ and non-responders having an L-allele frequency of $42 \% \quad(p=0.08)$. Similar trends were observed for remission although not statistically significant. The polymorphism in the HTR2A gene rs7997012 was associated in the combined sample with better treatment response. Discussion: Our results suggest that the L-allele of 5-HTTLPR at the SLC6A4 locus is associated with a better outcome after 6 months of treatment with venlafaxine $\mathrm{XR}$ in GAD patients. In addition, our results suggest that individuals with the HTR2A rs7997012 SNP G-allele show better treatment outcome over time. Future studies with larger sample sizes are necessary to further characterize the effect of these variants in treatment response to antidepressants in GAD.

Disclosure: F. Lohoff: None. T. Richardson: None. P. Bloch: None. R. Hodge: None. G. Doyle: None. B. Etemad: None. R. Gallop: None. K. Rickels: Part 4; NIMH,Pfizer, Otsuka, Lilly, PGx Health.

\section{Genetic Variation in the Circadian Clock is Associated with lithium Treatment Response in Bipolar Disorder and Gene Expression Differences \\ Michael McCarthy ${ }^{\star}$, Caroline M. Nievergelt, Daniel F. Kripke, Tatyana} Shekhtman, David Welsh, John Kelsoe

University of California, San Diego, La Jolla, CA

Background: The circadian clock is regulated by a family of interconnected signaling molecules, including Rev-Erb Alpha that together maintain rhythmic gene expression and behavioral rhythms over $\sim 24 \mathrm{hr}$ cycles. Bipolar disorder (BD) is a severe mental illness characterized not only by mood abnormalities, but also by disruptions in circadian rhythms such as sleep and daily activity. Interestingly, lithium alters circadian rhythms, and can correct both mood and circadian rhythm abnormalities in responsive BD patients. However, many patients fail to respond to lithium and needlessly suffer treatment delays or side effects. Lithium inhibits $\mathrm{GSK}_{3}$ Beta and in so doing, regulates Rev-Erb Alpha stability and affects the clock. We hypothesized that variation in circadian clock genes would be associated with lithium response in $\mathrm{BD}$ and furthermore, that associated genetic variants would confer differences in gene expression.

Methods: We conducted a candidate gene association study in 282 Caucasian BD patients previously treated with lithium. Each patient was retrospectively categorized as lithium responsive or nonresponsive. We genotyped the subjects for 23 variants in 13 circadian clock genes. Using lymphoblastoid cell lines from a subset of these patients, we performed gene expression studies using RT-PCR to measure the expression of Rev-Erb alpha and GSK3-Beta both at baseline and after lithium ( $1 \mathrm{mM})$ treatment for $72 \mathrm{hr}$.

Results: We found that a variant (rs2071427) in the promoter of the Rev-Erb Alpha gene was nominally associated with good lithium response $(\mathrm{p}<0.009, \mathrm{OR}=1.9)$ using a dominant transmission model. $\mathrm{GSK}_{3}$ Beta genotype was suggestive of a lithium response association, but not statistically significant by itself $(p<0.08, O R=1.8)$. No other variants had a lithium-response association. GSK3 Beta and Rev-Erb Alpha interact in a known signaling pathway within the clock. Interestingly, GSK 3 Beta and Rev-Erb Alpha genotype together were additive in predicting lithium response. Similarly, both the GSK3 Beta and Rev-Erb Alpha variants were associated with functional differences in gene expression at baseline $\left(\mathrm{GSK}_{3} \mathrm{Beta}\right)$ or in response to lithium (Rev-Erb Alpha).

Discussion: GSK3 Beta has a well-established role in the action of lithium. Our results suggest that Rev-Erb Alpha, a target of GSK3 Beta may also have an important role in the therapeutic response to lithium. Our data suggest that genetic variability in Rev-Erb Alpha may bias lithium treatment outcomes and that this variability is better explained when considering genetic variation in GSK3 Beta. Functional differences in gene expression may offer clues as to how genetic variation in the circadian clock system affects lithium treatment response.

Disclosure: M. McCarthy: None. C. Nievergelt: None. D. Kripke: None. T. Shekhtman: None. D. Welsh: None. J. Kelsoe: None.

\section{Variation In The TPH2 Gene Associated With Dorsal Raphe} Nucleus Diffusion Tensor Imaging Measurements

John McGeary*, Andrew Cohen, Valerie Knopik, Christopher Beevers, David Schnyer, Francisco Gonzalez-Lima

Providence VA/Brown University, Providence, RI

Background: The dorsal raphe nucleus (DRN) is a major source of serotonergic ennervation in the human brain. Tryptophan hydroxylase is the rate limiting enzyme for serotonergic production and two genes are known to code for this enzyme (TPH1 and $\mathrm{TPH} 2)$. Expression of tryptophan hydroxylase in the DRN is primarily from the $\mathrm{TPH} 2$ gene and variation in this gene has been associated with numerous psychiatric phenotypes related to serotonergic function (e.g., depression, suicide, etc.). Given the neuroanatomical importance of the DRN and the critical nature of $\mathrm{TPH}_{2}$ products in serotonin synthesis, diffusion tensor imaging techniques might identify differences in the DRN by $\mathrm{TPH} 2$ genotype.

Methods: Accordingly mean diffusivity and fractional anisotropy values in the DRN were calculated in 56 female participants. Participant DNA was genotyped for three single nucleotide polymorphisms and association analyses with these variants and the DTI measurements were conducted. 
Results: Fractional anisotropy and mean diffusivity values in the DRN were found to be associated with variation in one polymorphism in the $\mathrm{TPH}_{2}$ gene. These results represent one of very few imaging genetics measuring a brain region as ventral as the DRN.

Discussion: The differences in DTI values in this region may clarify the impact of $\mathrm{TPH}_{2}$ variation on psychiatric behaviors previously associated with these polymorphisms.

Disclosure: J. McGeary: None. A. Cohen: None. V. Knopik: None. C. Beevers: None. D. Schnyer: None. F. Gonzalez-Lima: None.

\section{The Pharmacogenetics Research Clinic: Towards the} Implementation of $\mathrm{CYP}_{2} \mathrm{D} 6$ and $\mathrm{CYP}_{2} \mathrm{C}_{19}$ Genotyping in Psychiatric Practice

Daniel J. Mueller*, Arun K. Tiwari, Olga Likhodi, Jessica Sturgess, Margaret A. Richter, James Kennedy

Centre for Addiction and Mental Health \& Dept. of Psychiatry, University of Toronto, Toronto, ON, Canada

Background: Antipsychotic and antidepressant medication continues to be the main treatment for many psychiatric conditions including schizophrenia, mood and anxiety disorders including obsessive-compulsive disorder (OCD) Two polymorphic enzymes, $\mathrm{CYP}_{2} \mathrm{D} 6$ and $\mathrm{CYP}_{2} \mathrm{C}_{19}$, metabolize a large number of these medications. Functional polymorphisms in these enzymes can confer altered enzymatic activity, potentially leading to toxic or subtherapeutic drug levels.

Methods: As part of the Toronto Roche AmpliChip (C) Study, 39 individuals with OCD were genotyped for $\mathrm{CYP}_{2} \mathrm{D} 6$ and $\mathrm{CYP}_{2} \mathrm{C}_{19}$. Abnormal CYP2D6 activity (i.e. non-extensive metabolizer) was significantly associated with non-response to antidepressants. Two individuals who were ultrarapid metabolibzers (UM) failed to respond with 9 antidepressants and showed only response with two trials, and one individual who was poor-responder did not respond to any SSRIs trial $(\mathrm{p}=.006)$. This data, including a thorough chart review, show that non-extensive metabolizers are significantly more likely to have complicated medication histories and suggest that CYP2D6 and $\mathrm{CYP}_{2} \mathrm{C}_{19}$ genotyping could have aided in selecting medications and dosages, potentially improving response and reducing side effects.

As part of a new study at our Pharmacogenetics Research Clinic, the first patients with a diagnosis of schizophrenia and mood disorders with complicated medication histories have been enrolled prospectively and genotyped for $\mathrm{CYP}_{2} \mathrm{D} 6$ and $\mathrm{CYP}_{2} \mathrm{C}_{19}$. At study entry, clients are informed in detail about potential advantages and limitations related to the genetic testing of liver enzyme activities. Patients participate in a structured diagnostic interview and are assessed of current and previous treatment response and occurrence of side effects. Physicians are then provided with an interpretation of the genotypic results and informed in detail about the potential clinical implications which they will discuss with their patients. Serum drug levels of the $\mathrm{CYP}_{2} \mathrm{D} 6$ and/or $\mathrm{CYP}_{2} \mathrm{C}_{19}$ metabolized drugs are also assessed and after 6 weeks the physician completes a questionnaire evaluating the usefulness of the genotypic information provided by the study. After 12 weeks, the clients are assessed again to monitor potential adjustments of medications and their overall treatment outcome. Selected case reports will be presented and discussed in detail at the conference.

Results: Overall, at the moment our pilot sample of 25 respondents have returned excellent feedback that the genotyping results have been helpful in allowing them to either select medications their patients are likely to better tolerate, or to adjust doses based on geneotype results and serum levels.

Discussion: Our findings suggest that $\mathrm{CYP}_{2} \mathrm{D} 6$ and $\mathrm{CYP}_{2} \mathrm{C}_{19}$ genotyping provides useful information that help physicians to improve pharmacotherapy for individual patients.

Disclosure: D. Mueller: None. A. Tiwari: None. O. Likhodi: None. J. Sturgess: None. M. Richter: None. J. Kennedy: None.
99. Fine-mapping Of The Corticotropin-releasing Hormone Receptor Gene

Amanda Myers*, Kerry Ressler, Bekh Bradley, Kristina Mercer, Todd Deveau, Alicia Smith, Charles Gillespie, Joseph Cubells, Elisabeth Binder, Charles Nemeroff

University of Miami, Miller School of Medicine, Miami, FL

Background: It is well known that hyperactivity of the hypothalamicpituitary-adrenal axis (HPA) is a hallmark of major depressive disorder (MDD) and in part due to a hyperactivity of corticotropinreleasing hormone $(\mathrm{CRH})$ circuits $^{1,2}$. Previous work has demonstrated that there is a synergistic effect between early life stress, variants in the corticotropin releasing hormone receptor 1 gene (CRHR1) and development of major depressive disorder ${ }^{3}$. The gene encoding CRHR 1 is located on chromosome 17q21.31. This area of the genome is unusual in that there is a large polymorphic inversion of $\sim 800 \mathrm{~kb}$ leading to a linkage disequilibrium feature which is $\sim 1.6 \mathrm{Mb}^{4}$. This inversion event contains 6 genes, including $C R H R 1$ and results in two major haplotypes $\left(\mathrm{H}_{1}, \mathrm{H}_{2}\right)$ one of which has limited geographic distribution ${ }^{5}$ and has been shown to be involved in the pathophysiology of certain neurologic diseases ${ }^{6,7,8}$. We will map the existing association results onto the background of the inversion event to determine the full extent of the risk haplotypes for CRHR1.

Methods: Because part of our cohort includes African Americans, which were not included in the original report mapping the inversion in different populations ${ }^{5}$, we used the Hapmap data (www. hapmap.org) to map whether the inversion was present in African Americans. The program Phase $^{9}$ was used to calculate haplotypes for the Hapmap data from the African Ancestry from the Southwest USA (ASW) panels as well as the CEU (Northern and Western European Ancestry, CEU) and the JPT (Japanese ancestry, used as a reference). We used these same cohorts to map the polymorphism in CRHR1 that was shown to be involved in the synergistic relationship between childhood trauma and MDD risk (rs110402) on the inversion haplotype background.

Results: The inversion haplotype was present in $\sim 19 \%$ of CEU samples and $\sim 10 \%$ of ASW samples. As expected, the inversion was virtually absent in the JPT sample. Additionally, as expected, mapping rs110402 to the inversion haplotype in either the African Ancestry cohorts or Caucasians showed that most of the CRHR1 variability mapped to the more common ( $\left.\mathrm{H}_{1}\right)$ background including the protective (A) allele.

Discussion: These results suggest that the complex haplotype structure previously mapped for the MAPT gene extends further on chromosome $17 \mathrm{q} 21.31$ and encompasses the CRHR1 gene as well. This finding has implications for the fine-mapping of CRHR1 haplotypes, in that risk haplotypes with occur both on the inverted and non-inverted backgrounds while the protective allele of rs110402 was absent from the inversion background. Further work will include imputation to determine whether the protective haplotypes formed by $\mathrm{rs} 7209436$, rs 4792887 along with rs110402 can be placed in the context of the inversion to fine map the functional variants on the protective haplotype.

References:

Nemeroff $\mathrm{CB}$ et al. Elevated concentrations of CSF corticotropinreleasing factor-like immunoreactivity in depressed patients. Science. 1984;226(4680):1342-1344.

2. Reul JM, Holsboer F. Corticotropin-releasing factor receptors 1 and 2 in anxiety and depression. Curr Opin Pharmacol. 2002;2(1):23-33. 3. Bradley RG et al. Influence of child abuse on adult depression: Moderation by the corticotropin-releasing hormone receptor gene. Arch Gen Psychiatry 2008. 65(2):190-200.

4. Zody MC et al Evolutionary toggling of the MAPT 17q21.31 inversion region. Nat Genet. 2008 Sep;40(9):1076-83.

5. Evans W, et al The tau $\mathrm{H}_{2}$ haplotype is almost exclusively Caucasian in origin. Neurosci Lett. 2004 Oct 21;369(3):183-5.

6. Pittman AM et al. Linkage disequilibrium fine mapping and haplotype association analysis of the tau gene in progressive supranuclear 
palsy and corticobasal degeneration. J Med Genet. 2005 Nov;42(11): 837-46.

7. Myers AJ et al. Hum Mol Genet. 2005 Aug 15;14(16):2399-404. 8. Myers AJ et al. The MAPT H1c risk haplotype is associated with increased expression of tau and especially of 4 repeat containing transcripts. Neurobiol Dis. 2007 Mar;25(3):561-70.

9. Stephens $M$ et al. A new statistical method for haplotype reconstruction from population data. Am J Hum Genet. 2001 Apr;68(4):978-89.

Disclosure: A. Myers: None. K. Ressler: None. B. Bradley: None. K. Mercer: None. T. Deveau: None. A. Smith: None. C. Gillespie: None. J. Cubells: None. E. Binder: None. C. Nemeroff: Part 1; AstraZeneca Pharmaceuticals, CeNeRx BioPharma, PharmaNeuroboost, Reevax Pharma, NovaDel Pharma, Inc., Corcept Therapeutics. Part 2; Cenerx, Pharmaneuroboost, Astrazeneca, Novadel.

100. Association Between SNPs in SCL6A2 and COMT and Response to LY2216684 in Patients with Major Depressive Disorder Laura Nisenbaum*, Kit Fun Lau, Sweta Dharia, Lauren Marangell, Leanne Munsie, Bonnie Fijal, Mary Anne Dellva, Kalpana Merchant, Sanjay Dube

Eli Lilly and Company, Indianapolis, IN

Background: LY2216684 is a novel selective norepinephrine reuptake inhibitor (NERI) under development for the treatment of major depression. Prior studies of NERI or dual serotonin/norepinephrine inhibitors suggest that SNPs in the norepinephrine transporter (SLC6A2) and catecholamine-o-methyltransferase (COMT) genes impact the efficacy of these compounds (Kim et al 2006, Perlis et al 2009, Uher et al 2009). The objective of the current analysis was to determine whether SNPs in SCL6A2 (rs1532701, rs2242447, rs4564560, rs5569) or COMT (rs1544325, rs165599, rs4680, rs740603) impact response to LY2216684.

Methods: Patients with major depressive disorder (MDD) were randomized in a double blind fashion to either LY2216684 (6-18 mg/day) or placebo for 10 weeks. 428 of the 495 randomized patients consented to DNA collection. Of these, 328 were Caucasians and were included in the pharmacogenomic analysis. The associations between the SNPs and change in Montgomery-Asberg Depression Rating Scale (MADRS) and Quick Inventory of Depressive Symptomatology-Self Rated (QIDS-SR-16) were tested using a mixed-effects model for repeated measures within each treatment group. Single Nucleotide Polymorphisms (SNP) were tested under recessive, dominant, and additive models. SNPs with 2-sided p-values $\leq 0.10$ in the LY2216684 arm were considered potentially associated with response to LY2216684 and worth examining in future studies.

Results: COMT rs165599 was modestly associated with change in MADRS and QIDS-SR-16 in both LY2216684 (p: 0.069 and 0.083 ) and placebo-treated (0.061 and 0.042$)$ patients. In both treatment arms, patients with the GG genotype responded better than other patients. In the LY2216684 arm, LS mean change \pm SE in MADRS for AA, AG, and GG patients were $-12.4 \pm 0.86(n=75),-13.1 \pm 0.90(n=68)$, and $-15.7 \pm 1.84(\mathrm{n}=16)$. In the placebo arm, LS mean change in MADRS for $\mathrm{AA}, \mathrm{AG}$, and GG patients were $-9.3 \pm 0.82(\mathrm{n}=91),-8.9 \pm 0.94(\mathrm{n}=64)$, and $-11.3 \pm(1.93)(n=14)$. LS mean change in QIDS-SR-16 showed a similar pattern of response. SLC6A2 rs1532701 was modestly associated with change in QIDS-SR-16 in LY2216684-treated (p: 0.072) but not placebo-treated (p: 0.519) patients. In the LY2216684 arm, LS mean change in QIDS-SR-16 for GG, GA, and AA patients were $-6.5 \pm 0.98$ $(n=27),-5.8 \pm 0.66(n=68)$ and $-5.9 \pm 0.83(n=38)$. In the placebo arm, LS mean change in QIDS-SR-16 for GG, GA, and AA patients were $-4.5 \pm 0.92(n=33),-5.4 \pm 0.67(n=64)$ and $-4.7 \pm 0.82(n=42)$. LS mean change in MADRS in LY2216684-treated patients showed a similar although non-significant trend (p: 0.151). LS mean change in MADRS for GG, GA, and AA LY2216684-treated patients were $-14.3 \pm 1.28(n=31), \quad-13.1 \pm 0.83 \quad(n=82)$ and $-12.1 \pm 1.05 \quad(n=46)$. SLC6A2 rs5569 was not significantly associated with change in MADRS or QIDS-SR-16 in either the LY2216684-treated patients (p: 0.612 and 0.810 ) or the placebo-treated patients (p: 0.537 and 0.742 ).

Discussion: In prior studies, the COMT rs165599 GG genotype has been associated with better response to duloxetine (Perlis et al, 2009) while the SLC6A2 rs1532701 G allele has been associated with better response to nortriptyline (Uher et al, 2009). However, neither study was placebo-controlled. In our analysis, the COMT rs165599 GG genotype was associated with better response to both LY2216684 and placebo. This result suggests that this SNP may identify "responders" irrespective of treatment intervention rather than patients who respond to a particular active treatment. Unlike the COMT rs165599 GG, the SLC6A2 rs1532701 GG genotype was associated with better response to LY2216684 but not placebo. This SNP may be useful in identifying patients with MDD who would particularly benefit from LY2216684 treatment. The association between SLC6A2 rs5569 and response, which was seen in Kim et al (2006), was not confirmed.

References:

Kim H, et al (2006) "Monoamine Transporter Gene Polymorphisms and Antidepressant Response in Koreans with Late-Life Depression" JAMA;296(13):1609-1618.

Perlis RH, et al (2009) "Variation in COMT is Associated with Duloxetine Response in a Clinical Trial for Major Depressive Disorder" Biol Psychiatry;65(9):785-791.

Uher R, et al (2009) "Genetic Predictors of Response to Antidepressants in the GENDEP Project" Pharmacogenomics J;9(4):225-233.

Disclosure: L. Nisenbaum: Part 5; Eli Lilly and Company. K. Lau: i3 Statprobe. S. Dharia: i3 Statprobe. L. Marangell: Eli Lilly and Company. L. Munsie: Eli Lilly and Company. B. Fijal: Eli Lilly and Company. M. Dellva: Eli Lilly and Company. K. Merchant: Eli Lilly and Company. S. Dube: Eli Lilly and Company.

101. DNA Methylation Signatures Of Human Cortical Samples Across The Lifespan

Shusuke Numata*, Barbara Lipska, Thomas Hyde, Tianzheng Ye, Xavier Guitart-Navarro, Ran Tao, Daniel Weinberger, Joel Kleinman

National Institute of Mental Health, Bethesda, MD

Background: DNA methylation is a major epigenetic modification playing a critical role in mammalian development. However, little is known about the DNA methylation signatures of the human brain, especially across the lifespan. The aim of this study was to examine the effects of age, sex, and genetic variation on DNA methylation status in the human dorsolateral prefrontal cortex in a large cohort of well characterized specimens across the entire life span, including fetal life. Deciphering the human epigenetic code throughout cerebral cortical development will be important for understanding pathological changes in regulation of gene expression and brain function.

Methods: The DNA methylation status of 27,578 CpG dinucleotides was assessed using Illumina Infinium microarray chips in the post-mortem dorsolateral prefrontal cortex (DLPFC) from 108 control (nonpsychiatric) subjects, ranging in age from the 2nd trimester of gestation $(\mathrm{n}=30)$ to late adulthood. Genome-wide single nucleotide polymorphism (SNP) genotyping was conducted on the same samples using Illumina BeadArrays, and a genome-wide association analysis of SNPs with each DNA methylation phenotype was performed. We used surrogate variable analysis (SVA) to identify covariates, including batch effects, influencing DNA methylation status. General linear model (GLM) was then used to examine the effects of the primary variables: sex, age, and developmental stage. Residuals from this analysis were used to investigate the effects of SNPs on DNA methylation levels (mQTLs).

Results: We found that across all subjects, $\sim 63 \%$ of sites were hypomethylated (beta $<0.2$ ), $\sim 13 \%$ were hypermethylated (beta $>0.8$ ) and $\sim 24 \%$ showed intermediate methylation status (beta $0.2-0.8$ ). DNA methylation levels were age- dependent, and the magnitude of DNA methylation changes differed according to the developmental life stage. The most rapid DNA methylation changes occurred in the 
prenatal period during six weeks of the 2nd trimester of gestation, followed by childhood and adulthood. During fetal period, out of 27,578 CpG sites, significant age-related changes in DNA methylation (FDR < 0.05) were observed at 865 sites, with $\sim$ twice as many sites showing a decline vs gain in methylation levels with increasing age. Later, during childhood and adulthood, the pattern of changes was opposite: of thousands of sites showing significant age-related changes in methylation, $\sim$ twice as many loci were progressively more methylated with aging vs those that showed diminished methylation. Sex-related differences in DNA methylation were observed at CpG sites on both the $\mathrm{X}$ chromosome and autosomes. Not surprisingly, given that DNA methylation is the prime mechanism of $\mathrm{X}$-chromosomal inactivation, a vast majority $(\sim 93 \%)$ of loci on the $\mathrm{X}$ chromosome demonstrated a sexually dimorphic pattern of DNA methylation, with females showing higher methylation levels at most sites. The magnitude of the methylation difference between females and males was far greater for the sites on X chromosome than at autosomes. Finally, and most importantly, DNA methylation was associated with genetic variance at numerous $\mathrm{CpG}$ sites. Significant Cis-associations were more frequent and more pronounced than trans-associations.

Discussion: Our results demonstrate that age, sex, and genetic variability are strong determinants of DNA methylation status in the human DLPFC at a large number of loci. Mapping the effects of these factors on DNA methylation and gene expression will be useful for understanding the molecular mechanisms of human brain development and pathophysiology of developmental brain disorders.

Disclosure: S. Numata: None. B. Lipska: None. T. Hyde: None. T. Ye: None. X. Guitart-Navarro: None. R. Tao: None. D. Weinberger: None. J. Kleinman: None.

102. Transciptional Control of Monocyte Gene Expression in Post-Traumatic Stress Disorder

Aoife O’Donovan*, Bing Sun, Steve Cole, Hans Rempel, Maryann Lenoci, Lynn Pulliam, Thomas Neylan

University of California San Francisco, San Francisco, CA

Background: Post-traumatic stress disorder (PTSD) confers an increased risk for disorders with an inflammatory etiology. Although the biological underpinnings of PTSD are poorly understood, dysregulation of immune system processes could mediate PTSDrelated increased risk for disease. In particular, PTSD-related dysregulation of the sympathetic nervous system (SNS) and hypothalamic-pituitary adrenal (HPA) axis and associated alterations in inflammatory signaling in immune cells may contribute to this increased risk. However, little is known about convergent SNS, HPA and inflammatory signaling at the level of the immune cell transcriptome in PTSD. To explore such signaling, we examined the prevalence of transcription factor binding motifs in the promoter regions of differentially expressed genes in monocytes from individuals with PTSD using the Transcription Element Listening System (http://www.telis.ucla.edu/).

Methods: Participants included 49 men (24 PTSD + and 25 agematched trauma-exposed controls) and 18 women (10 PTSD + and 8 age-matched controls). Differentially expressed genes were identified as those with $\geq 30 \%$ difference in levels of expression between participants with PTSD and controls. Analysis of inflammatory signaling was focused on the NF- $\kappa \mathrm{B} / \mathrm{Rel}$ family of pro-inflammatory transcription factors, specifically RelA/p65 (V\$NFKAPPAB65_01) and c-Rel (V\$CREL_01). Analysis of SNS adrenergic signaling was focused on the CREB/ATF family of transcription factors, specifically CREB-1 (V\$CREB_01) and ATF-1 (V\$ATF_01), which are elements responsive to adrenergic signals from catecholamines. Analyses for GR were focused on GR- $\alpha$ and GR- $\beta$ (V\$GR_Q6), which are responsive to glucorticoid signaling from cortisol. NF- $\kappa \mathrm{B} /$ Rel transcription factors generally covey pro-inflammatory signals, whereas CREB/ATF and GR can convey anti-inflammatory signals.
Results: Among men with PTSD, there was significant up-regulation of genes with response elements for the NF- $\kappa \mathrm{B} / \mathrm{Rel}$ transcription factors, RelA (1.55-fold difference in V\$NFKAPPAB65_01, $S E=.22, \mathrm{p}=.01)$ and c-Rel (1.42-fold difference in V\$CREL_01, SE $=.13, \mathrm{p}=.004)$. Second, there was significant up-regulation of genes with response elements for CREB-1 (1.50-fold difference in V\$CREB_01, SE =.18, $p=.006)$ and ATF-1 (1.79-fold difference in V\$ATF_01, SE $=.29, \mathrm{p}=.005)$. Finally, there was significant down-regulation of genes with response elements for GR- $\alpha$ and GR- $\beta$ (0.73-fold difference in V\$GR_Q6, SE $=.06$, $\mathrm{p}=.01$ ). Among women with PTSD, there was significant upregulation of genes with response elements for the NF- $\mathrm{KB} / \mathrm{Rel}$ family of transcription factors, RelA (1.65-fold difference in V\$NFKAPPAB65_01, $S E=0.10, p<.001)$ and c-Rel $(1.43$-fold difference in V\$CREL_01, $\mathrm{SE}=.13, \mathrm{p}=.008)$. Second, there was significant down-regulation of genes with response elements for CREB-1 (o.6o-fold difference in V\$CREB_01, SE $=.07, p=.002$ ) and ATF-1 (0.47-fold difference in V\$ATF_01, $\mathrm{SE}=.13, \mathrm{p}=.002)$. Although there were no significant differences in expression of genes bearing response elements for GR in the female sample, expression of such genes did tend to be down-regulated in women with PTSD compared with controls (o.70-fold difference in V\$GR_Q6, SE $=0.14$, $\mathrm{p}=.11)$.

Discussion: The present study represents the first demonstration of altered transcriptional control of immune cell gene expression in PTSD. Compared with trauma-exposed controls, men with PTSD had significant up-regulation of target genes for NF- $\mathrm{KB}$ and CREB/ATF and significant down-regulation of target genes for GR. These results indicate increased inflammatory and adrenergic signaling in conjunction with decreased glucocorticoid signaling in men with PTSD. Comparing our small sample of women with PTSD with the mixed sample of trauma-exposed and non-trauma-exposed controls, we found significant up-regulation of target genes for $N F-\kappa B$, but significant down-regulation of target genes for CREB/ATF and nonsignificant down-regulation of target genes for GR. These results indicate increased inflammatory and decreased adrenergic signaling in women with trauma exposure and PTSD. Differences in CREB/ATF signaling in males versus females could be due to gender effects on these systems or due to differences in characteristics of our control groups. In sum, altered transcriptional control of monocyte gene expression could contribute to exaggerated inflammatory activity in PTSD. Further understanding of such transcriptional control may lead to the development of targeted interventions to reduce physical disease risk in PTSD.

Disclosure: A. O’Donovan: None. B. Sun: None. S. Cole: None. H. Rempel: None. M. Lenoci: None. L. Pulliam: None. T. Neylan: None.

103. Relationship Between Genetic Variation in the Glutaminase Gene GLS1 and Brain Glutamine/Glutamate Ratio

Dost Öngür*, Stephen Haddad, Andrew Prescot, Eric Jensen, Richie Siburian, Bruce Cohen, Perry Renshaw, Jordan Smoller

McLean Hospital, Belmont, MA

Background: Abnormalities in glutamatergic neurotransmission are implicated in several psychiatric disorders, but studies of the glutamate system have been hampered by a lack of in vivo probes. Recent improvements in magnetic field strength and data acquisition/ analysis approaches have enabled quantification of glutamine and glutamate separately in proton magnetic resonance spectroscopy studies in vivo. Accumulating evidence indicates that the glutamine/glutamate ( $\mathrm{Gln} / \mathrm{Glu}$ ) ratio is an index of glutamatergic neurotransmission.

Methods: Here, we explored whether variation in GLS1 (the gene encoding the brain isoform of glutaminase which catalyzes Gln-to-Glu conversion) is associated with Gln/Glu measured in vivo in two brain regions (anterior cingulate cortex (ACC), parieto-occipital cortex (POC)). We used J-resolved proton magnetic resonance spectroscopy to measure the Gln/Glu ratio. 
Results: A specific haplotype of 4 single nucleotide polymorphisms within GLS1 was significantly associated with Gln/Glu in the POC in two independent MRS-genetics datasets.

Discussion: These findings suggest that genetic variation in a key component of glutamatergic machinery is associated with an in vivo index of glutamatergic neurotransmission. Thus, GLS1 genotype may provide insight into normal brain function and into the pathophysiology of many psychiatric conditions where glutamatergic neurotransmission has been implicated; it may also become a biomarker for predicting response to existing and novel therapeutic interventions in psychiatry that target glutamatergic neurotransmission.

Disclosure: D. Ongur: None. S. Haddad: None. A. Prescot: None. E. Jensen: None. R. Siburian: None. B. Cohen: None. P. Renshaw: None. J. Smoller: None.

104. Interferon-Gamma ( + 874) T/A Genotypes and Risk of Interferon-Alpha-Induced Depression

Gregory Oxenkrug*, Mary Perianaygam, Dennis Mikolich, Pura Requintina, Lawton Shick, Robin Ruthazer, Debbie Zucker, Paul Summergrad

Tufts University Medical School, Boston, MA

Background: Psychiatric complications of interferon (IFN)-alpha therapy are very often (up to 50\%) and may necessitate IFN-alpha dose reduction or even premature discontinuation of therapy; impair patients' ability to clear virus; interfere with patients' capacity to manage the requirements of everyday life; and negatively impact families and caregivers. There are no reliable tests to identify patientsat-risk for the development of IFN-alpha induced depression.

Methods: We retrospectively studied IFN-gamma $(+874)$ T/A genotypes in 178 hepatitis $\mathrm{C}$ patients (HCV) treated by IFN-alpha. Results: There were $80 \%$ more TA and $60 \%$ less AA carriers of IFNgamma (+874) gene among depressed than among not depressed HCV patients treated with IFN-alpha.

Discussion: This is the first observation of the different IFNG $(+874)$ T/A genotypes distribution between depressed and not-depressed HCV patients treated with IFN-alpha. Our data suggest that presence of high producer $(\mathrm{T})$ alleles might be a genetic risk factor for the development of IFN-alpha-induced depression. High producer ( $\mathrm{T}$ ) alleles are associated with the high production of IFN-gamma, the strongest inducer of indoleamine-2,3-dioxygenase (IDO), the rate-limiting enzyme of the kynurenine pathway of tryptophan metabolism. The present results are in line with the literature data suggesting that psychiatric side effects are triggered by IFN-induced up-regulation of IDO. Tryptophan - kynurenine pathway might be a new target for pharmacological prevention and treatment of IFN-alpha-induced psychiatric complications. Supported by NIH grant MHo83225 (GO). Disclosure: G. Oxenkrug: None. M. Perianaygam: None. D. Mikolich: None. P. Requintina: None. L. Shick: None. R. Ruthazer: None. D. Zucker: None. P. Summergrad: None.

105. Mutations in SLITRK1 Associated with the Obsessive-compulsive Disorder Spectrum Alter Neuronal Branch Length in Rat Embryonic Hippocampal Neurons

Uzoezi Ozomaro*, Alicia Pickrell, Donald McCorquodale, Gladys Montenegro, Jia Huang, Justin Price, Fan Zhang, Vance Lemmon, Stephan Zuchner

University of Miami Miller School of Medicine, Miami, FL

Background: Mutations in SLITRK1 have been positively associated with the obsessive-compulsive disorder spectrum (OCDS), and corresponding deleterious effects have been demonstrated for a truncation-causing variant and in SLITRK1 knockout animals. However, until recently, the functional impact of other relevant SLITRK1 missense variants has not been explored. This study screens OCDS cases and OCDS-negative controls for coding variants in
SLITRK1, then goes on to characterize the impact of overexpression of the variants in hippocampal neurons.

Methods: The present study evaluates SLITRK1 for coding variants more common in OCDS cases than in controls using direct sequencing. We screened 241 OCDS patients and 161 OCDS-negative controls for mutations in SLITRK1 and identified two novel mutations in the cases, N40oI and T418S. Subsequently, we explored the impact of overexpression of these variants and two OCDS-associated variants previously identified by our group, $\mathrm{R}_{584} \mathrm{~K}$, and $\mathrm{S}_{593} \mathrm{G}$, in in vitro embryonic rat hippocampal neurons compared to overexpression of the wild type gene and an empty control vector. At two days posttransfection, cells were stained with a number of markers and imaged with a laser-scanning microscope. Projecting neurites were quantified for length and branching by applying the ImageJ software package. Results: We identified and confirmed two novel mutations in SLITRK1 in OCDS cases and none in controls. A Fisher's exact test was used to compare the combined mutation load of OCDS cases in this and in our previous study ( 5 of 482 and 1 of 88 alleles, respectively) to that of OCDS controls (o of 322 alleles). The combined mutation load of SLITRK1 in OCDS cases compared to controls is significant $(\mathrm{p}=0.044)$. With regards to the overexpression experiments, a one-way ANOVA was used to test for differences in maximum neuron branch length in six groups of electroporated hippocampal neurons (dsRed-empty, SLITRK1-WT, SLITRK1-N40oI, SLITRK1T418S, SLITRK1-R584K, and SLITRK1-S593G). Maximum neuron branch length differed significantly across groups, $F(5,8)=13.79$, $\mathrm{p}=0.0009$. Dunnett's post hoc comparisons of the six groups indicate that the $\mathrm{N}_{400}$, $\mathrm{R}_{584} \mathrm{~K}$ and $\mathrm{S}_{593} \mathrm{G}$ variants had significantly longer maximum neuron branch lengths than the dsRed control vector (mean difference $(M D)=-17.57 \mathrm{um}, 95 \%$ CI $[-33.04,-2.098] ; \mathrm{MD}=-23.58 \mathrm{um}$, $95 \%$ CI $[-40.88,-6.289] ; \mathrm{MD}=-26.43 \mathrm{um}, 95 \%$ CI $[-43.73,-9.139]$, respectively). Neither the WT nor the T418S variant differed significantly from the dsRed control vector $(\mathrm{MD}=11.82 \mathrm{um}, 95 \% \mathrm{CI}[-$ $5.478,29.11], \mathrm{MD}=-.03333 \mathrm{um}, 95 \% \mathrm{CI}[-17.33,17.26])$. These analyses demonstrate a statistically significant difference between the control vector and the $\mathrm{N}_{400 I}, \mathrm{R}_{584}$ and $\mathrm{S}_{593} \mathrm{G}$ variants with respect to maximum neuron branch length.

Discussion: In this study, we identified two novel variants in SLITRK1. Including our previously identified variants, the combined SLITRK1 mutation load of OCDS cases compared to that of controls is significant $(p=0.044)$. With this follow-up study, we identified statistically significant differences in neuritic outgrowth in primary neurons in vitro in SLITRK1 mutants compared to controls. This further indicates specific functional consequences of SLITRK1 variants and underlines its potential role in psychiatric phenotypes.

Disclosure: U. Ozomaro: None. A. Pickrell: None. D. McCorquodale: None. G. Montenegro: None. J. Huang: None. J. Price: None. F. Zhang: None. V. Lemmon: None. S. Zuchner: None.

106. Parallel Independent Component Analysis Successfully Identifies Clusters of Interacting genes and Structural MRI Brain Regions from the Large-scale ADNI Alzheimer's/MCI Data Set

Shashwath Meda, Balaji Narayanan, Vince Calhoun, Jingyu Liu, Nora Bizzozero-Peron, Shannon Risacher, Li Shen, Andrew Saykin, Godfrey Pearlson*

\section{Institute of Living/Yale U, Hartford, CT}

Background: Liability for many neuropsychiatric disorders, including Alzheimer's disease (AD) is due to multiple interacting genes, plus environmental factors. Parallel independent component analysis (P-ICA)(refs 1,2$)$ is a novel multivariate statistical technique that can derive simultaneously linked imaging and genetic components (i.e. "clusters" of linked brain regions or SNPs) to reveal relationships between brain structure or function and genes. To date, P-ICA has been employed in smaller scale studies (typically $\sim 30$ subjects per group and $\sim 300$ SNP's). Because many studies now employ hundreds or thousands of subjects, up to millions of SNPs and hundreds of 
thousands of image voxels, it is important to know whether P-ICA can be used validly ("scaled up") on such high-dimensional data sets. Because some of the risk genes and all of the brain areas affected neuropathologically in $\mathrm{AD}$ are well known, using a large-scale $\mathrm{AD}$ data set such as the Alzheimer's Disease Neuroimaging Initiative (ADNI; ref 4) sample provides an ideal opportunity for such a proof-ofprinciple investigation for P-ICA.

Methods: We analyzed high-quality structural MRI (sMRI) data comprising of 86 different volumes of interest derived from VBM and 516,803 SNPs (after standard quality control analysis including removing bad calls, imputing missing values and removing SNP's with very low minor allele frequency) in the Caucasian participants in the ADNI study, consisting of 765 individuals (AD: Healthy Control: $\mathrm{MCI}=1: 1: 2)$. Genotyping was performed using an Illumina chip and sMRI data were analyzed in SPM as described in (ref 3,4) and P-ICA carried out as outlined in (refs 1-3).

Results: Of the derived genetic components, 36 correlated significantly with the 8 sMRI components. For example SNP component 2 was uniquely associated with hippocampal volume. The structural components contained all major areas known to be affected by the neuropathology of Alzheimer's disease, including hippocampus, parietal and temporal cortex. We selected the top 5 SNP components and applied an empirical dominant threshold of $\mathrm{Z}=2.5$. These components contained $\sim 9000$ SNPs associated with $\sim 4500$ known genes. Approximately $5 \%$ of these genes $(\sim 225)$ are already known to confer AD risk, including important candidates such as APoE4, ChAT, Presenilin-2, APP and GRIN2B. The genetic information was entered into the Ingenuity software suite in order to identify the relevant physiological groupings of all the identified genes.

Discussion: P-ICA, a novel multivariate analysis method, when used in a large-scale data set, identified sMRI components that comprised brain areas previously reported to be abnormal in $\mathrm{AD}$ and associated genetic components containing several well known $\mathrm{AD}$ risk genes. In addition multiple novel genes previously unassociated with AD were identified by the algorithm that comprised several physiological networks. Because both the location of the neuropathologic change and the identity of several major risk genes for late onset $\mathrm{AD}$ are known, their successful identification by the P-ICA algorithm provides strong evidence that the approach can be validly "scaled up". Additionally novel putative AD risk genes and physiologic pathways were identified.

References: 1). V Calhoun et al. 2009; Neuroimage 45; S163-172. 2). J Liu et al. 2009; Neuroimage 46; 809-816. 3). K Jagannathan et al. 2010; Biological Psychiatry 68: In press. 4). A Saykin et al. 2010; Alzheimer's and Dementia 6; 265-273.

Disclosure: S. Meda: None. B. Narayanan: None. V. Calhoun: None. J. Liu: None. N. Bizzozero-Peron: None. S. Risacher: None. L. Shen: None. A. Saykin: None. G. Pearlson: None.

107. TOMM40 Variants as Predictors of Lorazepam-Induced Memory Dysfunction in Healthy APOE4-Negative Elderly

Nunzio Pomara*, Davide Bruno, John J. Sidtis, Michael W. Lutz,

David J. Greenblatt, Ann M. Saunders, Allen D. Roses

Nathan Kline Institute/NYU School of Medicine, Orangeburg, NY

Background: Our group first demonstrated that healthy, cognitivelyintact elderly carriers of $\mathrm{APOE}_{4}$, an established risk factor for lateonset Alzheimer's disease (LOAD), experienced more pronounced and persistent memory deficits following acute administration of lorazepam, compared to non-E4 carriers, mostly with the APOE $3 / 3$ genotype. However, the latter showed considerable individual variability, which could not be ascribed to pharmacokinetic factors. Variants in the length of an intronic poly $\mathrm{T}$ repeat in the TOMM40 (the translocase of outer mitochondrial membrane 40 homolog) gene, which is in linkage disequilibrium with APOE, have recently been reported to modulate the age of LOAD onset in individuals with $\mathrm{APOE}_{3} / 3$. In this pilot pharmacogenetic study, we tested the hypothesis that healthy elderly non-E4 carriers, homozygous for the longer poly $\mathrm{T}$ sequences, would show greater sensitivity to lorazepam-induced cognitive toxicity as compared to those homozygous for the short TOMM40 poly $\mathrm{T}$ sequence.

Methods and Study Design: TOMM40 polymorphism genotyping for the variable length intronic poly $\mathrm{T}$ repeat (rs10524523) was performed on currently available DNA from subjects who completed our original lorazepam study. Out of a total sample of 52 healthy elderly subjects, we restricted our analysis to 14 individuals, who were non-e4 carriers, showed a high level of cognitive function (MMSE score of 28 or higher), and were free of confounding medical, neurologic and psychiatric conditions. The 14 subjects were divided into two groups: long TOMM40 (VL-VL \& L-VL, $\mathrm{n}=8$ ) and short TOMM40 $(S S, \mathrm{n}=6)$. All individuals, aged between 62 and 73, participated in three sessions, one week apart, during which they were administered oral doses of placebo or lorazepam (0.5 $\mathrm{mg}$ or $1 \mathrm{mg}$ ) in a double blind, randomized design. Each subject participated in all drug conditions and the order of the doses was counterbalanced. A repeated battery of psychometric tests, which included the Buschke Selective Reminding Task, was administered at baseline, 1 hour, 2.5 hours and 5 hours following drug administration. As our study focused on the maximum impact of lorazepam administration, we restricted our attention to $1 \mathrm{mg}$ doses at the time of greatest impairment (2.5 hours), which corresponds to peak plasma drug level. The study design had two independent variables: drug administration (placebo vs $1 \mathrm{mg}$; within-subjects) and TOMM-40 gene (short vs long; between-subjects). We evaluated these variables over the difference in total recall (total number of words recalled over seven trials) between 2.5 hours after drug administration and baseline.

Results: We conducted a 2 X 2 repeated measures ANOVA and, in addition to a main effect of drug administration $(p=.002)$, we observed an interaction between lorazepam and TOMM40 $[\mathrm{F}(1,12)=6.543, \mathrm{p}=.025]$. Post-hoc analyses indicated that TOMM40 length was inconsequential for the memory performance when placebo was administered $[\mathrm{F}(1,12) .05]$, but was associated with worse recall performance when lorazepam was administered $(p=.015)$, to the extent that the drop from baseline was twice as large for the long group than for the short group. The difference in performance between long and short TOMM40 groups, when lorazepam was administered, was not due to differences in plasma lorazepam concentrations $[\mathrm{t}(12)<1$, $\mathrm{p}=.447]$.

Conclusion: Our pilot data suggest that variants of TOMM40 may serve as pharmacodynamic predictors of drug-induced memory dysfunction in $\mathrm{E}_{4}$ negative individuals. Given that the frequency of $\mathrm{E}_{3} / \mathrm{E}_{3}$ in the general population is more than $70 \%$, this biomarker, if confirmed, could have important clinical implications.

Disclosure: N. Pomara: None. D. Bruno: None. J. Sidtis: None. M. Lutz: None. D. Greenblatt: None. A. Saunders: None. A. Roses: None.

108. Chimeric Genes in Schizophrenia

Caitlin Rippey*, Tom Walsh, Ming Lee, Alex Nord, Jack McClellan, Mary-Claire King

Univ of Washington, Seattle, WA

Background: Our lab has shown that individually rare, gene-disrupting copy number variants (CNVs) are significantly more prevalent in individuals with schizophrenia than in unaffected controls. Several groups have replicated and extended this finding, yet it remains an open question as to which individual events are pathogenic. A subset of CNVs connects components of two separate genes into novel, often deleterious chimeric genes. We hypothesize that some rare CNVs create brain-expressed chimeric genes that contribute to schizophrenia pathogenesis.

Methods: To test this hypothesis, we screened DNA from 124 individuals with schizophrenia using the Nimblegen $\mathrm{HD} 2$ array comparative genomic hybridization platform. We scanned genome-wide for CNVs greater than $30 \mathrm{~kb}$ in length that were present in at least one case 
and no controls $(\mathrm{n}=\sim 240$, run on the same platform), and did not coincide with events reported in the database of genomic variants. From these events, we selected only those predicted to delete or duplicate the $5^{\prime}$ end of one gene and the $3^{\prime}$ end of another, as these CNVs should produce chimeras.

Results: We detected four such events in cases, three resulting from tandem duplications and one from a deletion. We confirmed the breakpoints of each of these events by PCR and sequencing. We also confirmed that in all cases, at least one of the two genes in the predicted chimera was expressed in brain. In order to test whether these events produced stable chimeric transcripts in the patients' lymphoblasts, we targeted PCR primers to the predicted chimeric mRNA by placing a forward primer in an exon of the first gene and a reverse primer in an exon of the second. In three cases we were able to detect a stable transcript, confirmed by sequencing. In the fourth case, the gene contributing the $5^{\prime}$ end of the chimera had no detectable expression in lymphoblasts, while being highly expressed in adult and fetal brain, so we expect that this transcript is brain-specific. For the three chimeras with lymphoblast expression, we are performing western blots to ascertain protein stability.

Discussion: In conclusion, we have detected several rare copy number variants that lead to stable expression of chimeric mRNA in individuals with schizophrenia. We propose that these events are likely to lead to aberrant expression of fusion proteins in the brains of these patients, and contribute to schizophrenia. This represents a novel genetic mechanism for major mental illness, as well as implicating new genes and pathways in schizophrenia.

Disclosure: C. Rippey: None. T. Walsh: None. M. Lee: None. A. Nord: None. J. McClellan: None. M. King: None.

\section{The NF-kappa B Pathway in Schizophrenia Postmortem Brain} Tissue

Panos Roussos*, Pavel Katsel, Larry J. Siever, Vahram Haroutunian

\section{Mount Sinai School of Medicine, New York, NY}

Background: The etiology of schizophrenia remains unclear, however, evidence for the involvement of neuroinflammation and immunogenetics, mediated by cytokines has been accumulating. In the central nervous system, NF-kappa B acts as a regulator of growth, differentiation and adaptive response to extracellular signals, such as cytokines, oxidative stress, growth factors and glutamate. Neurons exhibit a constitutive level of NF-kappa B signaling and this pathway plays a significant role in neurite outgrowth, activity-dependent plasticity and cognitive function. The role of NF-kappa B signaling in schizophrenia has not been studied in postmortem tissue.

Methods: Brain tissue specimens were derived from the Brain Bank of the Department of Psychiatry of the Mount Sinai School of Medicine (New York, NY)/J.J. Peters VA Medical Center (Bronx, NY). For exploratory studies, ten cerebral cortical regions (BA 8, 21, 22, 23, 32, 38, Caudate, Hippocampus and Putamen) from 16 schizophrenic patients and 19 normal comparison subjects were analyzed using independent Affymetrix (Santa Clara, CA) HG-U133AB and HG-U133 Plus2 GeneChips. Hypothesis driven studies used qPCR to measure mRNA levels of NF-kappa B pathway genes in the superior temporal gyrus (BA 22) and primary visual cortex (BA 17) from an independent cohort of 22 persons with schizophrenia and 24 unaffected comparison subjects. We conducted similar studies in rats chronically exposed to haloperidol or placebo. Additionally, NF-kappa B p65 activation was examined by protein assays of nuclear extracts from BA 22. T-scores and fold changes (FC) were used as a standardized measure of gene expression change for each individual transcript. A two-tailed Student's t-test was used to compare relative mRNA expression in qPCR experiments and nuclear NF-kappa B assay.

Results: Microarray analysis showed an overall downregulation of the NF-kappa B pathway in temporal lobe, anterior cingulate cortex, basal ganglia and hippocampus, including genes that form the NF-kappa B complex, activate the NF-kappa B signaling pathway or are downstream target genes of NF-kappa B cascade, that are involved in apoptosis/cell survival, cellular response to inflammation and/or have a significant role during neurodevelopment. In BA 22 of the independent cohort, the brains of persons with schizophrenia had significantly reduced RELA [p65] $(\mathrm{p}=0.016 ; \mathrm{FC}=-1.56), \mathrm{MAP}_{3} \mathrm{~K}_{7}$ [TAK1] $(\mathrm{p}=0.007 ; \mathrm{FC}=-1.36)$ and $\mathrm{NFKB}_{1}[\mathrm{p} 50$ ] at a trend level $(p=0.1 ; F C=-1.19)$. In BA 17, schizophrenia patients had significantly increased $\mathrm{NFKB}_{1}\left[\mathrm{p}_{50}\right](\mathrm{p}=0.042 ; \mathrm{FC}=1.3)$ and RELA [p65] at a trend level $(\mathrm{p}=0.07 ; \mathrm{FC}=1.42)$. The $\mathrm{p} 50 / \mathrm{p} 65$ ratio of mRNA levels was higher in the brains of persons with schizophrenia in BA $22(p=0.025$; $\mathrm{FC}=1.3)$ but not BA $17(\mathrm{p}>0.8)$. In addition, two NF-kappa B targetgenes, were increased in patients in BA 17 [CCND1 $(p=0.006$; $\mathrm{FC}=1.63$ ) and NQO1 (po.6). NFKB1 and RELA mRNA levels were not altered in antipsychotic exposed rats ( $p>0.7$ in both comparisons). Transcription factor assay of p65 in BA 22 was significantly higher in controls $(\mathrm{p}=0.009)$ and correlated positively with the RELA expression levels in controls $(\mathrm{r}=0.67$, po.8).

Discussion: Using a multistep approach we provide strong evidence that NF-kappa B p65 signaling is downregulated in the superior temporal gyrus in schizophrenia. Neuroleptics do not alter the expression levels of $\mathrm{p}_{50}$ and $\mathrm{p} 65$ in rats. Reduced p65 mRNA expression levels and transcriptional activity might contribute to decreased expression of genes involved in cellular plasticity, neuronal survival/differentiation and neurite maintenance. Furthermore, increased p50/p65 stoichiometry contributes to the apoptotic program, while a neuroprotective effect is observed when p65 is expressed at high levels. Interestingly the p65 expression levels were correlated with GFAP, an astrocytic marker, in controls but not in persons with schizophrenia, providing evidence that NF-kappa B signaling might play a role in glial cell dysfunction in schizophrenia. We can further speculate, that the opposite results observed in occipital lobe might be part of a compensatory mechanism, which fails in superior temporal gyrus, a region reported to be dysfunctional in schizophrenia.

Disclosure: P. Roussos: None. P. Katsel: None. L. Siever: None. V. Haroutunian: None.

110. Identification of Genes and Biological Pathways Significantly Modulated by Cigarette Smoking in Human Peripheral Whole Blood Cells of Methamphetamine-dependent Subjects

Ju Wang, Tianhua Niu, Jennie Ma, Chamindi Seneviratne, Bankole Johnson, Ming Li ${ }^{\star}$

\section{University of Virginia, Charlottesville, VA}

Background: Cigarette smoking has been associated with many disorders and diseases. Although it is known that cigarette smoking can modulate the function of many genes/proteins and biological processes in multiple tissues, including the peripheral blood, our knowledge of gene expression patterns in the peripheral blood of smokers is limited. The goal of this study was to identify the genes and pathways differentially regulated in blood cells from smokers compared with non-smokers, as well as to investigate the genes and pathways whose changes in expression correlate with smoking quantity.

Methods: All RNA samples were extracted from the peripheral whole blood of methamphetamine-dependent subjects. Participants were classified as non-smokers (cigarette per day $[\mathrm{CPD}]=0 ; \mathrm{N}=10$ ), light smokers $(\mathrm{CPD} \leq 10 ; \mathrm{N}=22)$, moderate smokers $(\mathrm{CPD} \leq 20 ; \mathrm{N}=36)$, and heavy smokers $(\mathrm{CPD} \geq 21 ; \mathrm{N}=10)$. We compared genome-wide expression profiles of each smoking group with that of the nonsmoking group for the more than 40,000 genes on the Affymetrix Human Genome U133 Plus 2.0 GeneChip, and significantly regulated genes and biological pathways were identified.

Results: In single-gene analysis, 660, 516, and 686 differentially expressed genes were identified for light, moderate, and heavy smokers, respectively, at $\mathrm{p} \leq 0.05$. Among them, 126 genes were regulated in all three groups. Furthermore, the expression of 213 genes correlated significantly with smoking quantity, among which were 


\section{S350}

biomarkers of smoking-related diseases, such as lactotransferrin (LTF), lipocalin 2 (LCN2), and alpha synuclein (SNCA). We also identified a series of differentially enriched pathways in the smoking groups that are involved in immune function, metabolism, and neuronal function; e.g., T-cell receptor signaling pathway, apoptosis pathway, ubiquitin-mediated proteolysis, and axon guidance.

Discussion: Profiling gene expression in human peripheral blood can provide valuable information about biological responses to chronic cigarette smoking. We have identified a number of marker genes whose expression correlates with smoking quantity, as well as a series of biological pathways modulated by smoking. Furthermore, our study showed that peripheral blood may be used to develop a geneexpression-based approach to identify genes and biological processes in the neuronal system that are modulated by smoking.

Disclosure: J. Wang: None. T. Niu: None. J. Ma: None. C. Seneviratne: None. B. Johnson: Part 1; Johnson has served as a consultant to Johnson \& Johnson (Ortho-McNeil Janssen Scientific Affairs, LLC), Transcept Pharmaceuticals, Inc., D\&A Pharma, Organon, ADial Pharmaceuticals, Psychological. M. Li: Li serves as a scientific advisor to ADial Pharmaceuticals.

111. Most Replicated Addiction Vulnerability and Smoking Cessation Genome Wide Association Results

George Uhl*, Jed Rose, Catherine Johnson, Tomas Drgon, Frederique Behm

\section{NIH, Baltimore, MD}

Background: "Dark matter" has been postulated to account for the marked discrepancies between "genome wide significant" results from genome wide association studies for brain disorders and the heritable influences on these disorders, as identified in classical genetic studies. Family, adoption and twin data provide strong support for about 0.5 heritability for vulnerability to develop DSM dependence on an addictive substance, while twin data also supports possibly-overlapping 0.5 heritability for ability to quit smoking, largely through comparisons between current and former smokers. We and others have reported genome wide association data for these phenotypes with many results that achieve nominal $\left(\mathrm{p}<10^{-2}\right)$ significance and cluster in small genomic regions, though no result achieves reproducible genome wide $\left(\mathrm{p}<10^{-8}\right)$ significance. Increasing $\mathrm{n}$ by aggregating samples can increase variance in ways that mitigate the nominal increases in power for detection of variants with polygenic effects and substantial allelic heterogeneity.

Methods: We have, however, identified genes that display reproducible clusters of SNPs for which case $v s$ control $p$ values reach nominal significance in each of several studies.

Results: Several genes that we have initially identified in 100-50ok GWA studies for substance dependence and smoking cessation have now provided such levels of identification in more than 10 independent (or only modestly-overlapping) datasets. The genes that have been most frequently identified by studies from our groups have been among those most identified by data from others. Cumulative confidence in identification of these genes is thus much greater than $\mathrm{p}<10^{-8}$, even thought the result of no single study approaches this level of confidence.

Discussion: Repeated identification of gene variants in samples that provide modest variance among "case" groups and good matches between case and control groups on as many features as possible provide an alternative to searches for individual SNPs whose association reproducibly provides $\mathrm{p}<10^{-8}$ significance. Underlying polygenic genetic architectures with substantial allelic heterogeneity that differs based on modest differences in genetic background appear likely for many brain disorders. For substance dependence and ability to quit smoking, phenotypes for which there are no large, oligogenic effects and no clear negative selection that requires us to postulate contributions of many "new" allelic variants, such approaches provide atribution for some of this heritability for which "dark matter" would otherwise need to be postulated. Such nontemplate analyses should be useful in other brain disorders with similar underlying genetic architectures.

Disclosure: G. Uhl: None. J. Rose: Part 1; Lollilard, Inc. Part 2; P Morris USA. C. Johnson: None. T. Drgon: None. F. Behm: None.

\author{
112. Plasma Homocysteine After Methionine Loading in Patients with \\ Schizophrenia \\ Vadim Geller, Joseph Levine, Nurit Shalfman, RH Belmaker*
}

Ben Gurion University of the Negev, Beersheva, Israel

Background: A recent meta-analysis of 8 cross-sectional case control studies suggested that a $5 \mu \mathrm{Mol} / \mathrm{L}$ increase in homocysteine levels was associated with a $70 \%$ higher risk for schizophrenia (Muntjewerff et al. 2006). The methionine loading test may uncover latent cases of impaired homocysteine metabolism who have otherwise normal homocysteine levels. The methionine loading test evaluates the summation of a multitude of enzymatic pathways transforming methionine to homocysteine and degrading homocysteine. Den Heijer et al (2005) found the heritability of fasting homocysteine to be 0.21 , whereas the heritability of homocysteine levels after methionine loading was 0.67 .

Methods: We recruited outpatients with chronic schizophrenia (age 20-50), clinically stable for at least 3 months, all physically healthy and treated with an adequate dose of antipsychotic medication. The patients were given $100 \mathrm{mg} / \mathrm{kg}$ body weight oral dose of L-methionine dissolved in $200 c c$ of tea or fruit juice. Blood (10cc) for serum homocysteine levels are taken at baseline and 3 hours after L-methionine loading.

Results: This pilot study failed to detect a gross abnormality of plasma homocysteine level after acute methionine load in patients with schizophrenia.

Discussion: Elevations in plasma homocysteine reported in schizophrenia do not derive from a dynamic abnormality in methionine metabolism in this challenge paradigm.

Disclosure: V. Geller: None. J. Levine: None. N. Shalfman: None. R. Belmaker: None.

\section{A Combined Single Voxel and Proton Spectroscopic Imaging Study in Schizophrenia \\ Juan Bustillo*, Hongji Chen, Chris Abbott, Charles Gasparovics}

University of New Mexico, Albuquerque, NM

Background: A hypothesized process of glutamate-related excitotoxicity in schizophrenia can be potentially assessed in-vivo with H-MRS. Several H-MRS studies report reduced NAAc (a marker of neuronal viability) in mixed gray/white matter; however, abnormalities in glutamate (Glu), glutamine (Gln; the metabolite of synaptic Glu) and inositol (Ins; a glial marker), have not been consistently documented. Limitations in this H-MRS literature include poor spectral resolution (especially for glutamate and glutamine) and reduced spatial coverage (for all metabolites).

Methods: We are studying a large population of schizophrenia and age-matched healthy controls with chemical shift imaging (CSI; to improve coverage) and single-voxel H-MRS (to improve Glu and Gln resolution). We used an optimized PRESS sequence $(\mathrm{TE}=40 \mathrm{~ms}$, $\mathrm{TR}=1.5 \mathrm{~s}$ ) at 3 Tesla. With this sequence, we have documented reliable separation of Glu and Gln in the anterior dorsal cingulate cortex (ACcx, 2x2x3cc; Mullins, 2008). For CSI (an axial supraventricular tissue slab) 6 anatomical regions were identified (2 gray and 4 white matter). NAAc, Glu, Glx (Glu + Gln), Cre, Cho and Ins were fitted with LC model (CRLBs $<20$ for all voxels). The single voxel was acquired from the ACcx (CRLBs $<30$ for Gln, $<20$ for others). Partial volume and relaxation corrections were also implemented (Gasparovics, 2004) Results: $20 \mathrm{Sz}$ and $16 \mathrm{HNV}$ subjects were studied. Groups did not differ in age or gender. For the CSI data, there were diagnosis-by-tissue 
interaction for NAAc, $(\mathrm{F}(1,42)=7.96, \mathrm{p}=0.007$, with reduced NAAc in global WM in $\mathrm{Sz}(\mathrm{p}=0.007)$. For Glu there was was no diagnosisby-tissue interaction. However, a group main effect was present $(\mathrm{F}(1$, $42)=4.26, p=0.04$ ), with increased total Glu in Sz. For the SV data, there were no group differences in NAAc, Glu, Gln, Glx, Ins, Cre or Cho.

Discussion: In this preliminary analyses we detected reduced WM NAAc consistent with axonal dysfunction and increased Glu concentration across GM and WM in Sz. Elevations in synaptic Glu release can result from NMDA blockade, a hypothesized pathophysiological mechanism in Sz. Persistently elevated Glu may result in excitotoxicity, detected as reductions in NAAc. Understanding this process, may assist in the development of neuroprotective glutamate modulating agents, with the aim of preventing functional deterioration in Sz.

Disclosure: J. Bustillo: Part 1; Novartis study AQW051A2202 member of the Independent Data and Safety Minitoring Board. H. Chen: None. C. Abbott: None. C. Gasparovics: None.

114. Markedly Decreased $\left[{ }^{3} \mathrm{H}\right]$ pirenzepine Binding Defines a Sub-set of Subjects with Schizophrenia

Brian Dean*, Elizabeth Scarr

\section{Mental Health Research Institute, Parkville, Australia}

Background: A major impediment to understanding the pathophysiology of schizophrenia is that it is a syndrome, not a biochemically defined disease. We have consistently shown levels of cortical muscarinic M1 receptors (CHRM1) to be decreased in subjects with schizophrenia (Dean et al. Mol.Psychiatry 7, 1083-1091 (2002). We therefore wished to determine if a mutation in the CHRM1 gene may be i) associated with an altered risk of schizophrenia and ii) be a possible cause of decreased cortical CHRM1 levels in subjects with the disorder. Methods: We sequenced the CHRM1 gene using an $\mathrm{ABI}_{3100}$ genetic analyser and measured $\left[{ }^{3} \mathrm{H}\right]$ pirenzepine binding in Brodmann's area 9 from 74 controls and 84 subjects with schizophrenia.

Results: We identified 5 single nucleotide polymorphisms in the CHRM1 gene but no gene sequence gave a strong indication of being associated with an altered risk of schizophrenia. $\left[{ }^{3} \mathrm{H}\right]$ pirenzepine binding was significantly decreased in subjects with schizophrenia compared to controls (Mean \pm SEM: $133.9 \pm 7.25$ vs. $182.7 \pm 4.50 \mathrm{fmol} /$ mg ETE; p < 0.0001). Subsequent Kernel Density Analyses (Silverman, B.W. (1986) Density Estimation for Statistics and Data Analysis. Chapman and Hall, London) showed the radioligand binding data from the control subjects was best described as a single, binomially distributed, population. By contrast, the data from the subjects with schizophrenia was best described as two discrete populations. Using the populations as described by the Kernal Density Estimation, one population $(n=22)$ of subjects with schizophrenia was shown to have a marked reduction in $\left[{ }^{3} \mathrm{H}\right]$ pirenzepine binding (Mean \pm SEM: $44.3 \pm 6.88)$ whilst the other $(n=58)$ had levels of binding (Mean \pm SEM: $167.8 \pm 4.52 \mathrm{fmol} / \mathrm{mg}$ ETE) similar to that in controls. The population with the decreased $\left[{ }^{3} \mathrm{H}\right]$ pirenzepine could not be separated from the other subjects with schizophrenia based on any donor demographics, pharmacology, toxicology, cause of death or CNS collection parameter.

Discussion: Studies using cloned human CHRMs and CHRM knockout mice show, using our methodology, that $85 \%$ of the $\left[{ }^{3} \mathrm{H}\right]$ pirenzepine binding we measure is bound to CHRM1. Therefore our data suggests that approximately $25 \%$ of subjects with schizophrenia have lost the majority of their cortical CHRM1. These findings are similar to that of a neuroimaging study of pan-CHRMs levels where 3 of 12 subjects had levels of cortical CHRMs below that observed in any control subjects (Raedler, T.J. et al. (2003) Am.J.Psychiatr. 160: 118-127). Hence, a marked loss of cortical CHRM1 may be a potential biomarker for a sub-type of schizophrenia which, our early studies suggest, has a unique pathophysiology (Salah-Uddin, H. et al. (2009) Neuropsychopharmacol. 34: 2156-2166).

Disclosure: B. Dean: None. E. Scarr: None.
115. Age and Sex Associated Changes in Brain Metabolites in Young Marijuana Users

Christine Cloak*, Linda Chang, Daniel Alicata, Thomas Ernst

University of Hawaii; John A Burns School of Medicine, Honolulu, HI

Background: Marijuana use often begins during adolescence and precedes other illicit drug use. Brain imaging studies in adults with frequent marijuana use have shown abnormalities in regional cerebral blood flow, volumes, activation, chemistry, metabolism and diffusion measures. Less is known about the potential effects of marijuana use on the developing adolescent brain. The aim of this study is to longitudinally investigate the effects of marijuana use on adolescent brain development using proton magnetic resonance spectroscopy $\left({ }^{1} \mathrm{H}\right.$-MRS) to measure brain metabolites and corrolate findings with cognitive and psychiatric measures.

Methods: Fifty-five adolescent regular marijuana users (MJ: 13-23 years old, 30 males) and 40 adolescent controls (CON; 22 males) with minimal lifetime marijuana exposure were studied. All were screened and excluded if they had significant medical and psychiatric conditions. High-resolution MRI and localized ${ }^{1} \mathrm{H}-\mathrm{MRS}$ were performed on a Siemens 3 Tesla scanner. Spectra were obtained in five brain regions [anterior cingulate cortex, frontal white matter, basal ganglia (BG), cerebellum, and thalamus], and metabolite concentrations were calculated and analyzed with LCModel. Since brain metabolites vary with age, group comparisons of metabolites were performed using ANCOVAs (covaried for age), and followed up with regression analysis where appropriate.

Results: All individuals were healthy and had little or no exposure to other illicit drugs. At baseline, MJ users were $19 \pm 2$ years old. They began using at $14 \pm 2$ years of age, and smoked $2 \pm 4$ g/day, $6 \pm 2$ days/ week, for $48 \pm 23$ months, amounting to an estimated lifetime use of $2074 \pm 3475$ joints. CON subjects were $19 \pm 2$ years old, and 24 never used marijuana while others had $<20$ joints in their lifetime. Some participants used nicotine and or alcohol, but none met DSM-IV criteria for alcohol dependence. While there were no main effects of MJ at baseline, some metabolites showed interactions between MJ, sex and age. In the $\mathrm{BG}$, choline-containing compound concentrations $(\mathrm{CHO})$ decreased with age $(r=-0.46, p=0.03)$ only in the $M J$ females resulting in a 3 -way interaction $(\mathrm{p}=0.04)$, while myo-Inositol levels (MI) increased with age $(r=0.47, p=0.02)$ only in the MJ males (interaction $\mathrm{p}=0.05$ ). In the cerebellum, $\mathrm{N}$-acetyl aspartate concentrations (NAA) decreased with age $(r=-0.47, p=0.01)$ in the MJ males leading to a 3-way interaction $(\mathrm{p}=0.007)$ and the creatine levels decreased with age $(r=-0.53, p=0.04)$ only in the CON females (interaction- $p=0.03$ ). Cerebellar NAA in the male MJ users were lower with longer duration of $\mathrm{MJ}$ use $(\mathrm{r}=-0.43, \mathrm{p}=0.04)$. Thirteen participants (11 MJ users) had follow-up scans 1-2 years after baseline. BG CHO concentrations decreased $(-16 \%, \mathrm{p}=0.05)$ for MJ-users but not the CON relative to their baseline scans, while MI was unchanged in both groups. Cerebellar NA and creatine were also unchanged in both groups; however, cerebellar MI had increased $(+24 \%, \mathrm{p}=0.02)$ in MJ-users compared to baseline.

Discussion: Localized ${ }^{1} \mathrm{H}$-MRS demonstrated sex and age-related changes in brain metabolites of adolescent marijuana users. Our findings suggest that regular marijuana use may modestly impact the development of the BG and cerebellum in adolescents. The age-related increases in BG MI in the male MJ users, and the increased cerebellar MI from baseline to follow-up may reflect an ongoing glial response to injury or neurotoxicity associated with $\mathrm{MJ}$ use. In contrast, age-related decreases in BG CHO in the female MJ-users and the decreased BG $\mathrm{CHO}$ in all $\mathrm{MJ}$ users at follow-up suggest altered pruning or loss of cellularity. Lastly, age-associated decline in cerebellar NAA in the male MJ-users, more so in those with longer durations of MJ use, suggests greater neuronal damage to the cerebellum with longer duration of $\mathrm{MJ}$ use. The lower NAA in the cerebellum is in agreement with adult studies that show abnormal function and metabolism in this region. Since we did not observe cognitive deficits in this population, which are often detected in adult $\mathrm{MJ}$ users, these subtle metabolite changes 
may precede cognitive deficits. Continued follow-up of these subjects is underway to further evaluate the relationships between the duration of drug use and the development of psychiatric symptoms or cognitive deficits.

Support: Queen Emma Research Fund (033105), NIH (Ko1DA021203, K24DA16170, Ko2DA16991, 2U54NSo39406, U54NS56883, P2oRR11091, \& G12RRo03061), ONDCP, \& ACNP Faculty Research Fellowship (Eli Lilly).

Disclosure: C. Cloak: Part 1; ACNP Faculty Research Fellowship (Eli Lilly). L. Chang: None. D. Alicata: None. T. Ernst: None.

116. CSF 5-HIAA and Exposure to and Expression of Interpersonal Violence in Suicide Attempters

Jussi Jokinen*, Peter Nordström, Marie Åsberg

Karolinska Institutet, Stockholm, Sweden

Background: Serotonin is implicated in impaired impulse control, aggression and suicidal behaviour. Low cerebrospinal fluid (CSF) concentrations of the serotonin metabolite 5-hydroxyindoleacetic acid (5-HIAA) have been found in violent suicide attempters, suicide victims and in violent offenders. CSF 5-HIAA concentrations have both genetic and environmental determinants. Childhood trauma may have an effect on central monoamine function as an adult. The aim of this study was to assess the relationship of CSF 5-HIAA and the exposure to and the expression of violence in childhood and during adult life measured with the Karolinska Interpersonal Violence Scale (KIVS).

Methods: 42 medication free suicide attempters underwent lumbar puncture and were assessed with the Karolinska Interpersonal Violence (KIVS) scales to assess history of childhood exposure to violence and lifetime expressed violent behaviour.

Results: In women, but not in men, CSF 5-HIAA showed a significant negative correlation to exposure to violence during childhood. Furthermore, suicide attempters with low CSF 5-HIAA were more prone to commit violent acts as an adult if exposed to violence as a child compared to suicide attempters with CSF 5-HIAA above median. In the non-traumatized group, CSF 5-HIAA showed a significant negative correlation to expressed violent behaviour in childhood.

Discussion: Although central serotonergic function has important genetic determinants, exposure to childhood trauma may also affect serotonergic function. Low serotonergic function may facilitate impaired aggression control in traumatized suicide attempters.

Disclosure: J. Jokinen: None. P. Nordström: None. M. Åsberg: None.

\section{Altered Striatal Functional Connectivity in Adolescent Major} Depressive Disorder

Michael Milham, Vilma Gabbay*, Saroja Bangaru, Donald F. Klein, Benjamin Ely, Aviva Panzer, F. Xavier Castellanos

\section{NYU Child Study Center, New York, NY}

Background: While adolescent major depressive disorder (MDD) is a major public health concern due to its high prevalence and risk for suicide, research into MDD neurobiology in this age group remains limited. In the past decade, studies of adult MDD pathophysiology have increasingly utilized functional magnetic resonance imaging (fMRI) to examine abnormalities in the resting state functional connectivity (RSFC) of patients relative to healthy controls (HC). These studies have implicated altered RSFC of several neural networks in adult MDD, including decreased RSFC between cortico-limbicstriatal structures and altered RSFC in the default mode network (DMN). Our laboratory's studies of adolescent MDD, which utilize cutting-edge MR spectroscopy to non-invasively examine neurochemicals in vivo, have found increased total choline (tCho; biomarker for cell membrane turnover) in the left caudate of adolescent MDD subjects. Based on these findings, this study aimed to examine the role of the striatum in adolescent MDD and the neural networks associated with the striatum. We hypothesized that adolescents with MDD would exhibit altered RSFC within a striatum-based network and within the DMN. We also explored whether Beck Depression Inventory (BDI) and Children's Depression Rating Scale-Revised (CDRS-R) scores would be correlated with RSFC alterations.

Methods: Subjects: Seventeen adolescents with adolescent-onset MDD and sixteen HC (ages 13-19) were enrolled. A clinician met with parents and evaluated adolescents using a standard semi-structured interview, CDRS-R, and self-administered BDI. MDD subjects met full DSM-IVTR criteria, had episode durations of at least 8 weeks, CDRS-R scores of at least 40, and were psychotropic medication-free at least 3 months prior to the scan. HC did not meet criteria for any DSM-IV-TR diagnosis and were psychotropic medication-naïve. Data Acquisition: A 3.0 Tesla scanner was used to acquire 197 contiguous echo planar imaging functional volumes ( $\mathrm{TR}=2 \mathrm{~s}, 39$ slices) during rest with eyes open. A high-resolution $T_{1}$-weighted $3 \mathrm{D}$ anatomical image was also acquired using a magnetization prepared gradient echo sequence for spatial normalization and localization. Regions of Interest: Six previously defined bilateral basal ganglia seed regions were selected due to their ability to effectively differentiate striatal sub-regions: the dorsal caudate (DC), ventral caudate (VSs), ventral caudate/nucleus accumbens (VSi), dorsal rostral putamen, dorsal caudal putamen, and ventral rostral putamen. Preprocessing: Using FMRIB software library (FSL), slice time correction for interleaved acquisitions, motion correction, spatial smoothing, temporal bandpass filtering, and spatial normalization were performed. Nuisance signals were then removed and the residual mean time series for each seed was extracted. RSFC Analyses: Participant-Level: For each seed region of each participant, the time series was extracted and voxel-wise maps were generated, indicating correlation strength. Group-Level: MDD adolescents and HC were compared via FSL using a random-effects model covarying for age, handedness, and sex; Gaussian Random Field Theory was used to correct for multiple comparisons at the cluster level $(Z>2.3$; $p<0.05$, corrected). These analyses yielded within- and between-group thresholded Z-score maps of the positive and negative RSFC for each caudate seed.

Results: MDD Assessments: MDD subjects had a mean BDI score of $25.3 \pm 12.0$ and a mean CDRS-R score of $50.6 \pm 6.6$, while $\mathrm{HC}$ had a mean BDI score of $2.1 \pm 2.5$ and a mean CDRS-R score of $19.6 \pm 2.3$. RSFC: For all caudate seeds (VSs, VSi, DC), MDD subjects displayed greater RSFC with the prefrontal cortex and decreased RSFC with the occipital and right temporal lobes. Correlations: Symptom severities of MDD subjects, measured by $\mathrm{BDI}$ scores, were correlated with decreased RSFC between the DC, VSs, and right VSi seeds and several regions associated with the DMN, including the precuneus and posterior cingulate cortex.

Discussion: These data are consistent with our group's prior findings of caudate tCho abnormalities in adolescent MDD and with other groups' studies implicating the caudate in MDD. Interestingly, evidence of similar caudate-based RSFC abnormalities was recently reported in a study of adults with obsessive-compulsive disorder (OCD). These findings, which may indicate a link between the ritualbased ruminations of OCD and the negative self-ruminations of MDD, merit further study. Converging evidence of caudate dysfunction in adult and adolescent MDD forms a basis for improved diagnostics and targeted therapeutics.

Disclosure: M. Milham: None. V. Gabbay: None. S. Bangaru: None. D. Klein: None. B. Ely: None. A. Panzer: None. F. Castellanos: None.

118. Brain Reactivity to Smoking Cues Is Greater in Smokers with an Attentional Bias to Smoking Words

Amy C. Janes, Diego A. Pizzagalli, Sarah Richardt, Blaise deB. Frederick, Avram J. Holmes, Maurizio Fava, A. Eden Evins, Marc Kaufman*

McLean Hospital, Belmont, MA

Background: The World Health Organization reports that in the 21st century, tobacco use could contribute to the deaths of one billion 
people (World Health Organization, 2008), yet relapse rates remain high (1-3). During smoking quit attempts, most relapse episodes occur following exposure to smoking cues $(4,5)$. However, the link between relapse and smoking-cue induced craving is inconsistent (6). Attentional bias for smoking-related stimuli, as measured by the smoking emotional Stroop (SES) task, predicts treatment outcomes for tobacco smoking cessation $(7,8)$. Identifying relationships between smoking-related attentional bias and brain reactivity to smoking images using functional MRI (fMRI) may help identify neural substrates critical to relapse vulnerability.

Methods: Nicotine dependent women smokers $(n=28)$ performed a behavioral computerized SES task, based on work by Waters et al (7). The SES included neutral and smoking-related words displayed in red, green, or blue fonts. Participants were asked to identify the font color as quickly and accurately as possible, while ignoring word meaning. An attentional bias score was computed as the reaction time difference between smoking and neutral words (RTSmoking - RTNeutral), with higher values indicating increased interference effects associated with smoking-related words. Functional MRI was performed using a Siemens Trio 3 Tesla scanner (Erlangen, Germany), during which subjects viewed smoking or neutral images created by Gilbert and Rabinovich (International Smoking Image Series version 1.2. (with Neutral Counterparts), 9). Image analysis was conducted using Brain Voyager QX 1.10.4 (Brain Innovation, Maastricht, The Netherlands), following procedures previously described $(8,10)$. The relationship between brain reactivity to smoking $>$ neutral images and attentional bias (RTSmoking - RTNeutral) was assessed using a random effects analysis of covariates (ANCOVA), where SES performance was the covariate. The results were calculated on the whole-brain level and multiple comparisons were cluster corrected to $\mathrm{p}<0.05$.

Results: On average, smokers had an attentional bias score of $23.3 \pm 67.5 \mathrm{~ms}$ (range from 164 to $-57.5 \mathrm{~ms}$ ). Positive correlations were found between attentional bias and reactivity to smoking images in the amygdala, hippocampus, parahippocampal gyrus, insula, and occipital cortex.

Discussion: Heightened attentional bias was correlated with increased reactivity to smoking images in brain areas involved in emotion, memory, awareness of internal states, and visual processing. Thus, smokers with elevated smoking-stimuli attentional bias may shift attention toward smoking stimuli-induced internal states and emotional memories and away from other external stimuli. This attentional shift may explain why smokers with a greater attentional bias are more distracted by smoking cues, and why they may possibly be more vulnerable to cue-induced relapse. Treatments capable of inhibiting attentional shifts to smoking cues may lead to personalized cessation and relapse prevention treatment for smokers with heightened smoking-stimuli attentional bias. References: 1. Etter J-F, Stapleton JA (2006) Tob Control 15: 280-285. 2. Fiore MC, et al., (1994) JAMA 271: 1940-1947. 3. Garvey AJ, et al., (1992) Addict Behav 17: 367-377. 4. Ferguson SG, Shiffman S (2009) J Subst Abuse Treat 36: 235-243. 5. Shiffman S, et al., (1996) J Consult Clin Psychol 64: 366-379. 6. Perkins K (2009) Addiction 104:1610-1616. 7. Waters AJ, et al., (2003) Health psychology 22: 378-387. 8. Janes AC, et al., (2010) Biol Psychiatry 67:722-9. 9. Gilbert DG, Rabinovich NE (1999) Integrative Neuroscience Laboratory, Department of Psychology, Southern Illinois University. 10. Janes AC, et al., (2009) Exp Clin Psychopharmacol 17: 365-373. Acknowledgments: Supported by NIDA grants DA19378, DA017324, DA014013, DA015116, DA022276 \& DA15036.

Disclosure: A. Janes: None. D. Pizzagalli: Part 1; Advanced Neuro Technology, Astra Zeneca. Part 4; Advanced Neuro Technology. S. Richardt: None. B. Frederick: None. A. Holmes: None. M. Fava: Part 1; Affectis Pharmaceuticals AG, Clintara, LLC, Abbott Laboratories, Alkermes, Aspect Medical Systems, Astra-Zeneca, BioResearch, BrainCells, Inc, Bristol-Myers Squibb Company, Cephalon, Clinical Trial Soluctions, LLC, Eli Lilly \& Company, Forest Pharmaceuticals, Inc, Ganeden, GlaxoSmithKline, J\&J Pharmaceuticals, Lichtwer Pharma GmbH, Lorex Pharmaceuticals, NARSAD, NCCAM, NIDA, NIMH,
Norvartis, Organon Inc, PamLab, LLC, Pfizer, Inc, Pharmavite, Roche, Sanofi-Aventis, Shire, Solvay Pharmaceuticals, Inc, Synthelabo, WyethAyerst Laboratories, Amarin, Aspect Medical Systems, Auspex Pharmaceuticals, Inc, Bayer AG, Best Practice Project Management, Inc, BioMarin Pharmaceuticals, Inc, Biovail Pharmaceuticals, Inc, Cephalon, CNS Response, Compellis, Cypress Pharmaceuticals, Dov Pharmaceuticals, Eisai, Inc, EPIX Pharmaceuticals, Euthymics Bioscience, Inc, Fabre-Kramer Pharmaceuticals, Inc, Grunenthal GmBH, Janssen Pharmaceutica, Jazz Pharmaceuticals, Knoll Pharmaceutical Company, Labopharm, Lorex Pharmaceuticals, Lundbeck, MedAvante, Inc, Merck, Methylation Sciences, Neuronetics, Nutrition 21, PharmaStar, Precision Human Biolaboratory, PsychoGenics, Psylin Neuroscience, Inc, Ridge Diagnostics, Inc, Sepracor, Schering-Plough, Somaxon, Somerset Pharmaceuticals, Takeda, Tetragenex, TransForm Pharmaceuticals, Inc, Transcept Pharmaceuticals, Vanda Pharmaceuticals, Inc, Covidien, RCT Logic, LLC, CeNeRx BioPharma, GenOmind, LLC, Prexa Pharmaceuticals, Rexahn Pharmaceuticals, Sepracor, Inc. Part 4; Eli Lilly, GlaxoSmithKline, Briston-Myers Squibb Company, PamLab, LLC, Sanofi-Aventis, Pfizer, Inc, AstraZeneca, Johnson\&Johnson Pharmaceutical Research \& Development, Novartis, Healthcare Technologies, BrainCells, Inc. Part 1; EnVivo Pharmaceuticals. A. Evins: Pfizer, GSK. Part 4; Pfizer, GSK, NIDA. M. Kaufman: Part 1; NIH, GSK, Organon, Schering-Plough, Varian, Amgen, Novartis, NARSAD. Part 4; GSK, Organon, Schering-Plough, Varian, NARSAD.

119. The Effect of Varenicline on Cue-Induced Brain Activation and Drinking Parameters in Alcoholics Hugh Myrick*, Xingbao Li, Patrick Randall, Scott Henderson, Raymond Anton

Ralph H. Johnson VAMC/MUSC Department of Psychiatry, Charleston, SC

Background: Our group has demonstrated that taste and visual cues of alcohol could induce craving and regional brain activation in nontreatment seeking alcoholics during an fMRI procedure (Myrick et al., 2004) and that these cue-induced alterations can be attenuated by medication treatment (Myrick et al. 2008). The goal of the study was to utilize this paradigm to evaluate the effect of varenicline, an alpha4beta2 nicotinic receptor partial agonist, on cue-induced brain activation and drinking in alcoholics.

Methods: Non-treatment seeking alcoholics $(\mathrm{n}=40,23$ males 17 females, mean age 32.05) received either varenicline or identical placebo for 14 days prior to an alcohol cue induced brain fMRI imaging study. Subjects drank an average of 8.1 drinks per drinking day on $75 \%$ of days in the past 30 days prior to randomization (between group differences were non significant). In a Siemens 3.0 Tesla MRI scanner, subjects were given a sip of alcohol before viewing a 12 minute randomized presentation of pictures of alcoholic beverages, non-alcoholic beverages, and two different visual control tasks. During picture presentation, changes in regional brain activity were measured in 15 transverse $\mathrm{T}^{*}$-weighted BOLD slices. Subjects rated their urge to drink after each picture sequence. Differences in regional brain activity between viewing alcoholic beverage and nonalcoholic beverages were averaged over subjects and compared within groups and between groups. Alcohol use over the 14-day medication treatment was monitored.

Results: There was a reduction in drinks per drinking day, percent days abstinent and percent heavy drinking days during the treatment period though the differences between medication groups were not statistically significant. The varenicline group had significantly greater ventral striatum and medial prefrontal activation in response to alcohol cue stimuli.

Discussion: The study aims to provide both novel and valuable information regarding the effect of varenicline on cue-induced brain activation and voluntary drinking. While imaging trials by our group using medications that modulate dopaminergic pathways have found a blockade in ventral striatum activity, varenicline does not appear to act 
similarly. Further analysis correlating cue-induced brain activity with drinking behavior will be presented.

Disclosure: H. Myrick: None. X. Li: None. P. Randall: None. S. Henderson: None. R. Anton: None.

120. Midbrain dopamine differentially predicts neural response to happy and fearful facial expressions: ${ }^{18}$ FDOPA PET, fMRI, and MEG Findings

Tiffany Nash*, Mbemba Jabbi, Philip Kohn, Angela Ianni, Tom Holroyd, Frederick Carver, Jonathan S. Kippenhan, Richard Coppola, Karen Berman

National Institutes of Health, Bethesda, MD

Background: The midbrain ventral tegmental area (VTA) and substantia nigra (SN) are the main source of striatal and mesolimbic dopamine (DA). Though it is well-documented that DA is involved in motivational signaling ${ }^{1}$, its role in coding socially relevant emotional cues in humans remains largely unknown. Using ${ }^{18}$ FDOPA PET, fMRI, and MEG, we investigated the relationship between midbrain DA synthesis and neural response to happy and fearful facial expressions. In line with differential DA involvement in coding positive vs. negative signals in a time-dependent fashion ${ }^{2,3}$, we predicted dissociable influences of DA synthesis on neural responses to positive vs. aversive social stimuli.

Methods: Twenty-one participants ( 6 females; mean age $=31$ ) underwent 90-minute dynamic $16 \mathrm{mCi}{ }^{18} \mathrm{~F}$-DOPA PET scan. A voxel-wise Patlak method, with a cerebellar reference region, was used to determine FDOPA Ki, reflecting presynaptic DA synthesis, for every voxel in the brain. A midbrain volume of interest was manually delineated on each individual's structural MRI and coregistered to the native space FDOPA images for extraction of average midbrain Ki values. The same participants viewed emotional or neutral facial expressions that varied in length ( presentation dynamics (videos vs. static pictures) during event-related $3 \mathrm{~T}$ fMRI. In order to better capture the temporal dynamics of DA-mediated neural response, we repeated the same paradigm during MEG for 16 of the 21 participants ( 4 females; mean age =34.2). Data were acquired using a CTF 275 MEG system with a whole-head array of 275 radial 1 st order gradiometer/SQUID channels ${ }^{4}$. For fMRI, preprocessing and analysis were carried out using SPM5. For MEG, synthetic aperture magnetometry, a method for estimation of source power for each voxel of the brain using a beamformer, was carried out following coregistration of subjects' MEG scans to their anatomical MRIs. Second-level analyses were performed using midbrain Ki values as predictors of BOLD and MEG signal response. We focused on the gamma frequency band $(30-50 \mathrm{~Hz})$ given its reported role in emotional processing ${ }^{5}$; results are reported at $\mathrm{p}<0.001$, uncorrected.

Results: DA-Modulated Neural Response to Positive Stimuli During viewing of static happy stimuli, midbrain DA-BOLD coupling was observed in left fusiform, posterior insula, and hippocampus, as well as in the nucleus accumbens bilaterally. Further, DA predicted MEG gamma oscillatory response in anterior cingulate cortex for the static happy stimuli. For dynamic happy stimuli, there was positive DA-gamma coupling in left superior temporal sulcus, in line with this region's role in processing emotional dynamics. For brief presentations of happiness, DA predicted BOLD response in left fusiform, hippocampus, and insula, and in nucleus accumbens bilaterally, as well as MEG gamma response in the midbrain itself, possibly revealing a more transient reward related midbrain mechanism that may modulate upstream reward circuitry. DA-Modulated Neural Response to Negative Stimuli For static fear, there was a positive DA-BOLD coupling in the left fusiform. In contrast, dynamic fearful expressions led to a negative DA-BOLD coupling in bilateral temporal regions, as well as a negative DA-gamma coupling in the left caudate. During the viewing of brief fear, a negative midbrain DA-gamma coupling was shown in the left caudate and nucleus accumbens, perhaps representative of a role for DA in facilitating habituation (Fig1).

Discussion: DA differentially mediated neural response to environmentally relevant stimulus attributes in a valence-specific fashion. While there was a largely positive DA-mediated neural response for happy expressions, negative relations were apparent between DA and neural response for fearful stimuli. These results suggest that DA may positively mediate reward for positive stimuli, while serving an inhibitory role for negative stimuli. Our results support previous research on the role of DA in reward coding and fear processing ${ }^{6}$, and confirm a role for DA in mesolimbic modulation of emotions. The DA modulated gamma oscillatory findings may reflect faster, more transient neural coding of emotional information, which may not have been captured by fMRI's coarser temporal resolution? These differential findings for negative and positive emotions offer potential insight for studies of affective disorders.

\section{References:}

1. Wise R Neurotox Res 2008

2. Matsumoto, $\mathrm{M}$ et al. Nature 2009

3. Schultz, W Annu Rev Neurosci 2007

4. Fife A et al. Conf Biomagnetism 2002

5. Luo Q Neuroimage 2007

6. Wise R Nature Rev Neurosci 2004

7. Lachaux J et al. HBM 2007

Disclosure: T. Nash: None. M. Jabbi: None. P. Kohn: None. A. Ianni: None. T. Holroyd: None. F. Carver: None. J. Kippenhan: None. R. Coppola: None. K. Berman: None.

\section{Pharmacological Differentiation of the Novel Mu-Opioid Receptor Ligand GSK1521498 From Naltrexone Using [ $\left.{ }^{11} \mathrm{C}\right]$ carfentanil PET and fMRI}

Pradeep Nathan*, John Beaver, Edward Bullmore, Graham Searle, Christopher Long, Samuel Hill, Jonathan Howard, Paul Matthews, Roger Gunn, Eugenii Rabiner

GlaxoSmithKline Pharmaceuticals and University of Cambridge, Cambridge, United Kingdom

Background: The $\mu$ opioid receptor ( $\mu$-OR) plays a central role in hedonic and motivational processes, and is thus a potential therapeutic target for drug dependence and obesity. In vitro, GSK1521498 and naltrexone have similar affinity profiles for human $\mu$-OR (GSK1521498 $\mathrm{fpKi}=8.82$; naltrexone $\mathrm{fpKi}=8.70)$. We used $\left[{ }^{11} \mathrm{C}\right]$ carfentanil PET to compare the in vivo binding of GSK1521498 and naltrexone to $\mu$-OR in the brain of healthy human volunteers. For each compound we also evaluated the relationship between $\mu$-OR occupancy and pharmacodynamic effects, as measured by functional magnetic resonance imaging (fMRI) during receipt of highly palatable liquid food stimuli.

Methods: [11C]carfentanil PET scans were performed following administration of GSK1521498 (11 subjects; 0.4-100 mg p.o.) and naltrexone (11 subjects; 2-50 mg p.o.). Each subject received three $[11 \mathrm{C}]$ carfentanil PET scans, at baseline and at two time-points following administration of GSK1521498 (post-dose scan times $3-51 \mathrm{hrs}$ ) or naltrexone (post-dose scan times varied from 3-88 hrs). Doses and post-dose scan times were optimised to sample the dose-time-occupancy surface of each compound. fMRI measures of brain activation during receipt of liquid food stimuli were acquired following the baseline PET and the first post-dose PET scan. Simplified reference tissue model (SRTM), with occipital cortex as the reference region, was applied to dynamic $\mathrm{PET}$ data to generate regional binding potentials $\left(\mathrm{BP}_{\mathrm{ND}}\right) . \mu-\mathrm{OR}$ occupancies were calculated as the percent difference between baseline and post-drug $\mathrm{BP}_{\mathrm{ND}}$. Receptor occupancies were related to differences between baseline and post-drug fMRI endpoints using repeated measures regression.

Results: We found dose-dependent reductions in $\left[{ }^{11} \mathrm{C}\right]$ carfentanil $\mathrm{BP}_{\mathrm{ND}}$ for both compounds. A direct model best described the relationship between plasma concentrations of GSK152498 and $\mu-O R$ 
occupancy, with an $\mathrm{EC}_{50}=7.10 \mathrm{ng} / \mathrm{ml}$ (95\% CI: $5.96-8.25 \mathrm{ng} / \mathrm{ml}$ ). For naltrexone, a direct model did not fit the relationship between plasma concentrations and $\mu-O R$ occupancy. A significant inverse relationship was observed between GSK1521498 $\mu$-OR occupancy and brain activation to highly palatable liquid foods in the amygdala and hippocampus $(p s<.005)$. No significant relationships between $\mu$-OR occupancy by naltrexone and brain activation were observed.

Discussion: $\mu$-OR occupancy by GSK 1521498 was well predicted by plasma concentration at all time-points examined. For naltrexone, we demonstrated a changing relationship between plasma concentration and $\mu$-OR occupancy at different times post-dose (an indirect model). The reasons for dissociation between naltrexone plasma time-course and $\mu$-OR occupancy time-course are not clear, but may involve the activity of $6 \beta$-naltrexole, a known metabolite of naltrexone, at the $\mu$-OR. Finally, GSK1521498, but not naltrexone, attenuated brain activation to highly palatable liquid foods in the amygdala and hippocampus as a function of its $\mu$ opioid receptor occupancy. These findings suggest that despite having similar properties in vitro, GSK1521498 and naltrexone have different pharmacokinetic and pharmacodynamic characteristics in vivo.

Disclosure: P. Nathan: Part 5; GlaxoSmithKline Pharmaceuticals. J. Beaver: GlaxoSmithKline Pharmaceuticals. E. Bullmore: GlaxoSmithKline Pharmaceuticals. G. Searle: GlaxoSmithKline Pharmaceuticals. C. Long: GlaxoSmithKline Pharmaceuticals. S. Hill: GlaxoSmithKline Pharmaceuticals. J. Howard: GlaxoSmithKline Pharmaceuticals. P. Matthews: GlaxoSmithKline Pharmaceuticals. R. Gunn: GlaxoSmithKline Pharmaceuticals. E. Rabiner: GlaxoSmithKline Pharmaceuticals.

122. PET Imaging Reveals Sex Differences in CB1 Receptor Expression in Humans

Alexander Neumeister*, Yiyun Huang, Marc Normandin, Danielle

Piomelli, Christopher Bailey, Shannan Henry, James Murrough, Marc

Potenza, Richard Carson, Rachel Yehuda

\section{Mount Sinai School of Medicine, New York, NY}

Background: Relatively few imaging studies have been undertaken to systematically study gender differences in brain function in healthy people. Delineation of gender differences, however, appears critical before studying disease states. For example, an accumulating body of evidence suggests that gender differences exist in adaptive and maladaptive responses to stress and evidence from different disciplines suggests that acute and chronic stress-related mechanisms play an important role in the development of and the chronic, relapsing nature of a variety of psychiatric disorders including mood and anxiety disorders or addiction. An accumulating body of evidence suggests that the endocannabinoid (eCB) system participates in the stress response. Therefore, better understanding the eCB system and CB1 receptor function could provide a novel perspective on mechanisms of stress-response and could ultimately stimulate research targeting this system for prevention, pathophysiology and treatment purposes. Healthy human controls were each scanned with [11C]OMAR. Modeling of dynamic PET data using metabolite-corrected arterial input functions yielded regional estimates of distribution volume (VT), the concentration of tracer in tissue relative to plasma at equilibrium, assessments of plasma metabolite fraction and net tracer uptake in brain, and these data were assessed for differences between men and women.

Methods: Eleven healthy humans (6 male, 5 female) underwent one [11C]OMAR scan. PET data were acquired for $120 \mathrm{~min}$ on the HRRT and metabolite-corrected arterial input functions were measured. Injected activity was $18 \pm 1 \mathrm{mCi}$, specific activity was $4.7 \pm 1.5 \mathrm{mCi} /$ nmol, and mass dose was $0.03 \pm 0.01 \mu \mathrm{g} / \mathrm{kg}$. VT was estimated from tissue TACs using the metabolite-corrected arterial input functions. Data were described well by a two-tissue compartment model (2 T), whereas fits using a one-tissue model $(1 \mathrm{~T})$ were poor. Multilinear analysis (MA1) yielded good fits for $t^{*}>20 \mathrm{~min}$ and provided VT values in excellent agreement with $2 \mathrm{~T}$. Results reported here are from MA1 with $\mathrm{t}^{\star}=30 \mathrm{~min}$.

Results: VT values indicated highest binding in the pallidum, putamen, cortical subregions, amygdala, and hippocampus; low uptake was observed in white matter and diencephalon. For [11C]OMAR, parent fraction in plasma was greater in men than women. While regional brain uptake (SUV) is higher in men than women, SUV normalized by body weight and injected dose was lower in men than women.

Discussion: Plasma metabolite fraction and tracer uptake in brain differed between males and females. While tracer in brain (as measured by SUV) was greater in men than women, distribution volume was greater in women than men. These results suggest that analysis based on kinetic modeling with arterial input functions may lead to different interpretation of data than assessment of tracer uptake alone. Based on these findings, we recommend analysis using metabolite-corrected arterial input functions. Altogether, first, in healthy men and women we found global differences in $\mathrm{CB} 1$ receptor expression between men and women while we would expect gender by region between-group differences in psychiatric disorders based on the distribution of $\mathrm{CB} 1$ receptors in the brain and their functions which vary with brain region and the phenotype of various psychiatric disorders. Our results suggest that in studies of neuropsychiatric disorders group comparisons should be made using gender-matched control populations. Second, characterization of gender differences in major stress systems in healthy people is a critical first step to understand mechanisms of stress response in general. And third, we believe that clarification of the role of gender and better understanding of other contributing mechanisms to their function, i.e. glucocorticoid function, is important if the $\mathrm{eCB}$ system and $\mathrm{CB} 1$ receptors in particular will ever be considered as targets for treatment development or prevention.

Disclosure: A. Neumeister: None. Y. Huang: None. M. Normandin: None. D. Piomelli: None. C. Bailey: None. S. Henry: None. J. Murrough: None. M. Potenza: None. R. Carson: None. R. Yehuda: None.

123. Altered Anticipatory Amygdala Activity in Generalized Anxiety Disorder Is Linked to Genetic Variation of the Serotonin Transporter Jack Nitschke*, Desmond Oathes, Lori Hilt, Daniel Grupe

University of Wisconsin, Madison, WI

Background: Serotonin transporter gene variants and the amygdala are important candidates for investigating gene-brain interactions in anxiety disorders. To date, these biomarkers have not been investigated simultaneously in generalized anxiety disorder (GAD). Consistent with theories linking both GAD and worry to vigilance for threat (Mathews, 1990; Barlow, 2001; Borkovec et al., 2004), we have recently shown that GAD patients exhibit heightened amygdala responses during the anticipation of emotional pictures (Nitschke et al., 2009). Given the importance of the amygdala in anxiety and the link between amygdala activity and serotonin transporter promoter region polymorphisms, we sought evidence for an effect of the triallelic 5-HTTLPR on anticipatory amygdala activity in GAD patients.

Methods: A triallelic assay (5-HTTLPR and rs25531) was employed to assess genetic variation linked with altered serotonin signaling in 26 GAD patients (19 S/LG carriers, 7 LA homozygotes) and 21 nonpsychiatric controls (14 S/LG carriers, 7 LA homozygotes), as diagnosed using the Structured Clinical Interview for DSM-IV (SCID; First et al., 1996). Twelve GAD patients had no other current or past Axis I disorder, and 14 had current or past comorbid major depressive disorder, dysthymia, or social phobia. No participant was taking psychiatric medications during the study. All subjects were scanned using fMRI while performing an emotional anticipation task, during which subjects viewed anticipatory cues and emotional pictures and provided online ratings of their affective responses to the pictures. 


\section{S356}

An ' $\mathrm{X}$ ' cue signified that an aversive picture would be presented, an ' $O$ ' signified that a neutral picture would be presented, and a '?' indicated that either could be presented (half were followed by aversive and half were followed by neutral pictures). Subjects were instructed about all cue-picture pairings prior to scanning.

Results: Group differences were observed for genotype effects on anticipatory amygdala activity preceding both aversive and neutral pictures, as indicated by a diagnostic group $\mathrm{x}$ genotype interaction in the right dorsal amygdala for a voxelwise GLM analysis ( $\mathrm{p}<0.05$, corrected). Non-psychiatric controls with at least one copy of the $S$ or LG allele showed greater anticipatory amygdala activity preceding the pictures than LA homozygotes $(\mathrm{p}=0.04)$, consistent with prior reports of amygdala responses to emotional stimuli in healthy volunteers (Hariri et al., 2002, 2005; Smolka et al., 2007; Munafo et al., 2008). GAD patients showed the opposite pattern, with LA homozygotes showing hyperactive anticipatory responses in the amygdala $(\mathrm{p}=0.001)$, suggesting that the previously reported finding of heightened anticipatory amygdala activity in GAD (Nitschke et al., 2009) may be driven by LA homozygotes. Among LA homozygotes, GAD patients had more amygdala activity than controls $(\mathrm{p}=0.03)$. Among S/LG carriers, controls had more amygdala activity than GAD patients $(p=0.02)$. There were no other group, genotype, or interaction effects for anticipatory amygdala activity or for amygdala responses to the pictures. In addition, anticipatory amygdala hypoactivity in patients who were S/LG carriers was associated with increased depressive symptoms, as measured by the Ham-D $(\mathrm{r}=-0.51, \mathrm{p}=0.02)$. Discussion: Serotonin transporter genotype had opposite effects on anticipatory amygdala activity in patients with GAD as compared to healthy individuals. Patients with two LA alleles exhibited greater anticipatory amygdala activity preceding aversive and neutral pictures than patients with at least one copy of the S or LG allele, similar to recent findings in adolescents diagnosed with anxiety and depressive disorders (Lau et al., 2009). Conversely, healthy control participants who were S/LG carriers had more anticipatory amygdala activity than LA homozygotes, consistent with amygdala responses to emotional stimuli shown in numerous studies using nonpsychiatric samples (Hariri et al., 2002, 2005; Smolka et al., 2007; Munafo et al., 2008). In addition, these findings suggest that genetic variation associated with relatively greater serotonin signaling may be a contributing factor for the heightened anticipatory amygdala activity found in an independent sample of GAD patients (Nitschke et al., 2009). In sum, these findings link the serotonin transporter gene to anticipatory abnormalities in $\mathrm{GAD}$ and further suggest that genetic influences on amygdala activity are not uniform across psychiatric and healthy populations.

Disclosure: J. Nitschke: None. D. Oathes: None. L. Hilt: None. D. Grupe: None.

\section{Serotonin Regulates Delayed Reward Discounting via the Modulation of Striatum in Humans -from fMRI Studies After Acute Tryptophan Depletion and Loading \\ Yasumasa OKamoto*, Go Okada, Yoshihiko Kunisato, Yoshimura Shinpei, Saori Tanaka, Nicolas Schweighofer, Kenji Doya, Shigeto Yamawaki}

\section{Hiroshima University, Hiroshima, Japan}

Background: When hungry and looking for a restaurant, do you choose a well-reputed restaurant with many people waiting in line or a fast food restaurant where you can have a quick but perhaps less palatable meal? In our daily life, we constantly make such choices between actions leading to rewards of various sizes after different delays. "Delay discounting" is a theoretical concept in which the "value" of reward R after delay $D: V=R * G(D)$, where $R$ is the reward, and $G(D)$ is a discounting function that decreases with delay D. A steep rate of discounting results in impulsive choice, defined by an abnormally frequent choice of the more immediate reward. Serotonin (5-HT), a major ascending neuromodulator, is thought to be involved in temporal discounting. Previous studies have reported that decreased serotonin levels resulted in impulsive choices and that increased serotonin levels decreased impulsive choices. Lesions of specific parts of the cortico-basal ganglia loop also affect temporal discounting, whereas lesions in the orbitofrontal cortex promoted or reduced impulsive choices, and lesions in the dorsolateral part (core) of the nucleus accumbens resulted in impulsive choices. In our previous brain imaging study, we demonstrated differential involvement of the cortico-basal ganglia loops in impulsivity (Tanaka et al., 2004). Here, we hypothesized that central serotonin level has an effect on delayed reward discounting via the modulation of the cortico-basal ganglia loops in humans.

Methods: We examined the effects of three different dietary tryptophan levels on the prediction of delayed rewards in a counterbalanced, placebo-controlled, double-blind, within-subject study. Male subjects participated in experiments on three days with a minimum interval between experimental days of one week. Each day, a subject consumed one of three amino acid drinks: one containing a standard amount of tryptophan, one containing excess tryptophan, and one without tryptophan. Under each tryptophan condition, all subjects performed a multi-step delayed reward choice task. In this task, subjects chose between a white square indicating a small reward and a yellow square indicating a large reward. At the beginning of each trial, the white and yellow squares, occluded by variable numbers of black patches, were displayed side by side on a screen. After the fixation cross turned red, the subject selected either the white or yellow square by pressing a button on the corresponding side. The next set of squares was displayed 2.5 seconds after the previous one with a number of black patches removed from the selected square. When either square was completely exposed, a reward was delivered to complete the trial. A 1.5 Tesla scanner (Shimadzu-Marconi, MAGNEX ECLIPSE, Japan) was used to acquire both structural T1-weighted images and $\mathrm{T}_{2}{ }^{*}$ weighted echo planar images with BOLD contrast. We performed model-based data analyses based on an exponential discounting model which was supported in our previous delay discounting choice task (Tanaka et al., 2004). By using a computational model analysis, we estimated the discount factor $\gamma$ for each subject in each condition. We used SPM2 for preprocessing and statistical analyses.

Results: From the behavioral data, we found an increase of proportion in small reward choices, together with an increase in the rate of discounting of delayed rewards in the low-serotonin condition compared with the control and high-serotonin conditions. From neuroimaging data, a model-based analysis revealed that the activity of the ventral part of the striatum was correlated with reward prediction at shorter time scales, and this correlated activity was stronger at low serotonin levels. By contrast, the activity of the dorsal part of the striatum was correlated with reward prediction at longer time scales, and this correlated activity was stronger at high serotonin levels.

Discussion: The combined results suggest that serotonin may adjust the rate of delayed reward discounting via the modulation of striatum in corticobasal ganglia circuits. Our proposed role of serotonin may explain certain aspects of impulsivity in psychiatric disorders such as major depression, panic disorder or obsessive-compulsive disorder, that are known to effectively relieve symptoms by selective serotonin reuptake inhibitors. Future experiments using delayed reward paradigms could be designed to study impulsivity in these patients.

Disclosure: Y. OKamoto: None. G. Okada: None. Y. Kunisato: None. Y. Shinpei: None. S. Tanaka: None. N. Schweighofer: None. K. Doya: None. S. Yamawaki: None.

\section{Dissociable Patterns of Abnormal Cingulo-frontal-parietal Activity During Response Inhibition and Motor Control in Depressed Adolescents with and without a History of Suicidal Behavior Lisa Pan*, Silvia Batezati, Jorge Almeida, AnnaMaria Tomlanovich, Dalila Akkal, Stefanie Hassel, Sara Lakdawala, David Brent, Mary Phillips}

University of Pittsburgh, Pittsburgh, PA

Background: Impaired attentional and behavioral control are implicated in adult suicidal behavior. Little is known about the functional 
integrity of cingulo-fronto-parietal neural circuitry supporting these processes in suicidal behavior in adolescence.

Method: We employed fMRI in 15 adolescent suicide attempters with history of major depressive disorder (MDD; ATT), 15 adolescents with history of MDD but not suicide attempt (NAT), and 14 healthy controls (HC), during performance of a well-validated GoNoGo response inhibition and motor control task that measures attentional and behavioral control. Questionnaires assessed symptoms and standardized interviews characterized suicide attempt.

Results: A 3 group x 2 condition wholebrain analysis $(p<0.05$, corrected) revealed that NAT, but not ATT, showed significantly greater activity than $\mathrm{HC}$ in left-sided dorsolateral prefrontal cortex, inferior parietal cortex and posterior cingulate gyrus $(p=0.006)$ to GoNoGo response inhibition blocks. Both ATT and NAT showed significantly reduced activity than $\mathrm{HC}$ in right-sided premotor/ prefrontal cortex to Go, motor control, blocks $(\mathrm{p} \leq \mathrm{0.013})$. A parallel 2 group (ATT, NAT) by condition wholebrain analysis $(\mathrm{p}<0.05$, corrected), covarying for depression, anxiety, and age, revealed that ATT showed significantly reduced activity than NAT to GoNoGo response inhibition blocks in bilateral anterior cingulate gyri $(\mathrm{p}<0.025)$ and bilateral posterior cingulate gyri $(\mathrm{p}<0.015)$.

Discussion: ATT and NAT show differential patterns of neural activity during response inhibition. During adolescence, a failure to show elevated left-sided cingulo-frontal-parietal activity during response inhibition may represent a potential biological marker for suicide risk, distinct from that for MDD.

Disclosure: L. Pan: None. S. Batezati: None. J. Almeida: None. A. Tomlanovich: None. D. Akkal: None. S. Hassel: None. S. Lakdawala: None. D. Brent: None. M. Phillips: None.

126. High-dose Olanzapine/fluoxetine (O/F) Combination Therapy for Treatment-resistant Depression (TRD) Decreases Ventromedial Prefrontal and Right Amygdala Metabolism: Relationship to Clinical Response

Jose V. Pardo*, Graeme C. Schwindt, Sohail A. Sheikh, Christa Surerus, David Adson, Barry Rittberg, Faruk S. Abuzzahab, Scott M. Lewis, S. Hossein Fatemi

University of Minnesota \& VAMC, Minneapolis, MN

Background: Few studies have examined the brain effects of pharmacotherapy of TRD and their relationship to clinical response. This study examined regional cerebral glucose uptake before and six weeks after high-dose $\mathrm{O} / \mathrm{F}$ and its relationship to clinical response. Based on the published literature of depression responsive to drugs, the amygdala and ventromedial prefrontal cortex (VMPFC) were key regions of interest.

Methods: Nine TRD patients with numerous failed trials of various antidepressant therapies were scanned once during a drug-free phase and again after 8 weeks of O/F therapy (10-12 mg olanzapine and 30$45 \mathrm{mg}$ fluoxetine per day). Patients were considered responsive with a $50 \%$ decline in MADRS score. ${ }^{18} \mathrm{~F}$-FDG uptake was measured using a Siemens ECAT EXACT. Images were reconstructed using filtered-back projection to a final resolution of $12 \mathrm{~mm}$ FWHM. After whole-brain normalization and stereotactic warping to the Talairach \& Tournoux ('88) atlas using Neurostat (provided by Satoshi Minoshima, University of Washington), the data were analyzed voxel-wise (uncorrected threshold $\mathrm{P}<0.001$, typical for PET data at this resolution) and with regions of interest (ROI) including the amygdala, circumscribed BA 25, and a broader VMPFC region including portions of BA 32 and 33 .

Results: As expected, 2 X 2 ANOVA with a between-subjects factor of RESPONSE [responder (4) vs. non-responder (5)] and within-subject factor of TIME (pre- vs. post- trial) showed a significant main effect of TIME $[\mathrm{F}(1,7)=195.2, \mathrm{P}<0.001]$ and a RESPONSE $\mathrm{x}$ TIME interaction $[\mathrm{F}(1,7)=43.9, \mathrm{p}<0.001]$. From voxel-wise analysis, the only significant reduction in metabolism with treatment in responders localized to the right amygdala; increases surfaced in the left superior temporal gyrus, post-central gyrus, and the right precuneus. Non-responders showed no significant increases in metabolism; significant decreases localized to middle temporal gyrus, dorsal cingulate, lingual gyrus, thalamus, midbrain, and bilateral cerebellum. ROI analysis showed metabolic reduction in the right amygdala correlated positively $(\mathrm{r}=0.70, \mathrm{p}=0.03)$ with reduction in MADRS in the responders but not in the non-responders. In other words, successful treatment with $\mathrm{O} / \mathrm{F}$ was associated with reduced metabolism in the right amygdala. The BA 25 ROIs showed no significant changes in either group nor correlations with change in MADRS score. However, greater pretreatment right BA 25 metabolism (but not in left BA 25) predicted better response to $\mathrm{O} / \mathrm{F}$ therapy $(\mathrm{r}=0.68, \mathrm{p}=0.05)$. The broader right VMPFC (but not left VMPFC) in responders showed a significant decrease in metabolism $[\mathrm{t}(3)=4.18$, po.02] with treatment. Also, right VMPFC (but not left) pre-treatment metabolism correlated with greater MADRS score reduction after treatment $(r=0.83, p=0.01)$. In other words, the greater the right VMPFC activity at baseline, the greater the likelihood of responding.

Discussion: Successful O/F therapy in TRD produced metabolic changes similar to those previously reported in depression responsive to antidepressants: reduction of amygdala and VMPFC metabolism. These changes localized only to the right hemisphere, suggesting a remarkable degree of hemispheric asymmetry in these circuits. Examining pre-treatment predictors of response to O/F in TRD, greater activity in right BA 25 and right VMPFC predicted greater reductions in MADRS scores after treatment. It should be emphasized that this study was not designed to look for treatment effectiveness; it addressed only brain changes related to treatment. It had no placebo group. Despite the small sample size, robust statistical effects arose. Future work with larger samples should confirm these preliminary data and examine differences in functional connectivity in responders vs. non-responders before and after $\mathrm{O} / \mathrm{F}$ treatment to elucidate how treatment response differs between TRD and non-TRD.

Disclosure: J. Pardo: Part 4; Eli Lilly \& Co. G. Schwindt: None. S. Sheikh: Eli Lilly \& Co. C. Surerus: None. D. Adson: None. B. Rittberg: None. F. Abuzzahab: None. S. Lewis: None. S. Fatemi: None.

127. Lamotrigine Treatment Improves Prefrontal Activity in Pediatric Bipolar Disorder

Alessandra Passarotti*, John Sweeney, Mani Pavuluri

University of Illinois at Chicago, Chicago, IL

Background: This fMRI study examines the impact of 14-week lamotrigine treatment on the neural interface of working memory and affect in pediatric bipolar disorder (PBD). This is an important issue because working memory is key to daily activities at home and school and children with PBD constantly face emotional challenge together with working memory deficits. Recent research evidence indicates that along with emotional dysregulation PBD exhibits frontostriatal-parietal dysfunction (Passarotti et al., 2009; in press) that is related to persistent working memory deficits (Pavuluri et al., 2010). An affective fMRI n-back task was employed to characterize the neural circuitry at the interface of working memory and affect. We predicted that, together with symptom improvement, baseline dysfunction in fronto-limbic and fronto-striatal circuits in PBD relative to $\mathrm{HC}$ would improve with lamotrigine treatment.

Methods: Participants. Seventeen acutely ill and un-medicated patients with bipolar disorder Type I $(\mathrm{n}=8)$, Type II $(\mathrm{n}=8)$, and NOS $(\mathrm{n}=1)$ and 13 HC subjects, all matched for IQ and demographic measures. Task and fMRI session. Participants underwent a 7-min fMRI 2-back working memory task. On each trial a face stimulus (i.e., happy, angry or neutral face) was presented for $3 \mathrm{sec}$ and subjects responded by keypress if they saw the same face that was presented two trials earlier. Blocks of angry and neutral faces were alternated with blocks of happy and neutral faces according to a pseudorandom sequence. A 20 sec fixation in-between block was included to allow for emotional arousal to return to baseline. fMRI data acquisitions. We performed 


\section{S358}

gradient-echo echo-planar imaging (EPI) using a 3 Tesla GE scanner (25 axial slices; $\mathrm{TE}=25 \mathrm{~ms}$; flip angle $=90^{\circ}$; field of view $=20 \times 20 \mathrm{~cm} 2$; acquisition matrix $=64 \times 64 ; \mathrm{TR}=2.5 \mathrm{~s}$; slice thickness $=5 \mathrm{~mm}$ with $1 \mathrm{~mm}$ gap). Anatomical acquisitions were later co-registered with the functional data. fMRI Data Analyses. FIASCO software corrected for motion artifacts. Individual zr maps were obtained by contrasting activation for angry versus neutral as well as for happy versus neutral emotion. They were then imported in AFNI (Cox, 1996) and warped into Talairach space. A significant three-way interaction $[\mathrm{F}(1,28)=5.57$, $\mathrm{p}=.025]$ obtained from a whole-brain ANOVA was followed by t-tests on significant clusters from the interaction to elucidate group differences. AlphaSim (Ward, 2000) was used to correct for multiple comparisons and determine corrected significance levels $(p<.03)$.

Results: Clinical and Behavioral results: For PBD YMRS and CDRS scores improved significantly from baseline to follow-up $(p<.001)$. With regard to behavioral performance, the PBD group had significantly lower performance accuracy than HC $(\mathrm{p}<.004)$; RT was significantly faster at follow-up compared to baseline in both groups $(\mathrm{p}<.02)$. fMRI results: Group differences at Baseline. For the angry vs neutral face condition at baseline PBD exhibited reduced activation relative to HC in left ventrolateral prefrontal cortex (VLPFC), a key region for emotion regulation, and in right caudate, a region involved in working memory function. For the happy vs neutral face condition, PBD exhibited increased activity relative to $\mathrm{HC}$ in right amygdala, in right inferior frontal gyrus (IFG) and left VLPFC and in bilateral dorsolateral prefrontal cortex (DLPFC), a region involved in attention and working memory effort, as well as in left medial PFC and middle temporal gyrus, two regions involved in affect evaluation and face emotion processing, respectively. Group differences at Follow-up. At follow-up, for the angry vs neutral face condition PBD exhibited greater activation than HC in right amygdala and parahippocapal gyrus, and reduced activation in right temporoparietal regions. For happy vs neutral faces, PBD exhibited greater activation than HC in left amygdala and right middle temporal gyrus. Group differences at Follow-up vs Baseline. The PBD group showed greater changes over time than $\mathrm{HC}$ in ventromedial prefrontal (VMPFC), limbic and striatal regions involved in affect regulation and cognition for angry vs neutral faces, and in middle temporal gyrus for happy vs neutral faces.

Discussion: In summary, after treatment PBD showed improved clinical symptoms and enhanced functioning in fronto-striatal and fronto-limbic regions involved in emotion regulation and working memory. Amygdala hyper-activation persisted relative to HC. Our findings provide new evidence that lamotrigine treatment in PBD significantly improves not only clinical symptoms but also the dysfunctional interface of affect and working memory.

Disclosure: A. Passarotti: None. J. Sweeney: Part 1; Pfizer consultant. Part 4; Janssen research grant. M. Pavuluri: Part 1; Speakers Bureau: Bristol Myers Squibb, MC Neil.

128. Altered Error Processing in the Caudate Predicts Subsequent Emergence of Problem use with Amphetamine-type Stimulants in an At-risk Group of Stimulant Users

Martin Paulus*, Martina Reske, Jennifer Stewart, Taru Flagan, Susan Tapert

University of California San Diego, La Jolla, CA

Background: Amphetamine type stimulants are emerging as one of the most important groups of drugs of abuse world-wide. These drugs are used widely for enhancing performance but little is known about the factors that contribute to the risk of developing amphetamine abuse or dependence. We have previously shown that individuals who are using stimulants but do not have stimulant dependence show subtle neural processing abnormalities during error-related decision-making. This study examined a large cohort of individuals, who were followed up for three years to determine whether subtle neural processing abnormalities predict the emergence of problem use.
Methods: $\mathrm{n}=74$ stimulant using subjects were followed up over a three year time period. Each individual underwent a structured clinical interview in person or over the phone to determine diagnostic status at follow up. Moreover, detail time life follow back records were generated to quantify subsequent drug use. Based on the presence of abuse or dependence criteria during the follow up, individuals were categorized into problem and non-problem users as well as desisters, i.e. those individuals who did not use stimulants during the follow up period. These categorizations were used in a linear mixed effects model to determine brain activation differences at baseline during a simple two-choice prediction task. At baseline, each subject completed a fast event-related fMRI design of the two-choice prediction task with three error rate conditions $(20 \%, 50 \%$ or $80 \%$ errors) using an event-related fMRI paradigm. Subject-specific regressors were generated using the individual's response latency. Moreover, regressors were generated for the decision phase, i.e. the beginning of the trial until the subject made a choice and for the outcome phase, i.e. from the presentation of the result until the end of the trial. The resulting regressors were convolved with a hemodynamic response function and used to obtain estimates of the brain response to the different error rate conditions. Results: Relative to those individuals who did not develop problems during the three year follow-up period, problem users show altered brain activation - at baseline - in the caudate and the prefrontal cortex as a function of error-rate during the decision-phase. Specifically, problem users exhibited an exaggerated response to low error rates. These findings were specific for amphetamine-type stimulants and did not characterize individuals who were using predominately cocaine.

Discussion: This longitudinal follow up study provides the first evidence of altered brain processing underlying the risk for developing problem use with amphetamine type stimulants. The brain structures involved are critical for reward and error processing. Thus, individuals who use stimulants and who have problems processing correct and incorrect decisions properly may be at an increased risk for developing amphetamine abuse or dependence.

Disclosure: M. Paulus: None. M. Reske: None. J. Stewart: None. T. Flagan: None. S. Tapert: None.

129. This poster has been cancelled. 
longitudinally consistent with the reformatting to MRI-1. Gray matter/ white matter/CSF were segmented in both MRI-1 and MRI-2 SPGR using FSL FAST with tissue priors based also on the SRI24 atlas. Tissue volumes derived from the segmentations were quantified for the following brain regions: gray matter volumes for 7 cortical regions, tissue volumes (gray + white matter) for 10 subcortical and brain stem structures, and CSF volumes for ventricular regions and cortical sulci. For analysis, regional volumes were expressed as a percentage of change between MRIs for each subject.

Results: The supratentorial volume increased $0.9 \% \quad(\mathrm{p}<.0001)$. Cortical gray matter volumes declined significantly $(\mathrm{p} \leq .01)$ in anterior (lateral and medial frontal, precuneus, and parietal) but not posterior (occipital, calcarine) cortical regions. Although the anterior cingulate cortex declined in volume, the posterior cingulate, insular, and hippocampal-amygdala volumes did not change significantly. In contrast to the cortex, the caudate-putamen and thalamus expanded, but neither the globus pallidus nor cerebellum showed reliable changes over this $\sim 6$-month interval. All ventricular and sulcal volumes measured increased, with the largest percentage increases observed in cortical sulci. Although the average supratentorial volume of the boys was larger than that of the girls at both MRIs, observed changes in percentage volume over the 6-month interval were not related to sex, BMI, or Tanner Stage.

Discussion: These data present evidence that longitudinal MRI assessment is sufficiently sensitive to identify significant regional brain changes over even relatively short periods in boys and girls in early adolescence. Decline in anterior cortical volume was on average about $2 \%$, selected subcortical volume increase was about $1 \%$, and the patterns of change differed by brain region. Specifically, developmental changes were greater in anterior than posterior brain regions, suggesting remodeling of brain structure towards maturity, with the possible interpretation that frontal regions exhibit more active synaptic pruning than posterior regions during early adolescence. The active changes in anterior neocortex and allocortex may well parallel known developmental changes that occur in executive functions, including attention, behavioral regulation, judgment, and problem solving.

Support: AA016273, AA005965, AA017168, EBoo8381.

Disclosure: A. Pfefferbaum: None. T. Rohlfing: None. M. Padilla: None. E. Sullivan: None. I. Colrain: None.

Background: Early adolescence is a time of rapid change in anatomy, physiology, and sexual development. It is also the beginning of the age of risk for self-harmful behaviors, such as alcohol use and abuse. There are many unanswered questions about the effect of alcohol and other potentially harmful substances or events on the developing brain that can only be answered with longitudinal observation. The current study was designed to quantify regional brain structural development over a 6- to 12 -month period in normal, nonalcohol-using, early adolescent boys and girls.

Methods: The participants were recruited through local schools. Parents and children provided written permission to participate in this study. Participants scanned twice (average interval $=6.4$ months, range $=4-16$ months) and with adequate data for analysis were 17 boys (baseline age $=12.8 \pm .9,10.9$ to 13.9 years; Tanner Stage $=3.4$, range $=3$ to 4 ; grade $=7.1$, range $=5$ to 8 ) and 12 girls (baseline age $=12.4 \pm .7,11.2$ to 13.7 years; Tanner Stage $=2.3$, range $=1$ to 4 ; grade $=6.9,6$ to 8 ). MR data were collected on a GE 3 T Signa scanner with an 8-channel phased-array head coil. Brain volumes were derived from T1-weighted Inversion-Recovery Prepared SPoiled Gradient Recalled (SPGR) images $(\mathrm{TR}=7 \mathrm{~ms}, \mathrm{TE}=2.2 \mathrm{~ms}, \mathrm{TI}=300 \mathrm{~ms}$, Thick $=1.2 \mathrm{~mm}, 124$ Slices) and dual-echo Fast Spin-Echo (FSE) images $(\mathrm{TR}=8583 \mathrm{~ms}, \mathrm{TE}=13.5 / 108.3$, Thick $=2.5 \mathrm{~mm}, 62$ Slices $)$. Brain data analysis was conducted by registration-based parcellation. Each subject's MRI-2 SPGR was registered to that subject's MRI-1 SPGR data, again with nonrigid registration. After removal of non-brain signal using both the FSE and SPGR data, the SRI24 atlas was registered to each subject's MRI-1 SPGR brain data, again with nonrigid registration. The parcellated SRI24 atlas was then reformatted into native space for both MRI-1 and MRI-2 data sets. The MRI-1-toSRI24 and MRI-2-to-MRI-1 transformations were concatenated for a single reformatting operation of the atlas to MRI-2, which was

131. Surface Morphology of the Basal Ganglia Across the Life Span Kerstin J. Plessen*, Ravi Bansal, Juan Sanchez, Bradley Peterson

Columbia University, New York, NY

Background: Disturbances in the basal ganglia (BG) portions of cortico-striato-thalamo-cortical circuits likely contribute to the development of symptoms in several developmental neuropsychiatric disorders, such as attention-deficit/hyperactivity disorder, obsessivecompulsive disorder, eating disorder, and Tourette syndrome. We examined the morphologic features of the BG nuclei (caudate, putamen, and globus pallidus) in normally developing individuals to understand age-and gender related changes in these regions across lifespan. Although BG nuclei mediate the gross motor, cognitive, and emotional functions, data concerning its age-related changes across the human life span are scarce.

Methods: A total of 135 normally developing individuals, aged 7 to 79 years, were examined in a cross-sectional study using anatomical magnetic resonance imaging (MRI). Conventional volumes and the surface morphology for each BG nuclei were measured. Briefly, we used a rigid-body similarity transformation to register each subject brain to a template brain. The estimated parameters of the whole brain were applied to adjust regional volumes to overall brain size (global scaling) and transform the subject nuclei into the template space. The transformed BG nuclei for every participant were then individually and rigidly coregistered to the corresponding $\mathrm{BG}$ nuclei of the template brain to refine their registrations. In a last step, the nuclei were warped to the corresponding nuclei of the template brain using 


\section{S360}

a high-dimensional, nonrigid warping algorithm based on the dynamics of fluid flow. This detailed warping of the BG nuclei to the corresponding template nuclei precisely identified corresponding points (voxels) on the surfaces of the BG nuclei of each participant to those on the surface of the template BG nuclei. We computed the Euclidean distance between each point on the surfaces of the subject BG nuclei to the corresponding point on the surface of template BG nuclei. These distances were positive for outward deformation and negative for inward deformations in the subject surfaces as compared to the template surface. The computed Euclidean distances were correlated with age, quadratic age, and gender in each BG nuclei.

Results: Significant inverse correlations (P-value $<0.0001)$ of striatal surface distances (local volumes) with age were present bilaterally in the caudate nuclei and putamen. The inverse correlations were more prominent in the left caudate and right putamen. In the younger age group (under 22 years), however, we detected one localized region bilaterally with positive correlations within the dorsal part of the caudate nucleus, indicating local volume increases in that portion of caudate nuclei with age. Surface distances in the globus pallidus correlated positively with age, especially in anterior-medial surface and ventral-lateral surface of the globus pallidus. Inverse correlations clustered mainly in the right globus pallidus, stretching dorsally from the medial to the lateral side. Male participants, as compared to females, had more local volume reductions on all subregions of the striatum. This was especially prominent on the medial surface of both caudate nuclei and at the lateral side of the right caudate nucleus, whereas it was most prominent on the left putamen (both laterally and medially). In addition, males had more local volume reductions in the lateral mid-portion of the left globus pallidus and the mid-portion of the medial surface of the right globus pallidus. The main effects of sex overlapped with the effects of the interaction term sex $\mathrm{x}$ age, but extended those latter spatially. Consistently with correlations in the striatum, males had more local volume reductions in the globus pallidus, which were localized on the dorso-lateral portion of both globus pallidus. Discussion: Our findings strongly suggest that the volume and surface morphology of BG nuclei correlate with age in healthy developing individuals. Although correlations of surface distances with age are largely inverse, suggesting progressively greater decrements in local volumes with advancing age, small clusters of positive correlations in the dorso-anterior regions of the caudate that connect to dorsolateral prefrontal regions suggest that fronto-striatal connections may strengthen with age during adolescence. Moreover, surface analyses indicate that males compared to female participants have smaller local volumes in all BG nuclei. Mapping the correlations of surface with age and gender in healthy individuals will help to understand its deviations in children and adults with neuropsychiatric disorders.

Disclosure: K. Plessen: None. R. Bansal: None. J. Sanchez: None. B. Peterson: None.

132. Responses of IL-1 Family Cytokines to Experimental Pain and Relationships to Sex, Neuroticism and Opioid Receptor Gene Variants Alan Prossin*, Steven Zalcman, Alisa Koch, Phillip Campbell, Jon-Kar Zubieta

\section{University of Michigan, Ann Arbor, MI}

Background: Persistent pain represents a physical and emotional stressor with substantial morbidity, frequently presenting with co-occurring disorders. Genetic variants, female gender, pain catastrophizing and cooccurring conditions, such as the mood and anxiety disorders, have been associated with increased pain report and the development or maintenance of chronicity. However, current knowledge is limited regarding the interaction between these factors and objective measures of inflammation, such as peripheral levels of pro-inflammatory cytokines and the balance between pro- and anti-inflammatory factors. A potentially important factor that may contribute to the development of persistent inflammatory states is the response of the pro-inflammatory interleukin-1 (IL-1) family cytokines to stressful challenges. These cytokines have been investigated extensively in animal models for their interaction with peripheral and central opioid mechanisms (i.e., $\beta$-endorphin and mu-opioid receptors). IL-1 $\beta$ has been shown to have pro-nociceptive effects and IL-1 receptor antagonist (RA) to antagonize those effects. In addition, the IL-1 family of cytokines has been associated with opiate tolerance that frequently complicates pharmacological treatment of persistent pain states. Evidence relating IL-1 family cytokines to mood, pain, and central mu-opioid receptor measurements in humans is currently lacking, albeit supported by animal studies. Of further significance, it is not known if such interactions are sexually dimorphic. We examined the relationship between the response of IL-1 family cytokines and factors potentially associated with risk for chronicity.

Methods: Mu-opioid receptor availability centrally was quantified with $[11 \mathrm{C}]$ carfentanil, a mu-opioid selective radiotracer, and positron emission tomography (PET) in 36 healthy males and females during a sustained experimental pain challenge ( $5 \%$ saline intramuscularly over $20 \mathrm{~min}$ ) and a control condition ( $0.9 \%$ saline). IL-1 $\beta$ and IL-1RA concentrations were estimated using multiplexed ELISA techniques with commercially available kits (R\&D Systems, MN). The ratio of IL-1 $\beta$ to IL-1RA was used to determine the balance between pro- and antiinflammatory factors (IL-1 balance) as proposed in previous preclinical studies. Using independent samples t-tests we tested whether IL-1 cytokines differed between each of the following groups: male-female, low-high neuroticism, presence or absence of G-allele of the A118G polymorphism of the mu-opioid receptor gene. We then used SPM2 (Wellcome Trust, London, England) software and the simple regression model to determine if the IL-1 family of cytokines was associated with mu-opioid receptor binding potential (as determined via PET).

Results: Significantly greater plasma levels of IL-1 family cytokines were observed in the female sample during the control condition (IL-1 $\beta: t=6.07, p=0.000 ;$ IL-1RA: $t=3.83, p=0.001$; IL-1 balance: $\mathrm{t}=-1.97, \mathrm{p}=0.057)$ and during experimental pain (IL-1 $\beta: \mathrm{t}=5.31$, $\mathrm{p}=0.000$; IL-1RA: $\mathrm{t}=3.89, \mathrm{p}=0.000$ ). During the control condition, the IL-1 balance (i.e. IL-1 $\beta /$ IL-1RA) was positively associated with pain report $(\mathrm{r}=0.43, \mathrm{p}=0.01)$. Volunteers scoring in the upper median of the general population in Neuroticism scores showed significant increases in IL-1 $\beta(t=2.11, p=0.044)$ and marginally significant increases in IL-1RA $(t=1.87, p=0.072)$ in response to pain. The presence of the G-allele of the A118G polymorphism of the $\mu$-opioid receptor gene was associated with a greater IL-1 $\beta / \mathrm{IL}-1 \mathrm{RA}$ ratio $(\mathrm{t}=2.60, \mathrm{p}=0.014)$. Using a voxel-by-voxel regression analysis in SPM2, IL-1 balance correlated with $\mu$-opioid receptor availability at baseline in the left amygdala $(z=5.3, p<0.05$ after correction for multiple comparisons) and medial thalamus bilaterally (left: $\mathrm{z}=3.7$ $\mathrm{p}<0.05$ corrected; Right: $\mathrm{z}=3.0$ ), regions involved in the central regulation of pain and stress responses.

Discussion: The findings presented demonstrate effects of sex, a common genetic polymorphism of the mu-opioid receptor gene associated with alterations in central and peripheral opioid function and neuroticism, a risk factor for the development of mood and anxiety disorders, on pro- and anti-inflammatory cytokines responses to an experimental sustained pain challenge. Further we show a relationship between peripheral cytokines and central mu-opioid receptor measures, one of the principal antinociceptive mechanisms also involved in emotion regulation. Further examination of these processes in idiopathic pain states (e.g., fibromyalgia, temporomandibular pain) appears warranted.

Disclosure: A. Prossin: None. S. Zalcman: None. A. Koch: None. P. Campbell: None. J. Zubieta: None.

133. Neural Correlates of PTSD Symptom Dimensions During Emotional Processing: a Functional Magnetic Resonance Imaging Study Isabelle Rosso, William Killgore, Jennifer Britton, Melissa Weiner, Zachary Schwab, Scott Rauch ${ }^{\star}$

McLean Hospital, Belmont, MA

Background: The current Diagnostic and Statistical Manual (DSM-IV) defines post-traumatic stress disorder (PTSD) as a multifaceted 
psychiatric disorder that consists of three symptom clusters: re-experiencing, avoidance, and hyperarousal. Functional neuroimaging research suggests that both the categorical diagnosis of PTSD and its overall symptom severity are associated with heightened subcortical limbic, especially amygdala, activity and with diminished medial prefrontal activity in response to threatening stimuli. It is unclear, however, whether the different symptom dimensions of PTSD map onto similar versus distinct neural correlates.

Methods: Participants were 14 adults (4M:9F; $33 \pm 12$ years of age) who met criteria for PTSD based on the Structured Clinical Interview for DSM-IV. Symptom severity was assessed using the ClinicianAdministered PTSD Scale (CAPS). No participant was taking psychotropic medications at the time of study. Subjects underwent functional magnetic resonance imaging (fMRI) on a 3 Tesla Siemens whole-body scanner during a backward-masked emotional face perception paradigm. Subjects viewed alternating blocks of happy, fearful, and neutral faces (16 msec affective face followed by $184 \mathrm{msec}$ neutral face mask). Two runs of $5 \mathrm{~min} 36 \mathrm{sec}$ were completed, their order counterbalanced across subjects. The relationships between CAPS scores, total and subscale, and activation maps for the masked fear versus neutral contrast were evaluated using multiple regression within $\mathrm{SPM}_{5}$, with the whole brain intensity threshold set to $\mathrm{p}<.005$, and extent $(\mathrm{k})$ of at least 5 contiguous voxels.

Results: Total CAPS scores were significantly positively correlated with activation for fearful compared with neutral faces in the right amygdala and left insula, and significantly negatively correlated with activation in bilateral ventromedial prefrontal cortex (VMPC). Examination of symptom dimensions revealed that hyperarousal symptoms also correlated positively with right amygdala activation and negatively with right VMPFC activation, in addition to showing a positive correlation with left lateral orbital PFC activation. In contrast, re-experiencing symptoms showed a negative relationship with activation in the right amygdala and left insula, and a positive correlation with activation in the right VMPFC. Avoidance symptoms did not show any significant correlations with activation for fearful versus neutral faces.

Discussion: We found that hyper-arousal and re-experiencing symptoms of PTSD were associated with differential, and somewhat opposite, patterns of limbic-prefrontal neural activation during the processing of fearful faces. Specifically, hyper-arousal symptoms predicted increased limbic and decreased ventromedial prefrontal activation, whereas re-experiencing symptoms were associated with diminished limbic and heightened ventromedial prefrontal activation. These results suggest the importance of considering dimensional measures of PTSD psychopathology to parse its corresponding neurobiology.

Disclosure: I. Rosso: None. W. Killgore: None. J. Britton: None. M. Weiner: None. Z. Schwab: None. S. Rauch: Part 1; Medtronics Neurocircuitry, Baystate Medical, APPI, Oxford Press, Wolters Kluwer, ACNP. Part 2; None other than primary employer: McLean Hospital/ Partners Healthcare Inc. Part 3; None other than primary employer: McLean Hospital/Partners Healthcare Inc. Part 4; Cyberonics, Medtronic, Northstar Neuroscience.

134. OPRM11 A118G Genotype Predicts Mu Opioid Receptor Binding Potential in Healthy Subjects and Smokers

Riju Ray*, Andrew Newberg, Kosha Ruparel, Kanchana Jagannathan, James Loughead, Chaitanya Divgi, Jon Kar Zubieta, Julie Blendy, Caryn Lerman

University of Pennsylvania, Philadelphia, PA

Background: The OPRM1 A118G polymorphism is associated with multiple substance abuse phenotypes, including nicotine addiction. The minor $(\mathrm{G})$ allele is linked with reduced mRNA expression and mu opioid receptor (MOR) protein levels. Consistent with preclinical data, smokers who carry the $G$ allele exhibit reduced nicotine reward, decreased withdrawal severity, and greater ease of quitting smoking.
Clarifying whether this functional variant alters MOR binding in healthy subjects and smokers is a critical step toward elucidating the neurobehavioral mechanisms mediating genotype effects with substance abuse.

Methods: To examine the association of OPRM1 A118G with MOR binding potential in vivo, we conducted two experiments using $[11 \mathrm{C}]$ carfentanil positron emission tomography (PET) imaging. The first experiment included 20 non-smoking healthy subjects of European ancestry (10 AA; $10^{*} \mathrm{G}$, matched for sex) and the second experiment included 24 current smokers of European ancestry (12 AA and $12{ }^{*} \mathrm{G}$ matched for sex). To standardize nicotine exposure for experiment 2, all subjects abstained from smoking overnight, and smoked a standardized research cigarette approximately 10 minutes prior to the PET scan. Parametric maps of the binding potential (BPND) were obtained from the spatially normalized 30-50 min average images using tissue ratio method. The occipital cortex was used as the reference region. The BP images were smoothed by a Gaussian kernel (8-mm FWHM) and entered into whole brain voxelwise statistical parametric analysis using $\mathrm{SPM}_{5}$. The resulting SPM $\{\mathrm{T}\}$ maps were corrected for multiple comparisons using a cluster level correction at $\mathrm{p}=0.001$ threshold. Regional MOR BP levels were calculated from significant clusters.

Results: The voxel-based two sample $\mathrm{T}$ test analysis with SPM8 confirmed a genotype effect in the healthy subjects, with higher MOR BP $\left(A A>{ }^{*} \mathrm{G}\right.$ ) in cingulate gyrus (BA 24) and precentral gyrus (BA 4). In the smoker sample, higher $\mathrm{MOR} B P\left(A A>{ }^{*} \mathrm{G}\right)$ was observed in anterior cingulate gyrus (BA 32), cingulate gyrus (BA 24), superior temporal gyrus (BA 21), right caudate, insula (BA 13), thalamus and right medial frontal gyrus (BA 6). There were no regions in which MOR BP was higher in the ${ }^{*} \mathrm{G}$ group, compared to the AA group in either sample.

Discussion: These studies provide the first in vivo evidence that the reduced function OPRM1 A118G G allele is associated with reduced MOR binding potential. Common (e.g., cingulate gyrus) and unique regions for genotype effects were identified in healthy subjects and smokers, suggesting the presence of a constitutional effect as well as effects that may be modulated by nicotine exposure. The lower MOR $\mathrm{BP}$ in smokers carrying the $\mathrm{G}$ allele suggests a specific neurobehavioral mechanism through which this polymorphism may influence nicotine reward and relapse.

Disclosure: R. Ray: Part 4; Pfizer. A. Newberg: None. K. Ruparel: None. K. Jagannathan: None. J. Loughead: Pfizer, AstraZeneca. C. Divgi: None. J. Zubieta: Part 1; Eli Lilly, Merck. Part 2; Eli Lilly. Part 3; Eli Lilly. J. Blendy: None. C. Lerman: Part 1; Targacept. Part 4; GlaxoSmithKline, Pfizer, AstraZeneca.

135. COMT Val158Met Genotype and Cortical Maturation in Patients with Very Early-Onset Schizophrenia, Their Healthy Siblings and Typically Developing Children and Adolescents

Armin Raznahan*, Dede Greenstein, Yohan Lee, Bob Long, Liv Clasen, Peter Gochman, Anjene Addington, Jay Giedd, Judith Rapoport, Nitin Gogtay

\section{National Institute of Mental Health, BETHESDA, MD}

Background: Non-psychotic persons at increased risk for schizophrenia show alterations in fronto-striatal dopamine (DA) signaling and fronto-temporal cortical gray matter maturation reminiscent of those seen in schizophrenia. Abnormalities of both these phenotypes have been shown to index heritable risk for schizophrenia, and the possibility that they are inter-related is raised by evidence from animal studies that alterations of DA signaling can disrupt cortical development. It remains unclear however if genetically-determined variations in DA signaling can influence cortical maturation in typically developing humans, and if these influences are disrupted in persons at latent and manifest risk for schizophrenia. We sought to address this issue by relating a COMT val158met genetic polymorphism known to modulate DA signaling to longitudinal structural neuroimaging data 


\section{S362}

in healthy adolescent controls (HCs), probands with a severe childhood-onset form of schizophrenia (COS), and the healthy siblings (SIBs) of COS probands. Parallel study of COS probands and their SIBs allowed us to distinguish consequences of schizophrenia risk per se from the consequences of active illness. We hypothesized that (i) cortical maturation in HCs would vary according to the number of higher function Val alleles (thought to result in lower synaptic DA) within fronto-temporal brain regions relevant to schizophrenia neurobiology, (ii) COS and SIB groups would both show disruption of these typical gene-brain relationships, and (iii) this disruption would be more severe in probands with COS than their SIBs.

Methods: We included a total $7921.5 \mathrm{~T}$ structural MRI brain scans acquired longitudinally between ages 9 and 22 years from 208 HCs (475 scans), 83 COS (192 scans), and 62 of their healthy SIBs (124 scans). A fully automated and well-validated pipeline was used to generate estimates of gray matter (GM) cortical thickness at 80,000 points (vertices) on each cortical surface, We then used mixed linear models to relate age, Val allele dosage, clinical group membership, and the interaction of these terms to CT.

Results: Increasing Val allele dose attenuated the rate of GM loss amongst HCs within dorsolateral prefrontal, medial prefrontal and superior temporal cortices that are of established relevance to the biology of schizophrenia. However, these gene-brain relationships were completely inverted amongst both COS probands and their healthy SIBs, in whom increased Val allele load (proposed to aggravate cortical deficits in schizophrenia) was associated with greater GM loss in adolescence. This disruption of the normative relationship between val158met genotype and cortical maturation was maximal in dorsolateral and medial prefrontal cortices. There was also evidence that aberrant genetic regulation of cortical development was more pronounced in COS probands than their healthy SIBs. We also found fixed GM differences according to Val allele load that were inverted in HCs (reduced GM with greater Val allele load) relative SIBS (greater GM with greater Val load).

Discussion: This is the largest longitudinal schizophrenia neuroimaging study to date, and the first to provide longitudinal evidence linking genetic variants impacting dopamine signaling to GM deficits in those at latent risk for schizophrenia, as well the accentuation of these deficits in persons symptomatic of the disorder. These findings argue that disruption of the causal pathways linking variation in DA signaling to cortical maturation indexes primary risk factors for schizophrenia rather than solely resulting from the presence of active disease. This is consistent with the novel theory that abnormalities of DA signaling and cortical anatomy that have traditionally been separately considered to indicate the presence genetic risk for schizophrenia may be causally related to one another.

Disclosure: A. Raznahan: None. D. Greenstein: None. Y. Lee: None. B. Long: None. L. Clasen: None. P. Gochman: None. A. Addington: None. J. Giedd: None. J. Rapoport: None. N. Gogtay: None.

\section{Prefrontal Function during Working Memory Maintenance in Unmedicated First-Episode Schizophrenia and Psychotic Bipolar Disorder}

James Reilly, Anna-Maria D’Cruz, Sarah Keedy, Lisa Liu, Bruce McDonough, Peter Weiden, John Sweeney*

University of Illinois at Chicago, Chicago, IL, Univ. of IL Medical Center/Center for Cognitive Medicine, Chicago, IL

Background: A reduced ability to maintain information in working memory is an established neurocognitive deficit in schizophrenia. The specificity of this dysfunction to schizophrenia vs. psychotic bipolar disorder is unknown and is of interest given the appreciation for shared risk genes and neurobiological abnormalities between these disorders. In this study, we compared the neural substrate of working memory maintenance in untreated first-episode patients with these disorders.

Methods: We used event-related fMRI to compare patients with schizophrenia $(n=17)$ or psychotic bipolar disorder $(n=11)$ who were early in their course of illness and unmedicated at the time of testing to matched healthy individuals $(n=20)$ while performing an oculomotor delayed response task. This task requires maintenance of spatial location information in working memory over a 5-second delay period after which a saccade is made to the remembered location. Studies with nonhuman primates performing this task have established the relevant frontostriatal and parietal circuitry for maintaining information over delay periods. Activation among the patient and control groups during the delay period was contrasted with a passive central fixation period. Regions of interest included the middle frontal gyrus, frontal and parietal eye fields, dorsomedial thalamus and striatum.

Results: Compared to healthy individuals, schizophrenia and psychotic bipolar patients showed significant and comparable reduced activation in the middle frontal gyrus during the delay period while spatial location information was to be maintained in working memory. In contrast, only schizophrenia patients showed additional reduced activation in sensorimotor areas of frontal and parietal cortex and thalamus. No significant group differences in activation were observed in the striatum.

Discussion: Both first-episode schizophrenia and psychotic bipolar patients prior to treatment failed to activate dorsolateral prefrontal cortex while maintaining information in working memory. This finding suggests a shared prefrontal dysfunction underling working memory deficits between these disorders. For schizophrenia patients this dysfunction also included the broader thalamocortical working memory circuitry while for bipolar disorder it was restricted to prefrontal cortex.

Disclosure: J. Reilly: None. A. D'Cruz: None. S. Keedy: None. L. Liu: None. B. McDonough: None. P. Weiden: Part 1; Ortho-McNeil Janssen Scientific Affairs, AstraZeneca, Bristol-Myers Squibb/ Otsuka America Pharmaceutical, Eli Lilly, Forest, Merck, Novartis, Pfizer, Takeda, Vanda, Wyeth. Part 4; Ortho-McNeil Janssen Scientific Affairs. J. Sweeney: Part 1; Pfizer. Part 4; Ortho-McNeil Janssen Scientific Affairs.

137. The Tryptophan Hydroxylase-2 Risk Haplotype is Associated with a Differential Pattern of Laboratory Aggression-Associated Changes in Anterior Prefrontal Cortical Activity

Daniel R. Rosell, Erin A. Hazlett, Randall E. Newmark, Jane Zhang, Joseph Triebwasser, David Meyerson, Sophie Lazarus, Roanna Trisdorfer, Kim E. Goldstein, Marianne Goodman, Harold W. Koenigsberg, Janine D. Flory, Larry J. Siever, Monte Buchsbaum, Antonia S. New

\section{Mt. Sinai/Bronx VA, New York, NY}

Background: Evidence from our group and others has revealed that alleles of various genes of the serotonergic system exhibit a greater prevalence in patients with borderline personality disorder (BPD) compared to healthy controls, and appear to be related to the severity of various symptom dimensions of BPD. Specifically, we have described greater prevalence of the "risk" allele of the tryptophan hyroxylase-2 (THP2) gene in BPD patients relative to healthy controls. Moreover, the risk $\mathrm{TPH}_{2}$ haplotype was associated with greater aggression, affective lability, and (para)suicidal behaviors in patients with BPD. While the means by which these risk alleles may contribute to the development of BPD is important to characterize, it is also essential to understand how the presence or absence of these risk alleles within BPD may effect its pathophysiology. In other words, are there genetically-determined pathophysiologic subgroups of BPD? Therefore, we set out to determine whether the TPH2 risk allele affects the neural correlates of impulsive aggression in patients with BPD. We have previously described differential patterns of prefrontal cortical activity in BPD patients with intermittent explosive disorder (IED) compared to healthy controls using a laboratory-induced model of aggression. We describe here the effect of the $\mathrm{TPH}_{2}$ risk allele on the pattern of aggression-associated changes in prefrontal cortical activity in BPD-IED patients.

Methods: We employed the Point Subtraction Aggression Paradigm (PSAP) - a validated quantitative laboratory model of impulsive 
aggression - with 23 BPD-IED patients. Patients underwent positron emission tomgraphy (PET) scanning with 18 -fluoro-deoxyglucose on two occasions - once with a 'provoked' and once with an 'unprovoked' version of the PSAP. Mean relative glucose metabolic rate (rGMR) was calculated for gray and white matter of cortical regions, and differences between provoked and non-provoked conditions were scored. Provoked-minus-nonprovoked rGMR scores of gray and white matter were compared as a function of genotype for the $\mathrm{TPH} 2$ risk allele.

Results: BPD-IED patients homozygous for the risk $\mathrm{TPH}_{2}$ allele exhibited a significantly lower gray-white matter rGMR ratio compared to those homo- or heterozygous for the non-risk $\mathrm{TPH} 2$ allele $[F(2,20)=4.43$, Wilks $p=0.025]$ specifically in the anterior prefrontal cortical region.

Discussion: BPD-IED patients homozygous for the THP2 risk allele exhibited a different pattern of aggression-associated changes in metabolic activity in anterior prefrontal cortical regions compared to BPD-IED patients homo- or heterozygous for the non-risk allele. These findings may help to characterize the neural correlates of geneticallydetermined pathophysiologic subtypes of impuslive aggression in BPD. Such studies may lead to the identification of different pathophysiologic mechanisms that underlie the broader construct of BPD, and ultimately to greater diagnostic and therapeutic specificity for this disorder.

Disclosure: D. Rosell: None. E. Hazlett: None. R. Newmark: None. J. Zhang: None. J. Triebwasser: None. D. Meyerson: None. S. Lazarus: None. R. Trisdorfer: None. K. Goldstein: None. M. Goodman: None. H. Koenigsberg: None. J. Flory: None. L. Siever: None. M. Buchsbaum: None. A. New: None.

138. Local vs. Focal: The Evolving Functional Neuroanatomy of the Executive System in Adolescence

TD Satterthwaite*, J Loughead, Daniel Wolf, K Ruparel,

Kanchana Jagannathan, RT Garraty, Jeffery Valdez, E Yang,

Mark Elliott, J Richards, Monica Calkins, H Qiu, FD Mentch, PM

Sleiman, L Hermannsson, H Hakonarson, Ruben Gur, Raquel Gur

Univ of Pennsylvania, Philadelphia, PA

Background: Adolescence and early adulthood is a critical period for neurodevelopment and many neuropsychiatric disorders manifest during this period. There is evidence that frontal brain systems show the most pronounced development and dysfunction is associated with major disorders. However, linking brain-behavior measures the multiple facets of brain function requires large samples. We examined the development of the executive system with both task-based and resting-state functional magnetic resonance imaging (fMRI). This represents a preliminary report from a large ARRA NIMH study of neurodevelopmental trajectories that integrates functional and structural neuroimaging, genetic analyses, neurocognitive performance, and clinical phenotypic data.

Methods: fMRI blood oxygen level dependent (BOLD) response was examined at 3 Tesla during two functional runs in 250 subjects aged 8-21. Subjects performed a fractal n-back task with three levels of working memory load: o-back, 1-back, and 2-back. The contrast of interest was the differential BOLD response of 2-back greater than o-back. After standard preprocessing, the data were analyzed on a voxelwise basis across the whole brain using a bonfronni-corrected threshold of $z>7.00$. Age was included as a covariate of interest. Additionally, the BOLD response was measured at rest for 6 minutes. Timecourses from the resting BOLD data were extracted from 19 functionally defined regions of interest (ROIs) that displayed an abovethreshold response in the n-back task. To reduce spurious correlations, timecourses from the ventricles, subcortical white matter, and average whole brain signal were included as nuisance regressors. ROIs of each of these confound regressors was defined on an individual basis using segmentation algorithms. The timeseries from each ROI was correlated region by region for each subject, producing a 19x19 correlation matrix. To assess non-specific local connectivity, the timecourse from voxels within a $16 \mathrm{~mm}$ radius sphere around the peak of each seed region were extracted. Finally, the relationship between participant age and both inter-regional and local nonspecific connectivity was assessed.

Results: In the n-back task, 19 regions displayed a response that survived bonforonni correction. The regions (all bilateral if not midline) included the dorsal anterior cingulate, middle frontal gyrus, dorsolateral prefrontal cortex, superior parietal cortex, precuneus, frontal pole, anterior insula, thalamus, midbrain, and cerebellar crus I \& crus II. Notably, all regions displayed a positive correlation with age: older subjects demonstrated greater differential recruitment within the executive function network. The resting-state data revealed a remarkably similar pattern, demonstrating greater inter-regional connectivity with age. Notably, local non-specific correlation around each seed ROI diminished with age.

Discussion: Both the task-based and resting-state data demonstrated a strong relationship with age. The resting state results suggest that during development non-specific local connectivity is lost as the brain develops more focal connectivity within a functional network. These changes may relate to the augmented working memory response seen in the n-back task during development. Taken together, these data provides convergent evidence regarding the developmental trajectory of the executive function system. Examining the relationship of this trajectory to both genotype and phenotype has the potential to elucidate neuropsychiatric symptoms as aberrations in neurodevelopment.

Disclosure: T. Satterthwaite: None. J. Loughead: None. D. Wolf: None. K. Ruparel: None. K. Jagannathan: None. R. Garraty: None. J. Valdez: None. E. Yang: None. M. Elliott: None. J. Richards: None. M. Calkins: None. H. Qiu: None. F. Mentch: None. P. Sleiman: None. L. Hermannsson: None. H. Hakonarson: None. R. Gur: Part 1; Pfizer, AstraZeneca. R. Gur: Pfizer, AstraZeneca.

139. Reduced Infralimbic and Subgenual Anterior Cingulate Cortex Volumes in Healthy and Affectively Ill Carriers of the Met Allele of the BDNF Val66Met Variant

Jonathan Savitz ${ }^{*}$ Xinmin Liu, Alexander Neumeister, Allison Nugent, Dara Cannon, Nirmala Akula, Wendy Bogers, Carlos Zarate,

Dennis Charney, Francis McMahon, Wayne Drevets

LIBR, Tulsa, OK

Background: The methionine (met) allele of the functional val66met (rs6265) polymorphism of the brain-derived neurotrophic factor (BDNF) gene has been associated with reduced hippocampal volume, a potential endophenotye of major depressive disorder (MDD) and bipolar disorder (BD). However, the effect of rs6265 on the volume of two regions which are equally strongly implicated in mood disorders, the subgenual anterior cingulate cortex (sgACC) and the infralimbic cortex, which together make up the subcallosal gyrus, has not been measured.

Methods: High-resolution MRI (volumetric resolution $\approx 0.4 \mathrm{~mm} 3$ ) was conducted on a sample of unmedicated individuals with $\mathrm{BD}(\mathrm{n}=12)$, MDD $(n=29)$ and healthy controls $(n=42)$. MRI images were segmented blind to diagnosis and genotype by one rater. Data were analyzed using a two-step forced linear regression.

Results: After controlling for age, gender, handedness, diagnosis, and genetic ancestry we found no significant difference in absolute and normalized subcallosal gyrus volumes between $\mathrm{val} / \mathrm{met}$ (combined sample, $\mathrm{n}=20)$ heterozygotes and val/val homozygotes $(\mathrm{n}=63)$ $(\mathrm{p}>0.5)$. Post-hoc analyses showed no statistically significant effect of genotype for both the anterior sgACC and the IL ( $p>0.5)$. There was also no association between BDNF genotype and whole brain volume $(\mathrm{p}>0.3)$.

Discussion: Our results are not consistent with studies reporting an association between the met allele and volume reduction of the hippocampus and raise the possibility that the role of BDNF in 
neuronal plasticity does not extend to the subcallosal gyrus which regulates behavioral and endocrine responses to stress.

Disclosure: J. Savitz: None. X. Liu: None. A. Neumeister: None. A. Nugent: None. D. Cannon: None. N. Akula: None. W. Bogers: None. C. Zarate, Jr.: None. D. Charney: Part 1; Astro Zeneca, Bristol Myers Squibb, Cyberonics, Neurogen, Neuroscience Education Institute, Novartis, Orexin, Unilever. F. McMahon: None. W. Drevets: Pfizer.

140. Emotion Regulation and Pain in Borderline Personality Disorder - A Potential Link to the Understanding of Self-Injury Christian Schmahl*, Inga Niedtfeld, Sarah Reitz, Martin Bohus

Central Institute of Mental Health, Mannheim, Germany

Background: Patients with Borderline Personality Disorder (BPD) experience intense emotional stress and often show a deficiency of emotion regulation skills. Moreover, they display a high prevalence of self-injurious behavior (SIB). Patients report to engage in SIB because of its immediate relief effects on emotional tension. Pain in BPD has further been observed to lead to a reduction in neural activity in the amygdala and anterior cingulate cortex. This may be related to patients' subjective perception of relaxation.

Methods: To investigate the potential role of pain as a means of affect regulation in patients with $\mathrm{BPD}$, we conducted two studies: (1) an event-related fMRI study using picture stimuli to induce negative (versus neutral) affect for three seconds followed by thermal stimuli to induce heat pain (versus warmth perception) for nine seconds. 12 patients with BPD and 20 healthy controls (HC) were included in the study. (2) A study using incision-induced pain to model tissue damage and its influence on tension regulation. 14 patients with BPD and $18 \mathrm{HC}$ were included in this studies and a $4 \mathrm{~mm}$ wide incision with a scalpel as well as a "sham" treatment (touching the skin with the blunt end of the scalpel) were conducted by an investigator after a stress induction while subjective stress levels and heart rate were measured.

Results: In study (1), both negative and neutral pictures led to stronger activation of the amygdala, insula, and anterior cingulate cortex (ACC) in patients with BPD than in HC. Amygdala activation correlated with self-reported deficits in emotion regulation. During sensory stimulation, we found decreased amygdala and ACC activation, which was independent of painfulness. Pain led to increased activation of the left insula for $\mathrm{HC}$, but it did not lead to enhanced activation when the arousal was already high in BPD. In study (2), the incision resulted in an increase of subjective stress levels in HC, while stress levels decreased in the BPD group. Sham treatment led to a decrease of stress levels in both groups. In the BPD group, heart rate increased after the sham treatment, but decreased after the incision.

Discussion: The results replicate previous findings on emotional hyperactivity in BPD, and suggest that pain stimuli in BPD are processed differently depending on the arousal status. Our data preliminarily support the idea of a general mechanism of attentional shift underlying the stress-reducing effect of pain in BPD. Also, we could demonstrate that incision-induced pain may be suited as a model for the mechanism of tension reduction in the context of SIB. Disclosure: C. Schmahl: None. I. Niedtfeld: None. S. Reitz: None. M. Bohus: None.

141. Phenotypic Differences in Male Infants During the First Months of Life Predict Differential Reactivity of the Mmygdala to Novelty in Adulthood

Carl Schwartz*, Pratap Kunwar, Douglas Greve, Rachel Bloch, Lyndsey Moran, Nancy Snidman, Stuart Wallace

Massachusetts General Hospital, Charlestown, MA

Background: Temperament refers to a biologically based predilection for a distinctive pattern of behaviours, emotions and cognitions observed in infancy or early childhood. Previous studies have identified two temperamental categories in 2 year olds called behaviourally inhibited and uninhibited. Children with an inhibited temperament are timid with people, objects and situations that are novel or unfamiliar, whereas uninhibited children spontaneously approach these same incentives. Adults who had been categorized in the second year of life as inhibited, compared with those who had been categorized as uninhibited, showed greater amygdalar activation to unfamiliar neutral faces, suggesting some continuity at the level of neurobiology. Moreover, an inhibited temperament is a risk factor for the later development of social anxiety disorder (SAD) or social phobia. These discoveries in two year olds prompted the search for even earlier footprints of this temperamental profile. At four months of age high reactive (HR) infants demonstrate a temperamental profile in the laboratory characterized by vigorous motor activity and crying in response to specific unfamiliar visual, auditory, and olfactory stimuli, whereas low reactive (LR) infants show both low motor activity and low vocal distress to the same experimental stimuli. High reactive infants are biased to become behaviorally inhibited in the second year of life, whereas low reactive infants are biased to develop into uninhibited children. Given the central role of the amygdala in the processing of novelty and emotion in the brain, we hypothesized that there might be differences in the reactivity of the amygdala in adults associated with these contrasting high and low reactive infant temperaments.

Methods: Using fMRI, we measured amydgala reactivity to neutral faces in 135 subjects who were enrolled in an 18-year longitudinal study and had been characterized as high or low reactive infants at four months of age. ( $55 \mathrm{HR}-30$ males and 25 females- aged $18.19 \pm 0.1$, and 80 LR - 42 males and 38 females - aged $18.21 \pm 0.08$.

Results: Activity in the right amygdala during the familiarization phase of the protocol was significantly greater in adult subjects with the high reactive infant phenotype than the low reactive phenotype $[\mathrm{F}(1,125)=7.68, \mathrm{p}=.006])$, and greater in males than females $[\mathrm{F}(1,125)=6.62, \mathrm{p}=.01]$. Furthermore, there was a significant interaction between infant phenotype and gender $[\mathrm{F}(1,125)=4.65, \mathrm{p}=.03]$ in activity recorded from the right amygdala. Adult males who were high reactive infants show a high amygdala response to faces that does not decrease during the familiarization period. The mean bold signal was greater in male high reactives, compared both with males who had been low reactive infants $[\mathrm{t}(68)=-3.04, \mathrm{p}=.003]$ and with females of either infant phenotype [high reactive male $s$ vs. high reactive females $[\mathrm{t}(41)=-3.78, \mathrm{p}=.0005]$; high reactive males vs. low reactive females $[\mathrm{t}(60)=4.07, \mathrm{p}=.0001]$. In contrast, adult females showed no difference in their amygdala signal related to their infant phenotype. Furthermore, low reactive females did not differ from the low reactive males, who in turn were not different from high reactive females. During the second part of the paradigm that presented alternating blocks of novel and familiar faces, the BOLD signal recorded from the right amygdala was significantly greater in the subjects with the high reactive infant phenotype than the low reactive phenotype $[\mathrm{F}(1,125)=7.71, \mathrm{p}=.006]$, greater in males than females $[\mathrm{F}(1,125)=7.22, \mathrm{p}=.008]$, and greater to the novel faces than to the familiar ones $[\mathrm{F}(1,125)=22.34, \mathrm{p}<.0001]$. The hierarchy of responses in the subgroups defined by infant phenotype and gender was identical to that observed in the first part of the protocol.

Discussion: These data demonstrate that individual differences in reactivity of the amygdala to faces at maturity can be made solely on the basis of an infant behavioural phenotype observed at 4 months of age. Adult males who were high reactive infants showed particularly high levels of reactivity in the amygdala. Gender therefore appears to play an important role in predicting the impact of these infant temperaments on amygdalar reactivity in adulthood. This phenotype may shed light on the development of both normal and pathological anxiety in human beings and could be a fruitful target for genetic analysis.

Disclosure: C. Schwartz: None. P. Kunwar: None. D. Greve: None. R. Bloch: None. L. Moran: None. N. Snidman: None. S. Wallace: None. 
142. The 'Developmental Connectome' in Typically Developing Children and Its Disruption in Attention-Deficit/Hyperactivity Disorder

Philip Shaw*, Wendy Sharp, Francois Lalonde, Deanna Greenstein, Jay Giedd, Judith Rapoport

National Institute of Health, Bethesda, MD

Background: The delineation of connectivity within the human brain the 'connectome'- has been based on a synthesis of studies of cortical and white matter architecture and function using data which is almost exclusively cross-sectional and collected in adults . By the inclusion of in vivo neuroimaging data collected prospectively on typically developing youth, we add a developmental dimension to the 'connectome'. We ask if brain regions which are richly interconnected in adulthood also have homologous developmental trajectories in typically developing children. We further explore the extent to which disruptions in these synchronous developmental trajectories characterize attention-deficit/hyperactivity disorder (ADHD), one of the most common neuropsychiatric disorders of childhood.

Methods: 1758 neuroanatomic magnetic resonance scans were acquired prospectively on 664 typically developing youth (age range 4 to 26 years) and 179 children with DSM-IV defined ADHD. From this data, we defined the developmental trajectories of the cortex (using cortical thickness measured at 80,000 points), and striatal and medial temporal lobe structures. Through a process of matching developmental trajectories we delineated the cortical regions which developed in synchrony with each deep structure.

Results: We found similarity in developmental trajectories between the caudate and dorsolateral prefrontal cortex; between the putamen and premotor/motor cortex; and between the nucleus accumbens and cingulate cortex. These links appear to be the developmental homologues of the partly segregated cortico-striatal loops, defined through previous cross-sectional studies, which are pivotal in human cognition. The hippocampus and amygdala showed strikingly similar trajectories with each other and with the medial temporal cortex, stretching from the temporo-polar cortex, along rhinal and entorhinal cortex. This mirrors the pattern of anatomical connectivity in this region established early in prenatal development. In ADHD, there was anomalous synchronous development between the putamen and the parietal cortex, and between the hippocampus and the lateral prefrontal cortex. These anomalous conjoint trajectories were most prominent in those with a poor clinical outcome.

Discussion: We find that structures with rich anatomical and functional connectivity in adulthood develop in synchrony during typical childhood. Additionally, partial disruption to this coordinated pattern of development characterizes ADHD.

Disclosure: P. Shaw: None. W. Sharp: None. F. Lalonde: None. D. Greenstein: None. J. Giedd: None. J. Rapoport: None.

\section{Abnormalities of the Uncinate Fasciculus in First Episode} Schizophrenia Revealed by Diffusion Tensor Imaging

Jason Schneiderman, Thomas Whitford, Pelavin Paula, Diandra Lucia, Samuel Atwood, Raquelle Mesholam-Gately, Larry Seidman, Jill Goldstein, Robert McCarley, Marek Kubicki, Martha Shenton*

Brigham and Women's Hospital, Harvard Medical School, Boston, MA

Background: The uncinate fasciculus (UF) is the major direct connection between the frontal and temporal lobes and has been observed to be abnormal in patients with chronic schizophrenia (e.g., Kubicki, 2007). Using Diffusion Tensor MRI, a method that examines the tissue structure and connectivity of different regions of the brain by measuring the diffusion of water, this study explores the differences between patients with first episode schizophrenia and normal controls in the UF.

Methods: The UF of 18 patients with first episode schizophrenia (FESZ) and 20 controls (NC) were examined using diffusion tensor imaging on a 3 T MRI scanner. The UF was successfully extracted using two regions of interest, one placed in the frontal lobe and one in the temporal lobe, anterior to the temporal stem, to define the tract, and streamline fiber tractography was used to extract the fiber bundle.

Results: Results indicate increased trace (left $\mathrm{p}=.115$; right $\mathrm{p}=.028$ ), decreased FA (left $\mathrm{p}=.047$; right $\mathrm{p}=0.064$ ), and increased radial diffusivity (left $\mathrm{p}=.057$; right $\mathrm{p}=.026$ ) in FESZ compared to NC.

Discussion: FE patients showed significantly decreased FA compared to controls on the left. This decrease was also present on the right, but did not reach statistical significance. This suggests that FA is already decreased at first episode of schizophrenia. In overall diffusivity, FE patients showed increased trace diffusivity compared to their matched controls on the right hemisphere. This trend was also present on the left, but did not reach statistical significance. This increase in trace appears to have been primarily driven by an increase in radial diffusivity, which is likely associated with myelin abnormalities, since radial diffusivity is thought to be a putative marker of myelin.

Disclosure: J. Schneiderman: None. T. Whitford: None. P. Paula: None. D. Lucia: None. S. Atwood: None. R. Mesholam-Gately: None. L. Seidman: None. J. Goldstein: None. R. McCarley: None. M. Kubicki: None. M. Shenton: None.

\section{Combining PET and MRS to Study the Neurochemistry of} Obsessive-Compulsive Disorder

Helen Blair Simpson*, Lawrence Kegeles, Xiaoyan Xu, Xiangling Mao, James Bender, Michael Maher, Rena Staub, Olga Medina, Mark Slifstein, Dikoma Shungu, Anissa Abi-Dargham

New York State Psychiatric Institute/Columbia University, New York, NY

Background: Pharmacological evidence suggests abnormalities in the brain serotonin $(5-\mathrm{HT})$ system in obsessive-compulsive disorder (OCD). However, recent animal and human studies have implicated the glutamate system: animal studies find that disrupting glutamatergic signaling at cortico-striatal synapses leads to OCD-like behaviors; human genetic studies find associations between the glutamate transporter SLC1A1 and OCD; and open label trials demonstrate efficacy of glutamatergic agents in reducing OCD symptoms. This has led to the hypothesis that OCD symptoms result either directly or indirectly from increased glutamatergic signaling in cortico-striatal pathways. As $5-\mathrm{HT}_{2 \mathrm{~A}}$ receptors are located on both pyramidal cells and interneurons and may modulate glutamate and GABA transmission, we measured 5- $\mathrm{HT}_{2 \mathrm{~A}}$ receptor availability with $\mathrm{PET}$, and $\mathrm{Glx}$ (glutamate plus glutamine) and GABA levels with MRS in OCD patients and matched controls.

Methods: Twenty-one patients (ages $29 \pm 10$ years, 9 F, 12 M) and 21 healthy controls (ages $30 \pm 10$ years, $11 \mathrm{~F}, 10 \mathrm{M}$ ) completed the MRS studies; 19 in each group (matched for age, gender, and racial-ethnic group) also completed the PET scan. Patients were free of medications at the time of imaging and OCD was the primary diagnosis with no current Axis I comorbidity. MRS data were acquired using a $3 \mathrm{~T}$ GE system and an 8-channel phased-array head coil with the J-edited spin echo difference technique from an $18.8 \mathrm{cc}$ anterior cingulate (ACC) and a $9.6 \mathrm{cc}$ dorsolateral prefrontal cortex (DLPFC) voxel to generate GABA and Glx ratios to water (W). Test-retest reliability using these methods was previously shown to be high (percent coefficient of variation was $5.2 \%$, and intraclass correlation coefficient was 0.84 for GABA/W). $\left[{ }^{11} \mathrm{C}\right] \mathrm{MDL} 100907$ PET scans were acquired on an $\mathrm{HR}+$ camera with arterial input function and analyzed with two-tissue compartment kinetic modeling to generate $\left[{ }^{11} \mathrm{C}\right] \mathrm{MDL} 100907$ binding potential $\left(\mathrm{BP}_{\mathrm{P}}\right)$ in the ACC, DLPFC and other cortical and limbic ROIs. Results: There were no significant differences in Glx/W or GABA/W in the ACC or DLPFC between OCD and controls. However, GABA/W was $2.4 \pm .51$ in controls and $2.1 \pm .59$ in OCD in the ACC $(\mathrm{p}=0.08$, all ratios times $\left.10^{-3}\right)$. There were no significant group differences in $\left[{ }^{11} \mathrm{C}\right] \mathrm{MDL} 100907 \mathrm{BP}_{\mathrm{p}}$ in any of the ROIs. However, there were strong correlations between $\mathrm{ACC}$ GABA/W and $\left[{ }^{11} \mathrm{C}\right] \mathrm{MDL} 100907 \mathrm{BP}_{\mathrm{P}}$ in 


\section{S366}

several ROIs in OCD patients but not in healthy controls. For example, in ACC, where $\mathrm{BP}_{\mathrm{P}}$ values were $47.8 \pm 10.1 \mathrm{~mL}^{-3} \mathrm{~cm}^{-3}$ in $\mathrm{OCD}$ and $50.2 \pm 17.8 \mathrm{~mL} \mathrm{~cm}^{-3}$ in controls $(\mathrm{p}=0.6)$, Pearson $\mathrm{R}$ values between ACC $\mathrm{BP}_{\mathrm{P}}$ values and ACC GABA/W were $-0.16(\mathrm{p}=0.5)$ in controls and $0.66(\mathrm{p}=0.002)$ in $\mathrm{OCD}$. In $\mathrm{OFC}$, where $\mathrm{BP}_{\mathrm{P}}$ values were $37.4 \pm 8.4 \mathrm{~mL}^{-3} \mathrm{~cm}^{-3}$ in OCD and $38.0 \pm 14.4 \mathrm{~mL}^{\circ} \mathrm{cm}^{-3}$ in controls $(\mathrm{p}=0.9)$, Pearson $\mathrm{R}$ values between $\mathrm{OFC} \mathrm{BP}_{\mathrm{P}}$ values and ACC GABA/W were $-0.19(p=0.4)$ in controls and $0.67(p=0.002)$ in OCD. Discussion: We found no significant abnormalities in OCD in either Glx or GABA levels in the DLPFC or ACC or in the distribution of $5-\mathrm{HT}_{2 \mathrm{~A}}$ receptors in multiple cortical and limbic regions of interest. However, there was a trend toward lower GABA levels in the ACC in OCD that warrants further investigation in a larger sample. The strong correlations between PET and MRS data suggest heightened coupling in OCD between GABA and $5-\mathrm{HT}_{2 \mathrm{~A}}$ systems in certain brain regions. Further studies are needed to characterize the nature of this relationship and to explore its potential implications for treatment response.

Disclosure: H. Simpson: Part 1; Pfizer, Jazz Pharmaceuticals, Neuropharm, Janssen Pharmaceutica. Part 4; Neuropharm, Janssen Pharmaceutica. L. Kegeles: Part 1; Pfizer, Amgen. Part 4; Pfizer, Amgen. X. Xu: None. X. Mao: None. J. Bender: None. M. Maher: None. R. Staub: None. O. Medina: None. M. Slifstein: Part 1; GlaxoSmithKline, Amgen, Intra-Cellular Therapies. Part 4; Intra-Cellular Therapies. D. Shungu: None. A. Abi-Dargham: Part 1; BMS-Otsuka, Boehringer-Englheim, Lundbeck, Sepracor. Part 2; BMS-Otsuka. Part 3; BMS-Otsuka.

145. Medial Temporal Structural Neuroanatomy In Adolescents With Bipolar I Disorder

Manpreet Singh*, Layla Bararpour, Ryan Kelley, Kiki Chang, Allan Reiss

\section{Stanford University School of Medicine, Stanford, CA}

Background: Structural magnetic resonance imaging studies of children and adolescents bipolar disorder (BD) have consistently demonstrated volumetric differences in medial temporal regions including the amygdala and hippocampus, which are important in the regulation of emotion. However, it remains unclear whether such changes occur close to the onset of illness, or if they are due to prolonged mood symptoms or medication exposure. The goal of this study was to examine these structural differences in adolescents who recently experienced their first episode of mania to identify possible structural abnormalities that may be found early in the course of BD. Methods: Adolescents (13-18 years old) who were diagnosed as having their first episode of fully syndromal bipolar I disorder within the past 9 months $(\mathrm{N}=26)$ and adolescents without any personal or family history of a DSM-IV Axis I disorder $(\mathrm{N}=25)$ were examined for group differences in amygdala and hippocampus volumes after collection of high-resolution magnetic resonance structural images using a $3 \mathrm{~T}$ GE scanner. Diagnoses were determined by the Washington University in St. Louis Kiddie-Schedule for Affective Disorders and Schizophrenia (Geller et al. 1996), with established interrater reliability (kappa > 0.9). Manual tracing morphometric analyses were performed using Brain Image Java software (http://spnl.stanford.edu/tools/brainimagej.htm) by a rater blind to group status. SPM8 was used to segment into grey, white, and CSF partitions, and to measure total brain volumes (TBV). Results: Relative to controls, adolescents with BD showed significant increases in right amygdala volumes after covarying for total brain volume, gender, and age $(\mathrm{F}(47)=6.46, \mathrm{p}=0.01)$. No other structural differences were found between these groups in the left amygdala, or in the right or left hippocampus. Right amygdala volume increases within the $\mathrm{BD}$ group correlated significantly with higher total $(\mathrm{R}=0.51$, $\mathrm{p}=0.008)$, grey $(\mathrm{R}=0.46, \mathrm{p}=0.02)$ and white $(\mathrm{R}=0.50, \mathrm{p}=0.01)$ matter volumes. A trend for a positive correlation between increased clinical global functioning and increased right amygdala volumes $(\mathrm{R}=0.35, \mathrm{p}=0.078)$ was found. Symptoms of mania, depression, or exposure to lithium did not appear to correlate with any volumetric findings within either group.

Discussion: Amygdalar enlargement was seen in adolescents early in the onset of bipolar I mania, and appeared to be positively correlated with higher overall functioning. Among the most consistent neuroanatomical findings in pediatric $\mathrm{BD}$ is a reduction in amygdalar volume. This may not be seen in youth with BD-I until symptoms have progressed beyond the initial manic episode. Enlargement in amygdalar volume early in the course of illness in this study may reflect abnormal developmental pruning of medial temporal circuits, or a compensatory neuroprotective mechanism. Correlations to mood symptom ratings, and effects of medication exposure were considered and appeared to have limited impact on volumetric findings. Future longitudinal analyses examining the consequences of enlarged amygdala volumes in adolescents with BD-I on long-term clinical outcome are warranted.

Disclosure: M. Singh: None. L. Bararpour: None. R. Kelley: None. K. Chang: Part 1; GlaxoSmithKline, Eli Lilly and Company, Bristol-Myers Squibb, AstraZeneca Pharmaceuticals. A. Reiss: None.

146. Reward Circuit Functional Connectivity in Healthy Subjects Arielle Stanford ${ }^{\star}$, Bruce Luber, James Moeller, Dolores Malaspina, Sarah H Lisanby

Columbia University/New York State Psychiatric Institute, New York, NY

Background: Reward circuitry has been studied for a variety of psychiatric disorders including addiction and schizophrenia, both illnesses marked by changes in motivation. A commonly used task to study these circuits is the monetary incentive delay task (MID). The MID has been an effective way to identify regions responsible for anticipation, hedonia and motivated behavior. However studies have not examined functional connection between regions identified by MID. While GLM is a powerful tool for identifying meaningful associations between the brain and task performance, it lacks the power to extract circuits. In contrast, Ordinal Trends Analysis (OrT) is a multivariate covariance analysis based on the application of principal components analysis which can identify subject by condition by region interactions. These interactions produce activation pattern signatures within a distributed network, which can then be used to explore changes in brain activation associated with task measures as well as measures independent of the task. In this study, we examined whether self reports of subjective and behavioral aspects of reward processing were related to functional circuits associated with MID.

Methods: Eight subjects performed MID during event related fMRI acquisition. The MID has five task phases: 1) Cue signifying the amount of award possible to win, 2) variable Delay 1 (anticipation and motor planning), 3) Target indicating the need to respond, 4) variable Delay 2 (anticipation) and 5) Feedback (consumption) as to whether the subject won the trial. Functional and anatomical images were acquired using a 1.5-tesla twin speed General Electric magnetic resonance scanner with an 8 channel head coil capable of echo-planar imaging. Stimuli were presented using an LCD projector, which subjects viewed from a supine position in the magnet using mirrors attached to the head coil. Stimuli were generated and displayed using Presentation 11.o. To obtain $\mathrm{T} 2{ }^{*}$-weighted images, we acquired 24 axial slices along the AP axis using an echo-planar sequence. Preprocessing and first level analyses were performed on the data using SPM5 with conditions defined by the reward level and task phase. We performed OrT analysis for individual task phases as well as for different reward values across task phases using custom software. The analysis yielded a subject expression score for each principal component analysis pattern allowing the measurement of intersubject heterogeneity. We then examined relationships between task dependent and independent measures with our principal component analysis.

Results: Activations in the covariance pattern extracted from MID fMRI data during Delay 1 whose expression changed with decreasing 
reward value included SMA, thalamus, ACC, OFC, medial temporal lobes (MTL), visual cortex and ventral striatum and was expressed in all subjects. Subject scaling factors (SSF) from the first non-zero reward level during the target phase predict consummatory hedonia ratings at an $\mathrm{R} 2=0.61, \mathrm{p}<0.04$. SSF from the highest non-zero reward level during the target phase predict social hedonia at an $\mathrm{R}_{2}=0.62$, $\mathrm{p}<0.04$. The covariance pattern of activation across the task from Cue to Target was similar and also included posterior cingulate, but not MTL, with more activity for the higher reward condition. Two subjects did not demonstrate the change in the network with task phase for the no reward condition and there was greater subject variability in network expression. However, all 8 subjects expressed this pattern for the highest reward condition. The SSF from these activations also predicted social hedonia at $\mathrm{R} 2=.62, \mathrm{p}<.04$.

Discussion: These data suggest MID produces monotonic changes in activation of a network that includes reward anticipation (VS), consumption (OFC), motor preparation (SMA), and reward evaluation (IPL). Furthermore, they validate functional connections between regions activated during the delay phase and activated with changing reward value. In addition, functional circuits activated by the task were associated with self report measures of consummatory and social hedonia. This analysis demonstrates the ability to reveal heterogeneity between subjects.

Disclosure: A. Stanford: None. B. Luber: None. J. Moeller: None. D. Malaspina: None. S. Lisanby: Part 1; Northstar DSMB Chair (past), Astra Zenica support of CME event. Part 4; Cyberonics - grant, ANS/St. Jude Medical - grant, Neuronetics - grant, Brainsway - grant, Magstim equipment, MagVenture - equipment.

\section{Effects of Aripiprazole on Brain Circuitry in Youth with Pervasive Developmental Disorders}

Kimberly Stigler*, Yang Wang, Brenna McDonald, Andrew Saykin, Amit Anand, Christopher McDougle

\section{Indiana University School of Medicine, Indianapolis, IN}

Background: Pervasive developmental disorders (PDDs) are neuropsychiatric disorders often associated with functionally-impairing symptoms of irritability (tantrums, aggression, self-injury). Evidence to date supports the use of aripiprazole for the treatment of irritability in PDDs. However, its effects on brain circuitry that lead to symptom improvement are unknown. The purpose of this pilot functional magnetic resonance imaging (fMRI) study is to obtain preliminary data on the effects of aripiprazole versus placebo on brain activation in youth with PDDs.

Methods: Six children and adolescents (mean age, 11.3 years; mean IQ, 94) with PDDs and associated irritability received fMRI scans at baseline and after 8 weeks of double-blind treatment with aripiprazole or placebo. Scans were obtained on a Siemens Trio $3 \mathrm{~T}$ scanner with TIM using a 12 channel phased array RF coil. A high resolution anatomical scan (MPRAGE) was acquired. T2* BOLD scans were obtained with $3 \mathrm{~mm}$ isotropic resolution with a TR of $2 \mathrm{~s}$. Subjects completed a negative facial emotion task. Behavioral measures, including the Aberrant Behavior Checklist-Irritability subscale (ABCI) and Clinical Global Impressions-Improvement scale (CGI-I) were obtained to determine response to aripiprazole versus placebo. Image data were analyzed using Structural Parametric Mapping (SPM8) software and locally developed software programs.

Results: A total of 12 scans from 6 subjects, 3 per group, were completed. The mean task accuracy rate was $94 \%$; there was no significant difference in task accuracy rates between groups. Increased activation in response to negative pictures was found in right lateral prefrontal cortex (PFC) after treatment with aripiprazole, whereas no significant changes were found in the placebo-treated subjects $(\mathrm{p}<0.05)$. This change corresponded to improvement in behavioral measures with aripiprazole [baseline $\mathrm{ABC}-\mathrm{I}=27$, wk $8 \mathrm{ABC}-\mathrm{I}=12$; wk $8 \mathrm{CGI}-\mathrm{I}=1.4$ (much improved)] versus placebo [baseline $\mathrm{ABC}-\mathrm{I}=29$, wk 8 ABC-I $=23$; CGI-I $=4.8$ (no change)].
Discussion: These preliminary data suggest there is increased activation in PFC in children and adolescents with PDDs who respond to aripiprazole for irritability. The PFC, areas of which have reciprocal projections to the amygdala, plays an important role in inhibitory control and action monitoring. The findings suggest that aripiprazole treatment may enhance the modulatory influences of PFC in the regulation of irritability in ASDs. These encouraging initial results will be confirmed in a larger sample.

Disclosure: K. Stigler: Part 1; Bristol-Myers Squibb Co., Curemark, Eli Lilly \& Co., Forest Research Institute, Ortho-McNeil Janssen. Part 4; Bristol-Myers Squibb Co., Curemark, Eli Lilly \& Co., Forest Research Institute, Ortho-McNeil Janssen. Y. Wang: None. B. McDonald: None. A. Saykin: None. A. Anand: Part 1; AstraZeneca, Bristol-Myers Squibb Co., Eli Lilly \& Co., Forest Research Institute. Part 4; AstraZeneca, Bristol-Myers Squibb Co., Eli Lilly \& Co., Forest Research Institute. C. McDougle: Part 1; Bristol-Myers Squibb Co., Forest Research Institute. Part 2; Bristol-Myers Squibb Co. Part 3; Bristol-Myers Squibb Co. Part 4; Bristol-Myers Squibb Co.

\section{Disturbances in Resting State Functional Connectivity of Amygdala Subregions in Patients with Psychotic and Nonpsychotic} Major Depression

Keith Sudheimer*, Amit Etkin, Michael Greicius, Jennifer Keller, Alan Schatzberg

\section{Stanford University, Stanford, CA}

Background: In recent years resting state functional connectivity analyses have begun to elucidate a series of intrinsically-organized and interconnected brain networks that oscillate together at low frequencies $(.008-.01 \mathrm{~Hz})$ in the resting brain. Brain regions that oscillate together at these low frequencies are thought to have strong functional connections and a subset of these regions have direct anatomical connections. In the current study we investigate connectivity differences between healthy controls, patients with major depression but no psychotic features, and patients with major depression and psychotic features. Patients with depression often show abnormal amygdala structure and function in fMRI tasks. Uncovering connectivity differences between psychiatric patient populations holds great promise in advancing our understanding of disease and efficacy of treatment.

Methods: Healthy controls $(\mathrm{HC})(\mathrm{N}=16)$, patients with non psychotic major depression $(\mathrm{MDD})(\mathrm{N}=19)$, and patients with psychotic major depression $(\mathrm{PMD})(\mathrm{N}=10)$ underwent a 5 minute resting state scan as a part of a larger protocol (data presented elsewhere). A first level model was constructed for each subject. In these models a region of interest (ROI) time course was entered as a regressor along with a global signal estimate and 6 motion parameters. The basolateral nucleus (BLA) and centromedian nucleus (CMA) of the amygdala ROIs were defined and distinguished from each other using a probabilistic cytoarchitectonic maps of post-mortem data contained within the SPM Anatomy toolbox (Eickhoff et al. Neuroimage 2005). Appropriate ROI target regions were defined by the differential connectivity targets of the basolateral nucleus (BLA) or the centromedian nucleus (CMA) of the amygdala in a completely separate cohort of healthy controls $(\mathrm{N}=31$ Etkin et al. Arch. Gen. Psychiatry, 2009). The main target regions in this cohort for the BLA includes the occipital lobe, large extents of the ventral temporal lobe, superior temporal gyrus and sulcus, precentral and postcentral gyri, intraparietal sulcus, lateral posterior orbitofrontal cortex, dorsal and ventral medial prefrontal cortex (PFC), parahippocampal gyri, and a small region in the inferior lateral PFC. The target regions for the CMA includes the anterior cingulate, thalamus, insula, caudate, putamen, midbrain and cerebellum. Importantly the connectivity targets for the BLA and the CMA were mutually exclusive. Using BLA and CMA targets as the connectivity seeds in the present study, we were able to calculate the degree to which a subject's BLA and CMA is functionally connected to its appropriate targets and also the degree to which these regions were functionally connected to 
inappropriate targets. Measures of right and left BLA and CMA connectivity with their appropriate and inappropriate targets were extracted from first level contrast images using spmtools scripts (Adrian Imfeld). Extracted values from left and right BLA and CMA were then entered into a repeated measures ANOVA with side (laterality) as a within-subjects factor, group as a between-subjects measure and BLA and CMA as repeated measures using SPSS version 18 .

Results: An overall 3 level group effect of was observed $(p=0.03)$ and was driven by differences between $\mathrm{HC}$ and both MDD (BLA $\mathrm{p}=0.026$, CMA $\mathrm{p}=0.011)$ and PMD (BLA $\mathrm{p}=0.01, \mathrm{CMA} \mathrm{p}=0.017$ ) groups. No significant laterality effects or interactions between side and group for either BLA or CMA we observed. This indicates that both MDD and PMD subjects have perturbed connectivity between BLA and it's normal targets as well as CMA and it's normal targets. While differences between MDD and PMD patient groups were not significant it is notable that the PMD group had consistently more perturbed connectivity across each amygdala subregion.

Discussion: The BLA is heavily involved in forming emotion laden learning associations and the CMA is involved in integrating these associations and coordinating appropriate physiological responses. Disturbed connectivity between these nuclei and their appropriate targets may be responsible for the etiology and/or the maintenance of abnormal emotional processing in depression. CMA connectivity disturbances may interfere with normal physiological reactions to stimuli by reducing the coherence of the coordination of these reactions. Likewise BLA disturbances may serve to maintain a disease state by degrading the influence of new experiences on amygdala reactivity.

Disclosure: K. Sudheimer: None. A. Etkin: Part 1; Neostim. M. Greicius: None. J. Keller: None. A. Schatzberg: Corcept, Pfizer, Lilly, Neuronetics, BrainCells, Amnestix, PharmaNeuroBoost, Sanofi, Takeda, CeNeRx, CNS Response, Vivus, Roche, Xytis, GlaxoSmithKline, Somaxon. Part 2; APA, APPI, Stanford University, PharmaNeuroBoost, Neuronetics, BrainCells. Part 3; PharmaNeuroBoost, APA, APPI.

149. Parental Brain Function and Structure: Effects of Early Life Experience, Contemporaneous Breastfeeding, Correlations with Behavior and Changes in the Early Postpartum

James Swain*, Pilyoung Kim, Ruth Feldman, Linda Mayes, James Leckman

\section{University of Michigan, Ann Arbor, MI}

Background: Parenting constitutes an evolutionarily conserved set of attachment behaviors to assure infant survival, provide nurturance and care, and sooth the distressed infant. We are studying neural circuits and changes in thoughts and behaviors in new parents that respond to infant pictures and cries, particularly when those stimuli arise from their own infant. Animal models indicate that early life experiences predict later parenting behaviors, that certain key neuroendocrine factors such as oxytocin and limbic brain structures are critical hormone for adaptive parental behaviors, and that the structure and function of parenting systems changes in the postpartum. In humans, maternal sensitivity behaviors across the first year postpartum are linked with infant development, and certain limbicbrain as well as cortical regions are involved. Such brain-based systems allow adaptation to the postpartum infant's needs - providing appropriate emotional responses and organizing sensitive parenting behaviors by raising cognitive and emotional salience and reward derived from infant stimuli and creating contingencies between endocrine, autonomic, and sensory systems in parent and child. We hypothesize that neurobiological circuits that regulate parenting involve systems of neurotransmitters overlap with those that mediate caregiving, empathy as well as some aspects of anxiety, obsessivecompulsive disorder and addiction. In newest analyses, we aimed to determine if:
1) brain responses to own baby-cries in cortical-subcortical circuits correlate with levels of parental preoccupations involving anxious, intrusive, obsessive-compulsive-like thoughts and dyadic behaviors; 2) parent sub-grouped according to nursing status and early life experience show differences in brain activations;

3) brain structure varies as a function of early life experience as well as longitudinally during the first postpartum months.

Methods: We are studying parental attachment in several ways in $40+$ sets of parents: administering interview and self-report versions of the Yale Inventory of Parental Thoughts and Actions, mood and anxiety scales as well as an inventory of perceived quality of early life; making brief videos of parent-infant interaction to assess attachment and interpersonal synchrony; performing structural and functional magnetic resonance imaging (fMRI) of the brains of both mothers and fathers (using a Siemens $3 \mathrm{~T}$ Trio scanner) while listening to baby stimuli, including own and other baby cries as well pictures. All data are acquired longitudinally at 2 weeks \& 3 months postpartum, and analyzed with Brainvoyager and SPM analysis software. At 3-4 months postpartum, we are also acquiring brief free play videos involving mother plus infant, father plus infant, and mother plus father plus infant to assess attachment and interpersonal synchrony. Parents are grouped according to timing, experience, gender, psychometric measures, and early life experience. In order to determine the influence of the neuopeptide oxytocin, we also grouped parents according to breastfeeding and mode of delivery.

Results: The stable circuit that becomes active in response to ownbaby cry in mothers and fathers, including anterior cingulate and basal ganglia varies according to early life experience. In addition, brain density is determined by early life experience and varies with time in the first 3 months postpartum in medial frontal and temporal regions. Mothers who are breastfeeding vs. formula feeding were more sensitive to infant stimuli in amygdala, hypothalamus and putamen. Brain responses in basal ganglia and frontal lobes vary according to parental mood. Correlations of brain activity and parenting behavior appear in other arousal and emotion control regions.

Discussion: Human parenting involves brain circuits of emotion, motivation, salience, and habits, which are consistent with animal parenting work. The activity within parenting brain circuits varies as a function of early life experience, as well as contemporaneous breastfeeding and other dyadic behaviors. Also, the experience of parenting is affecting cortical gray matter density as part of parental adaptation. This work promises neuroimaging endophenotypes to test interventions for optimizing family bonding and perhaps prevent or treat perimartum mental illness toward improved child environments. Disclosure: J. Swain: None. P. Kim: None. R. Feldman: None. L. Mayes: None. J. Leckman: None.

150. A Diffusion Tensor Imaging Study of Gray and White Matter in Schizophrenia and Bipolar I Disorder

Dana Anderson, Babak Ardekani, Katherine Burdick, Delbert

Robinson, Serge Sevy, Anil Malhotra, Philip Szeszko*

Feinstein Institute for Medical Research, Manhasset, NY

Background: A dichotomy between schizophrenia and bipolar disorder was originally described by Kraepelin and continues to exist today in the diagnostic categories of the Diagnostic and Statistical Manual of Mental Disorders. It is increasingly recognized, however, that schizophrenia and bipolar disorder share certain epidemiological features such as age at onset, lifetime risk, incidence, and influence of sex. Few studies, however, have directly assessed similarities and differences in the gray or white matter in patients with schizophrenia compared to patients with bipolar disorder. The goal of this study was to compare measures of gray and white matter integrity using diffusion tensor imaging (DTI) in 35 patients with schizophrenia, 20 patients with bipolar I disorder (18 with psychotic features) and 56 healthy volunteers. 
Methods: Fractional anisotropy (FA) and mean diffusivity (MD) are measures that can be computed from DTI data that reflect the magnitude and anisotropy of the self-diffusion of water molecules in the brain. In this study FA was used as an index of white matter integrity. In addition, we examined MD within the gray matter as a surrogate marker for greater water diffusion in cerebrospinal fluid and concomitant gray matter loss. The dependent measures included average FA and MD within 44 regionsof-interest within the frontal, temporal, parietal, occipital and limbic lobes identified using the LPBA40 Atlas (Shattuck et al., 2008). Separate analyses examined FA within the white matter and MD within the gray matter in the frontal, temporal, parietal, occipital and limbic lobes. In each of these analyses the between subjects factors included group (patients with schizophrenia versus patients with bipolar disorder versus healthy volunteers) and sex. Significant group main effects for each lobe were followed by posthoc analyses investigating either FA or MD within each of the regions-of-interest comprising the lobes.

Results: Investigation of FA within the white matter revealed significant $(p<.05)$ main effects of group only for the temporal and occipital lobes. Posthoc analyses indicated that patients with schizophrenia had significantly lower FA compared to patients with bipolar disorder and healthy volunteers in the superior temporal, parahippocampal, superior occipital and middle occipital white matter. Patients with bipolar disorder did not differ significantly in FA from healthy volunteers in any of these white matter regions. Investigation of MD within the gray matter revealed significant $(\mathrm{p}<.05)$ main effects of group for the limbic and temporal lobes. Posthoc analyses indicated that both patient groups had higher MD within the superior temporal and parahippocampal gyri compared to healthy volunteers. Patients with schizophrenia had significantly higher MD within the hippocampus compared to healthy volunteers and significantly higher MD compared to healthy volunteers and patients with bipolar disorder in the inferior temporal gyrus gray matter.

Discussion: Our data implicate overlapping gray matter temporal lobe structural alterations in the neurobiology of schizophrenia and bipolar disorder, but suggest that temporal lobe white matter pathology may be an additional risk factor for schizophrenia. These findings may have relevance for diagnostic classification systems and future research focusing on the identification of susceptibility genes for these disorders. Disclosure: D. Anderson: None. B. Ardekani: None. K. Burdick: Part 1; Merck. D. Robinson: Janssen, BMS. S. Sevy: Abbott. A. Malhotra: Eli Lilly, Schering-Plough/Merck, Arbor Scientia (Sepracor, Inc.), Wyeth, PGx Health. Part 4; Eli Lilly. P. Szeszko: Part 1; Boehringer Ingelheim.

\section{Impact of the BDNF $\mathrm{Val}^{66}$ Met Polymorphism on White Matter} Tract Integrity in Healthy Humans

Heike Tost ${ }^{\star}$, Tajvar Alam, Matthew Geramita, Christine Rebsch, Bhaskar Kolachana, Herve Lemaitre, Alan S Barnett, Dwight

Dickinson, Daniel Weinberger, Stefano Marenco

\section{National Institute of Mental Health, Bethesda, MD}

Background: A frequent single nucleotide polymorphism (SNP) in the $5^{\prime}$ proregion of the $B D N F$ gene results in a valine (Val) to methionine (Met) substitution at codon $66\left(\mathrm{Val}^{66} \mathrm{Met}, \mathrm{rs6265}\right)$. While the Met allele has been previously linked to reductions of activity-dependent secretion of the neurothrophin and deficits in hippocampal structure, function, and cognition (Egan et al., 2003), meta-analyses suggest that the variant is protective for psychiatric disorders such as bipolar disorder (Fan and Sklar, 2008), which are characterized, among others, by working memory deficits and microstructural alterations of the corpus callosum (Bellani et al., 2009). Here, we examined the effects of the $B D N F \mathrm{Val}^{66}{ }^{\mathrm{Met}}$ polymorphism on the microstructural properties of the brain as measured with diffusion tensor imaging (DTI) and its relevance to cognitive function in healthy humans.

Methods: Eighty-five healthy volunteers without a history of psychiatric illness were included in this study (mean age $=33.5 \pm 9.6$ years, 46 males, $\mathrm{n}=50 \mathrm{Val} / \mathrm{Val}, \mathrm{n}=32 \mathrm{Val} / \mathrm{Met}, \mathrm{n}=3 \mathrm{Met} / \mathrm{Met}$ ). DTI data were acquired using a GE $1.5 \mathrm{~T}$ Signa scanner (Waukesha, WI) with an axial single shot echoplanar imaging sequence and cardiac gating for each slice ( $\mathrm{TE}=89 \mathrm{~ms}, \quad 80 \times 2 \mathrm{~mm}$ thick slices, $2 \times 2 \mathrm{~mm}$ in-plane resolution). Diffusion weighted gradients were applied in 120 independent directions (maximum b-value $=1127 \mathrm{sec} / \mathrm{mm}^{2}$ ). Data preprocessing was performed using the FMRIB Diffusion Toolbox and consisted of (1) affine registration to the reference $\left(b_{o}\right)$ image to correct head motion and eddy current distortions, (2) $b_{o}$ fieldmap unwarping, (3) brain extraction, and (4) diffusion tensor fitting. Voxel-wise statistical analysis of radial diffusivity (RD), axial diffusivity (AD), mean diffusivity (MD), and fractional anisotropy (FA) values was carried out using Tract-Based Spatial Statistic (TBSS) (Smith et al., 2006), and non-parametric permutation testing (500o permutations). The effects of genotype on white matter structure were examined in a multiple regression model with genotype as the covariate of interest and age and sex as nuisance covariates. Significance was measured at $p<0.05$, whole-brain corrected for multiple comparisons, using false discovery rate (FWE) and threshold-free cluster enhancement (TFCE). To relate our structural findings to behavior, working memory (WM) performance was assessed in a subset of 67 participants using the $n$-back task, a well-validated measure linked to prefrontal cortex function (Callicott et al., 1999). The main effects of genotype, $\mathrm{RD}$, and genotype by $\mathrm{RD}$ interaction on working memory performance were examined in the context of a multiple regression model with age and sex as nuisance covariates.

Results: We observed significant genotype-dependent white matter tract differences in $\mathrm{RD}$ (Val/Val $>$ Met carriers), which were particularly pronounced in the genu and body of the corpus callosum. Significant differences were also seen in the bilateral superior longitudinal fasciculus, left anterior thalamic radiation, and bilateral posterior thalamic radiation. No impact of the variant on measures of $\mathrm{MD}$ or $\mathrm{AD}$ was observed. Further analyses revealed a significant genotype by RD interaction effect on WM, suggesting that in $\mathrm{Val} / \mathrm{Val}$ subjects, individuals with higher diffusivity in the genu of the corpus callosum have worse working memory performance $(p<0.036)$. No significant main effects of genotype or callosal RD on working memory performance were observed. Post-hoc comparison of genotypestratified samples suggested a significant negative correlation of genu $\mathrm{RD}$ and working memory performance in Val/Val subjects (Pearsons's $r=-0.37, \mathrm{p}=0.018)$, but not the Met allele carriers $(r=.16, \mathrm{p}=0.43)$. Discussion: We observed significant effects of the BDNF $\mathrm{Val}^{6}{ }^{6} \mathrm{Met}$ polymorphism on white matter integrity in healthy human subjects, particularly in the genu and body of the corpus callosum. While the observed effects are not readily interpretable in the context of the known functionality of the SNP on BDNF secretion, our findings are in line with prior data suggesting microstructural deficits in the corpus callosum in disorders associated with this variant. The significant interaction between $B D N F$ genotype and $\mathrm{RD}$ underscores the behavioral relevance of our findings, suggesting deficits in working memory function in Val/Val subjects with higher callosal diffusivity. As increases in RD are sensititve markers for myelination deficits related to genetic (Song et al., 2002) and environmental (Song et al., 2005) factors, our finding might suggest a risk mechanism involving olygodendrocyte dysfunction.

Disclosure: H. Tost: None. T. Alam: None. M. Geramita: None. C. Rebsch: None. B. Kolachana: None. H. Lemaitre: None. A. Barnett: None. D. Dickinson: None. D. Weinberger: None. S. Marenco: None.

\section{Elucidating Functional Relationships between Ventral Striatal Dopamine Release and Prefrontal Cortical-striatal Activity in Human Reward Circuitry with fMRI and [11C]raclopride PET: Preliminary Data \\ Michael Travis*, Erika Forbes, N. Scott Mason, Jorge Almeida, Silvia Batezati, Natalie Velasquez, Eric Rodriguez, Michael Himes, Gordon Frankle, Rajesh Narendran, Mary Phillips}

Western Psychiatric Institute and Clinic, Pittsburgh, PA

Background: Alcohol and other substance use disorders, unipolar depression, bipolar disorder and attention-deficit/hyperactivity 


\section{S370}

disorder (ADHD) are amongst the most significant causes of morbidity and mortality. Abnormal function in reward systems is associated with these major psychiatric disorders. Dopamine (DA) release in the ventral and dorsal striatal regions, is important in reward processing. Understanding the relationship between functional activation and dopamine release will both help develop novel treatments and inform preventative strategies.

Methods: Subjects were 11 healthy volunteers, 2 male and 9 females, 18 to 35 years old. 8 subjects, ( 2 male, 6 female, mean age $23.2 \pm 2.7$ ), underwent an fMRI scan during a monetary reward task and four bolus plus constant infusion [11C]raclopride PET scans, each separated by a week: one at rest/baseline, one during a motor control task, one during the same monetary reward task as for the fMRI and a final $[11 \mathrm{C}]$ raclopride PET scan after administration of oral amphetamine $(0.5 \mathrm{mg} / \mathrm{kg}$ ) to displace [11C] raclopride. 3 subjects, (3 females, mean age $23 \pm 2$ years), underwent the same protocol but with no postamphetamine scan. The reward task was a block design for the fMRI comprising a card guessing game in which outcome was positive or negative for each trial. Upon receiving positive feedback subjects were required to respond via button press (either index or middle finger) to engage consummatory processes to elicit activation and DA release. There were also control blocks, during which participants were instructed to simply make alternating button presses during the presentation of an ' $\mathrm{x}$ '. This task reliably activates the ventral striatum (VST), head of caudate and the cortical region BA9 to reward. For the PET scans the task was split between two scans into the reward component, (reward and control), and the motor component, (control only). Both PET and fMRI data was analyzed as reward vs motor.

Results: fMRI - there were Significant BOLD signal changes in the VST bilaterally to reward ( 59 voxels, MNI co-ordinates $x=12, y=9, z=0$, $\mathrm{t}=7.57 ; \mathrm{z}=4.4, \mathrm{p}=0.001$, corr and MNI co-ordinates 44 voxels, $\mathrm{x}=-9$, $\mathrm{y}=6, \mathrm{z}=0, \mathrm{t}=4.77 ; \mathrm{z}=3.44, \mathrm{p}=0.002$, corr.) versus motor control task. Similar signal changes to reward versus control were also seen in the caudate head, ( 71 voxels, MNI co-ordinates $x=12, y=9, z=0$, $\mathrm{t}=7.57 ; \mathrm{z}=4.4, \mathrm{p}=0.001$ corr and 64 voxels, MNI co-ordinates $\mathrm{x}=-12$, $\mathrm{y}=9, \mathrm{z}=3, \mathrm{t}=4.77 ; \mathrm{z}=3.44, \mathrm{p}=0.003$, corr. $)$, and the putamen, (165 voxels, $M N I$ co-ordinates $x=15, y=9, z=0, t=6.8 ; z=4.18, p=0.003$ corr and 206 voxels, MNI co-ordinates $\mathrm{x}=-18, \mathrm{y}=18, \mathrm{z}=\mathrm{0}, \mathrm{t}=5.38$; $\mathrm{z}=3.69 \mathrm{p}=0.004$, corr.). PET - There was significantly increased $\left[{ }_{11 C} \mathrm{C}\right.$ raclopride displacement, (decreased $\left[{ }_{11} \mathrm{C}\right]$ raclopride binding), to the motor vs baseline in the left ventral striatum, $(-5.3 \pm 6.2 \% \mathrm{p}=0.02)$ and the left posterior putamen, $(-5.0 \pm 6.5, \mathrm{p}=0.02)$, but there was no significant displacement of $[11 \mathrm{C}]$ raclopride in the baseline vs reward condition. For motor vs reward there was a significant increase in $[11 \mathrm{C}]$ raclopride binding, $(5.4 \pm 7.3, \mathrm{p}=0.04)$ in the left anterior putamen and a numerical increase in $[11 \mathrm{C}]$ raclopride binding in all regions in this condition which did not reach significance There were no significant differences on the right. Importantly the degree of variability was high in all regions and conditions. Based on the neural circuitry of the reward system, we examined the relationship between the magnitude of the BOLD response in left BA9 and the motor vs reward $[11 \mathrm{C}]$ raclopride displacement in the left VST. There was a significant inverted $U$ shaped relationship between $\mathrm{BA}_{9}$ bold response and VST dopamine release, $(\mathrm{R}=0.62, \mathrm{p}=0.02)$, not present in other regions.

Discussion: These promising preliminary data suggest a complex relationship between VST dopamine response and magnitude of BA9 $\mathrm{BOLD}$ response during reward. When BA9 shows little response there is little or no DA release in the VST. As BA9 BOLD signal increases there is evidence that DA release in the VST initially increases but then decreases. The complexity of this relationship may underlie the conflicting findings from previous studies and may reflect interindividual differences in personality. Further work will include increased number of subjects, functional connectivity analysis of fMRI data, inclusion of pharmacological challenge data and determining the relationship between multi-modal imaging findings and impulsivity measures.
Disclosure: M. Travis: None. E. Forbes: None. N. Mason: None. J. Almeida: None. S. Batezati: None. N. Velasquez: None. E. Rodriguez: None. M. Himes: None. G. Frankle: None. R. Narendran: None. M. Phillips: None.

153. Effects of an Alpha 7-Nicotinic Agonist on Default Network Activity in Schizophrenia

Jason Tregellas*, Jody Tanabe, Donald Rojas, Shireen Shatti, Ann Olincy, Lynn Johnson, Laura Martin, Ferenc Soti, William Kem, Sherry Leonard, Robert Freedman

University of Colorado Denver School of Medicine, Denver, CO

Background: 3-(2,4-Dimethoxybenzylidene)-anabaseine (DMXB-A) is a partial agonist at $\alpha 7$ nicotinic acetylcholine receptors that has been evaluated clinically for treatment of schizophrenia. The current study examined the effects of DMXB-A on default network activity as a biomarker for drug effects on pathological brain function associated with schizophrenia.

Methods: Placebo and two doses of DMXB-A were administered in a randomized, double-blind crossover design during a one-month Phase-2 study of DMXB-A. Functional magnetic resonance imaging (fMRI) was performed on 16 non-smoking patients with schizophrenia while they performed a simple eye movement task. Independent component analysis was used to identify the default network component. Default network changes were evaluated in the context of a polymorphism in CHRNA7, the $\alpha 7$-nicotinic acetylcholine receptor subunit gene, which was previously found to be associated with schizophrenia.

Results: Compared to placebo, both $150 \mathrm{mg}$ and $75 \mathrm{mg}$ b.i.d. DMXB-A altered default network activity, including a reduction in posterior cingulate, inferior parietal cortex and medial frontal gyrus activity, and an increase in precuneus activity. The most robust difference, posterior cingulate activity reduction, was affected by CHRNA7 genotype.

Discussion: The observed DMXB-A-related changes are consistent with improved default network function in schizophrenia. Pharmacogenetic analysis indicates mediation of the effect through the $\alpha 7$-nicotinic receptor. These results further implicate nicotinic cholinergic dysfunction in the disease and suggest that default network activity may be a useful indicator of biologic effects of novel therapeutic agents.

Disclosure: J. Tregellas: None. J. Tanabe: None. D. Rojas: None. S. Shatti: None. A. Olincy: None. L. Johnson: None. L. Martin: None. F. Soti: None. W. Kem: None. S. Leonard: None. R. Freedman: None.

\section{Dopamine Transmission in Comorbid Schizophrenia and Substance Dependence \\ Nina Urban*, Mark Slifstein, Judy Thompson, Xiaoyan Xu, Ragy Girgis, Jill Harkavy-Friedman, Yael Beckerman, Beatriz Alvarez, Roberto Gil, Marc Laruelle, Anissa Abi-Dargham}

\section{Columbia University, New York, NY}

Background: $50-80 \%$ of patients with schizophrenia have a comorbid diagnosis of substance abuse or dependence, and this comorbidity predicts worse treatment outcome. Dopamine (DA) plays a role in the pathophysiology of both schizophrenia and addiction. Imaging studies have shown that DA is increased in the associative striatum (AST), and specifically in the precommissural caudate, in schizophrenia, while it is blunted in addiction, predominantly in the ventral striatum (VST). We hypothesized that subjects suffering from both schizophrenia and addiction may have both alterations. Here, we assessed D2 receptors and DA release in striatal subregions with $[11 \mathrm{C}]$ raclopride PET and the amphetamine challenge paradigm.

Methods: 12 medically healthy patients with schizophrenia and cannabis $(n=12)-$, alcohol $(n=11)-$, and/or cocaine $(n=2)$ depen- 
dence (DD, age $28.9 \pm 8.5$ years) and 12 healthy volunteers (HC, age $28.1 \pm 6.3$ years) matched for age, sex and ethnicity (2 F, 10 M) were included in the study. Clinical severity of psychopathology was rated with the positive and negative syndrome scale (PANSS) at baseline and after amphetamine. Patients were free from both medications and substances for at least 3 weeks prior to the study. Subjects were scanned with a $[11 \mathrm{C}]$ raclopride bolus plus constant infusion paradigm, using an HR + camera before and after injection of d-amphetamine $(0.3 \mathrm{mg} / \mathrm{kg})$ iv. Regions of interest (ROI) were drawn on each subject's MRI and transferred to the coregistered PET. ROIs included pre- and post commissural caudate and putamen (preDCA, preDPU, postCA, corresponding to the AST; and the postPU), the VST, and the striatum as a whole (STR). Cerebellum was used as a reference region. Equilibrium analysis was used to derive the specific to non-displaceable equilibrium partition coefficient BPND (unitless) as ROI activity/ CER activity -1 at steady-state. The percent change after amphetamine ( $\triangle B P N D)$ was analyzed by paired t-tests, a linear mixed model and RMANOVA. Relationship between clinical parameters and PET data were analyzed with Pearson-Product-Moment and Spearman's Rank Order correlation coefficients.

Results: There were no differences in injected dose or mass of the radiotracer between groups. D2 binding (BPND) was significantly lower in DD in preDPU and postPU compared to HC $(\mathrm{p}=0.05)$; when analyzed with RMANOVA, there was a trend to lower $\mathrm{D}_{2}$ binding in the sum of all regions $(\mathrm{p}=0.11)$. DA release $(\triangle \mathrm{BPND})$ compared between groups was significantly lower for DD than $\mathrm{HC}$ in preDPU, postPU, and AST, and at trend difference in all other ROIs except for VST:

preDPU: $-4 \% \pm 6 \%$ vs. $-10 \% \pm 6 \%, p=0.04$

postPU: $-10 \% \pm 6 \%$ vs. $-18 \% \pm 7 \%, \mathrm{p}=0.01$

AST: $-3 \% \pm 7 \%$ vs. $-9 \% \pm 5 \%, p=0.05$

VST: $-6 \% \pm 7 \%$ vs. $-10 \% \pm 9 \%, p=0.27$

STR: $-7 \% \pm 8 \%$ vs. $-12 \% \pm 6 \%, \mathrm{p}=0.12$

Linear mixed modeling with ROI as repeated variable ( 5 regions) and diagnosis as between subject variable showed a significant effect of diagnosis: $\mathrm{F}(1,22)=5.97, \mathrm{p}=0.02$ (RMANOVA: $\mathrm{F}(1,22)=5.975, \mathrm{p}=0.02$ ). As previously observed in patients with schizophrenia, and despite the low levels of dopamine release, the amphetamine induced change in positive symptoms correlated with the magnitude of dopamine release, $\triangle B P N D$, in the postCA $(\mathrm{r}=0.74, \mathrm{p}=0.02)$; and at trend level in the preDCA $(r=0.61, p=0.06)$. The age of onset of substance abuse was positively correlated with $\triangle B P N D$ in STR as a whole $(r=0.60$, $\mathrm{p}=0.04)$, and at trend level in preDPU $(\mathrm{r}=0.49, \mathrm{p}=0.10)$ and AST $(\mathrm{r}=0.51, \mathrm{p}=0.09)$. When controlling for "age" as a co-variate, the relationship became significant in the AST $(\mathrm{p}=0.04)$ and remained significant in the STR $(p=0.04)$. There was no correlation of illness duration (schizophrenia or substance use), nor time since last drug use with either BPND or $\triangle \mathrm{BPND}$.

Discussion: This report presents the first study of the striatal dopaminergic system in patients with schizophrenia and co-morbid substance dependence (dual diagnosis). Unlike schizophrenia, dual diagnosis was associated with low D2 in some, and low DA release in most striatal subregions, with VST least affected. Amphetamine induced worsening in psychosis was associated with greater DA release, as observed in patients with SCZ. These findings together indicate that despite overall blunted levels of release, patients may be sensitized to dopaminergic transmission. In addition, early onset of drug use was associated with more severe alterations in dopamine transmission, regardless of duration of use or age, possibly reflecting a negative effect of drug use on the adolescent brain. Further research is needed to characterize the time course of this decline in DA function in comorbid SCZ and drug dependence and its effects on treatment response.

Funded by NIDA.

Disclosure: N. Urban: None. M. Slifstein: Part 1; GlaxoSmithKline, Amgen, Intra-Cellular Therapies. J. Thompson: None. X. Xu: None. R. Girgis: Janssen, Lilly, Forest, Elsevier Science. Part 2; Lilly. J. HarkavyFriedman: None. Y. Beckerman: None. B. Alvarez: None. R. Gil: None. M. Laruelle: None. A. Abi-Dargham: Part 1; BMS-Otsuka, Boehringer-
Ingelheim, Lundbeck, Sepracor, Merck. Part 2; BMS-Otsuka. Part 3; BMS-Otsuka.

155. Effects of a Common Variant in $\mathrm{GSK}_{3} \beta$ on Hippocampal Volume in Healthy Human Volunteers

Beth A. Verchinski*, Becky Inkster, Catherine Gambale, Thomas E. Nichols, Paul M. Matthews, Brad Zoltick, Fengyu Zhang, Aaron L. Goldman, Venkata Mattay, Daniel Weinberger, Heike Tost

GCAP/NIMH/NIH, Bethesda, MD

Background: The glycogen synthase kinase- $3 \beta\left(\mathrm{GSK}_{3} \beta\right)$ gene, a candidate gene for major depressive disorder, schizophrenia, and the efficacy of neuropsychiatric drugs, is highly expressed in the mammalian hippocampus and temporal lobe. A common intronic polymorphism in $\mathrm{GSK}_{3} \beta$ (rs6438552) has been associated with alterations in gene transcription and disease-related brain structural phenotypes. Notably, while a previous study in MDD patients and healthy volunteers (Inkster et al., 2009 1) demonstrated a significant rs 6438552 genotype by group interaction effect on hippocampal gray matter volume, the observed data trend in the control subjects $(A A>A G>G G)$ failed to reach significance, an outcome that might relate to limitations in study power rather than to a lack of a true association. To examine this possibility, we studied the impact of rs6438552 on hippocampal gray matter volume in a large and independent sample of healthy volunteers using two different morphometric analysis techniques.

Methods: One-hundred-seventy-five healthy Caucasian volunteers without a history of psychiatric illness were included in this study (mean age $=33.0 \pm 11$ years, 78 males, 97 females, $\mathrm{n}=69 \mathrm{~A} / \mathrm{A}, \mathrm{n}=78$ $\mathrm{A} / \mathrm{G}, \mathrm{n}=28 \mathrm{G} / \mathrm{G})$. High-resolution structural MRI scans were acquired on a 1.5 Tesla General Electric scanner (Milwaukee, WI, USA) using a three-dimensional T1-weighted spoiled gradient recalled (SPGR) sequence with the following sequence specifications: repetition-time $(\mathrm{TR})=24 \mathrm{~ms}$, echo-time $(\mathrm{TE})=5 \mathrm{~ms}$, flip angle $(\mathrm{FA})=45^{\circ}$, matrix size $=256 \times 256$ pixels, field of view $(\mathrm{FOV})=24 \mathrm{~cm}, 124$ sagittal slices, $0.94 \times 0.94 \times 1.5 \mathrm{~mm} 3$ voxel resolution. Prior to data processing, all images were inspected both visually and quantitatively to ensure their quality. Automated image processing was performed using the software packages Freesurfer (release 4.5.0) and VBM5 (as implemented in the Statistical Parametric Mapping software, SPM5). Data preprocessing in Freesurfer consisted of (1) image resampling, (2) nonuniformity intensity normalization, (3) affine registration to the Montreal Neurological Institute (MNI) space, (4) intensity normalization and (5) skull stripping. Hippocampal volumes were extracted after delineating gross brain anatomy into cortical and subcortical labels using the combined information of image intensity, probabilistic atlas location, and the local spatial relationships between subcortical structures. For VBM, image preprocessing consisted of (1) image registration to MNI space, (2) tissue classification, (3) correction for image intensity non-uniformity, (4) noise correction, (5) modulation, and (6) smoothing using a $10 \mathrm{~mm}$ full-width-at-half-maximum (FWHM) isotropic Gaussian kernel. In both methods, effects of genotype on hippocampal volume were examined in a multiple regression model with genotype as the covariate of interest, and age, sex, and total gray matter volume as nuisance covariates. Significance was measured at $\mathrm{P}<0.05$ (for $\mathrm{VBM}$ : corrected for multiple comparisons using family-wise error, FWE, within the examined region of interest).

Results: In line with prior published results, our Freesurfer analysis provided evidence for a significant allele-load dependent decrease in total hippocampal volume in carriers of the minor allele in rs6438552 ( $A A>A G>G G ; t=-2.103, P=0.012)$. This finding was replicated in our voxel-based analysis, which demonstrated a significant effect of genotype on regional gray matter volume, with a peak voxel difference that mapped onto the left hippocampus (AA $>A G>G G ; t=3.1$, $\mathrm{P}=0.05$ FWE corrected, MNI coordinates: $\mathrm{x}=-29, \mathrm{y}=-20, \mathrm{z}=-12)$. Discussion: Consistent with prior data, we observed significant effects of a common variant in $\mathrm{GSK}_{3} \beta$ on hippocampal structure with a relative 
decrease of volume in carriers of the minor allele. The replication of the directionality of this association, as reported by Inkster et al., in a large and independent sample of subjects, underscores that the association of this variant with brain structural phenotypes in healthy humans is likely true. While the potential clinical implications of our data require further study, the replication of the finding with a second morphometric technique suggests that the reported effects are not an artifact of our statistical and image processing methods.

References

1. Inkster B, Nichols TE, Saemann PG, Auer DP, Holsboer F, Muglia P et al. Association of GSK3 beta polymorphisms with brain structural changes in major depressive disorder. Arch Gen Psychiatry 2009; 66(7): 721-728.

Disclosure: B. Verchinski: None. B. Inkster: None. C. Gambale: None. T. Nichols: None. P. Matthews: Part 5; GlaxoSmithKline. B. Zoltick: None. F. Zhang: None. A. Goldman: None. V. Mattay: None. D. Weinberger: None. H. Tost: None.

156. The BDNFval66met Polymorphism: Genetic Susceptibility for an Intermediate Phenotype Related to Alzheimer's Disease in Healthy Individuals

Aristotle Voineskos*, Jason Lerch, Daniel Felsky, Dielle Miranda,

Nancy Lobaugh, Benoit Mulsant, Bruce Pollock, James Kennedy

Centre for Addiction and Mental Health; University of Toronto, Toronto, ON, Canada

Background: The brain derived neurotrophic factor (BDNF) val66met polymorphism may predict risk for Alzheimer's disease. However, genetic association studies of the BDNF gene with Alzheimer's disease have produced equivocal results. Imaging-genetics strategies in healthy individuals can serve to characterize the effects of genetic risk variants on the brain.

Methods: Using high resolution magnetic resonance imaging and diffusion tensor tractography in 69 healthy individuals across the adult lifespan (19-82 years of age), we examined the interaction of the BDNF val66met variant with age in relation to cortical thickness, white matter tract integrity,and cognitive performance.

Results: The BDNF val66met interacted with age to predict: i) cortical thickness (prominently at entorhinal cortex and temporal gyri), ii) fractional anisotropy of white matter tracts, prominently at white matter tracts connecting to medial temporal lobe (cingulum bundle and inferior longitudinal fasciculus), and iii) episodic memory performance. For each of these findings, the pattern was similar: val/ val individuals in late-life were susceptible, and in early adult life, metallele carriers demonstrated susceptibility.

Discussion: The BDNF gene confers risk in an age-dependent manner on the brain structures and cognitive functions that are consistent with the neural circuitry vulnerable in the earliest stages of Alzheimer's disease. Our novel findings provide convergent evidence in vivo, for a BDNF genetic mechanism of susceptibility in an intermediate phenotype related to Alzheimer's disease.

Disclosure: A. Voineskos: Part 4; APA/APIRE Astra Zeneca Young Minds In Psychiatry Award. J. Lerch: None. D. Felsky: None. D. Miranda: None. N. Lobaugh: None. B. Mulsant: Bristol-Myers Squibb and Wyeth. B. Pollock: Part 1; Lundbeck International Neuroscience Foundation. J. Kennedy: None.

157. Decreased Striatal and Prefrontal Dopaminergic Responses in Active Cocaine Dependent Subjects

Gene-Jack Wang*, Nora Volkow, Frank Telang, Dardo Tomasi, Jean Logan, Christopher Wong, Millard Jayne, Panayotis Thanos, Joanna Fowler

\section{Brookhaven National Laboratory, Upton, NY}

Background: Cocaine addiction is associated with loss of control and compulsive drug use. We (Volkow et al., 1997) and others (Martinez et al, 2007) using PET and [C-11] raclopride (dopamine D2/3 receptor radioligand sensitive to competition with endogenous dopamine) have shown markedly blunted dopamine increases after intravenous stimulant administration in detoxified cocaine dependent subjects. Here we assessed brain dopamine responses in active cocaine dependent subjects.

Methods: Nineteen active cocaine dependent subjects $(45 \pm 3$ y/o) and 23 age- and gender-matched normal control subjects were evaluated with PET and [C-11] raclopride with placebo and the stimulant drug methylphenidate $\left(0.5 \mathrm{mg} / \mathrm{kg}\right.$, i.v.). $\mathrm{D}_{2} / 3$ receptor $\left(\mathrm{D}_{2} \mathrm{R}\right)$ availability (non-displaceable binding potential) was analyzed with the region of interest and statistical parameter mapping (SPM) methods. Self-report ratings (1-low to 10-high) of methylphenidate effect were recorded.

Results: Cocaine dependent subjects had lower D2R availability with placebo in caudate $(2.4 \pm 0.32)$, putamen $(2.87 \pm 0.32)$ and ventral striatum $(2.43 \pm 0.43)$ than controls (caudate: $2.65 \pm 0.38$, putamen: $3.26 \pm 0.49$, ventral striatum: $2.86 \pm 0.37$ ). Methylphenidate administration induced greater decrements in $\mathrm{D}_{2} \mathrm{R}$ availability (dopamine release) in controls (putamen: $-14.8 \pm 12.7 \%, p<0.004$; VS: $-18.6 \pm 17 \%$, $\mathrm{p}<0.0002)$ than in cocaine dependent subjects in whom changes were not significant (putamen: $-2.8 \pm 12.5 \%$; ventral striatum: $+1.8 \pm 15.9 \%$ ). Control subjects reported greater ratings of "high" ( +5.5 \pm 2.1 , $\mathrm{p}<0.0001)$ and restlessness $(+3.8 \pm 2.8, \mathrm{p}<0.00001)$ than cocaine dependent subjects $(+2.6 \pm 2.1,+0.4 \pm 1.4)$ after methylphenidate. The correlation analysis showed that methylphenidate-induced more intense in "high" was correlated with greater dopamine release in ventral striatum $(\mathrm{r}=0.37, \mathrm{p}<0.01)$ and changes in restlessness were correlated with greater dopamine release in putamen $(r=0.4$, $\mathrm{p}<0.009$ ). SPM corroborated the greater methylphenidate-induced dopamine release in striatum in controls than in cocaine dependent subjects ( $\mathrm{p}<0.005$, small volume correction, multiple comparisons). In addition, SPM revealed methylphenidate-induced dopamine release in prefrontal cortex (BA10, $\mathrm{p}<0.001$, cluster-level corrected for multiple comparisons) in controls but not in cocaine dependent subjects. The dopamine releases in controls were also greater than in cocaine dependent subjects in superior prefrontal cortex (BA10, $\mathrm{p}<0.009$ uncorrected). The correlations analysis showed that changes in high were correlated with methylphenidate-induced dopamine release in $\mathrm{BA} 10(\mathrm{r}=0.31, \mathrm{p}<0.05)$.

Discussion: These results in active cocaine dependent subjects expanded prior findings of decreased striatal dopamine responses in detoxified cocaine dependent subjects. They also identify attenuated responses in the prefrontal cortex, which are consistent with disrupted mesocortical dopamine function.

NIDA/NIAAA/GCRC, DOE/OBER (infrastructure support).

Disclosure: G. Wang: None. N. Volkow: None. F. Telang: None. D. Tomasi: None. J. Logan: None. C. Wong: None. M. Jayne: None. P. Thanos: None. J. Fowler: None.

158. Effects of Memantine and Donepezil Treatment on Longitudinal Changes of Hippocampal Structure in Dementia of the Alzheimer Type

Lei Wang*, Neha Mehta, Ilya Karagodin, M. Faisal Beg, John Morris, John Csernansky, James Galvin

Northwestern University Feinberg School of Medicine, Chicago, IL

Background: Treatment of moderate to severe Alzheimer Disease (AD) with a combination therapy of donepezil, a cholinesterase inhibitor, and memantine, an NMDA antagonist, has been shown to provide cognitive, functional, global outcome, and behavior symptomatic benefits, and is well tolerated. Other studies also suggest that memantine may provide benefits in mild stages of disease. Previously, we showed that hippocampal surface deformities were present early in the course of illness in individuals with very mild dementia of the Alzheimer type (DAT), and were correlated with a poor response to donepezil treatment, but treatment with donepezil did not alter the progression of hippocampal deformation in individuals with DAT as 
compared to untreated individuals. In this study, we compared longitudinal changes in the hippocampal structure in mild DAT patients treated with combination of memantine and donepezil, donepezil alone, untreated DAT patients and cognitively normal individuals.

Methods: Participants from the previously published study consisted of 56 cognitively normal individuals, 18 very mild DAT patients treated with donepezil alone and 14 untreated patients with mild DAT. Fourteen patients with mild DAT treated with combination of memantine and donepezil were included in this study. Drug treatment followed published recommendations with target does of $10 \mathrm{mg} /$ day donepezil and $20 \mathrm{mg} /$ day memantine based on tolerability. At each longitudinal assessment, the Clinical Dementia Rating (CDR) scale was used as the primary measure of dementia severity, and the Alzheimer Disease Assessment Scale-cognitive subscale (ADAS-Cog) total score was used as the primary clinical outcome measure. For all participants, MPRAGE sequences were collected approximately two years apart on 1.5-Tesla Siemens Vision systems. Longitudinal large-deformation diffeomorphic metric mapping (LDDMM) was applied to all scans to generate hippocampal surfaces and surface zones corresponding to the $\mathrm{CA1}$, subiculum and remainder (CA2-4, dentate gyrus) cellular subfields. Rate-of-change of total hippocampal volume and rates-of-change of subfield displacements were computed.

Results: At baseline, main group effects were significant for hippocampal volume and all subfield deformations. Between-group contrasts showed differences between DAT and cognitively normal individual; however, the 3 DAT groups (untreated, donepezil-treated and combination-treated) did not differ from each other on any of the hippocampal measures. Longitudinally, there were no significant main group effects in the rate of change of hippocampal volume or subfield deformation. Again, although DAT differed from cognitively normal individuals, the 3 groups of DAT patients did not differ from each other in rates of change of hippocampal volume and CA1 and subiculum subfield deformation. We further combined the DAT patients receiving all treatments together and grouped them into 3 subgroups (equal sample size) according to the rates of change of their ADAS-Cog scores. Comparing with the patients showing most negative ADAS-Cog rate of change (improving), patients with most positive ADAS-cog rate of change (worsening) showed significantly accelerated hippocampal volume atrophy over time, while patients with intermediate, near-zero ADAS-cog rate of change (stable) also accelerated hippocampal volume atrophy over time, although at a less severe level (trend).

Discussion: The progression of hippocampal deformity may be altered in DAT patients who respond to treatment with memantine and/or donepezil. Cognitive improvement and slowing of disease progression in $\mathrm{AD}$ as a result of drug treatment may be dependent upon factors that pre-determine who will respond to treatment. Understanding of these factors way lead to more appropriate therapy.

Disclosure: L. Wang: None. N. Mehta: None. I. Karagodin: None. M. Beg: None. J. Morris: Part 1; Wyeth Elan, AstraZeneca, Bristol-Myers Squibb, Genentech, Eli Lilly, Merck, Novartis, Pfizer, Schering Plough. J. Csernansky: Sanofi-Aventis, Eli Lilly. J. Galvin: Pfizer, Eisai, Novartis, Forest, Medivation, Avanir. Part 2; Washington University, New York University. Part 4; Novartis, Eisai, Pfizer.

159. Interaction between Brain Derived Neurotrophic Factor (BDNF) and Gonadal Steroid Hormones affects Hippocampal Function during Working Memory

Shau-Ming Wei*, Peter Schmidt, Erica Baller, Philip Kohn, Jonathan S. Kippenhan, Bhaskar Kolachana, David Rubinow, Daniel

Weinberger, Karen Berman

NIH, Bethesda, MD

Background: There is considerable evidence that the gonadal steroid hormones, estrogen and progesterone, influence activity in the CNS, particularly in the hippocampus, and that they modulate hippocampally-dependent cognitive functions 1,2. The neural effects of these ovarian hormones may be impacted by genetic variability that affects molecular pathways that interact with gonadal steroids, such as brain derived neurotrophic factor (BDNF). Preclinical experiments have demonstrated that the neurotropin BDNF mediates the actions of gonadal steroids via a number of common characteristics at the molecular and cellular levels 3,4. In BDNF transgenic rodents, BDNF genotype interacts with the ovarian cycle to affect spatial memory as well as BDNF expression in the hippocampus 5. While interactions between steroid hormones and BDNF are well documented on the molecular level, there is little behavioral and neural circuitry data in humans. To elucidate the relationship between the uniquely human BDNF Val66Met polymorphism and gonadal steroids in humans, we tested for BDNF-gonadal hormone interaction on the working memory network using positron emission tomography (PET) and an incisive pharmacologically-controlled hormonal manipulation protocol.

Methods: Thirty-four healthy, regularly menstruating women with no psychiatric history (23 Val homozygotes, mean age $=33.1 ; 11$ Met carriers, mean age $=38.9 ; \mathrm{p}=0.06)$ underwent PET scanning during each of three different hormone conditions: (1) ovarian suppression (i.e. temporary menopause) induced by the gonadotropinreleasing hormone agonist leuprolide acetate (Lupron), (2) Lupron plus estradiol replacement, and (3) Lupron plus progesterone replacement. BDNF Val66Met genotype was determined via TaqMan assay. Regional cerebral blood flow (rCBF) was measured with oxygen15 water PET (10mCi H215O/scan) during a series of fourteen 60 -second scans that alternated between a o-back (obk) sensorimotor control task and a 2-back (2bk) working-memory task. Following attenuation correction, anatomical normalization, and smoothing with a $10 \mathrm{~mm}$ Gaussian kernel, the obk scans and the $2 \mathrm{bk}$ scans of each subject were averaged separately and entered into group-level analyses using SPM5. A whole brain random-effects analysis was performed using subjects as a dependent measure and age as a nuisance covariate. Between-group differences in working memory activation (2bk-obk) were assessed with a statistical threshold of $\mathrm{p}<0.002$, uncorrected.

Results: There was no significant difference in working memory performance across hormone conditions or between the two genotype groups. However, an F-test on the PET data showed significant hormone-BDNF interactions on working memory activation in hippocampal/parahippocampal regions bilaterally. This effect was primarily attributable to genotype differences between Lupron alone and estrogen replacement conditions. Specifically, the Val homozygotes showed the typical working memory deactivation pattern in bilateral hippocampal/parahippocampal rCBF during temporary menopause (Lupron alone), but these regions showed abnormal engagement during estrogen replacement. However, the activity pattern was reversed for the Met carriers, showing typical deactivation during estrogen replacement and abnormal engagement during Lupron alone.

Discussion: Our data demonstrate interactions between gonadal hormone state and BDNF genotype in the hippocampal region during working memory. Previous studies have observed that BDNF Met carriers have abnormal hippocampal engagement during both working memory6 and episodic memory7, and our results suggest that these effects are further mediated by gonadal steroids. Hormonal levels influence the expression of BDNF and its receptor8, and our data are consistent with evidence that BDNF and gonadal steroids modulate similar neural mechanisms in the hippocampus. While the relationship between BDNF functional level and gonadal hormones is likely to be complex, this is the first study in humans to demonstrate a neurophysiologically-based BDNF genotype-hormonal interaction in a cognitive setting.

References:

1. Sherwin B et al. 1994; New York Acad Sciences. 743: 213-231. 2. Daniel J et al. 1997; Hormones and Behavior 32(3): 217-225. 


\section{S374}

3. Scharfman H E et al. 2006; Frontiers in Neuroendocrinology 27(4): 415-435.

4. Sohrabji F et al. 2006; Frontiers in Neuroendocrinology 27(4): 404-414.

5. Spencer JL et al. 2010; PNAS 107(9): 4395-400.

6. Egan MF et al. 2003; Cell 112(2): 257-269.

7. Hariri AR et al. 2003; J. Neurosci. 23: 6690-6694.

8. Gibbs RB et al. 1999; Brain Research 844(1-2): 20-27.

Disclosure: S. Wei: None. P. Schmidt: None. E. Baller: None. P. Kohn: None. J. Kippenhan: None. B. Kolachana: None. D. Rubinow: None. D. Weinberger: None. K. Berman: None.

160. Regional Effects of Acute Benzodiazepine Challenge on Resting Perfusion Measured with Magnetic Resonance Arterial Spin-Labeling Daniel Wolf*, Amy Pinkham, James Loughead, Theodore Satterthwaite, Eve Overton, Jeffrey Valdez, Mark Elliott, Gersham Dent, Mark Smith, Ruben Gur, Raquel E. Gur

\section{University of Pennsylvania, Philadelphia, PA}

Background: Functional MRI techniques can help characterize neural mechanisms of pharmacological effects, as part of drug discovery programs and investigations of psychiatric pathophysiology. MR perfusion using arterial spin-labeling (ASL) provides a non-invasive method for examining regional patterns of absolute cerebral blood flow. To date, most pharmacological MRI studies have used BOLD fMRI, and MR perfusion has been emphasized mainly as a secondary measure to evaluate perfusion confounds in BOLD results. However, regional perfusion may also be a primary variable of interest in understanding drug effects. Here we examine acute effects of the benzodiazepine alprazolam on MR perfusion in healthy controls and first-degree relatives of patients with schizophrenia. We hypothesized that alprazolam would alter resting perfusion in regions associated with anxiety, arousal, and reward. As schizophrenia is associated with GABAergic abnormalities, we anticipated that alprazolam's GABAergic effects would induce perfusion abnormalities in family members with a genetic predisposition to schizophrenia, relative to controls.

Methods: 47 subjects (20 first-degree family members of patients with schizophrenia and 27 controls) received an acute pharmacological challenge with $1 \mathrm{mg}$ of oral alprazolam or placebo in a double-blind counterbalanced crossover design, preceding identical 3T MRI sessions approximately 1 week apart. Whole brain pulsed Arterial Spin-Label (PASL) perfusion MRI was used to measure absolute rates of cerebral blood flow (CBF) at rest with eyes open viewing a black screen. Perfusion images generated by surround subtraction of label/control pairs were used to construct absolute $\mathrm{CBF}$ images that were averaged across time to produce one average CBF image per participant. Average CBF maps were then spatially transformed into MNI space and smoothed (6 mm FWHM isotropic). To account for individual differences in whole brain perfusion when examining regional effects, each subject's voxelwise CBF values were first normalized by whole brain $\mathrm{CBF}$, and normalized data tested for drug and group effects. Statistically significant clusters $(\mathrm{p}<.01)$ were identified using nonparametric monte carlo simulation (AlphaSim) to correct for multiple voxelwise comparisons.

Results: Across all subjects, alprazolam reduced resting CBF in a cluster spanning posterior midbrain and posterior thalamus, and increased it within the nucleus accumbens. Drug effects in accumbens and midbrain/thalamus were not correlated across subjects, suggesting they may reflect different aspects of drug action. Across drug conditions, family members showed reduced resting CBF in a cluster extending from anterior thalamus into left insula, orbitofrontal cortex, and amygdala. Family members showed increased resting CBF in bilateral posterior STG. While these group differences tended to be stronger under alprazolam than placebo, the group $\mathrm{x}$ drug interaction did not yield statistically significant clusters with this wholebrain approach. Under alprazolam treatment, subjects with higher accumbens perfusion at rest showed faster but less accurate responses in a subsequent facial emotion memory task $(r=-.37)$.

Discussion: Resting MR perfusion using ASL successfully identified region-specific benzodiazepine effects. Benzodiazepine-induced reduction of thalamic CBF has been reported previously and related to sedating effects. Increased perfusion by alprazolam focally within the bilateral nucleus accumbens is a novel finding that may result from the known GABAergic disinhibition of mesolimbic dopamine signaling, and may relate to the addictive properties of benzodiazepines. Family members showed regional abnormalities in perfusion, which tended to be greater under alprazolam, but group differences in drug-induced perfusion effects were not significant. Utilizing larger samples, more strongly schizotypal family members, or focused region of interest analyses could enhance sensitivity. The pattern of drug and group effects found during resting perfusion differed from that seen in prior analyses of BOLD fMRI data from the same subjects. ASL perfusion may prove useful for developing drugs targeting specific neural circuits to ameliorate psychiatric symptoms, providing complementary information to BOLD fMRI and a non-invasive alternative to PET CBF. Disclosure: D. Wolf: Part 4; AstraZeneca Pharmaceuticals. A. Pinkham: None. J. Loughead: AstraZeneca, Merck. T. Satterthwaite: None. E. Overton: None. J. Valdez: None. M. Elliott: None. G. Dent: Part 5; AstraZeneca Pharmaceuticals. M. Smith: AstraZeneca Pharmaceuticals. R. Gur: Part 4; AstraZeneca, Pfizer. R. Gur: AstraZeneca, Pfizer.

\section{1. $\left[{ }^{11} \mathrm{C}\right]-\mathrm{R} 05013853$ : a New PET Radioligand for the Glycine Transporter TYPE 1}

Dean Wong*, Susanne Ostrowitzki, Yun Zhou, Daniela Alberati, Emmanuel Pinard, Vanessa Raymont, Meret Martin-Facklam, Carsten Hofmann, Nikhat Parkar, James Brasic, John Hilton, Robert Dannals, Edilio Borroni

\section{Johns Hopkins University, Baltimore, MD}

Background: Various lines of evidence suggest that hypofunction of glutamatergic transmission via $\mathrm{N}$-methyl-D-aspartate (NMDA) receptors may be implicated in the pathophysiology of central nervous system (CNS) diseases such as schizophrenia. One strategy to normalize the reduced function of NMDA receptor neurotransmission is to increase the availability of the obligatory co-agonist glycine at its modulatory site on the receptor through inhibition of glycine transporter type 1 (GlyT1). The development of a GlyT1 positron emission tomography (PET) radioligand will allow the determination of drug-induced receptor occupancy. In this study we present the pharmacologic characterization of $\mathrm{RO}_{5013853}$ and its evaluation as PET ligand for GlyT1 in non-human primates and humans.

Methods: Affinity and kinetic properties of RO5013853 for human recombinant and rat native GlyT1 were determined using $\left[{ }^{3} \mathrm{H}\right]$ RO5013853; autoradiographic studies were performed on adult male Wistar rat brains. $\left[{ }^{11} \mathrm{C}\right]-\mathrm{RO}_{5013853}$ radiosynthesis employed ${ }^{11} \mathrm{C}$ methylation. Baboon and human PET included administration of $\left[{ }^{11} \mathrm{C}\right]-$ RO5013853 i.v., 90 minutes of scanning, separation of metabolites using high-performance liquid chromatography (HPLC), and determination of radial arterial input function. Baboon PET was performed using a high-resolution research tomography (HRRT) PET scanner. Human PET was performed using HRRT or GE Advance PET scanner. PET data were analyzed using a 2-tissue 5-parameter $\left(2 \mathrm{~T}_{5} \mathrm{P}\right)$ model, a 1tissue 3-parameter model, and a graphic reference region method (Logan plot). The outcome variables were transport rate constant from vascular space to brain tissue $\left(K_{1}\right)$ and volume of distribution $\left(V_{t}\right)$. Results: $\mathrm{RO}_{5013853}$ binds with high affinity to both human recombinant and rat native GlyT1 (dissociation constant $\left[K_{d}\right]$ was $2.8 \pm 0.6$ and $2.4 \pm 0.4 \mathrm{nM}$ for the human and the rat transporters, respectively). Kinetic analysis demonstrated the reversibility of the binding of RO5013853 to both human and rat GlyT1. Rat autoradiographic studies showed that the distribution of the binding sites of $\left[{ }^{3} \mathrm{H}\right]-\mathrm{RO}_{5013853}$ corresponded to the known distribution of GlyT1. Baboon PET showed 
rapid brain uptake of $\left[{ }^{11} \mathrm{C}\right]-\mathrm{RO} 5013853$ with the highest uptake in the brain stem, pons, thalamus, and cerebellum, whereas the lowest uptake was observed in the occipital $>$ parietal and hippocampus, consistent with rodent autoradiography. A $2 \mathrm{~T}_{5} \mathrm{P}$ model best described the baboon tracer tissue kinetics. Pre-treatment with RG1678, a potent and noncompetitive inhibitor of GlyT1, produced a plasma-concentrationdependent blockade of the tracer in most regions. Scans carried out in healthy male human volunteers $(n=7$; aged 23-56 years) confirmed the ability of $\left[{ }^{11} \mathrm{C}\right]-\mathrm{RO} 5013853$ to image regions expressing GlyT1. Administration of $\left[{ }^{11} \mathrm{C}\right]-\mathrm{RO} 5013853(\sim 30 \mathrm{mCi})$, with an average specific activity of $12905 \pm 7857 \mathrm{mCi} / \mu \mathrm{mol}$, was shown to be well tolerated. HPLC metabolites were $50 \%$ at 90 minutes. Regions of interest (ROIs) determined using co-registered magnetic resonance imaging included cerebellum, pons, caudate, putamen, thalamus, orbital frontal, prefrontal, superior frontal, parietal cortex, and cingulate. A $2 \mathrm{~T}_{5} \mathrm{P}$ model best described the human tracer tissue kinetics, which was consistent with primate imaging. $\mathrm{K}_{1}$ was fairly homogenous across all ROIs, ranging between 0.03 and $0.04 \mathrm{~mL} / \mathrm{min} / \mathrm{mL}$, with the highest values in thalamus and lowest values in cortical areas. $V_{t}$ was highest in the pons (mean \pm SD: $2.59 \pm 0.22$ ) and lowest in the temporal cortex $(0.85 \pm 0.04)$. To evaluate test-retest variability, each of the human subjects (either first-scan HRRT and second-scan GE Advance [n=2], or both scans GE Advance $[n=5]$ ) received two PET scans under the same conditions. Test-retest variability for $\mathrm{K}_{1}$ ranged from $13 \%$ to $18 \%$ (GE data only) across all regions, while $V_{t}$ had a test-retest variability of $3-6 \%$ across all regions. Values were uniform across both PET scanners.

Discussion: $\left[{ }^{11} \mathrm{C}\right]-\mathrm{RO}_{5013853}$ is a novel PET ligand for imaging of GlyT1 which may be used to support clinical development of GlyT1 inhibitors and occupancy studies investigating the role of GlyT1 in CNS diseases such as schizophrenia.

Support: Roche Pharmaceuticals

Disclosure: D. Wong: Part 2; Johns Hopkins University School of Medicine. Part 4; NIH, Amgen, Avid, DANA, Lilly, Merck, Intracellular, Otsuka, GE, Lundbeck, Roche, Sanofi Aventis. S. Ostrowitzki: Part 5; F. Hoffmann-LaRoche. Y. Zhou: None. D. Alberati: F. Hoffmann-LaRoche. E. Pinard: F. Hoffmann-LaRoche. V. Raymont: None. M. Martin-Facklam: F. Hoffmann-LaRoche. C. Hofmann: F. Hoffmann-LaRoche. N. Parkar: F. Hoffmann-LaRoche. J. Brasic: None. J. Hilton: None. R. Dannals: None. E. Borroni: F. Hoffmann-LaRoche.

162. Psychological Well Being and Life Satisfaction Predict Dopamine Release during a Stressor and the Activation of Motivational Networks During Expectation of Punishment in Healthy Males Sara Wright*, Tiffany Love, Barbara Fredrickson, Christian Stohler, Jon-Kar Zubieta

\section{University of Michigan, Ann Arbor, MI}

Background: Responsiveness of the dopaminergic system is known to occur during expectation of salient stimuli, including reward and punishment (Scott et al., 2007; Zald et al., 2004), and during response to stress (Pruessner et al., 2004). Well being and life satisfaction are known to predict response to stressors in behavioral studies (see Scheier et al., 2000) although the neural underpinnings of these relationships have not been measured directly. The current study related measures of well being and life satisfaction to the response of the dopaminergic system to a stressor and brain regional activation during expectation of monetary loss, using PET and fMRI, respectively, in the same subjects.

Methods: Volunteers were 22 healthy, right-handed males $26 \pm 2.9$ years of age. Subjects had no family history of heritable illnesses, psychiatric illness, or substance abuse. Volunteers were medicationfree for at least six months, were nonsmokers, and did not exercise more than one hour three times per week. DA D2/3 receptor availability in vivo (non-displaceable binding potential, BPND) was quantified with PET and [11C] raclopride at baseline (i.e., a non-painful control condition) and during a physical and emotional stressor (i.e., moderate levels of sustained pain). DA release in the basal ganglia was calculated as the reduction in regional DA D2 receptor availability in vivo from baseline to pain/stress conditions. Control and pain stress challenges were introduced for 20 minutes, at 5 and 45 minutes, respectively, after the administration of radiotracer by the infusion of medication-grade $5 \%$ hypertonic saline into the relaxed masseter muscle. Pain was maintained at a constant rate, at 40 VAS intensity units. DA D2/3 receptor measures were extracted for each volunteer from the nucleus accumbens (NAcc), for use in main analyses. All subjects also underwent BOLD fMRI while completing the Monetary Incentive Delay Task (MID) in the scanner. The MID was designed to activate motivational pathways during anticipation of monetary gains and losses, and has been related to DA release in previous work (Scott et al., 2007). Activation values were extracted from the NAcc during anticipation of small, medium, and large monetary loss and used in main analyses. All subjects completed a set of questionnaires designed to measure aspects of well-being and life satisfaction. Measures included the Emotional Well Being Scale (EWB), Psychological Well Being Scales (PWB; Ryff et al., 1995), Social Well Being Scales (SWB), Life Orientation Test-Revised (LOT-R; Scheier et al., 1984), Satisfaction with Life Scale (SWLS; Diener et al., 1985), and Ego Resiliency-89 (ER89; Block et al., 1965).

Results: NAcc DA release during the stressor was significantly negatively correlated with BOLD responses during anticipation of small $(\mathrm{r}=-.62, \mathrm{p}<.01)$, medium $(\mathrm{r}=--.50, \mathrm{p}=.02)$, and large $(\mathrm{r}=$ - -.48, $\mathrm{p}=.02$ ) loss, but only in the left hemisphere. DA release during the stressor was negatively correlated with PWB-Self Acceptance (left, $\mathrm{r}=-.53, \mathrm{p}=.01$; right, $\mathrm{r}=-.45, \mathrm{p}=.04$ ); PWB-Purpose in Life (right, $\mathrm{r}=-.53, \mathrm{p}=.01$ ), PWB-Environmental Mastery (left, $\mathrm{r}=-.50, \mathrm{p}=.02$; right, $\mathrm{r}=-.44, \mathrm{p}=.04)$, PWB-Positive Relationships (right, $\mathrm{r}=-.55, \mathrm{p}<.01$ ); SWB-Social Coherence (left, $\mathrm{r}=-.48, \mathrm{p}=.02$; right, $\mathrm{r}=-.61, \mathrm{p}<.01$ ); LOT-R (right, $\mathrm{r}=-.44, \mathrm{p}=.04$ ); and SWLS (left, $\mathrm{r}=-.48, \mathrm{p}=.02$ ). In analyses investigating relationships between measures of well being and activation to monetary loss during fMRI in the NAcc, significant correlations were found only between activation to small loss, and only in the left hemisphere for PWB-Self Acceptance (left, $\mathrm{r}=.44, \mathrm{p}=.04$ ); PWB-Positive Relationships (left, $\mathrm{r}=.48, \mathrm{p}=.03$ ); and SWLS (left $\mathrm{r}=.50, \mathrm{p}=.02$ ).

Discussion: Individuals with greater dopamine responses in the NAcc during a stress challenge (i.e., moderate levels of sustained pain) reported lower levels of psychological and social well being, optimism, and life satisfaction. Higher levels of BOLD NAcc activation during expectation of small monetary losses were associated with reported psychological well being and life satisfaction, albeit less robustly (across measures) than with DA release in response to stress. This study is the first to display the relationship between aspects of psychological well being with the responsiveness of the NAcc to salient negative stimuli, and supports a role of DA-mediated mechanisms in resiliency and vulnerability to adverse events.

Disclosure: S. Wright: None. T. Love: None. B. Fredrickson: None. C. Stohler: None. J. Zubieta: Part 1; Consultant, Eli Lilly, Merck. Part 2; Eli Lilly Lecture Bureau. Part 3; Eli Lilly Lecture Bureau.

\section{A Cognitive Intervention That Modulates HPA Responses to Pharmacological Activation May Be Ineffective with Psychosocial} Activation

James Abelson*, Stefanie Mayer, Hedieh Briggs, Israel Liberzon, Elizabeth Young, Samir Khan

\section{University of Michigan, Ann Arbor, MI}

Background: The hypothalamic-pituitary adrenal (HPA) response to stress is sensitive to cognitive/emotional factors (e.g. novelty, control, coping). A cognitive intervention targeting novelty reduction, coping, and sense of control can reduce HPA (cortisol) responses to pharmacological activation with pentagastrin, which directly stimulates ACTH release from the pituitary, but also produces physical side 


\section{S376}

effects and some anxiety. A similar intervention interacted with novelty and reduced ACTH response to low dose corticotropinreleasing hormone $(\mathrm{CRH})$, which also stimulates the pituitary but without subjectively discernible side effects. In this study, we examined the impact of a similar cognitive intervention (CI) on psychosocial activation of the HPA axis (using the Trier Social Stress Test, TSST). Methods: Healthy subjects were randomly assigned to receive either standard instructions or one of two interventions (provision of "control" over social exposure or a full CI parallel to those used in our pharmacological studies) prior to exposure to the social evaluative speech and arithmetic challenges of a TSST.

Results: Neither intervention had a significant impact on cortisol response to the TSST (group mean responses (mcg/dL $\pm \mathrm{SD}$ ): $5.6 \pm 5.0$, $5.7 \pm 4.3,4.0 \pm 3.9, p>.50$ ). Robust cortisol responses were seen in the majority of subjects regardless of condition (time effect in RMANOVA, p <.0001).

Discussion: In contrast to results using pharmacological activation, our cognitive intervention was not able to attenuate the HPA response to a psychosocial stressor. Some effects may yet be seen when ACTH samples are assayed, but psychological modulation of cortisol responses to the TSST has generally been difficult to demonstrate. Social evaluative threat may be a particularly potent activator of the HPA axis, perhaps because it feels inherently uncontrollable, despite our intervention. It may be that intervention attenuated responses to pituitary level activators in prior studies through top-down circuits that modulated psychological amplification of biologically driven responses, but is unable to modulate cortisol release when the activation itself involves the higher order processors involved in HPA responding to a purely psychosocial stimulus.

Disclosure: J. Abelson: None. S. Mayer: None. H. Briggs: None. I. Liberzon: None. E. Young: None. S. Khan: None.

164. Persistent Disturbance in HPA-Axis Activation and Sympathetic Reactivity to Stress in Euthymic Women with Histories of Major Depression and Postpartum Depression

Samantha Meltzer-Brody*, Susan Girdler, Brenda Pearson, Katherine Rinaldi, Robert Hamer, David Rubinow

University of North Carolina at Chapel Hill, Chapel Hill, NC

Background: There is increasing evidence that abnormalities in both the hypothalamic-pituitary adrenal (HPA) axis and sympathetic nervous system (SNS) play a key role in the pathogenesis of major depression (MDD) and postpartum depression (PPD).

Objective: We sought to examine HPA axis, blood pressure (BP) and heart rate (HR) reactivity to challenge in three groups of euthymic women: 1) those with a history of PPD, 2) those with a history of nonpuerperal MDD, and 3) control women without a history of PPD or MDD. We present preliminary results on the first subset of subjects $(\mathrm{N}=13)$ completing the study. Due to small cell sizes, we grouped the PPD and MDD women for analysis (history of depression group-DEP; $\mathrm{N}=6)$ and compared to controls $(\mathrm{N}=7)$.

Methods: Based on SCID interview, women met criteria for a history of PPD (a depressive episode meeting MDD DSM-IV criteria within 3 months of delivery; $n=3)$, a history of non-puerperal MDD $(n=3)$, or never depressed controls $(n=7)$. Both depression groups were euthymic at the time of testing. All women were tested between 12 and 24 months past their most recent childbirth, were medically healthy, had regular menstrual cycles, were not pregnant or lactating, and were not taking medications including oral contraceptives or psychotropics. Subjects were also free of a history of moderate to severe lifetime psychiatric illness (other than MDD or PPD). Prior data demonstrated that differential HPA axis stimulation in women with history of PPD could be elicited during high dose gonadal steroid replacement (Bloch et al, 2005). Here, we examined HPA activity under normal physiologic conditions, but during the luteal phase (days 6-10) when peak levels of gonadal steroids occur and HPA axis reactivity in women is maximal (Roca et al, 2003). On separate days we administered: 1) the Trier Social Stress Test (TSST; involving speech and mental arithmetic), during which BP, HR, plasma cortisol (CORT) and norepinephrine (NE) were sampled at rest and in response to stress; and 2) the standardized dexamethasone (DEX)/CRH pharmacological challenge (subjects took $1.5 \mathrm{mg}$ of DEX at 11pm the night before the $\mathrm{CRH}$ test and on day of testing received $1 \mathrm{ug} / \mathrm{kg}$ ovine $\mathrm{CRH}$ per I.V.). Plasma ACTH and CORT were assessed at minutes 5, 15, 30, 60,90 and 120 following the $\mathrm{CRH}$ push. Order of administration of DEX/CRH versus TSST was randomized.

Results: The mean age of participants was approximate 30 years and mean months postpartum $=18$. During the TSST, both groups showed significant increases in BP and HR in response to both the speech and mental arithmetic stressors (all Ps $<.05$ ). There were no group differences in resting BP or HR, but the DEP group showed blunted HR reactivity to the speech stressor relative to controls (deltas $=9.2 \mathrm{vs}$. $18.0 \mathrm{bpm})(\mathrm{F}(2,10)=3.4, \mathrm{p}=.07)$, and showed heightened, but nonsignificant, diastolic blood pressure (DBP) reactivity to both stressors, but especially the speech task, relative to controls (deltas $=15.3$ vs. $8.3 \mathrm{mmHg}$ ). In addition, despite similar baseline $\mathrm{NE}$, a stressor by DEP status effect emerged $(\mathrm{F}(2,10)=3.77, \mathrm{p}=.06)$, since the math task yielded greater NE reactivity in those with prior DEP (129 vs. $37 \mathrm{pg} / \mathrm{ml}$; $\mathrm{F}(1,12)=3.65, \mathrm{p}=.08)$. Furthermore, although groups did not differ in CORT reactivity to the TSST, absolute concentrations of plasma CORT were significantly lower in the DEP group at both time points $(\mathrm{F}(1,11)=4.8, \mathrm{p}=.05)$ (rest: 6.29 vs. 7.49 , ug/dl; and post-stress: $5.11 \mathrm{vs.}$ $7.86, \mathrm{ug} / \mathrm{dl})$. The DEX/CRH challenge revealed the same pattern of blunted HPA-axis activation, since both CORT and ACTH following the $\mathrm{CRH}$ push were consistently lower in the DEP group at all time points, though area under the curve analyses did not reach statistical significance.

Discussion: Our present small sample size generates a high likelihood of Type II errors. Nonetheless, our preliminary results suggest a persistent disturbance in both HPA and SNS axes in currently euthymic women with a history of MDD or PPD in the $12-24$ months postpartum. The combination of blunted HR but increased DBP reactivity seen in those with a history of DEP suggests diminished $\mathrm{B}$-adrenergic receptor (B-AR) responsivity, which may arise from excessive NE reactivity to stress. Similarly, the non-significant blunting of ACTH and CORT responses seen in our DEP group would be consistent with downregulated pituitary CRF receptors (in the absence of the postulated vasopressinergic drive in MDD). Whether these abnormalities reflect the prior experience of or vulnerability to MDD is currently unclear, as is the specificity of previously reported HPA axis abnormalities in women with PPD (compared with non-puerperal MDD) during exposure to high dose gonadal steroids.

Disclosure: S. Meltzer-Brody: Part 4; Astra Zeneca. S. Girdler: None. B. Pearson: None. K. Rinaldi: None. R. Hamer: None. D. Rubinow: None.

165. The Biology of Massage: Implications for Psychiatry Mark Rapaport*, Catherine Bresee, Pamela Schettler

Cedars-Sinai Medical Center, Los Angeles, CA

Background: Under a NCCAM-funded developmental grant, we investigated the neuro-endocrine and immune effects of Swedish massage therapy as compared to a light touch control condition in normal volunteers. Goals of the project were: (1) to determine the longterm biological effects of massage therapy on HPA and immune function, and (2) to discern whether there is a dosage difference between massage therapy once versus twice a week on biological measures.

Methods: Fifty healthy subjects were randomized to receive 5 weeks of massage therapy either once or twice a week, or 5 weeks of a light touch control condition, once or twice a week. Biological and psychological assessments were taken before and after the first and last therapy sessions.

Results: Over the first massage intervention and compared with the light touch control condition, there was a moderate increase in 
circulating CD25 + lymphocytes (effect size 0.50) and a decrease in salivary cortisol (effect size of -0.28). After 5 weeks of once-a-week treatment of massage, baseline levels of $\mathrm{CD}_{4}+, \mathrm{CD} 8+, \mathrm{CD} 25+$, and $\mathrm{CD} 56+$ lymphocytes were elevated as compared with the light touch control condition (effect sizes of $0.92,1.12,0.46$, and 0.87 respectively) while salivary cortisol levels were unchanged. There were large negative dose effect sizes on these measures when contrasting twicea-week to once-a-week massage therapy. The trends observed acutely at the first intervention visit were repeated and amplified when examining the cumulative effects from baseline to 60 minutes after the final therapeutic session in the once-a-week treatment groups. With twice-a-week treatment, the only notable cumulative biological effect over 5 weeks of treatment was a large increase in oxytocin levels for massage compared with the light touch control condition (effect size of 0.92). Using an in vitro mitogen stimulated blood sample cytokine assay, we determined that once-a-week massage therapy decreased IL-1b, IL-2, IL-5, IL-10, and IL-13 production levels, both acutely and cumulatively over 5 weeks of treatment, compared with our light touch control condition.

Discussion: These preliminary data suggest that massage therapy has biological effects, most notably on immune function, and these effects are magnified over the course of repeated treatments. A second finding from the study is that there is a dosage effect present with massage therapy. Once-a-week massage therapy seems to consistently produce more profound changes in immune function. Twice-a-week massage therapy seems to lead to a desensitization of immune function changes, but may have a slightly more profound effect on neuroendocrine measures. These results suggest that massage therapy may be useful in treating individuals with conditions involving dysregulation of immune or endocrine function, such as in anxiety or chronic fatigue syndrome.

Disclosure: M. Rapaport: Part 1; Wyeth, Dainippon-Sumitomo, Brain Cells, Inc., Astellas Pharma, Quintiles, Pfizer, Takeda Pharmacuticals. Part 4; National Institutes of Mental Health. C. Bresee: None. P. Schettler: Part 1; Brain Cells, Inc.

166. Vascular Endothelial Growth Factor (VEGF) in Individuals with Major Depressive Disorder Compared to Healthy Controls Zandra Walton, M. Taylor Dryman, Aparna Keshaviah, Maurizio Fava, Kwok-Kin Wong, Naomi M. Simon*

\section{Massachusetts General Hospital, Boston, MA}

Background: Vascular Endothelial Growth Factor (VEGF) has been identified as playing a key role in neurogenesis and neural plasticity, and may be linked to antidepressant efficacy in the brain in animal models of depression (e.g.,Warner-Schmidt \& Duman 2007, but not Greene et al. 2009). However, data examining VEGF as a potential peripheral biomarker in Major Depressive Disorder (MDD) are limited to small sample sizes and/or do not exclude antidepressant use or adjust for potential confounders such as medical illness (e.g., Ventriglia et al. 2009; Dome et al. 2008; Asberg et al. 2009).

Methods: Serum VEGF protein levels were measured from 67 antidepressant-free individuals with a DSM-IV diagnosis of depression compared to 67 age and gender matched controls. Serum samples were stored at $-80^{\circ} \mathrm{C}$ until the time of assay. Serum VEGF was captured with antibody-immobilized beads in the Milliplex MAP Human Cytokine/ Chemokine kit (Millipore, Billerica, MA) and the fluorescent reporter was read on a Luminex 200 System (Luminex Corporation, Austin, TX). All analyses were conducted in duplicate with an average intraassay coefficient of variation below $9 \%$. Positive and negative controls were included and performed as expected in every assay. VEGF levels were expressed in pg of protein per $\mathrm{mL}$ of serum as quantified using the kit-supplied standard curve. The average detection limit was 5.4 $\mathrm{pg} / \mathrm{mL}$. We also examined potential confounding by age, gender, smoking, Body Mass Index (BMI), exercise, education, stress (as measured by the Perceived Stress Scale), and medical comorbidity (as measured by the Cumulative Illness Rating Scale).
Results: After a log transformation of VEGF to achieve normality, univariate regression analyses found that serum from individuals with MDD had significantly lower VEGF levels than Controls (Mean(SD) $\mathrm{VEGF}=181(294)$ and $262(346) \mathrm{pg} / \mathrm{ml}$, respectively: $\mathrm{B}(\mathrm{SE})=-0.72(0.34)$, $\mathrm{p}<0.036)$. After assessment for potential confounding amongst all covariates as noted, a final multivariate analysis included covariates for education level, current smoking status, and exercise frequency. Results still suggested MDD subjects had lower VEGF levels than controls, though the differences were no longer statistically significant $(\mathrm{B}(\mathrm{SE})=-0.12(0.40), \mathrm{p}=0.76)$. Further, in multivariate analysis, significant predictors of VEGF level among MDD patients included BMI and perceived stress. In the MDD cohort, significantly lower VEGF level was associated with obesity (VEGF Mean $(S D)=223(361)$, 208(285), and 34(58) among normal, overweight, and obese patients, respectively: $\mathrm{p}=0.014)$ and lower perceived stress $(\mathrm{B}(\mathrm{SE})=0.11(0.04)$ : $\mathrm{p}=0.012$ )

Discussion: These data suggest individuals with a diagnosis of MDD have lower serum VEGF levels than age and gender matched healthy controls, but that these differences may be accounted for by differences in education (a marker of socioeconomic status), exercise frequency and current cigarette smoking, with a follow-up meditational analysis finding exercise to be a marginally significant mediator (Effect $=0.23$, $t(128)=1.85, p=0.067)$ of the effect of MDD on VEGF. Amongst those with depression, obesity predicted lower VEGF while higher stress predicted higher VEGF, suggesting that complex factors likely combine to determine peripheral VEGF levels. Study limitations include limited power for follow up analyses (e.g., $\mathrm{n}=13$ for MDD with obesity, defined as BMI > 30). It remains unclear whether serum VEGF may be a reasonable biomarker for MDD or treatment response and how closely peripheral measures of VEGF meaningfully reflect brain VEGF levels or associated function (Elving et al. 2010).

Disclosure: Z. Walton: None. M. Dryman: None. A. Keshaviah: None. M. Fava: Part 1; Abbott Laboratories, Adamed, Co., Advanced Meeting Partners, Affectis Pharmaceuticals AG, Alkermes, Inc., Amarin Pharma Inc., American Psychiatric Association, American Society of Clinical Psychopharmacology, Aspect Medical Systems, Astra Zeneca, Auspex Pharmaceuticals, Bayer AG, Belvoir Media Group, Best Practice Project Management, Inc., BioMarin Pharmaceuticals, Inc., BioResearch, Biovail Corporation, Boehringer Ingelheim $\mathrm{GmbH}$, BrainCells Inc., Bristol-Myers Squibb, CeNeRx BioPharma, Cephalon, Inc., Clinical Trials Solutions, LLC, Clintara, LLC, CNS Response, Inc., Compellis Pharmaceuticals, Covidien, Cypress Pharmaceutical, Inc., Dov Pharmaceuticals, Inc., Eisai Inc., Eli Lilly and Company, EnVivo Pharmaceuticals, Inc., EPIX Pharmaceuticals, Inc., Euthymics Bioscience, Inc., Fabre-Kramer Pharmaceuticals, Inc., Forest Pharmaceuticals, Inc., Ganeden Biotech, Inc., GenOmind, LLC, GlaxoSmithKline, Gruenthal GmbH, Imedex, LLC, Janssen Pharmaceutica, Jazz Pharmaceuticals, Inc., Johnson \& Johnson Pharmaceutical Research \& Development, Knoll Pharmaceuticals Corp., Labopharm Inc., Lichtwer Pharma GmbH, Lorex Pharmaceuticals, Lundbeck Inc., MedAvante, Inc., Merck \& Co., Inc., Methylation Sciences, MGH Psychiatry Academy/Primedia, MGH Psychiatry Academy/Reed Elsevier, NARSAD, NCCAM, Neuronetics, Inc., NIDA, NIMH, Novartis AG, Nutrition 21, Organon Pharmaceuticals, PamLab, LLC., Pfizer Inc., PharmaStar, Pharmavite ( LLC, Precision Human Biolaboratory, Prexa Pharmaceuticals, Inc., PsychoGenics, Psylin Neurosciences, Inc., RCT Logic, LLC, Rexahn Pharmaceuticals, Inc., Ridge Diagnostics, Inc., Roche, Sanofi-Aventis US LLC, Schering-Plough Corporation, Sepracor Inc., Shire, Solvay Pharmaceuticals, Inc., Somaxon Pharmaceuticals, Inc., Somerset Pharmaceuticals, Inc., Synthelabo, Takeda Pharmaceutical Company Limited, Tetragenex Pharmaceuticals, Inc., Transcept Pharmaceuticals, Inc., TransForm Pharmaceuticals, Inc., United BioSource, Corp., Vanda Pharmaceuticals, Inc., Wyeth-Ayerst Laboratories. Part 4; Astra Zeneca, Brain Cells, Inc., Bristol Meyers Squibb, Eli Lilly, GlaxoSmithKline, Healthcare Technologies, Johnson \& Johnson Pharmaceutical Research and Development, Novartis, PamLab, Pfizer, Sanofi-Aventis. K. Wong: Part 1; Abbot Pharmaceuticals, Agios Pharmaceuticals, Merrimack Pharmaceuticals, 


\section{S378}

Millennium Pharmaceuticals. Part 4; Agios Pharmaceuticals, GlaxoSmithKline, Merrimack Pharmaceuticals. N. Simon: Part 1; Astra Zeneca, Pfizer, MGH Psychiatric Academy. Part 4; American Foundation for Suicide Prevention, Astra Zeneca, Cephalon, Forest Laboratories, GlaxoSmithKline, Janssen, Lilly, NARSAD, NIMH, Pfizer, UCB-Pharma, Sepracor.

\section{Seasonality of Depression Risk and Suicidal Symptoms in the Postpartum Period}

Dorothy $\mathrm{Sit}^{*}$

University of Pittsburgh, Western Psychiatric Institute and Clinic, Pittsburgh, PA

Background: Postpartum depression (PPD) is the most common complication after delivery. A new episode of PPD affects 10 to $15 \%$ of mothers (Gaynes et al, 2005). Lasting episodes are related to maternal problems with infant attachment (England 1994), social conditions and relationships (Segre et al, 2007). Women with PPD respond robustly to serotonergic (sertraline) and noradrenergic (nortriptyline) drugs (Wisner et al, 2006). Evidence of reduced serotonin $(5 \mathrm{HT}) 1 \mathrm{~A}$ receptor binding by 20-28\% in depressed compared to healthy mothers (Moses-Kolko et al, 2008) suggests some postpartum patients may have lowered serotonergic tone. The effects of seasonal light on $5 \mathrm{HT}$ pathways may contribute to mood and suicidal symptoms. 5 HT metabolism varies across the seasons (Klompenhouwer et al, 1990) and is related to sunlight (Lambert et al, 2002). Suicide is associated with increased sunshine (Lambert et al, 2003), low levels of $5 \mathrm{H} 1 \mathrm{AA}$ and abnormal 5-HT activity (Asberg, 1997). We examine the relationship between seasonal variation, depression and suicidal ideation in postpartum women. We hypothesize the spring and fall would be associated with an increased depression risk and suicidality compared to other times of year.

Methods: The prospective study was approved by the University of Pittsburgh Institutional Review Board. Discharge nurses obtained a waiver of written informed consent for the phone screen. From 20062010, 9339 mothers ( $\geq 18 \mathrm{yrs}$ ) who were $4-6$ wks postpartum received PPD phone screens with the Edinburgh Postnatal Depression Scale (EPDS). The EPDS is a widely accepted tool to identify women with risk for PPD (sensitivity $=91 \%$, specificity $=76 \%$; Cox et al, 1987). An EPDS $\geq 10$ corresponds with concurrent diagnoses made by DSM-III-R or Research Diagnostic Criteria. The outcome variables were: EPDS $\geq 10$ (positive score $=$ PPD risk) and EPDS item $10 \geq 1$ (suicidal ideation-SI). Seasons were defined as spring-summer (April to September) vs. fall-winter (October to March). Analysis. Months were converted to radians $(1 \mathrm{yr}=2 \mathrm{pi})$. For the seasonal effect on positive EPDS $\geq 10$ and SI, we used periodic binomial regression analysis (Fourier Basis)(Stoffer, 1991). Results: We identified 1316 (14\%) mothers with positive EPDS scores and 294 (3\%) with SI. A positive EPDS score was associated significantly with SI $(\mathrm{OR}=41.3,95 \% \quad \mathrm{CI}=29.8-58.6$, Pearson's $\left.\chi^{2}=1243.9 \mathrm{df}=1 \mathrm{p}=2.2 \times 10^{-16}\right)$. Positive EPDS scores significantly peaked once (fall-winter) across 12-months (estimate $=0.117$, s.error $=0.052, \mathrm{z}$-value $=2.26 \mathrm{p}=0.03$ ). SI did not vary significantly across 12-months. SI was correlated with "the ability to laugh and see the funny side of things" (item1, Spearman's rank correlation $\sigma=0.26$ ); "look forward to enjoyment of things" (item2, $\sigma=0.27$ ); and "I have been so unhappy that I have had difficulty sleeping" (item $7, \sigma=0.27$ ).

Discussion: The rate of PPD risk (14\%) is consistent with published rates (Gaynes et al, 2005). The seasonal peak may be from a preexisting pattern of seasonal illness or low sunlight exposure. Seasonal mood symptoms are common in women with PPD $(n=62,29 \%)$ vs healthy controls $(n=62 ; 14 \%$; Corral et al, 2007). Childbirth in the darker months is associated with increased depression risk (ratio observed/ expected $=1.58,95 \% \mathrm{CI}=1.05-2.11$; Hiltunen et al, 2004). Low sunlight exposure in the fall and winter results in increased $5 \mathrm{HT}$ transporter binding, diminished synaptic $5 \mathrm{HT}$ levels and possible susceptibility to depressive symptoms (Praschak-Rieder et al, 2008) (Willeit et al, 2008). The frequency of SI (3\%) is lower than rates from population-based mothers $(n=386,8.3 \%$; Pinheiro et al, 2008). Depressed (rather than non-depressed) mothers are much more likely to have suicidal symptoms. Suicide is the leading cause of maternal death $(28 \%=68$ suicides $/ 242$ deaths)(Oates, 2003). Our patients with SI declare firm reasons for living (responsibility toward the family, religious beliefs). Reasons for living and proper depression treatment (Oquendo et al, 1999) can avert suicide risk (Malone et al, 2000). SI does not vary seasonally in postpartum patients. This diverges from prevailing reports of a peak incidence of suicide in the spring (Chew et al, 1995) and early summer. Some patients who complete suicide can overlap with but are distinct clinically from patients with SI or who attempt suicide (Gladstone et al, 2001). The effects of seasonal light variation on the $5 \mathrm{HT}$ pathways may contribute to increased risk for depressive symptoms. In the postpartum, suicidality may be related to maternal depression but not seasonal effects.

Disclosure: D. Sit: None.

168. Effect of Light Therapy upon Serotonin Transporter Binding in Prefrontal and Anterior Cingulate Cortex During Fall and Winter Using $\left[{ }^{11} \mathrm{C}\right]$ DASB Positron Emission Tomography

Sarah Harrison*, Sylvain Houle, Alan Wilson, Robert Levitan, Jose Nobrega, Jeff Meyer

\section{CAMH, Toronto, ON, Canada}

Background: Seasonal Affective Disorder (SAD) has a prevalence rate of $1-12 \%$ with higher incidence in northern latitudes and at least $25 \%$ of people are sensitive to seasonal changes. We previously discovered that healthy individuals show decreased regional serotonin transporter (5-HTT) binding in spring/summer as compared to fall/winter (Praschak-Rieder et al., Arch. Gen. Psych, 2008) and this finding tends to be replicated in studies of larger sample sizes. One possible cause of this change may be due to low light levels during winter months; however, no studies to date have examined the effects of light therapy on 5-HTT binding in the human brain. The objective of this study was to investigate the effects of light upon 5-HTT binding potential (BP) in the prefrontal and anterior cingulate cortex (PFC and ACC, respectively) in vivo in healthy individuals during fall and winter. Methods: 19 healthy, drug-naïve males and females, aged 19-39, received morning light therapy or placebo for 5 consecutive days followed by a $\left[{ }^{11} \mathrm{C}\right]$ DASB PET scan. Participants were also assessed with subjective and objective mood rating scales.

Results: No effects of light therapy upon PFC or ACC 5-HTT BP were observed in the group as a whole. However in the subgroup completing the protocol in the winter, 5 -HTT BP values in the ACC were $11 \%$ lower following light compared to the placebo condition $(\mathrm{n}=10, p=0.008)$. No significant changes were observed in the PFC in either group. Following 5 days of light therapy, subjects scanned in the winter scored significantly lower than baseline on the BDI and SIGH-ADS following light but not placebo ( $p=0.021$ and 0.025 , respectively).

Discussion: This is the first study to measure the effects of light therapy upon brain 5-HTT binding in humans. Our results indicate that 5-HTT binding in the ACC is reduced after light therapy in winter. Since the ACC is functionally associated with affect regulation, it is possible that light mediates some antidepressant effects through reductions in 5-HTT binding in this region.

Disclosure: S. Harrison: None. S. Houle: None. A. Wilson: None. R. Levitan: None. J. Nobrega: None. J. Meyer: None.

169. Subcallosal Cingulate Deep Brain Stimulation for TreatmentResistant Unipolar and Bipolar Depression Paul Holtzheimer*, Megan Filkowski, Mary Kelley, Robert Gross, Helen Mayberg

Emory University School of Medicine, Atlanta, GA

Background: Treatment-resistant depression (TRD) is a major public health problem. Subcallosal cingulate deep brain stimulation 
(SCC DBS) has shown preliminary safety and efficacy as a treatment for extreme TRD. The current study attempted to replicate these earlier results in unipolar TRD, determine the safety and efficacy of SCC DBS for bipolar TRD, and assess for a post-surgical placebo effect. Methods: All study procedures were approved by the Emory University Institutional Review Board (IRB), and conducted under an investigator-held Investigational Device Exemption from the U.S. Food and Drug Administration. Eligibility criteria included a current nonpsychotic major depressive episode (secondary to either Major Depressive Disorder [MDD] or Bipolar 2 Disorder [BP2]) of at least 12 months duration, failure of at least 4 adequate antidepressant treatments, failure or intolerance of electroconvulsive therapy (ECT), maximum baseline GAF of 50, screening 17-item Hamilton Depression Rating Scale (HDRS17) score $\geq 20$, no clinically significant medical or psychiatric comorbidity, no substance use disorder (within the past year), and no significant suicidality. Patients were allowed to remain on current psychiatric treatments but these were held constant during the 4 weeks prior to surgery and for at least 28 weeks following surgery. All patients received surgery to implant DBS leads into the bilateral SCC, followed by four weeks of single-blind, sham (off) stimulation, then 24 weeks of open, active chronic stimulation $(130 \mathrm{~Hz}$, $91 \mu$ s pulse width, 6-8 mA). Clinical status was assessed every 1-2 weeks during these phases. Patients were then invited to enter a 5 year naturalistic follow-up phase with assessment every 1-6 months.

Results: Seventeen patients (10 MDD, 7 BP2) received DBS implantation. A small but statistically significant decrease in HDRS17 score was seen after 4 weeks of sham stimulation compared to baseline, but this decrease generally occurred following surgery and prior to entering the blinded sham phase; no patient achieved response or remission with sham stimulation. With active stimulation, remission rates (HDRS $17 \leq 7$ ) were $18 \%$ at 6 months, $36 \%$ at 1 year and $58 \%$ at 2 years; response rates ( $\geq 50 \%$ decrease in HDRS17 score from baseline) were $41 \%$ at 6 months, $36 \%$ at 1 year and $92 \%$ at 2 years. There was no significant difference in remission/response rates between MDD and $\mathrm{BP} 2$ patients. Patients achieving remission and/or response generally remained well over time, and no patient achieving remission experienced a spontaneous relapse. Twelve serious adverse events occurred in 4 subjects; only 2 (both infections) were related to the device or surgery and these occurred in the same patient. There were 2 suicide attempts, and neither was related to stimulation. There were no adverse effects associated with either acute or chronic stimulation, and no patient experienced a hypomanic or manic episode.

Conclusions: These findings further support the safety and efficacy of chronic SCC DBS for TRD, and extend earlier work by showing safety and similar efficacy in $\mathrm{BP} 2$ patients. A placebo effect may account for a small portion of the improvements seen, but a major effect is highly unlikely given the general absence of improvement over the placebo phase (versus improvement seen post-surgery but prior to placebo stimulation), the consistent increase in remission over time and the very low relapse rate.

Disclosure: P. Holtzheimer: Part 1; St. Jude Medical Neuromodulation, Northstar, AvaCat Consulting, Oppenheimer \& Co., Shaw Science. Part 3; St. Jude Medical Neuromodulation. M. Filkowski: None. M. Kelley: None. R. Gross: Part 1; St. Jude Medical Neuromodulation, Medtronic, Boston Scientific, Bayer Healthcare, Neurovista. H. Mayberg: St. Jude Medical Neuromodulation. Part 2; St. Jude Medical Neuromodulation. Part 3; St. Jude Medical Neuromodulation.

170. Modulation of Adult Hippocampal Neurogenesis through HPA Axis Activity Determines the Divergent Effects of Distress and Eustress in the Induction of and Recovery from Depressive-like Behavior Michael Lehmann*, Rebecca Brachman, Keri Martinowich, Robert Schloesser, Miles Herkenham

\section{National Institute of Mental Health, Bethesda, MD}

Background: In animals, complex environments and physical exercise have been shown to reduce depressive behavior, increase hippocampal neurogenesis, and improve cognitive function. Previously, we showed that environmental enrichment facilitated the recovery from social defeat stress and enhanced stress resiliency. In a social conflict paradigm, repeated social defeat stress consistently yields a submissive phenotype and is an ethological means of inducing depression in mice. Social defeat stress and enriched environments are both strong modulators of hypothalamic-pituitary-adrenal (HPA) axis function. However, they have divergent effects on the HPA axis. Social defeat stress is a negative stressor (distress) and has been shown to induce HPA axis dysfunction, whereas physical exercise and enriched housing, both of which are positive stressors (eustress), render the HPA axis more adaptive. We hypothesized that the persistent arousal of HPA activity by defeat stress contributes to the pathogenesis of depression. We also hypothesized that HPA activity during enrichment is requisite for the restoration of normal phenotype in depressed mice. Because the hippocampus provides inhibitory control on HPA axis output, and because hippocampal neurogenesis has been implicated in the pathology of depression, we also hypothesized that divergent outcomes of eustress and distress exposure are hippocampal neurogenesis-dependant. We designed three experiments to test these hypotheses.

Methods \& Results: We first examined if adrenalectomy (ADX) coupled with basal corticosterone (CORT) replacement would alter how animals respond to social defeat. We exposed ADX and shamoperated male $\mathrm{C} 57 \mathrm{BL} 6 / \mathrm{J}$ mice to 14 days of chronic social defeat and measured the expression of maladaptive behaviors. We also examined the survival of newborn hippocampal neurons during social defeat. Mice adrenalectomized prior to social defeat showed increased stress resiliency (decreased anxiety and depressive-like behaviors) and increased survival of newborn hippocampal neurons compared to sham. Next, we examined the involvement of adrenal glucocorticoids in the restorative effects of environmental enrichment in defeated mice. Complex environments and exercise have been shown to induce brief intermittent glucocorticoid surges. We hypothesized that these surges are requisite for the ameliorative effects of enrichment after defeat stress. To test this hypothesis, male mice were exposed to social defeat for 2 weeks then either ADX (and basal CORT-replaced) or sham operated. Mice were then housed in enriched environments for 3 weeks and subsequently examined for anxious and depressive-like behaviors. Sham-operated mice showed decreased expression of anxiety- and depressive-like behaviors. However, if we adrenalectomized defeated mice prior to enrichment, the beneficial effects of enrichment disappeared, and hippocampal neurogenesis was decreased. It is noteworthy that changes in behavior correlate with changes in hippocampal neurogenesis and that ADX prevents the defeat stress-induced decline in survival of hippocampal neurons. We tested if decreased neurogenesis contributes to the etiology of social defeat-induced depression or if it is downstream from or tangential to changes in behavior. We hypothesized that defeat stress results in depressive-like behavior through a glucocorticoidinduced decline in surviving hippocampal neurons. We examined whether the protective effects of ADX in distress would persist in mice with conditionally suppressed neurogenesis (NG-). Suppression is achieved through the administration of ganciclovir to the diet of GFAP-HSVtk transgenic mice. NG- mice and their wildtype (WT) littermate controls were adrenalectomized and exposed to defeat stress as described in the first experiment. We found that ADX conferred stress resiliency to the WT mice, whereas ADX NG- mice lacked resiliency and developed depressive behavior. This suggests social defeat causes depressive behavior through CORT-induced declines in hippocampal neurogenesis. Moreover, the data suggest that decreased hippocampal neurogenesis, even in the absence of high levels of glucocorticoids, is sufficient to eliminate stress resiliency in mice.

Discussion: Overall, we have shown that the HPA axis is a key mediator of both defeat stress (distress)-induced depressive behavior and the reparative effects of environmental enrichment (eustress). Furthermore, we show that changes in hippocampal neurogenesis are 


\section{S380}

HPA-dependent. Lastly, we show the hippocampus to be a key mediator of stress resiliency, independent of glucocorticoid levels.

Disclosure: M. Lehmann: None. R. Brachman: None. K. Martinowich: None. R. Schloesser: None. M. Herkenham: None.

171. Selectively Activated Neurons in Ventral Medial Prefrontal Cortex Mediate Context-Induced Reinstatement of Heroin Seeking Jennifer Bossert*, Anna Stern, Florence Theberge, Eisuke Koya, Bruce Hope, Yavin Shaham

\section{IRP/NIDA/NIH/DHHS, Baltimore, MD}

Background: In humans, exposure to environmental contexts previously associated with heroin intake can provoke drug relapse. In rats, exposure to the heroin-associated context following extinction of drugreinforced responding in a different context reinstates heroin seeking. Here, we studied the role of dorsal and ventral medial prefrontal cortex (mPFC) in this reinstatement by using Fos (a neuronal activity marker) immunocytochemistry, reversible inactivation with muscimol + baclofen, and a novel "Dauno2 inactivation method" (Koya et al. NN 2009). In the Dauno2 method, selective neuronal inactivation is performed 90 min after performing a behavioral task by injecting a prodrug Dauno2 into a given brain area of c-fos-lacZ transgenic rats that express beta-galactosidase ( $\beta$-gal) in activated neurons. $\beta$-gal converts Dauno2 into daunorubicin which reduces neuronal excitability.

Methods: In adapting the Dauno2 method to context-induced reinstatement, we trained rats to self-administer heroin for 12 days in one context and extinguished lever responding in a different context. We then re-exposed rats to the heroin-associated context for $30 \mathrm{~min}$, and injected the rats 90 min later with Dauno2 or vehicle into ventral mPFC. Three days later, we tested rats for context-induced reinstatement.

Results: Context-induced reinstatement of heroin-seeking was associated with Fos induction in dorsal and ventral mPFC. Reversible inactivation of ventral, but not dorsal, mPFC by muscimol + baclofen $(0.1+1.0 \mathrm{mM} / \mathrm{side})$ decreased this reinstatement. This effect was mimicked by Dauno2 injections into ventral mPFC, which also decreased context-induced $\beta$-gal expression in ventral $\mathrm{mPFC}$.

Discussion: Results indicate that selectively activated ventral mPFC neurons mediate context-induced reinstatement of heroin seeking and demonstrate the feasibility of using the Dauno2 method to study the neuronal mechanisms of drug relapse.

Disclosure: J. Bossert: None. A. Stern: None. F. Theberge: None. E. Koya: None. B. Hope: None. Y. Shaham: None.

172. Circadian-Sleep Alignment in Bipolar Disorder: A Pilot Study Ellen Frank*, Jessica Levenson, Brant Hasler, Daniel Buysse, Francis Lotrich, Timothy Monk, David Kupfer

University of Pittsburgh, Pittsburgh, PA

Background: Ideally, in healthy individuals the whole brain is under the carefully controlled timing of a functioning circadian system, leading to the coordinated firing of structures that regulate reward and emotion processing. However, mounting evidence from both animal and human studies suggests that the synchronous functioning of this circuitry may be seriously challenged in individuals with bipolar disorder, leading to dysregulated mood and reward responsiveness. One possible strategy for exploring the extent to which circadian and sleep/wake regulation are misaligned in bipolar disorder is by examining the phase angle difference (PAD) between dim light melatonin onset (DLMO), a reliable marker of circadian phase, and the mid-point of sleep as established by wrist actigraphy. In normal controls, the PAD is typically about 6 hours.

Methods: In order to assess the viability of measuring PAD in bipolar patients, we conducted a pilot study in 10 currently euthymic individuals with a lifetime diagnosis of bipolar I disorder. We examined the timing of sleep over a period of 5 to 6 days, as assessed both via sleep diary and via wrist actigraphy. We then brought study participants into our Biological Rhythms Laboratory for a single night's stay, during which we sampled salivary melatonin from 4:30 pm until two hours past the participants' habitual bedtime to maximize the likelihood of identifying DLMO in all participants. Salivary samples were refrigerated as they were collected and then frozen at $-20^{\circ} \mathrm{C}$. Samples were analyzed in duplicate using the ELISA kit (ALPCO Corp.). DLMO was identified as the interpolated time at which melatonin exceeded 2 SD of the participant's running average value.

Results: Data for one participant was not considered valid since he had an average bedtime of 05:59 and melatonin was not sampled for a full two hours beyond that time. The remaining 2 males and 7 females, with a mean age of $44.6(\mathrm{SD}=9.7)$ had a mean age at onset of bipolar disorder of $19.2(\mathrm{SD}=5.0)$ years. When entering this pilot study, these euthymic patients had mean 17 - and 25 -item Hamilton Rating Scale for Depression Scores of $6.8(\mathrm{SD}=2.1)$ and $8.6(\mathrm{SD}=2.3)$, respectively, and a mean Young Mania Rating Scale Score of $2.6(\mathrm{SD}=2.3)$. Sampling from 4:30pm to 2 hours past participants' habitual goodnight time yielded an average of $18(\mathrm{SD}=2.7)$ samples/participant (range $=16$ to 23). Most participants showed relatively normal melatonin rise times between 20:00 h and 22:00 h (median $=21: 16 \mathrm{~h}$ ). Three of the nine participants had PAD values that deviated from the benchmark "normal" value of 6 hours by at least 45 minutes (mean $=54 \mathrm{~min}$, range $13 \mathrm{~min}$ to $3 \mathrm{hr} 4 \mathrm{~min}$ ). We observed marked night to night variability in the timing of sleep, with many participants' good-night times (mean $=24: 01 \mathrm{~h}, \mathrm{SD}=1: 14 \mathrm{~h}$ ) varying by as much as 2 hours from night to night and with comparable variability in goodmorning times (mean $=7: 45 \mathrm{~h}, \mathrm{SD}=1: 12 \mathrm{~h}$ ). Indeed the average withinsubject variability in good-night and good-morning times was 1:02 $\mathrm{h}$ and $55 \mathrm{~min}$ respectively.

Discussion: This small pilot study suggests the need for further study of the way that the circadian timing system and the sleep-wake system are aligned in individuals with bipolar disorder and the extent of dayto-day variability in both sleep and circadian parameters. The large within-subject variability in sleep-wake times point to the possibility that a single night of melatonin sampling may not be sufficient to precisely evaluate circadian-sleep misalignment in this population. Since DLMO values are likely to be comparatively stable, this would inevitably lead to aberrant PAD values on nights when a participant's bedtime varies substantially from his or her average. More than a single evening of sampling would be needed, though, to confirm DLMO stability in this patient population. Our previously published data (Frank et al, 2005; Miklowitz et al, 2007) suggest that treatments which emphasize more regular timing of daily routines are associated with improvement in bipolar depression and prevention of new episodes of mania and depression; however, the relationship between such treatments and objectively measured sleep-wake or circadian variables is yet to be elucidated.

Disclosure: E. Frank: Part 1; Servier International, Guilford Press, Forest Research Institute. Part 2; Servier International. Part 4; Forest Research Institute. J. Levenson: None. B. Hasler: None. D. Buysse: Part 1; Actelion, Arena, Cephalon, Eisai, Eli Lilly, GlaxoSmithKline, Merck, Neurocrine, Neurogen, Pfizer, Philips (Respironics), Sanofi-Aventis, Sepracor, Servier, Somnus Therapeutics, Stress Eraser, Takeda, Transcept Pharmaceuticals. F. Lotrich: None. T. Monk: None. D. Kupfer: None.

173. FACS Identifies Unique Gene Regulation In Adult Striatal Neuronal Ensembles Selectively Activated During Cocaine-induced Behavior

Danielle Guez-Barber*, Sanya Fanous, Brandon K. Harvey, Marina R. Picciotto, Bruce Hope

NIDA, Baltimore, MD

Background: Context-specific sensitization is due to a learned association between drug and stimuli in the administration environment. 
We had previously shown that this learned association is encoded by a pattern of sparsely distributed neurons called a neuronal ensemble. Until now, scientists have studied molecular neuroadaptations in brain homogenates without differentiating between activated neuronal ensembles and surrounding non-activated neurons. This likely obscures the changes seen only in the activated cells. Therefore, we developed a novel method for purifying activated neurons from rat striatal neuronal ensembles and assessing their unique set of cocaineinduced molecular neuroadaptations.

Methods: We used c-fos-lacZ transgenic rats to identify cocaineactivated neuronal ensembles in striatum. Electrophysiological activation of these neurons induces beta-galactosidase protein that can be labeled with a fluorescent antibody against beta-galactosidase. We then separated these fluorescently labeled activated neurons from the majority of non-activated neurons using Fluorescence Activated Cell Sorting (FACS). We used quantitative PCR and microarray to analyze gene expression in sorted cells.

Results: Compared to non-activated neurons from the same cocainetreated rats or all neurons from saline-treated rats, microarray analysis and quantitative PCR showed that activated neurons from cocainetreated rats had much higher expression levels of many immediate early genes including arc, fos, fos $\mathrm{B}$, and nr4az. Because several of these genes are transcription factors, they can influence the expression of many downstream genes, and thus produce a vastly different gene expression profile in the activated neurons. The activated betagalactosidase-expressing neurons also had increased prodynorphin mRNA (a marker of D1-type neurons) and decreased dopamine D2 receptor mRNA; thus, the majority of neurons activated by cocaine are of the D1-type. Finally, the beta-galactosidase-positive neurons likely have attenuated p38 MAPK signaling, because they expressed lower levels of the kinase that activates p38 (map2k6) and higher levels of the phosphatase that deactivates p38 (dusp1 or mkp1). Since p38 has been demonstrated to be involved in long term depression and to inhibit long term potentiation, these neurons may be primed for altered plasticity.

Discussion: We have shown that the neurons mediating contextspecific locomotor sensitization to cocaine have a broadly different gene expression profile than the surrounding non-activated neurons. This represents the first multi-gene analysis of a neuronal ensemble encoding a learned behavior.

Disclosure: D. Guez-Barber: None. S. Fanous: None. B. Harvey: None. M. Picciotto: None. B. Hope: None.

\section{Translational Information Improves Therapeutic Outcome in Inpatients with Depression, Somatoform Disorders, and Anxiety Disorders}

Dirk Hellhammer*, F. Gerhards, H. Thiart, T. Hero, M. Linden

\section{Trier University, Trier, Germany}

Background: Stress factors play an important role in mental and physical diseases. Given the tremendous complexity of factors affecting the crosstalk between the brain and the body, there is a broad heterogeneity in the pathogenesis of stress-related disorders among patients, even sharing similar symptoms. In addition, there is a missing co-variance between the psychological and biological stress response; thus, subjective patient reports are often misleading. We here introduce "Neuropattern", a newly developed diagnostic tool for clinical practice, allowing an individualized detection of stress effects in bodily diseases. Neuropattern analyses stress effects on neurobiological interfaces, participating in the communication between the brain and the body. Functional changes of each of these interfaces are assessed by characteristic patterns of concomitant biological, psychological, and symptomatic measures, occurring in consequence of stress. Stressed patients may differentially qualify for one or more of these neuropattern, thus facilitating highly individualized indications for therapeutic treatments.
Methods: Inpatients $(\mathrm{N}=106)$ suffering by stress related disorders (e.g. depression, somatoform disorders, and anxiety disorders) were randomly assigned to two groups: In the experimental group, physicians received information from Neuropattern diagnostics, while in the control group, these data were not available until discharge from the hospital. Improvements of symptoms in consequence of treatment were monitored by two self-rating scales, the SCL-90 and SF-12.

Results: Self reported symptomatology improved in both groups. However, the experimental group showed a significantly stronger reduction of symptoms. Subgroup analyses showed respective results for all subgroups, with best improvements of patients with somatoform and anxiety disorders.

Discussion: Treatment efficacy in patients with stress related disorders can be improved, once physicians and patients receive additional translational information. The specific role of the disease model and the respective therapeutic recommendations will be discussed in detail. Disclosure: D. Hellhammer: Part 1; Johnson \& Johnson, Cypress, Consumer Health, Daacro. Part 2; Johnson \& Johnson. F. Gerhards: None. H. Thiart: None. T. Hero: Daacro, Max-Planck, Univ of Trier. M. Linden: Part 1; Johnson \& Johnson, Pfizer.

\section{Clinical Differences among Schizophrenia Patient Subgroups Defined Using both Negative and Positive Symptom Trajectories} Lei Chen, Joseph Johnston, Bruce J. Kinon*, Virginia Stauffer, Glenn Phillips, Haya Ascher-Svanum

\section{Eli Lilly and Company, Indianapolis, IN}

Background: In the treatment of schizophrenia, changes in level of symptomatology has been typically measured by examining the change in negative and positive symptom aggregated total scores or change in symptom domains separately. In previous work retrospectively analyzing the interplay between negative and positive symptom trajectories in the naturalistic treatment of patients with schizophrenia, we observed three distinct patterns of symptom trajectories: (1) dramatic and sustained early improvement in both negative and positive symptoms (DSI); (2) mild and sustained improvement in negative and positive symptoms (MSI); and (3) no improvement in either negative or positive symptoms (NI) (Chen et al., Int J Neuropsychop 2010; 13: 1-268). In the present analysis, we explore clinical differences among these subgroups as a means of assessing the potential utility and construct validity of patient groups defined in this way. In particular, we examine whether baseline differences exist that might permit identification of patients likely to exhibit a particular symptom course a priori, as well as whether change in one-year functional and health-related quality of life outcomes exist and are consistent with patterns of symptom change.

Methods: This post hoc analysis used data from a randomized, openlabel, 1-year study of patients with schizophrenia who were treated with typical or atypical antipsychotics in usual clinical care settings. Participants met criteria for psychotic symptom exacerbation or had experienced an adverse event attributable to current antipsychotic therapy. Patients included in this analysis had complete one-year data on the Positive and Negative Syndrome Scale (PANSS). Three subgroups established from previous research - DSI $(\mathrm{N}=70)$, MSI $(\mathrm{N}=237)$ and NI $(\mathrm{N}=82)$ - were compared on baseline characteristics, patient perceived medication benefit (measured using the Rating of Medication Influence [ROMI] at 2 weeks of treatment), baseline to one-year change in global functioning (measured using the Global Assessment of Functioning [GAF]) and physical and mental component scores (measured using the Short Form -36 Health Interview [SF36] ). Statistical comparison for baseline characteristics and 2-week ROMI was made using analysis of variance [ANOVA] and Fisher's exact test. Within group changes from baseline to endpoint were analyzed using t-test. Between group differences in change from baseline were analyzed using analyses of co-variance [ANCOVA] adjusting the baseline value of the analyzed outcome variable. 
Results: At baseline, patients in the DSI group were least likely to have lifetime psychoactive substance use disorder (DSI: $19 \%$, MSI: $42 \%, \mathrm{NI}$ : $40 \%, \mathrm{p}<0.01)$ and least likely to have used both typical and atypical antipsychotics in the past year (DSI: $7 \%$, MSI: $25 \%$, NI: $20 \%, \mathrm{P}<0.05$ ). The NI group was more likely to have akathisia (DSI: $13 \%$, MSI: $9 \%$, NI: $23 \%$, NI vs. MSI: $\mathrm{p}<0.001$; NI vs. DSI: $\mathrm{p}=0.1$ ) and dyskinesia (DSI: $14 \%$, MSI: $13 \%$, NI: $28 \%, \mathrm{p}<0.05)$, and perceived significantly worse medication benefit compared to MSI and DSI patients $(\mathrm{P}<0.001)$ at two weeks of treatment. By one year, mean GAF scores significantly improved for both the DSI (from 49.6 [serious impairment] to 61.6 [mild impairment]) and MSI (from 48.9 [serious] to 56.9 [moderate]) groups (both $\mathrm{p}<0.001$ for within group change), but not for the NI group (baseline $=41.6$ to endpoint $=44.2$ [both serious], $\mathrm{p}$ value for change >0.05). The DSI and MSI groups also exhibited an improvements in SF-36 Mental Component Scores (+ 5.8 for DSI and MSI, $p<0.001$ for within group changes), while the NI group demonstrated no significant change $(+1.2, \mathrm{P}>0.05$ for within group change). No significant change was observed in Physical Component Score of SF-36 for any of the subgroups.

Discussion: Significant baseline differences among subgroups were observed, including lifetime substance use disorder, baseline extrapyramidal symptoms, and antipsychotic use pattern in the past year. These baseline differences, as well as the difference in perceived medication benefit after two weeks of treatment, could be used to predict patient symptom course during treatment. Differences in oneyear functional and health-related quality of life outcomes were directionally consistent with differences in subgroup symptom courses, and support the construct validity of the subgroups. These findings support the potential utility of patient subgroups defined using both negative and positive symptom trajectories. Further research work is warranted to study the underlying biological determinants of these response trajectory subgroups.

Disclosure: L. Chen: Part 5; Eli Lilly and Company. J. Johnston: Eli Lilly and Company. B. Kinon: Eli Lilly and Company. V. Stauffer: Eli Lilly and Company. G. Phillips: Eli Lilly and Company. H. AscherSvanum: Eli Lilly and Company.

176. Methylene Blue for Residual Symptoms and for Cognitive Dysfunction in Bipolar Disorder: Results of a Double-Blind Trial Martin Alda*, Glenda MacQueen, Margaret McKinnon,

Julie Garnham, Tomas Hajek, Claire O’Donovan, Marina Sokolenko

\section{Dalhousie University, Halifax, NS, Canada}

Background: Residual symptoms and cognitive impairment contribute significantly to disability in patients with bipolar disorder. They may be related to neuronal/glial cell loss and they often progress in the course of the illness. We hypothesized that methylene blue could potentially improve these symptoms due to its effect on nitric oxide synthase and guanylate cyclase that might play a neuroprotective role. Methods: We conducted an add-on double-blind cross-over study of methylene blue. As it stains urine, we used two doses of methylene blue - one low (15 mg daily, "placebo") and one high (195 mg daily, "active") - to facilitate blinding. Patients were allocated randomly to one of the treatment orders (placebo active or active-placebo). A total of 37 subjects were enrolled in a 26 -week trial. By design, all patients were treated with lamotrigine as their primary mood stabilizer; additional medications were allowed as long as they remained constant throughout the trial. The outcome measures included ratings of severity of depression (Montgomery-Asberg Depression Rating Scale, MADRS, and Hamilton Rating Scale for Depression, HAMD), mania (Young Mania Rating Scale, YMRS) and anxiety (Hamilton Anxiety Scale, HAMA), and cognitive assessments at baseline, cross-over (week 13), and at study termination (week 26). Statistical analyses (ANOVA for repeated measures with treatment order as a grouping variable) were conducted twice: 1) for all subjects enrolled using the lastobservation-carried-forward extrapolation, and 2) for completers only. In both cases the results were comparable with respect to significance of the findings. The trials was registered at http://clinicaltrials.gov (NCToo214877), and approved by Health Canada and by the local research ethics boards.

Results: Patients entered the trial euthymic $(n=20)$, mildly depressed $(\mathrm{n}=14)$, or mildly cycling $(\mathrm{n}=3)$. The MADRS scores at baseline were $11.1 \pm 7.7$, HAMD $5.5 \pm 5.0$, YMRS $2.8 \pm 3.2$, and HAMA $7.4 \pm 4.6$. Ten patients dropped out of the study mainly because of withdrawn consent and/or blue staining of urine. Compared to the placebo dose, the active dose of methylene blue improved symptoms of depression on the MADRS and HAMD scales by $6.6 \pm 14.2$ and $4.1 \pm 10.0$ points respectively (effect sizes of 0.47 and $0.42, p=0.02$ and 0.03 ). In addition, patients treated with methylene blue reported an improvement of anxiety on HAMA scale by $4.1 \pm 9.0$ points with an effect size of $0.46(p=0.004)$; ratings of mania remained low and constant throughout the study $(\mathrm{p}=\mathrm{ns})$. Methylene blue also improved several aspects of verbal memory performance on California Verbal Learning Test ( $\mathrm{p}$ values $\sim 0.07$ ), although no improvement was apparent on measures of executive functioning, selective attention, or early information processing. The medication was well tolerated with only transient and mild side effects; and there was no evidence of adverse effects of $\mathrm{MB}$ on cognitive functioning. In fact, most patients felt subjectively improved in the course of the study and six subjects decided to continue their treatment after completing the trial.

Discussion: Our results indicate that methylene blue used as an adjunct medication may improve residual symptoms of depression and anxiety in patients with bipolar disorder. In addition, treatment may be associated with improvement in certain aspects of verbal memory. It will be important to investigate the possible pharmacological mechanisms involved and whether other drugs with a similar mode of action could be also effective in bipolar disorder.

Disclosure: M. Alda: None. G. MacQueen: None. M. McKinnon: None. J. Garnham: None. T. Hajek: None. C. O'Donovan: None. M. Sokolenko: None.

\section{Effects of Marijuana Cues and a Marijuana "Prime" on Marijuana Craving and Self-Administration in Humans \\ Gillinder Bedi*, Ziva Cooper, Richard Foltin, Suzanne K. Vosburg, Margaret Haney}

\section{Columbia University, New York, NY}

Background: Relapse to drug use remains a critical problem for abstinent drug users. Although relapse triggers appear to be complex, both conditioned stimuli associated with drugs (drug cues) and small, "priming" drug doses reinstate drug-seeking in laboratory animals trained to self-administer drugs. In humans, cocaine-paired cues and exposure to a low dose of cocaine (a prime) increase cocaine selfadministration. However, little is known about the effects of marijuana (MJ) cues or priming MJ doses on MJ self-administration. Here, we assessed the effects of visual, olfactory, and gustatory MJ cues, with and without a priming dose of MJ, on MJ self-administration, craving, mood, and withdrawal symptoms in non-treatment seeking, daily MJ smokers.

Method: The design was within-subjects and double-blind. Participants $(\mathrm{N}=9$; all male) underwent a 10-day residential stay, with an initial day of investigator-administered active $\mathrm{MJ}$ ( $5.5 \% \mathrm{THC})$ to standardize exposure. They then underwent three, 3-day phases consisting of two days of abstinence followed by a single 'Relapse' day, in which participants could self-administer up to three puffs of active MJ (5.5\% THC) on six occasions over the day. To model relapse in non-treatment seekers, there was a financial incentive to remain abstinent on Relapse days, i.e., participants had to purchase selfadministered MJ using study earnings. The effects of three conditions on $\mathrm{MJ}$ relapse were assessed in counter-balanced order: cue, prime, and control. On the Cue day, participants were exposed to MJ cues without a priming dose. On the Prime day, they received both MJ cues and a prime. On the Control day, they received neither cues nor a prime. MJ cues consisted of an active MJ cigarette that participants 
smelt both lit and unlit, followed by a single inhalation from a placebo MJ cigarette. The MJ prime was the same as the cue condition, except that participants took a single inhalation from an active (5.5\% THC) MJ cigarette. Participants were blind to the doses of MJ tested in the Cue and Prime condition. The main outcomes were the amount of active MJ self-administered on Relapse days, and peak MJ craving change scores (maximal score on Relapse day minus the maximal score on the day prior). We also assessed effects on peak change scores on a range of subjective mood dimensions and on behavioral indicators of MJ withdrawal.

Results: There was no effect of MJ cues or a prime on MJ selfadministration. There was also no effect of cues or a prime on peak MJ craving change scores. On both Cue and Prime days, peak ratings of 'high' increased significantly compared to Control days. The MJ prime also increased peak ratings of 'stimulated', with no other effects on subjective mood observed. There was a trend $(p<0.08)$ for the MJ prime to reduce objectively-observed signs of MJ withdrawal.

Discussion: These data provide no evidence that MJ cues or a priming dose of MJ increase self-administration in non-treatment seeking participants. Surprisingly, the data also do not support an effect of MJ cues or a prime on MJ craving. However, although there was no effect on change in peak craving scores, $\mathrm{MJ}$ cues with or without a priming dose may have increased MJ craving in the period immediately after exposure; more detailed analysis will assess this possibility. MJ cues increased ratings of 'high', likely reflecting effects of conditioning or drug-related expectations. A priming dose of MJ produced expected subjective effects, and also appeared to alleviate withdrawal. Thus, unlike findings with cocaine, the experience of a MJ 'high' either due to expectancy effects following MJ-paired cues or due to a single puff of active MJ did not precipitate an increase in MJ self-administration. To date, there is no evidence that MJ cues or administration of a small amount of $\mathrm{MJ}$ result in increased $\mathrm{MJ}$ use.

Disclosure: G. Bedi: None. Z. Cooper: None. R. Foltin: None. S. Vosburg: None. M. Haney: None.

178. A Single Cross-Over, Open-Label Study of the Relative Bioavailability of Buprenorphine Implants versus Suboxone in Patients with Opioid Dependence

Katherine Beebe*, John Rotrosen, Walter Ling, Sunil P. Sreedharan, Rajesh Patel

Titan Pharmaceuticals, South San Francisco, CA

Background: Probuphine is a subdermal implant in Phase III development that delivers a low, continuous level of BPN for 6 months. This study evaluated the relative bioavailability of Probuphine versus SL-BPN by comparing plasma $\mathrm{BPN}^{\mathrm{AUC}_{\mathrm{O}-24}}$ at steady state.

Methods: Eleven opioid dependent adults were treated with SL-BPN ( $16 \mathrm{mg} /$ day) for at least 5 consecutive days prior to 24 -hour inpatient PK sampling, immediately followed by subdermal placement of four Probuphine implants and 24-hour re-sampling. Four weeks after implant insertion, steady-state 24-hour sampling was performed. At week 8, implants were removed and 24-hour PK sampling was repeated.

Results: BPN plasma levels post-implantation peaked on Day 1 and gradually reached steady state by Week 4. The mean peak plasma concentrations of BPN were significantly lower for Probuphine $(4.9 \pm 0.4 \mathrm{ng} / \mathrm{mL})$ compared to steady-state SL-BPN $(8.6 \pm 2.3 \mathrm{ng} / \mathrm{mL})$. At steady-state, Probuphine $\mathrm{AUC}_{\mathrm{o}-24}$ values $\left(19.6 \pm 1.2 \mathrm{ng}{ }^{\star} \mathrm{hr} / \mathrm{mL}\right)$ were significantly lower than SL-BPN $\left(66.3 \pm 12.0 \mathrm{ng}{ }^{\star} \mathrm{hr} / \mathrm{mL}\right)$. Twenty-four hours following implant removal, plasma BPN was virtually undetectable. Discussion: Results suggest that overall exposure to BPN is significantly reduced during treatment with Probuphine.

Disclosure: K. Beebe: Part 1; Senior Vice President, Titan Pharmaceuticals Inc. Part 2; Titan Pharmaceuticals Inc. Part 3; Titan Pharmaceuticals Inc. Part 5; Titan Pharmaceuticals Inc. J. Rotrosen: Part 1; Co-Investigator, Clinical Trial support for Probuphine studies administered through NYU and through Narrows Institute for
Biomedical Research \& Education. Some funding from AARA GO Grant from NIDA. Part 4; Co-Investigator, Clinical Trial support for Probuphine studies administered through NYU and through Narrows Institute for Biomedical Research \& Education. Some funding from AARA GO Grant from NIDA. W. Ling: Reckitt Benckiser, Hythiam, Titan Pharmaceuticals, (Research Grants). S. Sreedharan: Part 1; Consultant, Titan Pharmaceuticals Inc. Part 2; Titan Pharmaceuticals Inc. Part 3; Titan Pharmaceuticals Inc. Part 5; Titan Pharmaceuticals Inc. R. Patel: Part 1; Consultant, Titan Pharmaceuticals Inc. Part 2; Titan Pharmaceuticals Inc. Part 3; Titan Pharmaceuticals Inc. Part 5; Titan Pharmaceuticals Inc.

179. Interaction of Pimpamperone Augmentation of Citalopram and Genetic Variables in the Prediction of Antidepressant Response Elisabeth Binder*, Charles Nemeroff, Alan Schatzberg, Thomas Schlaepfer, Kees Bol, Ludo Haazen, Didier De Chaffoy, Erik Buntinx

Emory University School of Medicine, Atlanta, GA

Background: Individual variability observed in therapeutic response to antidepressant drug treatment is thought to be - in part - genetically determined. In a search for genetic predictors of antidepressant response in 1953 patients treated with citalopram (CIT) in the STAR ${ }^{*} \mathrm{D}$ trial, McMahon et al., reported that variation in the gene encoding the serotonin $2_{\mathrm{A}}$ receptor $\left(5 \mathrm{HTR}_{2 \mathrm{~A}}\right)$ was associated with antidepressant treatment outcome. Pipamperone (PIP) is an antipsychotic drug weak neuropleptic that not available in the US, but is widely used in Germany, Belgium and other EU countries. PIP is a highly potent and selective antagonist at the $5-\mathrm{HT}_{2 \mathrm{~A}}$ and $\mathrm{D}_{4}$ receptors when administered at very low doses ( 5 to $15 \mathrm{mg} /$ day). In a randomized double blind proofof-concept (POC) study in Major Depression (DSM-IV), PIP at those low doses was found to both accelerate and augment the antidepressant effects of CIT with no additional side effect burden.

Methods: As part of a separate protocol, approved by the responsible IRB, patients participating in the POC study were asked to participate in pharmacogenetic testing. Of the 165 patients enrolled into the POC study, 89 consented to this additional protocol, 44 of them receiving CIT and 45 PIPCIT. Pharmacogenetic assessments included polymorphisms in two genes influencing the pharmacokinetics of Cit and Pit: $\mathrm{CYP}_{2} \mathrm{D} 6$ and $\mathrm{ABCB} 1$ as well as polymorphisms in 5 genes known to be related to pharmacodynamic effects of selective serotonin receptor inhibitors: the serotonin transporter, BDNF, COMT, $5 \mathrm{HTR}_{2} \mathrm{~A}$ and $\mathrm{FKBP}_{5}$. In addition, the analysis included three putatively functional polymorphisms in genes regulating dopaminergic transmission: $\mathrm{DRD}_{4}, \mathrm{DRD}_{3}$ and the dopamine transporter gene. Here we present the results of an exploratory analysis on the relation of the assessed genetic variants and the onset of antidepressant effect, quantified by means of $\mathrm{T}_{50}$ values. T50 was defined as the time to $50 \%$ of the maximum observed antidepressant effect, i.e. $50 \%$ of the maximum drop in MADRS score and was obtained by means of population pharmacokinetic/pharmacodynamic (PK/PD) modeling.

Results: In this subsample, augmentation with PIP led to a highly significant reduction of $\mathrm{T}_{50}(\mathrm{p}<0.001)$, with the $\mathrm{T}_{50}$ being over 5 days shorter in patients treated with PIPCIT than patients treated with CIT alone. Of the 19 tested polymorphisms, only the BDNF $\mathrm{Val}_{66} \mathrm{Met}$ polymorphism had a significant main effect on $\mathrm{T}_{50}(\mathrm{p}=0.04$, genotypic model). Using a general linear model, we then tested the interaction of the genetic predictors with the treatment arm on $\mathrm{T}_{50}$ and observed a significant treatment by SNP interaction for the BDNF $\mathrm{Val}_{66}$ Met polymorphism $(\mathrm{p}=0.038)$ as well as for $\mathrm{r} 7997012$ within the ${ }_{5} \mathrm{HTR}_{2} \mathrm{~A}$ gene $(\mathrm{p}=0.024)$. In both cases, significant effects of the polymorphisms were only seen in CIT but not the PIPCIT group. In fact, for carriers of the BDNF Met risk allele, the improvement of T50 by the augmentation was 7.7 days on average vs. 4.2 days for the $\mathrm{Val} / \mathrm{Val}$ carriers. For carriers of the rs7997012 risk allele, the improvement was 6.6 days, vs. 1.6 days for the carriers of the rarer beneficial AA genotype. CYP2D6 metabolizer status did not have an effect on $\mathrm{T}_{50}$ in either treatment group. 
Discussion: Augmentation with PIP led to significant reduction in T50. Polymorphisms in $\mathrm{BDNF}$ and $5 \mathrm{HTR} 2 \mathrm{~A}$, previously reported to be associated with response to antidepressant treatment, predicted $\mathrm{T}_{50}$ only in the CIT but not the PIPCIT group. Improvement of $\mathrm{T}_{50}$ with PIP augmentation was much more pronounced in carriers of the previously defined non-response alleles. This suggests that patients carrying genetic polymorphisms known to be associated with poorer response to traditional antidepressant drugs would especially profit from PIP augmentation.

Disclosure: E. Binder: Part 1; PharmaNeuroBoost. C. Nemeroff: NovaDel Pharma, AstraZeneca Pharmaceuticals, CeNeRx BioPharma, PharmaNeuroboost, Corcept, Revaax Pharma. Part 2; Cenerx, Pharmaneuroboost, Astrazeneca, NovaDel Pharma. A. Schatzberg: Part 1; Lilly, Neuronetics, BrainCells, Corcept, Pfizer, PharmaNeuroBoost, Sanofi, Takeda, CeNeRx, CNS Response, Vivus, Roche, Xytis, GSK, Somaxon. Part 2; Corcept, PharmaNeuroBoost, Amnestix, BrainCells, Forest, Neuronetics, Pfizer, Merck, Neurocrine, Amylin, APA, APPI, Stanford. Part 3; PharmaNeuroBoost, APA, APPI. T. Schlaepfer: Part 1; PharmaNeuroBoost, Lundbeck. K. Bol: Part 5; Kinesis Pharma. L. Haazen: PharmaNeuroBoost. D. De Chaffoy: PharmaNeuroBoost. E. Buntinx: PharmaNeuroBoost.

\section{Risperidone-associated Prolactin Elevation And Markers Of Bone} Turnover Early In Treatment

Jeffrey Bishop*, Leah Rubin, Michael Akroush, James Reilly, Sunil Shrestha, Mani Pavuluri, John Sweeney

University of Illinois at Chicago, Chicago, IL

Background: Prolactin elevation has been proposed as a risk factor for low bone density and potentially osteoporosis in patients on long-term treatment with prolactin-elevating antipsychotics. We studied the acute effects of prolactin elevation on serum markers of bone formation and resorption in patients treated with risperidone.

Methods: Thirty-three participants (61\% male, $23.7 \pm 7.1$ years of age) meeting DSM-IV criteria for schizophrenia $(n=23)$, major depressive disorder with psychotic features $(n=5)$, bipolar disorder with psychosis $(n=5)$, or substance-induced psychosis $(n=1)$ were enrolled. At baseline, subjects were antipsychotic free for at least four half-lives of any prior medication, with $31 / 33$ having less than 4 weeks total lifetime exposure to antipsychotic medications. Subjects were evaluated before and after 4 weeks of risperidone treatment (median daily dose $=2 \mathrm{mg} / \mathrm{d}$, range $0.5-6 \mathrm{mg} / \mathrm{d}$ ). Assessments included symptom ratings, and a.m. blood draws to assess testosterone, estradiol, leptin, prolactin, osteocalcin (marker of bone formation), and n-telopeptide crosslinks (NTx marker of bone resorption). Primary analysis examined the impact of risperidone treatment on change on the bone markers and hormone levels from pre- to posttreatment. All analyses controlled for age, sex, and risperidone dose. Results: Prolactin levels increased significantly from pre- to posttreatment $(\mathrm{p}<0.001)$. NTx markers of bone resorption significantly decreased (improved) from pre- to post-treatment in the study sample as a whole $(\mathrm{p}<0.05)$. Subjects with the largest increases in prolactin after risperidone treatment had the greatest increases (worsening) in their NTx markers of bone resorption. Subjects with smaller increases in prolactin after beginning risperidone treatment had decreases (improvement) in bone resorption.

Discussion: This study suggests that risperidone-associated prolactin elevation has significant effects on bone physiology early in treatment. Interestingly, during the acute treatment phase, both positive and negative effects on bone resorption were observed, with higher doses potentially detrimental and lower doses associated with a reduction in bone resorption. The relationship between larger prolactin elevation and negative effects on bone resorption is consistent with prior studies showing increased risk for osteopenia associated with prolactinelevating antipsychotics. Our data indicate that these negative effects begin early in treatment in some patients which may have important implications for long term monitoring. However, the reduction of bone resorption observed in some patients illustrates that prolactin elevation is not uniformly negative in the acute phase of treatment. Disclosure: J. Bishop: Part 4; Ortho-McNeil Janssen. Part 1; Eli Lilly. L. Rubin: None. M. Akroush: None. J. Reilly: None. S. Shrestha: None. M. Pavuluri: Bristol-Meyers Squibb, Ortho-McNeil Janssen. J. Sweeney: Pfizer. Part 4; Ortho-McNeil Janssen.

\section{A Randomized, Double-Blind, Placebo-Controlled Trial of Citicoline for Bipolar and Unipolar Depression and Methamphetamine Dependence \\ E. Sherwood Brown*, Barry Gabrielson}

\section{UT Southwestern Medical Center, Dallas, TX}

Background: Amphetamine use disorders are common and severe problems. Persons with mood disorders, particularly bipolar disorder, have high rates of substance use disorders. Citicoline is a naturally occurring compound sold as an over-the-counter nutritional supplement in the United States and as drug in many other countries. Citicoline increases phospholipid incorporation into membranes, and enhances synthesis of structural phospholipids as well as increasing norepinephrine, dopamine, serotonin and acetyl choline levels in specific brain regions. Citicoline may have neuroprotective and cognitive enhancing properties. We previously reported promising findings on drug use, memory and study retention in patients with a history of mania and cocaine dependence given the nutritional supplement citicoline. In the current proof-of-concept study, we examined citicoline in bipolar or unipolar depression and methamphetamine dependence.

Methods: Sixty adults with bipolar depression or major depressive disorder and methamphetamine dependence were randomized to citicoline $(2000 \mathrm{mg} /$ day) for 12 weeks. Mood was assessed using Inventory of Depressive Symptomatology-Clinician Version (IDS-C), and cognition with the Hopkins Auditory Verbal Learning Test (HVLT), and Stroop Color Word Test. Drug use was assessed by self-report and urine drug screens. Between-group differences in baseline to exit change (intent-to-treat sample, defined as at least one post-baseline assessment on the outcome of interest, with last observation carried forward) in continuous outcomes were compared with ANCOVAs that used baseline scores of the outcome being assessed, gender, mood disorder diagnosis, age which differed between groups, baseline antidepressant use, and grams of methamphetamine use as covariates. Dichotomous data were compared using chi squares. Study survival was assessed using a KaplanMeier survival analysis. Significance was set at a $p$ value of $\leq 0.05$ for all comparisons.

Results: A total of 48 participants had a least one post-baseline visit and were used in the data analysis. These participants were $54 \%$ men, $77 \%$ Caucasian, and $65 \%$ had unipolar depression. An ANCOVA showed that those receiving citicoline had a statistically significantly greater improvement in IDS-C scores than those receiving placebo. Survival in the study was significantly longer and completion rates greater with citicoline $(41 \%)$ than with placebo $(15 \%)$. No significant differences were observed on cognition, or amphetamine-related outcomes. Citicoline was well tolerated.

Discussion: Citicoline was associated with an improvement in depressive symptoms, and longer study survival than placebo, with no differences in cognitive outcomes or drug use. Future studies of citicoline in mood disorder populations should target depressive symptoms. Future citicoline research in drug dependence should explore its use with other pharmacotherapeutic or psychosocial treatments to determine whether longer treatment retention with citicoline allows the other treatments to be more effective. This trial was supported by the Stanley Medical Research Institute.

Disclosure: E. Brown: Part 4; AstraZeneca, Forest. B. Gabrielson: None. 
182. Low 18F-Fallypride Binding Potential in Thalamus, Striatum, and Cortex Predicts Antipsychotic Treatment Response in Patients with Schizophrenia

Monte Buchsbaum*, Douglas Lehrer, Bradley Christian, Bingzhi Shi, Cemil Kirbas, Jonathan Entis, Meicheng Chiang, Shawn Sidhu, Holly Short, Holly Short, King-Wai Chu

\section{University of California, San Diego, San Diego, CA}

Background: Although many antipsychotic medications are available to patients with schizophrenia, their efficacy rates are limited to $\sim 50-60 \%$, highlighting the need for objective biomarkers that predict treatment response. We have found lower cortical and thalamic binding potential with the high-affinity $\mathrm{D}_{2} / \mathrm{D}_{3}$ dopamine PET ligand 18F-fallypride in never-medicated patients with schizophrenia. This suggests the hypothesis that individuals with low binding potential at baseline examination might show a greater subsequent response to antipsychotic therapy with risperidone or aripiprazole. This is an early report of our efforts to characterize potential biomarkers predictive of antipsychotic treatment response.

Methods: We acquired 18F-fallypride PET images from 9 unmedicated patients (6 medication naïve) with schizophrenia. Dopamine D2/D3 receptor levels were measured as binding potential (BP) using Logan analysis and a cerebellar reference. MRI images in standard Talairach position and segmented into gray and white matter were coregistered to fallypride images. A stereotaxic atlas of 40 Brodmann areas (BA) was applied, allowing calculation of BP in each BA. Subjects were then randomized into a 16-week double-blind aripiprazole $(n=3)$ or risperidone $(n=6)$ trial. Baseline vs. end-of-trial PANSS change scores were compared to BP values in an intent-to-treat analysis.

Results: Data were analyzed for the combined sample (aripiprazole + risperidone). $18 \mathrm{~F}$-fallypride $\mathrm{BP}$ in the parahippocampal gyrus (BA27 and $35)$, orbitofrontal cortex (BA47 and 12), BA28, and the putamen and caudate had correlations above $\mathrm{r}=0.58$ ( $\mathrm{p}<0.05$, 1-tailed) with PANSS change scores (general score and, in some instances, total score plus positive or negative symptom scores). The left thalamus (o.63) also showed a significant correlation with improvement on the negative scores. For the medial dorsal nucleus on the right (which had significantly lower BP in our larger cohort of 24 medication-naïve patients), the correlations were $0.64,0.50,0.76$, and 0.66 for PANSS total, positive, negative, and general symptoms, respectively, and $0.54,0.39,0.65,0.57$ for the left medial dorsal nucleus. Caudate values were $0.72,0.65,0.42,0.81$ for the left and $0.75,0.68,0.48,0.83$ for the right. The results were not dissimilar when analyzed only for the risperidone treatment group $(n=7)$.

Discussion: Dopamine receptor affinity and density estimated by BP appear to be an effective means to identify a testable biomarker associated with antipsychotic treatment response, particularly in temporal and subcortical regions of corticolimbic and corticostriatothalamic circuits previously implicated in the pathophysiology of schizophrenia. Results are consistent with the interpretation that lower binding of fallypride at baseline predicts greater clinical response to antipsychotic medication. Clearly, a larger sample is needed to extend these results, and importantly, to determine whether $18 \mathrm{~F}$-fallypride BP, as measured by PET, might provide a biomarker predictive of treatment response to antipsychotic drugs.

Disclosure: M. Buchsbaum: None. D. Lehrer: None. B. Christian: None. B. Shi: None. C. Kirbas: None. J. Entis: None. M. Chiang: None. S. Sidhu: None. H. Short: None. H. Short: None. K. Chu: None.

183. Efficacy of Adjunctive Aripiprazole in Major Depressive Disorder: Week 2 Early Response Predicts Remission

Daniel Casey*, Kimberly Laubmeier, Sabrina Marler, Robert Forbes, Ross Baker

Oregon Health \& Science University, Portland, OR, Bristol-Myers Squibb, Plainsboro, NJ

Background: The effect of antidepressant therapy (ADT) has conventionally been thought to occur after at least 2 to 3 weeks of treatment (1). Current American Psychiatric Association (APA) Guidelines are aligned with this delayed-onset theory, i.e., recommending re-evaluation of ADT pharmacotherapy if at least moderate improvement is not seen following 6-8 weeks of treatment (2). However, recent clinical evidence supports an early-onset theory of $\mathrm{ADT}$ action whereby an early pattern of response (within 2 weeks) is a significant predictor of subsequent clinical outcomes (3). Building from a previous time course of response analyses (4-6), we conducted a more detailed post-hoc examination of the early-onset theory of adjunctive aripiprazole using predefined quartiles of response (6).

Methods: Data were pooled from three nearly identical studies consisting of an 8-week prospective ADT phase to document inadequate response (Phase $\mathrm{B}$ ), and a 6-week, randomized, controlled phase (Phase $\mathrm{C}$ ) using adjunctive placebo or adjunctive aripiprazole (2-20 mg/day) $(7,8)$. Quartile response categories were defined based on percent reduction in the Montgomery-Asberg Depression Rating Scale (MADRS) total score at the end of Phase C, relative to the end of Phase B: [1] minimal response $(\leq 25 \%)$, [2] partial response $(>25 \%$ to $<50 \%)$, [3] moderate response $(\geq 50 \%$ to $<75 \%)$, and [4] robust response $(\geq 75 \%)$. The proportion of adjunctive placebo $(n=525)$ versus adjunctive aripiprazole $(\mathrm{n}=540)$ patients achieving each response category was compared for each category using the Cochran-Mantel-Haenszel test, and changes from baseline in mean MADRS score at Weeks 1 to 6 (Phase C) were compared between treatments using analysis of covariance. The predictive value of early response was calculated using univariate logistic regression on data from two trials and confirmed using data from the third trial.

Results: Compared with adjunctive placebo, adjunctive aripiprazole treatment was associated with a significantly greater proportion of patients achieving a partial response $(23.9 \%$ vs. $17.9 \%, \mathrm{p}=0.017)$, moderate response $(23.1 \%$ vs. $15.0 \%, \mathrm{p}<0.001)$, and robust response $(14.3 \%$ vs. $7.4 \%, \mathrm{p}<0.001)$ at the end of Week 6. Adjunctive aripiprazole treatment was associated with a significantly lower proportion of patients achieving a minimal response $(38.7 \%$ aripiprazole vs. $59.6 \%$ placebo, $\mathrm{p}<0.001)$ compared with adjunctive placebo. More adjunctive aripiprazole patients, including partial, moderate, and robust Week 6 responders, showed a marked improvement in their change from baseline MADRS total scores as early as Week 2 compared with those responding to adjunctive placebo. Among patients with an early response, $73 \%(80 / 110)$ in the adjunctive aripiprazole group and $57 \%(27 / 47)$ in the adjunctive placebo group achieved remission. In addition, Week 2 early response $(\geq 50 \%$ reduction in MADRS) was a significant predictor of Week 6 endpoint remission (MADRS $\leq 10$ and $\geq 50 \%$ decrease in MADRS) in both treatment groups.

Discussion: Consistent with the theory of early-onset of action for ADT, patients with moderate and robust responses to adjunctive aripiprazole showed substantial improvement in depressive symptoms within the first 2 weeks of treatment. Early response at week 2 was a significant predictor of endpoint remission. Taken together, these early response findings can be extremely helpful to both clinicians and patients in facilitating important treatment decisions early in the course of therapy.

References:

J Affect Disord 2010;120(1-3):16-23.

Am J Psychiatry 2000;157:1-45.

Progress in Neuro-Psychopharmacology \& Behavioral Psychiatry 2010;34:259-264.

Poster presented at the 63rd Annual Meeting of the Society for Biological Psychiatry, May 1-3, 2008, Washington DC.

J Clin Psychopharmacol 2010;30:300-305.

Poster presented at the 5oth Annual Meeting of the New Clinical Drug Evaluation Unit, June 14-17, 2010, Boca Raton, FL.

Prim Care Companion J Clin Psychiatry 2008;10(6):440-447.

CNS Spectr. 2009;14:197-206.

Disclosure: D. Casey: Part 1; Consulting relationship with: Lundbeck, Bristol-Myers Squibb, Janssen, Merck. K. Laubmeier: Part 5; Employee of Bristol-Myers Squibb. S. Marler: Employee of Bristol-Myers Squibb. 


\section{S386}

R. Forbes: Employee of Otsuka Pharmaceutical Development \& Commercialization, Inc. R. Baker: Employee of Bristol-Myers Squibb.

\section{Length of Prenatal Exposure to Selective Serotonin Reuptake Inhibitors (SSRI): Effects on Neonatal Adaptation and Psychomotor Development}

Regina Casper*

\section{Stanford University, Stanford, CA}

Background: This study evaluated the question whether length of in utero exposure to SSRI antidepressants might affect neonatal outcome and/or psychomotor development in infancy.

Methods: Birth outcome was determined in the offspring of 55 women with major depressive disorder who used SSRI medication for different durations during pregnancy. Children, average age 14 months, underwent a pediatric examination and an evaluation with the Bayley Scales of Infant Development (BSID-II).

Results: Duration of in utero exposure to SSRIs was negatively associated with total Apgar scores at 1 and 5 minutes, specifically the activity subscale. Odds ratios for a low score $(<2)$ on this scale were 3.8 [CI: 1.3, 10.6, $\mathrm{p}=.0121$ ] and 6.0 [CI: 1.3, 27.8, $\mathrm{p}=.0221]$ at 1 and 5 minutes, respectively. Newborns with longer exposure had more admissions to the Neonatal Intensive Care Unit. Longer duration of exposure was positively associated with the risk for lower psychomotor and behavioral rating scale scores in infancy $(p=.012$ and $p=.007$, respectively). Length of exposure was significantly associated with the BRS factor scales: Orientation/Engagement (95\% CI: 1.31-(-34); $\mathrm{p}<.001)$, Emotional Regulation (95\% CI: $-1.21-(-.21) ; \mathrm{p}<.005)$ and Motor Quality (95\% CI: -1.09-(-.08); p=.024).

Discussion: Newborns with longer duration of exposure to SSRI antidepressants had a higher risk for a transient adverse neonatal outcome, and lower scores in motor, but not mental, development in infancy. An influence of variability in depression severity as a contributing factor cannot be excluded. The observation that the children's motor function was found to be within the normal range offers reassurance to parents and physicians.

Disclosure: R. Casper: None.

\section{Contextual Cues Influence The Acute Effects Of D-amphetamine} Emma Childs*, Harriet De Wit

University of Chicago, Chicago, IL

Background: Contextual conditioning i.e., associations between psychoactive drug effects and environmental cues, is thought to play an integral role in the cycle of addiction. Environmental cues that are paired with drug experiences can acquire secondary reinforcing characteristics and the ability to control drug-seeking behavior. However, contextual conditioning has not been systematically studied in humans and it is not clear how drug effects interact with the environment in which they are experienced. In this experiment we aimed to investigate how the context in which d-amphetamine is administered influences the acute effects of the drug.

Methods: Healthy men and women underwent four double-blind conditioning sessions, with administration of amphetamine and placebo, in alternating order, counterbalanced between participants. One group of participants (paired group) always received amphetamine in one testing room and placebo in another testing room, while another group (unpaired group) received amphetamine and placebo, once in each testing room. During the sessions, participants completed subjective self-report questionnaires, and heart rate and blood pressure were monitored at $30 \mathrm{~min}$ intervals.

Results: Amphetamine produced its expected effects in all participants; it increased subjective ratings of stimulation, euphoria and feeling high. However, compared to the unpaired condition, the group who received amphetamine in the same environment during each session reported greater effects on the second occasion on certain measures of response to d-amphetamine (Drug Liking and Want More).

Discussion: These findings indicate that the context in which a drug is administered can influence the effects of a drug, perhaps through conditioning. This research is supported by NIDA Ro1 DA02812.

Disclosure: E. Childs: None. H. de Wit: None.

\section{Low Dose Naltrexone Increases Marijuana's Abuse Liability in} Daily Marijuana Smokers

Ziva Cooper*, Margaret Haney

\section{Columbia University, New York, NY}

Background: Findings from studies with laboratory animals provide compelling evidence of a bidirectional modulatory relationship between the opioidergic and cannabinoid systems, with opioid antagonists decreasing the reinforcing and rewarding behaviors elicited by cannabinoid receptor subtype 1 ( $\mathrm{CB} 1)$ agonists. However, studies in humans have generally shown an opposite pattern of effects. For example, a recent human laboratory study found that a broad range of naltrexone doses (12-10o $\mathrm{mg}$, po) increased the subjective and cardiovascular effects of a single strength of marijuana in daily marijuana smokers. The current study was designed to further clarify the interactions between the opioid and cannabinoid systems by determining how naltrexone shifts the dose-response function for marijuana-induced effects in human marijuana smokers. A marijuana smoking procedure was designed with the intent to characterize a dose-response relationship between marijuana exposure and its elicited effects. Over 8 sessions, naltrexone $(12 \mathrm{mg}$, po) or placebo was administered prior to smoking a range of marijuana puffs $(0,2,4$, or 6 puffs of active marijuana) under blind conditions. Marijuana's dose-dependent subjective and physiological effects were assessed in the absence and presence of naltrexone.

Methods: Marijuana smokers $(\mathrm{N}=18)$ were recruited for this withinsubject, randomized, double-blind, placebo-controlled study. During each session, participants smoked a total of 6 puffs from 3 marijuana cigarettes ( 2 puffs from each cigarette) according to verbal instructions 45 minutes after naltrexone $(12 \mathrm{mg})$ or placebo administration. The number of active $(6.2 \%$ or $5.5 \%$ THC) versus inactive (0.0\% THC) cigarettes smoked during each session varied according to the condition $(3$ inactive cigarettes $=0$ puffs; 1 active +2 inactive cigarette $=2$ puffs; 2 active +1 inactive cigarette $=4$ puffs; 3 active +0 inactive cigarette $=6$ puffs). Active cigarettes were always smoked before inactive. Subjective ratings of marijuana quality, mood, and physical symptoms were measured at 30-60 minute intervals after smoking, and vital signs (heart rate and blood pressure) were monitored throughout the sessions to assess physiological drug effects. Results: Compared to placebo, naltrexone increased the effects of inactive marijuana on subjective ratings of marijuana strength and 'High,' and also increased positive subjective ratings of drug effect ('Liking,' 'Good Effect,' 'Take Again,' and 'Stimulated'). Naltrexone alone had no effect on marijuana craving. Under placebo conditions, active marijuana increased subjective ratings of marijuana strength and 'High,' and also increased positive subjective ratings of drug effect ('Liking,' 'Good Effect,' 'Take Again,' and 'Stimulated') compared to inactive marijuana. These effects were not dose-dependent. However, active marijuana decreased marijuana craving in a dose-dependent fashion. Naltrexone administered before active marijuana (2, 4 and/or 6 puffs) further increased ratings of marijuana's positive drug effects, strength and 'High' relative to placebo. Naltrexone administered before active marijuana also increased marijuana craving. In terms of cardiovascular effects, naltrexone alone decreased heart rate relative to placebo. Marijuana alone dose-dependently increased heart rate relative to inactive marijuana, and naltrexone did not affect marijuanainduced increases in heart rate.

Discussion: The current marijuana-dosing paradigm failed to produce dose-dependent subjective effects while reliably producing dosedependent increases in heart rate. Given that active cigarettes were 
always smoked before inactive, participants may have had the expectation that all of cigarettes were active, thereby resulting in a flattened dose-response curve for $r$ subjective drug effect ratings. The results from this study confirm that among daily marijuana smokers, mu-opioid antagonism increases the subjective effects of marijuana associated with its increased abuse liability. These findings also demonstrate that under some conditions, increases in positive subjective drug effects do not predict a change in drug craving; though active marijuana alone and in combination with naltrexone increased positive subjective drug effects, marijuana alone dosedependently decreased craving whereas naltrexone in combination with marijuana increased craving. Though drug craving does not necessarily predict drug taking, these findings are particularly relevant when exploring potential pharmacotherapies for marijuana-use disorders.

Disclosure: Z. Cooper: None. M. Haney: None.

\section{Abstinent Cocaine-Dependent Subjects Have Reduced Affective Bias to Appetitive Cues}

Sarah Corcoran*, Samuel Fernandez-Carriba, Bruce Cuthbert, Jeff Hollis, Erica Duncan

\section{Atlanta VAMC/School of Medicine, Emory University, Atlanta, GA}

Background: Chronic cocaine use and subsequent abstinence leads to numerous neuroadaptations, including the dysregulation of dopamine systems associated with reward circuitry in the brain. A large body of literature reports decrements in reward directed behavior or anhedonia resulting from chronic psychostimulant exposure (Koob \& LeMoal 1997, 2001; Volkow et al 2004). Cocaine-associated cues as well as those associated with non-drug rewards can induce activation of brain systems associated with drugs of abuse. Childress et al. (2008) reported that even "unseen" images of sexual and cocaine-related content produced activation of limbic areas in cocaine-dependent subjects as measured with fMRI. Additionally, the increased activity in these limbic areas predicted more positive affective responses to "seen" versions of the same images in a later test session. The present study utilized an affective priming task modeled after the tasks used by Childress et al. (2008) and Fazio et al. (1995) to examine the effect of "unseen" priming images on reaction times (RT) for discrimination of positive or negative words. It is believed that the recognition of the word valence is facilitated or "primed" by prior presentation of congruent images (ie. positive word with a sexual image) whereas prior presentation of images that are incongruent to the word valence (ie. positive word with an aversive image) lead to slower responses or increased RT. The present study examined the effect of priming images, including cocaine-related images, on RT for word valence discrimination in abstinent cocaine-dependent and control subjects. Methods: 20 abstinent cocaine-dependent (mean days abstinent $=$ $78.2 \pm 110.8)$ and 13 age-, race- and sex- matched healthy control subjects performed an affective priming task in which they were asked to judge the valence (positive or negative) of 144 words (selected from the Affective Norms for English Words; Bradley and Lang, 1999) presented on a computer. The words were preceded by backwardmasked or "unseen" images from one of 4 categories (cocaine, sexual, aversive and neutral). Cocaine images were selected from our laboratory archive and all other images were selected from the International Affective Picture Systems (IAPS; Lang et al. 2001). During the task, subjects were briefly presented with a visual stimulus (the priming image) that was masked by a subsequent visual stimulus (the mask) such that the priming image was rendered invisible; these visual stimuli were followed by the presentation of the word target. Subjects were asked to respond on a button box to indicate whether the presented word was "good" or "bad". RT for the word valence discrimination was measured for the 8 different stimulus categories (4 types of images, each paired with a positive or negative word). Quantitative "affective bias scores" (Childress et al. 2008) were calculated for each of the four categories of images based on the equation: (meanRT(negative word trials)-meanRT(positive word trials)). Positive scores reflect a greater positive affect bias (faster RT for positive word trials), while negative scores reflect a greater negative affect bias (faster RT for negative word trials).

Results: In control subjects, the RT for identification of positive words was faster for trials with prior presentation of sexual images compared to negative words, thus resulting in a greater positive affect bias. However, the RT of the cocaine-dependent subjects remained relatively stable for all image categories, regardless of the valence of the word target, resulting in low affect bias scores. Specifically, abstinent cocaine-dependent subjects had significantly lower affect bias scores for sexual images as compared with control subjects $(F(1,32)=5.55$; $\mathrm{p}=0.025$ ). Cocaine-dependent subjects also failed to demonstrate any priming of positive word discrimination by the cocaine-related images. Discussion: The lack of facilitation of the discrimination of positive words by prior presentation with either cocaine or sexual images in abstinent cocaine-dependent subjects indicates a reduced affective response to both drug and non-drug appetitive cues. This may be a reflection of persistant hypodopaminergia and anhedonia associated with sustained abstinence following long-term cocaine addiction. Supported by NIDA 5RO1DA018294, E. Duncan.

Disclosure: S. Corcoran: None. S. Fernandez-Carriba: None. B. Cuthbert: None. J. Hollis: None. E. Duncan: Part 4; Janssen Pharmaceutica, Ortho-McNeil Janssen Scientific Affairs, Bristol-Myers Squibb and Company.

\section{Weight Gain and Metabolic Changes in Studies of Aripiprazole for the Treatment of Pediatric Bipolar I Disorder and Adolescent Schizophrenia \\ Christoph Correll*, Stephen Murray, Joan Zhao, George Manos,} Raymond Mankoski, Robert Forbes, Ronald Marcus, Robert McQuade

The Zucker Hillside Hospital, Glen Oaks, NY

Background: Second-generation antipsychotics (SGA) can cause weight gain and metabolic disturbances in pediatric patients, although differences exist among the SGAs. Studies of aripiprazole (ARI) in pediatric patients have shown that although weight gain can occur, there are usually minimal, if any, changes in metabolic parameters across the entire study population. This analysis investigates whether those subjects who do gain significant weight while taking ARI have a different metabolic profile than those who do not.

Methods: Subjects receiving ARI from short- and long-term trials (open-label and placebo-controlled) in pediatric patients with schizophrenia (SZ; 13-17 years) or a manic or mixed episode associated with bipolar I disorder (BPD, 10-17 years), were stratified according to whether or not they gained significant weight, defined as either an increase from baseline of $\geq 7 \%$ ( $+w t 7 \%$ ) or $\geq 0.5$ standard deviation in $\mathrm{Z}$-score $(+\mathrm{wtZ})$. Subjects who did not gain significant weight are designated as $-w t 7 \%$ or $-w t Z$. Fasting metabolic measures included glucose, total cholesterol, low-density lipoprotein (LDL), high-density lipoprotein (HDL), and triglycerides (all mg/dL). Group means ( \pm SE) and differences between groups in the change from baseline were derived using ANCOVA for the short-term studies and MMRM for the long-term studies.

Results: The numbers of subjects receiving ARI with significant weight gain were: SZ 6-week $(\mathrm{N}=196)$ : $+\mathrm{wt} 7 \%, \mathrm{n}=9(4.6 \%),+\mathrm{wtZ}, \mathrm{n}=3$ $(1.5 \%) ; \mathrm{SZ}$ 26-week $(\mathrm{N}=158)$ : $+\mathrm{wtZ}, \mathrm{n}=20 \quad(12.7 \%)$; BPD 4-week $(\mathrm{N}=190):+\mathrm{wt} 7 \%, \mathrm{n}=12(6.3 \%),+\mathrm{wtZ}, \mathrm{n}=6(3.2 \%) ;$ BPD 30-week $(\mathrm{N}=148),+w \mathrm{wZ}, \mathrm{n}=25(16.9 \%)$. For comparison, in the short-term placebo-controlled studies, the number of subjects receiving placebo with significant weight gain were: SZ 6-week $(\mathrm{N}=98)$ : + wt7 $\%, \mathrm{n}=1$ $(1.0 \%)$; BPD 4-week $(\mathrm{N}=92):+w t 7 \%, n=3(3.3 \%)$. In the short-term $\mathrm{SZ}$ trial, for subjects receiving ARI, the mean change in all metabolic measures for each group (with or without weight gain) was $\leq 5 \mathrm{mg} / \mathrm{dL}$. In the long-term SZ trial, mean changes were all $\leq 5.5$, with the exception of glucose: $+\mathrm{wtZ}=9.3(\mathrm{SE}=6.8, \mathrm{n}=12$, baseline $=90.6)$, $-w t Z=2.7(S E=1.7, n=67$, baseline $=86.5)$, and the $95 \%$ confidence 


\section{S388}

interval for the difference (CI) excluded zero. No individuals with weight gain shifted from a classification of "normal" to "high/ abnormal" on any metabolic parameter in either the short- or longterm SZ trials, with the exception of one subject who had an abnormal HDL at the last visit. In the short-term BPD trial, for subjects receiving ARI, the mean change in all metabolic measures for each group (with or without weight gain) was $\leq 5$ with two exceptions. For total cholesterol: $+\mathrm{wt} 7 \%=6.7(\mathrm{SE}=8.7, \mathrm{n}=7$, baseline $=164.6),-\mathrm{wt} 7 \%=$ $-1.2(\mathrm{SE}=1.9, \mathrm{n}=98$, baseline $=164.3)$, and $+\mathrm{wtZ}=5.3 \quad(\mathrm{SE}=13.9$, $\mathrm{n}=4$, baseline $=165.8)$, $-\mathrm{wtZ}=-0.9(\mathrm{SE}=1.9, \mathrm{n}=101$, baseline $=164.3)$; however, the $95 \% \mathrm{CI}$ in both groups included zero. For triglycerides: + $\mathrm{wt} 7 \%=95.6(\mathrm{SE}=40.6, \mathrm{n}=7$, baseline $=96.7),-\mathrm{wt} 7 \%=0.3(\mathrm{SE}=3.8$, $\mathrm{n}=98$, baseline $=99.5)$ and $+\mathrm{wtZ}=98.8 \quad(\mathrm{SE}=40.7, \mathrm{n}=4$, baseline $=76.3)$, $-\mathrm{wtZ}=3.0(\mathrm{SE}=4.6, \mathrm{n}=101$, baseline $=100.2)$; the $95 \%$ $\mathrm{CI}$ in both groups excluded zero. No individuals with weight gain shifted from a classification of "normal" to "high/abnormal" in any metabolic parameter except for triglycerides ( + wt $7 \%$, four of six with a normal baseline; + wtZ, two of four with a normal baseline). In the long-term BPD trial, the mean change in all metabolic measures was $\leq 5$ with the exception of triglycerides: $+\mathrm{wtZ}=10.9(\mathrm{SE}=10.6, \mathrm{n}=18$, baseline $=84.4),-w t Z=-14.0 \quad(S E=7.3, n=43$, baseline =109.4); the $95 \%$ CI included zero. Shifts from "normal" to "high/abnormal" were seen for LDL (four of 76) and triglycerides (two of 19).

Discussion: A minority of pediatric patients with schizophrenia or a manic or mixed episode associated with BPD gained significant weight in the 4-6 week short-term trials or the 26-30 week long-term trials. In general, subjects with SZ who gained significant weight did not develop short-term metabolic problems and the observed mean longterm changes in glucose were small. In BPD subjects who gained significant weight, increases in triglycerides were observed in the short-term, but these increases were smaller in the long-term. Potential small changes in LDL were also seen. It is important to note, for both the SZ and BPD studies, that a primary limitation of this analysis is the small sample sizes in the + wt groups, which can lead to unreliable point estimates and unbalanced baseline values for some metabolic parameters. Further analyses of demographic and illness predictors of significant cardiometabolic changes are warranted.

Disclosure: C. Correll: Part 1; Consultant for AstraZeneca, BristolMeyers Squibb, Eli Lilly, Hoffmann-La Roche, Otsuka, Pfizer, and Vanda; member of the advisory boards for Actelion, AstraZeneca, Bristol-Myers Squibb, IntraCellular. Part 3; Received honoraria from from Bristol-Myers Squibb, Cephalon, GlaxoSmithKline, Lundbeck, Janssen, Johnson \& Johnson, Otsuka and Supernus. S. Murray: Part 5; Employee for Bristol Myers Squib. J. Zhao: Otsuka Pharmaceutical Development \& Commercialization. G. Manos: Employee of BristolMyers Squibb. R. Mankoski: Employee for Bristol-Myers Squibb. R. Forbes: Employee for Otsuka Pharmaceutical Development \& Commercialization. R. Marcus: Employee of Bristol-Myers Squibb. R. McQuade: Employee of Ostuka Pharmaceutical Development \& Commercialization.

189. A Clinical Case Report Illustrating Genetically Guided Pharmacotherapy for Aggressive Behavior in a Patient with Schizophrenia, Epilepsy and Intellectual Impairment

Joseph Cubells*, Elizabeth DeOreo, Philip Harvey, Steven Garlow, Kathryn Garber, Margaret Adam, Christa Martin

Emory Univ. Sch. Med., Atlanta, GA

Background: Recent findings have established clear associations between several genomic copy-number variants (CNV) and mental illnesses including schizophrenia and autism. Clinical testing for such CNV would be indicated if it were useful for guiding treatment decisions.

Methods: We report here a case in which identification of an illnessassociated $\mathrm{CNV}$ in a patient with schizophrenia led directly to a novel and effective medication treatment.
Results: A 39 year-old male presented to our unit with tactile, visual and auditory hallucinations. He had previously received diagnoses of schizophrenia (age 18, during his first psychiatric hospitalization), seizure disorder (age 12), and intellectual impairment (age 5). The patient's mother reported frequent violent outbursts. Medications at admission were ziprasidone $120 \mathrm{mg}$ QHS, clonazepam $1 \mathrm{mg}$ BID $+2 \mathrm{mg}$ QHS, propranolol $80 \mathrm{mg} /$ day, fluoxetine $80 \mathrm{mg} /$ day, divalproex sodium $250 \mathrm{mg}$ TID, topiramate $100 \mathrm{mg}$ TID, and pregabalin $200 \mathrm{mg}$ BID. The patient's combination of psychosis, learning disabilities, seizure disorder and facial dysmorphology prompted a search for potential underlying genetic abnormalities. Array Comparative Genomic Hybridization (aCGH), performed using a $44 \mathrm{~K}$ custom-designed whole genome oligonucleotide array with a backbone resolution of $\sim 200$ kilobases $(\mathrm{kb})$, revealed an interstitial deletion of $\sim 1.6$ Megabases $(\mathrm{Mb})$ involving bands $15 \mathrm{q} 13.2$ to $15 \mathrm{q} 13.3$, which was confirmed by Fluorescence In Situ Hybridization (FISH). The patient's hallucinations resolved rapidly with an increase in ziprasidone and his cognition improved with discontinuation of divalproex sodium, but his aggressive behavior persisted. Following a 21-day hospitalization, he was discharged to his parent's home on ziprasadone $80 \mathrm{mg}$ BID, fluoxetine $80 \mathrm{mg} /$ day, and clonazepam $4 \mathrm{mg} /$ day in divided doses, for psychosis, anxiety and agitation; levetiracitam $500 \mathrm{mg}$ BID and pregabalin $200 \mathrm{mg}$ BID for seizure control; ropinirole $1 \mathrm{mg}$ /day for Parkinsonian symptoms; topirimate $50 \mathrm{mg}$ TID for neuropathic pain, and propranolol $80 \mathrm{mg} /$ day for hypertension. After discharge his hallucinations remained under control, but the patient's rage outbursts and aggressive behavior became more frequent and increasingly violent, leading to 8 additional hospitalizations in the 20 months following the index admission. Despite trials of several antipsychotic medications, benzodiazepines, lithium, and clonidine, the patient's aggressive outbursts continued. After the patient's seventh admission, the patient was placed in a host home with a retired law enforcement officer, who skillfully managed the patient's violent outbursts. Despite the clear benefits of more effective behavioral management, daily outbursts continued, and the patient was hospitalized again after a particularly intense and prolonged aggressive episode. At discharge back to the host home, the patient's medications were: haloperidol, $15 \mathrm{mg}$ QHS, lorazepam, $2 \mathrm{mg}$ QHS, benztropine $2 \mathrm{mg}$ BID, propranolol $60 \mathrm{mg}$ per day, levetiracitam $500 \mathrm{mg} \mathrm{BID}$, pregabalin $200 \mathrm{mg}$ BID, topirimate $50 \mathrm{mg}$ $\mathrm{BID}$, and omeprazol, $40 \mathrm{mg}$ per day. The patient's $15 \mathrm{q} 13.3$ deletion resulted in hemizygosity of several loci, including CHRNA7, encoding the $\alpha 7$ nicotinic cholinergic receptor (NChR). We hypothesized that deficient transmission at the $\alpha 7$ NChR contributed to the patient's aggressive behavior, and therefore considered strategies for enhancing transmission at that receptor. Galantamine, an FDAapproved treatment for cognitive deficits associated with Alzheimer disease, is a positive allosteric modulator of NChR function and inhibitor of acetylcholinesterase (AChE). We started $4 \mathrm{mg}$ p.o. per day, and titrated the dose over 2 weeks to $12 \mathrm{mg}$ BID. The frequency of the patient's rage episodes declined substantially, dropping from once or more per day to fewer than once per week. The patient has been on the galantamine regimen for approximately 8 months, with no need for hospitalization.

Discussion: The positive response of the current patient's rage outbursts to galantamine suggests that pharmacological enhancement of neurotransmission mediated by the $\alpha 7$ NChR could be an important therapeutic approach for treatment of aggressive behavior in psychotic patients carrying 15q13.3 deletions. This case illustrates the potential utility of testing for genomic disorders in psychotic patients.

Disclosure: J. Cubells: Part 4; Roche Pharmaceuticals, ScheringPlough. E. DeOreo: None. P. Harvey: Part 1; Eli Lilly, Abbott Labs, Shire Pharma, Johnson and Johnson, Solvay Pharma, Cypress BioScience, Wyeth Pharma, Merck and Company, Teva Pharma. Part 2; Sepracor Pharma. Part 4; Astra Zeneca. S. Garlow: Part 1; Eli Lilly. K. Garber: None. M. Adam: None. C. Martin: None. 
190. The Acetylcholinesterase Inhibitor Rivastigmine Reduces Methamphetamine-Induced "Desire METH" and "Likely to Use METH" in Methamphetamine-Dependent Volunteers

Richard De La Garza*, Thomas Newton, Chandra Nerumalla, Colin Haile, James Mahoney, Adel Aziziyeh

Baylor College of Medicine, Houston, TX

Background: Although changes in the dopamine (DA) system have been most extensively studied, cholinergic transmission is also altered by drugs of abuse and both DA and acetylcholine (ACh) may contribute to psychostimulant reinforcement. In the current study, we sought to characterize the effects of treatment with rivastigmine $(0,1.5$, or $3 \mathrm{mg}$, b.i.d.) on subjective and reinforcing effects produced by administration of methamphetamine (METH: 0,15 , and $30 \mathrm{mg}, \mathrm{IV}$ ) in non-treatment-seeking, METH-dependent volunteers.

Methods: This was a double-blind, placebo-controlled, within-subjects, inpatient study. Participants received METH on day 1 , and were then randomized to placebo or rivastigmine on days 2-8. METH dosing was repeated on day 6 . On day 7 , participants completed two sample sessions, each consisting of 10 non-contingent infusions. In one session doses of o mg METH were administered and in the other doses of $5 \mathrm{mg}$ METH were administered. On day 8, participants completed two choices sessions, each consisting of 10 choices between receiving METH and receiving nothing. In one session o mg METH was available and in the other session $5 \mathrm{mg}$ METH was available. Participants were discharged on day 9 then asked to return to complete the other rivastigmine dose conditions within 2-4 weeks.

Results: Participants $(\mathrm{N}=17)$ were primarily Caucasian males who were $34.4 \pm 8.3$ (mean \pm S.D.) years of age. Participants reported using METH for $10.2 \pm 6.2$ years and used METH $17.4 \pm 9.9$ days out of the last 30 days. The data indicate that METH, but not saline, increased subjective effects of "Any Drug Effect", "High", "Stimulated", "Desire METH" and "Likely to Use METH" $(p<0.05)$. Rivastigmine significantly attenuated METH-induced "Desire METH" and "Likely to Use METH". Self-administration data reveal that participants chose METH infusions more often than saline $(p<0.001)$, but rivastigmine did not alter total number of choices for METH. METH also increased heart rate and blood pressure $(p<0.05)$, as compared to saline, and rivastigmine did not alter these cardiovascular effects.

Discussion: The results indicate that rivastigmine treatment was well tolerated and there is evidence indicating efficacy for rivastigmine in attenuating some key subjective effects produced by METH. These data indicate that acetylcholinesterase inhibitors, and other drugs that target acetylcholine systems (e.g., varenicline), warrant further consideration as treatments for methamphetamine dependence.

Disclosure: R. De La Garza: None. T. Newton: None. C. Nerumalla: None. C. Haile: None. J. Mahoney: None. A. Aziziyeh: None.

191. Effects of High Schizotypy on Control of Eye Movements: Modulation by Antipsychotic Drugs and Nicotine Anne Schmechtig, Jane Lees, Gerry Dawson, Colin Dourish, Kevin Craig, Bill Deakin*, Lawrence Wilkinson, Steve Willliams, Ulrich Ettinger

\section{University of Manchester, Manchester, United Kingdom}

Background: Oculomotor dysfunction has been well documented within schizophrenia spectrum populations and has been proposed to be a sensitive biomarker for antipsychotic and cognitive enhancing compounds. This study examined the effects of two antipsychotics and nicotine on oculomotor performance and investigated whether individuals with subclinical levels of schizophrenia-like symptoms (called high schizotypy) show a differential profile of drug response to controls.

Methods: In a randomized double blind, placebo-controlled study 233 participants were randomly assigned to one of 4 drug groups (nicotine, risperidone, amisulpride, placebo) and performed prosaccade (PS), antisaccade (AS) and smooth pursuit eye movement (SPEM) tasks. Participants were classified into high and average schizotypy groups measured by the Schizotypal Personality Questionnaire.

Results: Main effects of drug were found for PS gain, PS velocity and AS velocity (all $\mathrm{p}<0.01$ ), indicating impaired performance with risperidone irrespective of schizotypy level. AS error rate showed a main effect of drug $(\mathrm{p}<0.01)$ as well as a drug by group interaction $(\mathrm{p}=0.035)$. This pattern indicated that the performance of high schizotypes tended to be worse than controls after placebo but better after antipsychotics. Both groups improved with nicotine treatment. For SPEM a trend for a main effect of drug was found for velocity gain $(\mathrm{p}=0.052)$, indicating reduced gain with risperidone irrespective of schizotypy level.

Discussion: In this study eye movement performance proved to be a sensitive measure of pharmacological treatment effects. The main findings were: (1) replication of previous findings of cognitive enhancing effects of nicotine on antisaccade performance; (2) risperidone caused a slowing of AS and PS peak saccade velocity, a reduction in PS spatial accuracy, and an impairment in the ability to match eye velocity to target velocity during SPEM; (3) for the key biomarker of antisaccade error rate, drug effects were modulated as predicted by schizotypy status - risperidone and amisulpride tended to disrupt saccade inhibition in controls but to improve it in high schizotypes. Together, these findings represent promising trends for future research investigating pharmacological influences on high risk and schizophrenia spectrum populations.

This study was supported by the P1vital CNS Experimental Medicine Consortium (members AstraZeneca, GlaxoSmithKline, Lundbeck, Organon (a subsidiary of Merck) and Pfizer).

Disclosure: A. Schmechtig: None. J. Lees: None. G. Dawson: Part 1; P1vital. Part 2; P1vital. Part 3; P1vital. Part 5; P1vital. C. Dourish: Part 1; P1vital. Part 2; P1vital. Part 3; P1vital. Part 5; P1vital. K. Craig: Part 1; P1vital. Part 2; P1vital. Part 3; P1vital. Part 5; P1vital. B. Deakin: Part 1; P1vital, Servier, Janssen, AstraZeneca. Part 4; P1vital, Servier, AstraZeneca. L. Wilkinson: Part 1; P1vital. Part 4; P1vital, Lilly (UK). S. Willliams: P1vital. U. Ettinger: P1vital, GE Healthcare.

192. Mood Changes and Nicotine Blood Levels After Tobacco Smoking Edward Domino*, Wendy Yang, Catherine Evans, Lisong Ni, Sally Guthrie, Jon-Kar Zubieta

\section{University of Michigan, Ann Arbor, MI}

Background: Few studies have determined the quantitative relationships between blood nicotine concentrations and mood changes, especially craving. In the past few years, our group has had a unique opportunity to provide quantitative results of venous plasma nicotine levels and craving reduction with both average nicotine (avnic) and denicotinized (denic) tobacco cigarettes. This study was part of a larger ongoing project involving tobacco smoking effects using positron emission tomography (PET). The protocol \# 2001-0924 was approved by the UM IRBMed Committee. Supported in part by NIH grant DA 18974-01-03(4).

Methods: A total of 23 male smokers were recruited for this study, mean age 25.8 , range $18-55$ years. After overnight abstinence the subjects smoked denic and later avnic cigarettes.

Results: Smoking both types of tobacco cigarettes had significant effects on craving and wakefulness, but not on relaxation, nervousness, or sickness. After denic cigarettes, subjects' plasma nicotine levels remained between $<1.0$ and $4.0 \mathrm{ng} / \mathrm{ml}$. After smoking avnic cigarettes, subjects' plasma nicotine levels increased from 2.92 to $35 \mathrm{ng} / \mathrm{ml}$. Seventeen of the 23 subjects had plasma increases after avnic cigarettes from 9.5 to $35 \mathrm{ng} / \mathrm{ml}$.

Discussion: These data were correlated with changes in craving and wakefulness. After cigarette smoking craving decreased with increased nicotine (Denic $\mathrm{r}=-0.38$, Avnic $\mathrm{r}=-0.59$ ). After cigarette smoking wakefulness correlated very poorly with increased plasma nicotine (Denic $r=+0.14$, Avnic $r=+0.28$ ). The relatively poor correlation 


\section{S390}

with plasma nicotine and craving especially with denic cigarettes indicates psychological factors obviously are more important than with avnic cigarette smoking.

Disclosure: E. Domino: None. W. Yang: None. C. Evans: None. L. Ni: None. S. Guthrie: None. J. Zubieta: None.

193. Pharmacodynamics of LY2216684: Evaluation of Peripheral and Central Norepinephrine Transporter Inhibition Using 3,4-Dihydroxyphenylglycol as a Biomarker of Pharmacologic Activity

William Kielbasa, Tonya Quinlan, Richard Lachno, David Bredt, Sanjay Dube*

University of Pittsburgh School of Medicine, Pittsburgh, PA and Lilly Research Laboratories, Eli Lilly and Company, Indianapolis, IN

Background: LY2216684 is a selective norepinephrine (NE) reuptake inhibitor being developed for treatment of major depressive disorder. An open-label study was conducted to characterize the pharmacodynamic (PD) effects of LY2216684 by examining changes in plasma and cerebrospinal fluid (CSF) concentrations of DHPG, an intraneuronal metabolite of NE.

Methods: LY2216684 was administered once daily to 27 healthy adult male subjects for 2 weeks at doses ranging from 1 - $9 \mathrm{mg}$. Plasma samples were collected in the supine and standing positions from subjects prior to baseline and following LY2216684 administration during the 2 week dosing period. The CSF samples were collected prior to baseline and at steady state after the last administration of LY2216684 at 4, 8 or 24 hours postdose via lumbar puncture performed in a standardized manner. Plasma and CSF samples were analyzed for LY2216684 using liquid chromatography with mass spectrometric detection and DHPG using high performance liquid chromatography with electrochemical detection. The plasma and CSF PK data and the supine DHPG data were analyzed by a population analysis approach using the software program NONMEM (Nonlinear Mixed Effects Modeling) version 5. A 2-compartment linear pharmacokinetic (PK) model with oral absorption was used to characterize the plasma and CSF PK profiles of LY2216684. An indirect inhibitory response model was used to characterize the effect of plasma concentrations of LY2216684 on the plasma and CSF DHPG response. Model parameters including baseline DHPG, Imax (maximum ability of LY2216684 to inhibit NE turnover to DHPG) and IC50 (LY2216684 plasma concentration producing $50 \%$ of maximum inhibition) were estimated.

Results: Dose-dependent increases in plasma and CSF LY2216684 exposures were observed and the median maximal plasma concentration was about 2 hours postdose. The baseline DHPG concentration was higher in CSF $(1850 \mathrm{pg} / \mathrm{mL}$; inter-subject variability $=23 \%)$ than plasma $(1210 \mathrm{pg} / \mathrm{mL}$; inter-subject variability $=17 \%)$. In general, the maximal reduction in plasma and CSF DHPG concentrations were observed by 4 to 8 hours postdose. The Imax and IC $_{50}$ based on plasma DHPG concentrations were $35 \%$ and $0.6 \mathrm{ng} / \mathrm{mL}$, and the Imax and IC50 based on CSF DHPG concentrations were $75 \%$ and $8 \mathrm{ng} / \mathrm{mL}$. These data show that higher LY2216684 doses/plasma concentrations lead to a greater maximal reduction of DHPG from baseline in CSF compared to plasma.

Discussion: Consistent with the physiological mechanism of NE reuptake inhibitors, administration of LY2216684 decreased peripheral and central concentrations of the DHPG metabolite. LY2216684 showed disparate peripheral and central biomarker effects suggesting that CSF DHPG might be a more sensitive and representative marker for central target engagement than plasma DHPG, and has potential to be a promising biomarker for NET inhibitors in drug development. The PK/PD model provided insights about LY2216684 pharmacology and may serve as a relevant predictor of an efficacious dose.

Disclosure: W. Kielbasa: Part 5; Eli Lilly and Company. T. Quinlan: Eli Lilly and Company. R. Lachno: Eli Lilly and Company. D. Bredt: Eli Lilly and Company. S. Dube: Eli Lilly and Company.
194. Glycine Transporter Inhibition Attenuates the Psychotomimetic Effects of Ketamine in Healthy Humans Subjects

Deepak C. D’Souza*, Nagendra Singh, Jacqueline Elander, Michelle Carbuto, Brian Pittman, Mohini Ranganathan, Joanna Udo de Haes, Magnus Sjogren, Pierre Peeters, Jacques Schipper

Yale University School of Medicine, West-Haven, CT

Background: Enhancing glutamate function by stimulating the glycine site of the NMDA receptor with glycine, D-serine or with drugs that inhibit glycine reuptake may have therapeutic potential in schizophrenia.

Methods: The effects of a single oral dose of ORG25935 a GLY-T Inhibitor and placebo pretreatment on ketamine-induced schizophrenia-like psychotic symptoms, perceptual alterations, and subjective effects were evaluated in 12 healthy male subjects in a randomized, counter-balanced, cross-over design. Two and a half hours after administration of the ORG25935 or placebo, subjects received a ketamine bolus and constant infusion lasting 100 minutes. Psychotic symptoms, perceptual and a number of subjective effects were assessed. These measures were collected in a repeated measures design before and several times during and after completion of ketamine administration.

Results: Ketamine produced psychotomimetic and subjective effects consistent with its known effects. ORG25935reduced the peak increase in ketamine-induced PANSS total, positive symptoms subscale and general symptoms subscale scores. ORG25935also reduced ketamineinduced perceptual alterations measured by the clinician-rated subscale of the clinician administered dissociative symptoms (CADSS) scale. The magnitude of the effect of GLY-T inhibition on ketamineinduced increases in total panss and CADSS Clinician-rated scores was 0.71 and 1.03, respectively. None of the behavioral effects of ketamine were increased by ORG25935 pretreatment.

Discussion: This exploratory study shows for the first time in humans that a GLT-T inhibitor reduces the psychotomimetic effects of the NMDA receptor antagonist ketamine. The findings of this study provide preliminary support for further study of the antipsychotic potential of GLY-T inhibitors.

Disclosure: D. D'Souza: Part 1; Pfizer Inc, Sanofi-Aventis, Astra Zeneca, Organon-Schering Plough-Merck, Cephalon, Eli Lilly, Abbott Labs. Part 2; Pfizer stock (wife). Part 4; Pfizer, Sanofi-Aventis, Astra Zeneca, Eli Lilly, Cephalon, Abbott Labs. N. Singh: None. J. Elander: None. M. Carbuto: None. B. Pittman: None. M. Ranganathan: None. J. Udo de Haes: Part 1; Organon/ScheringPlough/Merck. Part 2; Organon/ScheringPlough/Merck. M. Sjogren: Part 1; Organon/ScheringPlough/Merck. Part 2; Organon/ScheringPlough/Merck. P. Peeters: Part 1; Organon/ScheringPlough/Merck. Part 2; Organon/ScheringPlough/Merck. J. Schipper: Part 1; Organon/ScheringPlough/Merck. Part 2; Organon/ScheringPlough/Merck.

195. Daily Intranasal Oxytocin Improves Verbal Memory In Schizophrenia Patients

David Feifel*, Patrice Cobb, Kai MacDonald, Angel Nguyen, Arpi Minassian, William Perry

University of Caifornia, San Diego, San Diego, CA

Background: Findings have suggested that the peptide neurohormone oxytocin may disrupt non-social memory including verbal memory. These findings derive mainly from single dose administration of oxytocin whereas the effects of chronic oxytocin on cognition has not been systematically studied. We recently conducted a randomized control study that demonstrated that intranasal oxytocin given adjunctive to antipsychotics significantly reduced positive and negative symptoms of schizophrenia compared to placebo (Feifel et al, 2010. During that study some subjects were administered the California Verbal Leaning Test (CVLT), a well established verbal memory test, to assess the effects of chronic oxytocin on verbal memory. 
Methods: In a double-blind, placebo-controlled, randomized crossover trial, schizophrenia subjects with residual symptoms despite receiving a therapeutic dose of an antipsychotic for at least 4 weeks were administered daily intranasal oxytocin (80 IU divided in two doses) and placebo for three consecutive weeks adjunctive to their antipsychotics. The order of treatment was randomized with one week in between in which no intranasal drug was given. The CVLT was conducted at baseline and the final day (week 3) of each treatment arm. We are reporting on the 14 subjects who completed all designated CVLT assessments.

Results: Subjects performed significantly better after 3-weeks of adjunctive oxytocin treatment compared to placebo in multiple aspects of verbal memory as measured by the CVLT including: immediate recall, short delayed free recall, short delayed cued recall, total recall discriminability and total repetitions.

Discussion: These results suggest that chronic administration of intranasal oxytocin at the doses tested improves verbal memory in schizophrenia patients, a finding that stands in contrast to the oxytocin's general attribution as an amnesic agent for non-social memory.

Disclosure: D. Feifel: Part 1; Abbott Labs, Eli Lilly, Sepracor, Forest, Otsuka, Merck, Janssen, Shire, Alexza, Addrenex, AstraZeneca. Part 2; Eli Lilly. Part 3; Eli Lilly. Part 4; Abbot, Sepracor, Forest, Shire, Addrenex, AstraZeneca, Otsuka. P. Cobb: None. K. MacDonald: Part 1; Eli Lilly, Pfizer. Part 2; Eli Lilly, Pfizer. Part 3; Eli Lilly, Pfizer. A. Nguyen: None. A. Minassian: None. W. Perry: Part 1; Janssen, NSW family resiliency enterprise.

196. The Lithium Archive Project: The Role of Lithium in the Protection of Cardiovascular Disease

Ronald Fieve*, Michael Oliva, Monica Gilbert, Barbara Orlowski

New York State Psychiatric Institute, New York, NY

Background: Since the 1950's, lithium has been used worldwide in the successful treatment of bipolar disorders and there is a consensus in decades of previous literature that for these specific illnesses it remains the most researched and effective treatment yet discovered. While there has been a great amount of research dedicated to lithium and its effectiveness in the treatment of bipolar disorder and other diseases, there is a surprising dearth of literature investigating any potential relationships between lithium and cardiovascular disease with the exception of a previous examination by our team. The following represents an extension of the existing literature regarding lithium by examining the incidence of cardiovascular disease in patients diagnosed with a mood disorder who were treated with lithium compared to those who were not treated with lithium and the general population. The data for this research has been gathered as part of The Lithium Archives Project, which is based on systematic chart reviews of over 8,000 patients with mood disorders treated at the New York State Psychiatric Institute's Lithium Clinic (NYSPI) and two affiliate lithium clinics of Columbia University (CUMC) and the Foundation for Depression and Manic Depression, now known as the Foundation for Mood Disorders (FMD) over the past 40 years. This is a retrospective naturalistic study which includes over 100 variables that can be analyzed collectively or autonomously. This database has been used at various points of development for a number of different studies which include an analysis of the family history of anxiety in Bipolar I and II patients as well as presentations on the role of lithium in neuroprotection at the CINP and ACNP annual conferences.

Methods: The research design used in this study was a retrospective, random paper-based patient medical chart review conducted by a trained research scientist. The charts of patients who received outpatient treatment for Bipolar and Unipolar mood disorders were examined to determine patterns in the prevalence of cardiovascular diseases among those treated with lithium and those who were not treated with lithium in comparison to the prevalence of these disorders in the general population. Pilot data of demographic, cardiovascular disease, and lithium exposure variables was analyzed and entered from randomly selected medical charts into a standard statistical software program by a skilled statistician. Three data analyses have been carried out thus far. While the sample size was based on available data, power is sufficient to meet the guidelines addressed in other studies.

Results: A total of 6 patients in the non-lithium group suffered MIs compared with none in the lithium treated group. Given the significance level of $5 \%(\alpha=.05)$, t-tests revealed that the patients receiving lithium treatment had a significantly lower proportion of myocardial infarction $(\mathrm{n}=178)$ than patients not receiving lithium treatment $(n=426, p=.014)$ and the general population $(\mathrm{p}<.01)$, despite comparable levels of critical risk factors between the two groups of patients. The differences in the prevalence of other types of cardiovascular diseases between the three groups were not statistically significant.

Discussion: The current analysis is very promising and indicates that in this patient population, lithium may play a role in reducing the probability of myocardial infarction. Further research should include an analysis of cardiovascular related variables to gain a greater understanding of the possible causes of this finding and in the longer term, a prospective study is encouraged to attempt to replicate these findings.

Disclosure: R. Fieve: None. M. Oliva: None. M. Gilbert: None. B. Orlowski: None.

197. The Effect of Sertraline on Psychopathic Traits Boadie Dunlop, Jared DeFife, Lauren Marx, Steven Garlow*, Philip Ninan, Charles Nemeroff, Scott Lilienfield

Department of Psychiatry and Behavioral Science, Emory University, GA, Emory University School of Medicine, Atlanta, GA

Background: There is growing interest in how personality traits are affected by treatment with antidepressants. However, to date no research has explored the effect of antidepressants on psychopathic personality traits.

Methods: Data for this analysis were collected as part of a doubleblind, placebo-controlled 8-week trial exploring the efficacy of combination treatment with flexibly-dosed sertraline $(50-200 \mathrm{mg} / \mathrm{d})$ plus either tri-iodothyronine $\left(\mathrm{T}_{3}, 25-50 \mu \mathrm{g} / \mathrm{d}\right)$ or matching placebo in adult outpatients with major depressive disorder. Depression severity was assessed by the Hamilton Depression Rating Scale, 21-item version (HDRS), and Montgomery-Asberg Depression Rating Scale (MADRS). At the baseline and week 8 visits, patients completed the short form of the Psychopathic Personality Inventory (PPI), a self-report measure assessing two major factors of psychopathy: Fearless Dominance (PPII), and Self-centered Impulsivity (PPI-II). Change in PPI scores were assessed using paired t-tests for all subjects who completed a baseline and post-randomization PPI.

Results: Ninety patients were eligible for the analysis (84 completers and 6 early terminating patients). Men scored significantly higher at baseline than women on both PPI factors (PPI-I $\mathrm{p}<.01$, PPI-II $\mathrm{p}<.05$ ). Correlations of PPI factors with depression rating scale scores at baseline were small and not significant. Both PPI factors changed significantly from baseline to endpoint. The mean score on the Fearless Dominance factor, PPI-I, increased significantly during treatment [Baseline: 44.7 (9.4); Final: 47.8 (10.6), $\mathrm{p}<.05$ ], with Cohen's $d=.32$. This change was mildly correlated with change in depression scores (HDRS $r=-.32$; MADRS $r=-.29$, both $\mathrm{p}<.05$ ). In contrast, the mean score on the Self-Centered Impulsivity factor, PPI-II, decreased significantly [Baseline: 55.4 (9.8); Final: 51.2 (9.1), p<.001], with Cohen's $\mathrm{d}=-.44$; these changes were not correlated with depression score changes.

Discussion: Antidepressants have mixed effects on psychopathic traits in depressed patients. These changes are largely independent from changes in depressive symptoms. Study findings suggest that in conjunction with depressive symptom improvements, antidepressants increase more adaptive traits traditionally observed in psychopathic 
individuals as assessed by PPI factor I, such as increased social charm and reduced anxiety and interpersonal sensitivity. Independent of symptomatic improvements, antidepressants appear to reduce aspects captured by the PPI factor II, including self-directed rumination, dysregulated impulsivity, and externalization. Further studies of the effects of antidepressants on personality traits are warranted, including in individuals meeting full criteria for Psychopathic personality.

Disclosure: B. Dunlop: Part 1; AstraZeneca, Evotec, Forest, GSK, NIH, Novartis, Ono Pharmaceuticals, Takeda, Pfizer, Digitas, Imedex LLC, MedAvante. Part 4; AstraZeneca, Evotec, Forest, GSK, NIH, Novartis, Ono Pharmaceuticals, Takeda, Pfizer. J. DeFife: None. L. Marx: None. S. Garlow: Part 1; Eli Lilly Co. P. Ninan: Pfizer. Part 5; Pfizer. C. Nemeroff: Part 1; AstraZeneca, Forest, Quintiles, Janssen/Ortho-McNeil, PharmaNeuroboost, Mt Cook Pharmaceuticals, Corcept, Revaax, NovaDel Pharma, CsNeRx, AFSP, NARSAD, George West Mental Health Foundation. S. Lilienfield: Psychological Assessment Resources.

\section{Differential Modulation of Platelet Aggregation in Response} to Escitalopram in Depression

Angelos Halaris*, Evangelos Litinas, John Piletz, Jawed Fareed, Edwin Meresh, Debra Hoppenstead, James Sinacore

Loyola University Chicago, Maywood, IL

Background: Platelet activation-a process central to the pathophysiology of atherosclerosis and thrombus formation-involves a series of steps including serotonin release prior to aggregation. Platelets exposed ex vivo to SSRIs have shown attenuated aggregation as a short-term response. However, the effects of SSRIs in vivo on platelet aggregation after months of treatment have not been adequately characterized. We hypothesized that over extended SSRI treatment of patients with major depressive disorder, without cardiovascular disease, adaptive regulation in platelet aggregation parameters will occur.

Methods: Depressed patients $(\mathrm{N}=35)$ were treated with escitalopram (ESC). Platelet aggregation was measured in response to saline (autoaggregation), epinephrine (EPI), ADP, collagen and arachidonic acid. In Study-1 platelets were harvested at o, 4 and 8 weeks and in Study-2 at 0,8 , and 12 week of treatment.

Results: After 4 weeks on ESC fewer platelets aggregated in response to ADP and EPI $(\mathrm{p}=0.1 ; \mathrm{n}=13)$. At 8 and 12 weeks the number of platelets that aggregated was normal in response to both agonists. However, the slope in response to ADP (1.25 microM) was steeper at 8 $(\mathrm{p}=0.02 ; \mathrm{n}=20)$ and 12 weeks $(\mathrm{p}=0.03 ; \mathrm{n}=17)$. Neither the trend observed at week 4 (in maximum aggregation to both agonists) nor the sensitization to ADP observed at weeks 8 and 12 were significantly correlated with the scores of mood improvement. However, complete symptom remission correlated with a higher ADP slope at week 12. No differences were noted in response to arachidonic acid or collagen.

Discussion: SSRI-induced serotonin depletion may initially lead to decreased platelet aggregation, but in a compensatory manner by $8-12$ weeks the platelets are restored to aggregate, and do so faster selectively in response to ADP than before treatment. These results, if confirmed in larger studies, may be of clinical significance in the treatment of depressed cardiovascular patients with known risk factors and who are also exposed to anti-platelet therapies.

Disclosure: A. Halaris: Part 1; Boehringer Ingelheim, Takeda. Part 2; Merck, Pfizer. Part 4; AtraZeneca. E. Litinas: None. J. Piletz: AstraZeneca. J. Fareed: None. E. Meresh: None. D. Hoppenstead: None. J. Sinacore: None.

199. C-Reactive Protein: A Marker of Inflammation in Depression Jawed Fareed, Angelos Halaris*, John Piletz, Edwin Meresh, Debra Hoppensteadt, Laila Al-Duwaisan, James Sinacore

Loyola University Chicago, Maywood, IL

Background: Major depressive disorder (MDD) and cardiovascular disease (CVD) are highly prevalent and frequently co-morbid conditions. They singly or jointly represent major causes of morbidity and mortality worldwide. Several mechanisms have been considered as being at least partially responsible for this co-morbidity. Amongst those mechanisms inflammatory processes have received most of the research emphasis in as much as they are common in both MDD and CVD. Cardiac risk has been associated with C-reactive protein (CRP), a non-specific acute phase protein synthesized in the liver and stimulated by pro-inflammatory cytokines, notably IL-1 and IL-6. There is also evidence that CRP itself is active in the inflammatory process. A number of studies have provided evidence that CRP is elevated in MDD but not all studies agree. Similarly, there is insufficient evidence that acute treatment of depression with antidepressants reverses CRP elevations. The present study was undertaken in an effort to clarify whether high sensitivity CRP (hs-CRP) is elevated in MDD patients in the absence of any identifiable cardiovascular condition or risk. Additionally we sought to determine whether treatment with escitalopram would reverse any CRP abnormalities observed at pretreatment.

Methods: A cohort of 47 patients diagnosed with MDD (Mean age: of $38.0 \pm 11$, BMI: $30.2 \pm 6.0$ ), and 16 healthy controls (Mean age: $37.1 \pm 11.2$, BMI: $27 \pm 6.2$ ) were recruited. All subjects were carefully assessed and determined to be free of cardiovascular disease and other inflammatory conditions. Additionally, healthy control serum (NHP), and serum from a pool of patients with cardiovascular pathology (PP) was included for comparison purposes. Patients were diagnosed by the MINI structured interview. Depressed patients were treated with escitalopram (ESC) for a period of 12 weeks (dose range: 10-40 mg/ daily). Severity of depression was assessed using the Hamilton depression rating scale (HAM-D). Plasma hs-CRP levels were measured using high-sensitivity ELISA. The difference in mean baseline hs-CRP was analyzed using covariance (ANCOVA), controlling for age, sex, and BMI. Endothelial dysfunction was assessed by means of applanation tonometery using Augmentation Index (AIx) as a measure of arterial stiffness.

Results: Levels of plasma hs-CRP at baseline were significantly higher in MDD patients $(3.54 \mathrm{ng} / \mathrm{ml})$ than healthy controls $(1.71 \mathrm{ng} / \mathrm{ml}$, $\mathrm{p}=0.054$ ) after adjusting for age, sex, and BMI. Levels of hs-CRP in serum from an independently-pooled healthy control population (NHP) were similar to hs-CRP levels in our own healthy control cohort $(1.43 \mathrm{ng} / \mathrm{ml}$, and $1.71 \mathrm{ng} / \mathrm{ml}$, respectively). Hs-CRP levels in the pathological pool were significantly higher than our depressed cohort (6.5 ng/ml, vs. $3.54 \mathrm{ng} / \mathrm{ml}$, respectively). Differences in hs-CRP levels were not observed at 8 and 12 weeks of ESC treatment $(p=.610$ and .981 , respectively). The baseline augmentation index significantly correlated with age $(\mathrm{p}<.0005)$, but not with sex, BMI, or baseline CRP. Discussion: These data confirm findings in the literature that indicate increased baseline hs-CRP levels in patients with MDD. Our results highlight the presence of low grade inflammation in MDD patients. While our patient cohort improved significantly after twelve weeks of treatment with escitalopram, with the majority of patients achieving remission of symptomatology, we failed to observed normalization of hs-CRP levels at the end of the acute treatment phase. A prolonged course of treatment, at least with an SSRI, may be required to achieve normalization of this inflammation biomarker. Our findings differ from at least one report in the literature indicating CRP normalization after a few weeks of treatment with flouxetine. Although further studies will be required to definitively prove that antidepressant drug therapy can reverse the inflammatory status of MDD patients, routine measurement of hs-CRP may be a useful laboratory test to be conducted in this patient population.

Disclosure: J. Fareed: None. A. Halaris: None. J. Piletz: None. E. Meresh: None. D. Hoppensteadt: None. L. Al-Duwaisan: None. J. Sinacore: None. 
200. Proton MRS to Evaluate the Neuroprotective and Procognitive Potential of Davunetide in Schizophrenia

Jeffrey Lieberman*, L. Fredrik Jarskog, Zhengchao Dong, Alayar Kangarlu, Tiziano Colibazzi, Ragy Girgis, Deanna Barch, Robert Buchanan, John Csernansky, Donald Goff, Daniel Javitt, Richard Keefe, Robert McMahon, Bradley Peterson, Stephen Marder

Columbia University/New York State Psychiatric Institute, New York, NY, University of North Carolina at Chapel Hill, Chapel Hill, NC

Background: Schizophrenia is characterized in part by subtle reductions in cortical neuropil and persistent neurocognitive deficits. $\mathrm{N}$-acetylaspartate (NAA) is a putative marker of neuronal integrity and proton magnetic resonance spectroscopy ( $1 \mathrm{H}-\mathrm{MRS}$ ) has previously found reduced NAA/ creatine in dorsolateral prefrontal cortex (DLPFC) in schizophrenia. Choline is thought to represent a measure of membrane phospholipid turnover and elevated choline levels have been found in classic neurodegenerative disorders. Davunetide is an 8-amino acid peptide (an active derivative of activity-dependent neuroprotective protein) that functions as a stabilizer of microtubular structure with efficacy in several rodent models of neurodegeneration and cognitive impairment. In a 12week study, low dose adjunctive davunetide was found to improve the functional co-primary outcome measure (UPSA composite) in stable outpatients with schizophrenia. $1 \mathrm{H}$-MRS was used to test the hypothesis that davunetide can increase NAA/creatine in schizophrenia DLPFC. Choline/creatine was also determined as a potential measure of membrane turnover and plasticity.

Methods: 12-week, randomized, double-blind, placebo-controlled multi-site trial of 2 doses of davunetide $(5$ and $30 \mathrm{mg} / \mathrm{d}$ i.n.) in 63 patients with schizophrenia on stable antipsychotic treatment. 18 subjects at 4 sites had ${ }_{3} \mathrm{~T} 1 \mathrm{H}$-MRS performed at baseline and at 12 weeks. NAA/creatine and choline/creatine was measured in an $8 \mathrm{cc}$ voxel placed in left DLPFC. Cognition was assessed using the MATRICS Consensus Cognitive Battery (MCCB).

Results: NAA/creatine for davunetide (low and high doses combined, $\mathrm{n}=11$ ) was $1.769+0.300$ at Week 12 compared to $1.664+0.258$ at baseline, a $6.3 \%$ increase that was not significant at $\mathrm{p}=0.122$ by Wilcoxon rank sum test. Baseline NAA/creatine correlated with attention/vigilance $(p=0.003)$ and verbal learning $(p=0.005)$, with a trend for the composite MCCB score $(p=0.053)$. Choline/creatine for davunetide (low and high doses combined) was $0.896+0.166$ at Week 12 compared to $0.842+0.145$ at baseline, a trend for a $6.4 \%$ increase $(\mathrm{p}=0.069)$ by Wilcoxon rank sum test. Cognitive tests did not correlate with choline/creatine ratios.

Discussion: Davunetide represents a potentially useful pro-cognitive adjunctive agent for clinically stable patients with schizophrenia. Although this MRS substudy was only powered to detect large effect sizes, the results are remarkably consistent in demonstrating modest increases for both NAA and choline measures after 12 weeks of davunetide. As a marker of neuronal integrity, increased NAA would be consistent with davunetide as a neuroprotective agent. The modest effect on choline is of uncertain significance but could signal a neuroplastic effect of davunetide. Larger studies are needed to better understand associations between davunetide, cognitive performance and functional outcomes.

Disclosure: J. Lieberman: None. L. Jarskog: Part 1; Novartis, GSK. Z. Dong: None. A. Kangarlu: None. T. Colibazzi: None. R. Girgis: None. D. Barch: None. R. Buchanan: None. J. Csernansky: None. D. Goff: None. D. Javitt: None. R. Keefe: None. R. McMahon: None. B. Peterson: None. S. Marder: None.

201. Efficacy of Valproic Acid, Lithium Carbonate and Carbamazepine in Maintenance Phase of Bipolar Disorder-A Naturalistic Study Shannon Stepan, Eric Peselow*, Nunzio Pomara

Maimonides Medical Center, Brooklyn, NY

Background: Bipolar disorder is a lifelong illness for $90 \%$ of patients who experience a manic episode. Maintenance treatment is usually recommended. Our goal was to look at the efficacy of lithium, depakote and tegretol as maintenance treatment of bipolar disorder in a naturalistic setting.

Methods: 225 outpatients with bipolar disorder were followed for up to 124 months, or until they had a manic or depressive episode, or dropped out of the study well 98 patients $(43.6 \%)$ were taking lithium, $77(34.2 \%)$ depakote, and $50(22.2 \%)$ tegretol.

Results: A total of 103 patients $(45.8 \%)$ had either a manic or depressive episode during the study period. Across the medication groups, $36.7 \%$ of the patients taking lithium $(\mathrm{N}=36), 54.5 \%$ of the patients taking depakote $(\mathrm{N}=42)$, and $50 \%$ of the patients taking tegretol $(\mathrm{N}=25)$ had either a manic or depressive episode at some point during the 124 month study period. The median survival time was 45.76 months for the entire sample, 36 months the depakote group, 42 months tegretol, and 81 months for lithium. 52 patients dropped out of the study well and 7o remained in the study well. A cox regression model evaluating the probability of having a manic or depressive episode among the three medications after controlling for several covariates showed that patients taking depakote had a significantly higher risk of having a manic or depressive episode than patients taking lithium. For patients taking depakote the hazard of having a manic or depressive episode was 1.63 (CI $1.01-2.63$ ) times higher vs. patients taking lithium. Thus, the hazard of becoming unstable was $38.5 \%$ lower in patients taking lithium compared to those taking depakote. There was a non-significant trend for greater maintenance efficacy of lithium vs tegretol and tegretol vs depakote.

Discussion: Lithium patients had statistically better maintenance than the depakote group and a trend toward better maintenance vs.the tegretol group in this naturalistic setting

Disclosure: S. Stepan: None. E. Peselow: None. N. Pomara: None.

\section{Determinants of Intravenous Alcohol Self-Administration in Social Drinkers \\ Vijay Ramchandani*, Megan Cooke, Vatsalya Vatsalya, Julnar Issa,} Ulrich Zimmermann, Sean O’Connor, Daniel Hommer, Markus Heilig

NIH/NIAAA, Bethesda, MD

Background: Computer-Assisted Self-infusion of Ethanol (CASE) is a method of intravenous (IV) alcohol administration that provides individuals with choices for self-administering alcohol in a laboratory setting, while controlling the breath alcohol concentration (BrAC) using a physiologically-based pharmacokinetic (PBPK) model-based algorithm. Previous studies have demonstrated sensitivity of IV alcohol self-administration measures to the infusion rate and family history of alcoholism. The objective of this study was (1) to evaluate the influence of recent drinking history, retrospective measures of alcohol sensitivity, and personality assessments on IV alcohol selfadministration; and (2) to examine the relationship between subjective measures of alcohol effects and IV alcohol self-administration, using the CASE system, in male and female social drinkers.

Methods: Thirty-eight healthy social drinkers (17 females, 21 males) were included in the study. Subjects completed two identical study sessions, between 3 and 30 days apart. Each session consisted of an initial 25-min priming phase during which the subjects were prompted to push a button to receive individually standardized alcohol infusions. This was followed by a 2-hour "open bar" phase during which subjects had ad-lib access to standardized alcohol infusions, and were instructed to self-administer alcohol to their typical level of exposure in social settings. Serial BrAC measurements were obtained throughout the study session. Primary self-administration measures included total number of button presses (NBP), peak BrAC (PEAK) and average BrAC (AVG). Subjective measures, obtained repeatedly throughout the study session, included Drug Effects Questionnaire (DEQ), Biphasic Alcohol Effects Scale (BAES) and Alcohol Urge Questionnaire (AUQ). Recent drinking history was measured using Time-Line Follow-Back. Measures of alcohol sensitivity included the Self-Report of the Effects of Alcohol (SRE) and the Alcohol Effects Questionnaire (AEFQ). 


\section{S394}

Personality measures included the NEO-PI-R, Barratt's Impulsivity Scale (BIS) and Sensitivity to Punishment and Sensitivity to Reward Questionnaire (SPSRQ).

Results: Correlations between measures within each session demonstrated a high level of internal consistency (NBP vs. PEAK: R2 $=0.82$; NBP vs. AVG: $\mathrm{R} 2=0.80$ ). There was a significant association between recent drinking history measures and self-administration measures. Stepwise multiple regression analyses revealed drinks per drinking day to be the strongest predictor of NBP, PEAK and AVG (all p values $<$ 0.001). There were no sex differences in self-administration measures. The SRE total score, a retrospective measure of level of response to alcohol, was significantly associated with self-administration measures (NBP: $\mathrm{R} 2=0.20, \mathrm{p}=0.006$; PEAK: $\mathrm{R} 2=0.14, \mathrm{p}=0.028$; AVG: $\left.\mathrm{R}_{2}=0.16, \mathrm{p}=0.017\right)$. Sensitivity to reward was robustly correlated with self-administration measures (NBP: $\mathrm{R} 2=0.49$, $\mathrm{p}=0.003$; PEAK: $\mathrm{R} 2=0.36, \mathrm{p}=0.013 ; \mathrm{AVG}$ : $\mathrm{R} 2=0.36, \mathrm{p}=0.014)$. The AEFQ, NEO-PI-R and BIS measures did not show any significant correlations with self-administration measures. DEQ measures of "liking drug effects" and "wanting more drug" obtained following the priming phase were significantly associated with NBP, PEAK and AVG. AUQ total score following the priming phase was also significantly associated with self-administration measures (NBP: $\mathrm{R} 2=0.20, \mathrm{p}=0.005 ;$ PEAK: $\mathrm{R} 2=0.18, \mathrm{p}=0.008 ; \mathrm{AVG}: \mathrm{R} 2=0.23$, $\mathrm{p}=0.003)$. There was a strong association between peak alcohol effects of "feeling drug effects", liking drug effects", intoxication", "stimulation" during the open-bar phase and self-administration measures (all $\mathrm{p}<0.015)$.

Discussion: IV alcohol self-administration measures were significantly associated with measures of recent drinking history with heavier drinkers showing higher alcohol self-administration. Individuals with high SRE scores, indicative of a low responsiveness to alcohol, also demonstrated higher alcohol self-administration. Individuals who reported greater reward sensitivity demonstrated higher alcohol selfadministration. Measures of drug liking, wanting, and alcohol urges following priming were predictive of ad lib alcohol self-administration. There was a strong association between alcohol self-administration and alcohol-induced intoxication and stimulation. The CASE method demonstrates sensitivity to the rewarding effects of alcohol and is being used to examine the effect of medications being developed for alcoholism pharmacotherapy.

Disclosure: V. Ramchandani: None. M. Cooke: None. V. Vatsalya: None. J. Issa: None. U. Zimmermann: None. S. O'Connor: None. D. Hommer: None. M. Heilig: None.

\section{Epigenetic and Gene Expression Analysis in a Rat Model of In Utero Antidepressant Exposure and Maternal Depression Michael Owens*}

\section{Emory University, Atlanta, GA}

Background: The treatment of maternal depression during pregnancy is important for both mother and child well-being. The risks and benefits of untreated illness versus fetal antidepressant medication exposure have not been fully delineated. We developed a rat model of chronic antidepressant treatment and stress during pregnancy to examine epigenetic and gene expression changes in adult male offspring.

Methods: Adult female rats were implanted with osmotic minipumps delivering clinically relevant concentrations of the SSRI escitalopram (confirmed by laboratory assay) beginning 5 days before mating and continuing throughout the entire course of pregnancy. After mating, half of the females were exposed to a chronic unpredictable mild stress model on gestational days 8-20. The offspring were divided into four groups based upon their in utero environment: control, stress, escitalopram, and stress + escitalopram. Maternal care was monitored continuously for the first 10 days following parturition.

Results: Offspring growth rates were monitored from birth to adulthood with no differences between the groups. At adulthood (aged 90-95 days), basal endocrine measurements showed a very small but statistically significant increase in serum corticosterone in escitalopram-exposed rats and a decrease in serum insulin in prenatal stress-exposed rats although neither observation was considered to be physiologically relevant. Chromatin immunoprecipitation assays (ChIP) were completed studying two histone modifications (dimethylated $\mathrm{H}_{3} \mathrm{~K}_{2} 7$ and acetylated $\mathrm{H}_{3} \mathrm{~K}_{9}$ ) and 4 promoter regions of several genes (BDNF, CRF, GR, and SERT) that are often modulated by stress or antidepressant exposure in the hippocampal regions of adult male rats. Analyses did not reveal any significant changes associated with in utero escitalopram exposure. In contrast, ChIP assays indicate that long-term GR and BDNF protein expression could be repressed in prenatally stressed male rats independent of antidepressant administration. Specifically, we observed an increased presence of dimethylated $\mathrm{H}_{3} \mathrm{~K}_{4}$ at the promoter 3 region of $\operatorname{BDNF}(\mathrm{p}<0.032)$ and a decreased presence of acetylated $\mathrm{H}_{3} \mathrm{~K}_{9}$ at exon 17 (a promoter region) of GR $(\mathrm{p}<0.018)$. We did not observe any significant changes in the histone modification at the promoter regions of CRF and SERT. The physiological relevance of these findings is under examination. Separate microarray analyses of both hippocampus and hypothalamus revealed no major differences in gene expression across treatment groups.

Discussion: Continued analyses are underway to examine distinct pathways regulated by prenatal treatments however the current results show that alterations in the in utero environment modeled on maternal depression and its treatment do not result in long-term effects on the transcriptome in adult offspring.

Supported by NIH P50 MHo77928, T32ESo12870

Disclosure: M. Owens: Part 1; Research Grants: Eli Lilly, Lundbeck A/S, Cyberonics, Ortho-McNeil Janssen, AstraZeneca, Dainippon Sumitomo Pharma, SK Life Sciences., Consultant: H. Lundbeck A/S., Patent: Method of assessing antidepressant drug therapy via transport inhibition of monoamine neurotransmitters (US 7,148,027 B2). Part 4; Eli Lilly, Lundbeck A/S, Cyberonics, Ortho-McNeil Janssen, AstraZeneca, Dainippon Sumitomo Pharma, SK Life Sciences.

\section{Effects of Naltrexone on Alcohol Consumption in Adolescent} Rats

Ashwin Patkar*, Cynthia Kuhn, Nicole Schramm-Sapyta, Sean Pat Oswald, Ting-Kai Li

\section{Duke University Medical Center, Durham, NC}

Background: There are currently no approved pharmacotherapies to treat adolescent alcohol abuse, nor have any of the approved pharmacotherapies been tested in adolescent animal models. One challenge in conducting such trials in rodent models is the difficulty of achieving high levels of alcohol intake during adolescence, which is brief. Our lab adopted a novel approach that leads to rapid onset of alcohol intake in adolescent rats to test alcohol consumption in a population of outbred Sprague Dawley rats.

Methods: Outbred Sprague Dawley rats were obtained from a local supplier (Charles River, Raleigh, NC), and housed under reversed light-dark cycle so consumption during the early dark phase could be easily monitored. In study 1 , adolescent rats (postnatal day 28, PN 28) were given a choice between water and $20 \%$ ethanol solution in a " 4 days on-3 days off," 2 bottle choice paradigm. Alcohol consumption was monitored for 7 cycles, and then rats received two more cycles (starting on PN 80) during which they received saline or naltrexone $(10 \mathrm{mg} / \mathrm{kg}$ ) once daily in a randomized pattern (half animals received saline first, half received naltrexone first). In study 2, adolescents $\left(\mathrm{PN}_{28}\right.$ ) rats received saline or naltrexone on cycles 2 and 3, (while animals were still adolescent, and before ethanol consumption was long-established). Ethanol and water bottles were weighed each morning and at noon to compute consumption over 24 hours, and during the early dark phase, when animals drank the fastest. Ethanol consumption was analyzed by repeated measures ANOVA with treatment and day as within subjects factors. In addition, total 
consumption over the 4 day cycle was analyzed by repeated measures ANOVA with treatment as a within subjects factor. Data from experiment 1 were also analyzed by linear regression correlating consumption for individuals at the end of the trial with consumption during initial stages. Total consumption during naltrexone and saline trials were compared for experiment 1 and 2 by 2 -way ANOVA (age $\mathrm{x}$ treatment).

Results: Adolescent rats rapidly established a consistent pattern of ethanol consumption across the 4-day cycle. They exhibited high intake on day 1 , lower intake on days 2 and 3, and high intake again on day 4. Alcohol consumption varied significantly by day during the first 7 cycles $(\mathrm{F}=18.8, \mathrm{p}<.0001)$. Individual differences in consumption were significant and consistent: a median split of the data showed that high consuming animals maintained high intake over the 7 weeks, while the lower half decreased intake after the first three weeks. Alcohol consumption on day 15 of exposure predicted consumption at the end of choice consumption $\left(\mathrm{r}^{2}=.71\right)$. Naltrexone treatment significantly decreased consumption relative to saline treatment during the morning (first 3 hours after injection) $(\mathrm{F}=7.3, \mathrm{p}<.02$ for an effect of treatment). Total consumption in the morning across the 4 days of trial was significantly decreased by naltrexone $(\mathrm{f}=7.82, \mathrm{p}<.017$ for effect of treatment, naltrexone vs. saline). Twenty-four hour consumption levels were not affected by naltrexone treatment, likely due to rapid clearance of naltrexone. In experiment 2, with the shorter duration of ethanol exposure before naltrexone testing, total ethanol consumption was again significantly less in naltrexone-treated than in saline-treated rats $(\mathrm{F}=8.4, \mathrm{p}<.014)$. However, 2-way ANOVA (day $\mathrm{x}$ treatment) did not show a significant effect of naltrexone over the days of the trial. Finally, 2-way ANOVA comparing naltrexone response in adult, long-term ethanol-exposed animals versus adolescent, short-term ethanol exposed-animals (experiment 1 vs. experiment 2) showed significant effects of age and treatment $(\mathrm{p}<.0001$ and .0006 respectively) but no age $\mathrm{x}$ treatment interaction. The younger rats on experiment 2 drank significantly more alcohol, but naltrexone decreased consumption in both.

Discussion: These data show that intermittent alcohol exposure causes rapid establishment of individually-consistent patterns of ethanol intake in adolescent rats. While some animals maintain high intake into adulthood, others suppress intake as they mature. Naltrexone decreased intake both in adult animals with a prolonged experience of intermittent alcohol exposure and in adolescents after brief exposure. These results suggest that naltrexone may be useful in treating adolescent alcohol abusers.

Supported by DA019114 to CMK, AA 016473 to AAP and DA020729 to NLSS.

Disclosure: A. Patkar: Part 1; Alkermes, Pfizer, Bristol-Myers Squibb/ Otsuka, Gilead, Reckitt Benckiser. Part 2; Pfizer, Bristol-MyersSquibb/ Otsuka Pharmaceuticals. Part 4; Titan Pharmaceuticals, Lundbeck, Cephalon, Pfizer, Merck, Bristol-Myers Squibb, Jazz, Forest Research Institute. C. Kuhn: None. N. Schramm-Sapyta: None. S. Oswald: None. T. Li: None.

205. Lurasidone Pharmacokinetics: Assessment of Potential for DrugDrug Interactions

Sheldon Preskorn*, Yu-Yuan Chiu, Donald Sarubbi, Masaaki Ogasa, Josephine Cucchiaro, Antony Loebel

\section{Clinical Research Institute, Wichita, KS}

Background: Lurasidone is a new psychotropic agent, under development for the treatment of schizophrenia, with high affinities for dopamine $\mathrm{D}_{2}$, serotonin 5-HT7, 5- $\mathrm{HT}_{2} \mathrm{~A}, 5-\mathrm{HT}_{1} \mathrm{~A}$, and noradrenaline $\alpha_{2} \mathrm{C}$ receptors. Lurasidone exhibits little or no affinity for histamine $\mathrm{H} 1$ or acetylcholine M1 receptors. In vitro data suggest that lurasidone is metabolized primarily by cytochrome $\mathrm{P} 450$ 3A4. Lurasidone's activity is primarily due to the parent drug and to a lesser extent to the active metabolites ID-14283 and ID-14326, which represent $25 \%$ and $3 \%$ of the parent exposure, respectively. The objective of the studies summarized here was to evaluate the potential risk for drug-drug interactions (DDIs) with lurasidone.

Methods: 7 studies were conducted in subjects to evaluate potential pharmacokinetic (PK) interactions with single or multiple-dose lurasidone. The PK of lurasidone was evaluated when co-administered with potent or moderate $\mathrm{CYP}_{3} \mathrm{~A}_{4}$ inhibitors (ketoconazole or diltiazem); with the $\mathrm{CYP}_{3} \mathrm{~A}_{4}$ inducer rifampin; and with lithium. Plasma concentration changes in midazolam, a CYP3 44 substrate and in an oral contraceptive (ethinyl estradiol/norgestimate combination) were evaluated in the presence of lurasidone. The effect of lurasidone on digoxin, a P-gp substrate was also investigated.

Results: Concomitant administration of lurasidone and ketoconazole, a strong $\mathrm{CYP}_{3} \mathrm{~A}_{4}$ inhibitor, resulted in increased lurasidone AUCo-72 (9.3-fold) and Cmax (6.8-fold) relative to lurasidone administered alone. Co-administration of lurasidone and diltiazem, a moderate $\mathrm{CYP}_{3} \mathrm{~A}_{4}$ inhibitor, resulted in increased lurasidone AUC (2-fold) and Cmax (2.1-fold) relative to lurasidone alone. Concomitant administration of rifampin, a strong $\mathrm{CYP}_{3} \mathrm{~A}_{4}$ inducer, resulted in a 5.5 -fold decrease in AUC and 6.8-fold decrease in Cmax relative to lurasidone administered alone. Lurasidone $(120 \mathrm{mg}$ ) at steady state exhibited weak $\mathrm{CYP}_{3} \mathrm{~A}_{4}$ inhibition on the $\mathrm{CYP}_{3} \mathrm{~A}_{4}$ substrate midazolam, while a single-dose of $120 \mathrm{mg}$ lurasidone exhibited no meaningful $\mathrm{CYP}_{3} \mathrm{~A}_{4}$ inhibition. No PK interaction was observed after concomitant administration of lithium $600 \mathrm{mg}$ BID and steady state dosing of lurasidone compared to lurasidone alone. Concomitant administration of lurasidone had no effect on digoxin (a P-gp substrate) suggesting that lurasidone is not associated with $\mathrm{P}$-gp inhibition. Concomitant administration of lurasidone and the ethinyl estradiol/norgestimate combination, an oral contraceptive, resulted in equivalent AUCo-tau and Cmax compared to when ethinyl estradiol/norgestimate was administered alone.

Discussion: Lurasidone exposure is significantly increased when coadministered with a potent inhibitor of cytochrome $\mathrm{P}_{450}{ }_{3} \mathrm{~A} 4$ (ketoconazole) and decreased when co-administrated with a strong $\mathrm{CYP}_{3} \mathrm{~A}_{4}$ inducer, rifampin. Conversely, lurasidone did not inhibit either $\mathrm{CYP}_{3} \mathrm{~A}_{4}$ or P-gp to a clinically significant degree. No interaction was observed between lithium and lurasidone.

Disclosure: S. Preskorn: Part 1; See list in part 4. Part 2; Pfizer, Boehringer Ingelheim. Part 4; Abbott, AstraZeneca, Biovail, Boehringer Ingelheim, Cetero, Dainippon Sumitomo Pharma, Eli Lilly \& Company, EnVivo, Evotec, Link Medicine, Mylan, Pfizer, Quintiles, Roche, Takeda, Targacept, Wyeth. Part 2; Wyeth. Y. Chiu: Part 5; Sunovion, Inc., Ft. Lee, NJ. D. Sarubbi: Sunovion, Inc., Ft. Lee, NJ. M. Ogasa: Sunovion, Inc., Ft. Lee, NJ. J. Cucchiaro: Sunovion, Inc., Ft. Lee, NJ. A. Loebel: Sunovion, Inc., Ft. Lee, NJ.

206. Is Valproate's Brain Target in Treating Bipolar Disorder an Arachidonic Acid-Selective Acyl-CoA Synthetase?

Hiren Modi, Jakob Shimshoni, Mireille Basselin, Lei Li, Rosalind Coleman, Stanley Rapoport*

National Institute on Aging. NIH, Bethesda, MD

Background: Each of three FDA-approved mood stabilizers, lithium, carbamazepine and valproate, when given chronically to rats to produce plasma concentrations therapeutically relevant to bipolar disorder, selectively downregulates arachidonic acid (AA, 20:4n-6) but not docosahexaenoic acid (DHA, 22:6n-3) turnover in brain phospholipids, as well as brain activity of cyclooxygenase (COX)-2 and the concentration of an AA metabolite formed via COX-2, prostaglandin E2. Downregulation of AA turnover by lithium and carbamazepine, but not by valproate, corresponds to reduced transcription and activity in brain of calcium-dependent cytosolic phospholipase A2 (cPLA2 Type IVA), which selectively releases AA from membrane phospholipid. We hypothesized that valproate targets another enzyme that regulates AA turnover, namely a brain acyl-CoA synthetase (Acsl) that can convert AA to AA-CoA. Such targeting could account for valproate's selective 


\section{S396}

reduction of AA turnover in brain phospholipids of unanesthetized rats, and contribute to its antibipolar action.

Methods: Recombinant rat Acsl3-, Acsl6v1- and Acsl6v2-, and Acsl4flag proteins were expressed in E. coli. The ability of VPA to inhibit the activation of AA, DHA and palmitic acid (16:0) to acyl-CoA by each of the recombinant Acsl enzymes was characterized in vitro using Michaelis-Menten kinetics.

Results: Valproate uncompetitively inhibited Acsl4-mediated conversion of AA and of DHA but not of palmitic acid to its respective acylCoA, but did not affect AA conversion to AA-CoA by Acsl3, Acsl6vi or Acsl6v2. Acsl4-mediated conversion of AA to AA-CoA showed substrate inhibition, but at an in vitro $\mathrm{Ki}$ 10-times the estimated therapeutic brain concentration of valproate. The catalytic efficiency of conversion of AA to AA-CoA was 10-times higher than the catalytic efficiency of its conversion of DHA to DHA-CoA. Butyrate, octanoate, or lithium did not inhibit AA activation by Acsl4.

Discussion: Assuming that the discrepancy between the in vitro $\mathrm{Ki}$ and the therapeutic brain valproate concentration can be explained by the reported ability of brain cells to accumulate valproate and to other differences between in vivo and in vitro measurements, valproate's ability to inhibit Acsl4 activation of AA and of DHA to their respective acyl-CoAs in vitro, when taking into account the higher catalytic efficiency of AA than of DHA conversion, may account for VPA's selective reduction of AA turnover in brain phospholipids in unanesthetized rats. This inhibition may contribute to valproate's proven efficacy against bipolar disorder. Other agents, perhaps less hepatotoxic and teratogenic than valproate, which are shown to inhibit Acsl-4 mediated conversion of AA in vitro by recombinant Acsl4, could be screened in unanesthetized rats for their ability to also reduce brain AA turnover. Those that do would be potential agents for clinical trails in bipolar disorder. Refs: M. C. Chang et al., J. Neurochem. 77, 796 (2001); S. I. Rapoport, M. Basselin, H.-W. Kim, J. S. Rao, Brain Res. Rev. 61185 (2009); S. I. Rapoport, F. Bosetti, Arch. Gen. Psychiatry 59, 592 (2002); R. P. Bazinet, M. T. Weis, S. I. Rapoport, T. A. Rosenberger, Psychopharmacology (Berl) 184, 122 (Jan, 2006). This work was supported in part by the Intramural Program of the National Institute on Aging, NIH.

Disclosure: H. Modi: None. J. Shimshoni: None. M. Basselin: None. L. Li: None. R. Coleman: None. S. Rapoport: None.

\section{Distinct Effects Of Escitalopram And Lithium On G Protein Signaling \\ Lanqiu Zhang, Surabhi Panda, Huailing Zhong, Connie Sanchez,} Mark Rasenick ${ }^{*}$

\section{U. Illinois Chicago, Chicago, IL}

Background: Lipid rafts are specialized membrane domains rich in cholesterol and intimately associated with cytoskeletal components. $\mathrm{G}$ protein signaling is influenced by these domains, but, depending upon the receptor, $G$ protein, and effector enzyme, they either facilitate or attenuate signaling. We have demonstrated that, for Gs and Gs-coupled receptors ( $\beta$-adrenergic, VPAC and 5 HT-4, -6, -7), lipid rafts attenuate signaling by separating Gs $\alpha$ from adenylyl cyclase. This is supported by data obtained in cells treated with cholesterol chelating agents or in which the lipid-raft protein caveolin is knocked down. Furthermore, caveolin knockout mice show a similar effect. Activated $G s \alpha$ is internalized in lipid raft vesicles, but release of Gs $\alpha$ from these vesicles increases microtubule dynamics and this leads to increased neurite outgrowth and increased formation of dendritic spines in primary neurons. Several lines of investigations from different laboratories suggest a post-synaptic effect of chronic antidepressants and a possible postsynaptic target for these drugs. Data from rats, cultured neural and glial cells, and postmortem brain tissue from depressed subjects all suggest that the localization of the $\mathrm{G}$ protein, $\mathrm{Gs} \alpha$, in lipid rafts is modified by chronic treatment with a number of antidepressant compounds (SSRI, MAOI and tricyclic).
Methods: In this study, we sought to compare escitalopram and its enantiomeric counterpart, R-citalopram, in the translocation of Gs $\alpha$ from lipid rafts. We also compared these compounds with the mood stabilizer lithium in a model system using C6 glioma cells. Raft association of Gs $\alpha$ was determined by detergent extraction and cell fractionation, and the mobility of Gs $\alpha$ in the plasma membrane was determined by Fluorescence Recovery After Photobleaching (FRAP) using either confocal (lateral mobility) or Total Internal Reflectance Fluorescence (TIRF) microscopy ("horizontal" mobility).

Results: As seen for other antidepressants, chronic escitalopram treatment decreased the association of Gs $\alpha$ with lipid rafts and decreased "membrane-anchoring" as determined by FRAP. Gs $\alpha$ mediated activation of adenylyl cyclase was augmented by chronic escitalopram treatment. R-citalopram was no different from the control and did not antagonize any of the effects of escitalopram. Surprisingly, lithium treatment had the opposite effects of escitalopram in translocating $\mathrm{Gs} \alpha$ from lipid rafts.

Conclusions: It appears that chronic escitalopram treatment increases Gs $\alpha /$ cAMP signaling, in part, by releasing Gs $\alpha$ from lipid rafts. The mood stabilizer lithium appears to have the opposite effect.

Disclosure: L. Zhang: None. S. Panda: None. H. Zhong: Part 5; Lundbeck. C. Sanchez: Lundbeck. M. Rasenick: Part 1; Eli Lilly, Lundbeck, Sepracor. Part 3; Pax Neuroscience. Part 4; Eli Lilly, Lundbeck.

\section{Analgesic Synergy of NTS2 Receptor Agonist (NT79) and Morphine}

Mona Boules, Hannah Johnston, Jessica Tozy, Kristin Smith, Zhimin Li, Elliott Richelson*

Mayo Clinic, Jacksonville, FL

Background: Neurotensin (NT) is a tridecapeptide with naloxone independent analgesic action. NT exerts its effects through three molecularly cloned receptor subtypes, NTS1, NTS2 and NTS3. The analgesic efficacy of NT agonists depends on their activation of NTS 1 and/or NTS2. NT79 is an NTS2 receptor agonist that produces analgesic effects in animal models of visceral (writhing) but not thermal (hot plate) pain without inducing hypothermia and hypotension. This study extends previous work with NT79 to test its efficacy in an animal model of persistent pain (formalin test) and to determine if there is analgesic synergy between NT79 (N-methyl-Arg, L-Arg, L-Pro, D-3,1-Nal, tert-Leu, L-Leu) and morphine in visceral and persistent pain models.

Methods: Independent groups of 4-7 Sprague-Dawley rats (200-250 g) were divided into 4 groups (saline/saline, saline/NT79, saline/ morphine, and NT79/morphine) and tested in two animal models for pain (writhing and formalin-induced pain). Writhing was induced by injecting the rats intraperitoneally (i.p.) with $0.5 \mathrm{ml}$ of a $2 \%$ aqueous solution of acetic acid. The number of writhes was counted during a $60 \mathrm{~min}$ observation period and presented as mean \pm SEM. Formalin-induced pain was done by injecting $50 \mu \mathrm{l}$ of $5 \%$ formalin subcutaneously into the plantar surface of the left paw of the rat. The time in seconds per 5-min interval the rat spent lifting/biting in a $1 \mathrm{~h}$ observation period was recorded and presented as mean \pm SEM.

Results: NT79 and morphine attenuated acetic acid-induced writhing and formalin-induced lifting and biting during the inflammatory phase. Upon testing each individual nociceptive behavior in the formalin test, NT79 and morphine significantly blocked the lifting but not the biting response, which involves the activity of the spinal nociceptive circuits. The combination of NT79 and morphine attenuated both lifting and biting responses, indicating both spinal and supraspinal modulation of persistent nociception.

Discussion: Data show analgesic synergism between NT79 and morphine thus providing promising therapy for visceral and persistent pain while minimizing adverse effects associated with morphine use. (Supported by Mayo Foundation for Medical Education and Research.) 
Disclosure: M. Boules: Part 1; Sarentis Therapeutics. H. Johnston: None. J. Tozy: None. K. Smith: None. Z. Li: None. E. Richelson: OrthoMcNeill Janssen. Part 2; Astra-Zeneca. Part 3; Sarentis Therapeutics.

\section{Preclinical Profile of the Novel mGluR5 Negative Allosteric Modulator GRN-529: A case for potential efficacy in Treatment Resistant Depression}

Zoe A Hughes, Sarah J Neal, Deborah L Smith, Stacey J Sukoff Rizzo, Claudine M Pulicicchio, Sue Lotarski, Dianne Bryce, S Lu, Jason M Dwyer, Mike Olsen, Corey N Bender, Evguenia Kouranova, Dane Springer, D Li, Steven V O'Neil, Terrance H Andree, Garth T Whiteside, Sarah K Leonard, Robert H Ring*

Pfizer, Groton, CT

Background: Patients suffering from major depressive disorder (MDD) who do not respond to one or more lines of pharmacotherapy may be classified as suffering from Treatment Resistant Depression (TRD). The proportion of patients diagnosed with MDD who fall into this refractory population has been estimated to be as high as $30-50 \%$, highlighting the significant unmet medical needs of TRD. The origin if treatment resistance in depression is unknown, but clues to an underlying endophenotype may be found in the higher incidence of co-morbid symptoms such as anxiety and pain in TRD patients. Recognizing that current standards of chemical antidepressant management either directly or indirectly target monoaminergic systems, it is reasonable to hypothesize that mechanisms of action (MOAs) targeting non-monoaminergic systems may offer promise as novel approaches for future drug discovery efforts. Multiple lines of evidence have implicated enhanced glutamatergic activity in TRD, which has led to an emerging hypothesis that agents acting to negatively modulate central glutamatergic systems may deliver novel therapeutic MOAs for the treatment of TRD. Within this target space, the metabotropic glutamate receptor $5\left(\right.$ mGluR $\left._{5}\right)$ has emerged as a putative target. In addition to depression, $\mathrm{mGluR}_{5}$ has been prosecuted as a target for numerous CNS indications including anxiety disorders, fragile X, autism, Parkinson's disease, and pain. Here we report a broad spectrum profile of preclinical efficacy for a novel mGluR5 negative allosteric modulator (NAM), GRN-529, which supports the case for mGluR5 as a potential target for TRD.

Methods: The in vitro pharmacological profile of GRN-529 at mGluR 5 was determined using cell-based pharmacological assays. In this study, to examine the antidepressant-like effects of GRN-529, we investigated effects on immobility time in the mouse tail suspension test (TST) and forced swim test (FST). Anxiolytic-like efficacy of GRN-529 was investigated by measuring the effects of drug on punished crossings in the mouse four plate test (FPT), stress induced hyperthermia (SIH) in mice, and punished responding in the rat Vogel test. To investigate the antinociceptive effects of GRN-529 on mechanical hyperalgesia, paw withdrawal thresholds were determined in the rat FCA model of inflammatory pain, and sciatic nerve ligation (SNL) model of neuropathic pain. For all studies, the relationship between central target engagement and efficacy was determined using ex vivo receptor occupancy (RO). The potential for antidepressant-induced sexual sideeffects was also examined in a novel preclinical animal model (Sukoff Rizzo SJ, 2008). All animal procedures were approved by the Institutional Animal Care and Welfare Committee.

Results: We report that GRN-529 is a potent mGluR5 NAM (Ki $6.5 \mathrm{nM}$, IC50 $1.7 \mathrm{nM}$ ) in cell based pharmacology assays. Acute oral administration of GRN-529 was efficacious in preclinical antidepressant and anxiolytic models. In the mouse TST and FST, GRN-529 produced dose dependent decreases in immobility time. GRN-529 was also active in mouse anxiolytic models, attenuating a stress-induced hyperthermic response and increasing punished crossings in the four plate test. GRN-529 also increased punished responding in the rat Vogel test. A strong correlation between ex vivo receptor occupancy and efficacy was observed in these anxiety paradigms. In rat pain models GRN-529 produced a dose-dependent increase in paw withdrawal threshold in the FCA and SNL models of mechanical hyperalgesia. In these studies, RO results again demonstrated a strong correlation between target engagement and efficacy. As sexual dysfunction represents a leading cause of non-compliance with current standards of antidepressant therapy, GRN-529 was evaluated in a rat model of antidepressantinduced sexual dysfunction. GRN-529 was dosed for 14 days at doses which occupied $>75 \%$ of receptors. At these doses GRN-529 did not affect sexual behavior in sexually experienced male rats, whereas SSRIs and SNRIs have been shown to cause marked effects in the same assay. Discussion: Our results indicate that GRN-529 is a potent, selective mGluR5 NAM with a broad spectrum profile of efficacy in preclinical assays sensitive to antidepressant-like effects of test compounds. Moreover, GRN-529 demonstrate efficacy in anxiety and pain paradigm, which suggest the potential to address prominent co-morbidities in TRD patients. The broad spectrum of preclinical efficacy observed with GRN-529 in these studies suggest that the mGluR5 NAM mechanism of action may hold promise as a non-monoaminergic treatment approach to TRD.

Disclosure: Z. Hughes: Part 5; Pfizer Global R\&D. S. Neal: Pfizer Global R\&D. D. Smith: Pfizer Global R\&D. S. Sukoff Rizzo: Pfizer Global R\&D. C. Pulicicchio: Pfizer Global R\&D. S. Lotarski: Pfizer Global R\&D. D. Bryce: Pfizer Global R\&D. S. Lu: Pfizer Global R\&D. J. Dwyer: Pfizer Global R\&D. M. Olsen: Pfizer Global R\&D. C. Bender: Pfizer Global R\&D. E. Kouranova: Pfizer Global R\&D. D. Springer: Pfizer Global R\&D. D. Li: Pfizer Global R\&D. S. O’Neil: Pfizer Global R\&D. T. Andree: Pfizer Global R\&D. G. Whiteside: Pfizer Global R\&D. S. Leonard: Pfizer Global R\&D. R. Ring: Pfizer Global R\&D.

\section{The Change of Brain-Derived Neurotrophic Factor and TrkB Receptor on Platelet in Depression}

Toshikazu Saito*, Eri Hashimoto, Wataru Ukai, Kimihiko Watanabe, Ippei Watanabe, Tomohiro Shirasaka, Hiroo Kaneta, Masayasu Kigawa, Toshihiro Yoshinaga, Masaru Tateno, Tae woo Park, Takao Ishii

Sapporo Medical University, Sapporo, Japan

Background: Brain-derived neurotrophic factor (BDNF) is part of a family of neurotrophins and is important for neuronal development and synaptic plasticity. It has been reported that BDNF plays a role in reversing the hippocampal deficit often observed in depressed patients through the promotion of neurogenesis activated by antidepressant therapy. Recently, a decreased serum BDNF level has been implicated in the pathophysiology of depression. It has been suggested that most of the BDNF in blood is pooled in the platelet and released into the serum by an unknown mechanism. However, there have been few reports about the function of platelet BDNF release, especially concerning its role in the pathophysiology of depression. In one of our previous studies, we suggested that antidepressants could cause increased release of BDNF from platelets via tyrosine kinase receptor $B$ (TrkB; BDNF receptor) signaling-dependent mechanisms.

Methods: In the present study, we investigated the in vitro BDNF release in platelets from depression-model rats focusing on the change in TrkB expression. The depression model condition was created by repeatedly injecting rats with corticosterone for 21 days $(20 \mathrm{mg} / \mathrm{kg} /$ day). The washed platelet and serum samples were prepared from control and experimental rats and used for analysis of BDNF release, TrkB receptor expression and the amount of BDNF platelets.

Results: When both groups were treated with various antidepressants, platelets from depression-model rats had lower BDNF release compared to platelets from control rats. We also found that BDNF platelet release was inhibited by $\mathrm{K}_{252 \mathrm{a}}$ (a TrkB inhibitor) and that the expression of TrkB was decreased in the depression-model rats.

Discussion: Our findings suggest that depressive states can cause changes in BDNF release and TrkB expression in platelets which may lead to blood and brain BDNF level changes in depressed patients. Disclosure: T. Saito: None. E. Hashimoto: None. W. Ukai: None. K. Watanabe: None. I. Watanabe: None. T. Shirasaka: None. 


\section{S398}

H. Kaneta: None. M. Kigawa: None. T. Yoshinaga: None. M. Tateno: None. T. Park: None. T. Ishii: None.

211. Increasing the Speed of Intravenous Cocaine Delivery Leads to Greater Drug Intake and Greater Motivation for the Drug Anne-Noël Samaha*, Irina Gheoda, Ellie-Anna Minogianis

University of Montreal, Montreal, QC, Canada

Background: Much research has focused on genetic, biological and environmental factors that can promote or retard the evolution from drug use to drug addiction. In addition to such factors, certain characteristics of the drug itself can modulate the transition to addiction. One such characteristic is the speed at which the drug reaches its sites of action in the brain. Drugs, formulations and routes of administration that achieve a rapid rate of rise of drug levels in the brain are thought to increase the potential for addiction. Why this might be is not known. We hypothesized here that the rapid entry of drugs into the brain might promote patterns of drug-taking that define addiction. Specifically, we hypothesized that increasing the speed of intravenous (i.v.) cocaine infusions [delivered over 5 versus 90 seconds (s)] would 1) promote increased drug consumption when prolonged daily access to drug was permitted and 2) increase the motivation to obtain the drug.

Methods: Male Wistar rats were implanted with chronically indwelling jugular catheters. They were then trained to self-administer i.v. injections of $0.5 \mathrm{mg} / \mathrm{kg}$ cocaine delivered over $5 \mathrm{~s}$ under a fixed ratio schedule of reinforcement in standard operant cages. The number of injections, active lever presses and inactive lever presses were recorded on each session. The time-out period between infusions was progressively increased in separate daily sessions (20, 45, 65 and 85 s). This is because we ultimately wished to study the effects of 5-versus 90-s cocaine infusions on self-administration behaviour, and an 85-s time out period ensured that regardless of infusion speed, all rats could take only one injection every $90 \mathrm{~s}$. Following training, rats were divided into two groups. The first group self-administered cocaine infusions delivered over $5 \mathrm{~s}$ (using a 3.33 RPM syringe pump motor), and the second group received cocaine over $90 \mathrm{~s}$ (using a $0.1 \mathrm{RPM}$ motor), under short-access conditions ( $1 \mathrm{~h} /$ session), for $2-3$ sessions. Access to cocaine was then increased to $6 \mathrm{~h} /$ session for a total of 12 sessions, as this would allow us to assess potential changes in drug consumption in the 5-s versus the 90-s group. Finally, we assessed the motivation for cocaine in both groups by studying breakpoints for the drug under a progressive ratio (PR) schedule. Breakpoints were determined as a function of dose (0.5, 0.25 and $0.125 \mathrm{mg} / \mathrm{kg} /$ injection) and speed of infusion ( 5 and $90 \mathrm{~s}$ ). Varying the speed of infusion during PR testing allowed us to determine whether potential differences in breakpoints for cocaine are attributable to variations in the speed of drug delivery in the past versus variations in the speed of drug delivery during PR testing.

Results: When cocaine was only available for $1 \mathrm{~h} /$ session, the 5 and $90 \mathrm{~s}$ groups took an equivalent number of injections. Under extended access conditions, both groups increased their cocaine intake compared to what they took during short-access sessions, but this increase was of greater magnitude in the $5 \mathrm{~s}$ group. The speed of drug infusion also influenced the motivation for cocaine as assessed by the $\mathrm{PR}$ procedure. The dose-response curve for breakpoints for cocaine was shifted upward in the $5 \mathrm{~s}$ relative to the $90 \mathrm{~s}$ group. In addition, while breakpoints decreased with decreasing dose in the $5 \mathrm{~s}$ group, breakpoints were insensitive to dose in the $90 \mathrm{~s}$ group. Lastly, breakpoints were greater in the $5 \mathrm{~s}$ group relative to the $90 \mathrm{~s}$ group, regardless of whether cocaine was delivered over 5 or $90 \mathrm{~s}$ during PR testing.

Discussion: These findings demonstrate that increasing the speed at which cocaine is delivered leads to greater drug intake and an increased willingness to expend effort to obtain the drug. Thus, drugs, routes and formulations that rapidly deliver drug to the brain might have greater addictive potential because they promote a loss of control over drug taking and increased motivation for drug. The challenge now is to determine at a cellular level how the intake of rapid versus more sustained cocaine infusions impacts upon the brain systems that regulate motivation for drug.

Disclosure: A. Samaha: None. I. Gheoda: None. E. Minogianis: None.

\section{The NK1 Antagonist L822429 Inhibits Stress-induced} Reinstatement of Alcohol Seeking Behavior in Rats Jesse Schank*, Kelly Rowe, Kejun Cheng, Kenner Rice, Annika Thorsell, Markus Heilig

National Institutes of Health, Bethesda, MD

Background: Substance P (SP) and its preferred neurokinin-1 (NK1) receptor play a significant role in stress and anxiety-related behaviors. While the influence of the $\mathrm{NK}_{1}$ receptor on stress responses is well documented, less is known about its role in addiction. Previous studies have suggested an important role of the SP/NK1 system in the rewarding properties of opiates. More recently, our lab was the first to demonstrate that alcohol consumption is decreased by genetic deletion or pharmacological inhibition of the $\mathrm{NK}_{1}$ receptor in mice. We have also reported that administration of an $\mathrm{NK} 1$ antagonist to alcoholics can alleviate craving.

Methods: Pharmacological studies of $\mathrm{NK}_{1}$ antagonism in animal models of alcohol related behaviors have presented a challenge, as receptor affinity shows significant interspecies variation and antagonists developed for the human receptor have limited affinity for the rat receptor. $\mathrm{L} 822429$ is an $\mathrm{NK} 1$ antagonist specifically engineered to possess high affinity for the rat NK1 receptor, and has previously been reported to produce anxiolytic-like effects in rat models when administered systemically. Here, we evaluated the effects of L822429 on alcohol self-administration and reinstatement of alcohol seeking in rats, a model of relapse-like behavior.

Results: L822429 had little effect on baseline alcohol self-administration or cue-induced reinstatement of alcohol seeking behavior. In contrast, it dose-dependently blocked the expression of stress-induced reinstatement. Behavioral specificity was further supported by observations that L822429 lacked effects on locomotor activity or self-administration of a sucrose solution.

Discussion: To our knowledge, this is the first exploration of the effects of NK1 receptor antagonists on alcohol-related behaviors in rats. Taken together, these results indicate $\mathrm{NK}_{1}$ antagonism as a promising candidate mechanism for pharmacotherapy for alcoholism. Future studies will explore the impact of $\mathrm{NK}_{1}$ antagonism in alcohol dependent rats and attempt to identify the neuroanatomical substrates that mediate the effects of $\mathrm{NK}_{1}$ antagonists.

Disclosure: J. Schank: None. K. Rowe: None. K. Cheng: None. K. Rice: None. A. Thorsell: None. M. Heilig: None.

\section{Dopamine Regulation of Risky Decision-Making}

Barry Setlow ${ }^{\star}$, Nicholas Simon, Blanca Beas, Karienn Montgomery, Rebecca Haberman, Jennifer Bizon

University of Florida, Gainesville, FL

Background: Individuals are faced with daily choices among competing alternatives, some of which are accompanied by adverse consequences. Most people are able to accurately assess the risks and rewards of such alternatives and decide adaptively; however, individuals suffering from several pathological conditions (including ADHD, schizophrenia, and addiction) display maladaptive decision making, such that choices are biased toward risky options. This type of decision-making is commonly studied in laboratory tasks in humans; however, there have been few animal models that examine how risks of adverse consequences (punishment, as opposed to reward omission) influence decision-making. Our lab recently developed such a model, in which rats choose between small "safe" rewards and large rewards accompanied by varying risks of punishment. Here we report the 
results of studies using this task in which we examined how dopamine modulates risky decision-making.

Methods: Male Long-Evans rats were given discrete trials in which they could press levers to choose between a small, "safe" food reward and a large food reward associated with risk of punishment (footshock). Each test session consisted of 5 blocks of 10 choice trials, with punishment probability (risk) increasing with each consecutive block $(0,25,50,75,100 \%)$. Preference for the large, "risky" reward typically decreases with increased punishment probability (i.e. - risk of punishment "discounts" the value of the large reward). In Experiment 1 , we examined the effects on risky decision-making of acute administration of dopamine receptor agonists and antagonists. In Experiment 2, we examined the relationship between individual differences in risky decision-making and forebrain D1 and D2 dopamine receptor mRNA expression using in situ hybridization.

Results: We showed previously that acute systemic amphetamine attenuates risky choice in this task. In Experiment 1, neither the D1-like antagonist SCH23390 nor the D1-like agonist SKF81297 affected risky choice. The D2-like antagonist eticlopride also had no effect on risky choice; however, the D2-like agonist bromocriptine reduced risky choice in a manner similar to amphetamine. Furthermore, coadministration of eticlopride with amphetamine abolished amphetamine's effects on risky choice, whereas amphetamine's effects were unaffected by coadministration of $\mathrm{SCH}_{23} 390$. In Experiment 2, there was a linear relationship between D1 receptor mRNA expression and risky choice in insular cortex and nucleus accumbens shell, such that higher levels of D1 mRNA predicted greater preference for the large risky reward. There were also non-linear relationships between D2 receptor mRNA expression and risky choice in orbital and medial prefrontal cortex. Interestingly, these relationships took the form of Ushaped and inverted U-shaped curves, respectively: high levels of D2 mRNA in orbital prefrontal cortex were associated with both high and low levels of risky choice, whereas low levels of D2 mRNA were associated with moderate levels of risky choice. This relationship was reversed in medial prefrontal cortex, such that low levels of D2 mRNA were associated with both high and low levels of risky choice, whereas high levels of D2 mRNA predicted moderate levels of risky choice. In dorsal striatum, high levels of D2 mRNA were associated with low levels of risky choice (risk aversion).

Discussion: Data from the pharmacological experiments suggest that activation of D2-like receptors may be particularly important for modulation of risky decision-making, and the in situ hybridization data suggest that dorsal striatum may be a locus through which D2 receptor-acting drugs attenuate risky choice. Although the pharmacological experiments do not support a role for D1-like receptors in risky decision-making, the in situ data suggest that further investigation of an insular cortex-nucleus accumbens circuit may be warranted.

Disclosure: B. Setlow: None. N. Simon: None. B. Beas: None. K. Montgomery: None. R. Haberman: None. J. Bizon: None.

214. Omega-3 Fish Oil Enhances Bioavailability and Anti-Amyloidogenic Properties of EGCG inTg2576 Mice

R. Douglas Shytle*, Brian Giunta, Hou Houyan, Yuyan Zhu, Jon Salemi, Amanda Ruscin, Cindy Sanberg, Paul Sanberg, Jun Tan

University of South Florida, Tampa, FL

Background: Extracellular plaques of $\beta$-amyloid $(A \beta)$ peptides are implicated in Alzheimer's Disease (AD) pathogenesis. $A \beta$ formation is precluded by $\alpha$-secretase, which cleaves within $A \beta$ generating soluble APP- $\alpha$ (sAPP- $\alpha)$, a neurotrophic metabolite. Thus, $\alpha$-secretase upregulation appears to be a favorable target for $\mathrm{AD}$ therapy. We previously reported that orally administered green tea derived flavonoid, EGCG, improved cognitive function and decreased A $\beta$ levels in the brains $\mathrm{AD}$ transgenic mice. However, the comparable effective dose of EGCG in humans may exceed clinical convenience and/or safety. Epidemiological studies suggest that moderate consumption of omega- 3 fish oil is associated with reduced dementia risk.
Methods: Here we investigated if oral combination of fish oil $(8 \mathrm{mg} / \mathrm{kg} /$ day $)$ and EGCG $(62.5 \mathrm{mg} / \mathrm{kg} /$ day or $12.5 \mathrm{mg} / \mathrm{kg} /$ day $)$ would reduce $\mathrm{AD}$-like pathology in $\mathrm{Tg} 2576$ transgenic mice.

Results: In vitro, co-treatment of N2a cells transfected with a human Swedish AD mutation with fish oil and EGCG enhanced sAPP- $\alpha$ production compared to either compound alone $(\mathrm{P}<0.001)$. In vivo, fish oil enhanced the bioavailability of EGCG and had additive therapeutic effects on inhibition of cerebral $A \beta$ deposits $(\mathrm{P}<0.001)$.

Discussion: These findings suggest that moderate consumption of EGCG with omega-3 fish oil may have complimentary therapeutic potential for the treatment of AD.

Acknowledgements: This work was supported, in part, by NIH grants: NIA (R21AGo31037) to RDS, NIMH Clinical Scientist Career Development Award (1Ko8MHo82642-01A1) to BG, and NCCAM (1R43AToo4871-01) to JT.

Disclosure: R. Shytle: Part 1; Natura Therapeutics, Inc., Pfizer, Inc., Targacept, Inc., Forest Laboratories, Inc., Yaupon Therapeutics, Inc. Part 2; Targacept, Inc., Natura Therapeutics, Inc. Part 3; Targacept, Inc. Part 4; Natura Therapeutics, Inc. B. Giunta: None. H. Houyan: None. Y. Zhu: None. J. Salemi: None. A. Ruscin: None. C. Sanberg: Part 1; Natura Therapeutics, Inc., Saneron, Inc. Part 2; Natura Therapeutics, Inc., Saneron, Inc. Part 3; Natura Therapeutics, Inc. Part 4; Natura Therapeutics, Inc. Part 5; Natura Therapeutics, Inc. P. Sanberg: Part 1; Saneron, Inc., AstraZeneca, Inc., Targacept, Inc., Johnson \& Johnson, Inc., Natura Therapeutics, Inc., Cryopraxis, Inc., Cell Therapeutics, Inc. Part 2; Saneron, Inc., Cryopraxis, Inc., Targacept, Inc., Cell Therapeutics, Inc., Natura Therapeutics, Inc. Part 3; Targacept, Inc., Saneron, Inc. J. Tan: Part 1; Natura Therapeutics, Inc., Saneron, Inc. Part 2; Natura Therapeutics, Inc., Saneron, Inc. Part 4; Natura Therapeutics, Inc., Saneron, Inc.

\section{Membrane Lipid Rafts Are Required For D2 Dopamine Receptor} Signaling

David R. Sibley*, Lisa A. Hazelwood, R. Benjamin Free, Rebecca A. Roof, Yang Han, Jonathan A. Javitch

\section{NINDS/NIH, Bethesda, MD}

Background: Lipid rafts are specialized membrane microdomains (10$200 \mathrm{~nm}$ ) enriched in cholesterol and sphingolipids and are important in the organization of GPCR-protein complexes and the regulation of signaling. Given the emerging significance of lipids with respect to GPCR structure and activation, we have investigated the role of lipid rafts and membrane cholesterol on $\mathrm{D}_{2}$ dopamine receptor (DAR) localization and function.

Methods: D2 DAR constructs were expressed in HEK293T cells. Sucrose density fractionation resolved lipid rafts from other membrane components. Methyl-beta-cyclodextrin (MCD) was used to deplete membrane cholesterol and to disrupt lipid rafts. Receptor signaling activities were determined as described below.

Results: Detergent solubilization followed by sucrose gradient centrifugation resolved buoyant lipid rafts from heavier membrane fractions. Cholesterol and flotillin, markers for lipid rafts, peaked in factions 4-5 whereas the transferrin receptor and total protein, markers for non-raft membranes, peaked in fractions 9-11. Identification of the D2 DAR required its immunoprecipitation revealing it to be approximately equally distributed amongst fractions 4-11. Pretreatment with MCD eliminated cholesterol and flotillin from fractions 4-5 with no effect on transferrin receptor or total protein localization. MCD also eliminated D2 DAR localization from fractions 4-9, although the receptor was still found in heavier membrane fractions 10-11. GM1 ganglosides are components of lipid rafts that can be visualized through labeling with GFP-tagged cholera toxin (CTX) subunit B. Confocal fluorescence microscopy of GFP-CTX-treated cells revealed discreet puncta that were eliminated with MCD treatment. Taken together, these data show that the D2 DAR is present in lipid rafts, as well as other membrane fractions, and that MCD treatment effectively disrupts lipid rafts in HEK293T cells. We also found that MCD 
treatment had no effect on total or cell surface D2 DAR expression whereas receptor-mediated inhibition of cAMP accumulation was totally abolished. In contrast D1 DAR or forskolin-stimulated cAMP accumulation was unaffected by MCD treatment. MCD treatment was also found to abolish M2 muscarinic receptor-mediated inhibition of cAMP accumulation as well as $\mathrm{D}_{2} \mathrm{DAR}$ and $\mathrm{M}_{4}$ muscarinic receptor activation of GIRK channels - all Gi/o mediated signaling events. Subsequent to MCD treatment, cholesterol repletion was found to restore D2 DAR-mediated inhibition of cAMP accumulation indicating that that this effect is reversible. As MCD is known to extract cholesterol from membranes and proteins, we wished to determine if the effect of MCD on D2 DAR signaling was due to a physical separation of D2 DAR and Gi/o proteins, perhaps via lipid raft disruption, or due to cholesterol depletion from the receptor per se. To address this, we created a receptor construct where the alpha subunit of Gi1 was tethered to the D2 DAR's C-terminus. The Gi1 alpha subunit was further mutated such that it was refractory to pertussis toxin (PTX) inactivation. This construct behaved in an identical fashion as the wild-type (WT) D2 DAR when expressed in HEK293 T cells and retained its ability to inhibit cAMP accumulation subsequent to PTX inactivation of endogenous Gi/o proteins (WT D2 DAR signaling was abolished by PTX treatment). Notably, in contrast to the WT D2 DAR, MCD treatment had virtually no effect on the ability of the D2 DAR-Gi tethered construct's ability to inhibit cAMP accumulation.

Discussion: Our current results show that the D2 DAR is distributed in multiple membrane microdomains, including cholesterol-rich buoyant lipid rafts. We found that extraction of cholesterol led to a disruption of lipid rafts and also to an abolishment of D2 DAR-mediated signaling through Gi/o protein-mediated pathways. Notably, Gs-mediated signaling was unaffected. As cholesterol is known to bind directly to some membrane proteins, including GPCRs and transporters, we wondered if potential extraction of cholesterol from the receptor led to its uncoupling or if a physical separation of D2 DARs and Gi/o proteins was responsible for the loss of signaling. Our observation that the Gi/o-mediated signaling of a fused D2 DAR-Gi construct was unaffected by cholesterol extraction argues that cholesterol disruption of lipid rafts leads to a physical uncoupling of the D2 DAR and Gi/o. As similar results were observed with the Gi/o-coupled $\mathrm{M}_{2}$ and $\mathrm{M}_{4}$ muscarinic receptors, this may be a general mechanism for Gi/omediated signaling. We thus hypothesize that lipid rafts provide a signaling platform for the D2 DAR as well as other Gi/o-coupled receptors.

Disclosure: D. Sibley: None. L. Hazelwood: None. R. Free: None. R. Roof: None. Y. Han: None. J. Javitch: None.

\section{Biodegradable Ropinirole Implants (NP201) Yield Sustained Reversal of Parkinsonian Symptoms in MPTP-treated Monkeys without Concomitant Periods of Hyperactivity seen Following Oral Treatment}

Steven Siegel*, Carol O’Neill, Mark Pierce, Lori Langhamer, Jeffery Kordower

\section{University of Pennsylvania, Philadelphia, PA}

Background: Most current treatments for PD require dosing of one or more times per day. This leads to a cycle of brief remission of symptoms interspersed among periods of bradykinesia and dyskinesia. Additionally, clinically effective doses of dopamine agonists can lead to intermittent periods of iatrogenic psychosis at peak concentrations. Consequently, there is a critical need for long-acting treatments that avoid fluctuations in dopamine activity and motor function as well as reducing psychiatric side effects of intermittent treatments.

Objective: To compare the efficacy and potential side effects of NP201, a biodegradable implant that delivers the dopamine agonist ropinirole for 40-60 days, with oral ropinirole in treating Parkinsonian Rhesus monkeys. The secondary objective was to assess plasma levels of TID oral ropinirole and the long acting NP201 implant.
Methods: Rhesus monkeys were treated with MPTP until they displayed stable Parkinsonian behaviours with a Clinical Rating Scale (CRS) between 7.0 - 20.5. Before implantation of NP201, the optimal TID oral ropinirole dose was defined for each subject as the dose that resulted in a $50 \%$ decrease in CRS. One group of monkeys $(n=4)$ continued on their predefined optimal TID ropinirole dose whereas another group $(n=4)$ received NP201 implants corresponding to 1/9th of their own optimal daily ropinirole dose. A placebo group $(n=4)$ was included. CRS, continuous activity levels, global dyskinesia rating scale, fine motor pick-up test and skin irritation were measured.

Results: Both oral ropinirole and NP201, at 1/9th of the oral dose, showed a CRS measure that was statistically superior to placebo $(p<0.05)$. There was no significant difference in CSR $(p>0.05)$ between NP2O1 and oral ropinirole for the period of time during which serum levels were comparable. This corresponded to 11-53 days post implantation. Activity data suggest that $\mathrm{NP}_{201}$ restored a pattern of normal, pre-MPTP levels of activity, while oral ropinirole yielded alternating periods of very high activity interspersed with normal levels. Furthermore, oral ropinirole treated animals were indistinguishable from placebo on weekend days, when they did not receive active agent, while NP2O1 animals displayed continuously normal activity throughout the active phase of the study. There was much greater variability for CRS scores, activity and plasma levels in the oral ropinirole group relative to $\mathrm{NP}_{2} \mathrm{O1}$. There were no signs of irritation at the site of implantation following either NP201 or placebo implants made of polymer alone.

Discussion: NP201 was clinically effective at 1/9th of the lowest effective oral dose and restored the pre-MPTP pattern and levels of activity, while oral ropinirole yielded alternating periods of very high and normal activity. Since dopamine agonist-induced hyperactivity in animals is predictive of psychotic effects in humans, low dose NP201 has the potential to provide clinical improvement in bradykinesia with less "off" periods and lower risk for medication induced psychosis.

Disclosure: S. Siegel: Part 1; NuPathe, Merck. Part 2; NuPathe. Part 4; AstraZenca. C. O'Neill: Part 1; NuPathe. Part 2; NuPathe. Part 5; NuPathe. M. Pierce: Part 1; NuPathe. Part 2; NuPathe. Part 5; NuPathe. L. Langhamer: None. J. Kordower: Part 1; NuPathe, Ceregene, Brainstorm. Part 2; Ceregene and Brainstorm. Part 4; NuPathe.

217. Ex-vivo Analysis of Adrenergic $\alpha_{1}$ and $\alpha_{2}$ and Serotonin $5-\mathrm{HT}_{1 \mathrm{~A}}$ and $5-\mathrm{HT}_{2 \mathrm{~A}}$ Receptor Occupancy by Asenapine, Olanzapine, and Risperidone

Yong Kee Choi, Brian Henry, Mohammed Shahid, Frank Tarazi*

\section{Harvard Medical School, Belmont, MA}

Background: Asenapine is approved in the United States for acute treatment of schizophrenia and of manic or mixed episodes associated with bipolar I disorder with or without psychotic features in adults. Although it is postulated that adrenergic $\left(\alpha_{1}\right.$ and $\left.\alpha_{2}\right)$ and serotonergic $\left(5-\mathrm{HT}_{1 \mathrm{~A}}\right.$ and $\left.5-\mathrm{HT}_{2 \mathrm{~A}}\right)$ receptors play a role in the efficacy and tolerability of antipsychotics, ex-vivo receptor occupancy of these receptors by asenapine and other established antipsychotics in different brain regions remains to be investigated. In this study, we analyzed $\alpha_{1}, \alpha_{2}, 5-\mathrm{HT}_{1 \mathrm{~A}}$, and $5-\mathrm{HT}_{2 \mathrm{~A}}$ receptor occupancy induced by asenapine, olanzapine, and risperidone.

Methods: Adult male rats $(\mathrm{N}=6$ /group) were injected subcutaneously (s.c.) with 6 doses of asenapine $(0.003-1.0 \mathrm{mg} / \mathrm{kg})$ or vehicle $(1 \mathrm{ml} / \mathrm{kg})$, or intraperitoneally (i.p.) with 6 doses of olanzapine $(0.1-30 \mathrm{mg} / \mathrm{kg}$ ), risperidone $(0.01-3 \mathrm{mg} / \mathrm{kg})$, or vehicle $(1 \mathrm{ml} / \mathrm{kg})$; these doses approximate clinically-relevant doses in humans. Sixty minutes following treatment, brains were collected and processed for ex-vivo receptor autoradiography using $\left[{ }^{3} \mathrm{H}\right]$ prazosin for $\alpha_{1},\left[{ }^{3} \mathrm{H}\right] \mathrm{RX} 821002$ for $\alpha_{2}$, $\left[{ }^{3} \mathrm{H}\right] 8-\mathrm{OH}-\mathrm{DPAT}$ for $5-\mathrm{HT}_{1 \mathrm{~A}}$, and $\left[{ }^{3} \mathrm{H}\right]$ ketanserin for $5-\mathrm{HT}_{2 \mathrm{~A}}$ receptors. Adrenergic and serotonin receptor occupancies were measured in medial prefrontal cortex (MPC), dorsolateral frontal cortex (DFC), nucleus accumbens (NAc), lateral and medial caudate putamen (CP-L or CP-M), hippocampus (HIPP), and entorhinal cortex (EC). 
Results: Acute treatment with different doses of asenapine, olanzapine, and risperidone induced dose-related increases in $\alpha_{1}, \alpha_{2}, 5-\mathrm{HT}_{1 \mathrm{~A}}$, and $5-\mathrm{HT}_{2 \mathrm{~A}}$ receptor occupancy in different forebrain regions. Maximum $\alpha_{1}$ receptor occupancy levels were similar across agents in MPC and DFC $(\geq 95 \%)$, but were lower with asenapine in NAc (75\%), CP-M (74\%), CP-L (68\%), HIPP (85\%), and EC (87\%) compared with olanzapine and risperidone ( $\geq 95 \%$ for both agents across all regions). Comparable levels of $\alpha_{2}$ receptor occupancy were observed across all agents in the CP-M and CP-L (range, $81 \%-84 \%$ ), but occupancy levels were higher with asenapine in MPC (96\%), DFC (93\%), NAc (88\%), HIPP (95\%), and EC ( $96 \%)$ compared with olanzapine (MPC, $88 \%$; DFC, $84 \%$; NAc, $73 \%$; HIPP, $82 \%$; EC, 92\%) and risperidone (MPC, 86\%; DFC, $86 \%$; NAc, $74 \%$; HIPP, $82 \%$; EC, $89 \%$ ). Extrapyramidal levels of 5 -HT ${ }_{1 A}$ receptors were too low to adequately assess occupancy, but maximum $5-\mathrm{HT}_{1 \mathrm{~A}}$ receptor occupancy levels were higher with asenapine $(\geq 95 \%$ in MPC, DFC, HIPP, and EC) than with olanzapine and risperidone (78\%-85\% in MPC, DFC, HIPP, and EC). Maximum 5- $\mathrm{HT}_{2 \mathrm{~A}}$ receptor occupancy levels were similar for all agents in MPC and DFC $(\geq 95 \%)$, but were lower with asenapine in NAc (72\%), CP-M (64\%), CP-L (65\%), HIPP (89\%), and EC (84\%) compared with olanzapine and risperidone ( $\geq 93 \%$ for both agents across all regions). Asenapine was also more potent than olanzapine and risperidone, with $\mathrm{ED}_{50}$ values in $\mathrm{mg} / \mathrm{kg}$ for asenapine ( $\alpha_{1}$ range, 0.010-0.194; $\alpha_{2}$ range, 0.003-0.039) being consistently lower than those of olanzapine ( $\alpha_{1}$ range, $0.614^{-}$ $0.796 ; \alpha_{2}$ range, $\left.0.581-1.081\right)$ and risperidone ( $\alpha_{1}$ range, $0.045-0.199 ; \alpha_{2}$ range, 0.056-0.146) across all forebrain regions examined. In addition, asenapine displayed higher affinity for cortical and limbic $\alpha_{1}\left(\mathrm{ED}_{50}\right.$ in mg/kg: MPC, 0.013; DFC, 0.010; HIPP, 0.012; EC, 0.015) and $\alpha_{2}\left(\mathrm{ED}_{50}\right.$ in $\mathrm{mg} / \mathrm{kg}$ : MPC, 0.003; DFC, 0.003; HIPP, 0.005; EC, 0.008) receptors versus the same receptor subtypes in extrapyramidal brain regions $\left(\alpha_{1}\right.$ : NAc, 0.134; CP-M, 0.194, CP-L, 0.148; $\alpha_{2}$ : NAc, 0.039; CP-M, 0.032, CP$\mathrm{L}, 0.023)$; a finding that may contribute to previous reports of asenapine-induced increases in $\alpha_{1}$ and $\alpha_{2}$ receptors in cerebral cortex. All agents displayed a preferentially higher potency for $5-\mathrm{HT}_{2 \mathrm{~A}}$ receptors in $\mathrm{MPC}\left(\mathrm{ED}_{50}\right.$ in $\mathrm{mg} / \mathrm{kg}$ : asenapine, 0.008; olanzapine, 0.344 ; risperidone, 0.04$)$, $\mathrm{DFC}\left(\mathrm{ED}_{50}\right.$ in $\mathrm{mg} / \mathrm{kg}$ : asenapine, 0.009; olanzapine, 0.376; risperidone, 0.039), and HIPP ( $\mathrm{ED}_{50}$ in $\mathrm{mg} / \mathrm{kg}$ : asenapine, 0.02; olanzapine, 0.34; risperidone, 0.042) compared with the CP-M (ED ${ }_{50}$ in $\mathrm{mg} / \mathrm{kg}$ : asenapine, 0.204; olanzapine, 0.955; risperidone, 0.124$)$ and $\mathrm{CP}-\mathrm{L}\left(\mathrm{ED}_{50}\right.$ in $\mathrm{mg} / \mathrm{kg}$ : asenapine, 0.126 ; olanzapine, 0.796 ; risperidone, 0.123 ).

Discussion: The profile of asenapine-induced $\alpha_{1}, \alpha_{2}, 5-\mathrm{HT}_{1 \mathrm{~A}}$, and $5-\mathrm{HT}_{2 \mathrm{~A}}$ receptor occupancy provides new insights into the mechanisms of action of asenapine and further supports its unique psychopharmacologic properties. Specifically, differences in the receptor profiles of these agents may contribute to their differential ability to alleviate negative symptoms and cognitive deficits of schizophrenia.

Disclosure: Y. Choi: None. B. Henry: Part 5; MSD, Translational Medicine Research Centre, Singapore. M. Shahid: MSD, Newhouse, Lanarkshire, UK. F. Tarazi: Part 1; Pfizer, Merck, GlaxoSmithKline. Part 4; Organon, Pfizer, Janssen, Forest Labs.

\section{Glycine Transporter Type 1 Inhibitor RG1678: Pharmacological} Characterization of a Novel Agent for the Treatment of Schizophrenia Daniela Alberati, Jean-Luc Moreau, Emmanuel Pinard, Edilio Borroni, Dominik Hainzl, Judith Lengyel, Nicole Hauser, Roland Mory, Frederic Knoflach, Joseph G. Wettstein*

\section{F. Hoffmann-La Roche, Basel, Switzerland}

Background: More than 20 years ago, observations that noncompetitive N-methyl-D-aspartate (NMDA) receptor antagonists such as phencyclidine and ketamine produced schizophrenia-like effects in normal subjects and worsened psychotic symptoms in patients led to the development of the glutamate hypothesis of schizophrenia. It is now thought that increasing NMDA receptor function pharmacologically will compensate for the hypofunctional receptor signalling and may provide a way to better manage the symptoms of schizophrenia.
One approach to normalize NMDA receptor neurotransmission is to increase the availability of the obligatory co-agonist glycine at its modulatory site on the receptor through inhibition of glycine transporter type 1 (GlyT1). Described herein are the pharmacological characteristics of $\mathrm{RG} 1678$, a GlyT1 inhibitor recently shown to have a beneficial effect in a Phase II trial in patients with schizophrenia.

Methods: For biochemical studies on the transporter, tritiated glycine uptake was determined in $\mathrm{CHO}$ cells expressing human and mouse GlyT1b in the presence of RG1678. For neurochemistry, levels of glycine were measured using microdialysis with a probe in the striatum in freely moving Sprague-Dawley rats treated with RG1678. Glycine was also measured in rat cerebrospinal fluid (CSF) by $1 \mathrm{H}-\mathrm{NMR}$ spectroscopy. For electrophysiological experiments, NMDA-dependent long-term potentiation (LTP) was performed in rat hippocampal slices by stimulating the Schaffer collaterals and recording from CA1 pyramidal cells. Behavioral studies focused on locomotor activity in either NMRI mice or Wistar rats: in mice, graded doses of RG1678 were administered prior to d-amphetamine or L-687414, a glycine site antagonist at the NMDA receptor complex; rats were treated for 14 days with PCP and, after the last dose, given RG1678 followed by damphetamine (a paradigm for NMDA receptor hypofunction).

Results: RG1678 inhibited human GlyT1 with an IC50 of $25 \mathrm{nM}$; kinetic analysis revealed that $\mathrm{RG} 1678$ blocked the transporter in a noncompetitive manner, decreasing the maximal velocity (V-max) of the transporter without influencing its affinity for glycine $(\mathrm{Km})$. In vivo, over a dose range of $1-10 \mathrm{mg} / \mathrm{kg}, \mathrm{RG1678}$ dose-dependently increased glycine in brain extracellular space as well as in the CSF of rats. In hippocampal CA1 pyramidal cells, treatment of the slices with RG1678 produced an enhancement of LTP at $100 \mathrm{nM}$ but not $300 \mathrm{nM}$. In the behavioral experiments, RG1678, ranging from $0.1-10 \mathrm{mg} / \mathrm{kg}$, attenuated to different degrees the hyperlocomotion induced by d-amphetamine or L-687414 in mice and the hyperresponse to $\mathrm{d}$-amphetamine challenge in rats treated chronically with PCP.

Discussion: The biochemical and neurochemical data demonstrate that RG1678 is a highly efficacious, non-competitive GlyT1 inhibitor in vitro and in vivo. Data from electrophysiology and behavioral studies also show that RG1678 can modulate both glutamatergic and dopaminergic transmission. Together, the results support the advancement of RG1678 into clinical studies in patients with schizophrenia.

Disclosure: D. Alberati: Part 5; F. Hoffmann-La Roche. J. Moreau: F. Hoffmann-La Roche. E. Pinard: F. Hoffmann-La Roche. E. Borroni: F. Hoffmann-La Roche. D. Hainzl: F. Hoffmann-La Roche. J. Lengyel: F. Hoffmann-La Roche. N. Hauser: F. Hoffmann-La Roche. R. Mory: F. Hoffmann-La Roche. F. Knoflach: F. Hoffmann-La Roche. J. Wettstein: F. Hoffmann-La Roche.

219. Targeting Phosphodiesterase-4 (PDE4) to Reduce Ethanol Intake in Rodents

Wei Hu, Rui-Ting Wen, Ying Huang, Rolf Hansen, Wang Chuang, Qiang Wu, Marco Conti, Jian-Hui Liang, Han-Ting Zhang*

West Virginia University Health Sciences Center, Morgantown, WV

Background: Phosphodiesterase-4 (PDE4) plays a critical role in the regulation of cyclic AMP (cAMP) signaling via hydrolysis of intracellular cAMP in the brain. Given that cAMP-protein kinase A (PKA) signaling is negatively correlated with ethanol consumption, it was of interest to know whether PDE4 was involved in ethanol intake. Methods: Ethanol intake was evaluated using the two-bottle choice paradigm in $\mathrm{C}_{57} \mathrm{BL} / 6 \mathrm{~J}$ mice, high-alcohol preferring (HAP) mice, and alcohol preferring fawn-hooded $(\mathrm{FH})$ rats, which were treated with the selective $\mathrm{PDE}_{4}$ inhibitor rolipram at different doses. Mice deficient in $\mathrm{PDE}_{4} \mathrm{~A}, \mathrm{PDE}_{4} \mathrm{~B}$, or $\mathrm{PDE}_{4} \mathrm{D}$ were also examined for ethanol intake in order to identify the involvement of $\mathrm{PDE}_{4}$ subtypes.

Results: Treatment (i.p.) with rolipram, at doses of $0.1-0.5 \mathrm{mg} / \mathrm{kg}$ (for mice) or $0.1-2.0 \mathrm{mg} / \mathrm{kg}$ (for rats), decreased ethanol intake in a dosedependent manner; it also increased water intake, leading to unchanged total fluid consumption. Mice deficient in $\mathrm{PDE}_{4} \mathrm{~B}$, which 
is one of the four PDE4 subtypes (PDE4A-D) and is the predominant subtype expressed in the striatum and amygdala, two brain regions important for the regulation of ethanol consumption, displayed decreases in ethanol intake, compared to wild-type controls. In contrast, mice deficient in $\mathrm{PDE} 4 \mathrm{~A}$ or $\mathrm{PDE} 4 \mathrm{D}$ did not show similar changes in ethanol intake. In addition, PDE4B deficient mice exhibited decreases in ethanol intake in the drinking-in-the-dark paradigm. Further, inhibition of the remaining $\mathrm{PDE}_{4}$ subtypes (i.e. PDE4A and $\mathrm{PDE} 4 \mathrm{D})$ in $\mathrm{PDE} 4 \mathrm{~B}$ deficient mice did not alter decreased ethanol intake.

Discussion: Inhibition of $\mathrm{PDE}_{4}$ produced promising reduction of ethanol intake in various alcohol drinking models. This appears to be involved primarily by the $\mathrm{PDE}_{4} \mathrm{~B}$ subtype. Overall, the results suggest that $\mathrm{PDE}_{4}$ is a novel target for drugs that reduce ethanol intake; selective inhibitors of $\mathrm{PDE}_{4}$, in particular $\mathrm{PDE}_{4} \mathrm{~B}$, may be used for treatment of alcohol dependence (This work was supported by research grants from WVU Research Development Funding and NIH/NIA).

Disclosure: W. Hu: None. R. Wen: None. Y. Huang: None. R. Hansen: None. W. Chuang: None. Q. Wu: None. M. Conti: None. J. Liang: None. H. Zhang: Part 1; Lundbeck Pharmaceuticals.

220. Do Mood Disorders Alter Vascular Function and Stiffness? Jess G. Fiedorowicz ${ }^{\star}$, William H. Coryell, Lois L. Warren, Robyn J. Netz, John P. Rice, Nora S. Metz, Caroline E. Drain, William Haynes

The University of Iowa, Iowa City, IA

Background: Individuals with major depression or bipolar disorder appear more likely to have risk factors for and disproportionately die from vascular disease. In prior analyses from the Collaborative Depression Study (CDS), hypomanic/manic, but not depressive, symptoms showed a dose-response relationship with cardiovascular mortality. We sought to assess the effects of symptom burden and treatment exposure on measures of vascular function.

Methods: The National Institute of Mental Health CDS rigorously assessed participants' phenomenological and treatment states for more than 25 years. At a separate visit, a small subsample of participants consented for a metabolic and vascular function assessment. The primary outcomes, endothelium-dependent and endothelium-independent vascular function of conduit vessels, were assessed noninvasively via ultrasound measurement of brachial artery diameter following flow-mediated and nitroglycerin-mediated vasodilation. Measures of vascular stiffness from pulse wave analysis and pulse wave velocity served as secondary outcomes. Body mass index, body fat percentage, fasting lipids, leptin, C-reactive protein, interleukin-6, insulin, and glucose levels were measured. The proportion of weeks with clinically significant hypomanic, manic, minor depressive, or major depressive symptoms during follow-up quantified symptom burden. To minimize risk of Type I error on multiple comparisons, treatment exposures were lumped into two broad classes: mood stabilizers/antipsychotics and antidepressants. Analyses of more refined medication classes followed any significant findings. To minimize the impact of outliers and to maximize the likelihood that residuals were normally distributed in this small sample, exposures of interest were modeled as the logit transformation of the proportion of time exposed. Linear regression models determined the effect of symptom burden and treatment exposure on vascular function, controlling for age, gender, and tobacco exposure (pack*years). Because symptom burden and respective treatments were highly correlated in this small sample, symptom burden and treatment exposure were modeled separately to mitigate multicollinearity and overfitting.

Results: 35 participants from the University of Iowa (33) and Washington University (2) sites of the CDS consented for a crosssectional vascular and metabolic assessment at the University of Iowa. The mean (SD) age of participants was $61(8), 83 \%$ were female, and
$57 \%$ had a diagnosis of bipolar disorder. The mean (SD) body mass index was 30 (6) and $63 \%$ smoked tobacco. In multivariate linear regression, controlling for age, gender, and tobacco exposure, manic symptom burden was associated with lower flow-mediated dilation $\left(\mathrm{t}=-2.15\right.$, partial $\left.\mathrm{R}^{2}=0.15, p=0.04\right)$. Depressive symptom burden was not associated with any of the vascular function measures. Subsequent exploratory analyses associated manic symptom burden with hypertriglyceridemia, higher body mass index, larger waist circumference, and insulin resistance. Cumulative exposure to antipsychotics and mood stabilizers was associated with elevated augmentation pressure $\left(\mathrm{t}=2.44\right.$, partial $\left.\mathrm{R}^{2}=0.18, p=0.02\right)$ and mean aortic systolic blood pressure $\left(\mathrm{t}=2.25\right.$, partial $\left.\mathrm{R}^{2}=0.15, p=0.03\right)$. First generation antipsychotics were the only medication class significantly associated with these findings and were also associated with elevated brachial systolic blood pressure. Hypertriglyceridemia and insulin resistance were associated with cumulative exposure to second generation antipsychotics and valproic acid derivatives. Valproic acid derivative exposure was also associated with low high density lipoprotein cholesterol.

Discussion: These results provide evidence that mood disorders may contribute to vasculopathy in a dose-dependent fashion. Patients with more hypomanic/manic symptoms had poorer endothelial function, perhaps as a consequence of hypertriglyceridemia, obesity, or insulin resistance. First generation antipsychotic exposure was associated with arterial stiffness, evidenced by higher augmentation pressure and aortic systolic pressure. Long-term exposure to antipsychotics and valproic acid derivatives were associated with several vascular risk factors. Analyses could not reliably disentangle the effects of symptom burden independent of treatment exposure and vice versa. Vascular phenotyping methods may nonetheless provide a promising means of elucidating the mechanisms linking mood disorders to vascular disease.

Disclosure: J. Fiedorowicz: None. W. Coryell: None. L. Warren: None. R. Netz: None. J. Rice: None. N. Metz: None. C. Drain: None. W. Haynes: None.

\section{Progressive and Lasting Amplification of Accumbal Signals Related to Cocaine-predictive Behavior and Cues \\ Laura Peoples*, Karine Guillem}

University of Pennsylvania School of Medicine, Philadelphia, PA

Background: It is hypothesized that the development and chronicity of cocaine addiction is mediated by progressive and persistent changes in cortico-striatal neuronal signaling. Identifying these changes in neuronal activity is important for understanding and treating cocaine addiction. In the present investigation we tested for progressive and lasting changes in the neuronal activity of single accumbal (NAc) neurons across phases of cocaine SA, abstinence, and re-exposure. Methods: Animals $(n=10)$ were implanted with an intravenous jugular catheter and an array of mircrowires in the NAc. Following habituation to the operant chamber, rats were trained to self-administer (SA) cocaine $(0.75 \mathrm{mg} / \mathrm{kg} /$ infusion $)$ in short daily training sessions. Thereafter rats were exposed to the following: 14 days of long-access (6-hr) cocaine SA, 30 days of abstinence, and 8 days of re-exposure to cocaine SA. During the cocaine SA and re-exposure periods, each daily SA session included a pre-session baseline phase, a conditioned reinforcement phase, and a cocaine + cue reinforcement phase. During the conditioned reinforcement phase, animals responded on a Fixed-Ratio 1 (FR1) schedule for cue reinforcement. During the cocaine + cue reinforcement phase animals responded on a FR1 schedule of cocaine + cue reinforcement. Animals remained in the home cage during the abstinence period. Extracellular recordings of single NAc neurons were made on the following days: the last habituation day, the 2nd and 16th day of cocaine SA, the 3oth day of cocaine abstinence, and the 2nd and 8 th day of re-exposure. Recording sessions were conducted as were normal daily training sessions except for an additional cue-presentation phase which occurred between the baseline and cue-reinforcement phases. The strength of drug-seeking behavior was characterized 
during progressive-ratio sessions that were conducted pre-abstinence and post-re-exposure. Additionally, animals' resistance to punishment of cocaine SA was tested at the end of the re-exposure phase. Behavior during the SA sessions, the progressive-ratio session, and the punishment session were used to calculate a score for the development of addiction-like behaviors for each animal. An additional group of rats was treated similarly except that subjects were trained to selfadminister sucrose.

Results: During the 2nd cocaine SA day a sub-group of neurons (25\%) exhibited a phasic increase in firing to either or both the cocainepredictive cues and the operant (referred to as task-activated neurons). Most (70\%) other neurons showed no phasic response during the cocaine-predictive behaviors and cues (referred to as task-nonactivated neurons). Between-session comparisons showed that taskactivated neurons underwent no change in either phasic or average firing between the 2nd and 16th day of initial SA, but increased in prevalence across the 30-day period of abstinence. This increased prevalence persisted across 8 days of re-exposure to cocaine SA. On the other hand, task-non-activated neurons showed a decrease in average baseline and SA firing rates across the first 3 recording sessions (i.e., pre-session baseline, 2nd SA session, and 16th SA session). Average firing rates of the same neurons returned to normal by the 3oth day of abstinence, but decreased again during re-exposure. These observations show that neither the task-activated nor the tasknon-activated neurons showed a progressive and lasting change across the three successive phases. However, in combination, the plasticity patterns exhibited by the two groups of neurons were associated with an increase in the average firing rate of NAc neurons during cocainepredictive behavior and cues, which developed progressively, and persisted across the SA, abstinence, and re-exposure periods. The magnitude of the increase was predictive of between-subject differences in the behavioral addiction score measure. The neural adaptations did not occur in animals with a history of sucrose SA, abstinence, and re-exposure.

Discussion: The present findings show that the firing of NAc neurons exhibits activity-dependent (i.e., firing-pattern-dependent) and timedependent (i.e., SA vs abstinence) plasticity. The plasticity causes a progressive and persistent net increase in the strength of NAc signaling associated with cocaine-predictive events, which is correlated with addiction-like behaviors. The NAc neuroplasticity potentially contributes to progressive and persistent behavioral changes that define cocaine addiction.

Disclosure: L. Peoples: None. K. Guillem: None.

\section{It's All About the Reward: Mesolimbic Dopamine Release in Adolescent Rats}

Donita Robinson*, Dawnya Zitzman, Katherine Smith, Randall Ung, Linda Spear

\section{University of North Carolina, Chapel Hill, NC}

Background: Dopamine transients (fast dopamine release events) arise from burst firing of dopamine neurons and can coincide with unexpected rewards as well as cues that predict rewards. Multiple aspects of dopamine transmission, including firing activity and receptor expression, are dynamic during adolescence. We recently measured dopamine transients during adolescence at baseline, at the presentation of unexpected novel stimuli, and during social interaction with another rat. We found that adolescent rats were less likely than their adult counterparts to exhibit dopamine transients at unexpected novel stimuli, but more likely to emit transients during repeated interaction with another rat. As rats are known to be more sensitive to various drug and social rewards during adolescence relative to other developmental periods, one interpretation of these data is that adolescent rats are more sensitive to rewards (social interactions) than non-reward stimuli. To further explore the dopaminergic response to rewards in adolescent rats, we trained adolescents and adults on a conditioned Pavlovian approach paradigm and then measured dopamine release during cue and reward presentations.

Methods: Adolescent and adults rats underwent two pre-exposure sessions of reward delivery ( $0.1 \mathrm{ml}$ chocolate Ensure $\AA)$, followed by six training sessions in which a cue (light + lever extension) occurred during the $8 \mathrm{~s}$ prior to reward delivery. On the 7 th and/or 8 th training day we used fast-scan cyclic voltammetry to measure real-time dopamine release events in the nucleus accumbens core.

Results: Both adolescent and adult rats learned cue-reward association, as evidenced by approach to the cue and/or dispensing cup soon after cue onset. However, across the training sessions adolescent rats spent more time focused on the dispensing cup and less time focused on the cue than their adult counterparts. During voltammetric recording, dopamine transients were time-locked to both the cue onset and reward delivery in both age groups. Interestingly, while dopamine release at the cue onset was similar in adolescent and adult rats, dopamine transients coincident with reward delivery were significantly larger in the adolescents.

Discussion: These data illustrate a developmental difference in the dopaminergic encoding of reward versus the anticipation of reward. Previous studies in adult animals (e.g., Schultz et al., 1997, Science 275:1593; Day et al., 2007, Nat Neurosci 10:1020) show that dopamine neuronal firing and subsequent dopamine release often occur to an unexpected reward. However, as a cue-reward association is learned, the burst firing and dopamine release to the predictive cue emerge over training, while the dopaminergic response to the predicted reward attenuates. Thus, the present data suggest that adolescent rats learn the predictive value of the cue, as indicated by their behavior as well as dopamine release at the cue onset. However, the larger dopamine response on days 7 and 8 of training in adolescents versus adults indicates a developmental variation in reward encoding that may reflect differences in learning rates or reward sensitivity. Ultimately, these differences in reward processing within the mesolimbic pathway may underlie developmental differences in reward-related behaviors such as drug self-administration and social interaction.

Supported by NIDA Ro1 019071 to L.P.S. and UNC Bowles Center for Alcohol Studies.

Disclosure: D. Robinson: None. D. Zitzman: None. K. Smith: None. R. Ung: None. L. Spear: None.

\section{Impaired Hippocampal-Prefrontal Synchrony in a Genetic Mouse} Model of Schizophrenia

Torfi Sigurdsson ${ }^{\star}$, Kimberly Stark, Maria Karayiorgou, Joseph Gogos, Joshua Gordon

Columbia University, New York, NY

Background: Abnormalities in functional connectivity between brain areas have been postulated as an important pathophysiological mechanism underlying schizophrenia. In particular, neuroimaging and electroencephalographic studies suggest that functional connectivity between the prefrontal cortex and the temporal lobe, including the hippocampus, is altered in schizophrenia patients. However, it remains unclear whether such dysconnectivity relates to the aetiology of the illness, and how it is manifested in the activity of neural circuits. Because schizophrenia has a strong genetic component, animal models of genetic risk factors are likely to aid our understanding of the pathogenesis and pathophysiology of the disease. Here we study $D f(16) A^{+/-}$mice, which model a microdeletion on human chromosome 22 (22q11.2) that constitutes one of the largest known genetic risk factors for schizophrenia. We examined functional connectivity in these mice by measuring the synchronization of neural activity between the hippocampus and the prefrontal cortex while animals performed a task requiring working memory, which is one of the cognitive functions disrupted in schizophrenia.

Methods: Recording electrodes targeting the prefrontal cortex and the hippocampus were surgically implanted in $D f(16) A^{+/-}$mice and their wild-type littermates under anesthesia. Following recovery from 
surgery, the activity of individual neurons in the prefrontal cortex was recorded, as well as local field potentials in both structures, while animals performed a spatial working memory task in a T-maze. On each trial of the task, the animals were first directed to enter one of the arms of the T-maze. Following a short delay, the animals had to choose between the two arms and enter the arm not previously visited to obtain reward. Synchrony between the hippocampus and prefrontal cortex was measured using two independent methods: the modulation of prefrontal neuron spiking by hippocampal theta-frequency (4-12$\mathrm{Hz}$ ) oscillations (phase-locking) and the coherence of prefrontal and hippocampal local field potentials in the theta-frequency range.

Results: In wild-type mice, hippocampal-prefrontal synchrony increased during working memory performance, consistent with previous reports in rats. However, $D f(16) A^{+/-}$mice, which are impaired in the acquisition of the task, showed drastically reduced synchrony, measured both by phase-locking of prefrontal cells to hippocampal theta oscillations and by the coherence of prefrontal and hippocampal local field potentials. Furthermore, the magnitude of hippocampal-prefrontal coherence at the onset of training could be used to predict the time it took the $D f(16) A^{+/-}$mice to learn the task. We also observed that during training, hippocampal-prefrontal synchrony increased in parallel with performance in both genotypes. However, these changes occured more slowly in the $D f(16) A^{+/-}$mice. In contrast to the impairments in theta synchrony between the hippocampus and prefrontal cortex, theta synchrony within either of these structures was normal in the $D f(16) A^{+/-}$mice.

Discussion: These data demonstrate that hippocampal-prefrontal synchrony is impaired in $D f(16) A^{+/-}$mice, which model the 22q11.2 microdeletion, a major genetic risk factor for schizophrenia. The data also suggest that the impairments in hippocampal-prefrontal synchrony contribute to the deficits in spatial working memory in these mice. In particular, the delayed increase in hippocampal-prefrontal synchrony during learning may reflect impaired plasticity in this circuit; further experiments are needed to address this possibility. Our data are of relevance to understanding schizophrenia as they demonstrate how the deficits in functional connectivity observed in patients with schizophrenia may be realized at the single neuron level. Our findings further argue that impaired long-range synchrony of neural activity is one consequence of the 22q11.2 deletion and may be a fundamental component of the pathophysiology underlying schizophrenia.

Disclosure: T. Sigurdsson: None. K. Stark: None. M. Karayiorgou: None. J. Gogos: None. J. Gordon: None.

\section{Dysregulation of Locus Coeruleus Neuronal Activity in CRF-Overexpressing Female Mice: Potential Mechanism Underlying Sex Differences in Affective Disorders}

Debra Bangasser*, Beverly Reyes, David Piel, Veraaj Rarach, Xiao-Yan Zhang, Elisabeth Van Bockstaele, Sheryl Beck, Rita Valentino

The Children's Hospital of Philadelphia Philadelphia, PA

Background: Affective disorders, such as depression and anxiety, occur twice as frequently in women as in men, however the basis for this disparity remains largely unknown. These disorders have been linked to stress and hypersecretion of the stress neuromediator, corticotropin-releasing factor (CRF). Therefore, sex differences in CRF expression or sensitivity could underlie differences in vulnerability to affective disorders. Supporting this, we previously identified sex differences in $\mathrm{CRF}_{1}$ receptor function that were expressed as increased sensitivity of female brain norepinephrine neurons to CRF and stress. Because transgenic CRF-overexpressing mice model some aspects of the CRF hypersecretion proposed to occur in affective disorders, these mice were used to test the hypothesis that CRF hypersecretion may have a greater impact on the brain norepinephrine system of females. Methods: Adult (3-5 month) male and female CRF overexpressing (CRF-OE) mice and wild type (WT) littermates were derived and purchased from Jackson laboratories. CRF-OE mice were generated with the use of a chimeric CRF transgene comprising the methallothionein protomer driving the rat $\mathrm{CRF}$ gene and backcrossed onto the $\mathrm{C}_{57} \mathrm{BL} / 6$ mouse strain (Stenzel-Poore et al., 1992). For whole-cell locus coeruleus (LC) electrophysiology recordings, mice were weighed, decapitated, brains were rapidly removed, and 200 micron thick slices were prepared. Current clamp recordings were made with biocytinfilled microelectrodes to obtain active and passive membrane characteristics and subsequently localize the recorded neuron in the LC using tyrosine hydroxylase immunohistochemistry. For immunoelectron microscopy studies, separate groups of mice were transcardially perfused and LC tissue was processed for electron microscopy. Compartmental localization of $\mathrm{CRF}_{1}$ receptor within LC dendrites was visualized with silver intensified $\mathrm{CRF}_{1}$ receptor immunogold labeling. The ratio of cytoplasmic to total silver grains was calculated to quantify the degree of $\mathrm{CRF}_{1}$ receptor internalization.

Results: CRF overexpression increased the body weight of female mice selectively $(21 \pm 0.4 \mathrm{~g}, \mathrm{n}=24$ WT vs. $31 \pm 1.3 \mathrm{~g}, \mathrm{n}=16$ CRF-OE $)$ $[\mathrm{F}(1,69)=28.7, \mathrm{p}<0.001$, post-hoc $\mathrm{p}<0.001]$. Male mice, regardless of genotype had similar body weights $(28 \pm 1.0 \mathrm{~g}, \mathrm{n}=16 \mathrm{WT}$ vs. $29 \pm 0.9 \mathrm{~g}, \mathrm{n}=17$ CRF-OE; $\mathrm{p}>0.05)$ and these were greater than those of female WT mice $(\mathrm{p}<0.001)$. CRF overexpression resulted in a tonic elevation of LC neuronal activity selectively in female mice $[\mathrm{F}(1,68)=5.4, \mathrm{p}<0.05$, post-hoc $\mathrm{p}<0.05]$. LC spontaneous discharge rate was $1.8 \pm 0.4 \mathrm{~Hz}$ in female CRF-OE mice $(\mathrm{n}=20$ cells) vs. $0.7 \pm 0.2 \mathrm{~Hz}$ in female WT mice $(\mathrm{n}=15$ cells). In contrast LC discharge rates were comparable in male mice regardless of genotype $(\mathrm{p}>0.05)$; $0.7 \pm 0.2 \mathrm{~Hz}$ for both male CRF-OE ( $\mathrm{n}=20$ cells) and WT ( $\mathrm{n}=18$ cells) mice. CRF overexpression had opposing effects on the cellular localization of $\mathrm{CRF}_{1}$ receptors in male and female mice $[\underline{F}(1,15)=75.2, \underline{p}<0.001]$. Male CRF-OE mice had increased cytoplasmic labeling compared to male WT mice, indicating that CRF overexpression resulted in receptor internalization. In contrast, increased plasma membrane labeling was observed in CRF-OE females compared to their WT counterparts.

Discussion: Here the CRF-OE mouse was used to model excessive CRF function that is thought to occur in stress-related psychiatric disorders. CRF overexpression distinctly affected body weight, LC neuronal activity, and $\mathrm{CRF}_{1}$ receptor trafficking in females. The CRF receptor internalization observed in LC neurons of male CRF-OE mice may be a compensatory mechanism that maintains neuronal firing rates to $\mathrm{WT}$ levels. The finding that $\mathrm{CRF}_{1}$ receptors were more prominent on the plasma membrane of female CRF-OE mice is consistent with our previous studies demonstrating a lack of stressinduced $\mathrm{CRF}_{1}$ receptor internalization in LC neurons of female rats. This sex difference in receptor trafficking can account for tonically elevated LC discharge rates in female CRF-OE mice. As tonic activation of the LC-norepinephrine system has been implicated in anxiety and in the arousal components of depression, the present findings suggest a cellular mechanism that can contribute to the increased vulnerability of females to affective disorders. Importantly, these findings suggest that females may be particularly sensitive to conditions that result in excessive CRF release in brain.

Disclosure: D. Bangasser: None. B. Reyes: None. D. Piel: None. V. Rarach: None. X. Zhang: None. E. Van Bockstaele: None. S. Beck: None. R. Valentino: None.

225. Response to a Psychological Stressor and a Physiological Stressor in Male and Female Smoked Cocaine Users

Stephanie Collins Reed*, Richard Foltin, Suzette Evans

Columbia University Medical Center, New York, NY

Background: Stress has been shown to impact cocaine use and to produce different effects in males and females but sex differences in the effects of various types of stressors in male and female cocaine users has not been well characterized. Thus, we examined the response to two different laboratory stressors: 1) a psychological stressor, the Trier Social Stress Test (TSST) and 2) a physiological stressor, the Cold 
Pressor Test (CPT) compared to a No Stress Condition in male and female non-treatment seeking smoked cocaine users.

Methods: Seventeen cocaine users (10 male and 7 female) experienced the TSST, CPT and No Stress Condition on separate days. Participants were administered the stress tests between $2-4 \mathrm{pm}$, to control for diurnal variation in salivary cortisol levels. The TSST consisted of a 5 min public speaking task and a 5 min math task. The CPT consisted of immersing the forearm in cold water for as long as possible (maximum of 3 minutes). Dependent measures included self-reported anxiety and perceived stress, cocaine craving, and cardiovascular effects.

Results: Both the TSST and CPT produced greater cardiovascular and subjective stress response than the No Stress Condition, and the TSST also produced greater cardiovascular and subjective stress response than the CPT (TSST $>$ CPT $>$ NS). There was little effect of any stress condition on self-reported cocaine craving. When examining the data as a function of sex, females had a greater increase in heart rate during the TSST than the CPT and No Stress Condition, whereas males had a greater increase in heart rate during the CPT than the No Stress Condition. However, there were no differences in cardiovascular and subjective anxiety and stress response between males and females within any test condition.

Discussion: Overall, both a psychological and physiological stressor produce a stress response in cocaine users, but the psychological stressor appears to have more of an impact than a physiological stressor, especially in female cocaine users. However, laboratory stressors in general may not be good predictors of the effects of stress on cocaine craving in this population of cocaine users. Supported by NIDA grants $\mathrm{P}_{50}$ DAoog236 and Ko1 DA022282.

Disclosure: S. Collins Reed: None. R. Foltin: None. S. Evans: None.

226. A Gender Analysis of the NIMH Study of the Pharmacotherapy of Psychotic Depression (STOP-PD)

Kristina M. Deligiannidis*, Anthony Rothschild, Bruce A. Barton, Barnett S. Meyers, Alastair J. Flint, Ellen M. Whyte, Benoit H. Mulsant

\section{Univ. of Massachusetts Medical School, Worcester, MA}

Background: Gender differences exist in clinical characteristics and treatment response of psychiatric disorders. The NIMH STOP-PD trial reported higher remission rates after 12 weeks of treatment with olanzapine/sertraline (OLZ/SERT) than olanzapine/placebo (OLZ/ PBO) in 259 randomized subjects aged 18-93. The primary aims were to determine if gender differences existed in the sociodemographic and clinical characteristics of patients with psychotic depression (MDpsy) and if menopausal status was associated with change in HAM-D 17 score. The secondary aim was to determine if gender and menopausal status was a predictor of treatment-associated weight gain.

Methods: The sociodemographic and clinical characteristics of subjects were compared according to gender, treatment and age in order to verify the similarity of groups at baseline by using $\chi^{2}$ tests for categorical variables and t tests for continuous variables. An age group of 18-59 years was used to approximate pre/perimenopausal status and age $\geq 60$ years for postmenopausal status. For cross-sectional data, we used a t-test for comparison of means and, a $\chi^{2}$ test for comparison of proportions. We analyzed the longitudinal weight and HAM-D measures in the STOP-PD trial using generalized estimating equation methods. This approach allowed us to analyze all available data for each patient while controlling for the inherent correlation among the data collected at Weeks $0,4,8$, and 12. Both main effects and interaction terms were investigated. Reported p-values are either for a test that a single regression coefficient was equal to o or for an overall test of any difference among the levels of a factor. Because of sample size, the study had limited power to detect significant interactions.

Results: Female gender was associated with divorced or widowed marital status $(\mathrm{p}=0.0023)$. Probable or definite presence of hallucinations was significantly associated with female gender $(\mathrm{p}=0.0247)$, as were auditory $(\mathrm{p}=0.0297)$, somatic or tactile $(\mathrm{p}=0.0144)$ and olfactory hallucinations $(\mathrm{p}=0.0056)$ as measured by the Scale for the Assessment of Positive Symptoms. Females less often reported severe and persistent diminished sexual desire than males $(p=0.048)$. There was a trend for females to report less moderate and persistent orgasmic dysfunction than males $(p=0.0662)$. Compared to baseline, there was an overall increase in weight of $7.5 \mathrm{lbs}$ to Week $4,11.0 \mathrm{lbs}$ to Week 8, and $12.9 \mathrm{lbs}$ to Week 12 $(\mathrm{p}<0.0001)$. Males were $24.8 \mathrm{lbs}$ heavier than females $(\mathrm{p}<0.0001)$ with no significant variation by visit $(\mathrm{p}=0.58)$ and, compared to patients $\geq 60$ years, patients $\leq 59$ years were 23.9 lbs. heavier on average $(\mathrm{p}<0.0001)$ with increasing weight gain in that group by length of time on study $(\mathrm{p}<0.0001)$. There was no significant difference in weight by treatment at baseline $(\mathrm{p}=0.90)$ and a marginal treatment differential change in weight during treatment $(\mathrm{p}=0.03)$ with the OLZ/SERT group gaining weight more slowly at Week $4(-3.65 \mathrm{lbs}$ compared to OLZ/PBO, $\mathrm{p}=0.014)$, but non-significant at Week $8(\mathrm{p}=0.10)$ and Week $12(p=0.94)$. There were no significant interactions between treatment and gender $(\mathrm{p}=0.35)$ or menopausal status $(\mathrm{p}=0.14)$ in weight change during treatment between the treatment groups. Compared to baseline, there was an overall decrease of 14.9 points to Week $4,18.3$ points to Week 8 , and 18.8 points to Week 12 in the HAM$\mathrm{D}$ measures on average in both treatment groups $(\mathrm{p}<0.0001)$ with a significant additional decrease of 2.0 points on average in the OLZ/ SERT treatment group compared to the OLZ/PBO group $(p=0.0042)$. There was no difference in change in HAM-D by menopausal status, either overall $(p=0.40)$ or by visit $(p=0.36)$ or by gender, either overall $(p=0.73)$ or by visit $(p=0.15)$. There were no significant interactions between treatment and age $(\mathrm{p}=0.21)$ or gender $(\mathrm{p}=0.31)$, indicating that the treatment effect on HAM-D was consistent across age groups and for both genders.

Discussion: To our knowledge this is the first analysis of gender and menopausal status as predictors of treatment outcome and treatment-associated weight gain in MDpsy. Gender differences exist in sociodemographic and clinical characteristics of patients with MDpsy. Men and pre/perimenopausal women $\leq 59$ years of age had greater weight gain than men and postmenopausal women $\geq 60$ years of age, but neither gender nor menopausal status was associated with change in HAM-D 17 score. Additional gender analyses from the STOP-PD study will be presented. Further studies may need larger sample sizes to detect potential gender differences in treatment outcome and treatment-associated weight gain in MDpsy.

Disclosure: K. Deligiannidis: Part 1; Grant support from the Worcester Foundation for Biomedical Research, Investigator-initiated grant support from Forest Research Institute, US Patent of the US Dept. of Health and Human Services/National Institutes of Health/National Institute of Child Health and Human Development. Part 4; Grant support from the Worcester Foundation for Biomedical Research, Investigator-initiated grant support from Forest Research Institute. A. Rothschild: Part 1; Pfizer, GlaxoSmithKline, Forest, Lilly, Astra-Zeneca. Part 4; Cyberonics, St. Jude Medical, Takeda. B. Barton: None. B. Meyers: Part 1; AstraZeneca, Cyberonics, Eli Lilly, Forest Laboratories, Organon, Pfizer. Part 4; Cyberonics, Eli Lilly, Forest Laboratories, Organon, Pfizer. A. Flint: Part 1; Janssen, Lundbeck. E. Whyte: Pfizer, Forest Laboratories, Ortho-McNeil, National Institute of Mental Health, National Institute of Child Health and Human Development/National Center for Medical Rehabilitation Research. Part 4; Pfizer, Forest Laboratories, Ortho-McNeil, National Institute of Mental Health, National Institute of Child Health and Human Development/National Center for Medical Rehabilitation Research. B. Mulsant: Part 1; Board of Directors Centre for Addiction and Mental Health, Bristol-Myers Squibb, Canadian Institutes of Health Research, Eli Lilly, National Institutes of Health, Pfizer, Wyeth. Part 4; BristolMyers Squibb, Canadian Institutes of Health Research, Eli Lilly, National Institutes of Health, Pfizer, Wyeth. 
227. Sex Differences In The Effects Of Social and Environmental Enrichment On The Behavioral And Neurochemical Responses To Cocaine Reward

Sari Izenwasser*, Ellen Unterwald

Univ Miami Miller School of Medicine, Miami, FL

Background: Adolescence is a vulnerable period associated with a high incidence of drug abuse initiation and an increased risk for developing dependence and addiction. There are numerous factors, including social and environmental enrichment, that modulate the effects of drugs of abuse during this critical period of development. These studies were done to determine whether there are sex differences in response to social and environmental enrichment on drug reward and on underlying neurochemical systems during adolescence.

Methods: In these studies, rats were housed under conditions where both social (number of rats per cage) and environmental (availability of toys) factors were manipulated starting on postnatal day (PND) 23 . Socially isolated rats were housed alone impoverished with no toys (II) or enriched with toys (IE). Social rats were housed 2 rats/cage with no toys (SI2) or with toys ( $\left.\mathrm{SE}_{2}\right)$, or 3/cage with $\left(\mathrm{SE}_{3}\right)$ or without $\left(\mathrm{SI}_{3}\right)$ toys. During adolescence, on PND 43, cocaine conditioned place preference (CPP) sessions began with the post-test done on PND 47.

Results: In males, cocaine CPP was highest in II rats and lowest in the rats living three/cage, regardless of toys. Enriched housing $\left(\mathrm{SE}_{3}\right)$ alone (without drug) increased dopamine transporter (DAT) protein in the nucleus accumbens compared to II. Binding to DAT (measured by autoradiography) was increased in response to enrichment only in the shell, but not the core of the nucleus accumbens, suggesting that the shell may be the region that mediates the effects of environment. Cocaine increased DAT binding in both the core and shell, but only in II rats. Similarly, there were differential effects of cocaine on tyrosine hydroxylase depending on housing, with increases in the nucleus accumbens in II but not $\mathrm{SE}_{3}$ rats. In females, opposite effects were observed. The greatest reward was seen in $\mathrm{SE}_{3}$ rats and the lowest in isolated rats. In addition, unlike in the males, environmental and social enrichment interacted to alter cocaine reward. In the presence of environmental enrichment, cocaine reward was greater with an increased number of rats in the cage, while social conditions alone had no effect on CPP. In females, tyrosine hydroxylase was increased by enrichment, whereas cocaine treatment had no effect in either II or $\mathrm{SE}_{3}$ rats, in contrast to the males.

Discussion: Social and environmental enrichment differentially alter cocaine reward in males and females during adolescence and these changes are mediated by distinct neurochemical changes. These factors should be taken into account when developing preventions and/or treatments for cocaine abuse in adolescent males and females. (Supported by NIDA/ORWH grants P5oDA024584, Ro1DA15119, Ro1DA09580, P3oDA13429)

Disclosure: S. Izenwasser: None. E. Unterwald: None.

\author{
228. Obesity, BMI and Measures Of Psychopathology in Urban \\ Minority Adolescent Males and Females \\ Lawrence Maayan*, Allison Larr
}

Nathan Kline Institute/NYU Child Study Center, Orangeburg, NY

Background: The prevalence of child and adolescent obesity in the US has more than tripled since 1970 predicting a public health problem of considerable personal and societal costs in the coming decades. The psychological correlates of obesity have received recent attention with data showing decreased volume in orbito-frontal cortex and worsened executive function in obese children and large epidemiologic work showing associations between weight and ADHD as well as depression in youth. Relatively little of this work, however, has been performed in an urban minority population. We examined a population of African American and Latino youth from 2 New York City Public Schools to examine correlations between race, BMI, gender and psychopathology on the Child Behavior Checklist
Methods: We evaluated 254 adolescents, $76.1 \%$ Hispanic, $16.3 \%$ African-American and $6.4 \%$ other ethnicity, ages 14 to 20 from 2 New York city public schools. Psychological measures were evaluated with the Child Behavior Checklist/ Youth Self Report. Weight was assessed with a Detecto balance beam scale and height with an accompanying stadiometer. The primary outcome measures were effects on weight and body mass index (BMI) as well as change in subsection score on CBCL.

Results: This cohort had an average BMI of $30.1 \pm 5.6$ (range 20.1-49.9). Girls scored higher than boys on self reports of somatic complaints $68.5 \pm 16.4$ vs $63.8 \pm 14.4 \mathrm{p}=$, o1, interenalizing symptoms $59.3 \pm 26.0$ vs $53.0 \pm 26.1$ and total problems $61.1 \pm 25.9$ vs $53.4 \pm 27.9$ ). When looking at the effect of BMI, positive associations were found in boys between BMI and somatic complaints $(r=.193, p=.035)$ and total psychiatric problems $(\mathrm{r}=.204, \mathrm{p}=.026)$ but not in girls. In addition, only boys were sensitive to the effects of obesity (defined as BMI > 30.0) with significant increases in scores of Anxiety/ Depression $(62.5 \pm 16.3$ vs $56.7 \pm 16.7)$. There were statistically significant gender by $\mathrm{BMI}$ interactions for somatic complaints $(\mathrm{r}=-0.128, \mathrm{p}=.04)$ and total problems $(\mathrm{r}=-.121, \mathrm{p}=.05)$. Race did not predict CBCL score nor did it interact with gender or BMI to predict CBCL psychopathology scores.

Discussion: While measures of psychopathology were higher overall for girls, obesity was a vulnerability factor for boys in our largely overweight primarily Hispanic population. Only boys had significant correlations between increased BMI and higher self report of somatic symptoms and total problems. Obese boys also scored significantly higher on self ratings of anxiety/depression. Research has shown obesity to have clear neurobiological effects as well as social and psychological effects. In our sample weight and obesity was a vulnerability factor for boys but not for girls. Whether this is an effect of obesity interacting with hormonal or cultural factors cannot be interpreted in this cross sectional design, however this study provides preliminary data on the relationship between gender, psychiatric and metabolic dysregulation. Further exploration is needed to determine endocinologic, psychological and sociocultural factors that may underlie these trends as well as longitudinal work to help explain the temporal direction of these correlations.

Disclosure: L. Maayan: None. A. Larr: None.

229. Clinical Course of Bipolar Disorder During the Late Menopausal Transition: Comparison with Reproductive Age and Post-Menopausal Women

Wendy Marsh*, Terrence Ketter, Sybil Crawford, Julia Johnson, Anthony Rothschild

University of Massachusetts, Worcester, MA

Background: Times of endocrinological fluctuation can be associated with mood episodes in women. The postpartum period, time of rapid gonadal hormone decline, is well known to be associated with an increased risk of severe mood episodes in women with bipolar disorder. In women without bipolar disorder the menopausal transition is a time of increased risk of depression. While small studies show an increased or high rate of depression in menopausal age women with bipolar disorder, they do not report menstrual status. We report on the clinical mood course of late menopausal transition women compared to reproductive age and post-menopausal women with bipolar disorder.

Methods: Consented reproductive age women (menstruating 28-38 year olds, 42 yo, $>365$ days since menstruation) with bipolar I, II, NOS or schizoaffective bipolar type were selected from the prospectively collected systematic clinical data from the multi-site Systematic Treatment Enhancement Program for Bipolar Disorder (STEP-BD). The three reproductive groups were evaluated for differences in the proportion of clinic visits meeting DSM-IV criteria for major depressive episode or syndromal mood elevation (mania or hypomania) by chi square analysis. 
Results: 521 reproductive age, 107 menopausal transition and 145 post menopausal (with 24 women converting from peri to post menopause) women of whom $88 \%$ were white/ Caucasian met inclusion criteria. Of these women $61 \%(\mathrm{n}=474)$ were bipolar I, $32 \%(n=237)$ bipolar II, $6 \%(n=44)$ NOS and $1 \%(n=8)$ schizoaffective disorder bipolar type were followed for and average of $18( \pm 15)$ months with average $0.8( \pm 0.7)$ clinic visits a month. Twelve percent or reproductive age women, $19 \%$ of menopausal transition, and $35 \%$ of postmenopausal women were using female sexual hormone therapy at clinic entry. There were significant differences between reproductive age, menopausal transition and post-menopausal women in the proportion of clinic visits in different mood states $\left(\mathrm{X}_{2}=57, \mathrm{df}=10, \mathrm{p}<0.0001\right)$. The proportion of visits in a depressed state differed significantly between the three groups $\left(\mathrm{X}_{2}=10.5\right.$, $\mathrm{df}=2, \mathrm{p}<0.005)$ with reproductive age women experiencing $18.1 \%$ of visits in depression, menopausal transition $20.5 \%$ of visits and post-menopausal $21.6 \%$. Likewise, the proportion of visits in a syndromal mood elevation episode differed significantly between the three groups $\left(\mathrm{X}_{2}=19.9, \mathrm{df}=2, \mathrm{p}<0.0001\right)$ with reproductive women experiencing $5.3 \%$ of visits in a syndromal mood elevation episode, menopausal transition $4.8 \%$ of visits and postmenopausal $2.6 \%$.

Discussion: Similar to previous results that the menopausal transition may be a time of increased risk of depression in women with bipolar disorder, our findings show that compared to reproductive age women (but not compared to post menopausal women of similar or older age), women in the late menopausal transition experience a greater proportion of mood episodes in the depressed state. These results are congruent with worsening of depressive mood course over time. Being in the late menopausal transition, when gonadal hormones are at their steepest rate of decline, did not confer a greater proportion of depressed visits compared to post menopause when gonadal hormones are around their nadir. The proportion of visits in a mood elevation was greatest in the reproductive age women and least in the postmenopausal women, with the menopausal transition group in between. Thus the decline in reproductive hormones in the late menopausal transition was associated with a decreased rather than increased risk of mood elevation compared to younger reproductive age women. The low levels of gonadal hormones in the post-menopause may be associated with lower mood elevation risk. The strengths of this study include prospective mood assessment, a relatively large sample size, patients with well-characterized DSM defined mood episodes, and longitudinal menstrual status assessment in mood in women with bipolar disorder during the menopausal transition. The limitations of this study include that clinic visit frequency likely increases if patients are in syndromal mood episodes, a high level of missing menstrual cycle data, and lack of assessment of estradiol or follicular stimulating hormone. Future work will include analyzing whether factors such as hormone therapy use, history of menstrual cycle worsening of mood and/or postpartum mood episodes are associated with risk of mood episode rate (depression or elevation) during the menopausal transition.

Disclosure: W. Marsh: None. T. Ketter: Part 1; Abbott Laboratories, Inc. AstraZeneca Pharmaceuticals LP Bristol-Myers Squibb Company Cephalon Inc. Eli Lilly and Company GlaxoSmithKline Pfizer Inc. Consultant AstraZeneca Pharmaceuticals LP Astellas. Part 2; Financial relationships in Part I with value greater than $\$ 10,000$ per year - for Terence Ketter only GlaxoSmithKline for 2009; for Nzeera Ketter only Johnson \& Johnson for each year. Part 3; Financial relationships in Part I which constitute more that $5 \%$ of personal income - for Terence Ketter none; for Nzeera Ketter only Johnson \& Johnson for each year. Part 4; IRUSQUE To463 (SPO \# 41037) (Ketter PI). Astra Zeneca. "The Long Term Effectiveness of Quetiapine plus LTG Therapy in Bipolar Patients" - 02/27/07 - 09/30/10 - \$34,404 PROTOCOL\#F1D-US-X279 (SPO \# 3024. S. Crawford: None. J. Johnson: None. A. Rothschild: Part 1; Pfizer, GlaxoSmithKline, Forest, Lilly, Astra-Zeneca. Part 4; Cyberonics, St. Jude Medical, Takeda.
230. Sex Differences in Orexin 1 Receptor Mediation of Cocaineseeking in Rats

Ronald See*, Luyi Zhou

Medical University of South Carolina, Charleston, SC

Background: Prior studies have demonstrated sex differences in reward-associated behaviors with drugs of abuse, including cocaine. The orexin/hypocretin system has recently been implicated in the conditioned-cued reinstatement of cocaine-seeking in male rats. Although female rats express higher levels of orexin A peptide and orexin 1 receptor in hypothalamus when compared to males, sex differences in orexin-dependent drug-seeking have not been explored. Here, we assessed the effects of the selective orexin 1 receptor antagonist, SB-334867, on responding during cue- and stress-induced reinstatement to cocaine-seeking following chronic cocaine selfadministration in female and male rats.

Methods: Female and male Sprague-Dawley rats experienced daily sessions ( $2 \mathrm{hr} /$ day for 10-14 days) of lever pressing for intravenous cocaine $(0.6 \mathrm{mg} / \mathrm{kg} /$ infusion) paired with the presentation of stimulus cues (light + tone). Following self-administration, rats underwent daily extinction trials for at least 7 days, whereby responding had no programmed consequences. Reinstatement of cocaine-seeking was then triggered by presentation of previously cocaine-paired cues, injection of the pharmacological stressor, yohimbine $(2.5 \mathrm{mg} / \mathrm{kg}, \mathrm{IP})$, or a combination of cues + yohimbine. Thirty min prior to each reinstatement trial, rats received SB-334867 (o, 10, 20, or $30 \mathrm{mg} / \mathrm{kg}, \mathrm{IP})$. Results: Both males and females readily acquired cocaine selfadministration, extinguished to criterion, and showed reinstatement of responding to cues alone, yohimbine alone, or cues + yohimbine. SB-334867 pretreatment significantly attenuated cue-induced reinstatement in males, but not females. Surprisingly, while orexin 1 receptor blockade failed to affect cue-induced reinstatement in females, SB-334867 dose-dependently decreased yohimbine stress-induced reinstatement, and abolished the potentiative effects of cues + yohimbine.

Discussion: Our results show a clear sexual dimorphism in orexinmediated control of cocaine-seeking behavior, whereby orexin regulation of cocaine-seeking produced by conditioned cues occurs in males, but not females. However, stress activated reinstatement of cocaine-seeking in females is under the control of the orexin system. These sex differences may be due to intrinsic differences in hypothalamic orexin-mediated signaling in males vs. females. A greater understanding of sex differences in the orexin/hypocretin system will lead to improved treatment of cocaine addiction. These studies were conducted in accordance with the Guide for the Care and Use of Laboratory Animals, as adopted and promulgated by the National Institutes of Health. This research was supported by NIH grant P50 DA16511 (Specialized Center of Research on Sex and Gender Factors Affecting Women's Health).

Disclosure: R. See: None. L. Zhou: None.

231. Gender Differences in the Topology of Functional Brain Networks Dardo Tomasi*, Nora Volkow

National Institutes of Health, Upton, NY

Background: Recent diffusion tensor imaging studies reported that cortical networks have greater anatomical connectivity and more efficient organization in the female than in the male brain ${ }^{1}$. The potential gender effects on functional connectivity, however, are largely unknown. We hypothesized that data-driven analyses of BOLD image time series collected in resting conditions would reveal brain networks that would have higher functional connectivity strength for women than for men and that his dimorphism would vary across networks.

Methods: "Resting-state" functional connectivity datasets of 966 healthy subjects ( 550 females, age: $25.5 \pm 10.0$ years; 416 males, age: $26.4 \pm 9.7$ y) from 21 research sites of the "1000 Functional 
Connectomes" Project ${ }^{2}$ and ultrafast data-driven functional connectivity density mapping $(\mathrm{FCDM})^{3}$ were used to locate the main functional connectivity hubs (highly interconnected regions) in the brain with high spatial resolution (3-mm isotropic). The hub locations were then used as "seed" regions in standard analyses of functional connectivity to map the networks associated with the hubs. Complementary VBM analyses were conducted on structural MRI datasets from a large fraction of the subjects (336 females and 225 males) to evaluate potential gray matter differences between the genders. SPM ANOVA was used to evaluate differences in functional connectivity (local functional connectivity density, $l$ FCD, and strength of connectivity with the hubs) and gray matter between the genders. Pearson correlation, was used to evaluate the coupling between BOLD signals. Statistical significance was set at $\mathrm{P}<0.05$, corrected for multiple comparisons at the cluster level.

Results: Women had up to $28 \%$ higher $l$ FCD than men in parietal, prefrontal, and temporal cortices, limbic lobe, insula and thalamus, and up to $5 \%$ higher gray matter density than men in these regions. These differences were maximal in the ventral anterior nucleus of the thalamus (bilateral) and there were no significant laterality patterns in $l \mathrm{FCD}$ nor in the gender differences in $l$ FCD or gray matter density, which suggest minimal gender effects on hemispheric differentiation. The BOLD signals in the four major cortical hubs (posterior cingulate/ventral precuneus, PC-VP; inferior parietal; cuneus, and postcentral) were minimally coupled and associated to four independent (largely cortical) networks (defaultmode, DMN; dorsal-attention, DAN; visual; and somatosensory). The strength of the functional connectivity in the PC-VP, inferior parietal, and cuneus hub networks was higher for females than for males; the functional connectivity strength in the postcentral hub network was much weaker than that for the remaining cortical networks, and was the only one that had higher connectivity for males than for females. The BOLD signals in the three major subcortical hubs (cerebellum, thalamus, and amygdala) were significantly coupled and associated to three overlapped (largely subcortical) networks that had higher functional connectivity strength for females than for males.

Discussion: Using an ultrafast data driven approach that can reduce the complexity of the data by a factor of $10^{4}$ and resting state datasets from 966 healthy humans, we identified the location of the major functional connectivity hubs in cortical and subcortical regions and the seven bilateral networks, which cover $80 \%$ of the gray matter volume, that were associated to these hubs. Our results are consistent with the females' higher global anatomical connectivity reported by diffusion tensor imaging studies ${ }^{1}$. Women had $28 \%$ higher $l F C D$ than men, even after controlling for brain volume differences, suggesting that $l$ FCD-differences reflect brain organization differences between genders rather than gray matter density effects. These gender differences included default mode network regions that show high glucose metabolic rate at rest and seem to be among the first to show metabolic decrements in Alzheimer's disease (AD), probably reflecting the high energetic requirements of these hub regions. Thus, the female's higher connectivity could help explain why female $\mathrm{AD}$-patients show greater impairments than male patients for the equivalent reduction in cerebral metabolic rate.

References: 1- Gong (2009). J Neurosci 29: 15684-93; 2- Biswal (2010). PNAS 107: 4734-9; 3- Tomasi (2010) PNAS 107: 9885-90.

Disclosure: D. Tomasi: None. N. Volkow: None.

232. Dopamine D2 Receptor Polymorphism Modulates Prefrontal Function and Connectivity During Reward and Emotion Processing Marta Pecina*, Brian Mickey, Heng Wang, Tiffany Love, Shen Pei-Hong, Scott Langenecker, David Hsu, Tal Shafir, Sandra Villafuerte, Sara Wright, David Goldman, Jon-Kar Zubieta

University of Michigan, Ann Arbor, MI

Background: Dopamine and D2 receptors have been implicated in reward, cognitive and emotional processing. The dorsolateral and medial prefrontal cortices, as well as the ventral striatum are thought to play a central role in these three dimensions. Individual differences in the dopaminergic system that modulate these processes and their underlying circuits are still unknown but seem to play a critical role in understanding individual susceptibility for dopamine related neuropsychiatric illness.

Methods: Using functional magnetic resonance imaging in healthy humans, we assessed the effect of 14 DRD2 SNPs on hemodynamic responses during anticipation of monetary gains and losses (45 subjects) and processing of positive and negative emotional words (82 subjects). Task-related brain regions were further evaluated using a seed-based functional connectivity analysis for the monetary anticipation task (45 subjects). Gene effects on the NEO-PI-R (Costa \& McCrae 1992) personality inventory and behavioral measures were also assessed. BOLD contrasts were analyzed using the SPM2 canonical hemodynamic response function. Statistical analysis proceeded in two stages. At first level, parameter estimates of block- or event-related activity were established for each voxel, and contrast images were calculated by applying appropriate linear contrasts to the parameter estimates of each event type to produce statistical parametric maps. At second level, interindividual, random-effects analyses were performed using SPM2. Statistical analysis consisted of an ANCOVA model between the three genotypes, using age and sex as nuisance covariates. The functional connectivity analysis was performed using the "conn" toolbox for SPM8 (http://www.nitrc.org/projects/conn). Statistical thresholds of significance were set at $\mathrm{p}<0.001$ uncorrected with an extent of $>55$ voxels.

Results: We found that an intronic single nucleotide polymorphism within the DRD2 gene (rs4274224) predicted hemodynamic responses during both reward and emotional processing. During anticipation of reward, heterozygous $\mathrm{A} / \mathrm{G}$ subjects showed a greater response in the left dorsolateral prefrontal cortex (DLPFC) during both losses and gains. During emotion processing, homozygous A/A subjects showed greater responses to negative words in the subgenual and pregenual anterior cingulate cortex (ACC). A different pattern of negative functional connectivity during anticipation of monetary gains and losses between the left DLPFC and the subgenual ACC was also observed: A/A subjects showed greater negative connectivity for both gains and losses. Finally, A/G individuals showed higher Openness to Experience $(\mathrm{F}=[[$ Unsupported Character - Codename \&shy; $]] 4.014$, $\mathrm{p}=0.023$, ANOVA, $71 \mathrm{df}$ ) on the NEO-PI-R personality inventory.

Discussions: Our findings suggest that DRD2 rs4274224 is a candidate to understand individual differences in dopaminergic-mediated processes, such as reward, cognition and emotional processing, including the functional relationship among the regions implicated. It has already been described that increasing reward levels and cognitive task demands correlates with increased blood oxygenation level-dependent (BOLD) response in the DLPFC (BA 9/46) (Pochon et al., 2002; Gilbert and Fiez, 2004) and that the regulation of negative emotions involves increased activation of the subgenual AC (Elliott et al., 2000; Shafritz et al., 2006). We observed that DRD2 rs4274224 plays a role in activating the DLPFC during anticipation of gains and losses and the sgAC during processing negative emotions. Moreover, the connectivity data obtained suggests that DRD2 rs4274224 modulates the functional relationship between these regions during anticipation of gains and losses. A dopaminergic dependent, altered relationships between regions engaged during emotion and reward processing may contribute to interindividual variations in risk for disorders related to emotion processing (e.g., mood and anxiety disorders) and reward (e.g., substance use disorders, gambling).

Disclosure: M. Pecina: None. B. Mickey: None. H. Wang: None. T. Love: None. S. Pei-Hong: None. S. Langenecker: None. D. Hsu: None. T. Shafir: Part 1; Board member of Teva Pharmaceuticals. S. Villafuerte: None. S. Wright: None. D. Goldman: None. J. Zubieta: Consultant, Elli Lilly, Merck. Part 2; Elli Lilly. Part 3; Elli Lilly. 